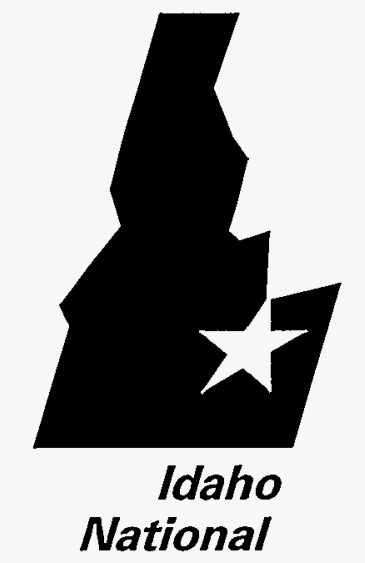

Engineering Laboratory
INEEL/EXT-97-01396 -

Vol.2

February 1998

\section{ICPP Calcined Solids Storage Facility Closure Study}

\section{Volume II}

Cost Estimates, Planning Schedules, Yearly Cost Flowcharts, and LifeCycle Cost Estimates

\section{MASTER}

CARTRISUTION OF TIS DOCUMENT IS UNLMITED

L OCKHEED MATTIN 
This volume contains draft information on cost estimates, planning schedules, yearly cost flowcharts, and life-cycle costs for the four options described in Volume I, Section 2:

Option 1: Risk-Based Clean Closure; NRC Class C fill

Option 2: Risk-Based Clean Closure; Clean fill

Option 3: Closure to Landfill Standards; NRC Class C fill

Option 4: Closure to Landfill Standards; Clean fill

Cost estimates were also prepared for Risk-Based Clean Closure; NRC Class A fill and Closure to Landfill Standards; NRC Class A fill. These cost estimates are not included in this volume. Further analysis has shown, however, that the CSSF cannot be filled with NRC Class A grout following Closure to Landfill Standards, as the concentration of various radionuclides exceed the concentration limits for Class A type waste. Additional decontamination cycles, not included in the original cost estimate, would be required to fill the bins with Class A type waste following Risk-Based Clean Closure. Estimates were done for the additional decontamination cycles and are given in EDF-BSC-016, Cost Estimate for RBCC; NRC Class A Landfill, February 1998.

This volume is divided into two portions. The first portion contains the cost and planning schedule estimates, while the second half contains life-cycle costs and yearly cash flow information for each option.

NOTE: The options numbers in the Life-Cycle Costs (LCC) do not match the final option numbers in the report, as these estimates were prepared prior to determining that it is not feasible to create an NRC Class A landfill following Risk-Based Clean Closure (RBCC) or Closure to Landfill Standards (CLFS).

For the purposes of the LCC section only, the following key is provided:

Option 1: Risk-Based Clean Closure; NRC Class C fill (Option 1 in LCC) Option 2: Risk-Based Clean Closure; Clean fill (Option 3 in LCC)

Option 3: Closure to Landfill Standards; NRC Class C fill (Option 4 in LCC)

Option 4: Closure to Landfill Standards; Clean fill (Option 6 in ICC)

\section{DISCLAIMER}

This report was prepared as an account of work sponsored by an agency of the United States Government. Neither the United States Government nor any agency thereof, nor any of their employees, makes any warranty, express or implied, or assumes any legal liability or responsibility for the accuracy, completeness, or usefulness of any information, apparatus, product, or process disclosed, or represents that its use would not infringe privately owned rights. Reference herein to any specific commercial product, process, or service by trade name, trademark, manufacturer, or otherwise does not necessarily constitute or imply its endorsement, recommendation, or favoring by the United States Government or any agency thereof. The views and opinions of authors expressed herein do not necessarily state or reflect those of the United States Government or any agency thereof. 
Date: January 28,1998

$\begin{array}{llll}\text { To: } & \text { B. C. Spaulding } & \text { MS } 3765 & 6-8633 \\ \text { From: } & \text { S. L. Coward \&C } & \text { MS } 3655 & 6-0399\end{array}$

Subject: ICPP BIN SET CLOSURE (EIS STUDY) - SLC-05-97

Cost Estimating has prepared Planning Cost Estimates for the subject project. These estimates were based on information received from you and your design team through December 17, 1997, as well as any subsequent comments from the review team.

The Total Project Costs (TPC) are broken down into present day (unescalated) and escalated costs, as follows:

Unescalated

Option 1 Risk Based Clean Closure; Class C Fill

Option 2 Risk Based Clean Closure; Class A Fill

Option 3 Risk Based Clean Closure; Clean Fill

Option 4

Option 5

Closure to RCRA Landfill Standards; Class C Fill

Option 6

Closure to RCRA Landfill Standards; Class A Fill

Closure to RCRA Landfill Standards; Clean Fill

$\$ 255,000,000$

$\$ 249,000,000$

$\$ 227,000,000$

$\$ 162,000,000$

$\$ 155,000,000$

$\$ 132,000,000$

Escalated

Option 1 Risk Based Clean Closure; Class C Fill

Option 2 Risk Based Clean Closure; Class A Fill

Option 3 Risk Based Clean Closure; Clean Fi11

Option 4

Option 5

Closure to RCRA Landfil1 Standards; Class C Fill

Closure to RCRA Landfill Standards; Class A Fi1l

$\$ 514,000,000$

$\$ 501,000,000$

$\$ 460,000,000$

Option 6

Closure to RCRA Landfill Standards; Clean Fill

$\$ 327,000,000$

$\$ 316,000,000$

$\$ 271,000,000$

The actual estimates were generated in several parts in order to mix and match component processes. The totals from these estimates make up the TPC and are shown on the Cost Estimate Summary Sheets. These costs include permitting costs, direct and indirect costs, Engineering costs, Inspection costs, Project and Construction Management costs, and associated procurement fees. G\&A and PIF have been included in this estimate. In addition, contingency and escalation to the midpoint of the activities have been included. For more detailed information, please refer to the Cost Estimate Support Data Recapitulation forms. 
January 28, 1998

SLC-05-98

Page 2 of 2

Attached for your review are the Summary Estimate Sheets, the Cost Estimate Support Data Recapitulation forms, Detailed Estimate sheets, Contingency Analysis sheets, and Estimate Schedules for the twelve different options.

If you have any questions or comments, please do not hesitate to contact me at 526-0399 or Office Vision - ID SQJ.

SLC

Attachments

cc: Estimate File \#2423

S. L. Coward File 
Lockheed Martin Idaho Technologies Co.

(Rev. 06/90)

\section{COST ESTIMATE SUPPORT DATA . RECAPITULATIOH}

Project Title: ICPP BIN SET CLOSURE (EIS Study)

Type of Estimate: Planning File No: 2423

Estimator: Sherry Coward Date: January $28 / / 997$ Approved By:

I. SCOPE OF WORK: Brief description of the proposed project.

Scope of work includes the closure of the seven Calcined Solids Storage Facilities (CSSFs). located at the ICPP following after calcine retrieval. The estimates are broken down into six different options, as follows:

Risk Based Clean Closure - Clean Fill

Risk Based Clean Closure - Class C Fill

Risk Based Clean Closure - Class A Fill

Closure to RCRA Landfill Standards - Clean Fill

Closure to RCRA Landfill Standards - Class C Fi11

Closure to RCRA Landfill Standards - Class A Fil1

Each option inciudes component type estimates for the various functions within the option.

II. BASIS OF THE ESTIMATE: Drawings, Design Report, Engineers notes, and/or other documentation upon which the estimate is originated.

1. Verbal information obtained from Bryan Spauiding and the design team

2. Quantities of vaults, bins, calcine, etc. are derived from Engineering Design File (EDF) \#EDF-BSC-00I

3. Costs for the tractor robot, pipe crawler, and Light Duty Utility Arm (LDUA) are derived from Engineering Design FiTe (EDF) \#EDF-BSC-014

III. ASSUMPTIONS: Condition statements accepted or supposed true without proof or demonstration. An assumption has a direct impact on total estimated cost.

- It was assumed that Project Management and Construction Management will be $10 \%$ of construction costs respectively, Engineering and Design - Title I and II will be 20\% of construction costs, Engineering and Design - Conceptual will be $5 \%$ of construction costs, and Inspection will be $5 \%$ of construction costs for all estimates with the exception of the D\&D of equipment. In this estimate, the D\&D cost is all inclusive. 
Lockheed Martin Idaho Technologies Co.

\section{COST ESTIMATE SUPPORT DATA RECAPITULATION \\ (CONTINUATION).}

File No: 2423

Page 2 of 7

III. ASSUMPTIONS: (Continued)

- Indirect markups were assumed to be:

- Consumables and small tools - 3\% of labor

- Construction equipment (cranes, etc.) - $10 \%$ of 7 abor

- NQA-1 QuaTity requirements - $25 \%$ of material

- DOE/RH/0333P Quality standards - 10\% of material

- Subcontractor procurement costs - $1 \%$ of equipment and material

- Material and equipment overhead and profit - 5\% of material and equipment

- General contractor's profit on Subcontracts - 10\% of Subcontracts

- Bond - $2 \%$ of Construction Costs•

- Labor Indirects, Overhead and Profit - 50\% of 1abor

- LMITCO G\&A has been applied at $23 \%$ of construction and GFE, with a $\$ 500,000$ ceiling applied, and the Performance Incentive Fee (PIF) has been applied at $5.5 \%$. Procurement fees have been assumed at $1 \%$, as procurement support to DOE, rather than the normal $3 \%$ fee.

- Permitting and documentation for the separate options were included in a separate estimate. These estimates were broken down into one estimate to cover "clean" fill and one estimate to cover "Class C" or "Class A" fill.

- It was assumed that the pre-engineered metal containment enclosure and Ventilation, Instrumentation, and Control (VIC) building that will be constructed at each CSSF for the calcine retrieval process will remain and can be used during the closure process also.

- Assume HVAC equipment required during the calcine retrieval process will remain and be used during the grout installation process.

- Assume all monitoring equipment for the containment enclosures and VICs will remain in place from the retrieval process and can be used for the closure process.

- In most cases, unit costs were derived from the cost estimate for the Tank Farm at ICPP. 
Lockheed Martin Idaho Technologies Co.

COST ESTIMATE SUPPORT DATA RECAPITULATION

(CONTINUATION)

FiTe No: 2423

Page 3 of 7

III. ASSUMPTIONS: (Continued)

- Estimates for the various options are included at both present-day costs which include the Cost Estimate Summary, Detail Sheets, and. Contingency Analysis, and escalated costs to the midpoint of the activities. Only the Summary Sheet and Contingency Analysis have been provided for the escalated estimates. For more information, the Detail Sheets can be referenced. In addition, the D\&D Model showing the facility ratings and assumptions is included for present-day costs for the D\&D of equipment.

Assumptions relating to the vault estimates:

- Assume that four $18^{n}$ diameter access holes with shielding will be required to obtain access to the vaults to install the grout. It was assumed that four holes will be required in order to keep the grout level during the 4 ' 7 ifts and to fill any voids.

- It was assumed that the subcontractor would be required to purchase a grout pump, air compressor, etc. for the grout installation of the vaults. To assure coverage when the component estimates were compiled, the costs for these items were included in the estimates for the bin options also. Therefore, these costs may be inflated by two in the Summary.

- It was assumed that the grout manifold and cleaning system would be cleaned and reused.after each vautt.

- It was assumed that the grout delivery piping would be cleaned after each lift within each vault.

- It was assumed that the grout drop tubes and accessories purchased would be the quantity required to complete the distance for the tallest. vault times 4 tubes, and then could be cleaned and reused for the next vault, etc.

- It was assumed that the grout delivery piping required would be a single run of 200 ! plus 4 lines times $1 / 2$ of the maximum height of the vaults (assume $1 / 2$ of the vaults are below grade). 
Lockheed Martin Idaho Technologies Có.

COST ESTIMATE SUPPORT DATA RECAPITULATION

(CONTINUATION)

File No: 2423

Page 4 of 7

III. ASSUMPTIONS: (Continued)

Assumptions relating to the bin estimates:

- Assume that each bin will require two $18^{\text {" }}$ diameter access holes for the robots to be placed within the bins. The risers and the $18^{n}$ holes will be completed during the calcine retrieval process. However, the holes will require capping during this closure process.

- It. was assumed that four (4) radiation monitors per bin will be installed at the base of the tanks, except on CSSF \#1. On CSSF \#1, radiation monitors. will be installed on the outer bin of the 4 tanks. However, it was assumed that this function will require no excavation due to previous excavations. Gravel will be placed around the monitors.

- Assume that access to the bins for installation of the grout will be available through previously existing or constructed risers installed during the retrieval. process. No penetrations into the bins will be required for this project.

- Quantities for the bin heights required to estimate grout drop tubes were obtained from the above referenced EDF.

- It was assumed that the grout manifold and cleaning system would be cleaned and reused after each CSSF is complete.

- It was assumed that the grout delivery piping from the manifold to the grout tubes would be cleaned after each 4' lift within each bin.

- It was assumed that the grout drop tubes and accessories purchased would be the maximum quantity required for one CSSF. This quantity will be adequate for reuse in the remaining CSSFs after cleaning.

- It was assumed that the grout delivery piping would include a single run of $200^{\prime}$ pipe from the grout delivery point to the manifold plus additional piping lines running verticaliy up the vaults to the drop tubes. (Assume $1 / 2$ of vaults will be below grade)

- Continued on Page 5- 
Lockheed Martin Idaho Technologies Co.

\section{COST ESTIMATE SUPPORT DATA RECAPITULATION \\ (CONTINUATION)}

File No: 2423

Page 5 of 7

III. ASSUMPTIONS: (Continued)

- It was assumed that the NRC Class $C$ grout will be poured in $4^{\prime}$ lifts and then wait 7 days before the next lift can be poured. In some cases, a waiting period might be required for the scheduling of the next pour.

- It was assumed that an existing pipe control-center trailer will be available at the site for the Class $C$ grout pour.

- The retrieval tubes for each bin will not be reused on a subsequent bin, but will be bagged and disposed of after filling each bin with Class $C$ grout.

- The delivery pipe and manifolds will be bagged and disposed of after each CSSF is completed with Class C grout pour.

- Assume that 1,600 CY of Class C, NRC grout can be pumped per year.

- It was assumed that the installation of the NRC Class C grout will be performed continuousiy around the clock until the CSSFs are completed.

Assumptions relating to the retrieval of calcine with robots:

- Costs were based on the information provided in the EDF-BSC-014 for the tractor robot, pipe crawler, and the robotic arm (LDUA). The base costs were derived from the INEEL costs shown therein and doubled to equal the IS Robotics cost for the initial unit, since this cost is all inclusive. The duplicate units for the tractor were based on IS Robotics' cost for duplicate units. The same basis and ratio of duplicate units were used for the LDUAs and the pipe crawlers.

- An allowance was included for fabrication of the additional robotic units to design and make modifications specifically needed for each CSSF. 
Lockheed Martin Idaho Technologies Co.

\section{COST ESTIMATE SUPPORT DATA RECAPITULATION (CONTINUATION)}

File No: 2423

Page 6 of 7

III. ASSUMPTIONS: (Continued)

- Costs for the actual operation of the robotic units to retrieve the remaining $5 \%$ of calcine from the CSSFs were included in this estimate. The estimated costs were based on calculations derived from the engineer and include making 3 passes, which will involve continuously working around the clock, for the risk-based estimates. The RCRA closure estimates are based on only 1 pass and 1 shift/day.

- It was assumed that the tractor units for cleaning the floor will be left in the bins, but the LDUAs used for cleaning the walls and the pipe crawlers will be pulled out and reused on the other bins within

- It was assumed that costs for the supervisor, training, and the two radcon technicians could share their time between the work for the cleaning of the piping and the walls since it will be done at the same time. Therefore, the costs were split equally between the two estimates.

- The costs for the actual operation of the robotic units to retrieve the remaining $5 \%$ of calcine from the bin walls and piping were shared equally between the piping cleaning estimate and the wall cleaning estimate.

- Costs to construct an area for shielding when pulling the LDUAs and the pipe crawlers out of the bins are included in the pipe crawler/cleaning estimate.

Assumptions relating to the retrieval of calcine with robots:

- The costs associated with the D\&D Model include all costs associated with the D\&D of facilities or structures. These costs include project Management, Construction Management, Engineering, Inspection, etc. Therefore, no additional costs were applied, other than escalation, to the D\&D estimate.

- The costs derived from the D\&D model are based on assumed facility ratings for the equipment and assumed square footages that would require $D \& D$. 
Lockheed Martin Idaho Technologies Co.

\section{COST ESTIMATE SUPPORT DATA RECAPITULATION \\ (CONTINUATION)}

File No: 2423

Page 7 of 7

IV. CONTINGENCY GUIDELINE IMPLEMENTATION:

Because of the level of development and the lack of information for this project, generally contingency was applied at 35\%, which is at the upper range of the guidelines suggested in the INEL cost Estimating Guide. This was deemed reasonable because of the major risks involved with contaminated areas, and the fact that much of the equipment will require development. 
ESCALATION SCHEDULE

\begin{tabular}{|c|c|c|c|c|c|c|c|c|c|c|c|c|c|c|c|c|c|c|c|c|}
\hline \multicolumn{21}{|c|}{ MATAIX - MIDPOINT OF ACTIVITY } \\
\hline \multirow[t]{3}{*}{ OPTIONS } & \multicolumn{3}{|c|}{$\begin{array}{l}\text { Grout "Clean" } \\
\text { in Vaults }\end{array}$} & \multicolumn{3}{|c|}{$\begin{array}{c}\text { Grout "Clean" } \\
\text { in Bins } \\
\end{array}$} & \multicolumn{3}{|c|}{$\begin{array}{c}\text { NRC Class A and C } \\
\text { in Bins }\end{array}$} & \multicolumn{3}{|c|}{$\begin{array}{c}\text { Cloaning with Robots } \\
\text { Walls \& Piping }\end{array}$} & \multicolumn{3}{|c|}{$\begin{array}{c}\text { Cleaning with Robots } \\
\text { Floors }\end{array}$} & \multicolumn{2}{|c|}{$\begin{array}{c}\text { Permit/Doc. } \\
\text { RCRA/Risk Bas } \\
\text { Grout "Clean" }\end{array}$} & \multicolumn{2}{|c|}{$\begin{array}{c}\text { Permit/Doc } \\
\text { RCRA/Rlsk Bas } \\
\text { NRC Class A\&C }\end{array}$} & \multirow{3}{*}{$\begin{array}{c}\text { D\&D } \\
\text { of } \\
\text { Equip } \\
2423 \\
\end{array}$} \\
\hline & \multicolumn{3}{|c|}{ 2423-A2 } & \multicolumn{3}{|c|}{ 2423-A1 } & \multicolumn{3}{|c|}{ 2423-B \& B1 } & \multicolumn{3}{|c|}{ 2423-D\&E } & \multicolumn{3}{|c|}{$2423-\mathrm{C}$} & \multicolumn{2}{|c|}{ 2423-A1D } & \multicolumn{2}{|c|}{ 2423-BD } & \\
\hline & ED\&1 & Mgmt. & Constr & ED\&1 & Mgmt. & Constr. & ED\&! & Mgmt. & Constr. & ED\&! & Mgmt. & Constr. & ED\&! & Mgmt. & Constr. & Mgmt. & Pemilt & Mgmt. & Permit & \\
\hline RCRA Closure-Clean & $1 / 1 / 14$ & $6 / 1 / 23$ & $6 / 1 / 24$ & $10 / 1 / 13$ & 10/1/23 & $1 / 1 / 25$ & --- & --- & --- & $-\cdots$ & --- & --- & $1 / 1 / 13$ & $8 / 1 / 22$ & $8 / 1 / 24$ & $7 / 1 / 13$ & $7 / 1 / 13$ & --- & --- & 2035 \\
\hline RCAA Closure-NRC Class C & $7 / 1 / 13$ & 9/1/22 & $9 / 1 / 23$ & -- & -- & $-\infty$ & $7 / 1 / 22$ & $6 / 1 / 27$ & $12 / 1 / 28$ & -- & -- & --- & $7 / 1 / 12$ & $10 / 1 / 21$ & $10 / 1 / 23$ & --- & -- & $1 / 1 / 12$ & $1 / 1 / 12$ & 2035 \\
\hline RCRA Closure-NRC Class A & $7 / 1 / 13$ & $9 / 1 / 22$ & 9/1/23 & --- & -- & $-\cdots$ & $7 / 1 / 22$ & $6 / 1 / 27$ & $12 / 1 / 28$ & --- & -- & --- & $7 / 1 / 12$ & $10 / 1 / 21$ & $10 / 1 / 23$ & --- & --- & $1 / 1 / 12$ & $1 / 1 / 12$ & 2035 \\
\hline Alsk Based-Clean Grout & $7 / 1 / 14$ & $6 / 1 / 23$ & 6/1/24 & $1 / 1 / 14$ & $7 / 1 / 24$ & $11 / 1 / 25$ & $-\infty$ & -- & --- & $1 / 1 / 13$ & $8 / 1 / 22$ & $8 / 1 / 24$ & $4 / 1 / 13$ & $6 / 1 / 23$ & $6 / 1 / 25$ & $7 / 1 / 13$ & $7 / 1 / 13$ & --- & --- & 2035 \\
\hline Bisk Based-NRC Class C & $7 / 1 / 13$ & 9/1/22 & 9/1/23 & --- & $-m$ & $-\cdots$ & $7 / 1 / 22$ & $11 / 1 / 27$ & $7 / 1 / 29$ & $7 / 1 / 12$ & $10 / 1 / 21$ & $10 / 1 / 23$ & $10 / 1 / 12$ & 12/1/22 & $1 / 1 / 24$ & --- & -- & $1 / 1 / 12$ & $1 / 1 / 12$ & 2035 \\
\hline Risk Based-NRC Class A & $7 / 1 / 13$ & 9/1/22 & 9/1/R3 & --- & --- & --- & 7/1/22 & $11 / 1 / 27$ & $7 / 1 / 29$ & $7 / 1 / 12$ & $10 / 1 / 21$ & $10 / 1 / 23$ & $10 / 1 / 12$ & $12 / 1 / 22$ & $1 / 1 / 24$ & --- & --- & $1 / 1 / 12$ & $1 / 1 / 12$ & 2035 \\
\hline & & & & & & & & & & & & & & & & & & & & \\
\hline
\end{tabular}

ASSUME: Class A has Samo Mildpoint of Activities as Class C 
움 
COST ESTIMATE SUMMARY

UNESCALATED

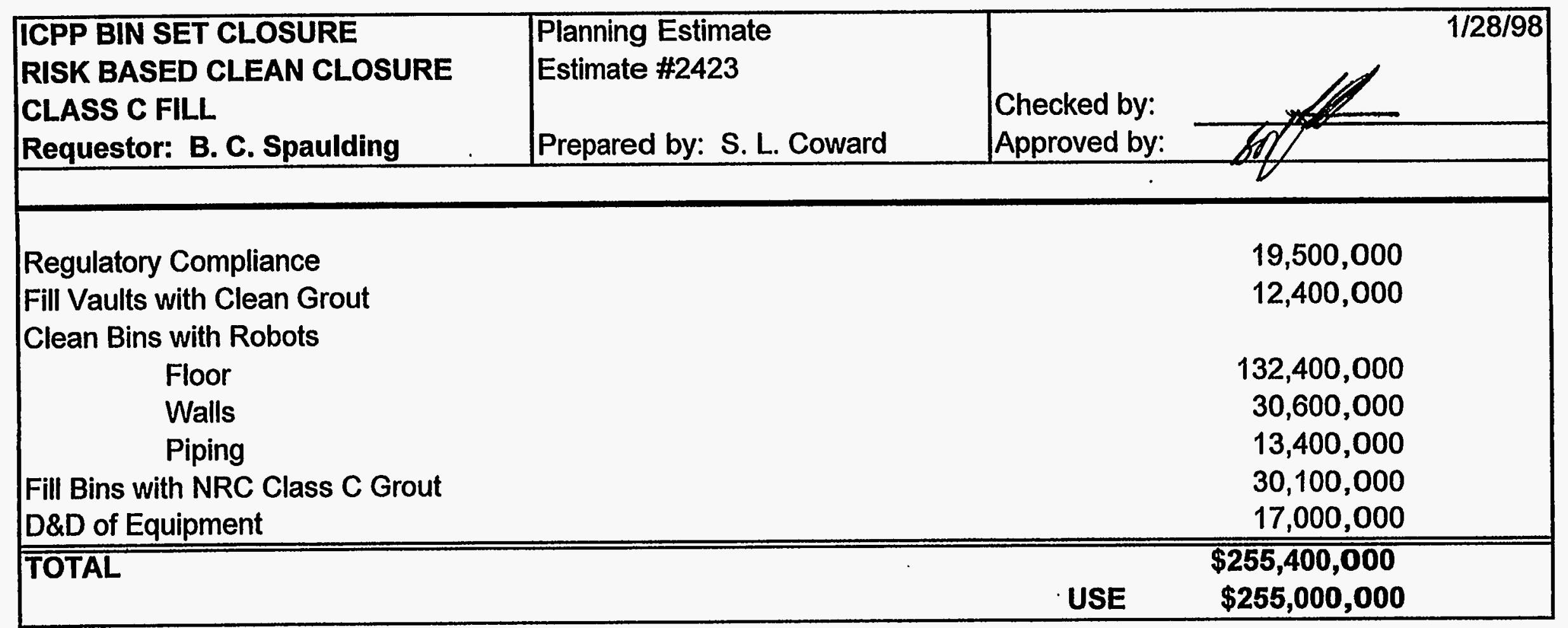


RISK BASED CLEAN CLOSURE - NRC CLASS C GROUT

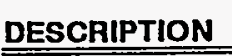

BIN SET \#-1

BIN SET \#5

BIN SET \#7

BIN SET \#6

BIN SET \#

$(8 / 1 / 28)$

BIN SET \#4

(2/1/31)

BIN SET \#2

ASSUME: Wait 6 Months until Start of Closure

$(1 / 1 / 14)$

$(4 / 1 / 18)$

(12/1/22)

(11/1/26)

$(8 / 4 / 28)$

$(5 / 1 / 31)$

Pormitting (5 Yoars)

$7 / 1 / 9-7 / 1 / 14$

Grout Vaults "Cloan" (Assume 1 Yoar)

\begin{tabular}{ll}
\hline ED\&l (2 Yrs) & $7 / 1 / 12-7 / 1 / 14$ \\
Management & $7 / 1 / 12-11 / 1 / 32$
\end{tabular}

Construction

$7 / 1 / 14-7 / 1 / 15 \quad 10 / 1 / 18-10 / 1 / 19 \quad 6 / 1 / 23-6 / 1 / 24 \quad 5 / 1 / 27-5 / 1 / 28$

$2 / 1 / 29-2 / 1 / 30$

$8 / 1 / 31-8 / 1 / 32$

$11 / 1 / 31-11 / 1 / 32$

Clean Bin Walls/Piping with Robot

(3 Months)

(15 Months)

(25 Months)

(24 Months)

(17 Months)

(7 Months)

(13 Months)

\begin{tabular}{ll}
\hline ED\&l (4 Yrs) & $7 / 1 / 10-7 / 1 / 14$ \\
Management & $7 / 1 / 10-12 / 1 / 32$
\end{tabular}

Construction

$7 / 1 / 14-10 / 1 / 14 \quad 10 / 1 / 18-1 / 1 / 20 \quad 6 / 1 / 23-7 / 1 / 25 \quad 5 / 1 / 27-5 / 1 / 29$

$2 / 1 / 29-7 / 1 / 30$

$8 / 1 / 31-3 / 1 / 32$

$11 / 1 / 31-12 / 1 / 32$

Clean Bin Floors with Robot

\begin{tabular}{ll}
\hline ED\&l (4 Yrs) & $10 / 1 / 10-10 / 1 / 14$ \\
Management & $10 / 1 / 10-1 / 1 / 34$
\end{tabular}

Management

(3 Months)

(15 Months)

(25 Months)

(24 Months)

(17 Months)

(7 Months)

(13 Months)

Construction

$10 / 1 / 14-1 / 1 / 15 \quad 1 / 1 / 20-4 / 1 / 21$

$7 / 1 / 25-8 / 1 / 27$

$5 / 1 / 29-5 / 1 / 31$

$7 / 1 / 30-12 / 1 / 31 \quad 3 / 1 / 32-10 / 1 / 32 \quad 12 / 1 / 32-1 / 1 / 34$

\section{RCAA CLOSUAE}

\section{Grout Bins "NRC Class C"} ED\&l (3 Yrs)

Management

Construction
(3 Months)

(11 Months)

(18 Months)

(17 Months)

(12 Months)

(6 Months)

(10 Months)

$1 / 1 / 21-1 / 1 / 24$

$1 / 1 / 21-8 / 1 / 34$

\section{ASSUMPTIONS:}

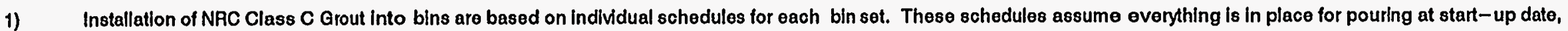
and allow no flexibillty for error or downtime.

2) More than 1 crow could be utlized simultaneously for pouing the "clean" grout into the vaults.

3) Moe than 1 crew could be utllized simuitaneously for cleaning the separate bins. However, cleaning of the floors will require scheduling after cleaning the walls/plping.

4) Cleaning of bin floors are based on indlvidual bin pro-rated calcine retrieval volumes to total volume. These schedules assume mob/demob, installation of robotle units, and any modifleations of blns will be completed and bins will be ready for retrieval.

5) Because of the difficulty of oleaning of bln walls/plping, it was assumed that It would take the same duration as the cleaning of the bin floors for each bin.

6) Installation of "clean" grout into vaults will average 1 year per vault. 
Lockheed Martin Idaho Technologies Co. Rov. 6-96 PROJECT NAME: Permitting/Documentation Risk Based - NRC Class C INEELMCPP

LOCATION 1: REQUESTOR:
COST. ESTIMATE SUMMARY

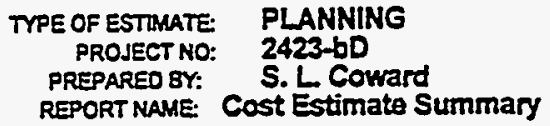

ATE:

TINE

CHECXED BY:

APPRO BY:

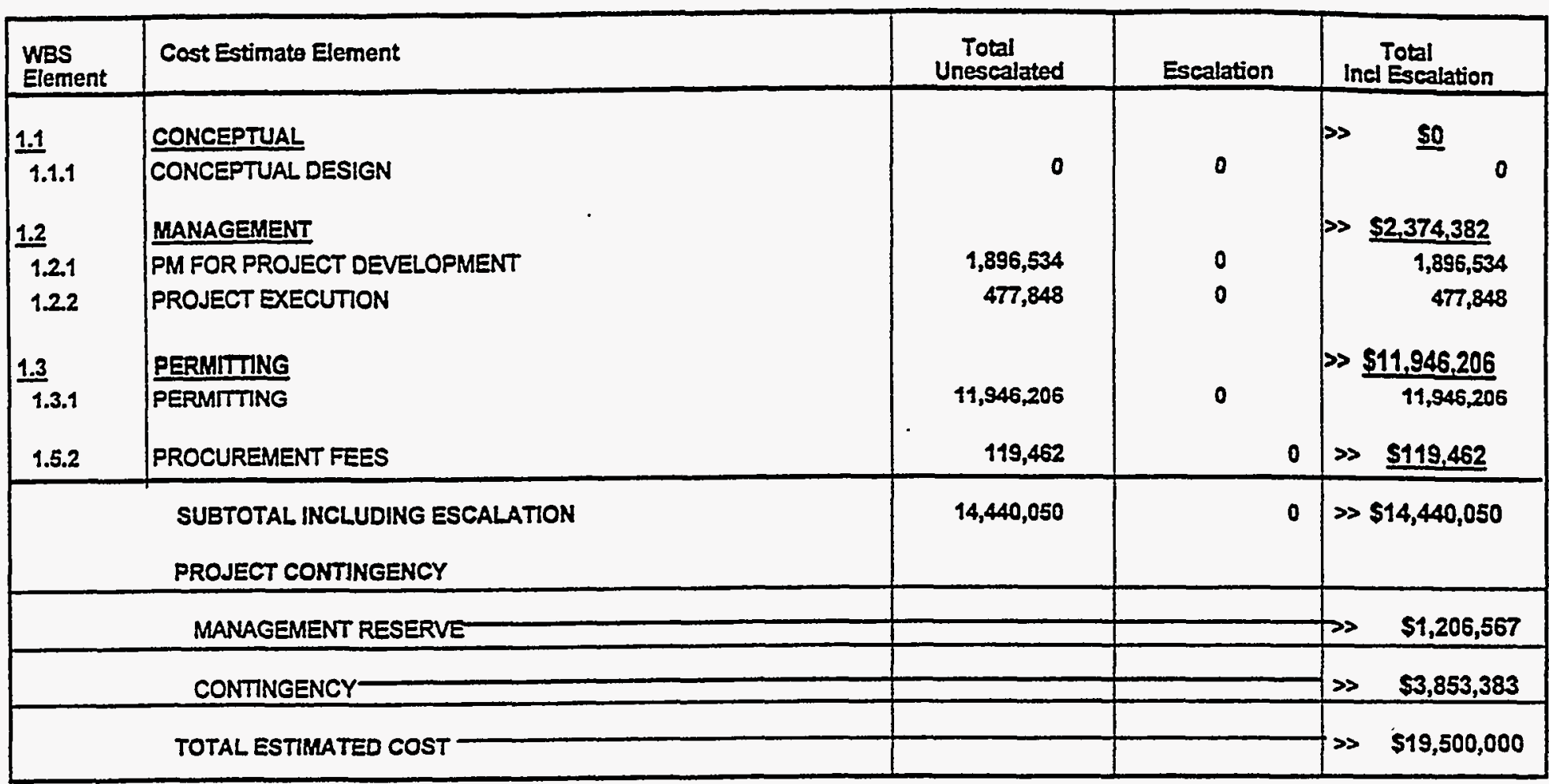

PROJECT COST PARAMETERS

EDI AS A \% OF CONST. + GFE $=0.00 \%$ 
Lockheed Martin Idaho Technologles Co. Rav 6.96

'ROJECT NAME: Permitting/Documentation Risk Based - NRC Class C

INEELICPP

IEQUESTOR: Bryan Spaulding
DETAILED COST ESTIMATE SHEET

TYPE OF ESTIMATE: PLANNING

PROJECT NO: 2423.bD

PREPARED BY: S. L. Coward
PAGE * 1

DATE 27-Jan-1998

TIME: 19:51:09

REPORT NAME: Dotall Cost Estimate Sheot

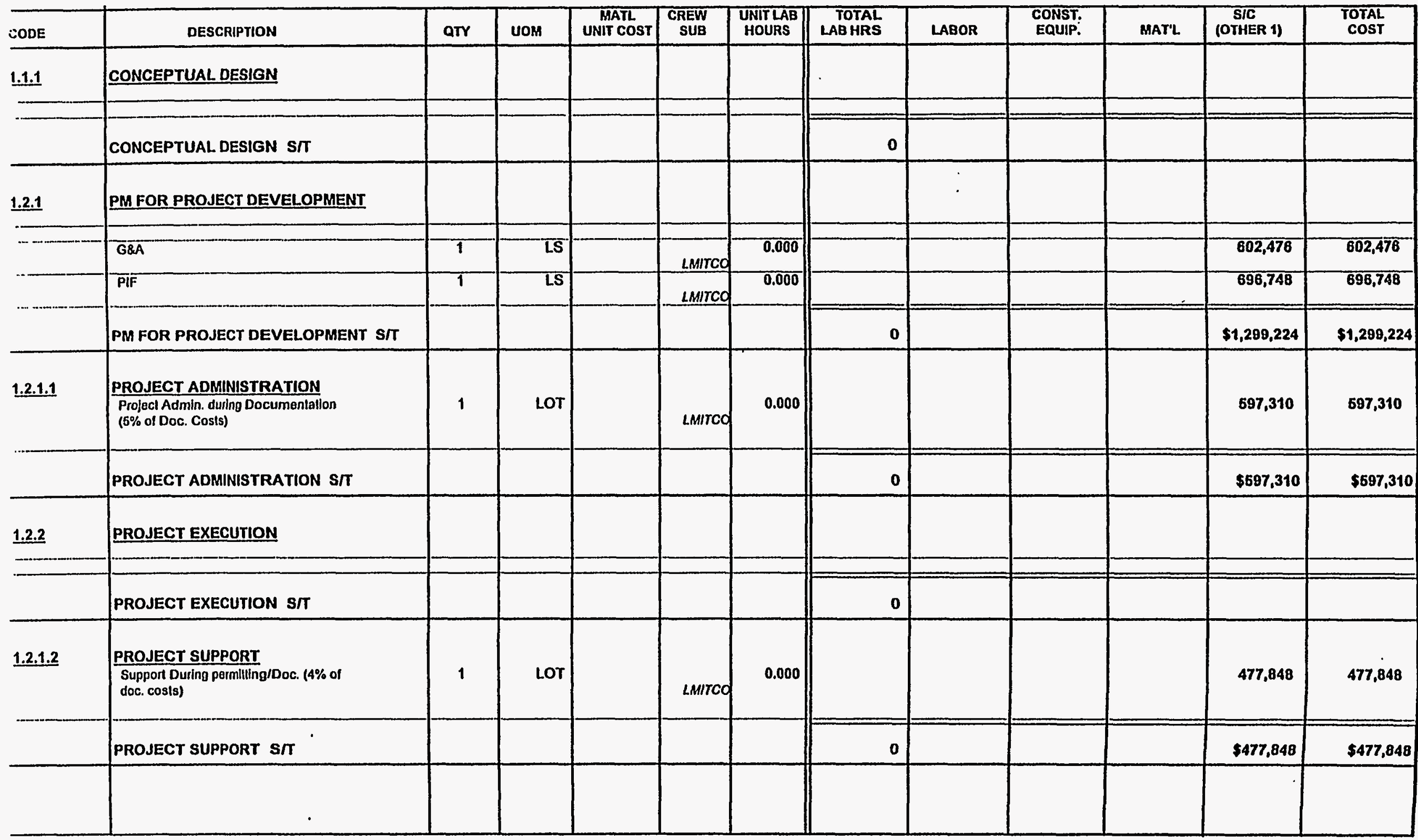


ockheed Martin Idaho Technologles Co.

$$
\text { Rov } 6.00
$$

OJECr NAME. Permitting/Documentation RIsk Based - NRC Class C. INEELIICPP

:oueston Bryan Spaulding

\section{DETAILED COST ESTIMATE SHEET}

TYPE Of estimate: PLANNING

PROJECT NO.: 2423-bD

PREPARED BY: S. L. Coward
PAGE 2

DATE 27 Jan-198B

TIME: 19:61:09

AEPORT NAME: Detall Cost Estimate Sheot

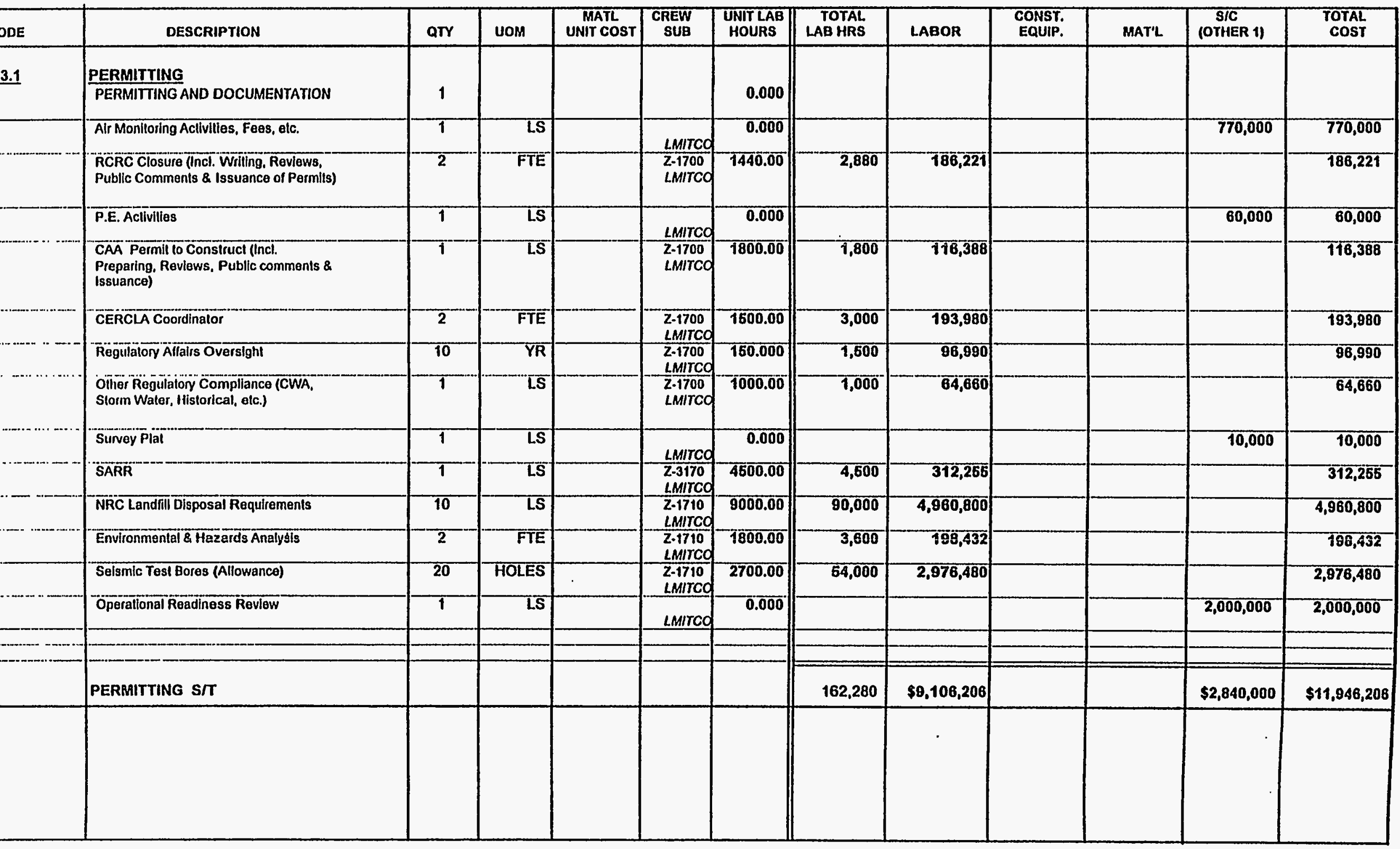




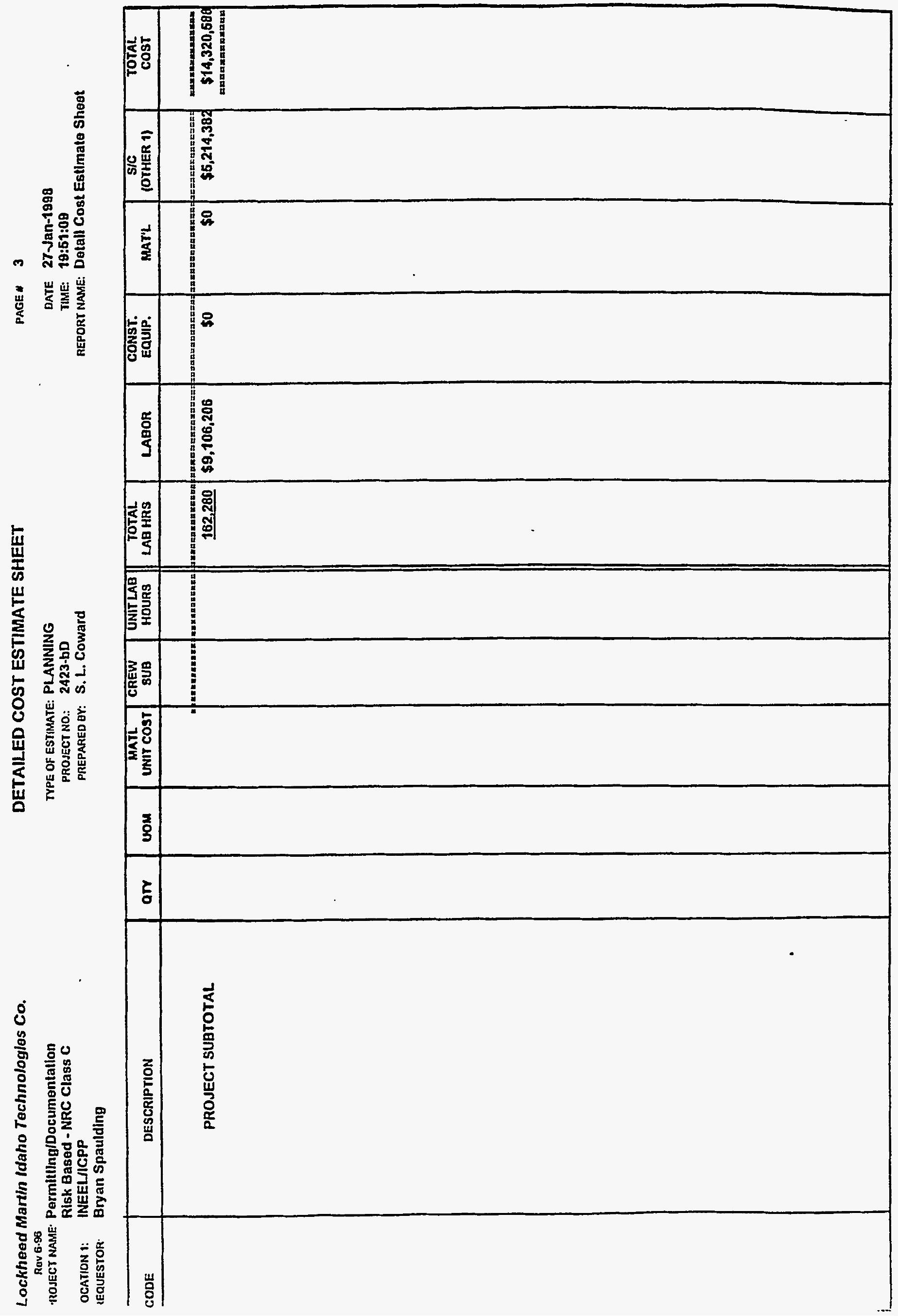


Lockheed Martin Idaho Technologies Co.

Rev 6 -96

PROJECT NAME: Permitting/Documentation

Risk Based - NRC Class C

LOCATION $\%$ INEELICPP

REQUESTOR: Bryan Spaulding

CONTINGENCY ANALYSIS

TYPE OF ESTIMATE: PLANNING

PROJECT NO: 2423-bD

PREPAREO BY: S. L Coward
DATE: 27 Jan-1998

TIME: 19:51:03

REPORT NAME Contingency Analysis

\begin{tabular}{|c|c|c|c|c|c|c|c|c|c|c|c|}
\hline \multicolumn{9}{|c|}{ PROBABLE \% VARIATION } & \multicolumn{2}{|c|}{$\begin{array}{l}\text { PROJECT } \\
\text { CONTINGENCY }\end{array}$} & \multirow{3}{*}{$\begin{array}{l}\text { SUMMARY } \\
\text { Total cost } \\
\text { by Eement }\end{array}$} \\
\hline $\begin{array}{l}\text { WBS } \\
\text { Element }\end{array}$ & \multirow{2}{*}{ Cost Estimate Element } & \multirow{2}{*}{$\begin{array}{l}\text { Total Cost wfo } \\
\text { Contingency }\end{array}$} & \multirow[t]{2}{*}{$\begin{array}{l}\% \text { Total } \\
\text { Cost }\end{array}$} & \multicolumn{2}{|c|}{$\begin{array}{l}\text { Prob. \% Var. } \\
\text { From Est. }\end{array}$} & \multicolumn{2}{|c|}{ Wh \% of Prob. } & \multirow{2}{*}{ Contingency } & \multirow{2}{*}{$\%$} & \multirow{2}{*}{ Cost } & \\
\hline & & & & - & + & - & + & & & & \\
\hline 1.1 .1 & CONCEPTUAL DESIGN & 0 & 0.00 & 10 & 40 & 0.00 & 0.00 & $0.000 \%$ & $0.00 \%$ & 0 & 0 \\
\hline 121 & PM FOR PROJECT DEVELOPMENT & $1,896,534$ & 13.13 & 10 & 40 & 1.31 & 5.25 & $4597 \%$ & $13.13 \%$ & 684,566 & $2,561,100$ \\
\hline 1.22 & PROJECT EXECUTION & 477,848 & 3.31 & 10 & 40 & 0.33 & 1.32 & $1.158 \%$ & $3.31 \%$ & 167,443 & 645,291 \\
\hline 1.3 .1 & PERMITIING & $11,946,206$ & 82.73 & 10 & 40 & 8.27 & 33.09 & $28.955 \%$ & $8273 \%$ & $4,186,080$ & $16,132,286$ \\
\hline 1.52 & PROCUREMENT FEES & 119,462 & 0.83 & 10 & 40 & 0.08 & 0.33 & $0.290 \%$ & $0.83 \%$ & 41,861 & 161,323 \\
\hline & ESCALATION & 0 & 0.00 & 0 & 0 & 0.00 & 0.00 & $0.000 \%$ & $0.00 \%$ & 0 & 0 \\
\hline & SUBTOTAL & $14,440,050$ & 100.00 & & & & & $35.000 \%$ & & & \\
\hline & CALCULATED CONTINGENCY & $5,054,017$ & & & & & & & & & \\
\hline & RESULTANT TEC & $19,494,067$ & & & & & & - & & & \\
\hline & ROUNDED TEC & $19,500,000$ & & & & & & & & & \\
\hline & PROJECT CONTINGENCY & $5,059,950$ & & & & & & $35.04 \%$ & & & \\
\hline & MANAGEMENT RESERVE & $1,206,567$ & & 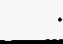 & & & & & & & \\
\hline & CONTINGENCY & $3,853,383$ & & & & & & & & & \\
\hline & TOTAL ESTIMATED COST & $19,500,000$ & & & & & & & & $5,059,950$ & $19,500,000$ \\
\hline
\end{tabular}

CONFIDENCE LEVEL AND ASSUMED RISKS:

The Lockheed idaho Technologies Co. Cost Estimate Contingency Analysis Model is based on the applied contingency and the assumptions upon which

the estimate was predicated. The model is applied with a suggested risk level of $18 \%$ and a level of confidence of $90 \%$ the estimate will fall within the bid range. The Contingency Analysis is based on a weighted average to provide a

$90 \%$ probability of underrun and a $10 \%$ probability of overnun.
CONTINGENCY ANALYSIS GUIDE BY TYPE OF ESTTMATE

Guidelines established by DOERFM 50 , Cost Estimating Guide, Vol. 6 , Cost Guide, and as presented in the INEL Cost Estimating Guide.
PLANNING
Experimentalspecial Conditions.
$20 \%-30 \%$
Conceptual
TITLEI
TITLE II
TITLE IIIAFC
$15 \%-25 \%$
$10 \%-20 \%$
$5 \%-15 \%$
Market Conditions 


\section{G\&A/PIF ADDER CALCULATION SHEET \\ ICPP BIN SET CLOSURE \\ RISK BASED CLEAN CLOSURE; CLASS C FILL; UNESCALATED}

REGULATORY COMPLIANCE

PROCUREMENT FEE:

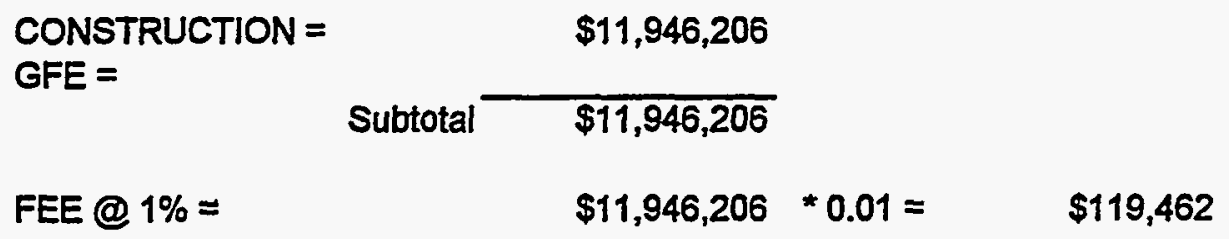

G\&A @ 23\% (with a ceiling of $\$ 500,000$ imposed per year , 5 Years)

CONSTRUCTION OR

CEILING $• 5$ YEARS $=\quad \$ 2,500,000$

GFE =

PROCUREMENT FEE = $\$ 119,462$

Subtotal

$\$ 2,619,462$

FEE @ 23\%=

$\$ 2,619,462 * 0.23=$

$\$ 602,476$

PIF @ 5.5\%

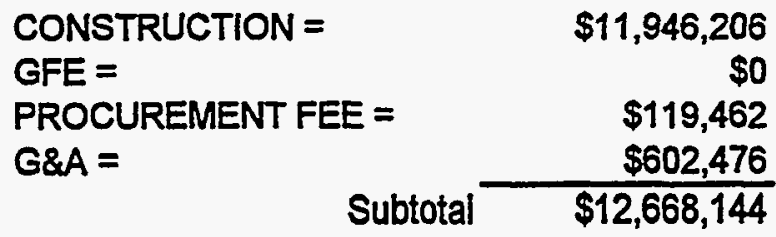

FEE @ 5.5\% =

$\$ 12,668,144 * 0.055=$

$\$ 696,748$

TOTAL PROCUREMENT FEE:

$\$ 119,462$

TOTAL G\&A FEE:

$\$ 602,476$

TOTAL PIF:

$\$ 696,748$ 
Lockheed Martin Idaho Technologies Co. Rov. 6-96 PROJECT NAME: ICPP Bin Set Closure (EIS Stud Place Clean Grout in Vault LOCATION 1: REQUESTOR: ICPP Bryan Spaulding
COST ESTIMATE SUMMARY

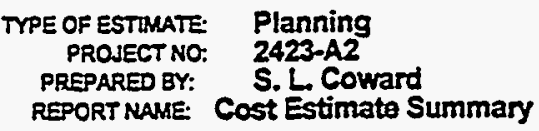

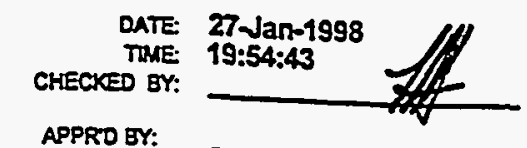

APPRO BY:

\begin{tabular}{|c|c|c|c|c|}
\hline $\begin{array}{l}\text { WBS } \\
\text { Element }\end{array}$ & Cost Estimate Element & $\begin{array}{c}\text { Total } \\
\text { Unescalated }\end{array}$ & Escalation & $\begin{array}{l}\text { Total } \\
\text { Incl Escalation }\end{array}$ \\
\hline $\begin{array}{l}\frac{1.1}{1.1 .1} \\
1.1 .2 \\
\frac{1.2}{1.2 .1} \\
1.2 .2 \\
\frac{1.3}{1.3 .1} \\
1.3 .2 \\
1.3 .3 \\
1.5 .2\end{array}$ & $\begin{array}{l}\text { ENGINEERING, DESIGN AND INSPECTION } \\
\text { DESIGN ENGINEERING } \\
\text { TITLE III INSPECTION } \\
\text { MANAGEMENT COSTS } \\
\text { PROJECT MANAGEMENT } \\
\text { CONSTRUCTION MANAGEMENT } \\
\text { CONSTRUCTION } \\
\text { GENERAL CONDITIONS } \\
\text { SITEWORK } \\
\text { CONCRETE } \\
\text { PROCUREMENT FEES }\end{array}$ & $\begin{array}{r}1,324,812 \\
264,962 \\
\\
1,686,432 \\
529,925 \\
\\
1,067,791 \\
0 \\
4,231,459 \\
52,992 \\
\end{array}$ & $\begin{array}{l}0 \\
0 \\
0 \\
0 \\
0 \\
0 \\
0\end{array}$ & 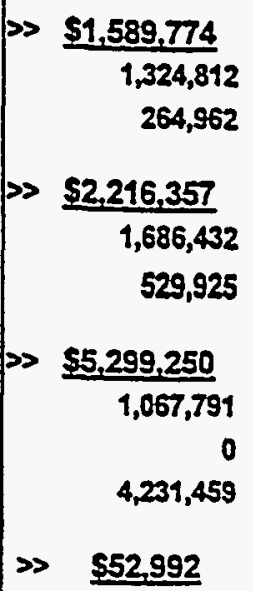 \\
\hline & $\begin{array}{l}\text { SUBTOTAL INCLUDING ESCALATION } \\
\text { PROJECT CONTINGENCY }\end{array}$ & $9,158,373$ & 0 & $\gg>\$ 9,158,373$ \\
\hline & MANAGEMENT RESERVE & & & $\$ 535,224$ \\
\hline & CONTINGENCY - & & & $\Rightarrow \quad \$ 2,706,403$ \\
\hline & TOTAL ESTIMATED COST - & & & $\$ 12,400,000$ \\
\hline
\end{tabular}

PROOJECT COST PARAMETERS

EDI AS A \% OF CONST. + GFE $=30.00 \%$ 
Lockheod Martin Idaho Technologles Co.

Rov 6.96

ROATE: ICPP Bin Sot Closure (EIS Study)

Place Clean Grout in Vault

OCATION I: ICPP

IEQUESTOR Bryan Spaulding

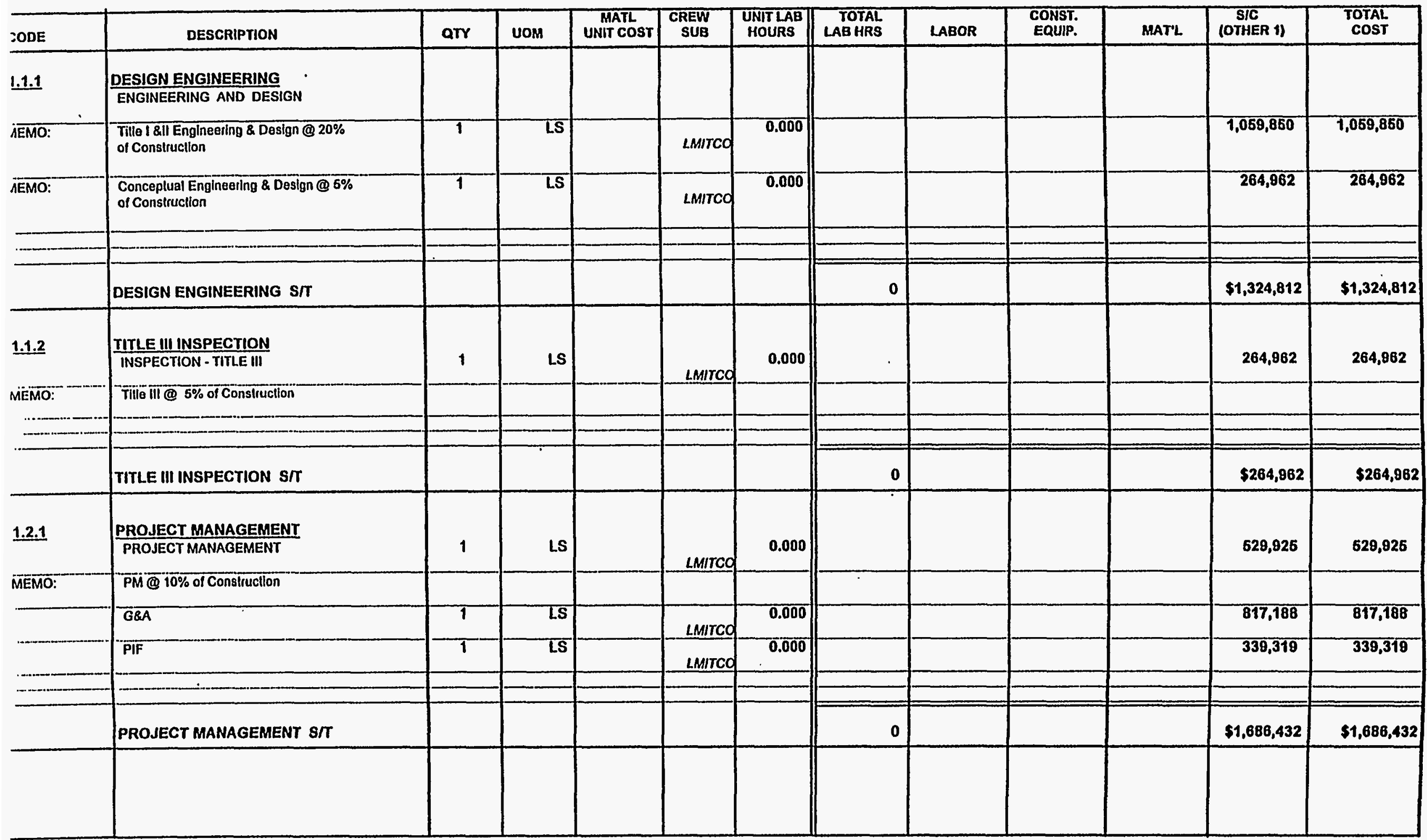

DETAILED COST ESTIMATE SHEET

IYPE Of estlmate: Planning

PROJECT NO: 2423-A2

PRepared ay: S. L. Coward
PAGE * 1

DATE 27-Jan-1988

TIME: 19:64:46

REPORT NAME: Detall Cost Estlmate Sheet 
Ickheed Martin Idaho Technologles Co.

Rov 6.06
JJECT NAME. ICPP Bin Set Closure (EIS Study) Place Clean Grout in Vault

ICPP

ZUESTOR Bryan Spaulding
DETAILED COST ESTIMATE SHEET

TYPE OF estimate: Planning

PROJECT NO: 2423-A2

PREPARED BY: S. L. Coward
PAGE* 2

DATE 27-Jan-1898

TIME: 19:64:46

REPORT NAME: Detall Cost Estimato Sheot

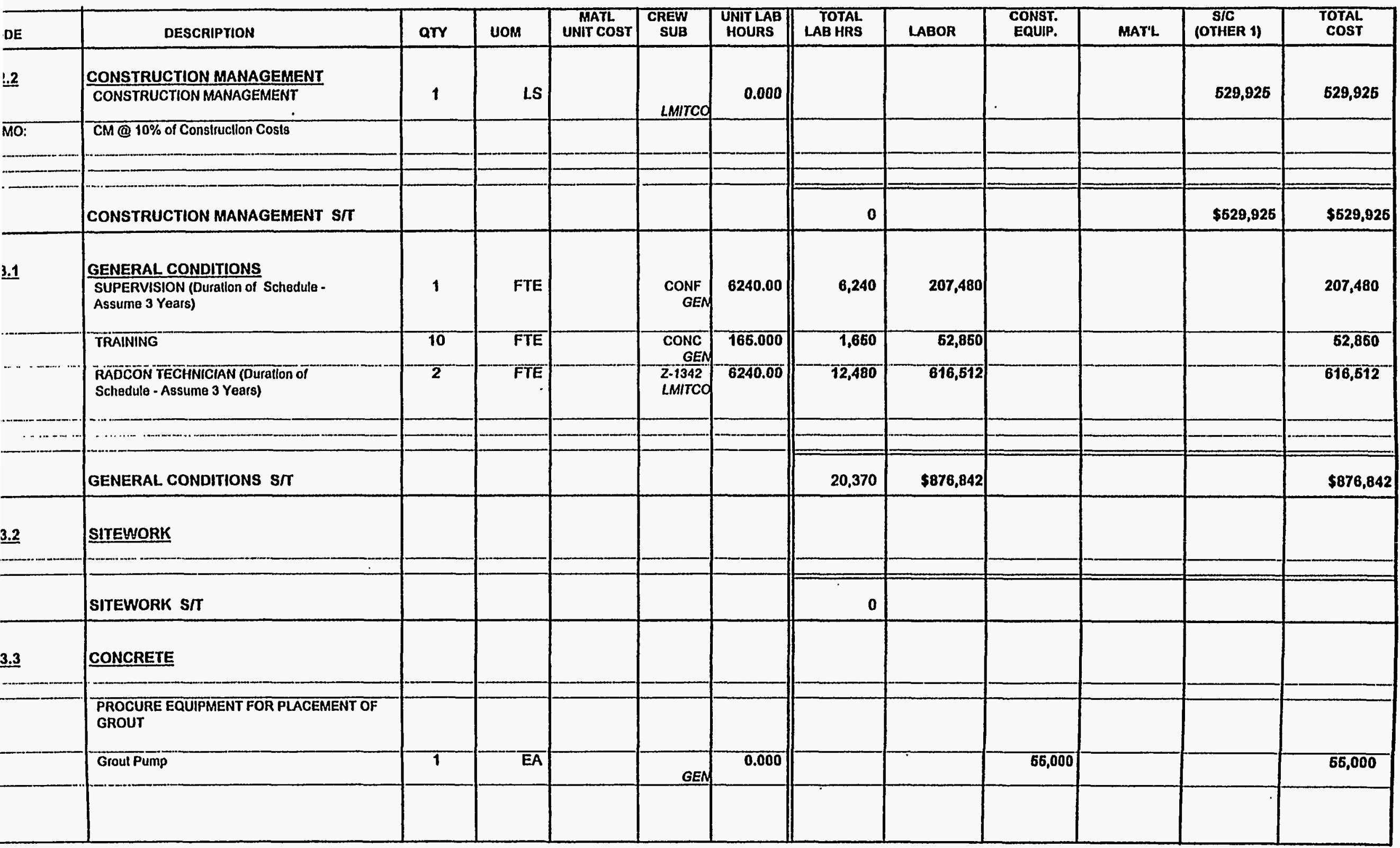


Lockheed Martin Idaho Technologies Co.

PROJECT MAME: ICPP Bin Set Closuro (EIS Stud Place Clean Grout in Vault

LOCATION 1:

REQUESTOR: ICPP

Bryan Spaulding
CONTINGENCY ANALYSIS

$\begin{array}{cl}\text { TYPE OF ESTINAATE: } & \text { Planning } \\ \text { PROJECT NO: } & 2423-A 2 \\ \text { PREPARED BY: } & \text { S. L Coward }\end{array}$

DATE: 27 Jan-1998

TRME: 19:54:39

REPORT NAME: Contingency Analysis

\begin{tabular}{|c|c|c|c|c|c|c|c|c|c|c|c|}
\hline & PROE & BLE \% VARIAT & ON & & & & & & $\begin{array}{l}\text { PRO } \\
\text { CONT }\end{array}$ & $\begin{array}{l}\text { CT } \\
\text { SENCY }\end{array}$ & SUMMARY \\
\hline WBS & Cost Estimato Element & Total Cost wto & $\begin{array}{l}\% \text { Total } \\
\text { Cost }\end{array}$ & & $\begin{array}{l}\text { \% Var. } \\
\text { nEst. }\end{array}$ & $m \%$ & Prob. & Contingency & $\%$ & Cost & Total cost \\
\hline & & Contingency & & - & + & - & + & & & & by Eement \\
\hline 1.1.1 & DESIGN ENGINEERING & $1,324,812$ & 14.47 & 10 & 40 & 1.45 & 5.79 & $5.053 \%$ & $14.47 \%$ & 468,920 & $1,793,732$ \\
\hline 1.1 .2 & TIIE III INSPECTION & 264,962 & 2.89 & 10 & 40 & 0.29 & 4.16 & $1.013 \%$ & $289 \%$ & 93,784 & 358,746 \\
\hline 1.21 & PROJECT MANAGEMENT & $1,686,432$ & 18.41 & 10 & 40 & 1.84 & 7.37 & $6.445 \%$ & $18.44 \%$ & 596,916 & $2,283,348$ \\
\hline 1.2 .2 & CONSTRUCTION MANAGEMENT & 529,925 & 5.79 & 10 & 40 & 0.58 & 239 & $2025 \%$ & $5.79 \%$ & 187,568 & 717,493 \\
\hline 1.3 .1 & GENERAL CONDMTONS & $1,067,791$ & 11.56 & 10 & 40 & 1.17 & 4.66 & $4.081 \%$ & $11.65 \%$ & 377,947 & $1,445,738$ \\
\hline 1.3 .2 & STIEWORK & 0. & 0.00 & 0 & 0 & 0.00 & 0.00 & $0.000 \%$ & $0.00 \%$ & 0 & 0 \\
\hline 1.3 .3 & CONCRETE & $4,231,459$ & 46.20 & 90 & 40 & 4.62 & 18.48 & $16.171 \%$ & $46.20 \%$ & $1,497,735$ & $5,729,194$ \\
\hline 1.5 .2 & PROCUREMENT FEES & 52,992 & 0.58 & 10 & 40 & 0.06 & 0.23 & $0.203 \%$ & $0.58 \%$ & 18,757 & 71,749 \\
\hline & ESCALATION & 0 & 0.00 & 0 & 0 & 0.00 & 0.00 & $0.000 \%$ & $0.00 \%$ & 0 & 0 \\
\hline & SUBTOTAL & $9,158,373$ & 100.00 & & & & & $35.000 \%$ & & & \\
\hline & CALCULATED CONTINGENCY & $3,205,431$ & & & & & & & & & \\
\hline & RESULTANT TEC & $12,363,804$ & & & & & & & & & \\
\hline & ROUNDED TEC & $12,400,000$ & & & & & & & & & \\
\hline & PROJECT CONTINGENCY & $3,241,627$ & & & & & & $35.40 \%$ & & & \\
\hline & MANAGEMENT RESERVE & $\mathbf{5 3 5 , 2 2 4}$ & & & & & & & & & \\
\hline & CONTINGENCY & $2,706,403$ & & & & & & & & & \\
\hline & TOTAL ESTIMATED COST & $12,400,000$ & & & & & & & & $3,241,627$ & $12,400,000$ \\
\hline
\end{tabular}

CONFIDENCE LEVEL AND ASSUMED RISKS:

The Lockheed Idaho Technologies Co. Cost Estimate Contingency Analysis Model is based on the applied contingency and the assumptions upon which

the estimate was predicated. The model is applied with a suggested risk level of $18 \%$ and a level of confidence of $90 \%$ the estimate will fall within the bid range. The Contingency Analysis is based on a weighted average to provide a $90 \%$ probability of underrun and a $10 \%$ probability of overrun.
CONTINGENCY ANALYSIS GUIDE BY TYPE OF ESTIMATE

Guidelines established by DOE/FM 50. Cost Estimating Guide, Vol. 6. Cost Guide, and as presented in the INEL Cost Estimating Guide.
PLANNING
Experimental/Special Conditions............. Up to $50 \%$
Conceptual
ExperimentaVSpecial Conditions.............. Up to $40 \%$
TITLE I
TITLE II
TIILE IUAFC
$10 \%-20 \%$
$5 \%-15 \%$
Market Conditions 


\section{G\&A/PIF ADDER CALCULATION SHEET ICPP BIN SET CLOSURE \\ RISK BASED CLEAN CLOSURE; CLASS C FILL; UNESCALATED}

FILL VAULTS W/ CLEAN GROUT

PROCUREMENT FEE:

$\begin{aligned} & \text { CONSTRUCTION } \\ & \text { GFE }=\end{aligned} \quad \begin{aligned} & \$ 5,299,250 \\ & \text { Subtotal }\end{aligned} \frac{\$ 5,299,250}{}$

FEE @ 1\%=

$\$ 5,299,250 * 0.01=$

$\$ 52,993$

G\&A @ 23\% (with a ceiling of $\$ 500,000$ imposed per year, 7 yrs)

CONSTRUCTION OR

CEILING $\bullet 7$ YEARS $=$

$\$ 3,500,000$

GFE =

$\$ 0$

PROCUREMENT FEE $=$

$\$ 52,993$

Subtotal $\$ 3,552,993$

FEE @ 23\% =

$\$ 3,552,993 * 0.23=$

$\$ 817,188$

PIF@ $0.5 \%$

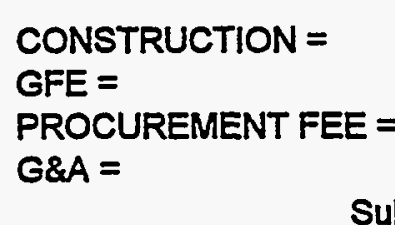

$\$ 5,299,250$

CONSTRUCTION =

$\$ 817,188$

Subtotal

$\$ 6,169,431$

FEE @ $5.5 \%=$

$\$ 6,169,431 \cdot 0.055=$

$\$ 339,319$

TOTAL PROCUREMENT FEE:

$\$ 52,993$

TOTAL G\&A FEE:

$\$ 817,188$

TOTAL PIF:

$\$ 339,319$ 
Lockheed Martin Idaho Technologies Co. Rev. 6.96 PROJECT NAME: ICPP Bin Set Closuro (EIS Stud LOCATION 1: Tractor (Risk Based Estimates) REQUESTOR:
COST ESTIMATE SUMMARY

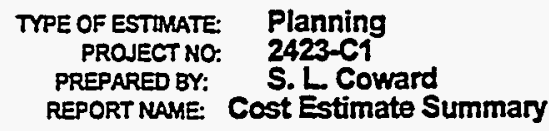

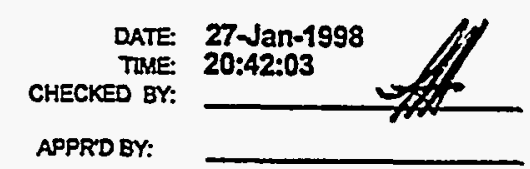

\begin{tabular}{|c|c|c|c|c|}
\hline $\begin{array}{l}\text { WBS } \\
\text { Element }\end{array}$ & Cost Estimate Element & $\begin{array}{c}\text { Total } \\
\text { Unescalated }\end{array}$ & Escalation & $\begin{array}{c}\text { Total } \\
\text { Incl Escalation }\end{array}$ \\
\hline $\begin{array}{l}\frac{1.1}{1.1 .1} \\
1.1 .2 \\
\frac{1.2}{1.2 .1} \\
1.2 .2 \\
\frac{1.3}{1.3 .1} \\
1.3 .13 \\
1.5 .2\end{array}$ & $\begin{array}{l}\text { ENGINEERING, DESIGN AND INSPECTION } \\
\text { DESIGN ENGINEERING TITLE I \& } \| \\
\text { TITLE III INSPECTION } \\
\text { MANAGEMENT COSTS } \\
\text { PROJECT MANAGEMENT } \\
\text { CONSTRUCTION MANAGEMENT } \\
\text { CONSTRUCTION } \\
\text { GENERAL CONDITIONS } \\
\text { SPECIAL CONSTRUCTION } \\
\text { PROCUREMENT FEES }\end{array}$ & $\begin{array}{r}9,900,244 \\
3,300,081 \\
11,639,957 \\
6,600,163 \\
\\
6,840,414 \\
59,161,216 \\
660,016\end{array}$ & $\begin{array}{l}0 \\
0\end{array}$ & $\begin{array}{r}\text { P> } \begin{array}{r}\$ 13,200,325 \\
9,900,244 \\
3,300,081\end{array} \\
\gg \frac{\$ 18,240,120}{11,639,957} \\
6,600,163 \\
\gg \frac{\$ 66,001,630}{6,840,414} \\
59,161,216\end{array}$ \\
\hline & $\begin{array}{l}\text { SUBTOTAL INCLUDING ESCALATION } \\
\text { PROJECT CONTINGENCY }\end{array}$ & $98,102,091$ & 0 & > $\$ 98,102,091$ \\
\hline & MANAGEMENT RESERVE- & & & $\$ 6,666,165$ \\
\hline & CONTINGENCY - & & & $\gg \quad \$ 27,631,744$ \\
\hline & TOTAL ESTIMATED COST - & & & $\gg \$ 132,400,000$ \\
\hline
\end{tabular}

\section{PROJECT COST PARAMETERS}

EDI AS A $\%$ OF CONST. + GFE $=20.00 \%$ CONTINGENCY $=34.96 \%$ 


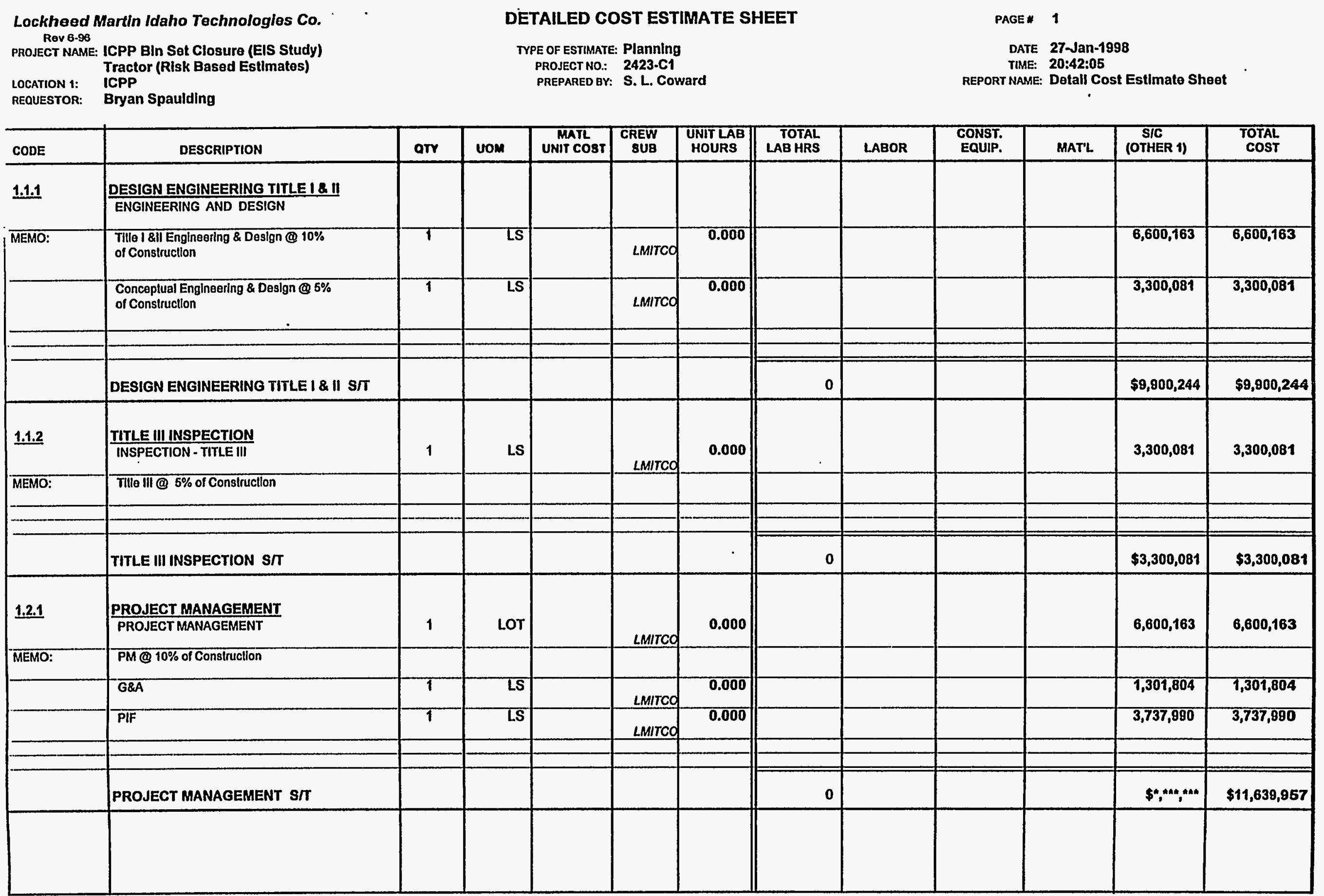


Lockheed Martin Idaho Technologies Co.

Rov 6-96

PROJECT NAME: ICPP Bin Set Closure (EIS Stud Tractor (Risk Based Estimates)

LOCATION 1: REQUESTOR:
icPp

Bryan Spaulding

\section{CONTINGENCY ANALYSIS}

$\begin{aligned} \text { TYPE OF ESTIMATE: } & \text { Planning } \\ \text { PROJECT NO: } & \text { 2423-C1 } \\ \text { PREPARED BY: } & \text { S. L. Coward }\end{aligned}$

DATE: 27-Jan-1998

TMEE: 20:42:00

REPORT NAME: Contingency Analysis

\begin{tabular}{|c|c|c|c|c|c|c|c|c|c|c|c|}
\hline \multicolumn{9}{|c|}{ PROBABLE \% VARIATION } & \multicolumn{2}{|c|}{$\begin{array}{l}\text { PROJECT } \\
\text { CONTINGENCY }\end{array}$} & \multirow{3}{*}{$\begin{array}{l}\text { SUMMARY } \\
\begin{array}{l}\text { Total cost } \\
\text { by Element }\end{array}\end{array}$} \\
\hline \multirow[t]{2}{*}{$\begin{array}{l}\text { WBS } \\
\text { Element }\end{array}$} & \multirow{2}{*}{ Cost Estimate Element } & \multirow{2}{*}{$\begin{array}{l}\text { Total Cost wro } \\
\text { Contingency }\end{array}$} & \multirow[t]{2}{*}{$\begin{array}{l}\text { \% Total } \\
\text { Cost }\end{array}$} & \multicolumn{2}{|c|}{$\begin{array}{l}\text { Prob. \% Var. } \\
\text { From Est. }\end{array}$} & \multicolumn{2}{|c|}{ Wh $\%$ of Prob. } & \multirow{2}{*}{ Contingency } & \multirow{2}{*}{$\%$} & \multirow{2}{*}{ Cost } & \\
\hline & & & & - & + & - & + & & & & \\
\hline 1.1 .1 & DESIGN ENGINEERING TIILE $1 \& \|$ & $9,900,244$ & 10.09 & 10 & 40 & 1.01 & 4.04 & $3.532 \%$ & $10.09 \%$ & $3,461,268$ & $13,361,512$ \\
\hline 1.1 .2 & IITLE III INSPECTION & $3,300,081$ & 3.36 & 10 & 40 & 0.34 & 1.35 & $1.177 \%$ & $3.36 \%$ & $1,153,756$ & $4,453,837$ \\
\hline 121 & PROJECT MANAGEMENT & $11,639,957$ & 11.87 & 10 & 40 & 1.19 & 4.75 & $4.153 \%$ & $11.87 \%$ & $4,069,497$ & $15,709,454$ \\
\hline 1.22 & CONSTRUCTION MANAGEMENT & $6,600,163$ & 6.73 & 10 & 40 & 0.57 & 2.69 & $2355 \%$ & $6.73 \%$ & $2,307,512$ & $8,907,675$ \\
\hline 1.3 .1 & GENERAL CONDITIONS & $6,840,414$ & 6.97 & 10 & 40 & 0.70 & 2.79 & $2440 \%$ & $6.97 \%$ & $2,391,508$ & $9,231,922$ \\
\hline 1.3 .13 & SPECIAL CONSTRUCTION & $59,161,216$ & 60,31 & 10 & 40 & 6.03 & 24.12 & $21.107 \%$ & $60.31 \%$ & $20,683,616$ & $79,844,832$ \\
\hline \multirow[t]{2}{*}{1.5 .2} & PROCUREMENT FEES & 660,016 & 0.67 & 10 & 40 & 0.07 & 0.27 & $0.235 \%$ & $0.67 \%$ & 230,751 & 890,767 \\
\hline & ESCALATION & 0 & 0.00 & 0 & 0 & 0.00 & 0.00 & $0.000 \%$ & $0.00 \%$ & 1 & 1 \\
\hline & SUBTOTAL & $98,102,091$ & 100.00 & & & & & $35.000 \%$ & & & \\
\hline & CALCULATED CONTINGENCY & $34,335,732$ & & & & & & & & & \\
\hline & RESULTANT TEC & $132,437,823$ & & & & & & & & & \\
\hline & ROUNDED TEC & $132,400,000$ & & & & & & & & & \\
\hline & PROSECT CONTINGENCY & $34,297,909$ & & & & & & $34.96 \%$ & & & \\
\hline & MANAGEMENT RESERVE & $6,666,165$ & & & & & & & & & \\
\hline & CONTINGENCY & $27,631,744$ & & & & & & & & & \\
\hline & TOTAL ESTIMATED COST & $132,400,000$ & & & & & & & & $34,297,909$ & $132,400,000$ \\
\hline
\end{tabular}

\section{CONFIDENCE LEVEL AND ASSUMED RISKS:}

The Lockheed Idaho Technologies Co. Cost Estimate Contingency Analysis Model is based on the applied contingency and the assumptions upon which the estimate was predicated. The model is applied with a suggested risk level

of $18 \%$ and a level of confidence of $90 \%$ the estimate will fall within the bid range.

The Contingency Analysis is based on a weighted average to provide a

$90 \%$ probability of underun and a $10 \%$ probability of ovemun.
CONTINGENCY ANALYSIS GUIDE BY TYPE OF ESTIMATE

Guidelines established by DOE/FM 50, Cost Estimating Guide, Vol. 6,

Cost Guide, and as presented in the INEL Cost Estimating Guide. PLANNING $20 \%-30 \%$ ExperimentaVSpecial Conditions............Up to 50\% Conceptual $15 \%-25 \%$ Experimental/Special Conditions.............Up to $40 \%$ TITLE TITLE II TITLE IVAFC
$10 \%-20 \%$ Market Conditions 


\section{G\&APIF ADDER CALCULATION SHEET \\ ICPP BIN SET CLOSURE \\ RISK BASED CLEAN CLOSURE; CLASS C FILL; UNESCALATED \\ CLEAN BINS W/ ROBOTS - FLOOR}

PROCUREMENT FEE:

$\begin{aligned} & \text { CONSTRUCTION }= \\ & \text { GFE }=\end{aligned} \quad \begin{aligned} & \$ 66,001,630 \\ & \text { Subtotal } \\ & \text { FEE @ } 1 \%=\end{aligned} \quad \$ 66,001,630$

G\&A @ 23\% (with a ceiling of $\$ 500,000$ imposed per year, 10 yrs)

CONSTRUCTION OR

CEILING 10 YEARS $=$

$\$ 5,000,000$

GFE =

\$0

PROCUREMENT FEE $=$ $\$ 660,016$

Subtotal $\$ 5,660,016$

FEE @ $23 \%=$

$\$ 5,660,016 \cdot 0.23=\$ 1,301,804$

PIF @ 5.5\%

CONSTRUCTION $=$

GFE =

PROCUREMENT FEE =

$G \& A=$

FEE @ 5.5\%=
$\$ 66,001,630$

$\$ 0$

$\$ 660,016$

$\$ 1,301,804$

$\$ 67,963,450$

Subtotal

$\$ 67,963,450 \cdot 0.055=$

$\$ 3,737,990$

TOTAL PROCUREMENT FEE:

$\$ 660,016$

TOTAL G\&A FEE:

$\$ 1,301,804$

TOTAL PIF:

$\$ 3,737,990$ 
Lockheed Martin Jdaho Technologies Co. Rov, 6.96 PROJECT NAME: ICPP Bin Set Closure (EIS Stud LOCATION 1:

LDUA (Risk Based Estimates) REQUESTOR:
Bryan Spauiding

\section{COST ESTIMATE SUMMARY}

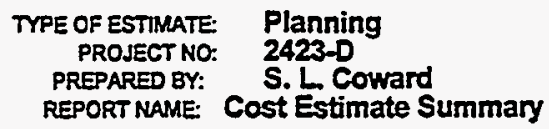

CHECKED BY:

APPRD BY:

\begin{tabular}{|c|c|c|c|c|}
\hline $\begin{array}{l}\text { WBS } \\
\text { Element }\end{array}$ & Cost Estimate Element & $\begin{array}{c}\text { Total } \\
\text { Unéscalated }\end{array}$ & Escalation & $\begin{array}{l}\text { Total } \\
\text { Incl Escalation }\end{array}$ \\
\hline $\begin{array}{l}\frac{1.1}{1.1 .1} \\
1.1 .2 \\
1.2 \\
1.2 .1 \\
1.2 .2 \\
\frac{1.3}{1.3 .1} \\
1.3 .13 \\
1.5 .2\end{array}$ & $\begin{array}{l}\text { ENGINEERING, DESIGN AND INSPECTION } \\
\text { DESIGN ENGINEERING TITLE I \& } \| \\
\text { TITLE III INSPECTION } \\
\text { MANAGEMENT COSTS } \\
\text { PROJECT MANAGEMENT } \\
\text { CONSTRUCTION MANAGEMENT } \\
\text { CONSTRUCTION } \\
\text { GENERAL CONDTIONS } \\
\text { SPECIAL CONSTRUCTION } \\
\text { PROCUREMENT FEES }\end{array}$ & $\begin{array}{r}2,083,513 \\
694,504 \\
3,407,558 \\
2,463,481 \\
\\
1,831,085 \\
12,059,014 \\
138,901\end{array}$ & $\begin{array}{l}0 \\
0\end{array}$ & 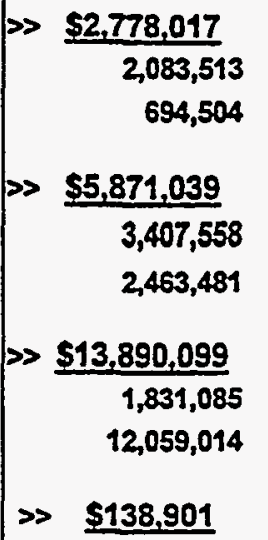 \\
\hline & $\begin{array}{l}\text { SUBTOTAL INCLUDING ESCALATION } \\
\text { PROJECT CONTINGENCY }\end{array}$ & $22,678,056$ & 0 & $\gg \$ \$ 22,678,056$ \\
\hline & MANAGEMENT RESERVE- & & & $\$ 1,402,900$ \\
\hline & CONTINGENCY- & & & $\$ 6,519,044$ \\
\hline & TOTAL ESTIMATED COST - & & & $\$ 30,600,000$ \\
\hline
\end{tabular}

\section{PROJECT COST PARAMETERS}

EDI AS A $\%$ OF CONST. + GFE $=20.00 \%$ 
Lockheed Martin Idaho Technologles Co.

$$
\text { Rev } 6.96
$$

PROJECT NAME: ICPP Bin Set Closure (EIS Study)

LDUA (RIsk Based Estimates)

LOCATION i: ICPP

REQUESTOR: Bryan Spaulding
DETAILED COST ESTIMATE SHEET

TYPE OF ESTIMATE: Planning

PROJECTNO:: 2423-D

PREPARED BY: S. L. Coward
PAGE* 1

DATE 27-Jan-1998

TIME: 20:47:35

Report name: Detall Cost Estimate Sheot

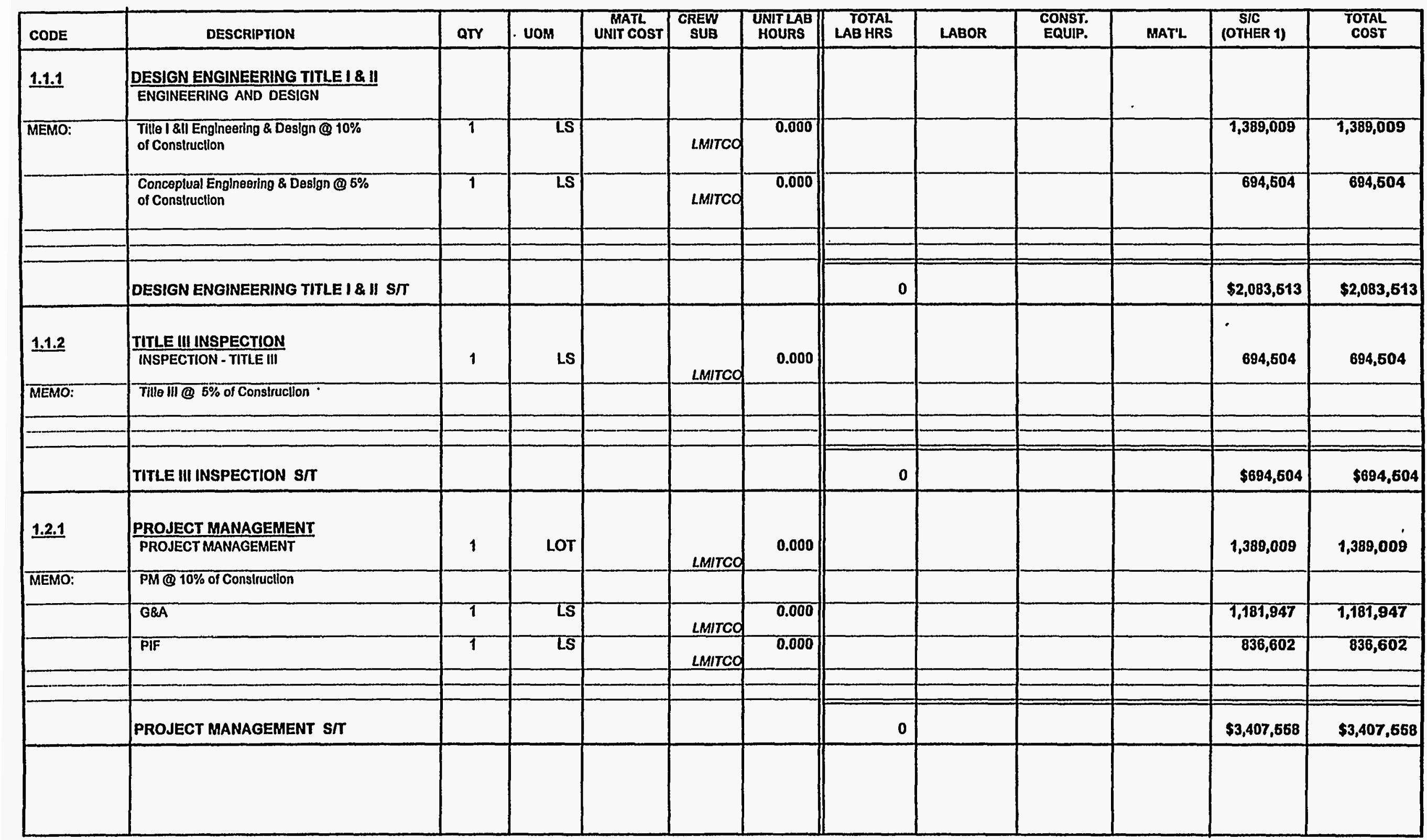


Lockhoed Martin Idaho Technologies Co. Rov 6.96

PROJECT NAME: ICPP Bin Set Closuro (EIS Study) LDUA (Risk Based Estimates)

ICPP

$\begin{array}{ll}\text { LOCATION 1: } & \text { ICPP } \\ \text { REQUESTOR: } & \text { Bryan Spaulding }\end{array}$
DETAILED COST ESTIMATE SHEET

TYPE Of estimate: Planning

PROJECT NO:: 2423-D

PREPARED BY: S. L. Coward
PAGE \# 2

DATE 27-Jan-1998

TIME: 20:47:35

REPORT NAME: Detall Cost Estimate Sheot

\begin{tabular}{|c|c|c|c|c|c|c|c|c|c|c|c|c|}
\hline CODE & DESCRIPTION & aTr & Uom & $\begin{array}{l}\text { MATL } \\
\text { UNIT COST }\end{array}$ & $\begin{array}{l}\text { CREW } \\
\text { SUB }\end{array}$ & $\begin{array}{l}\text { UNITLAB } \\
\text { HOURS }\end{array}$ & $\begin{array}{l}\text { TOTAL } \\
\text { LAB HRS }\end{array}$ & LABOR & $\begin{array}{l}\text { CONST. } \\
\text { EQUIP. }\end{array}$ & MAT'L & $\begin{array}{c}\text { S/C } \\
\text { (OTHER 1) }\end{array}$ & $\begin{array}{l}\text { TOTAL } \\
\text { COST }\end{array}$ \\
\hline 1.2 .2 & $\frac{\text { CONSTRUCTION MANAGEIMENT }}{\text { CONSTRUCTION MANAGEMENT }}$ & 1 & LOT & & LMITCO & 0.000 & & & & & $2,463,481$ & $2,463,481$ \\
\hline MEMO: & CM @ $10 \%$ of Constructlon Costs & & & • & & & & & & & & \\
\hline & & & & & . & & & & & & & \\
\hline & CONSTRUCTION MANAGEMENT S $/ T$ & & & & & & $\mathbf{0}$ & . & & & $\$ 2,463,481$ & $\$ 2,463,481$ \\
\hline$\underline{1.3 .1}$ & $\begin{array}{l}\text { GENERAL CONDITIONS } \\
\text { SUPERVISION (Assume Share wilh PIplng } \\
\text { EsI. (10 Yrs/2) - Duratlon of } \\
\text { Schedule) }\end{array}$ & 1 & FTE & & $\begin{array}{l}\text { PIPF } \\
\text { GEN }\end{array}$ & 10400.0 & 10,400 & 419,848 & & & & 418,848 \\
\hline & TRAINING & 8 & FTE & & $\begin{array}{r}\text { SKWK } \\
\text { GEN }\end{array}$ & 165.000 & 1,320 & 43,706 & & & & 43,705 \\
\hline & $\begin{array}{l}\text { RADCON TECHNICIAN SUPPORT (Assume } \\
\text { Share wilh PIping Est (10 YIs/2) - } \\
\text { Duratlon) }\end{array}$ & 2 & FTE & & $\begin{array}{l}\text { Z-1342 } \\
\text { LMITCO }\end{array}$ & 10400.0 & 20,800 & $1,027,620$ & & & & $1,027,520$ \\
\hline & & & & & & & & & & & & \\
\hline & GENERAL CONDITIONS ST & & & & & & 32,520 & $\$ 1,491,073$ & & & & $\$ 1,481,073$ \\
\hline 1.3 .13 & SPECIAL CONSTRUCTION & & & & & & & & & & & \\
\hline & DESIGN AND DEVELOPMENT OF LDUA & & & & & & & & & & & \\
\hline & $\begin{array}{l}\text { Deslgn and Fabricale trst LOUA, } \\
\text { including: }\end{array}$ & 1 & EA & & GEN & 0.000 & & & & & $6,000,000$ & $6,000,000$ \\
\hline & $\begin{array}{l}\text { Deslgn, Approvals, Mock-up, Proof of } \\
\text { Process, otc. }\end{array}$ & & & & & & & & & & & \\
\hline & Fabrication of Add"l Units & 6 & EA & & $C r$ & 0.000 & & & & & $2,650,000$ & $2,560,000$ \\
\hline & & & & & & & & & & & & \\
\hline
\end{tabular}


Lockheod Martin Idaho Technologies Co. Rov 6.96 PROJECT NAME: ICPP Bin Set Closure (EIS Study) LOCATION 1: LDUA LDUA (RIsk Based Estlmates)

REQUESTOR: Bryan Spaulding
DETAILED COST ESTIMATE SHEET

TYPE OF ESTIMATE: Planning

PROJECT NO:: 2423-D

PREPARED BY: S. L. Coward
Page * 3

DATE 27 Jan-1998

TIME: 20:47:35 REPORT NAME: Detall Cost Estimate Sheat

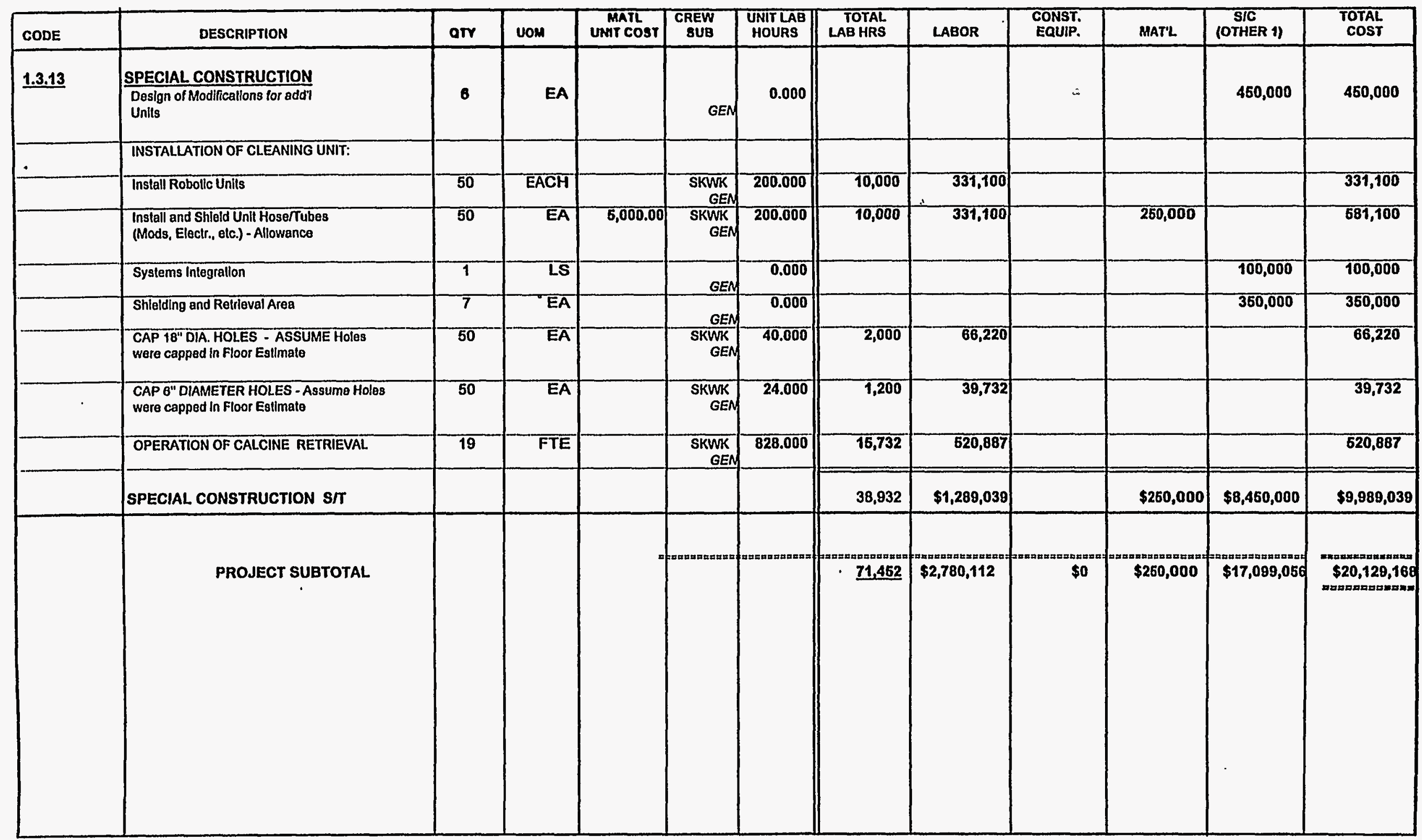


Lockheed Martin Jdaho Technologies Co.

Rev 6-95

PROJECT NAME: ICPP Bin Set Closure (EIS Stud

LOCATION 1: ICPP

REQUESTOR: Bryan Spaulding
CONTINGENCY ANALYSIS

$\begin{array}{cl}\text { TYPE OF ESTIMATE: } & \text { Planning } \\ \text { PROJECT NO: } & 2423-D \\ \text { PREPARED BY: } & \text { S.L. Coward }\end{array}$

DATE: 27 Jan-1998

TIME: 20:47:30

REPORT NAME: Contingency Analysis

\begin{tabular}{|c|c|c|c|c|c|c|c|c|c|c|c|}
\hline \multirow{3}{*}{$\begin{array}{l}\text { WBS } \\
\text { Eement }\end{array}$} & \multicolumn{8}{|c|}{ PROBABLE \% VARIATION } & \multicolumn{2}{|c|}{$\begin{array}{l}\text { PROJECT } \\
\text { CONTINGENCY }\end{array}$} & \multirow{3}{*}{$\begin{array}{l}\text { SUMMARY } \\
\text { Total Cost } \\
\text { by Element }\end{array}$} \\
\hline & \multirow{2}{*}{ Cost Estimato Element } & \multirow{2}{*}{$\begin{array}{l}\text { Total Cost w/o } \\
\text { Contingency }\end{array}$} & \multirow{2}{*}{$\begin{array}{l}\text { \% Total } \\
\text { Cost }\end{array}$} & \multicolumn{2}{|c|}{$\begin{array}{l}\text { Prob. \% Var. } \\
\text { From Est. }\end{array}$} & \multicolumn{2}{|c|}{ Wt $\%$ of Prob. } & \multirow{2}{*}{ Contingency } & \multirow{2}{*}{$\%$} & \multirow{2}{*}{ Cost } & \\
\hline & & & & - & \pm & - & + & & & & \\
\hline 1.1 .1 & DESIGN ENGINEERING TTTLE $\& \|$ & $2,083,513$ & 9.19 & 10 & 40 & 0.92 & 3.67 & $3.216 \%$ & $9.19 \%$ & 727,817 & $2,811,330$ \\
\hline 1.1 .2 & TITLE III INSPECTION & 694,504 & 3.06 & 10 & 40 & 0.31 & 122 & $1.072 \%$ & $3.06 \%$ & 242,606 & 937,110 \\
\hline 1.2 .1 & PROJECT MANAGEMENT & $3,407,558$ & 15.03 & 10 & 40 & 1.50 & 6.01 & $5.259 \%$ & $15.03 \%$ & $1,190,335$ & $4,597,893$ \\
\hline 1.22 & CONSTRUCTION MANAGEMENT & $2,463,481$ & 10.86 & 10 & 40 & 1.09 & 4.35 & $3.802 \%$ & $10.85 \%$ & 860,548 & $3,324,029$ \\
\hline 1.3 .1 & GENERAL CONDITONS & $1,831,085$ & 8.07 & 10 & 40 & 0.81 & 323 & $2826 \%$ & $8.07 \%$ & 639,638 & $2,470,723$ \\
\hline 1.3 .13 & SPECLAL CONSTRUCTION & $12,059,014$ & 53.17 & 10 & 40 & 5.32 & 21.27 & $18.611 \%$ & $53.17 \%$ & $4,212,479$ & $16,271,493$ \\
\hline \multirow[t]{2}{*}{1.5 .2} & PROCUREMENT FEES & 138,901 & 0.61 & 10 & 40 & 0.06 & 0.24 & $0.214 \%$ & $0.61 \%$ & 48,521 & 187,422 \\
\hline & ESCALATION & 0 & 0.00 & 0 & 0 & 0.00 & 0.00 & $0.000 \%$ & $0.00 \%$ & 0 & 0 \\
\hline & SUBTOTAL & $22,678,056$ & 100.00 & & & & & $35.000 \%$ & & & \\
\hline & CALCULATED CONTINGENCY & $7,937,320$ & & & & & & & & & \\
\hline & RESULTANT TEC & $30,615,376$ & & & & & & & & & \\
\hline & ROUNDED TEC & $30,600,000$ & & & & & & & & & \\
\hline & PROJECT CONTINGENCY & $7,921,944$ & & & & & & $34.93 \%$ & & & \\
\hline & MANAGEMENT RESERVE & $1,402,900$ & & & & & & & & & \\
\hline & CONTINGENCY & $6,519,044$ & & & & & & & & & \\
\hline & TOTAL ESTIMATED COST & $30,600,000$ & & & & & & & & $7,921,944$ & $30,600,000$ \\
\hline
\end{tabular}

CONFIDENCE LEVEL AND ASSUMED RISKS:

The Lockheed Idaho Technologies Co. Cost Estimate Contingency Analysis Model is based on the applied contingency and the assumptions upon which the estimate was predicated. The model is applied with a suggested risk level of $18 \%$ and a level of confidence of $90 \%$ the estimate will fall within the bid range. The Contingency Analysis is based on a weighted average to provide a 90 \% probability of underrun and a $10 \%$ probability of overnu.
CONTINGENCY ANALYSIS GUIDE BY TYPE OF ESTIMATE

Guidelines established by DOE FM 50 . Cost Estimating Guide, Vol. 6 ,

Cost Guide, and as presented in the INEL Cost Estimating Guide.
PLANNING
ExperimentalSpecial Conditions
$20 \%-30 \%$
Conceptual ...Up to $50 \%$
Exper
TITLE I
TITLE IUAFC
$15 \%-25 \%$
.....Up to $40 \%$
$10 \%-20 \%$
$5 \%-15 \%$
Market Conditions 


\section{G\&APIF ADDER CALCULATION SHEET ICPP BIN SET CLOSURE \\ RISK BASED CLEAN CLOSURE; CLASS C FILL; UNESCALATED \\ CLEAN BINS W/ ROBOTS - WALLS}

PROCUREMENT FEE: CONSTRUCTION $=$
GFE $=$

FEE @ 1\%=
$\$ 13,890,099$

Subtotal

$\$ 13,890,099$

$\$ 13,890,099 * 0.01=$

G\&A@ $@ 23 \%$ (with a ceiling of $\$ 500,000$ imposed per year, 10 yrs)

CONSTRUCTION OR

CEILING * 10 YEARS =

GFE =

$\$ 5,000,000$

PROCUREMENT FEE =

$$
\$ 138,901
$$

Subtotal

$\$ 138,901$

FEE @ 23\% =

$\$ 5,138,901 \cdot 0.23=$

$\$ 1,181,947$

PIF@5.5\%

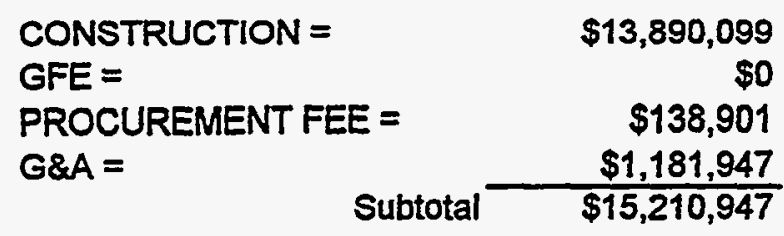

FEE @ $5.5 \%=$

$\$ 15,210,947 * 0.055=$

$\$ 836,602$

TOTAL PROCUREMENT FEE:

$\$ 138,901$

TOTAL G\&A FEE:

$\$ 1,181,947$

TOTAL PIF:

$\$ 836,602$ 
Lockheed Martin Idaho Technologies Co. Rov.6-96

PROJECT MAME: ICPP Bin Set Closure (EIS Stud Pipe Crawier (Risk Based Estim

LOCATION 1: ICPP

REQUESTOR: Bryan Spaulding
COST ESTIMATE SUMMARY

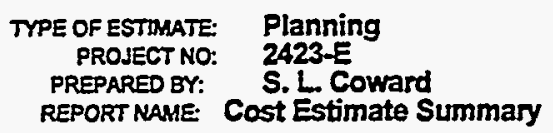

APPRD BY:

\begin{tabular}{|c|c|c|c|c|}
\hline $\begin{array}{l}\text { WBS } \\
\text { Element }\end{array}$ & Cost Estimate Element & $\begin{array}{c}\text { Total } \\
\text { Unescalated }\end{array}$ & Escalation & $\begin{array}{c}\text { Total } \\
\text { Incl Escalation }\end{array}$ \\
\hline $\begin{array}{l}\frac{1.1}{1.1 .1} \\
1.1 .2 \\
\frac{1.2}{1.2 .1} \\
1.2 .2 \\
\frac{1.3}{1.3 .1} \\
1.3 .13 \\
1.5 .2\end{array}$ & $\begin{array}{l}\text { ENGINEERING, DESIGN AND INSPECTION } \\
\text { DESIGN ENGINEERING } \\
\text { TITE III INSPECTION } \\
\text { MANAGEMENT COSTS } \\
\text { PROJECT MANAGEMENT } \\
\text { CONSTRUCTION MANAGEMENT } \\
\text { CONSTRUCTION } \\
\text { GENERAL CONDITIONS } \\
\text { SPECIAL CONSTRUCTION } \\
\text { PROCUREMENT FEES }\end{array}$ & $\begin{array}{r}889,168 \\
296,389 \\
2,149,702 \\
592,779 \\
\\
1,831,085 \\
4,096,709 \\
59,278\end{array}$ & $\begin{array}{l}0 \\
0 \\
0 \\
0\end{array}$ & $\begin{array}{r}\text { P1,185,557 } \\
889,168 \\
296,389\end{array}$ \\
\hline & $\begin{array}{l}\text { SUBTOTAL INCLUDING ESCALATION } \\
\text { PROJECT CONTINGENCY }\end{array}$ & $9,915,110$ & 0 & $\gg \$ 9,915,110$ \\
\hline & MANAGEMENT RESERVE- & & & $\$ 598,707$ \\
\hline & CONTINGENCY - & & & $\$ 2,886,183$ \\
\hline & TOTAL ESTIMATED COST & & & $\gg \quad \$ 13,400,000$ \\
\hline
\end{tabular}

\section{PROJECT COST PARAMETERS}

EDI AS A $\%$ OF CONST. + GFE $=20.00 \%$ 
Lockheed Martin Idaho Technologles Co.

$$
\text { Rev 6-96 }
$$

PROJECt NAME: ICPP Bin Set Closure (EIS Study) Pipe Crawler (Risk Based Estlmates) ICPP

LOCATION 1:

REQUESTOR:

Bryan Spaulding

\section{DETAILED COST ESTIMATE SHEET}

TYPE Of estimate: Planning

PROJECT NO:: 2423-E

PREPARED BY: S. L. Coward
PAGE* 1

DATE 27-Jan-1998

TIME: 20:52:35

REPORT NAME: Detall Cost Estimate Shoot

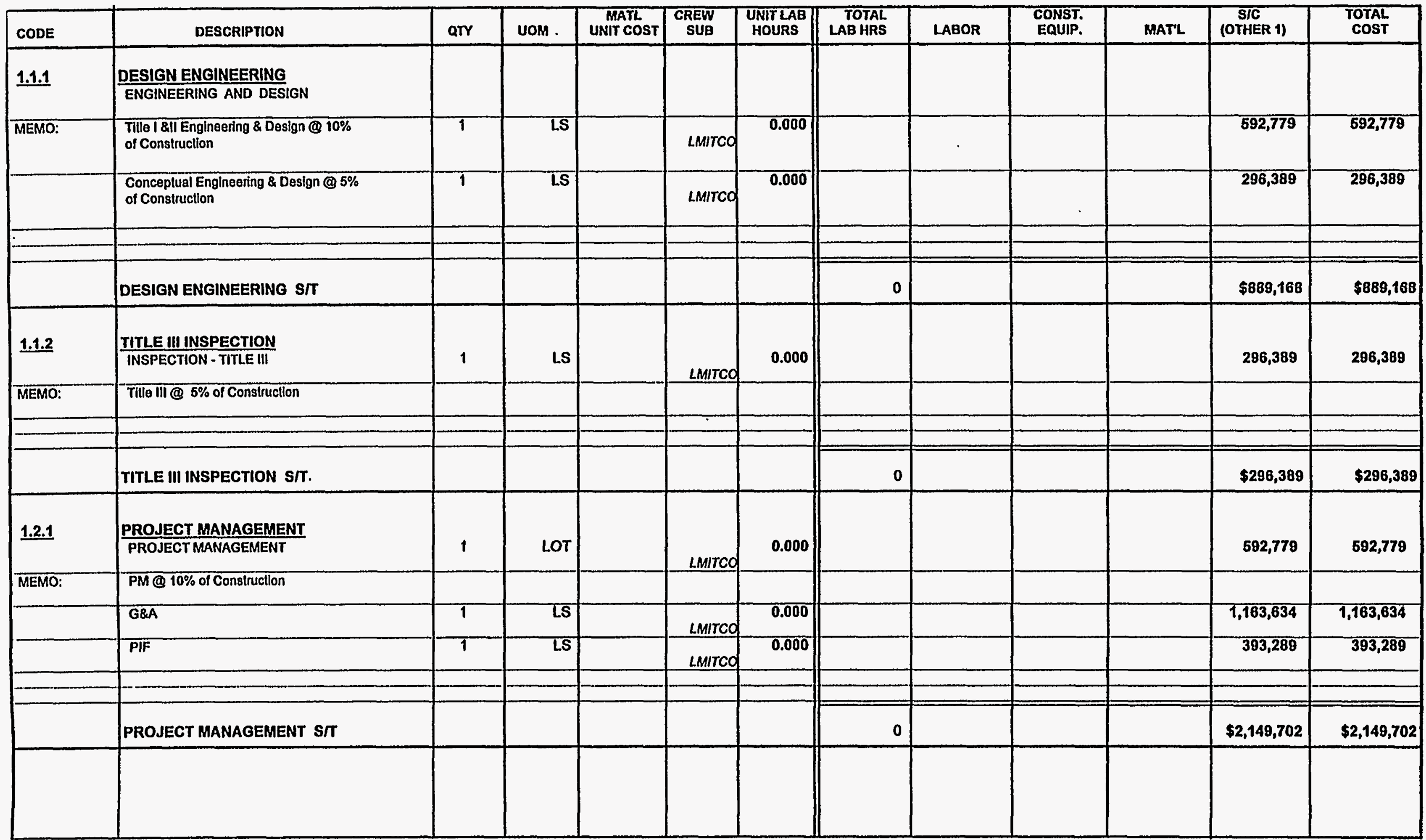


Lockhoed Martin Idaho Technologles Co.

Rev 8.96

JROJECT NAME: ICPP Bin Sot Closure (EIS Study)

Pipe Crawler (Risk Based Estimates)

ICPP

OCATION 1:

Bryan Spaulding

\section{DETAILED COST ESTIMATE SHEET}

TYPE OF Estimate: Planning

PROJECT NO.: 2423-E

PREPARED BY: S.L. Coward
PAGE 2

DATE 27-Jan-1998

TIME: 20:62:36

REPORT NAME: Detall Cost Estimate Sheot

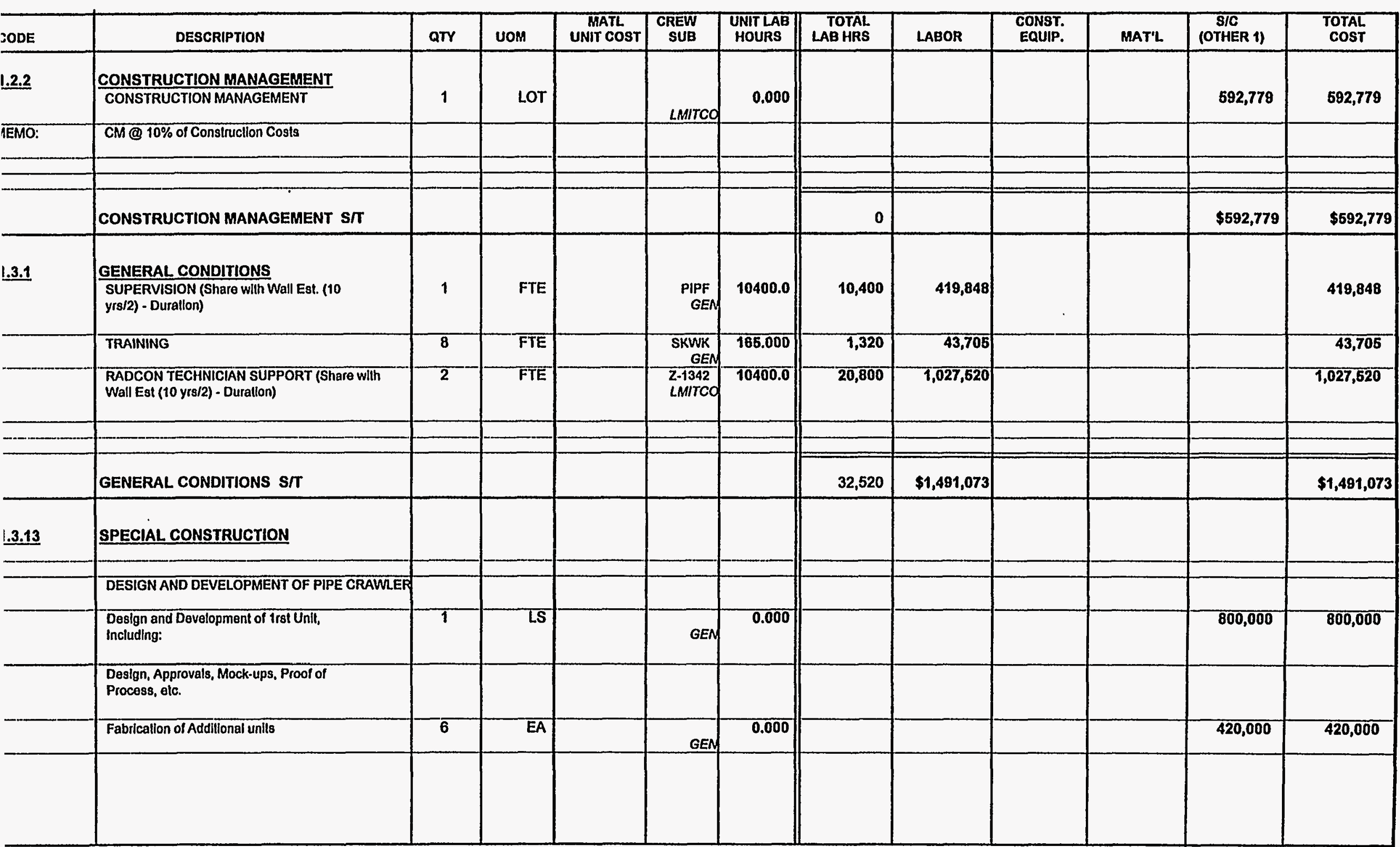


Lockheed Martin Idaho Technologies Co.

PROJECT NAME: ICPP Bin Sot Closure (EIS Stud

LOCATION 1:

REQUESTOR:
Pipe Crawler (Risk Based Estim icPp

Bryan Spaulding
CONTINGENCY ANALYSIS

$\begin{array}{cl}\text { TYPE OF ESTIMATE: } & \text { Planning } \\ \text { PROJECT NO: } & \text { 2423-E } \\ \text { PREPARED BY: } & \text { S. L. Coward }\end{array}$

DATE: 27-Jan-1998

TIME: 20:52:30

\begin{tabular}{|c|c|c|c|c|c|c|c|c|c|c|c|}
\hline \multicolumn{9}{|c|}{ PROBABLE \% VARIATION } & \multicolumn{2}{|c|}{$\begin{array}{l}\text { PROJECT } \\
\text { CONTINGENCY }\end{array}$} & \multirow{3}{*}{$\begin{array}{l}\text { SUMMARY } \\
\begin{array}{l}\text { Total Cost } \\
\text { by Element }\end{array}\end{array}$} \\
\hline \multirow{2}{*}{$\begin{array}{l}\text { WBS } \\
\text { Element }\end{array}$} & \multirow{2}{*}{ Cost Estimate Element } & \multirow{2}{*}{$\begin{array}{l}\text { Total Cost w/o } \\
\text { Contingency }\end{array}$} & \multirow[t]{2}{*}{$\begin{array}{l}\text { \% Total } \\
\text { Cost }\end{array}$} & \multicolumn{2}{|c|}{$\begin{array}{l}\text { Prob. \% Var. } \\
\text { From Est. }\end{array}$} & \multicolumn{2}{|c|}{ Wt \% of Prob. } & \multirow{2}{*}{ Contingency } & \multirow{2}{*}{$\%$} & \multirow{2}{*}{ Cost } & \\
\hline & & & & 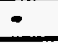 & + & - & + & & & & \\
\hline 1.1.1 & DESIGN ENGINEERING & 889,168 & 8.97 & 10 & 40 & 0.90 & 3.59 & $3.139 \%$ & $8.97 \%$ & 312,518 & $1,201,686$ \\
\hline 1.12 & TITE III INSPECTION & 296,389 & 299 & 10 & 40 & 0.30 & 120 & $1.046 \%$ & $299 \%$ & 104,173 & 400,562 \\
\hline 1.2 .1 & PROJECT MANAGEMENT & $2,149,702$ & 21.68 & 10 & 40 & 217 & 8.67 & $7.588 \%$ & $21.68 \%$ & 755,561 & $2,905,263$ \\
\hline 1.22 & CONSTRUCTION MANAGEMENT & 592,779 & 5.98 & 10 & 40 & 0.60 & 239 & $2.092 \%$ & $5.98 \%$ & 208,346 & 801,125 \\
\hline 1.3 .1 & GENERAL CONDITIONS & $1,831,085$ & 18.47 & 10 & 40 & 1.85 & 7.39 & $6.464 \%$ & $18.47 \%$ & 643,576 & $2,474,661$ \\
\hline 1.3 .13 & SPECLAL CONSTRUCTION & $4,096,709$ & 41.32 & 10 & 40 & 4.13 & 16.53 & $14.461 \%$ & $41.32 \%$ & $1,439,889$ & $5,536,590$ \\
\hline \multirow[t]{2}{*}{1.5 .2} & PROCUREMENT FEES & 59,278 & 0.60 & 10 & 40 & 0.06 & 0.24 & $0.209 \%$ & $0.50 \%$ & 20,835 & 80,113 \\
\hline & ESCALATION & 0 & 0.00 & 0 & 0 & 0.00 & 0.00 & $0.000 \%$ & $0.00 \%$ & 0 & 0 \\
\hline & SUBTOTAL & $9,915,110$ & 100.00 & & & & & $35.000 \%$ & & & \\
\hline & CALCULATED CONTINGENCY & $3,470,289$ & & & & & & & & & \\
\hline & RESULTANT TEC & $13,385,399$ & & & & & & & & & \\
\hline & ROUNDED TEC & $13,400,000$ & & & & & & & & & \\
\hline & PROJECT CONTINGENCY & $3,484,890$ & & & & & & $35.15 \%$ & & & \\
\hline & MANAGEMENT RESERVE & 598,707 & & & & & & & & & \\
\hline & CONTINGENCY & $2,886,183$ & & & & & & & & & \\
\hline & TOTAL ESTIMATED COST & $13,400,000$ & & & & & & & & $3,484,890$ & $13,400,000$ \\
\hline
\end{tabular}

CONFIDENCE LEVEL AND ASSUMED RISKS:

The Lockheed Idaho Technologies Co. Cost Estimate Contingency Analysis Model is based on the applied contingency and the assumptions upon which

the estimate was predicated. The model is applied with a suggested risk level

of $18 \%$ and a level of confidence of $90 \%$ the estimate will fall within the bid range.

The Contingency Analysis is based on a weighted average to provide a

$90 \%$ probability of underrun and a $10 \%$ probability of ovemun.
CONTINGENCY ANALYSIS GUIDE BY TYPE OF ESTIMATE

Guidelines established by DOE/FM 50, Cost Estimating Guide, Vol. 6 ,

Cost Guide, and as presented in the INEL Cost Estimating Guide.

PLANNING

Experimental/Special Conditions.

Conceptual

Experimentalspecial Conditions............. 15 p to $40 \%$

TITLE

TTIL U

TITLE IUAFC
$10 \%-20 \%$

$5 \%-15 \%$

Market Conditions 


\section{G\&A/PIF ADDER CALCULATION SHEET ICPP BIN SET CLOSURE}

RISK BASED CLEAN CLOSURE; CLASS C FILL; UNESCALATED

CLEAN BINS W/ ROBOTS - PIPING

PROCUREMENT FEE:

CONSTRUCTION $=$

$\$ 5,927,794$

GFE =

Subtotal $\longdiv { \$ 5 , 9 2 7 , 7 9 4 }$

FEE @ 1\%=

$\$ 5,927,794 * 0.01=$

G\&A @ 23\% (with a ceiling of $\$ 500,000$ imposed per year, 10 yrs)

CONSTRUCTION OR

CEILING 10 YEARS =

$\$ 5,000,000$

GFE =

$\$ 0$

PROCUREMENT FEE $=$

$\$ 59,278$

Subtotal $\$ 5,059,278$

FEE @ $23 \%=$

$\$ 5,059,278 \cdot 0.23=$

$\$ 1,163,634$

PIF @ 5.5\%

$$
\begin{aligned}
& \text { CONSTRUCTION = } \\
& \text { GFE = } \\
& \text { PROCUREMENT FEE = } \\
& \text { G\&A }=
\end{aligned}
$$

FEE @ $5.5 \%=$
$\$ 5,927,794$

$\$ 0$

$\$ 59,278$

$\$ 1,163,634$

Subtotal

$\$ 393,289$

$\$ 7,150,706 \cdot 0.055=$

$\$ 59,278$

TOTAL PROCUREMENT FEE:

$\$ 1,163,634$

TOTAL G\&A FEE:

$\$ 393,289$ 
Lockheed Martin Idaho Technologies Co. Rov. 6-96 PROJECT NAME: ICPP Bin Set Closure (EIS Stud Place NRC Class C Grout LOCATION1: ICPP REQUESTOR: Bryan Spaulding
COST ESTIMATE SUMMARY

TYPE OF ESTIMATE: Planning

PROJECT NO: 2423-B

PREPARED BY: S. L Coward

REPORT NAME: Cost Estimate Summary

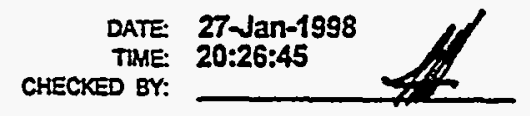

APPRD BY:

\begin{tabular}{|c|c|c|c|c|}
\hline $\begin{array}{l}\text { WBS } \\
\text { Element }\end{array}$ & Cost Estimate Element & $\begin{array}{c}\text { Total } \\
\text { Unescalated }\end{array}$ & Escalation & $\begin{array}{c}\text { Total } \\
\text { Incl Escalation } \\
\end{array}$ \\
\hline $\begin{array}{l}\frac{1.1}{1.1 .1} \\
1.1 .2 \\
1.2 \\
1.2 .1 \\
1.2 .2 \\
1.3 \\
1.3 .1 \\
1.3 .2 \\
1.3 .3 \\
1.3 .15 \\
1.3 .16 \\
1.5 .2\end{array}$ & $\begin{array}{l}\text { ENGINEERING, DESIGN AND INSPECTION } \\
\text { DESIGN ENGINEERING TITLE I \& II } \\
\text { TITLE III INSPECTION } \\
\text { MANAGEMENT COSTS } \\
\text { PROJECT MANAGEMENT } \\
\text { CONSTRUCTION MANAGEMENT } \\
\text { CONSTRUCTION } \\
\text { GENERAL CONDITIONS } \\
\text { STEWORK } \\
\text { CONCRETE } \\
\text { MECHANICAL } \\
\text { ELECTRICAL } \\
\text { PROCUREMENT FEES }\end{array}$ & $\begin{array}{r}3,282,538 \\
656,507 \\
\\
2,923,531 \\
1,301,490 \\
\\
3,437,150 \\
0 \\
3,876,603 \\
3,365,584 \\
2,450,822 \\
131,302\end{array}$ & $\begin{array}{l}0 \\
0 \\
0 \\
0 \\
0\end{array}$ & $\begin{array}{r}\text { >> } \begin{array}{r}\$ 3,939,045 \\
3,282,538 \\
656,507\end{array} \\
>>\frac{\$ 4,225,021}{2,923,531} \\
1,301,490 \\
>>\$ 13,130,159 \\
3,437,150 \\
0 \\
3,876,603 \\
3,365,584 \\
2,450,822\end{array}$ \\
\hline & $\begin{array}{l}\text { SUBTOTAL INCLUDING ESCALATION } \\
\text { PROJECT CONTINGENCY }\end{array}$ & $21,425,527$ & 0 & $\gg \$ 21,425,527$ \\
\hline & MANAGEMENT RESERVE- & & & $\$ 1,326,146$ \\
\hline & CONTINGENCY - & & & $\$ 7,348,327$ \\
\hline & TOTAL ESTIMATED COST - & & & $\gg \quad \$ 30,100,000$ \\
\hline
\end{tabular}

\section{PROJECT COST PARAMETERS}

EDI AS A \% OF CONST. + GFE $=30.00 \%$

CONTINGENCY $=40.49 \%$ 


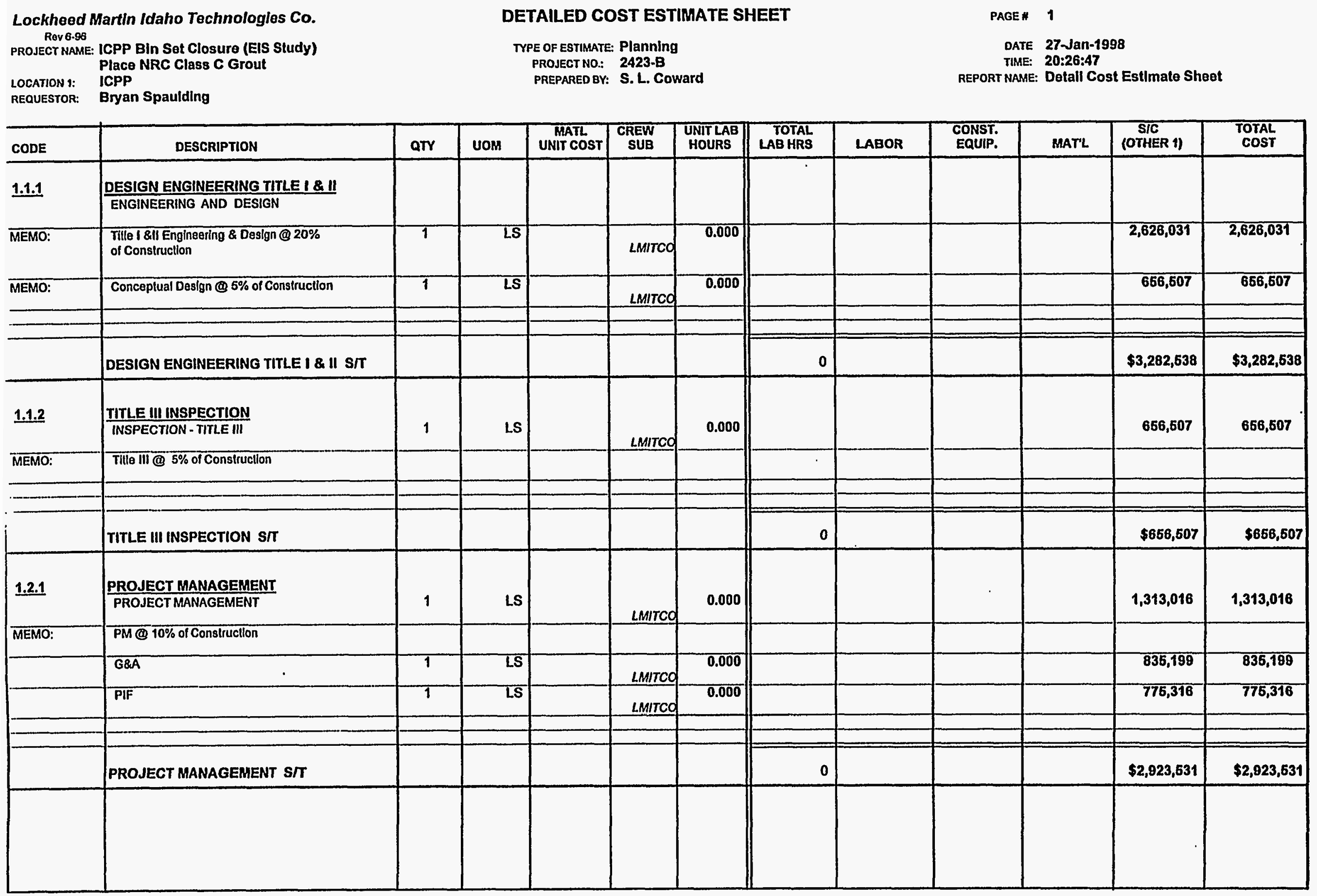


Lockhoed Martin Idaho Technologies Co.

$$
\text { Rov } 6.86
$$

PROJECT NAME: ICPP Bin Sot Closuro (EIS Study)

Place NRC Class C Grout

LOCATION 1:

ZEQUESTOR:

ICPP

Bryan Spaulding

\section{DETAILED COST ESTIMATE SHEET}

TYPE OF ESTIMATE: Plannlng

PROJECT NO.: 2423-B

PREPARED BY: S. L. Coward
PAGE \# 2

DATE 27-Jan-1998

TIME; 20:26:47

REPORT NAME: Detall Cost Estimate Sheot

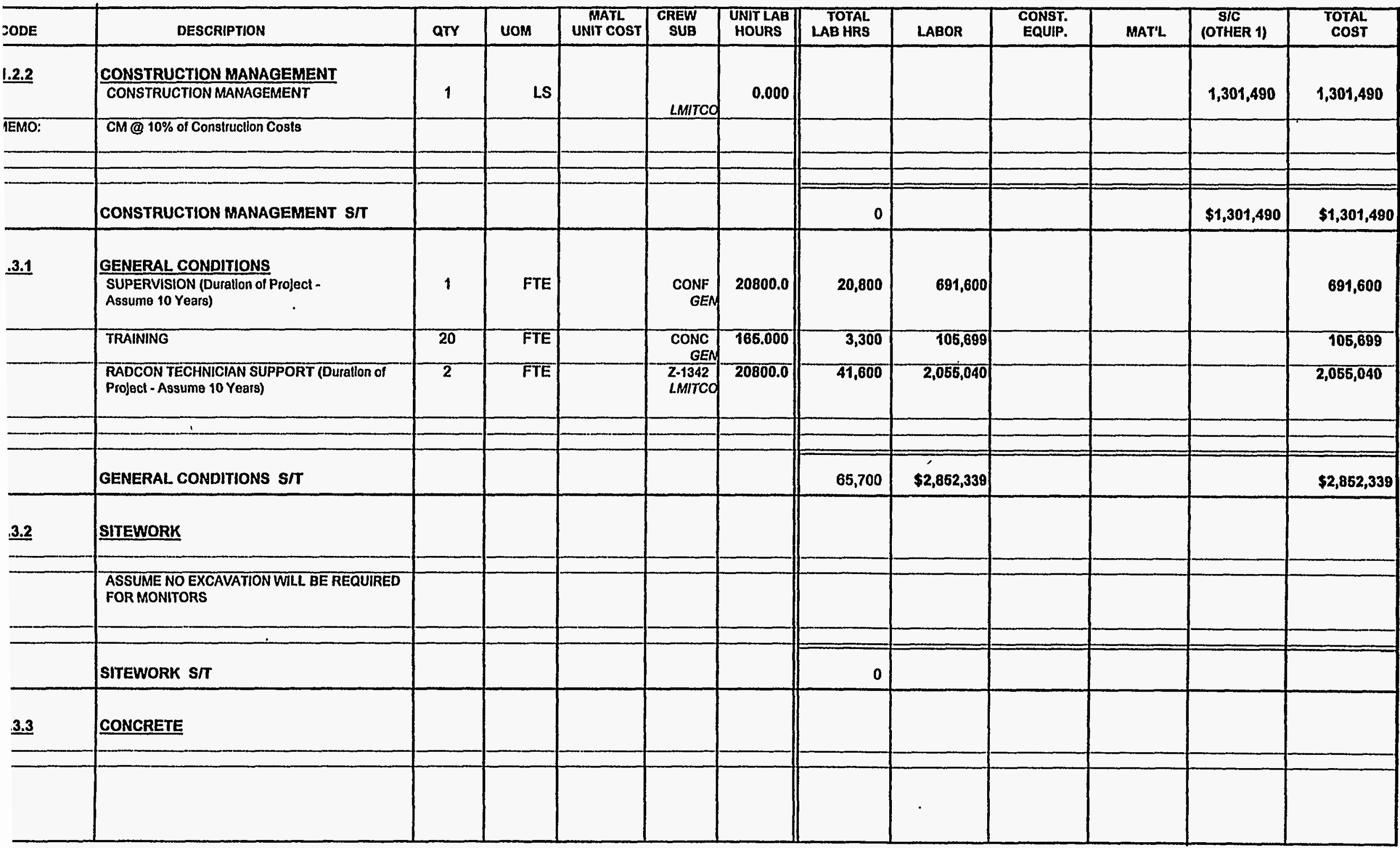


Lockheed Martin Idaho Technologies Co. Rev 6.96

PROJECT NAME: ICPP BIn Set Closure (EIS Study) Place NRC Class C Grout

ICPP

REQUESTOR: Bryan Spaulding

\section{DETAILED COST ESTIMATE SHEET}

TYPE OF Estimate: Planning

PROJECTNO: 2423-B

PREPARED BY: S. L. Coward
PAGE* 3

DATE 27-Jan-1998

TIME: 20:26:47

REPORT NAME: Dotall Cost Estimate Shoot

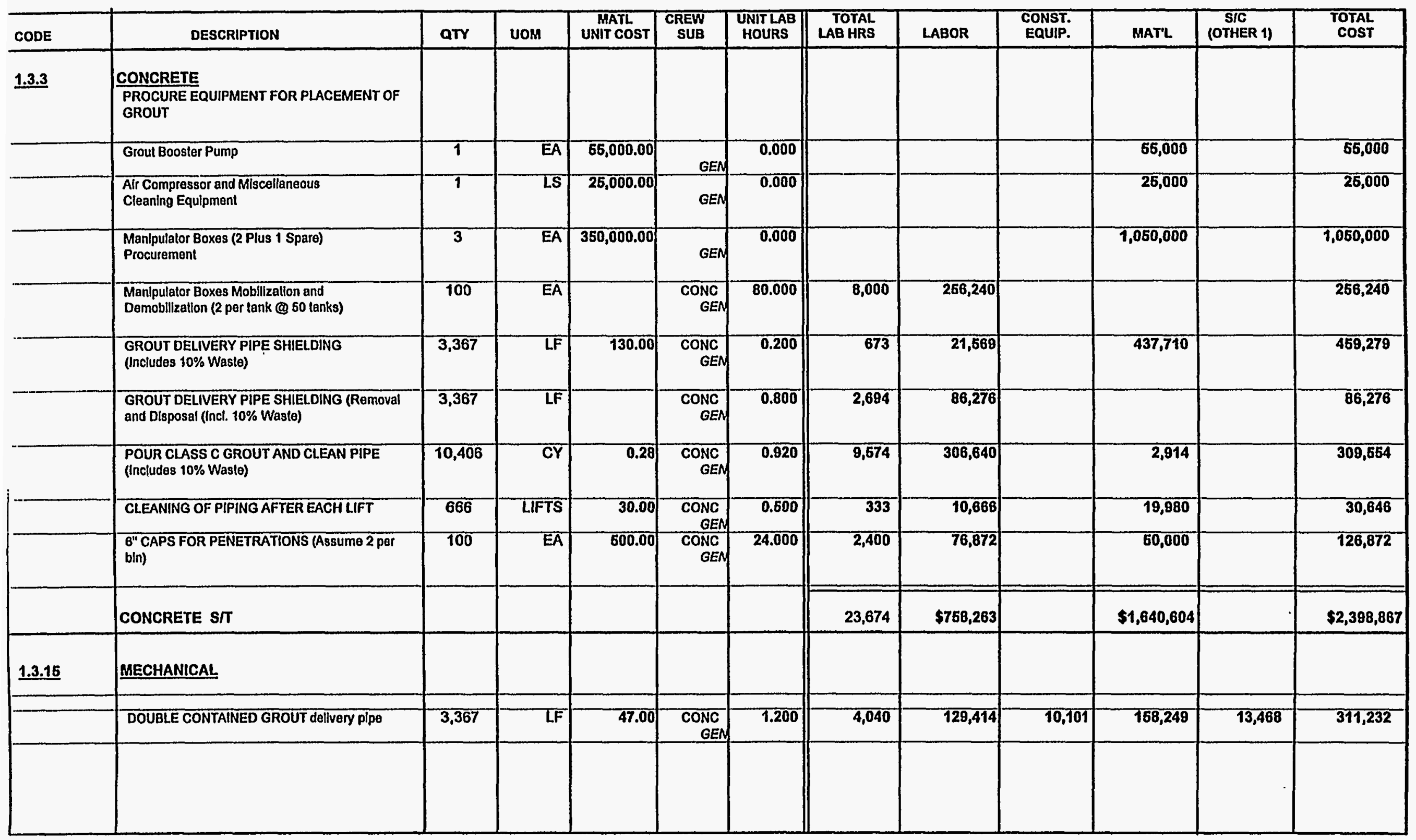


Lockheed Martin Idaho Technologies Co.

Rov 6.96
PROJECT NAME: ICPP Bin Sot Closure (EIS Study) Place NRC Class C Grout

LOCATION 1: ICPP

REQUESTOR: Bryan Spaulding

\section{DETAILED COST ESTIMATE SHEET}

TYPE OF EstiMATE: Planning

PROJECT NO: 2423-B

PREPARED BY: S. L. Coward
PAGE \# 5

DATE 27-Jan-1998

TIME: 20:26:47

REPORT NAME: Dotall Cost Estlmate Sheot

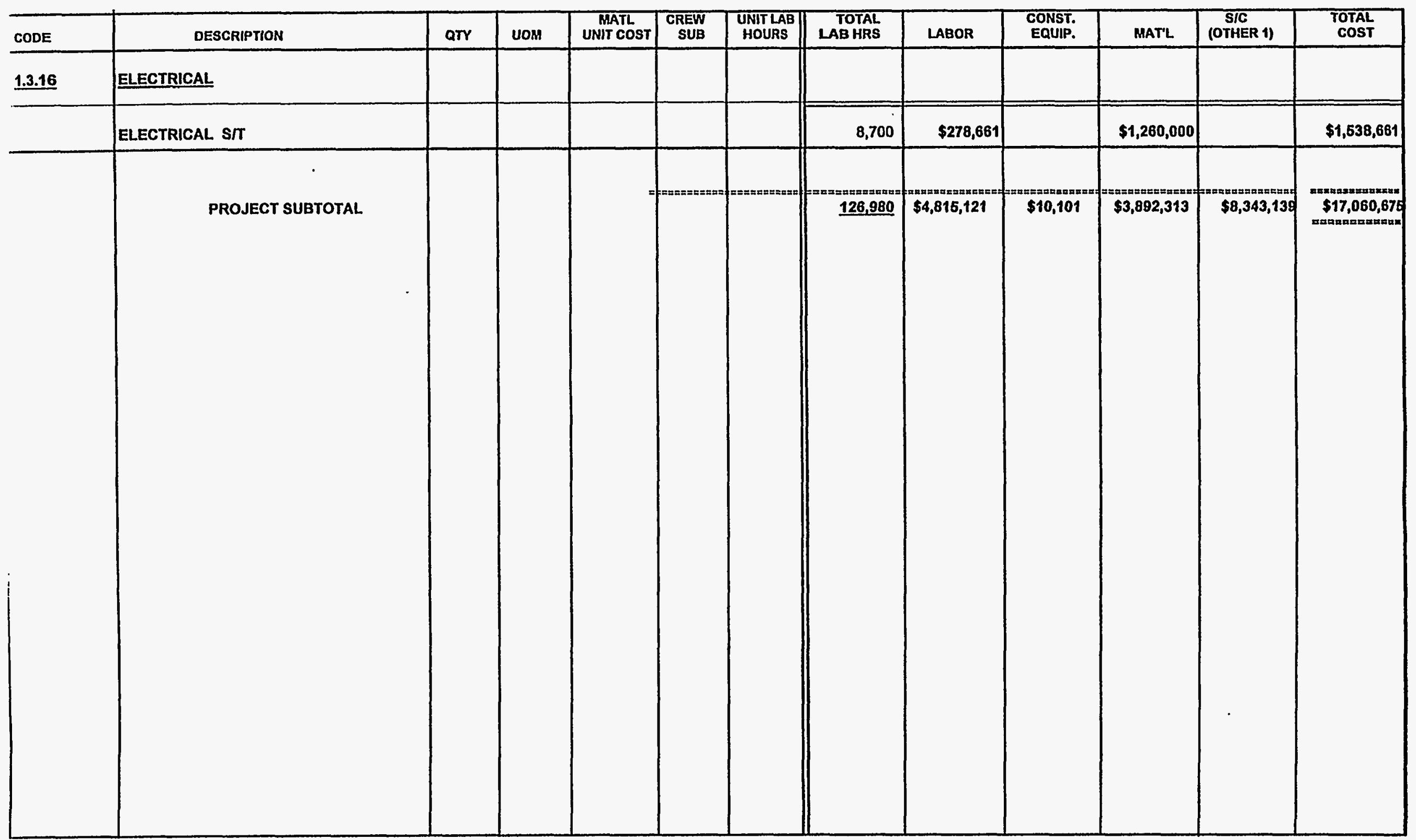


Lockheed Martin Jdaho Technologies Co.

PROJECT NAME: ICPP Bin Set Closure (EIS Stud Place NRC Class C Grout

ICPP

LOCATION 1:

REQUESTOR:
Bryan Spaulding
CONTINGENCY ANALYSIS
TYPE OF ESTIMATE: Planning PROJECT NO: 2423-B

PREPARED BY:
DATE: 27-Jan-1998

TME: 20:26:41

REPORT NAME: Contingency Analysis

\begin{tabular}{|c|c|c|c|c|c|c|c|c|c|c|c|}
\hline \multicolumn{9}{|c|}{ PROBABLE \% VARIATION } & \multicolumn{2}{|c|}{$\begin{array}{l}\text { PROJECT } \\
\text { CONTINGENCY }\end{array}$} & \multirow{3}{*}{$\begin{array}{l}\text { SUMMARY } \\
\text { Total Cost } \\
\text { by Element }\end{array}$} \\
\hline \multirow[t]{2}{*}{$\begin{array}{l}\text { WBS } \\
\text { Element }\end{array}$} & \multirow{2}{*}{ Cost Estimate Element } & \multirow{2}{*}{$\begin{array}{l}\text { Total Cost w/o } \\
\text { Contingency }\end{array}$} & \multirow[t]{2}{*}{$\begin{array}{l}\text { \% Total } \\
\text { Cost }\end{array}$} & \multicolumn{2}{|c|}{$\begin{array}{l}\text { Prob. \% Var. } \\
\text { From Est. }\end{array}$} & \multicolumn{2}{|c|}{ Wit. \% of Prob. } & \multirow{2}{*}{ Contingency } & \multirow[t]{2}{*}{$\%$} & \multirow{2}{*}{ Cost } & \\
\hline & & & & - & + & - & + & & & & \\
\hline 1.1 .1 & DESIGN ENGINEERING TITLE I\&\| & $3,282,538$ & 15.32 & 10 & 40 & 1.53 & 6.13 & $5362 \%$ & $13.08 \%$ & $1,134,503$ & $4,417,041$ \\
\hline 1.1 .2 & TITLE III INSPECTION & 656,507 & 3.06 & 10 & 40 & 0.31 & 123 & $1.072 \%$ & $2.62 \%$ & 226,900 & 883,407 \\
\hline 1.21 & PROJECT MANAGEMENT & $2,923,531$ & 13.65 & 10 & 40 & 1.36 & 5.46 & $4.776 \%$ & $11.65 \%$ & $1,010,424$ & $3,933,955$ \\
\hline 1.22 & CONSTRUCTION MANAGEMENT & $1,301,490$ & 6.07 & 10 & 40 & 0.61 & 243 & $2126 \%$ & $5.15 \%$ & 449,818 & $1,751,308$ \\
\hline 1.3 .1 & GENERAL CONDITIONS & $3,437,150$ & 16.04 & 10 & 50 & 1.60 & 8.02 & $7.059 \%$ & $1722 \%$ & $1,403,409$ & $4,930,559$ \\
\hline 1.3 .2 & SITEWORK & 0 & 0.00 & 0 & 0 & 0.00 & 0.00 & $0.000 \%$ & $0.00 \%$ & 0 & 0 \\
\hline 1.3 .3 & CONCRETE & $3,876,603$ & 18.09 & 10 & 50 & 1.81 & 9.05 & $7.961 \%$ & $19.42 \%$ & $1,684,348$ & $5,560,951$ \\
\hline 1.3 .15 & MECHANICAL & $3,365,584$ & 15.71 & 10 & 50 & 1.57 & 7.85 & $6.912 \%$ & $16.86 \%$ & $1,452,315$ & $4,827,899$ \\
\hline 1.3 .16 & ELECTRICAL & $2,450,822$ & 11.44 & 10 & 50 & 1.14 & 5.72 & $5.033 \%$ & $12.28 \%$ & $1,064,859$ & $3,515,681$ \\
\hline \multirow[t]{2}{*}{1.5 .2} & PROCUREMENT FEES & 131,302 & 0.61 & 10 & 40 & 0.06 & 0.25 & $0.214 \%$ & $0.52 \%$ & 45,380 & 176,682 \\
\hline & ESCALATION & 0 & 0.00 & 0 & 0 & 0.00 & 0.00 & $0.000 \%$ & $0.00 \%$ & 102,517 & 102,517 \\
\hline & SUBTOTAL & $21,425,527$ & 100.00 & & & & & $40.515 \%$ & & & \\
\hline & CALCULATED CONTINGENCY & $8,680,649$ & & & & & & & & & \\
\hline & RESULTANT TEC & $30,106,176$ & & & & & & & & & \\
\hline & ROUNDED TEC & $30,100,000$ & & & & & & & & & \\
\hline & PROJECT CONTINGENCY & $8,674,473$ & & & & & & $40.49 \%$ & & & \\
\hline & MANAGEMENT RESERVE & $1,326,146$ & & & & & & & & & \\
\hline & CONTINGENCY & $7,348,327$ & & & & & & & & & \\
\hline & TOTAL ESTIMATED COST & $30,100,000$ & & & & & & & & $8,674,473$ & $30,100,000$ \\
\hline
\end{tabular}

CONFIDENCE LEVEL AND ASSUMED RISKS:

The Lockheed Idaho Technologies Co. Cost Estimate Contingency Analysis Model is based on the applied contingency and the assumptions upon which the estimate was predicated. The model is applied with a suggested risk level of $18 \%$ and a level of confidence of $90 \%$ the estimate will fall within the bid range. The Contingency Analysis is based on a weighted average to provide a

$90 \%$ probability of underrun and a $10 \%$ probability of overrun.
CONTINGENCY ANALYSIS GUIDE BY TYPE OF ESTIMATE

Guidelines established by DOEJFM 50, Cost Estimating Guide, Vol. 6 , Cost Guide, and as presented in the INEL Cost Estimating Guide.

PLANNING

Experimental/Special Conditions

$20 \%-30 \%$ Conceptual

TITLEI

TITLE II

TITLE IVAFC
$15 \%-25 \%$

$10 \%-20 \%$

$5 \%-15 \%$

Market Conditions 


\section{G\&A/PIF ADDER CALCULATION SHEET ICPP BIN SET CLOSURE \\ RISK BASED CLEAN CLOSURE; CLASS C FILL; UNESCALATED}

FILL BINS W/ NRC CLASS C GROUT

PROCUREMENT FEE:

$$
\begin{aligned}
& \text { CONSTRUCTION = } \\
& \text { GFE }=
\end{aligned}
$$

FEE @ $1 \%=$
$\$ 13,130,159$

Subtotal

$\$ 13,130,159$

$\$ 13,130,159 * 0.01=$

G\&A @ 23\% (with a ceiling of $\$ 500,000$ imposed per year , 7 Years)

CONSTRUCTION OR

CEILING * 7 YEARS =

GFE =

$\$ 3,500,000$

PROCUREMENT FEE $=$

Subtotal $\$ 131,302$

FEE @ $23 \%=$

PIF @ 5.5\%

CONSTRUCTION =
GFE =
PROCUREMENT FEE =
G\&A $=$

Subtotal
$\$ 13,130,159$

$\$ 0$

$\$ 131,302$

$\$ 835,199$

$\$ 14,096,660$

$\$ 14,096,660 \cdot 0.055=$

$\$ 775,316$

TOTAL PROCUREMENT FEE:

$\$ 835,199$

TOTAL G\&A FEE:

$\$ 775,316$

TOTAL PIF: 
Lockheed Martin Idaho Technologies Co. Rev, 6-56 PROLECT MUME: ICPP Bin Set Closure D\&D of Equipment LOCATION 1: INEEL / KCPP

REQUESTOR: B. C. Spaulding
COST ESTIMATE SUMMARY

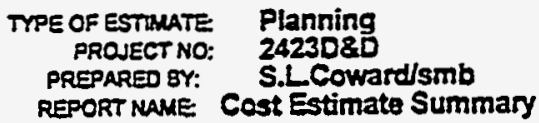

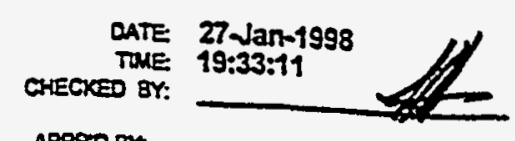

Appro BY.

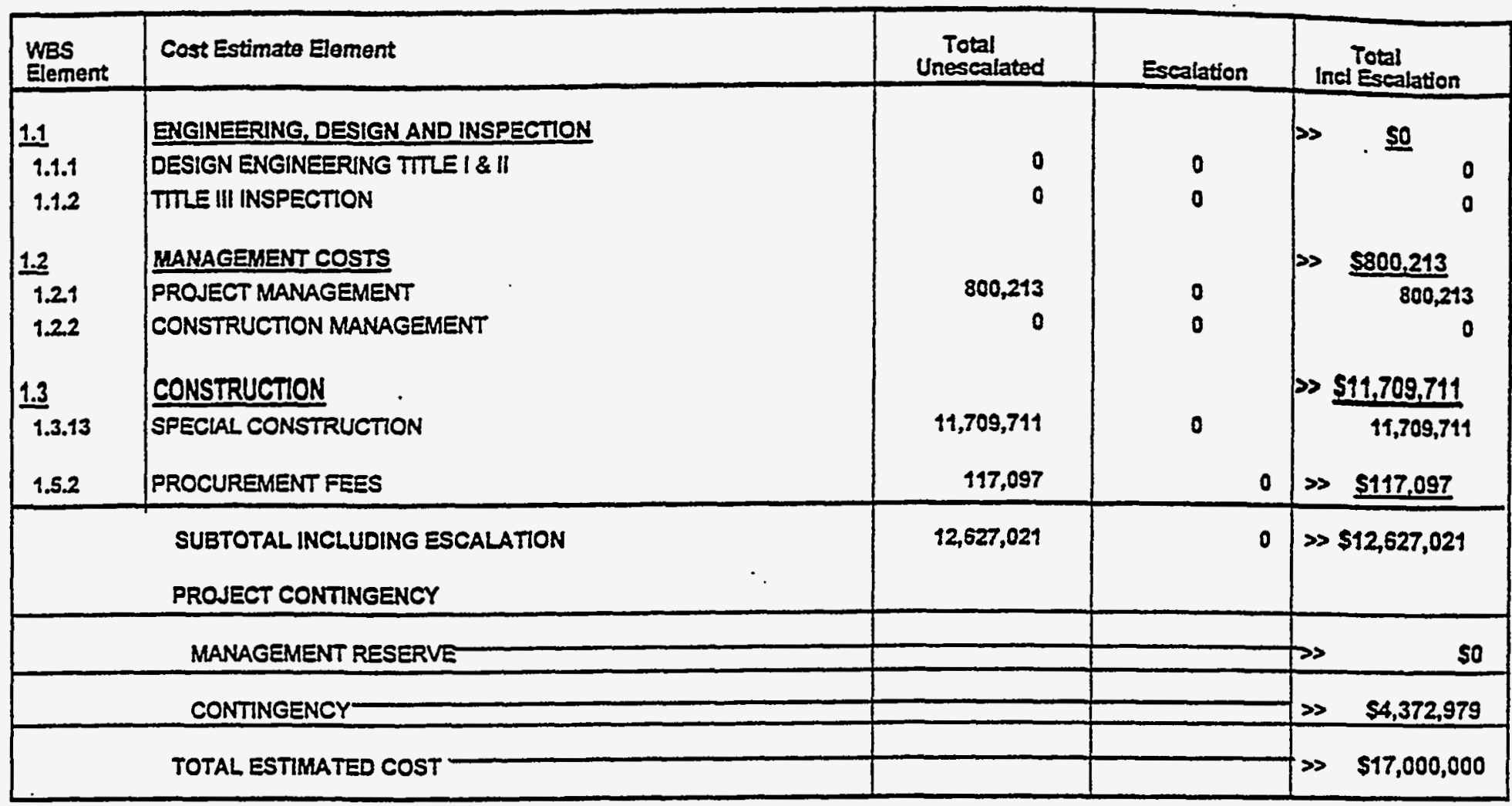

PROJECT COST PARAMETERS

EDI AS A $\%$ OF CONST. + GFE $=0.00 \%$

CONTINGENCY $=34.63 \%$ 


\begin{tabular}{|c|c|c|c|c|c|c|c|c|c|c|c|c|}
\hline \multicolumn{3}{|c|}{ 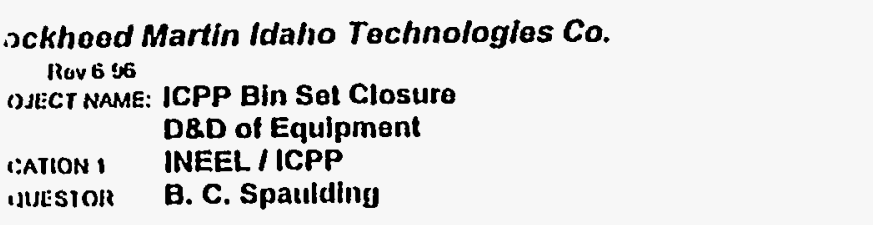 } & \multicolumn{5}{|c|}{ 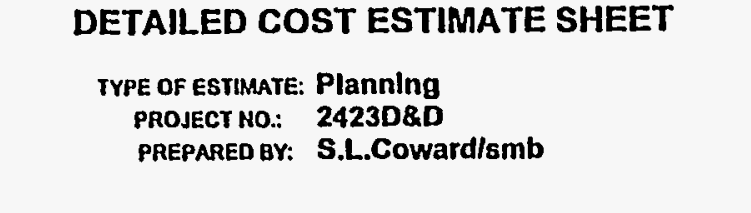 } & \multicolumn{2}{|c|}{$\begin{array}{r}\text { PAGE: } \\
\text { DATE } \\
\text { TIME: } \\
\text { REPORT MAME: }\end{array}$} & \multicolumn{2}{|c|}{$\begin{array}{l}1 \\
\text { 27-Jan-1998 } \\
\text { 19:30:67 } \\
\text { Detall Cost Estimate Sheot }\end{array}$} & \multirow[b]{2}{*}{$\begin{array}{l}\text { TOTAL } \\
\text { COST }\end{array}$} \\
\hline JDE & DESCRIPTION & ary & UOM & $\begin{array}{c}\text { MATL } \\
\text { UNIT COST }\end{array}$ & $\begin{array}{r}\text { CREW } \\
\text { SUB }\end{array}$ & $\begin{array}{l}\text { UNITLAB } \\
\text { HOURS }\end{array}$ & $\begin{array}{l}\text { TOTAL } \\
\text { LAB HRS }\end{array}$ & LABOR & $\begin{array}{l}\text { CONST. } \\
\text { EquIP. }\end{array}$ & MAT'L & $\begin{array}{l}\text { SIC } \\
\text { (OTHER 1) }\end{array}$ & \\
\hline \multirow[t]{4}{*}{$\underline{1.1}$} & DESIGN ENGINEERING TIMLE $1 \& \|$ & & & & & & & & & & & \\
\hline & & & & & & & & & & & & \\
\hline & $\begin{array}{l}\text { Costs lor Design aclivilies Included } \\
\text { in Special Construction }\end{array}$ & & & & & & & & & & & \\
\hline & DESIGN ENGINEERING TITLE I \& $\| \mathrm{S} \pi$ & & & & & & $\mathbf{0}$ & & & & & \\
\hline \multirow{4}{*}{\begin{tabular}{cc|}
1.2 & \\
$\ldots \ldots$ & $\ldots$ \\
$\ldots \ldots \ldots \ldots$
\end{tabular}} & TITLE III INSPECTION & & & & & & & & & & & \\
\hline & 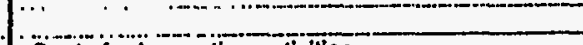 & & & & & & & & & & & \\
\hline & $\begin{array}{l}\text { Cossts los linpectlon acclvilles } \\
\text { included in Special Construction }\end{array}$ & & & & & & & & & & & \\
\hline & TITLE III INSPECTION SIT & & & & & & $\mathbf{0}$ & & & & & \\
\hline \multirow[t]{3}{*}{.2 .1} & PROJECT MANAGEMENT & & & & & & & & & & & \\
\hline & & & & & & ............. & & $-{ }^{2}$ & . & & $-\infty$ & $\ldots$ \\
\hline & 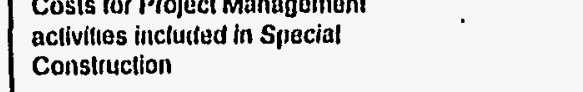 & & & & & & & & & & & \\
\hline \multirow[t]{2}{*}{$\cdots \cdots+\cdots$} & $G B A$ & 1 & TS & & LMITCO & 0.000 & & & & & 141,932 & 141,932 \\
\hline & PIF & 1 & LS & & LMIrCO & 0.000 & & & & & 658,281 & 668,281 \\
\hline 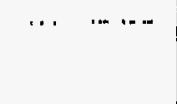 & PROJECT MANAGEMENT STT & & & & & & $\mathbf{0}$ & & & & $\$ 800,213$ & $\$ 800,213$ \\
\hline !.2.2 & CONSTRUCTION MANAGEMENT & & & & & & & & & & & \\
\hline -................ & (- & & & & & & & & & & & \\
\hline & & & & & & & & & & & & \\
\hline & & & & & & & & & & & & \\
\hline
\end{tabular}


ckheod Martin Idaho Technologles Co. Ruv 6.46 ict Name. ICPP Bin Set Closure D\&D of Equipinent INEEL / ICPP

Butston Spaulding

\section{DETAILED COST ESTIMATE SHEET}

TYPE OF Estimate: Planning

PROJECT NO: 2423D\&D

PREPARED BY: S.L.Coward/smb
PAgE 2

DATE 27 -Jan-1898

TIME: 18:30:67

Report name: Detall Cost Estimate Sheot

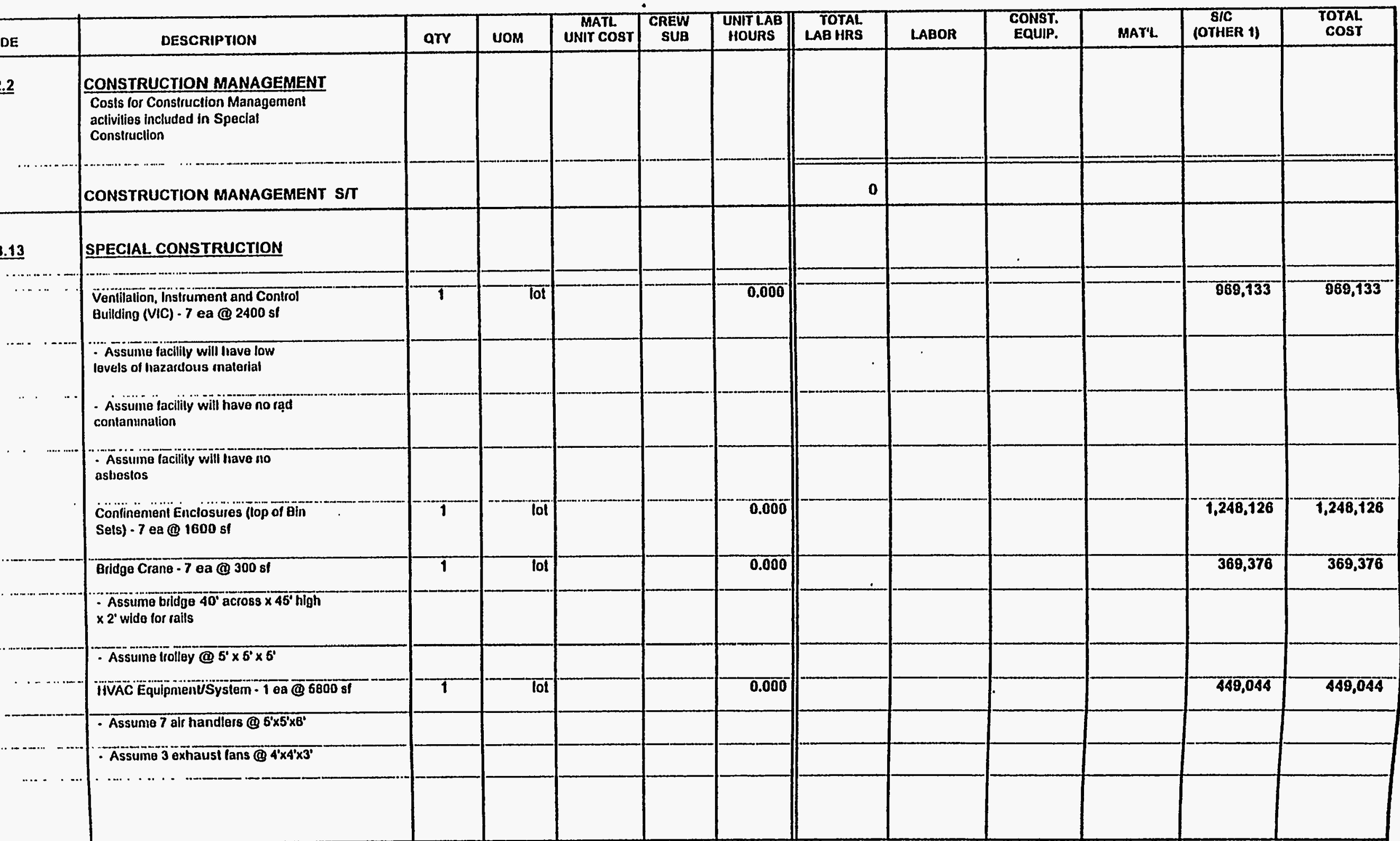


ockheed Martin Idaho Technologles Co. Rav 6.96 (O)JECT NAME ICPP Bin Sot Closuro D\&D of Equipment INEEL I ICPP

CAllon! UUESTOR B. C. Spaulding
DETAILED COST ESTIMATE SHEET

TYPE OF EstIMATE: Plannlng

PROJECT NO:: 2423D\&D

PREPARED QY: S.L.Coward/smb page: 3

DATE 27-Jan-1998

TMME: 19:30:67

Report mane: Dotall Cost Est/mato Sheot

one

DESCRIPTION

(1)

.3 .13

SPECIAL CONSTRUCTION

- Assume 63 HEPA fillers @ 6'x6'x2'

Jumper Retrleval Piping (Shielded) - 1

ea (1) 420 st

- Assume $210^{-1}$ long $\times 2$ wide concrele

Remole Core Drilling Plallorm/Saw.

etc. 1 ea (1) 250 st

$\because$ Assume platiorm (e) $5 \times 6^{\prime} \times 6^{\prime}$ and saw is $\mathbf{1 0 0}$ si

Bivinolo Woilding and Culling Equipment Allowanco 1 ea (a) 500 sl

Veiticai Deployment Appartus - 7 ea@ 150 st

- Assumi $5^{\circ} \times 5^{\circ} \times 6^{\circ}$

siniaiting äniser plitos - 100 ea (C) 4 si

- Assume 2' $\times 2$

Cö2 Blasting Equipment - Allowance 2 ea @ 600 s!

Retrievaitines - T ea (1) 2310 st

- Assuino 2310 iong $\times 107$ wide.

Contiol Consoles, Cameras, LIghting -

Allowanco.1 0a @ 1000 8!

Groulino Maniloids - Clean - Allowanco

- 8 ea (1) 300 sl

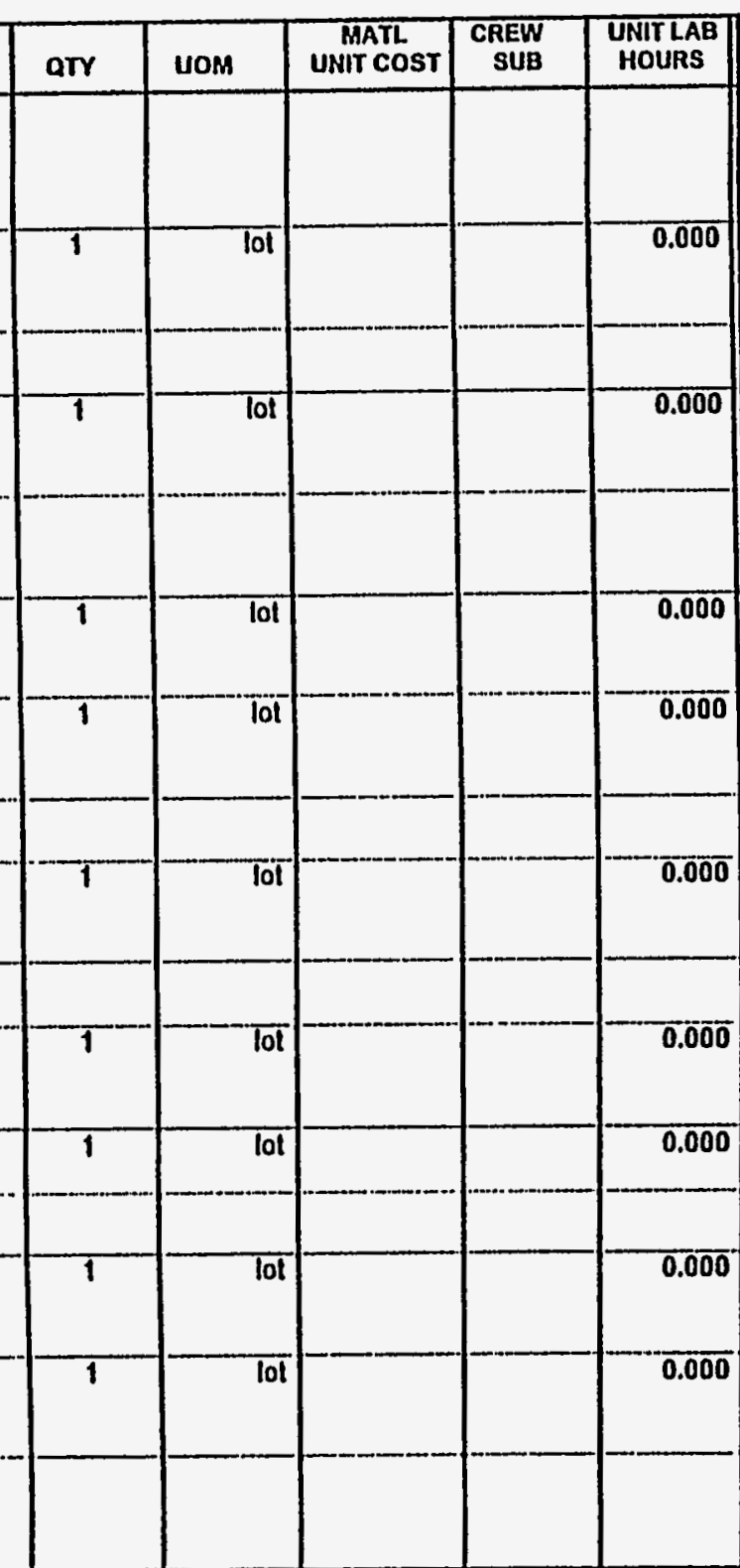

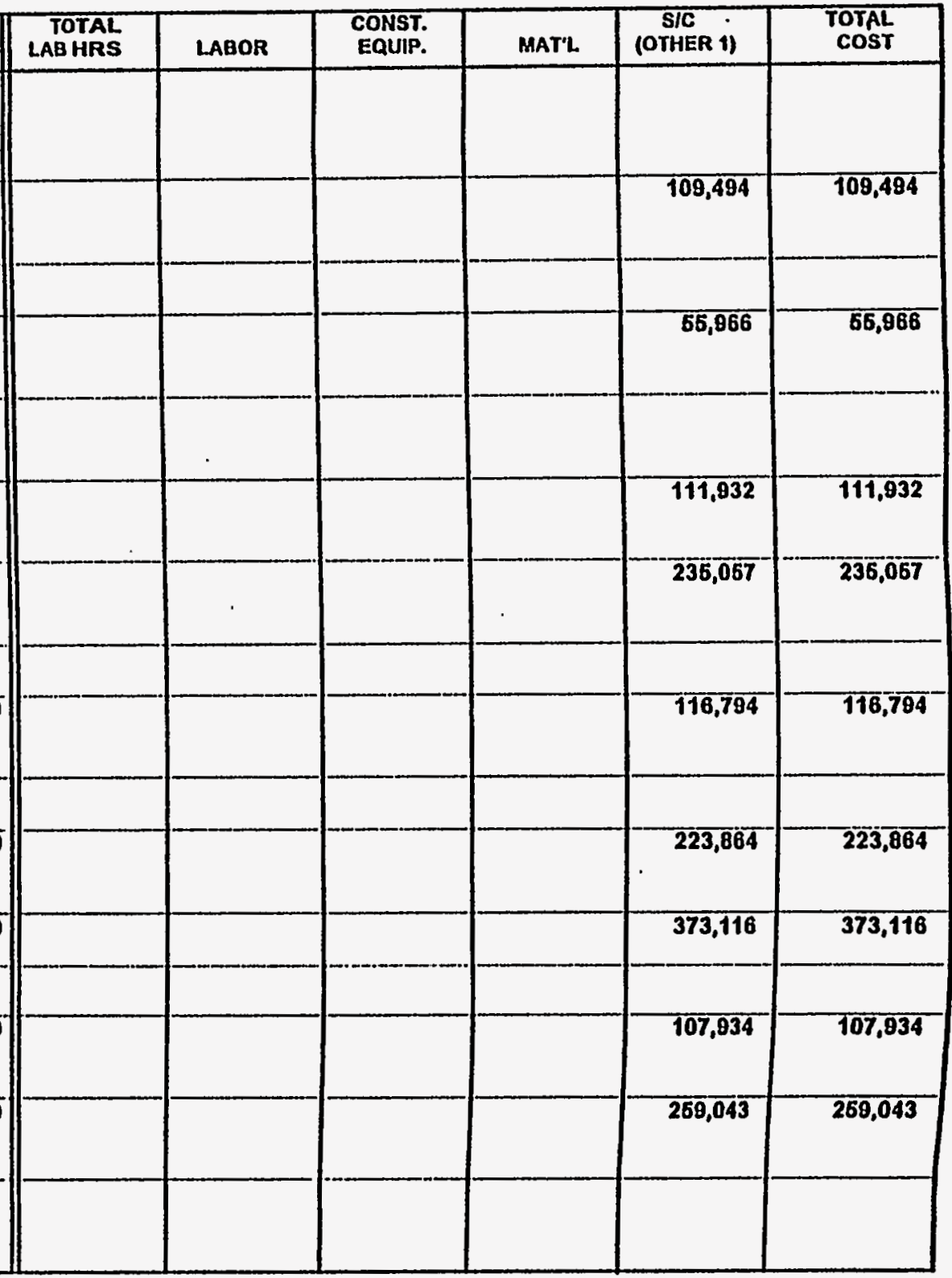


ckheod Martin Idaho Technologles Co.

Rev 6.56

ECI NAME. ICPP BIn Sot Closure

D\&D of Equipment

INEEL / ICPP

B. C. Spaulding
DETAILED COST ESTIMATE SHEET

TYPE OF EsTIMATE: Planning

PROJECT NO.: 2423D\&D

PREPARED BY: S.L.Coward/smb
PAGE : 4

DATE 27-Jan-1988

TIME: $\quad$ 19:30:57

neport name: Detall Cost Estlmate Sheot

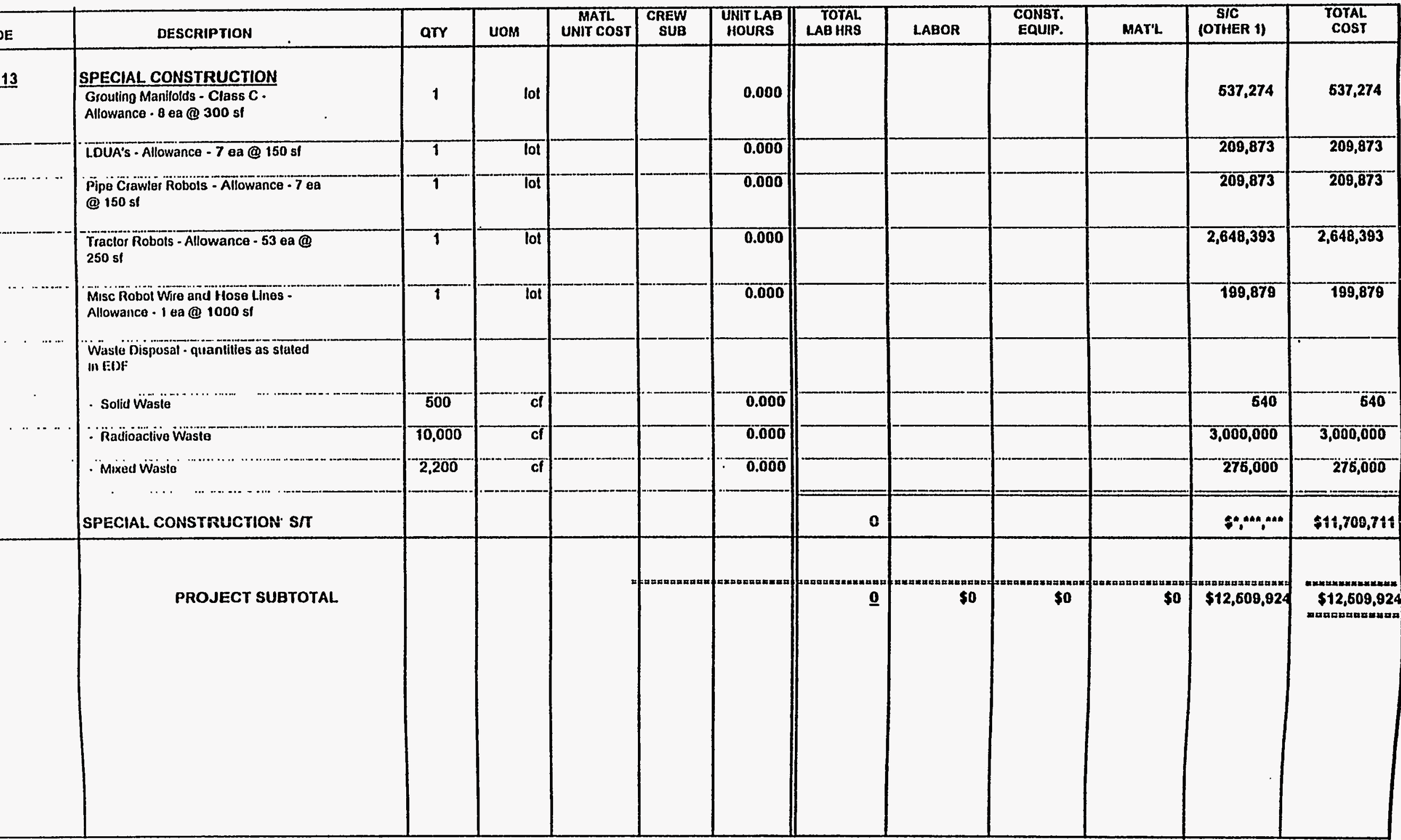



Lockheed Martin Idaho Technologies Co. PROSECT NAME: ICPP Bin Sot Closure D\&D of Equipment

LOCATION 1: REQUESTOR:
CONTINGENCY ANALYSIS

TYPE OF ESTULATE:
PROJECT NO:

PROJECT NO: Planning

FREPAREO BY: S.LCOWard/smb
DATE 27-Jan-1998

TRE 19:33:04

report raue Contingency Analysis

\begin{tabular}{|c|c|c|c|c|c|c|c|c|c|c|c|}
\hline \multicolumn{9}{|c|}{ PROBABLE \% VARIATION } & \multicolumn{2}{|c|}{$\begin{array}{l}\text { PROJECT } \\
\text { CONTINGENCY }\end{array}$} & \multirow{3}{*}{$\begin{array}{l}\text { SUMMARY } \\
\text { Totl Cost } \\
\text { by Eement }\end{array}$} \\
\hline $\begin{array}{l}\text { WBS } \\
\text { Eement }\end{array}$ & \multirow{2}{*}{ Cost Estimate Element } & \multirow{2}{*}{$\begin{array}{l}\text { Total Cost w/o } \\
\text { Contingency }\end{array}$} & \multirow[t]{2}{*}{$\begin{array}{l}\% \text { Total } \\
\text { Cost }\end{array}$} & \multicolumn{2}{|c|}{$\begin{array}{c}\text { Prob. \% Vap. } \\
\text { From Est. }\end{array}$} & \multicolumn{2}{|c|}{ Wh of Prob. } & \multirow[t]{2}{*}{ Contungeney } & \multirow{2}{*}{$\%$} & \multirow{2}{*}{ Cost } & \\
\hline & & & & - & + & - & \pm & & & & \\
\hline 1.1 .1 & DESIGN ENGINEERING TILE | \& II & 0 & 0.00 & 0 & 0 & 0.00 & 0.00 & $0.000 \%$ & $0.00 \%$ & 이 & 0 \\
\hline 1.12 & TITLE III INSPECTION & 0 & 0.00 & 0 & 0 & 0.00 & 0.00 & $0.000 \%$ & $0.00 \%$ & of & 0 \\
\hline 1.21 & PROJECT MANAGEMENT & 800,213 & 6.34 & 10 & 40 & 0.53 & 253 & $2218 \%$ & $6.34 \%$ & 277,120 & $1,077,342$ \\
\hline 122 & CONSTRUCTION MANAGEMENT & 0 & 0.00 & 0 & 0 & 0.00 & 0.00 & $0.000 \%$ & $0.00 \%$ & 0 & 0 \\
\hline 1.3 .13 & SPECLAL CONSTRUCTION & $11,709,711$ & 92.74 & 10 & 40 & 9.27 & 37.09 & $32457 \%$ & $9274 \%$ & $4,055,297$ & $15,765,008$ \\
\hline \multirow[t]{2}{*}{1.52} & PROCUREMENT FEES & 117,097 & 0.93 & 10 & 40 & 0.09 & 0.37 & $0.325 \%$ & $0.93 \%$ & 10,553 & 157.650 \\
\hline & ESCALATTON & 0 & 0.00 & 0 & 0 & 0.00 & 0.00 & $0.000 \%$ & $0.00 \%$ & 0 & 0 \\
\hline & SUBTOTAL & $12,627,021$ & 100.00 & & & & & $35.000 \%$ & & & \\
\hline & CALCULATED CONTINGENCY & $4,419,457$ & & & & & & & & & \\
\hline & RESULTANT TEC & $17,046,478$ & & & & & & & & & \\
\hline & ROUNDED TEC & $17,000,000$ & & & & & & & & & \\
\hline & PROJECT CONTINGENCY & $4,372,979$ & & . & & & & $34.63 \%$ & & & \\
\hline & MANAGEMENT RESERVE & 0 & & & & & & & & & \\
\hline & CONTNGENCY & $4,372,979$ & & & & & & & & & \\
\hline & TOTAL ESTIMATED COST & $17,000,000$ & & & & & & & & $4,372,979$ & $17,000,000$ \\
\hline
\end{tabular}

CONFIDENCE LEVEL AND ASSUMED RISKS:

The Lockheed Idaho Technologies Co. Cost Estimate Contingency Analysis Model is based on the applied contingency and the assumptions upon which

the estimate was predicated. The model is applied with a suggested risk level

of $18 \%$ and a level of confidence of $90 \%$ the estimate will fall within the bid range.

The Contingency Analysis is based on a weighted average to provide a

$90 \%$ probability of undernu and a $10 \%$ probability of overnu.
CONTINGENCY ANALYSIS GUIDE BY TYPE OF ESTIMATE

Guidelines established by DOEJFM 50, Cost Estimating Guide. Vol. 6 ,

Cost Guide, and as presented in the INEL Cost Estimating Guide.

$$
\text { PLANNING }
$$

Conceptual

TITE

TTLE II

TIRE IUAFC

$20 \%-30 \%$

$15 \%-25 \%$

Up to $40 \%$

$10 \%-20 \%$

$5 \%-15 \%$

Market Conditions 

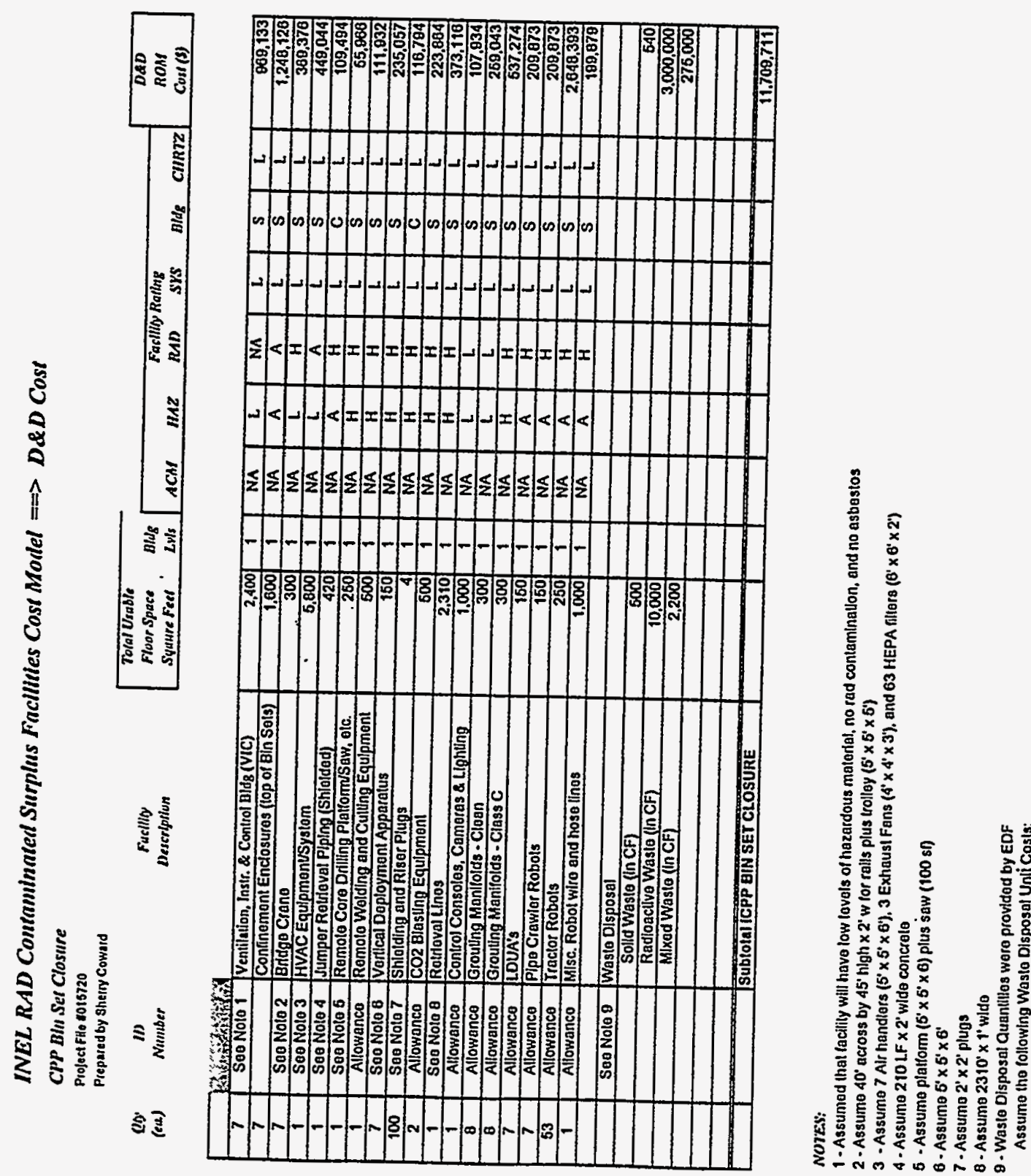


\section{G\&APIF ADDER CALCULATION SHEET ICPP BIN SET CLOSURE \\ RISK BASED CLEAN CLOSURE; CLASS C FILL; UNESCALATED \\ D\&D OF EQUIPMENT}

PROCUREMENT FEE:

CONSTRUCTION = $\$ 11,709,711$

GFE =

Subtotal $\$ \$ 11,709,711$

FEE @ 1\%=

$\$ 11,709,711 * 0.01=$

$\$ 117,097$

G\&A @ 23\% (with a ceiling of $\$ 500,000$ imposed per year, 1 yr)

CONSTRUCTION OR

CEILING * 1 YEARS =

GFE =

$\$ 500,000$

PROCUREMENT FEE $=$

Subtotal $\$ \frac{\$ 17,097}{}$

FEE @ 23\% =

$\$ 617,097 * 0.23=$

$\$ 141,932$

PIF @ 5.5\%

CONSTRUCTION =

GFE =

$\$ 11,709,711$

PROCUREMENT FEE $=$

$G \& A=$

Subtotal $\$ \frac{\$ 1,968,740}{\$ 141,932}$

FEE @ $5.5 \%=$

$\$ 658,281$

TOTAL PROCUREMENT FEE:

$\$ 117,097$

TOTAL G\&A FEE:

$\$ 141,932$

TOTAL PIF: 


\section{COST ESTIMATE SUMMARY}

\section{ESCALATED}

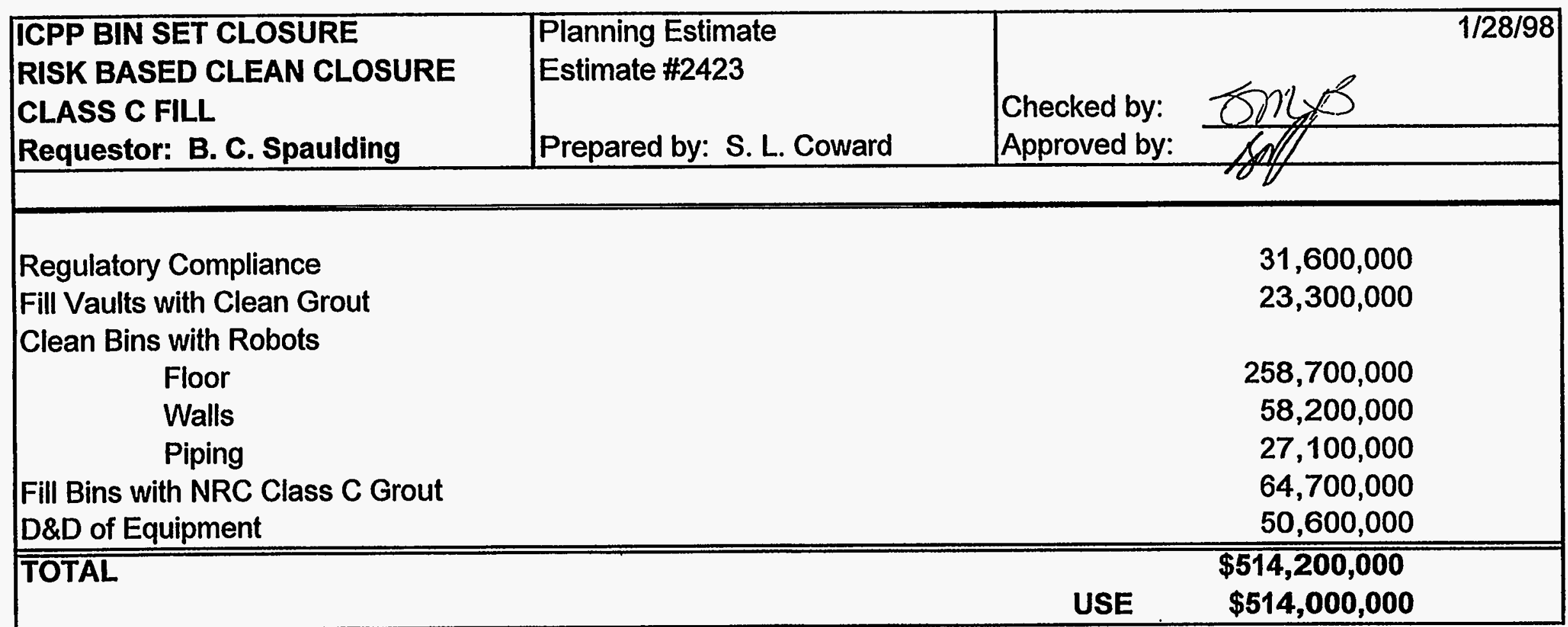


Lockheed Martin Idaho Technologies Co. Rev, 6-96

PROJECT NAME: Permitting/Documentation

Risk Based - NRC Class C

INEELICPP

LOCATION 1:

REQUESTOR:
COST ESTIMATE SUMMARY

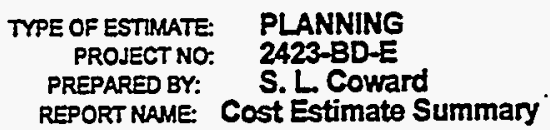

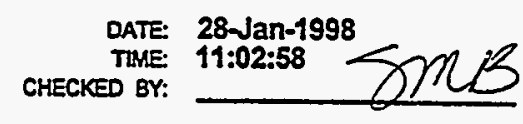

APPRD BY:

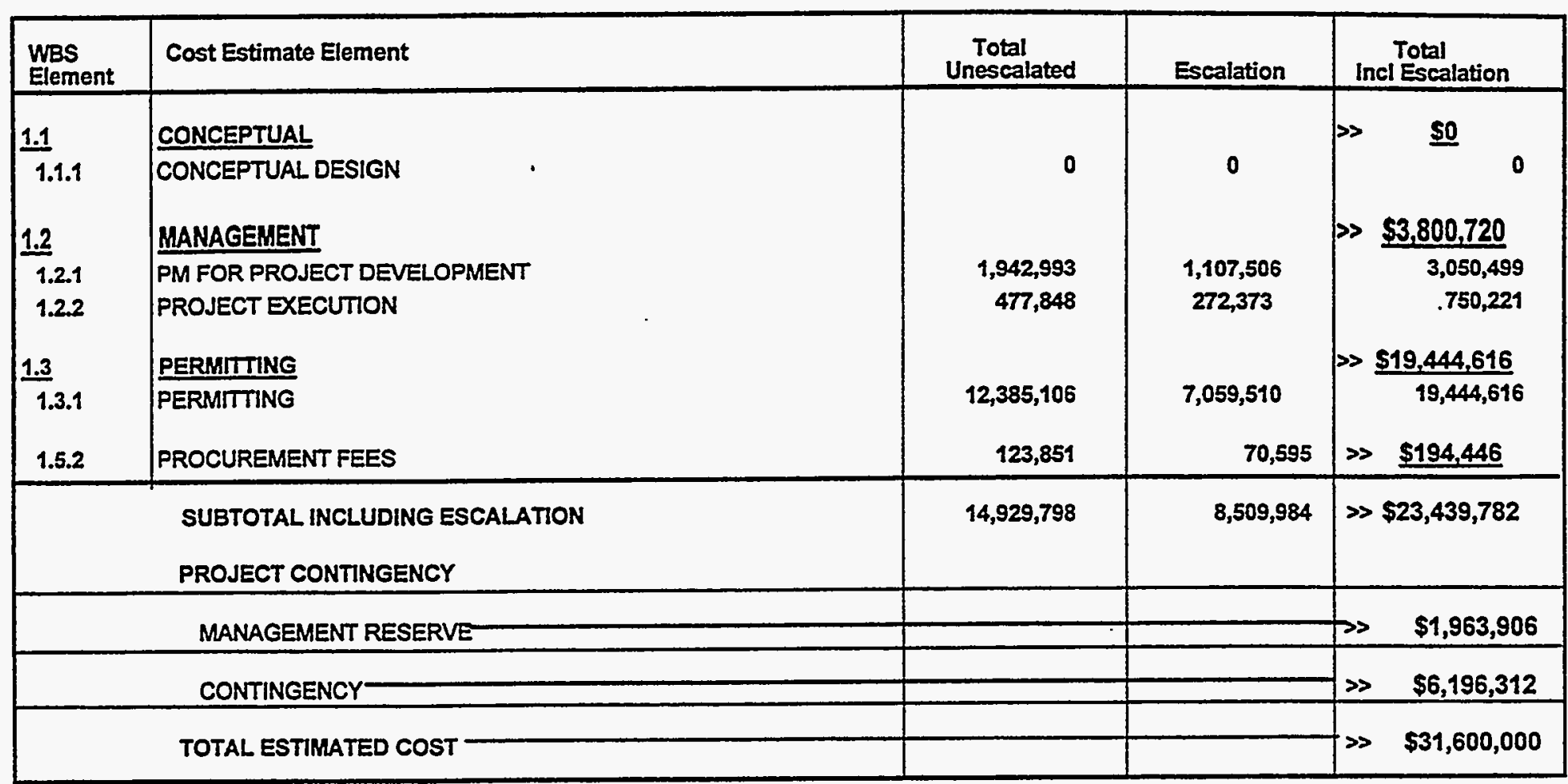


Lockheed Martin Idaho Technologies Co.

Rov 6.96

PROJeCt NAME: Permitting/Documentation

Risk Based - NRC Class C

INEEL/ICPP

REQUESTOR: Bryan Spaulding

\section{DETAILED COST ESTIMATE SHEET}

TYPE OF ESTIMATE: PLANNING

PROJECT NO:: 2423-BD-E

PREPARED BY: S. L. Coward
PAGE\# 1

DATE 28-Jan-1998

TIME: 11:03:00

REPORT NAME: Detall Cost Estimate Sheet

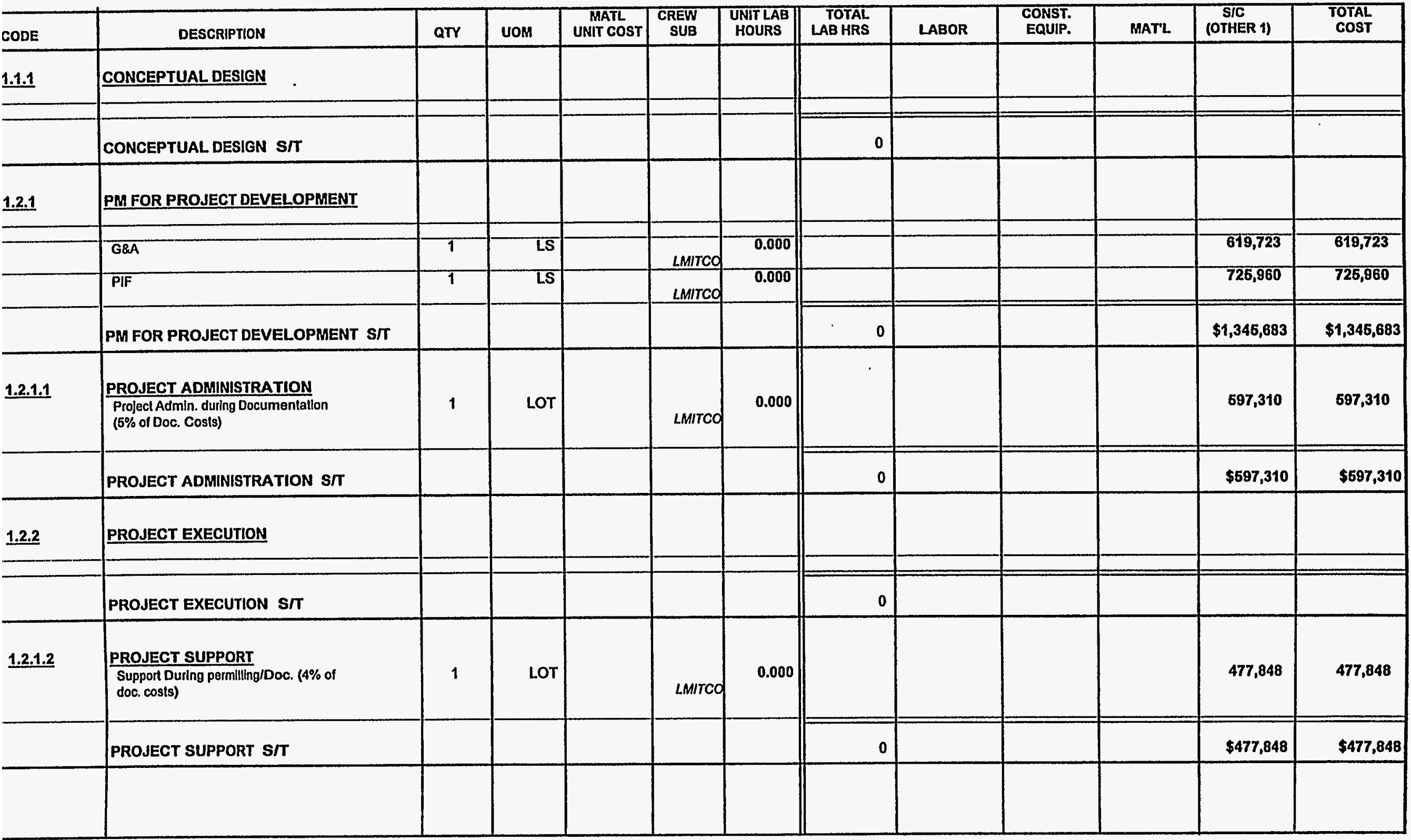


Lockheed Martin Idaho Technologies Co.

Rov 6-96

PROJECT NAME: Permitting/Documentation Risk Based - NRC Class C

LOCATION 1:

INEELICPP

REQUESTOR: Bryan Spaulding

CONTINGENCY ANALYSIS

TYPE OFESTIMATE: PLANNING

PREPARED BY: S. L. Coward
DATE. 28-Van-1998

TRME: 11:02:47

REPORT NAME: Contingency Aralysis

\begin{tabular}{|c|c|c|c|c|c|c|c|c|c|c|c|}
\hline \multicolumn{9}{|c|}{ PROBABLE \% VARIATION } & \multicolumn{2}{|c|}{$\begin{array}{l}\text { PROJECT } \\
\text { CONTINGENCY }\end{array}$} & \multirow{3}{*}{$\begin{array}{l}\text { SUMMARY } \\
\begin{array}{l}\text { Total Cost } \\
\text { by Element }\end{array}\end{array}$} \\
\hline \multirow[t]{2}{*}{$\begin{array}{l}\text { Wes } \\
\text { Element }\end{array}$} & \multirow{2}{*}{ Cost Estimate Element } & \multirow{2}{*}{$\begin{array}{l}\text { Total Cost w/o } \\
\text { Contingency }\end{array}$} & \multirow[t]{2}{*}{$\begin{array}{l}\text { \% Total } \\
\text { Cost }\end{array}$} & \multicolumn{2}{|c|}{$\begin{array}{l}\text { Prob. \% Var. } \\
\text { From Est. }\end{array}$} & \multicolumn{2}{|c|}{ Wt. \% of Prob. } & \multirow{2}{*}{ Contungency } & \multirow[t]{2}{*}{$\%$} & \multirow[t]{2}{*}{ Cost } & \\
\hline & & & & - & + & - & + & & & & \\
\hline 1.1 .1 & CONCEPTUAL DESIGN & 0 & 0.00 & 10 & 40 & 0.00 & 0.00 & $0.000 \%$ & $0.00 \%$ & 0 & 0 \\
\hline 1.21 & PM FOR PROJECT DEVELOPMENT & $1,942,993$ & 8.29 & 10 & 40 & 0.83 & 3.32 & $2904 \%$ & $829 \%$ & 676,425 & $2,619,418$ \\
\hline 1.2 .2 & PROJECT EXECUTION & 477,848 & 2.04 & 10 & 40 & 0.20 & 0.82 & $0.714 \%$ & $2.04 \%$ & 166,356 & 644,204 \\
\hline 1.3 .1 & PERMITIING & $12,385,106$ & 52.84 & 10 & 40 & 5.28 & 21.14 & $18.493 \%$ & $5284 \%$ & $4,311,694$ & $16,696,800$ \\
\hline \multirow[t]{2}{*}{1.5 .2} & PROCUREMENT FEES & 123,851 & 0.53 & 10 & 40 & 0.05 & 0.21 & $0.185 \%$ & $0.53 \%$ & 43,117 & 166,968 \\
\hline & ESCALATTON & $8,509,984$ & 36.31 & 10 & 40 & 3.63 & 14.52 & $12.707 \%$ & $36.31 \%$ & $2,952,626$ & $11,472,610$ \\
\hline & SUBTOTAL & $23,439,782$ & 100.00 & & & & & $.35 .000 \%$ & & & \\
\hline & CALCULATED CONTINGENCY & $8,203,924$ & & & & & & & & & \\
\hline & RESULTANT TEC & $31,643,706$ & & & & & & & & & \\
\hline & ROUNDED TEC & $31,600,000$ & & & & & & & & & \\
\hline & PROJECT CONTINGENCY & $8,160,218$ & & & & & & $34.81 \%$ & & & \\
\hline & MANAGEMENT RESERVE & $1,963,906$ & & & & & & & & & \\
\hline & CONTINGENCY & $6,196,312$ & & & & & & & & & \\
\hline & TOTAL ESTIMATED COST & $31,600,000$ & & & & & & & & $8,160,218$ & $31,600,000$ \\
\hline
\end{tabular}

CONFIDENCE LEVEL AND ASSUMED RISKS:

The Lockheed Idaho Technologies Co. Cost Estimate Contingency Analysis Model is based on the applied contingency and the assumptions upon which

the estimate was predicated. The model is applied with a suggested risk level

of $18 \%$ and a level of confidence of $90 \%$ the estimate will fall within the bid range.

The Contingency Analysis is based on a weighted average to provide a

$90 \%$ probability of underrun and a $10 \%$ probability of overnun.
CONTINGENCY ANALYSIS GUIDE BY TYPE OF ESTIMATE

Guidelines established by DOE/FM 50 , Cost Estimating Guide, Vol. 6 ,

Cost Guide, and as presented in the INEL Cost Estimating Guide.

PLANNING

Conceptual ....Up to $50 \%$ TITLEI

TITLE II

TITLE IUAFC
$10 \%-20 \%$

$5 \%-15 \%$

Market Conditions 


\section{G\&APIF ADDER CALCULATION SHEET ICPP BIN SET CLOSURE \\ RISK BASED CLEAN CLOSURE; CLASS C FILL; ESCALATED \\ REGULATORY COMPLIANCE}

PROCUREMENT FEE:

$\begin{aligned} & \text { CONSTRUCTION }= \\ & \text { GFE }=\end{aligned}$
Subtotal
FEE @ $1 \% \doteq$

G\&A @ 23\% (with a ceiling of $\$ 500,000$ imposed per year, 5 yrs)

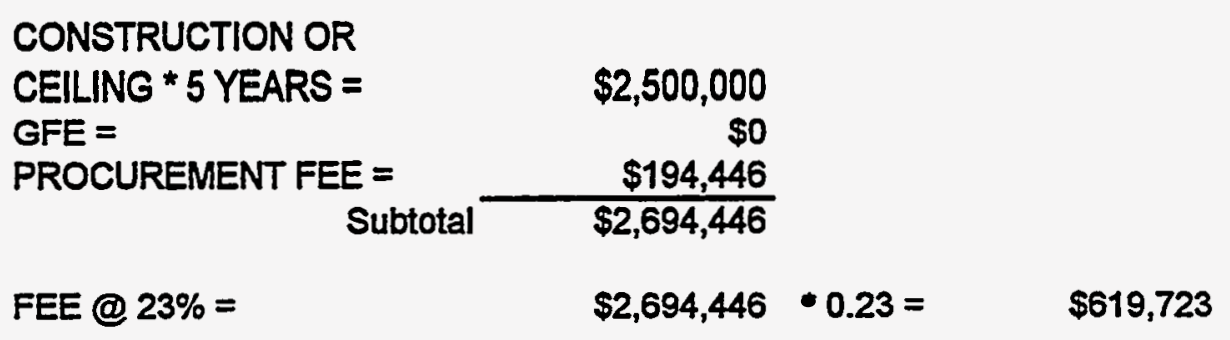

PIF @ 5.5\%

\begin{tabular}{lr} 
CONSTRUCTION $=$ & $\$ 12,385,106$ \\
GFE $=$ & $\$ 0$ \\
PROCUREMENT FEE $=$ & $\$ 194,446$ \\
G\&A $=$ & $\$ 619,723$ \\
\hline & Subtotal
\end{tabular}

FEE @ 5.5\% =

$\$ 13,199,275 \cdot 0.055=$

$\$ 725,960$

TOTAL PROCUREMENT FEE:

$\$ 194,446$

TOTAL G\&A FEE:

$\$ 619,723$

TOTAL PIF:

$\$ 725,960$ 
Lockheed Martin Idaho Technologies Co. PROJECT NAME: ICPP Bin Set Closure (EIS Stud Place Clean Grout in Vault LOCATION 1: ICPP REQUESTOR: Bryan Spaulding
COST ESTIMATE SUMMARY

TPPE OF ESTMMATE: Planning

PROJECT NO: 2423-A2-E1

PREPARED BY: S. L. Coward

REPORT NAME: Cost Estimate Summary

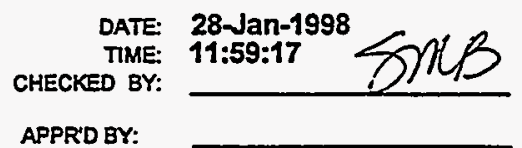

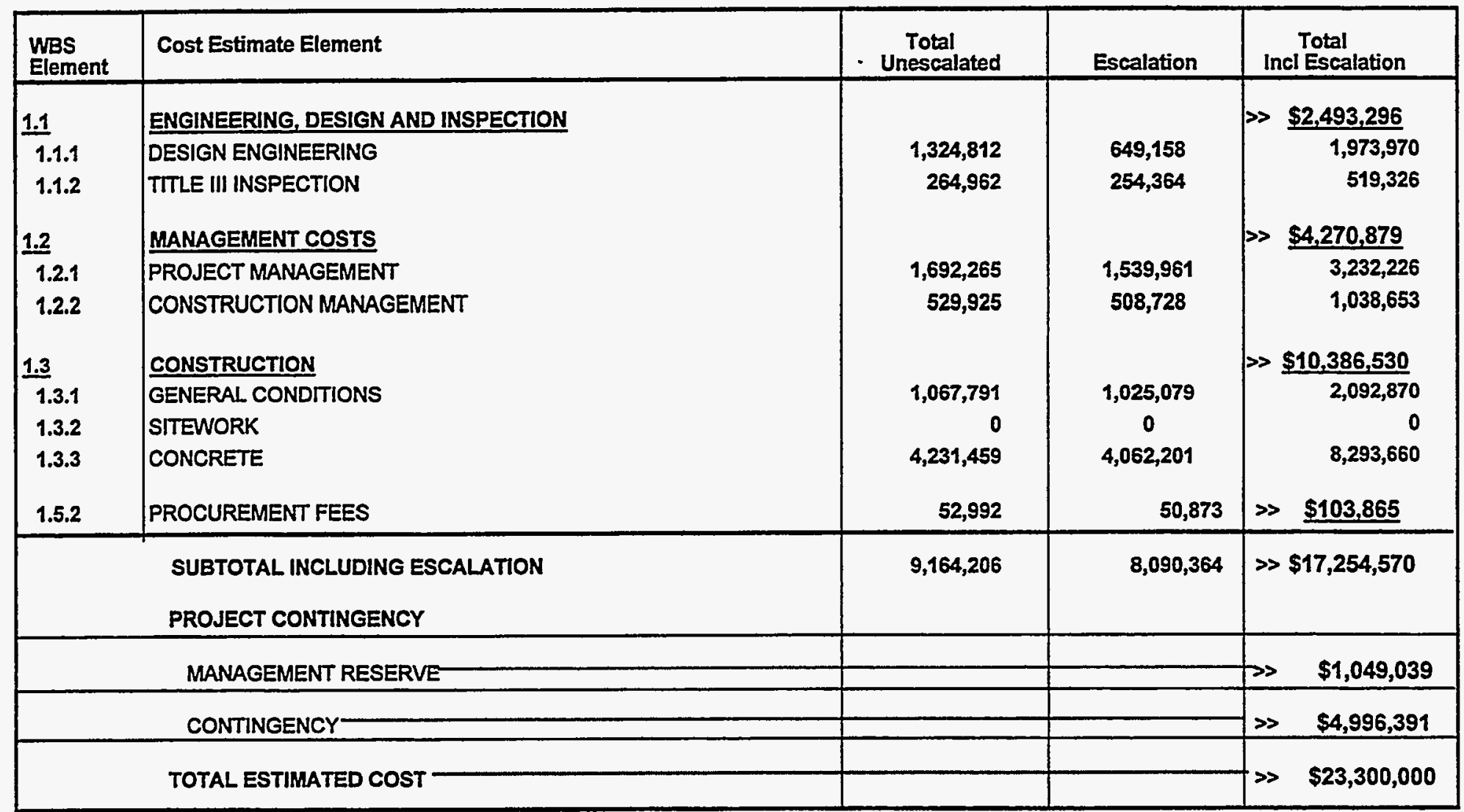

\section{PROJECT COST PARAMETERS}

EDI AS A \% OF CONST. + GFE $=24.00 \%$ 
Lockheed Martin Idaho Technologies Co. Rov 6.08 PROJECT NAME: ICPP Bin Set Closure (EIS Study) Place Clean Grout In Vault

ICPP

REQUESTOR: Bryan Spaulding

\section{DETAILED COST ESTIMATE SHEET}

TYPE OF Estimate: Planning

PROJECT NO.: 2423-A2-E1

PREPARED BY: S. L. Coward
PAGE \# 2

DATE 28-Jan-1998

TIME: 11:59:19

REPORT NAME: Detall Cost Estimate Sheet

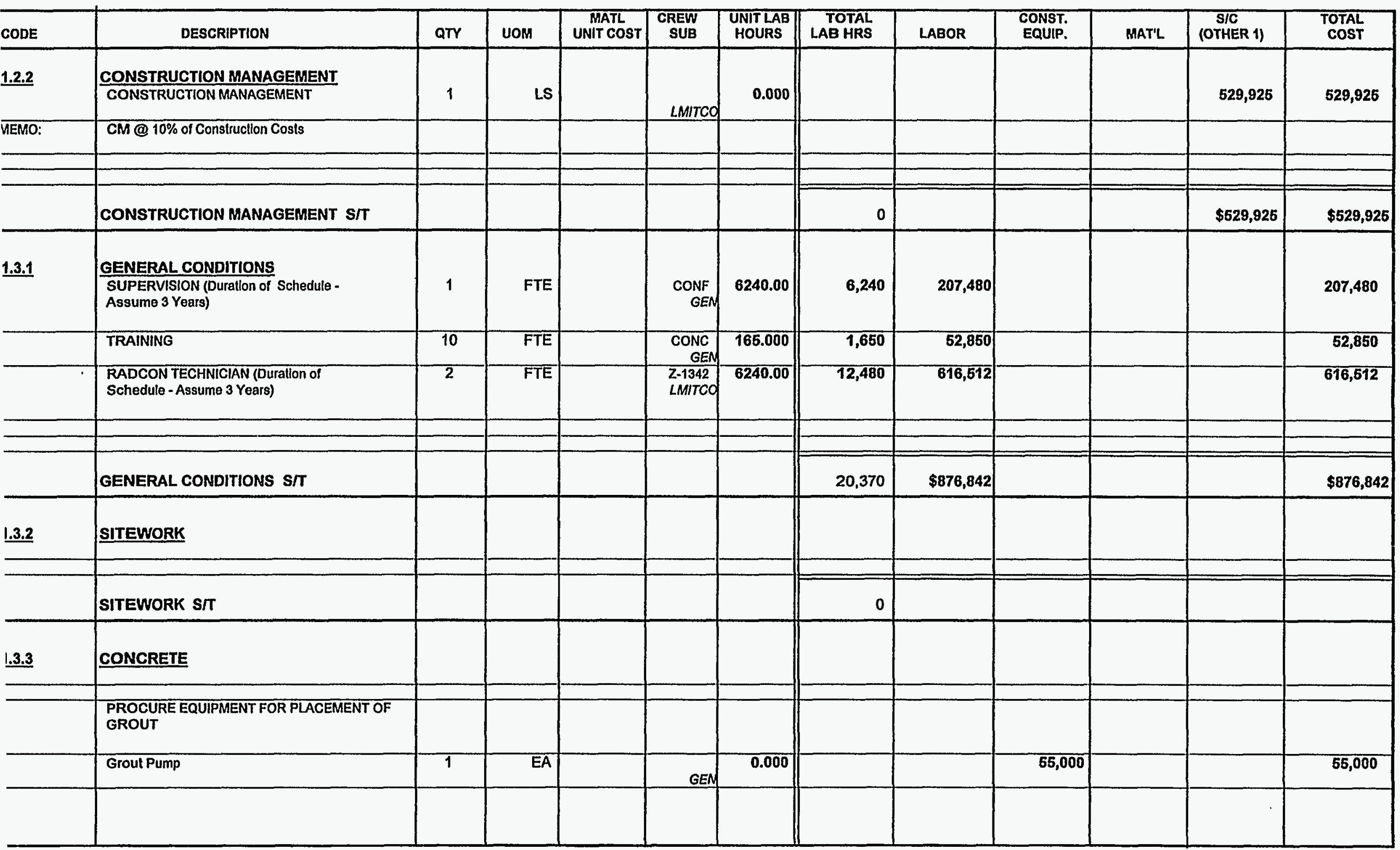


Lockheed Martin Idaho Technologios Co. Rev 6.96

PROJECT NAME: ICPP BIn Set Closure (EIS Study) Place Clean Grout in Vault

LOCATION 1: ICPP

REQUESTOR: Bryan Spaulding

\section{DETAILED COST ESTIMATE SHEET}

TYPE OF ESTIMATE: Planning

PROJECT NO.: 2423-A2-E1

PREPARED BY: S. L. Coward
PAGE\# 3

DATE 28-Jan-1998

TIME: 11:59:19

REPORT NAME: Detall Cost Estimate Sheet

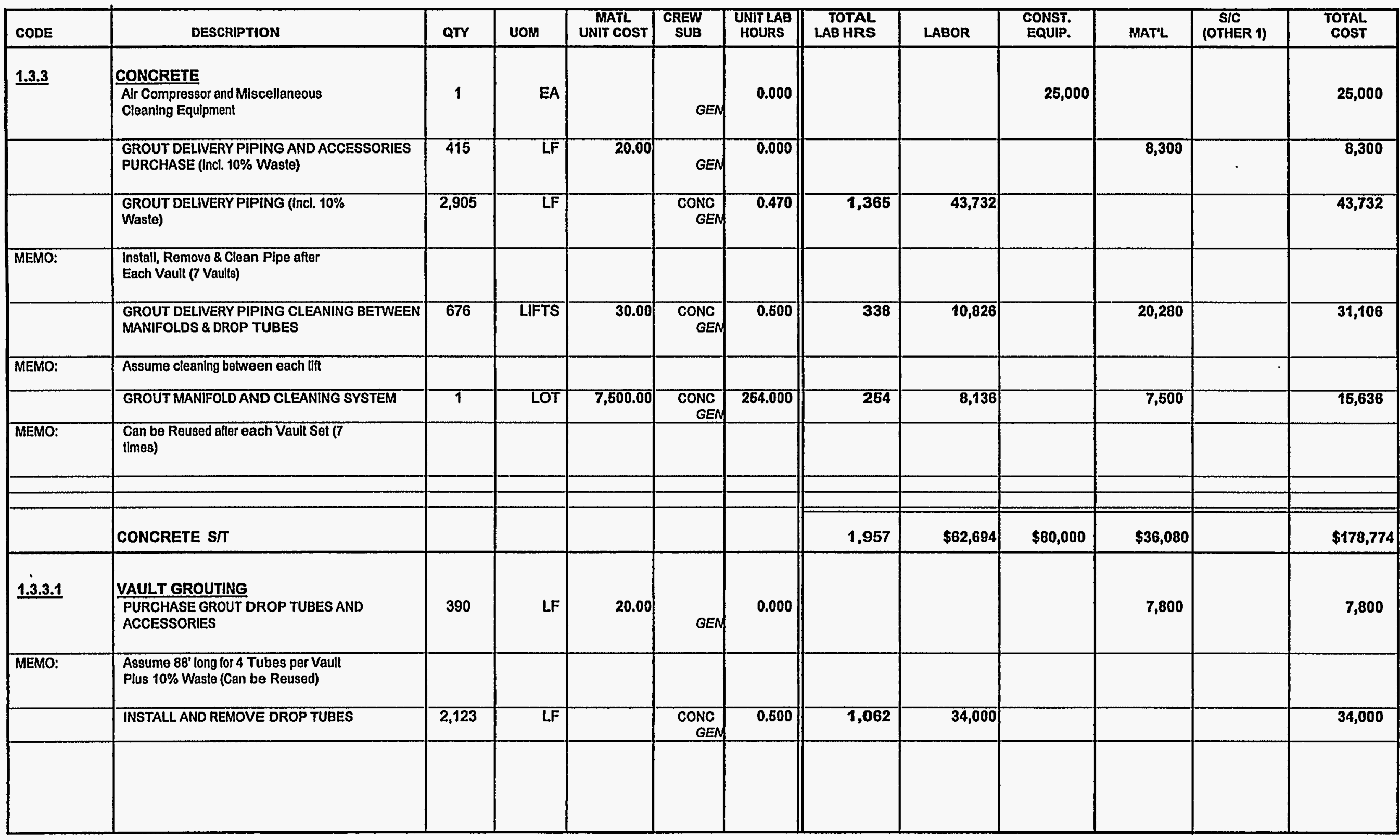


Lockheed Martin Idaho Technologies Co. Rev 6-S6

PROJECT NAME: ICPP Bin Set Closure (EIS Stud Place Clean Grout in Vault

LOCATION 1:

ICPP

REQUESTOR: Bryan Spaulding
CONTINGENCY ANALYSIS
TYPE OF ESTIMATE:

PROJECT NO PREPARED BY:
Planning

S. L. Coward
DATE: 28-Jan-1998

TIME: 11:58:53
REPORT NAME: Contingency Analysis

\begin{tabular}{|c|c|c|c|c|c|c|c|c|c|c|c|}
\hline \multicolumn{9}{|c|}{ PROBABLE \% VARIATION } & \multicolumn{2}{|c|}{$\begin{array}{l}\text { PROJECT } \\
\text { CONTINGENCY }\end{array}$} & \multirow{3}{*}{$\begin{array}{l}\text { SUMMARY } \\
\begin{array}{l}\text { Total Cost } \\
\text { by Element }\end{array}\end{array}$} \\
\hline \multirow{2}{*}{$\begin{array}{l}\text { WBS } \\
\text { Element }\end{array}$} & \multirow{2}{*}{ Cost Estimate Element } & \multirow{2}{*}{$\begin{array}{c}\text { Total Cost w/o } \\
\text { Contingency }\end{array}$} & \multirow[t]{2}{*}{$\begin{array}{l}\text { \% Total } \\
\text { Cost }\end{array}$} & \multicolumn{2}{|c|}{$\begin{array}{l}\text { Prob. \% Var. } \\
\text { From Est. }\end{array}$} & \multicolumn{2}{|c|}{ Wt. \% of Prob. } & \multirow{2}{*}{ Contingency } & \multirow{2}{*}{$\%$} & \multirow{2}{*}{ Cost } & \\
\hline & & & & - & + & - & + & & & & \\
\hline 1.1 .1 & DESIGN ENGINEERING & $1,324,812$ & 7.68 & 10 & 40 & 0.77 & 3.07 & $2.687 \%$ & $7.68 \%$ & 464,170 & $1,788,982$ \\
\hline 1.1 .2 & TITLE III INSPECTION & 264,962 & 1.54 & 10 & 40 & 0.15 & 0.61 & $0.537 \%$ & $1.54 \%$ & 92,834 & 357,796 \\
\hline 1.2 .1 & PROJECT MANAGEMENT & $1,692,265$ & 9.81 & 10 & 40 & 0.98 & 3.92 & $3.433 \%$ & $9.81 \%$ & 592,914 & $2,285,179$ \\
\hline 1.2 .2 & CONSTRUCTION MANAGEMENT & 529,925 & 3.07 & 10 & 40 & 0.31 & 1.23 & $1.075 \%$ & $3.07 \%$ & 185,668 & 715,593 \\
\hline 1.3 .1 & GENERAL CONDITIONS & $1,067,791$ & 6.19 & 10 & 40 & 0.62 & 2.48 & $2.166 \%$ & $6.19 \%$ & 374,119 & $1,441,910$ \\
\hline 1.3 .2 & SITEWORK & 0 & 0.00 & 0 & 0 & 0.00 & 0.00 & $0.000 \%$ & $0.00 \%$ & 0 & 0 \\
\hline 1.3 .3 & CONCRETE & $4,231,459$ & 24.52 & 10 & 40 & 2.45 & 9.81 & $8.583 \%$ & $24.52 \%$ & $1,482,563$ & $5,714,022$ \\
\hline \multirow[t]{2}{*}{1.5 .2} & PROCUREMENT FEES & 52,992 & 0.31 & 10 & 40 & 0.03 & 0.12 & $0.107 \%$ & $0.31 \%$ & 18,567 & 71,559 \\
\hline & ESCALATION & $8,090,364$ & 46.89 & 10 & 40 & 4.69 & 18.76 & $16.411 \%$ & $46.89 \%$ & $2,834,595$ & $10,924,959$ \\
\hline & SUBTOTAL & $17,254,570$ & 100.00 & & & & & $35.000 \%$ & & & \\
\hline & CALCULATED CONTINGENCY & $6,039,100$ & & & & & & & & & \\
\hline & RESULTANT TEC & $23,293,670$ & & & & & & & & & \\
\hline & ROUNDED TEC & $23,300,000$ & & & & & & & & & \\
\hline & PROJECT CONTINGENCY & $6,045,430$ & & & & & & $35.04 \%$ & & & \\
\hline & MANAGEMENT RESERVE & $1,049,039$ & & & & & & & & & \\
\hline & CONTINGENCY & $4,996,391$ & & & & & & & & & \\
\hline & TOTAL ESTIMATED COST & $23,300,000$ & & & & & & & & $6,045,430$ & $23,300,000$ \\
\hline
\end{tabular}

CONFIDENCE LEVEL AND ASSUMED RISKS:

The Lockheed Idaho Technologies Co. Cost Estimate Contingency Analysis Model is based on the applied contingency and the assumptions upon which the estimate was predicated. The model is applied with a suggested risk level of $18 \%$ and a level of confidence of $90 \%$ the estimate will fall within the bid range. The Contingency Analysis is based on a weighted average to provide a

$90 \%$ probability of undemun and a $10 \%$ probability of overnun.
CONTINGENCY ANALYSIS GUIDE BY TYPE OF ESTIMATE

Guidelines established by DOE/FM 50, Cost Estimating Guide, Vol. 6 , Cost Guide, and as presented in the INEL Cost Estimating Guide.

PLANNING Conceptual

$15 \%-25 \%$

TITLE I

TITLE II

TITLE IIIAFC
.... Up to $40 \%$

$10 \%-20 \%$

$5 \%-15 \%$

Market Conditions 


\section{G\&APIF ADDER CALCULATION SHEET \\ ICPP BIN SET CLOSURE \\ RISK BASED CLEAN CLOSURE; CLASS C FILL; ESCALATED}

FILL VAULTS WI CLEAN GROUT

PROCUREMENT FEE:

$\begin{aligned} & \text { CONSTRUCTION }= \\ & \text { GFE }=\end{aligned} \quad \begin{aligned} & \$ 10,386,530 \\ & \text { Subtotal } \\ & \text { FEE @ 1\%= }\end{aligned} \quad \$ 10,386,530$

G\&A @ 23\% (with a ceiling of $\$ 500,000$ imposed per year, 7 yrs)

CONSTRUCTION OR

CEILING $\bullet 7$ YEARS $=$

GFE $=$

$\$ 3,500,000$

PROCUREMENT FEE =

$\$ 103,865$

Subtotal $\$ 3,603,865$

FEE @ 23\% =

$\$ 3,603,865 * 0.23=$

$\$ 828,889$

PIF @ 5.5\%

CONSTRUCTION =
GFE =
PROCUREMENT FEE =
G\&A =

Subtotal
$\$ 5,129,992$

\$0

$\$ 103,865$

$\$ 828,889$

$\$ 6,062,746$

FEE @ 5.5\% =

$\$ 6,062,746 \cdot 0.055=$

$\$ 333,451$

TOTAL PROCUREMENT FEE:

$\$ 103,865$

TOTAL G\&A FEE:

$\$ 828,889$

TOTAL PIF:

$\$ 333,451$ 
Lockheed Martin Idaho Technologies Co. Rov. 6-96 PROJECT NAME: ICPP Bin Set Closure (EIS Stud LOCATION 1: Tractor (Risk Based Estimates) REQUESTOR
COST ESTIMATE SUMMARY

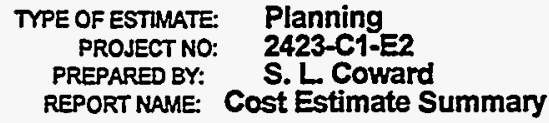

TMM

CHECKED BY:

APPR'D BY

\begin{tabular}{|c|c|c|c|c|}
\hline $\begin{array}{l}\text { WBS } \\
\text { Element }\end{array}$ & Cost Estimate Element & $\begin{array}{c}\text { Total } \\
\text { Unescalated }\end{array}$ & Escalation & $\begin{array}{c}\text { Total } \\
\text { Incl Escalation }\end{array}$ \\
\hline $\begin{array}{l}\frac{1.1}{1.1 .1} \\
1.1 .2 \\
\frac{1.2}{1.2 .1} \\
1.2 .2 \\
\frac{1.3}{1.3 .1} \\
1.3 .13 \\
1.5 .2\end{array}$ & $\begin{array}{l}\text { ENGINEERING, DESIGN AND INSPECTION } \\
\text { DESIGN ENGINEERING TITLE I \& II } \\
\text { TITLE III INSPECTION } \\
\text { MANAGEMENT COSTS } \\
\text { PROJECT MANAGEMENT } \\
\text { CONSTRUCTION MANAGEMENT } \\
\text { CONSTRUCTION } \\
\text { GENERAL CONDITIONS } \\
\text { SPECIAL CONSTRUCTION } \\
\text { PROCUREMENT FEES }\end{array}$ & $\begin{array}{r}9,900,244 \\
3,300,081 \\
\\
11,840,340 \\
6,600,163 \\
\\
6,840,414 \\
59,161,216 \\
660,016\end{array}$ & $\begin{array}{r}4,455,110 \\
3,366,083 \\
10,774,709 \\
6,732,166 \\
\\
6,977,222 \\
60,344,441 \\
673,217\end{array}$ & $\begin{array}{r}\gg>\begin{array}{r}\$ 21,021,518 \\
14,355,354 \\
6,666,164\end{array} \\
\gg>\frac{\$ 35,947,378}{22,615,049} \\
13,332,329 \\
\\
\Rightarrow>\frac{\$ 133,323,293}{13,817,636} \\
119,505,657 \\
\end{array}$ \\
\hline & $\begin{array}{l}\text { SUBTOTAL INCLUDING ESCALATION } \\
\text { PROJECT CONTINGENCY }\end{array}$ & $98,302,474$ & $93,322,948$ & $\gg>\$ 191,625,422$ \\
\hline & MANAGEMENT RESERVE- & & & $\$ 13,465,653$ \\
\hline & CONTINGENCY- & & & $\gg \quad \$ 53,608,925$ \\
\hline & TOTAL ESTIMATED COST - & & & $\gg \$ 258,700,000$ \\
\hline
\end{tabular}

PROJECT COST PARAMETERS

EDI AS A \% OF CONST. + GFE $=16.00 \%$

CONTINGENCY $=35.00 \%$ 
Lockheed Martin Idaho Technologies Co.

Rev 6.96

PROJECT NAME: ICPP BIn Set Closure (EIS Study) Tractor (Risk Based Estimates)

LOCATION 1: ICPP

REQUESTOR: Bryan Spaulding

\section{DETAILED COST ESTIMATE SHEET}

TYPE OF ESTIMATE: Planning PROJECT NO:: 2423-C1-E2 PREPARED BY: S. L. Coward
PAGE \# 3

DATE 28-Jan-1998

TIME: 11:15:47 REPORT NAME: Detall Cost Estimate Sheet

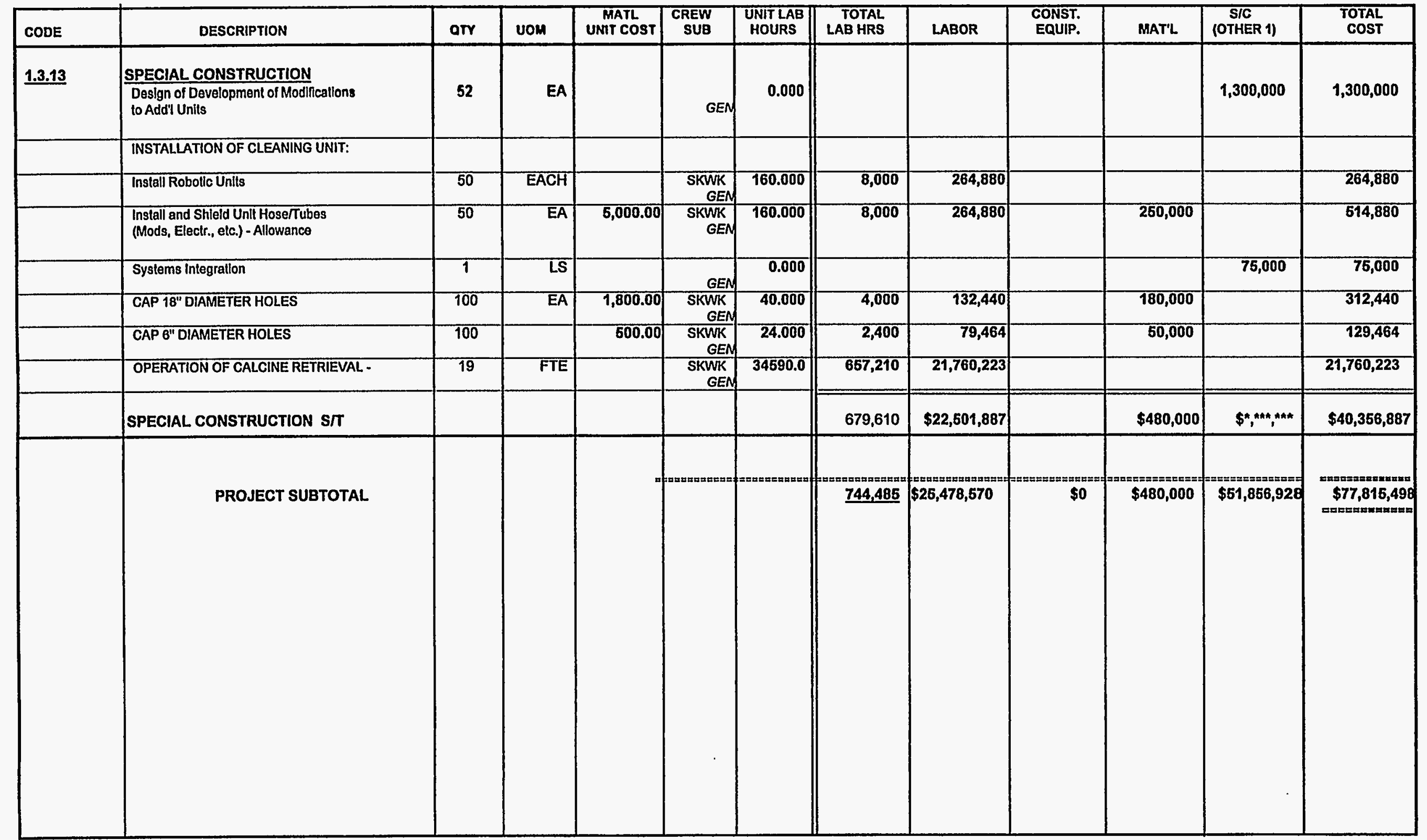


Lockheed Martin Idaho Technologies Co. Rev6.96 PROJECT NAME: ICPP Bin Set Closure (EIS Stud

LOCATION 1: Tractor (Risk Based Estimates) REQUESTOR: ICPP

Bryan Spaulding
CONTINGENCY ANALYSIS

$\begin{array}{cl}\text { TYPE OF ESTIMATE: } & \text { Planning } \\ \text { PROJECT NO: } & \text { 2423-C1-E2 } \\ \text { PREPARED BY: } & \text { S.L. Coward }\end{array}$

DATE: 28-Jan-1998

TME: 11:15:42

REPORT NAME: Contingency Analysis

\begin{tabular}{|c|c|c|c|c|c|c|c|c|c|c|c|}
\hline \multicolumn{9}{|c|}{ PROBABLE \% VARIATION } & \multicolumn{2}{|c|}{$\begin{array}{c}\text { PROJECT } \\
\text { CONTINGENCY }\end{array}$} & \multirow{3}{*}{$\begin{array}{l}\text { SUMMARY } \\
\begin{array}{l}\text { Total Cost } \\
\text { by Element }\end{array}\end{array}$} \\
\hline \multirow[t]{2}{*}{$\begin{array}{l}\text { WBS } \\
\text { Element }\end{array}$} & \multirow[t]{2}{*}{ Cost Estimate Element } & \multirow{2}{*}{$\begin{array}{c}\text { Total Cost w/o } \\
\text { Contingency }\end{array}$} & \multirow[t]{2}{*}{$\begin{array}{l}\text { \% Total } \\
\text { cost }\end{array}$} & \multicolumn{2}{|c|}{$\begin{array}{l}\text { Prob. \% Var. } \\
\text { From Est. }\end{array}$} & \multicolumn{2}{|c|}{ Wt. \% of Prob. } & \multirow[t]{2}{*}{ Contingency } & \multirow[t]{2}{*}{$\%$} & \multirow[t]{2}{*}{ Cost } & \\
\hline & & & & - & + & - & + & & & & \\
\hline 1.1.1 & DESIGN ENGINEERING TITLE I \& $\|$ & $9,900,244$ & 5.17 & 10 & 40 & 0.52 & 2.07 & $1.808 \%$ & $5.17 \%$ & $3,465,379$ & $13,365,623$ \\
\hline 1.1 .2 & TITLE III INSPECTION & $3,300,081$ & 1.72 & 10 & 40 & 0.17 & 0.69 & $0.603 \%$ & $1.72 \%$ & $1,155,126$ & $4,455,207$ \\
\hline 1.2 .1 & PROJECT MANAGEMENT & $11,840,340$ & 6.18 & 10 & 40 & 0.62 & 2.47 & $2.163 \%$ & $6.18 \%$ & $4,144,470$ & $15,984,810$ \\
\hline 1.2 .2 & CONSTRUCTION MANAGEMENT & $6,600,163$ & 3.44 & 10 & 40 & 0.34 & 1.38 & $1.206 \%$ & $3.44 \%$ & $2,310,253$ & $8,910,416$ \\
\hline 1.3 .1 & GENERAL CONDITIONS & $6,840,414$ & 3.57 & 10 & 40 & 0.36 & 1.43 & $1.249 \%$ & $3.57 \%$ & $2,394,348$ & $9,234,762$ \\
\hline 1.3 .13 & SPECIAL CONSTRUCTION & $59,161,216$ & 30.87 & 10 & 40 & 3.09 & 12.35 & $10.806 \%$ & $30.87 \%$ & $20,708,179$ & $79,869,395$ \\
\hline \multirow[t]{2}{*}{1.5 .2} & PROCUREMENT FEES & 660,016 & 0.34 & 10 & 40 & 0.03 & 0.14 & $0.121 \%$ & $0.34 \%$ & 231,025 & 891,041 \\
\hline & ESCALATION & $93,322,948$ & 48.70 & 10 & 40 & 4.87 & 19.48 & $17.045 \%$ & $48.70 \%$ & $32,665,798$ & $125,988,746$ \\
\hline & SUBTOTAL & $191,625,422$ & 100.00 & & & & & $35.000 \%$ & & & \\
\hline & CALCULATED CONTINGENCY & $67,068,898$ & & & & & & & & & \\
\hline & RESULTANT TEC & $258,694,320$ & & & & & & & & & \\
\hline & ROUNDED TEC & $258,700,000$ & & & & & & & & & \\
\hline & PROJECT CONTINGENCY & $67,074,578$ & & & & & & $35.00 \%$ & & & \\
\hline & MANAGEMENT RESERVE & $13,465,653$ & & & & & & & & & \\
\hline & CONTINGENCY & $53,608,925$ & & & & & & & & & \\
\hline & TOTAL ESTIMATED COST & $258,700,000$ & & & & & & & & $67,074,578$ & $258,700,000$ \\
\hline
\end{tabular}

\section{CONFIDENCE LEVEL AND ASSUMED RISKS:}

The Lockheed Idaho Technologies Co. Cost Estimate Contingency Analysis Model is based on the applied contingency and the assumptions upon which the estimate was predicated. The model is applied with a suggested risk level of $18 \%$ and a level of confidence of $90 \%$ the estimate will fall within the bid range. The Contingency Analysis is based on a weighted average to provide a $90 \%$ probability of underrun and a $10 \%$ probability of overrun.
CONTINGENCY ANALYSIS GUIDE BY TYPE OF ESTIMATE Guidelines established by DOE/FM 50 , Cost Estimating Guide, Vol. 6 , Cost Guide, and as presented in the INEL Cost Estimating Guide. PLANNING ExperimentauSpecial Conditions $20 \%-30 \%$ Conceptual Experimental/Special Conditions. TITLE I

TITLE II TITLE IVAFC
...Up to $40 \%$ $10 \%-20 \%$

$5 \%-15 \%$

Market Conditions 


\section{G\&A/PIF ADDER CALCULATION SHEET \\ ICPP BIN SET CLOSURE \\ RISK BASED CLEAN CLOSURE; CLASS C FILL; ESCALATED \\ CLEAN BINS W/ ROBOTS - FLOOR}

PROCUREMENT FEE:

$\begin{aligned} & \text { CONSTRUCTION }= \\ & \text { GFE }=\end{aligned}$
Subtotal

G\&A @ 23\% (with a ceiling of $\$ 500,000$ imposed per year, 10 yrs)

CONSTRUCTION OR

CEILING 10 YEARS $=\quad \$ 5,000,000$

GFE $=$

PROCUREMENT FEE =

$\$ 1,333,233$

Subtotal $\$ 6,333,233$

FEE @ 23\% =

$\$ 6,333,233 * 0.23=$

$\$ 1,456,644$

PIF @ 5.5\%

\begin{tabular}{|c|c|}
\hline $\begin{array}{l}\text { CONSTRUCTION = } \\
\text { GFE = } \\
\text { PROCUREMENT FEE = } \\
\text { G\&A = }\end{array}$ & $\begin{array}{r}\$ 66,001,630 \\
\$ 0 \\
\$ 1,333,233 \\
\$ 1,456,644 \\
\$ 68,791,507\end{array}$ \\
\hline FEE @ 5.5\%= & $\$ 68,791,507 \cdot 0.055=$ \\
\hline
\end{tabular}

TOTAL PROCUREMENT FEE:

$\$ 1,333,233$

TOTAL G\&A FEE:

$\$ 1,456,644$

TOTAL PIF:

$\$ 3,783,533$ 
Lockheed Martin Idaho Technologies Co. Rev. 6-96

PROJECT NAME: ICPP Bin Set Closure (EIS Stud

LOCATION 1: LDUA (Risk Based Estimates)

REQUESTOR: Bryan Spaulding
COST ESTIMATE SUMMARY

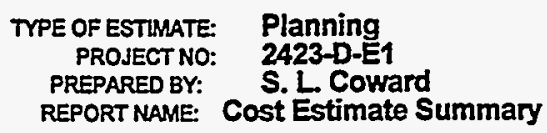

DATE: 28-Jan-1998

CHECKED BY:

APPR'DBY:

\begin{tabular}{|c|c|c|c|c|}
\hline $\begin{array}{l}\text { WBS } \\
\text { Element }\end{array}$ & Cost Estimate Element & $\begin{array}{c}\text { Total } \\
\text { Unescalated }\end{array}$ & Escalation & $\begin{array}{c}\text { Total } \\
\text { Incl Escalation }\end{array}$ \\
\hline $\begin{array}{l}\frac{1.1}{1.1 .1} \\
1.1 .2 \\
\frac{1.2}{1.2 .1} \\
1.2 .2 \\
\frac{1.3}{1.3 .1} \\
1.3 .13 \\
1.5 .2\end{array}$ & $\begin{array}{l}\text { ENGINEERING, DESIGN AND INSPECTION } \\
\text { DESIGN ENGINEERING TITLE I \& II } \\
\text { TITLE III INSPECTION } \\
\text { MANAGEMENT COSTS } \\
\text { PROJECT MANAGEMENT } \\
\text { CONSTRUCTION MANAGEMENT } \\
\text { CONSTRUCTION } \\
\text { GENERAL CONDITIONS } \\
\text { SPECIAL CONSTRUCTION } \\
\text { PROCUREMENT FEES }\end{array}$ & $\begin{array}{r}2,083,513 \\
694,504 \\
\\
3,447,249 \\
2,463,481 \\
\cdot \\
1,831,085 \\
12,059,014 \\
138,901\end{array}$ & $\begin{array}{r}937,581 \\
666,724 \\
2,964,634 \\
2,364,942 \\
\\
1,757,841 \\
11,576,653 \\
133,345\end{array}$ & $\begin{array}{r}\gg>\begin{array}{r}\$ 4,382,322 \\
3,021,094 \\
1,361,228\end{array} \\
\gg>\begin{array}{r}\$ 11,240,306 \\
6,411,883 \\
4,828,423\end{array} \\
\gg>\frac{\$ 27,224,593}{3,588,926} \\
23,635,667\end{array}$ \\
\hline & $\begin{array}{l}\text { SUBTOTAL INCLUDING ESCALATION } \\
\text { PROJECT CONTINGENCY }\end{array}$ & $22,717,747$ & $20,401,720$ & >> \$43,119,467 \\
\hline & MANAGEMENT RESERVE- & & & $\$ 2,749,684$ \\
\hline & CONTINGENCY - & & & $\gg \$ 12,330,849$ \\
\hline & TOTAL ESTIMATED COST - & & & $\$ 58,200,000$ \\
\hline
\end{tabular}

PROJECT COST PARAMETERS

EDI AS A \% OF CONST. + GFE $=16.00 \%$

CONTINGENCY $=34.97 \%$ 
Lockheed Martin Idaho Technologies Co.

Rev 6.96

PROJECT NAME: ICPP Bin Set Closure (EIS Study)

LDUA (Risk Based Estimates)

LOCATION 1: ICPP

REQUESTOR: Bryan Spaulding

\section{DETAILED COST ESTIMATE SHEET}

TYPE OF EStIMATE: Planning

PROJECT NO: 2423-D-E1

PREPARED BY: S. L. Coward
PAGE \# 1

DATE 28-Jan-1998

TIME: 11:25:30

REPORT NAME: Detall Cost Estimate Sheet

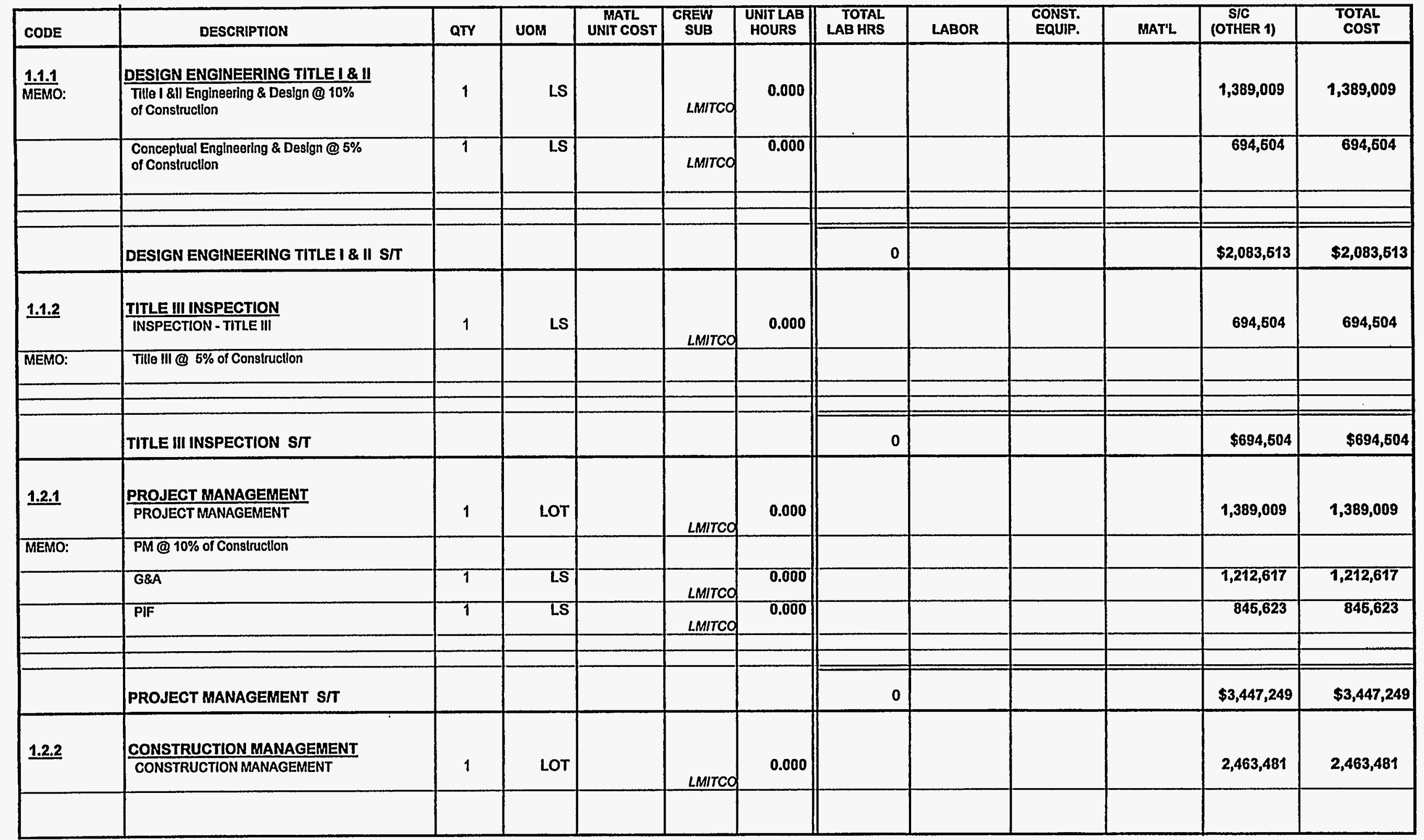


Lockheed Martln Idaho Technologies Co. Rav6.96

PROJECT NAME: ICPP BIn Set Closure (EIS Study) LDUA (Risk Based Estimates)

LOCATION 1: ICPP

ICPP

REQUESTOR: Bryan Spaulding

\section{DETAILED COST ESTIMATE SHEET}

TYPE OF ESTIMATE: Planning

PROJECTNO:: 2423-D-E1

PREPARED BY: S. L. Coward
PAGE\# 3

DATE 28-Jan-1998

TIME: 11:25:30

REPORT NAME: Detall Cost Estimate Sheet

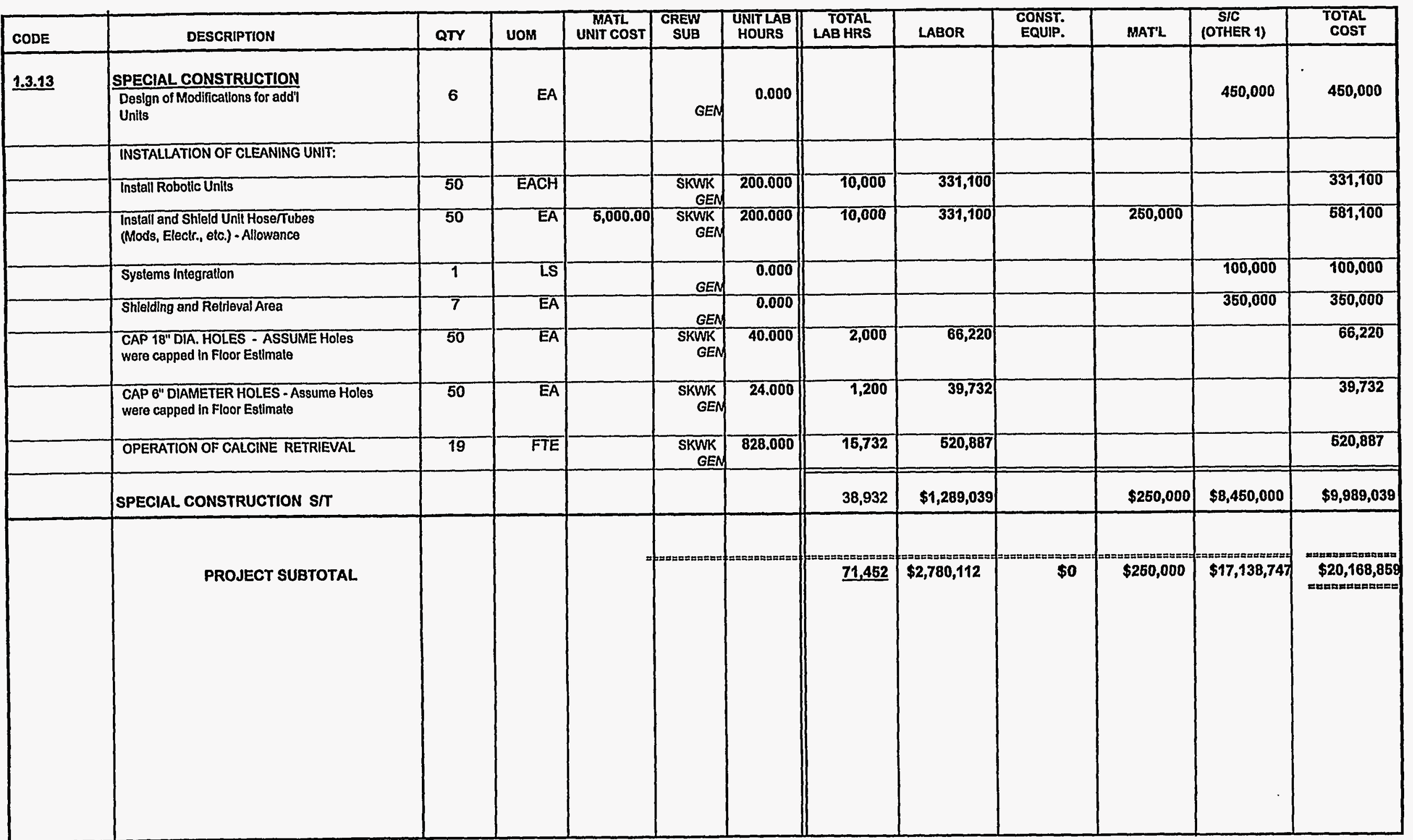


Lockheed Martin Idaho Technologies Co. PROJECT NAME: ICPP Bin Set Closure (EIS Stud

LDUA (Risk Based Estimates)

LOCATION 1: REQUESTOR:
Bryan Spaulding
CONTINGENCY ANALYSIS

\author{
TYPE OF ESTIMATE: \\ PROJECTNO: Planning \\ PREPARED BY: \\ 2423-D-E1 \\ S. L. Coward
}

DATE: 28-Jan-1998

TIME: 11:25:25

REPORT NAME: Contingency Analysis

\begin{tabular}{|c|c|c|c|c|c|c|c|c|c|c|c|}
\hline & PROE & BLE \% VARIA & 1ON & & & & & & $\begin{array}{l}\text { PRO } \\
\text { CONT }\end{array}$ & $\begin{array}{l}\text { CT } \\
\text { IENCY }\end{array}$ & SUMMARY \\
\hline $\begin{array}{l}\text { WBS } \\
\text { Element }\end{array}$ & Cost Estimate Element & Total Cost w/o & $\begin{array}{l}\% \text { Total } \\
\text { Cost }\end{array}$ & & $\begin{array}{l}\% \text { Var. } \\
\text { m Est. }\end{array}$ & Wt. \% & Prob. & Contingency & $\%$ & Cost & Total Cost \\
\hline & & Contingency & & - & + & - & + & & & & by Element \\
\hline 1.1 .1 & DESIGN ENGINEERING TITLE I \& II & $2,083,513$ & 4.83 & 10 & 40 & 0.48 & 1.93 & $1.691 \%$ & $4.83 \%$ & 728,684 & $2,812,197$ \\
\hline 1.1 .2 & TITLE III INSPECTION & 694,504 & 1.61 & 10 & 40 & 0.16 & 0.64 & $0.564 \%$ & $1.61 \%$ & 242,895 & 937,399 \\
\hline 1.2 .1 & PROJECT MANAGEMENT & $3,447,249$ & 7.99 & 10 & 40 & 0.80 & 3.20 & $2.798 \%$ & $7.99 \%$ & $1,205,635$ & $4,652,884$ \\
\hline 1.2 .2 & CONSTRUCTION MANAGEMENT & $2,463,481$ & 5.71 & 10 & 40 & 0.57 & 2.20 & $2.000 \%$ & $5.71 \%$ & 861,574 & $3,325,055$ \\
\hline 1.3 .1 & GENERAL CONDITIONS & $1,831,085$ & 4.25 & 10 & 40 & 0.42 & 1.70 & $1.486 \%$ & $4.25 \%$ & 640,401 & $2,471,486$ \\
\hline 1.3 .13 & SPECLAL CONSTRUCTION & $12,059,014$ & 27.97 & 10 & 40 & 2.80 & 11.19 & $9.788 \%$ & $27.97 \%$ & $4,217,500$ & $16,276,514$ \\
\hline 1.5 .2 & PROCUREMENT FEES & 138,901 & 0.32 & 10 & 40 & 0.03 & 0.13 & $0.113 \%$ & $0.32 \%$ & 48,579 & 187,480 \\
\hline & ESCALATION & $20,401,720$ & 47.31 & 10 & 40 & 4.73 & 18.93 & $16.560 \%$ & $47.31 \%$ & $7,135,265$ & $27,536,985$ \\
\hline & SUBTOTAL & $43,119,467$ & 100.00 & & & & & $35.000 \%$ & & & \\
\hline & CALCULATED CONTINGENCY & $15,091,813$ & & & & & & & & & \\
\hline & RESULTANT TEC & $58,211,280$ & & & & & & & & & \\
\hline & ROUNDED TEC & $58,200,000$ & & & & & & & & & \\
\hline & PROJECT CONTINGENCY & $15,080,533$ & & & & & & $34.97 \%$ & & & \\
\hline & MANAGEMENT RESERVE & $2,749,684$ & & & & & & & & & \\
\hline & CONTINGENCY & $12,330,849$ & & & & & & & & & \\
\hline & TOTAL ESTIMATED COST & $58,200,000$ & & & & & & & & $15,080,533$ & $58,200,000$ \\
\hline
\end{tabular}

CONFIDENCE LEVEL AND ASSUMED RISKS:

The Lockheed Idaho Technologies Co. Cost Estimate Contingency Analysis Model is based on the applied contingency and the assumptions upon which the estimate was predicated. The model is applied with a suggested risk level of $18 \%$ and a level of confidence of $90 \%$ the estimate will fall within the bid range. The Contingency Analysis is based on a weighted average to provide a

$90 \%$ probability of underrun and a $10 \%$ probability of overrun.
CONTINGENCY ANALYSIS GUIDE BY TYPE OF ESTIMATE

Guidelines established by DOE/FM 50 , Cost Estimating Guide, Vol. 6 , Cost Guide, and as presented in the INEL Cost Estimating Guide.

PLANNING Conceptual

$15 \%-25 \%$

TITLE

TITLE II

TITLE IVAFC
$10 \%-20 \%$

$5 \%-15 \%$

Market Conditions 


\section{G\&APIF ADDER CALCULATION SHEET ICPP BIN SET CLOSURE}

RISK BASED CLEAN CLOSURE; CLASS C FILL; ESCALATED

CLEAN BINS W/ ROBOTS - WALLS

PROCUREMENT FEE:

$\begin{aligned} & \text { CONSTRUCTION }= \\ & \text { GFE }=\end{aligned}$ Subtotal $\frac{\$ 27,224,593}{\$ 27,224,593}$

FEE @ 1\%= $\$ 27,224,593 * 0.01=$

G\&A @ 23\% (with a ceiling of $\$ 500,000$ imposed per year, 10 yrs)

CONSTRUCTION OR

CEILING * 10 YEARS $=\quad \$ 5,000,000$

GFE = \$0

PROCUREMENT FEE $=$ $\$ 272,246$

Subtotal $\$ 5,272,246$

FEE @ 23\%=

PIF @ 5.5\%

\begin{tabular}{lr} 
CONSTRUCTION $=$ & $\begin{array}{r}\$ 13,890,099 \\
\text { GFE }=\end{array}$ \\
PROCUREMENT FEE $=$ & $\$ 0$ \\
G\&A $=\quad \begin{array}{r}\$ 272,246 \\
\end{array} \quad$ Subtotal & $\$ 1,212,617$ \\
\cline { 2 - 2 } & $\$ 15,374,961$
\end{tabular}

FEE @ $5.5 \%=$ 
Lockheed Martin Idaho Technologies Co.

Rov. 6-56

PROJECT NAME: ICPP Bin Sot Closure (EIS Stud

LOCATION 1:

REQUESTOR:
ICPP

Bryan Spaulding
COST ESTIMATE SUMMARY

TYPE OF ESTIMATE: Planning

PROJECT NO: 2423-E-E1

PREPARED BY: S. L Coward

REPORT NAME: Cost Estimate Summary

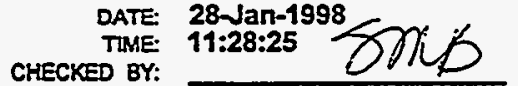

APPRD BY:

\begin{tabular}{|c|c|c|c|c|}
\hline $\begin{array}{l}\text { WBS } \\
\text { Element }\end{array}$ & Cost Estimato Element & $\begin{array}{c}\text { Total } \\
\text { Unescalated }\end{array}$ & Escalation & $\begin{array}{l}\text { Total } \\
\text { Incl Escalation }\end{array}$ \\
\hline $\begin{array}{l}\frac{1.1}{1.1 .1} \\
1.1 .2 \\
\frac{1.2}{1.2 .1} \\
1.2 .2 \\
\frac{1.3}{1.3 .1} \\
1.3 .13 \\
1.5 .2\end{array}$ & $\begin{array}{l}\text { ENGINEERING, DESIGN AND INSPECTION } \\
\text { DESIGN ENGINEERING } \\
\text { TITLE III INSPECTION } \\
\text { MANAGEMENT COSTS } \\
\text { PROJECT MANAGEMENT } \\
\text { CONSTRUCTION MANAGEMENT } \\
\text { CONSTRUCTION } \\
\text { GENERAL CONDITIONS } \\
\text { SPECLAL CONSTRUCTION } \\
\text { PROCUREMENT FEES }\end{array}$ & $\begin{array}{r}1,778,336 \\
296,389 \\
2,166,640 \\
592,779 \\
\\
1,831,085 \\
4,096,709 \\
59,278\end{array}$ & $\begin{array}{r}800,251 \\
284,533 \\
1,863,310 \\
569,068 \\
\\
1,757,841 \\
3,932,840 \\
56,907\end{array}$ & $\begin{array}{r}\text { \3,159,509} \\
2,578,587 \\
580,922\end{array}$ \\
\hline . & $\begin{array}{l}\text { SUBTOTAL INCLUDING ESCALATION } \\
\text { PROJECT CONTINGENCY }\end{array}$ & $10,821,216$ & $9,264,750$ & $\gg \$ 20,085,966$ \\
\hline & MANAGEMENT RESERVE- & & & $\gg \quad \$ 1,173,466$ \\
\hline & CONTINGENCY - & & & $\$ 5,840,568$ \\
\hline & TOTAL ESTIMATED COST - & & & $\$ 27,100,000$ \\
\hline
\end{tabular}

\section{PROJECT COST PARAMETERS}

EDI AS A \% OF CONST. + GFE $=27.00 \%$ 
ockheed Martin Idaho Technologies Co. Rav 6-98
ROJECT NAME: ICPP BIn Sot Closure (EIS Study) Pipe Crawler (Risk Based Estlmates) ICPP EQUESTOR: Bryan Spaulding

\section{DETAILED COST ESTIMATE SHEET}

TYPE OF ESTIMATE: Planning

PROJECT NO: 2423-E-E1

PREPARED BY: S. L. Coward
PAGE 1

DATE 28-Jan-1998

TIME: 11:28:28

REPORT NAME: Detall Cost Estimate Sheot

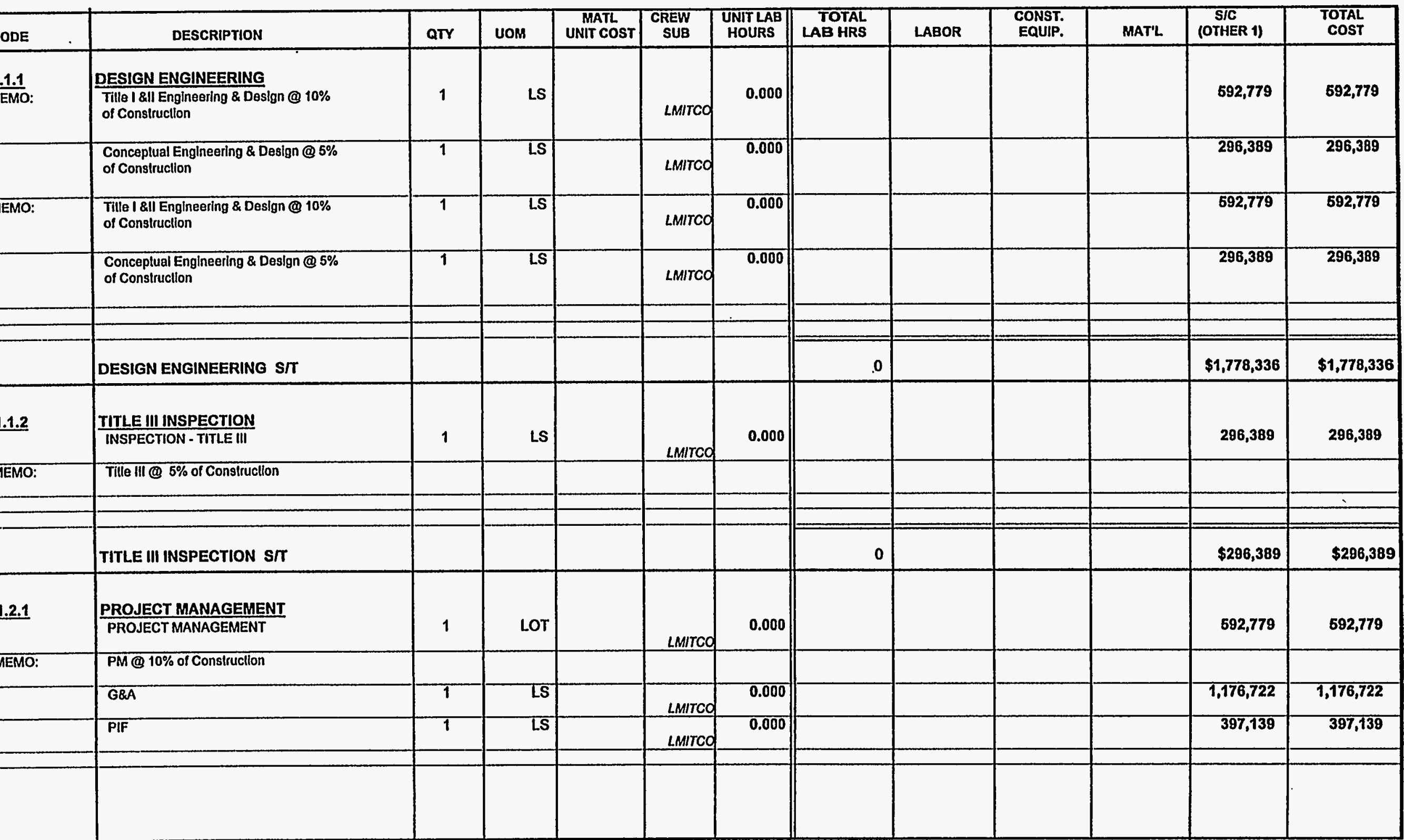


Lockheed Martin Idaho Technologies Co. Rev 6.96 PROJECT NAME: ICPP BIn Sot Closure (EIS Study) Plpo Crawler (RIsk Based Estimates) ICPP

LOCATION 1:

REQUESTOR: Bryan Spaulding
DETAILED COST ESTIMATE SHEET

TYPE OF ESTIMATE: Planning PROJECT NO.: 2423-E-E1

PREPARED BY: S. L. Coward
PAGE \# 3

DATE 28-Jan-1998

TIME: 11:28:28

REPORT NAME: Dotall Cost Estimate Shoet

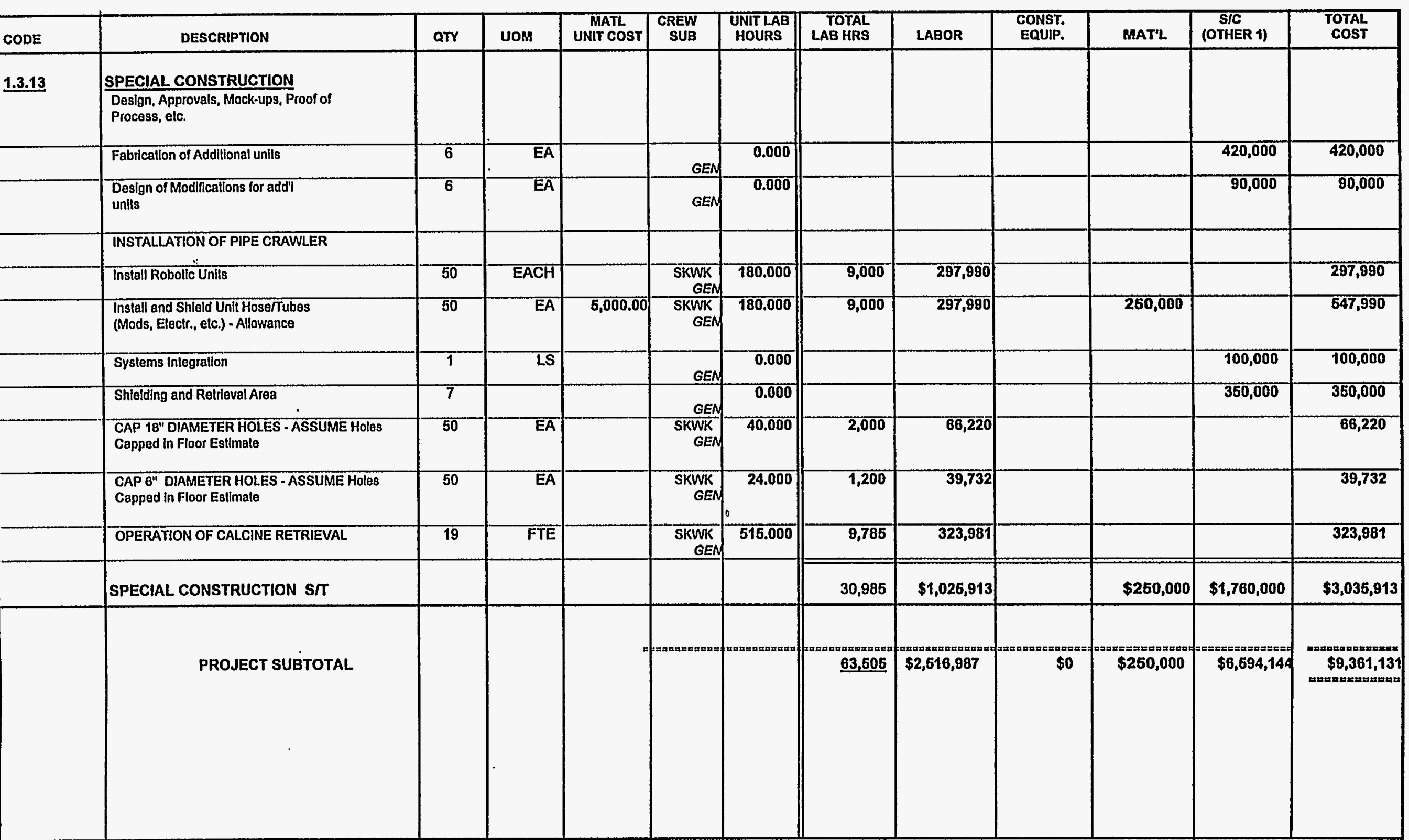


Lockheed Martin Idaho Technologies Co.

PROJECT NAME: ICPP Bin Set Closure (EIS Stud

LOCATION 1: ICPP

REQUESTOR: Bryan Spaulding
CONTINGENCY ANALYSIS
TYPE OF ESTIMATE: PROJECT NO: PREPARED BY:
Planning

2423-E-E1

S. L. Coward
DATE: 28-Jan-1998

TIME: 11:28:22
REPORT NAME: Contingency Analysis

\begin{tabular}{|c|c|c|c|c|c|c|c|c|c|c|c|}
\hline \multicolumn{9}{|c|}{ PROBABLE \% VARIATION } & \multicolumn{2}{|c|}{$\begin{array}{l}\text { PROJECT } \\
\text { CONTINGENCY }\end{array}$} & \multirow{3}{*}{$\begin{array}{l}\text { SUMMARY } \\
\begin{array}{l}\text { Total Cost } \\
\text { by Element }\end{array}\end{array}$} \\
\hline \multirow[t]{2}{*}{$\begin{array}{l}\text { WBS } \\
\text { Element }\end{array}$} & \multirow{2}{*}{ Cost Estimate Element } & \multirow{2}{*}{$\begin{array}{c}\text { Total Cost w/o } \\
\text { Contingency }\end{array}$} & \multirow[t]{2}{*}{$\begin{array}{l}\text { \% Total } \\
\text { Cost }\end{array}$} & \multicolumn{2}{|c|}{$\begin{array}{l}\text { Prob. \% Var. } \\
\text { From Est. }\end{array}$} & \multicolumn{2}{|c|}{ Wt $\%$ of Prob. } & \multirow[t]{2}{*}{ Contingency } & \multirow[t]{2}{*}{$\%$} & \multirow[t]{2}{*}{ Cost } & \\
\hline & & & & - & + & - & + & & & & \\
\hline 1.1 .1 & DESIGN ENGINEERING & $1,778,336$ & 8.85 & 10 & 40 & 0.89 & 3.54 & $3.099 \%$ & $8.85 \%$ & 620,996 & $2,399,332$ \\
\hline 1.1 .2 & TITLE III INSPECTION & 296,389 & 1.48 & 10 & 40 & 0.15 & 0.59 & $0.516 \%$ & $1.48 \%$ & 103,499 & 399,888 \\
\hline 1.21 & PROJECT MANAGEMENT & $2,166,640$ & 10.79 & 10 & 40 & 1.08 & 4.31 & $3.775 \%$ & $10.79 \%$ & 756,592 & $2,923,232$ \\
\hline 1.22 & CONSTRUCTION MANAGEMENT & 592,779 & 2.95 & 10 & 40 & 0.30 & 1.18 & $1.033 \%$ & $2.95 \%$ & 206,999 & 799,778 \\
\hline 1.3 .1 & GENERAL CONDITIONS & $1,831,085$ & 9.12 & 10 & 40 & 0.91 & 3.65 & $3.191 \%$ & $9.12 \%$ & 639,416 & $2,470,501$ \\
\hline 1.3 .13 & SPECLAL CONSTRUCTION & $4,096,709$ & 20.40 & 10 & 40 & 2.04 & 8.16 & $7.139 \%$ & $20.40 \%$ & $1,430,574$ & $5,527,283$ \\
\hline \multirow[t]{2}{*}{1.5 .2} & PROCUREMENT FEES & 59,278 & 0.30 & 10 & 40 & 0.03 & 0.12 & $0.103 \%$ & $0.30 \%$ & 20,700 & 79,978 \\
\hline & ESCALATTON & $9,264,750$ & 46.13 & 10 & 40 & 4.61 & 18.45 & $16.144 \%$ & $46.13 \%$ & $3,235,258$ & $12,500,008$ \\
\hline & SUBTOTAL & $20,085,966$ & 100.00 & & & & & $35.000 \%$ & & & \\
\hline & CALCULATED CONTINGENCY & $7,030,088$ & & & & & & & & & \\
\hline & RESULTANT TEC & $27,116,054$ & & & & & & & & & \\
\hline & ROUNDED TEC & $27,100,000$ & & & & & $\dot{-}$ & & & & \\
\hline & PROJECT CONTINGENCY & $7,014,034$ & & & & & & $34.92 \%$ & & & \\
\hline & MANAGEMENT RESERVE & $1,173,466$ & & & & & & & & & \\
\hline & CONTINGENCY & $5,840,568$ & & & & & & & & & \\
\hline & TOTAL ESTIMATED COST & $27,100,000$ & & & & & & & & $7,014,034$ & $27,100,000$ \\
\hline
\end{tabular}

\section{CONFIDENCE LEVEL AND ASSUMED RISKS:}

The Lockheed idaho Technologies Co. Cost Estimate Contingency Analysis Model is based on the applied contingency and the assumptions upon which the estimate was predicated. The model is applied with a suggested risk level of $18 \%$ and a level of confidence of $90 \%$ the estimate will fall within the bid range. The Contingency Analysis is based on a weighted average to provide a $90 \%$ probability of underrun and a $10 \%$ probability of overun.

\section{CONTINGENCY ANALYSIS GUIDE BY TYPE OF ESTIMATE}

Guidelines established by DOEIFM 50, Cost Estimating Guide, Vol. 6. Cost Guide, and as presented in the INEL Cost Estimating Guide.
PLANNING Experimental/Special Conditions............. up to $50 \%$ Conceptual

Experimental/Special Conditions.............. Up to $40 \%$ TITLEI

TITLE II

TITLE IIIAFC
$15 \%-25 \%$

$10 \%-20 \%$

$5 \%-15 \%$

Market Conditions 


\section{G\&APIF ADDER CALCULATION SHEET \\ ICPP BIN SET CLOSURE \\ RISK BASED CLEAN CLOSURE; CLASS C FILL; ESCALATED \\ CLEAN BINS W/ ROBOTS - PIPING}

PROCUREMENT FEE:

CONSTRUCTION $=$
GFE $=$
FEE @ $1 \%=$

G\&A @ 23\% (with a ceiling of $\$ 500,000$ imposed per year, $10 \mathrm{yrs}$ )

CONSTRUCTION OR

CEILING * 10 YEARS =

GFE =

PROCUREMENT FEE =

Subtotal
$\$ 5,000,000$

\$0

$\$ 116,185$

$\$ 5,116,185$

$\$ 5,116,185 \cdot 0.23=\quad \$ 1,176,722$

PIF @ 5.5\%

FEE @ 23\% =

$\$, 927,794$
$\$ 0$

CONSTRUCTION =

$\$ 116,185$

PROCUREMENT FEE =

$\$ 1,176,722$

Subtotal

$\$ 7,220,701$

FEE @ $5.5 \%=$

$\$ 7,220,701 * 0.055=$

$\$ 397,139$

TOTAL PROCUREMENT FEE:

$\$ 116,185$

TOTAL G\&A FEE:

$\$ 1,176,722$

TOTAL PIF:

$\$ 397,139$ 
Lockheed Martin Idaho Technologies Co. Rov, 6-96

PROJECT NAME: - ICPP Bin Set Closure (EIS Stud

LOCATION 1: Place NRC Class C Grout ICPP

REQUESTOR: Bryan Spaulding
COST ESTIMATE SUMMARY

$\begin{array}{cc}\text { TYPE OFESTIMATE: } & \text { Planning } \\ \text { PROJECT NO: } & 2423-\text { B-E1 } \\ \text { PREPARED BY: } & \text { S. L Coward } \\ \text { REPORT NAME: Cost Estimate Summary }\end{array}$

CHECKED BY:

DATE: 28-Jan-1998

TIME: 11:33:51

APPRO BY

\begin{tabular}{|c|c|c|c|c|}
\hline $\begin{array}{l}\text { WBS } \\
\text { Element }\end{array}$ & Cost Estimate Element & $\begin{array}{c}\text { Total } \\
\text { Unescalated }\end{array}$ & Escalation & $\begin{array}{c}\text { Total } \\
\text { Incl Escalation }\end{array}$ \\
\hline 1.1 & ENGINEERING, DESIGN AND INSPECTION & & & $\gg \quad \$ 7,746,789$ \\
\hline 1.1 .1 & DESIGN ENGINEERING TITLE I \& II & $3,282,538$ & $2,987,110$ & $6,269,648$ \\
\hline 1.1 .2 & TITLE III INSPECTION & 656,507 & 820,634 & $1,477,141$ \\
\hline 1.2 & MANAGEMENT COSTS & & & $\gg \quad \$ 9,437,874$ \\
\hline 1.2 .1 & PROJECT MANAGEMENT & $2,972,384$ & $3,537,137$ & $6,509,521$ \\
\hline 1.2 .2 & CONSTRUCTION MANAGEMENT & $1,301,490$ & $1,626,863$ & $2,928,353$ \\
\hline 1.3 & CONSTRUCTION & & & $>\$ 29.542 .856$ \\
\hline$\overline{1.3 .1}$ & GENERAL CONDITIONS & $3,437,150$ & $4,296,437$ & $7,733,587$ \\
\hline 1.3.2 & SITEWORK & $\mathbf{0}$ & 0 & $\mathbf{0}$ \\
\hline 1.3 .3 & CONCRETE & $3,876,603$ & $4,845,753$ & $8,722,356$ \\
\hline 1.3.15 & MECHANICAL. & $3,365,584$ & $4,206,980$ & $7,572,564$ \\
\hline 1.3.16 & ELECTRICAL & $2,450,822$ & $3,063,527$ & $5,514,349$ \\
\hline \multirow[t]{3}{*}{1.5 .2} & PROCUREMENT FEES & 131,302 & 164,127 & $\$ 295,429$ \\
\hline & SUBTOTAL INCLUDING ESCALATION & $21,474,380$ & $25,548,568$ & \multirow[t]{2}{*}{$\gg \$ 47,022,948$} \\
\hline & PROJECT CONTINGENCY & & & \\
\hline \multicolumn{5}{|r|}{ 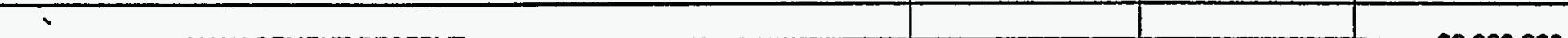 } \\
\hline$\cdot$ & MANAGEMENT RESERVE & & & $\$ 2,983,829$ \\
\hline \multicolumn{4}{|c|}{ CONTINGENCY } & $\gg \quad \$ 14,693,223$ \\
\hline \multicolumn{4}{|c|}{ TOTAL ESTIMATED COST - } & $\gg \quad \$ 64,700,000$ \\
\hline
\end{tabular}

PROJECT COST PARAMETERS

EDI AS A $\%$ OF CONST. + GFE $=26.00 \%$ CONTINGENCY $=37.59 \%$ 
Lockheed Martin Idaho Technologies Co.

Rov 6.96

PROJECT NAME: ICPP Bin Set Closure (EIS Study)

Place NRC Class C Grout

LOCATION 1: ICPP

REQUESTOR: Bryan Spaulding

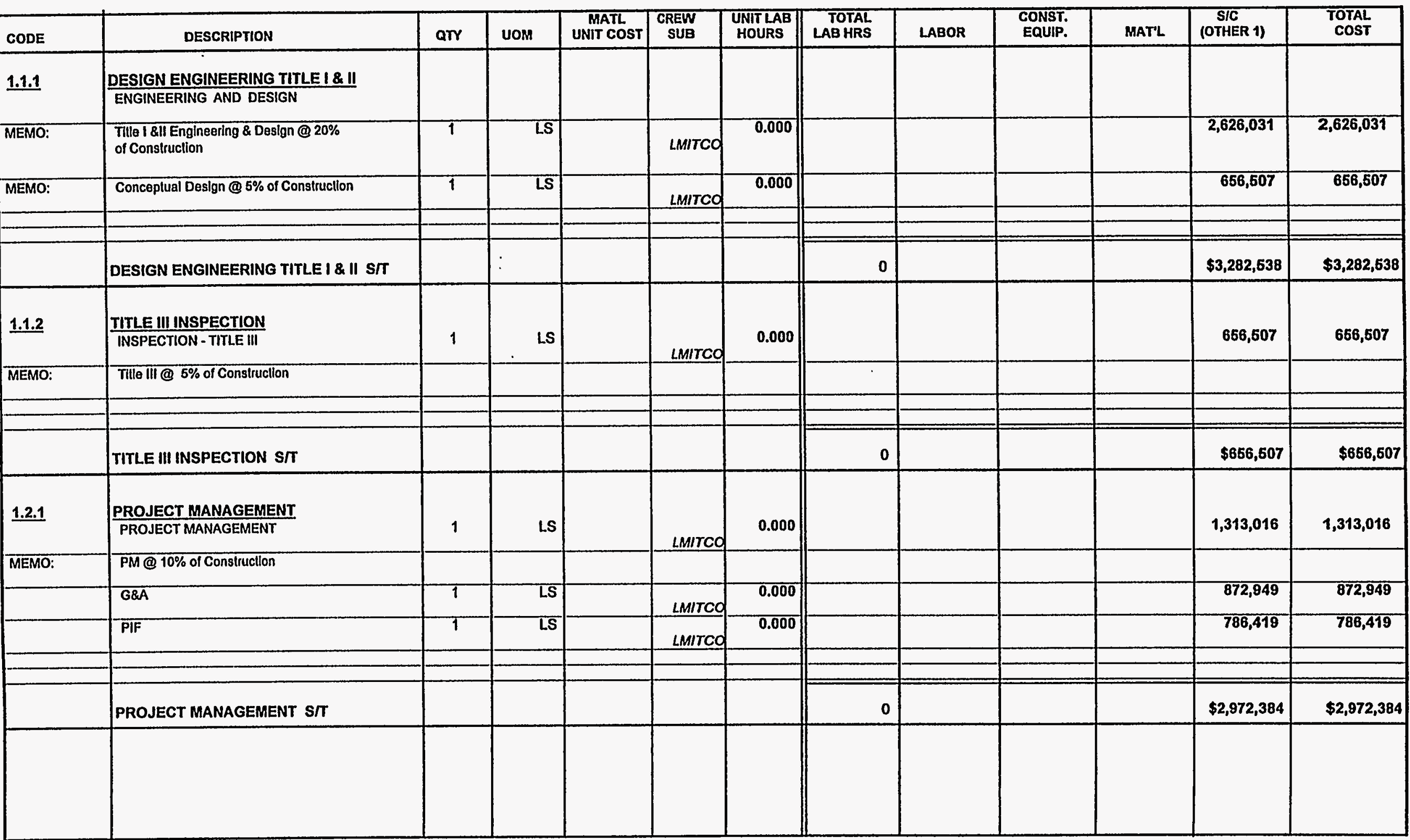

TYPE OF ESTIMATE: Planning

PROJECT NO: 2423-B-E1

PREPARED BY: S. L. Coward

\section{DETAILED COST ESTIMATE SHEET}

PAGE \# 1

TIME: 11:33:53

REPORT NAME: Detail Cost Estimate Sheot
DATE 28 Jan-1998 
Lockheed Martin Idaho Technologies Co. Rov 6.96

PROJECT NAME: ICPP Bin Set Closuro (EIS Study) Place NRC Class C Grout

ICPP

LOCATION: Bryan Spaulding

\section{DETAILED COST ESTIMATE SHEET}

TYPE OF ESTIMATE: Planning

PROJECT NO: 2423-B-E1

PREPARED BY: S. L. Coward
PAGE \# 3

DATE 28-Jan-1998

TIME: 11:33:63

REPORT NAME: Dotall Cost Estimate Sheot

\begin{tabular}{|c|c|c|c|c|c|c|c|c|c|c|c|c|}
\hline CODE & DESCRIPTION & aTr & UOM & $\begin{array}{l}\text { MATL } \\
\text { UNIT COST }\end{array}$ & $\begin{array}{l}\text { CREW } \\
\text { SUB }\end{array}$ & $\begin{array}{l}\text { UNIT LAB } \\
\text { HOURS }\end{array}$ & $\begin{array}{l}\text { TOTAL } \\
\text { LAB HRS }\end{array}$ & LABOR & $\begin{array}{l}\text { CONST. } \\
\text { EQUIP. }\end{array}$ & MAT'L & $\begin{array}{c}\text { SIC } \\
\text { (OTHER 1) }\end{array}$ & $\begin{array}{l}\text { TOTAL } \\
\text { COST }\end{array}$ \\
\hline \multirow[t]{11}{*}{1.3 .3} & $\begin{array}{l}\text { CONCRETE } \\
\text { PROCURE EQUIPMENT FOR PLACEMENT OF } \\
\text { GROUT }\end{array}$ & & & & & & & & & & & \\
\hline & Grout Booster Pump & 1 & $E A$ & $55,000.00$ & $\left.C_{0}\right)$ & 0.000 & & & & 55,000 & & 66,000 \\
\hline & $\begin{array}{l}\text { Air Compressor and Miscellaneous } \\
\text { Cleaning Equipment }\end{array}$ & 1 & $\overline{L S}$ & $25,000.00$ & GEN & 0.000 & & & & 25,000 & & 25,000 \\
\hline & $\begin{array}{l}\text { Manlpulator Boxes (2 Plus } 1 \text { Spare) } \\
\text { Procurement }\end{array}$ & 3 & $\overline{E A}$ & $350,000.00$ & GEN & 0.000 & & & & $1,050,000$ & & $1,050,000$ \\
\hline & $\begin{array}{l}\text { Manipulator Boxes Mobilizallon and } \\
\text { Demobllizallon (2 per tank @ } 50 \text { tanks) }\end{array}$ & 100 & EA & & $\begin{array}{r}\text { CONC } \\
\text { GEN }\end{array}$ & 80.000 & 8,000 & 266,240 & & & & 256,240 \\
\hline & $\begin{array}{l}\text { GROUT DELIVERY PIPE SHIELDING } \\
\text { (includes } 10 \% \text { Waste) }\end{array}$ & 3,367 & LF & 130.00 & $\begin{array}{r}\text { CONC } \\
\text { GEN }\end{array}$ & 0.200 & 673 & 21,569 & & $\mathbf{4 3 7 , 7 1 0}$ & & 459,279 \\
\hline & $\begin{array}{l}\text { GROUT DELIVERY PIPE SHIELDING (Removal } \\
\text { and Dlsposal (Incl. 10\% Waste) }\end{array}$ & 3,367 & LF & & $\begin{array}{r}\text { CONC } \\
\text { GEN }\end{array}$ & 0.800 & 2,694 & 86,276 & & & & 86,276 \\
\hline & $\begin{array}{l}\text { POUR CLASS C GROUT AND CLEAN PIPE } \\
\text { (includes } 10 \% \text { Waste) }\end{array}$ & 10,406 & $\overline{C Y}$ & 0.28 & $\begin{array}{c}\text { CONC } \\
\text { GEN }\end{array}$ & 0.920 & 9,674 & 306,640 & & 2,914 & & 309,564 \\
\hline & CLEANING OF PIPING AFTER EACH LIFT & 666 & LIFTS & 30.00 & $\begin{array}{l}\text { CONC } \\
\text { GEN }\end{array}$ & 0.500 & $\overline{333}$ & 10,666 & & 19,880 & & 30,646 \\
\hline & $\begin{array}{l}\text { 6" CAPS FOR PENETRATIONS (Assume } 2 \text { per } \\
\text { bin) }\end{array}$ & 100 & $\overline{E A}$ & 500.00 & $\begin{array}{r}\text { CONC } \\
\text { GEN }\end{array}$ & 24.000 & 2,400 & 76,872 & & 60,000 & & 126,872 \\
\hline & CONCRETE S/T & & & & & & 23,674 & $\$ 768,263$ & & $\$ 1,640,604$ & & $\$ 2,398,867$ \\
\hline 1.3 .16 & MECHANICAL & & & & & & & & & & & \\
\hline & & & & & & & & & & & & \\
\hline & DOUBLE CONTAINED GROUT delivery plpe & 3,367 & $\mathrm{LF}$ & $\mathbf{4 7 . 0 0}$ & $\begin{array}{c}\text { CONC } \\
\text { GEN }\end{array}$ & 1.200 & 4,040 & 129,414 & 10,101 & 168,249 & 13,468 & 311,232 \\
\hline & & & & & & & & & & & & \\
\hline & & & & & & & & & & & & \\
\hline
\end{tabular}


okheod Martin Idaho Technologles Co.

Rov 6.96
DJECT NAME: ICPP Bin Set Closure (EIS Study) Place NRC Class C Grout

ATION 1: ICPP

QUESTOR: Bryan Spaulding
DETAILED COST ESTIMATE SHEET

TYPE OF Estimate: Planning

PROJECT NO: 2423-B-E1

PREPARED BY: S. L. Coward
PAge 4

DATE 28-Jan-1988

TIME: 11:33:53

REPORT NAME: Detall Cost Estimate Sheot

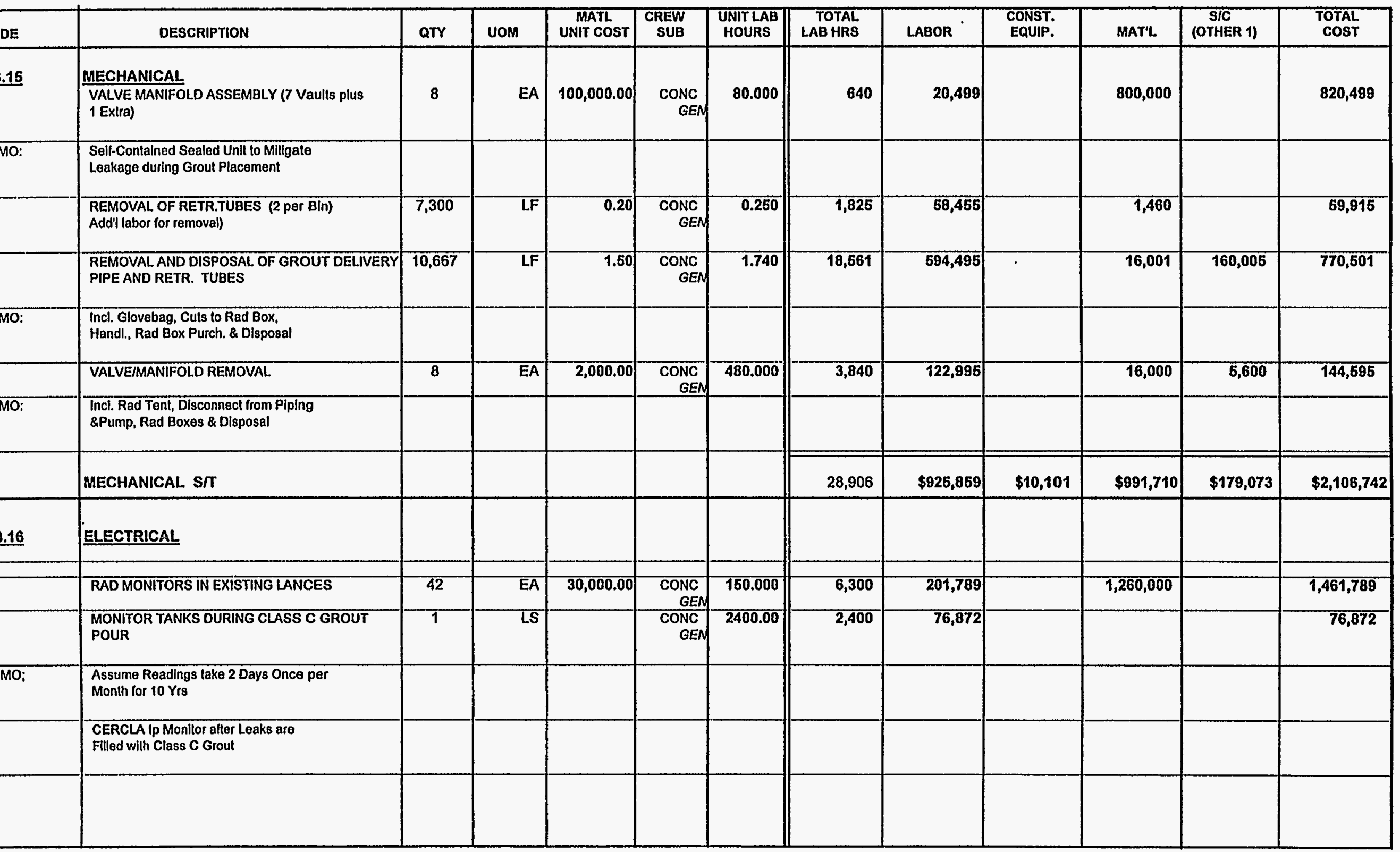


Lockheed Martin Idaho Technologies Co. Reversed

PROJECT NAME: ICPP Bin Set Closure (EIS Stud Place NRC Class C Grout ICPP

LOCATION 1:

REQUESTOR: Bryan Spaulding

CONTINGENCY ANALYSIS

TYPE OF ESTTMATE:

PROJECT NO:

PREPARED BY:
Planning

2423-B-E1

S. L Coward
DATE: 28-Jan-1998

TIME: 11:33:48

REPORT NAME: Contingency Analysis

\begin{tabular}{|c|c|c|c|c|c|c|c|c|c|c|c|}
\hline & PROB & BLE \% VARIA & ION & & & & & & $\begin{array}{r}\text { PRC } \\
\text { CON7 }\end{array}$ & $\begin{array}{l}\text { CT } \\
\text { SENCY }\end{array}$ & SUMMARY \\
\hline WBS & Cost Estimate Element & & $\begin{array}{c}\% \text { Total } \\
\text { Cost }\end{array}$ & & $\begin{array}{l}\% \text { Var. } \\
\text { m Est. }\end{array}$ & $w_{t} \%$ & Prob. & Contingency & $\%$ & Cost & Total Cost \\
\hline & $\cdot$ & Contingency & & - & + & - & + & & & & by Element \\
\hline 1.1 .1 & DESIGN ENGINEERING TITLE I \& $\|$ & $3,282,538$ & 6.98 & 10 & 40 & 0.70 & 2.79 & $2.443 \%$ & $5.96 \%$ & $1,053,402$ & $4,335,940$ \\
\hline 1.1 .2 & TITLE III INSPECTION & 656,507 & 1.40 & 10 & 40 & 0.14 & 0.56 & $0.489 \%$ & $1.19 \%$ & 210,680 & 867,187 \\
\hline 1.2 .1 & PROJECTMANAGEMENT & $2,972,384$ & 6.32 & 10 & 40 & 0.63 & 253 & $2212 \%$ & $5.40 \%$ & 953,870 & $3,926,254$ \\
\hline 1.2 .2 & CONSTRUCTION MANAGEMENT & $1,301,490$ & $2 \pi 7$ & 10 & 40 & 0.28 & 1.11 & $0.969 \%$ & $236 \%$ & 417,662 & $1,719,152$ \\
\hline 1.3 .1 & GENERAL CONDITONS & $3,437,150$ & 7.31 & 10 & 50 & 0.73 & 3.65 & $3216 \%$ & $7.84 \%$ & $1,386,651$ & $4,823,801$ \\
\hline 1.3 .2 & SITEWORK & 0 & 0.00 & 0 & 0 & 0.00 & 0.00 & $0.000 \%$ & $0.00 \%$ & 0 & 0 \\
\hline 1.3 .3 & CONCRETE & $3,876,603$ & 8.24 & 10 & 50 & 0.82 & 4.12 & $3.627 \%$ & $8.85 \%$ & $1,563,940$ & $5,440,543$ \\
\hline 1.3 .15 & MECHANICAL & $3,365,584$ & 7.16 & 10 & 50 & $0 . \overline{72}$ & 3.58 & $3.149 \%$ & $7.68 \%$ & $1,357,779$ & $4,723,363$ \\
\hline 9.3 .16 & ELECTRICAL & $2,450,822$ & 5.21 & 10 & 50 & 0.52 & 2.61 & $2293 \%$ & $5.59 \%$ & 988,737 & $3,439,559$ \\
\hline 1.52 & PROCUREMENT FEES & 131,302 & 0.28 & 10 & 40 & 0.03 & 0.11 & $0.098 \%$ & $0.24 \%$ & 42,136 & 173,438 \\
\hline & ESCALATION & $25,548,568$ & 54.33 & 10 & 40 & 5.43 & 21.73 & $19.016 \%$ & $46.38 \%$ & $9,702,195$ & $35,250,763$ \\
\hline & SUBTOTAL & $47,022,948$ & 100.00 & & & & & $37.513 \%$ & & & \\
\hline & CALCULATED CONTINGENCY & $17,639,746$ & & & & & & & & & . \\
\hline & RESULTANT TEC & $64,662,694$ & & & & & & & & & \\
\hline & ROUNDED TEC & $64,700,000$ & & & & & & & & & \\
\hline & PROJECT CONTINGENCY & $17,677,052$ & & & & & & $37.59 \%$ & & & \\
\hline & MANAGEMENT RESERVE & $2,983,829$ & & & & & & & & & \\
\hline & CONTINGENCY & $14,693,223$ & & & & & & & & & \\
\hline & TOTAL ESTIMATED COST & $64,700,000$ & & & & & & & & $17,677,052$ & $64,700,000$ \\
\hline
\end{tabular}

CONFIDENCE LEVEL AND ASSUMED RISKS:

The Lockheed Idaho Technologies Co. Cost Estimate Contingency Analysis Model is based on the applied contingency and the assumptions upon which the estimate was predicated. The model is applied with a suggested risk level of $18 \%$ and a level of confidence of $90 \%$ the estimate will fall within the bid range. The Contingency Analysis is based on a weighted average to provide a

$90 \%$ probability of undermun and a $10 \%$ probability of overrun.
CONTINGENCY ANALYSIS GUIDE BY TYPE OF ESTIMATE

Guidelines established by DOE/FM 50, Cost Estimating Guide, Vol. 6. Cost Guide, and as presented in the INEL Cost Estimating Guide.

\section{PLANNING}

Conceptual $20 \%$ - $30 \%$ $15 \%-25 \%$

TITLE

TITLE II

TITLE IUAAFC
Up to $40 \%$

$10 \%-20 \%$

$5 \%-15 \%$

Market Conditions 


\section{G\&APIF ADDER CALCULATION SHEET ICPP BIN SET CLOSURE \\ RISK BASED CLEAN CLOSURE; CLASS C FILL; ESCALATED}

FILL BINS W/ NRC CLASS C GROUT

PROCUREMENT FEE:

CONSTRUCTION $=$
GFE $=$ Subtotal $\frac{\$ 29,542,856}{\$ 29,542,856}$

FEE @ $1 \%=$

$\$ 29,542,856 * 0.01=$

$\$ 295,429$

G\&A @ 23\% (with a ceiling of $\$ 500,000$ imposed per year, 7 yrs)

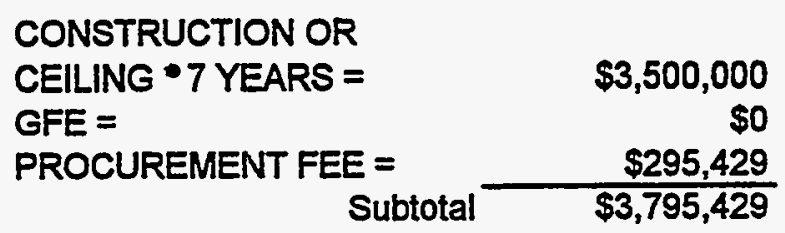

FEE @ $23 \%=$

$\$ 3,795,429 * 0.23=$

$\$ 872,949$

PIF @ 5.5\%

\begin{tabular}{lr} 
CONSTRUCTION $=$ & $\begin{array}{r}\$ 13,130,159 \\
\text { GFE }=\end{array}$ \\
PROCUREMENT FEE $=$ & $\$ 0$ \\
G\&A $=\quad \begin{array}{r}\$ 295,429 \\
\end{array} \quad$ Subtotal & $\$ 872,949$ \\
\cline { 2 - 2 } & $\$ 14,298,536$
\end{tabular}

FEE @ 5.5\% =

TOTAL G\&A FEE:

$\$ 872,949$

TOTAL PIF:

$\$ 786,419$ 
Lockheed Martin Jdaho Technologies Co. Rev, 6-96 PROJECT NAME: ICPP Bin Set Closure D\&D of Equipment

LOCATION 1: REQUESTOR:
COST ESTIMATE SUMMARY

$\begin{array}{cc}\text { TYPE OF ESTMATE: } & \text { Planning } \\ \text { PROJECT NO: } & \text { 2423D\&D } \\ \text { PREPARED BY: } & \text { S.LCOWard/Smb } \\ \text { REPORT NAME: CoSt Estimate Summary }\end{array}$

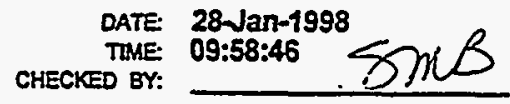

APPRD BY:

\begin{tabular}{|c|c|c|c|c|}
\hline $\begin{array}{l}\text { WBS } \\
\text { Element }\end{array}$ & Cost Estimate Element & $\begin{array}{c}\text { Total } \\
\text { Unescalated }\end{array}$ & Escalation & $\begin{array}{l}\text { Total } \\
\text { Incl Escalation }\end{array}$ \\
\hline $\begin{array}{l}\frac{1.1}{1.1 .1} \\
1.1 .2 \\
\frac{1.2}{1.2 .1} \\
1.2 .2 \\
1.3 \\
1.3 .13 \\
1.5 .2\end{array}$ & $\begin{array}{l}\text { ENGINEERING, DESIGN AND INSPECTION } \\
\text { DESIGN ENGINEERING TITLE I \& II } \\
\text { TITLE III INSPECTION } \\
\text { MANAGEMENT COSTS } \\
\text { PROJECT MANAGEMENT } \\
\text { CONSTRUCTION MANAGEMENT } \\
\text { CONSTRUCTION } \\
\text { SPECIAL CONSTRUCTION } \\
\text { PROCUREMENT FEES }\end{array}$ & $\begin{array}{r}868,178 \\
0 \\
11,709,711 \\
117,097\end{array}$ & $\begin{array}{c}1,692,947 \\
0 \\
\\
22,833,936 \\
228,339\end{array}$ & $\begin{array}{l}\gg \quad \begin{array}{l}\$ 0 \\
0\end{array} \\
\gg \frac{\$ 2,561,125}{2,561,125} \\
0 \\
\gg \frac{\$ 34,543,647}{34,543,647} \\
\gg \quad \$ 345,436\end{array}$ \\
\hline & $\begin{array}{l}\text { SUBTOTAL INCLUDING ESCALATION } \\
\text { PROJECT CONTINGENCY }\end{array}$ & $12,694,986$ & $24,755,222$ & $\gg \$ 37,450,208$ \\
\hline & MANAGEMENT RESERVE- & & & \$o \\
\hline & CONTINGENCY- & & & $\$ 13,149,792$ \\
\hline & TOTAL ESTIMATED COST - & & & $\$ 50,600,000$ \\
\hline
\end{tabular}

\section{PROJECT COST PARAMETERS}

EDI AS A $\%$ OF CONST. + GFE $=0.00 \%$ 
Lockheed Martin Idaho Technologies Co.

Rav 6.96

PROJECT NAME: ICPP Bin Set Closure

D\&D of Equipment

INEEL IICPP

B. C. Spautding
DETAILED COST ESTIMATE SHEET

TYPE OF ESTIMATE: Planning

PROJECT NO: 2423D\&D

PREPARED BY: S.L.Coward/smb
PAGE\# 1

DATE 28-Jan-1998

TIME: 10:13:24

REPORT NAME: Detall Cost Estimate Sheet

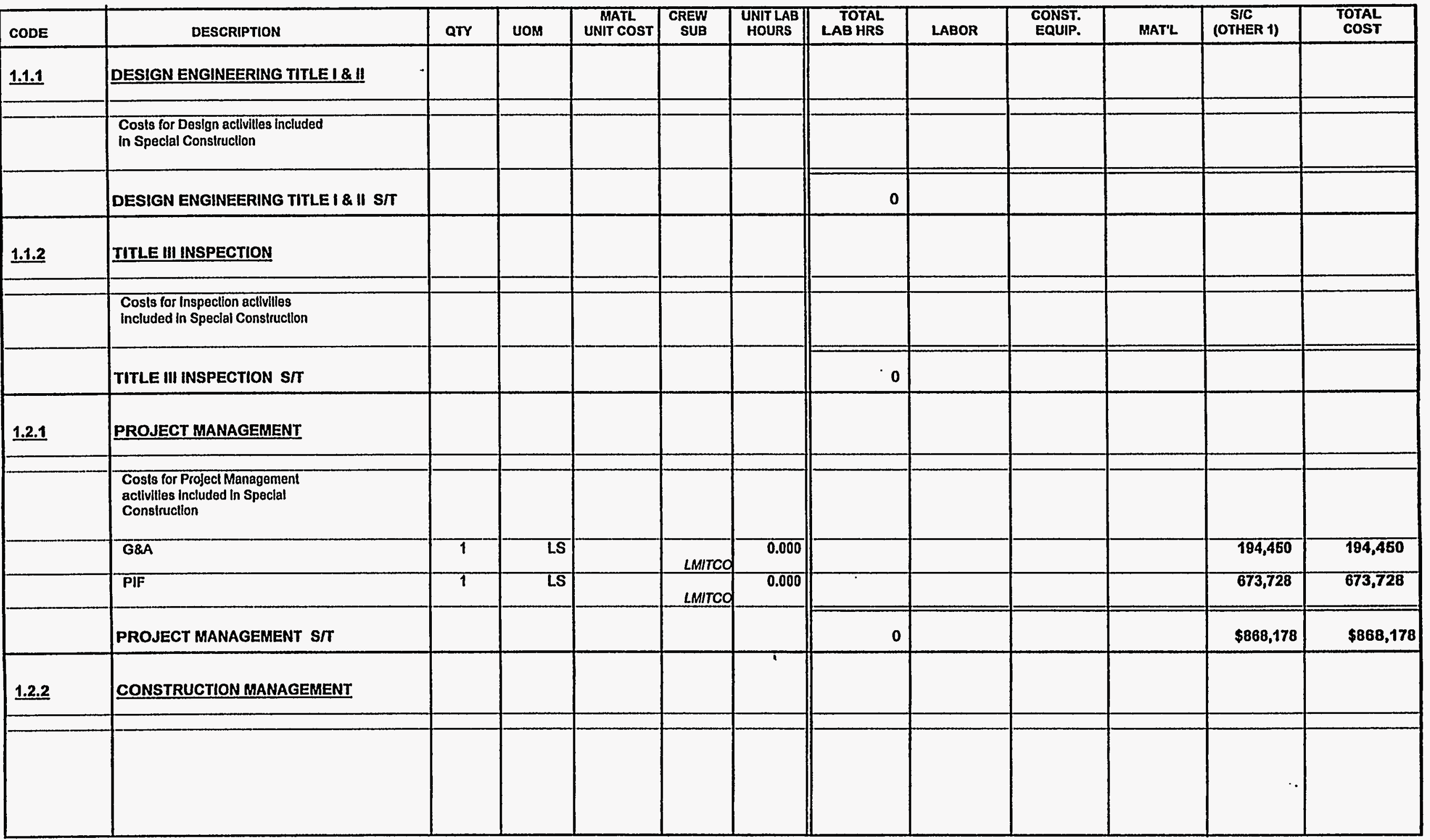


Lockheed Martin Idaho Technologies Co.

\section{Rov 6.96}

RAME: ICPP Bin Sot Closure

D\&D of Equipment

INEEL / ICPP

B. C. Spaulding

\section{DETAILED COST ESTIMATE SHEET}

TYPE OF ESTIMATE: Planning

PROJECT NO.: 2423D\&D

PREPARED BY: S.L.Coward/smb
PAGE 2

DATE 28van-1998

TIME: 10:13:24

REPORT nAME: Detall Cost Estlmate Sheot

\begin{tabular}{|c|c|c|c|c|c|c|c|c|c|c|c|c|}
\hline ODE & DESCRIPTION & QTY & UOM & $\begin{array}{l}\text { MATL } \\
\text { UNIT COST }\end{array}$ & $\begin{array}{l}\text { CREW } \\
\text { SUB }\end{array}$ & $\begin{array}{l}\text { UNIT LAB } \\
\text { HOURS }\end{array}$ & $\begin{array}{l}\text { TOTAL } \\
\text { LAB HRS }\end{array}$ & LABOR & $\begin{array}{l}\text { CONST. } \\
\text { EQUIP. }\end{array}$ & MAT'L & $\begin{array}{c}\text { S/C } \\
\text { (OTHER 1) }\end{array}$ & $\begin{array}{l}\text { TOTAL } \\
\text { COST }\end{array}$ \\
\hline 2.2 & $\begin{array}{l}\text { CONSTRUCTION MANAGEMENT } \\
\text { Cosis for Constructlon Management } \\
\text { actlvittes included in Special } \\
\text { Construction }\end{array}$ & & & & & & & & & & & \\
\hline & CONSTRUCTION MANAGEMENT SI & & & & & & 0 & & & & & \\
\hline 3.13 & SPECIAL CONSTRUCTION & & & & & & & & & & & \\
\hline & Ventilation, Instrument and Control & 1 & Tot & & & 0000 & & & & & & \\
\hline & Building (VIC) - 7 ea @ 2400 of & & & & & 0.000 & & & & & 969,133 & 968,133 \\
\hline & $\begin{array}{l}\text { - Assume facllly will have low } \\
\text { levels of hazardous material }\end{array}$ & & & & & & & & & & & \\
\hline & $\begin{array}{l}\text { - Assume facillty will have no rad } \\
\text { contamination }\end{array}$ & & & & & & & & & & & \\
\hline & $\begin{array}{l}\text { - Assume facillty will have no } \\
\text { asbestos }\end{array}$ & & & & & & & & & & & \\
\hline & $\begin{array}{l}\text { Confinement Enclosures (top of Bin } \\
\text { Sets) -7 ea @ (1600 sf }\end{array}$ & 1 & Tot & & & 0.000 & & & & & $1,248,126$ & $1,248,126$ \\
\hline & Bridge Crane - 7 ea (300 sl & 1 & tot & & & 0.000 & & & & & 369,376 & 369,376 \\
\hline & $\begin{array}{l}\text { - Assume bridge } 40^{\prime} \text { across } \times 45^{\prime} \text { high } \\
\times 2 \text { wlde for ralls }\end{array}$ & & & & & & & & & & & \\
\hline & - Assume trolley @ $6^{2} \times 5^{1} \times 5^{1}$ & & & & & & & & & & & \\
\hline & HVAC EquipmenVSystem - 1 ea @ 5800 sf & 1 & lot & & & 0.000 & & & & & $\overline{449,044}$ & 449,044 \\
\hline & - Assume 7 alr handlers @ 5 $\times 5^{\prime} \times \theta^{\prime}$ & & & & & & & & & & & \\
\hline & 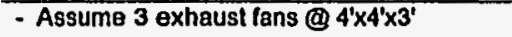 & & & & & & & & & & & \\
\hline & & & & & & & & & & & & \\
\hline & & & & & & & & & & & & \\
\hline
\end{tabular}


Lockheed Martin Idaho Technologles Co. Rev 6.96 PROJECT NAME: ICPP Bin Set Closure D\&D of Equlpment

INEEL / ICPP

REQUESTOR: B. C. Spaulding
DETAILED COST ESTIMATE SHEET

TYPE OF ESTIMATE: Planning

PROJECT NO.: 2423D\&D

PREPARED BY: S.L.Coward/smb
PAGE* 3

DATE 28-Jan-1998

TIME: 10:13:24

REPORT NAME: Dotall Cost Estimate Sheot

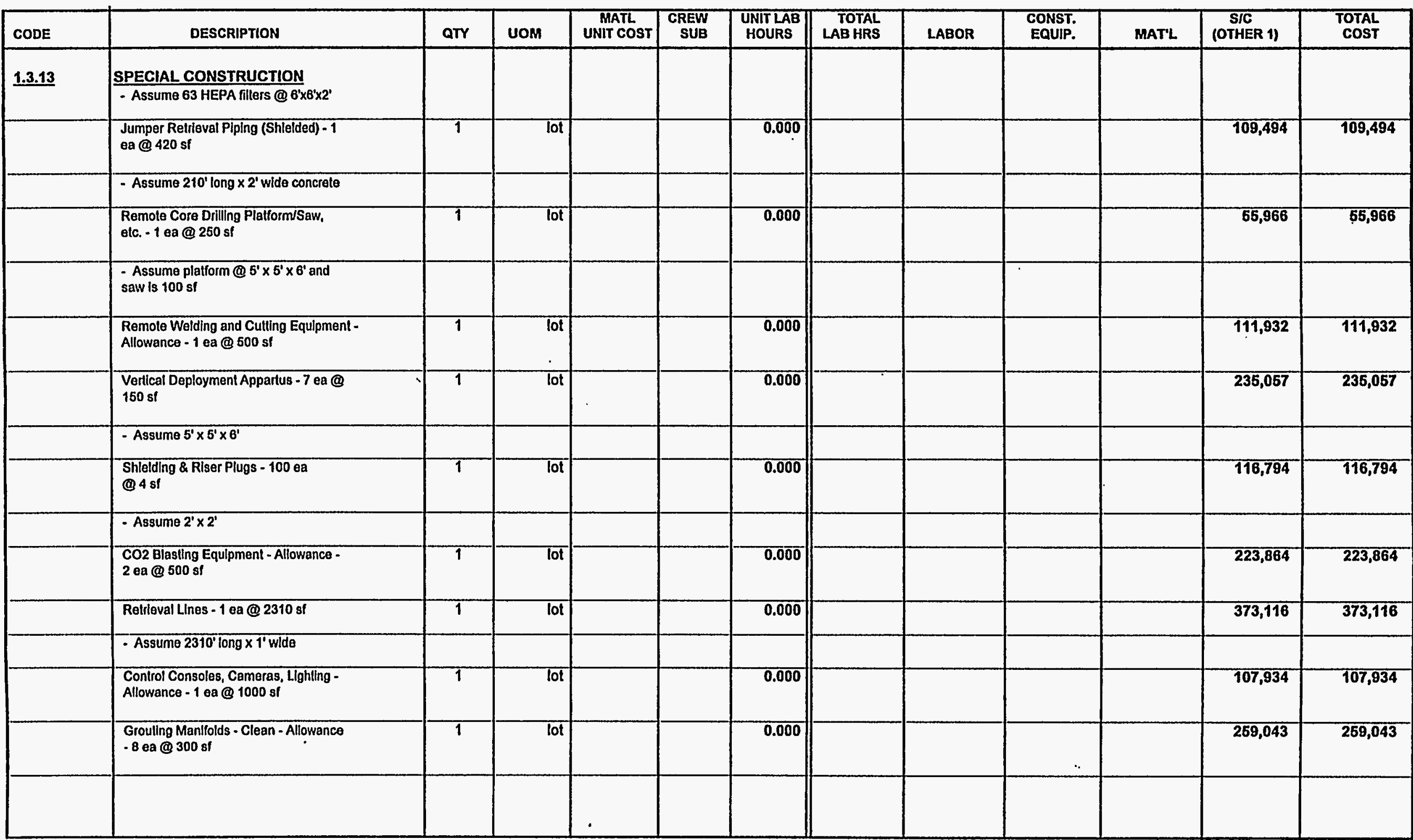


Lockhoed Martin Idaho Technologles Co. Rov 6-96

'ROJECT NAME: ICPP Bin Set Closure D\&D of Equipment INEEL / ICPP IEOUESTOR: B. C. Spaulding

\section{DETAILED COST ESTIMATE SHEET}

TYPE OF estimate: Planning

PROJECT NO.: 2423D\&D

PREPARED BY: S.L.Coward/smb
PAGE A

DATE 28-Jan-1998

TIME: 10:13:24

REPORT NAME: Detall Cost Estimate Sheot

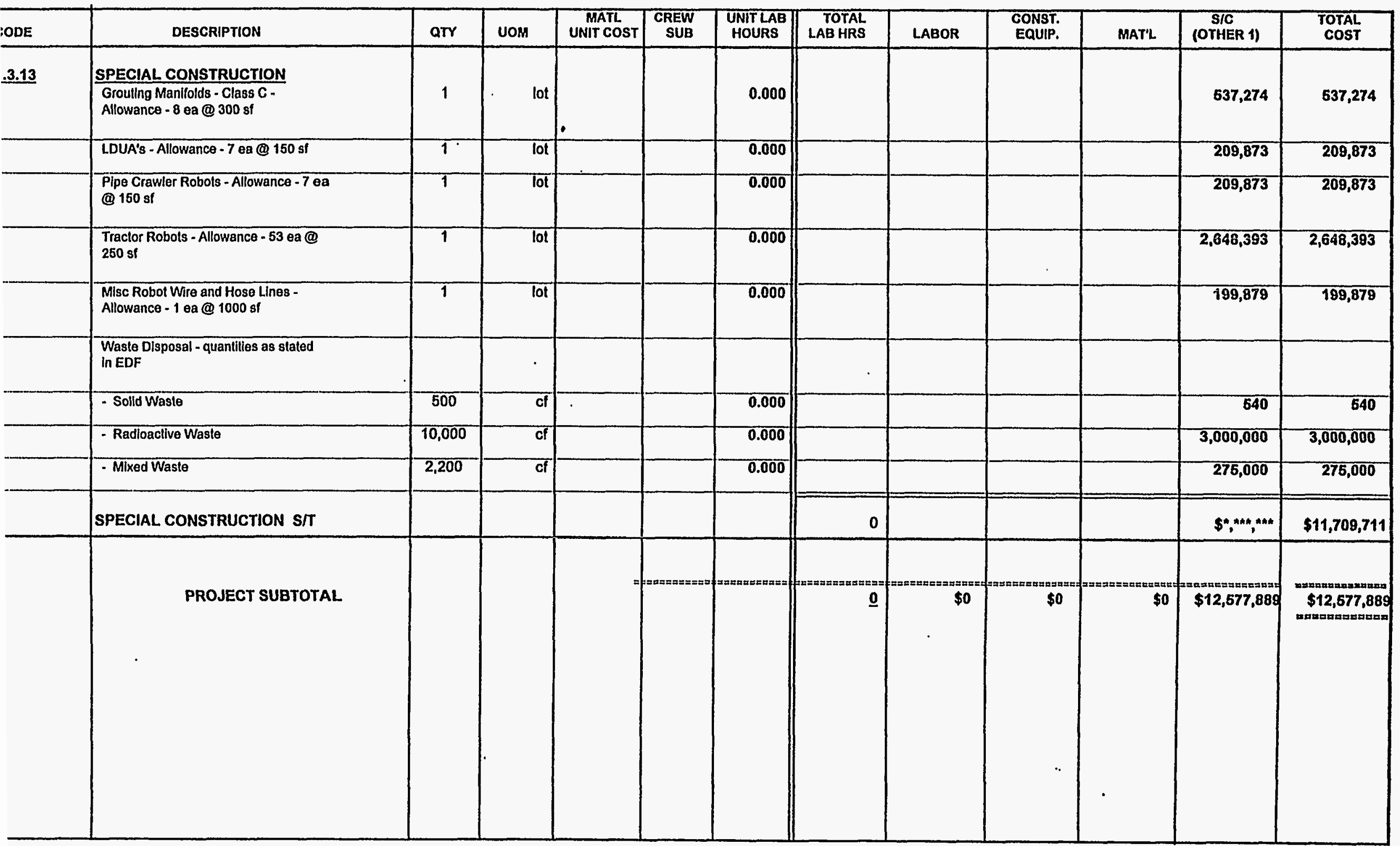


Lockheed Martin Idaho Technologies Co. Rov 6-95

PROJECT NAME: ICPP Bin Set Closure

DED of Equipment

LOCATION 1: INEEL/ICPP

REQUESTOR: B. C. Spaulding
CONTINGENCY ANALYSIS

$\begin{array}{cl}\text { TYPE OF ESTIMATE: } & \text { Planning } \\ \text { PROJECT NO: } & \text { 2423D\&D } \\ \text { PREPARED BY: } & \text { S.LCOWard/smb }\end{array}$

DATE: 28-Jan-1998

TIME: 09:58:43

REPORT NAME: Contingency Analysis

\begin{tabular}{|c|c|c|c|c|c|c|c|c|c|c|c|}
\hline \multicolumn{9}{|c|}{ PROBABLE \% VARIATION } & \multicolumn{2}{|c|}{$\begin{array}{l}\text { PROJECT } \\
\text { CONTINGENCY }\end{array}$} & \multirow{3}{*}{$\begin{array}{l}\text { SUMMARY } \\
\text { Total Cost } \\
\text { by Element }\end{array}$} \\
\hline \multirow[t]{2}{*}{$\begin{array}{l}\text { WBS } \\
\text { Element }\end{array}$} & \multirow{2}{*}{ Cost Estimate Element } & \multirow{2}{*}{$\begin{array}{l}\text { Total Cost w/o } \\
\text { Contingency }\end{array}$} & \multirow[t]{2}{*}{$\begin{array}{l}\% \text { Total } \\
\text { cost }\end{array}$} & \multicolumn{2}{|c|}{$\begin{array}{c}\text { Prob. \% Var. } \\
\text { From Est. }\end{array}$} & \multicolumn{2}{|c|}{ Wt \% of Prob. } & \multirow{2}{*}{ Contingeney } & \multirow[t]{2}{*}{$\%$} & \multirow{2}{*}{ Cost } & \\
\hline & & & & - & + & - & + & & & & \\
\hline 1.1 .1 & DESIGN ENGINEERING TILE $1 \& \|$ & 0 & 0.00 & 0 & 0 & 0.00 & 0.00 & $0.000 \%$ & $0.00 \%$ & o) & 0 \\
\hline 1.1 .2 & TITLE III INSPECTON & 0 & 0.00 & 0 & 0 & 0.00 & 0.00 & $0.000 \%$ & $0.00 \%$ & 0 & 0 \\
\hline 1.2 .1 & PROJECT MANAGEMENT & 868,178 & 232 & 10 & 40 & 0.23 & 0.93 & $0.811 \%$ & $2.32 \%$ & 304,841 & $1,173,019$ \\
\hline 1.22 & CONSTRUCTION MANAGEMENT & 0 & 0.00 & 0 & 0 & 0.00 & 0.00 & $0.000 \%$ & $0.00 \%$ & 0 & 0 \\
\hline 1.3 .13 & SPECIAL CONSTRUCTION & $11,709,711$ & 31.27 & 90 & 40 & 3.13 & 12.51 & 10.944\% & $31.27 \%$ & $4,111,600$ & $15,821,311$ \\
\hline \multirow[t]{2}{*}{1.5 .2} & PROCUREMENT FEES & 117,097 & 0.31 & 10 & 40 & 0.03 & 0.13 & $0.109 \%$ & $0.31 \%$ & 41,116 & 158,213 \\
\hline & ESCALATION & $24,755,222$ & 66.10 & 10 & 40 & 6.61 & 26.44 & $23.136 \%$ & $66.10 \%$ & $8,692,235$ & $33,447,457$ \\
\hline & SUBTOTAL & $37,450,208$ & 100.00 & & & & & $35.000 \%$ & & & \\
\hline & CALCULATED CONTINGENCY & $13,107,573$ & & & & & & & & & \\
\hline & RESULTANT TEC & $50,557,781$ & & & & & & & & & \\
\hline & ROUNDED TEC & $50,600,000$ & & & & & & & & & \\
\hline & PROJECT CONTINGENCY & $13,149,792$ & & & & & & $35.11 \%$ & & & \\
\hline & MANAGEMENT RESERVE & 0 & & & & & & . & & & \\
\hline & CONTINGENCY & $13,149,792$ & & & & & & & & & \\
\hline & TOTAL ESTIMATED COST & $50,600,000$ & & & & & & & & $13,149,792$ & $50,600,000$ \\
\hline
\end{tabular}

\section{CONFIDENCE LEVEL AND ASSUMED RISKS}

The Lockheed ldaho Technologies Co. Cost Estimate Contingency Analysis Model is based on the applied contingency and the assumptions upon which the estimate was predicated. The model is applied with a suggested risk level of $18 \%$ and a level of confidence of $90 \%$ the estimate will fall within the bid range. The Contingency Analysis is based on a weighted average to provide a $90 \%$ probability of undermun and a $10 \%$ probability of overmun.

\section{CONTINGENCY ANALYSIS GUIDE BY TYPE OF ESTIMATE}

Guidelines established by DOEFM 50, Cost Estimating Guide, Vol. 6 ,

Cost Guide, and as presented in the INEL Cost Estimating Guide. PLANNING $20 \%-30 \%$ ExperimentalSpecial Conditions............ Up to $50 \%$ Conceptual

Experimentalspecial Conditions............. $10 \%-20 \%$ TITLE!

TITLE II

TITLE IIIAFC
$5 \%-25 \%$

$5 \%-15 \%$

Market Conditions 


\section{G\&A/PIF ADDER CALCULATION SHEET \\ ICPP BIN SET CLOSURE \\ RISK BASED CLEAN CLOSURE; CLASS C FILL; ESCALATED \\ D\&D OF EQUIPMENT}

PROCUREMENT FEE:

\begin{tabular}{|c|c|c|c|}
\hline $\begin{array}{l}\text { CONSTRUCTION = } \\
\text { GFE = }\end{array}$ & & $\$ 34,543,647$ & \\
\hline & Subtotal & $\$ 34,543,647$ & \\
\hline FEE @ 1\% = & & $\$ 34,543,647$ & $\star 0.01=$ \\
\hline
\end{tabular}

G\&A@ $@ 23 \%$ (with a ceiling of $\$ 500,000$ imposed per year, 1 yr)

CONSTRUCTION OR

CEILING 11 YEARS $=\quad \$ 500,000$

GFE = $\$ 0$

PROCUREMENT FEE $=\quad \$ 345,436$

Subtotal $\$ 845,436$

FEE @ $23 \%=\quad \$ 845,436 \cdot 0.23=\quad \$ 194,450$

PIF @ 5.5\%

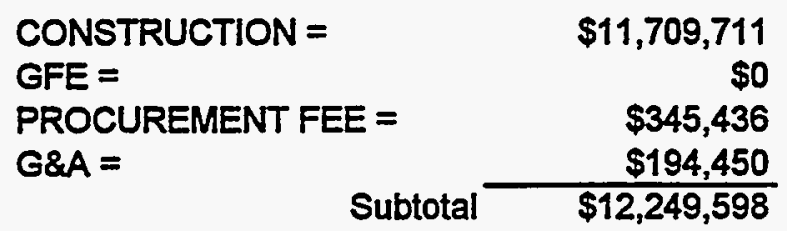

FEE @ $5.5 \%=\quad \$ 12,249,598 \cdot 0.055=\quad \$ 673,728$

TOTAL PROCUREMENT FEE: $\quad \$ 345,436$

TOTAL G\&A FEE: $\quad \$ 194,450$

$\begin{array}{ll}\text { TOTAL PIF: } & \$ 673,728\end{array}$ 


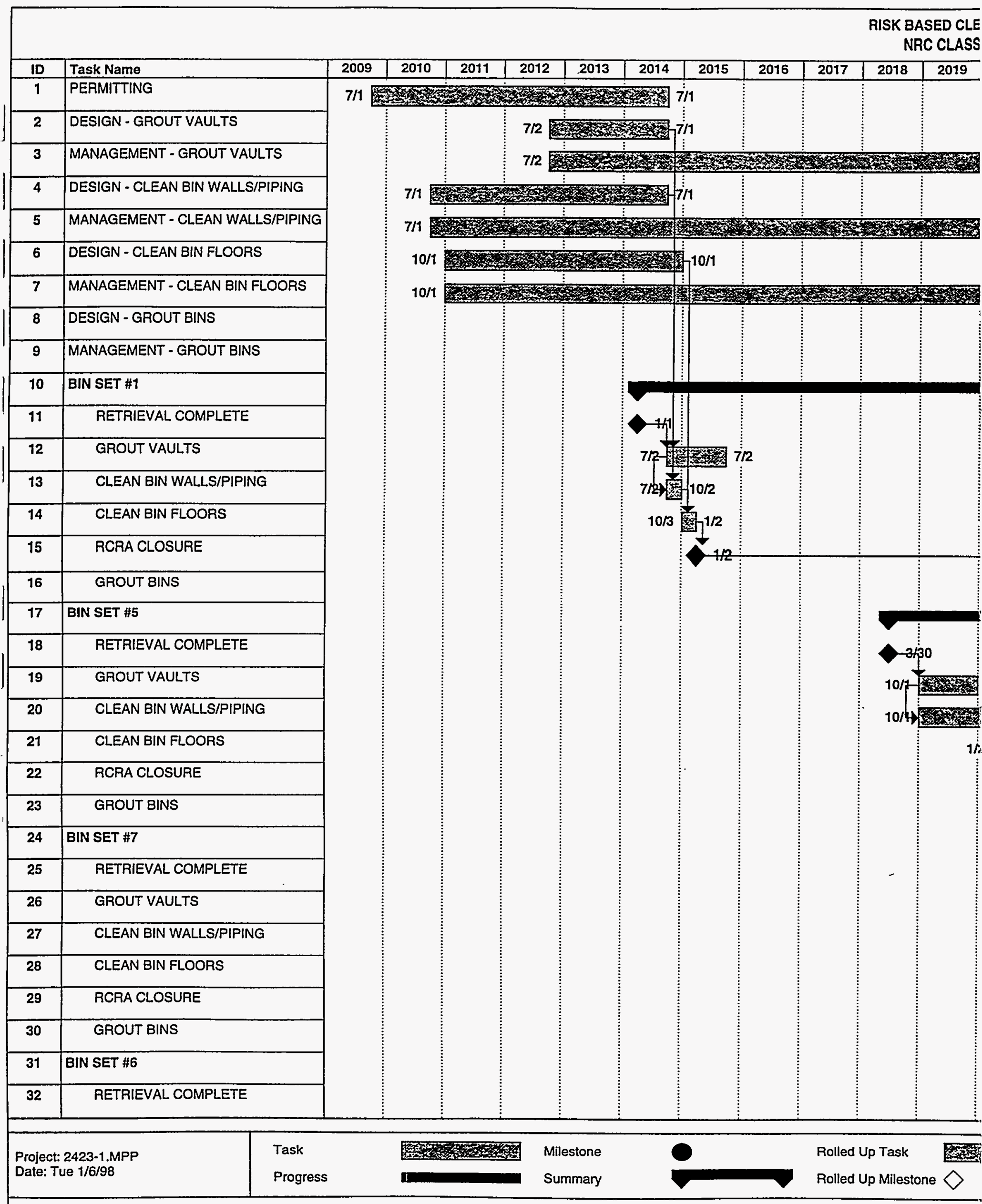


COST ESTIMATE SUMMARY

UNESCALATED

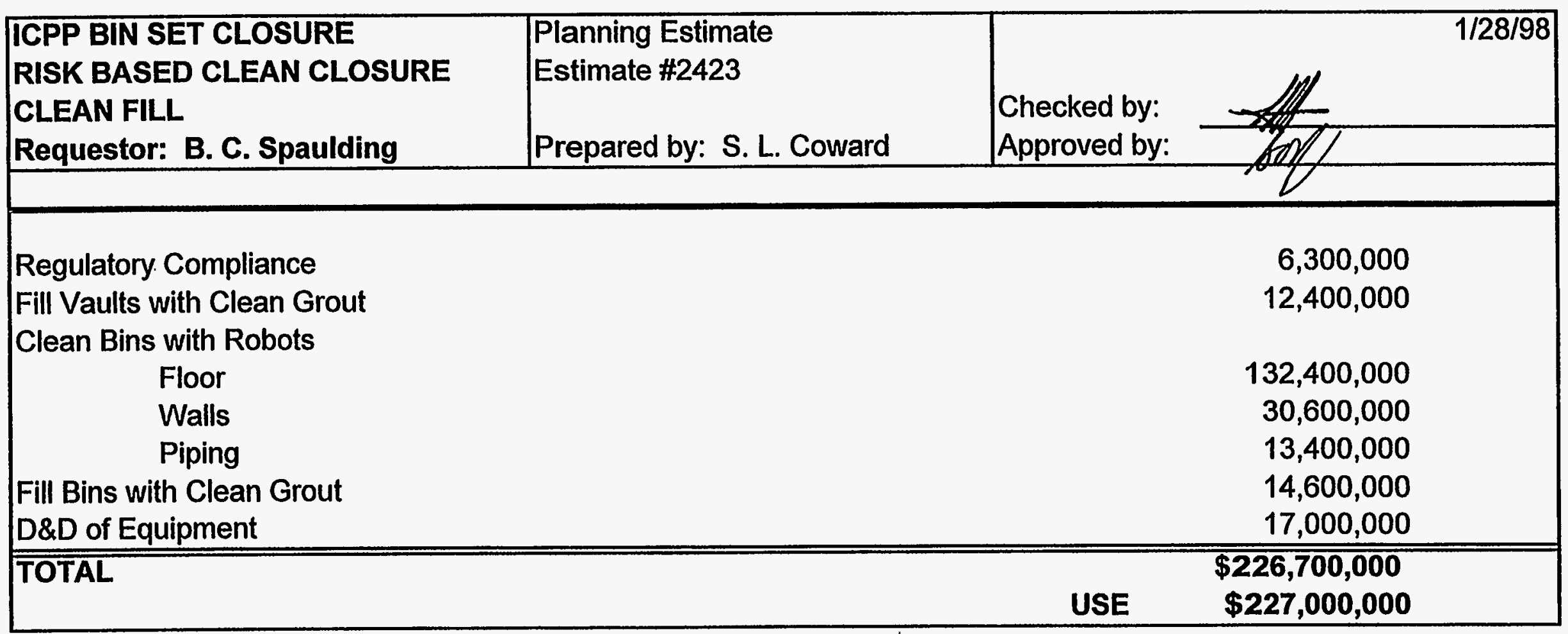


Risk based clean closure - 'Clean' gRoUt

\begin{tabular}{|c|c|c|c|c|c|c|c|}
\hline DESCRIPTION & BIN SET \#1 & BIN SET \#5 & BIN SET \#6 & BIN SET \#2 & BIN SET \#7 & BIN SET \#4 & BIN SET \#3 \\
\hline Scheduled Retrieval Completion & $(1 / 1 / 14)$ & $(3 / 1 / 18)$ & $(8 / 1 / 22)$ & $(2 / 1 / 27)$ & $(2 / 1 / 27)$ & $(2 / 1 / 29)$ & $(10 / 1 / 31)$ \\
\hline
\end{tabular}

AssumE: Wait 1 Year untll Start of Closure

Pormitting (3 Yoars)

$1 / 1 / 12-1 / 1 / 15$

Grout Vaults "Clean" (Assume 1 Yoar)

\begin{tabular}{|c|c|c|c|c|c|c|c|c|}
\hline ED\&l (2 Yrs) & $1 / 1 / 13-1 / 1 / 15$ & & & & & & & \\
\hline Management & $1 / 1 / 13-10 / 1 / 33$ & & & & & & & \\
\hline Construction & & $1 / 1 / 15-1 / 1 / 16$ & $3 / 1 / 19-3 / 1 / 20$ & $8 / 1 / 23-8 / 1 / 24$ & $2 / 1 / 28-2 / 1 / 29$ & $2 / 1 / 28-2 / 1 / 29$ & $2 / 1 / 30-2 / 1 / 31$ & $10 / 1 / 32-10 / 1 / 33$ \\
\hline Clean Bin Walls/Piping with R & lobot & (3 Months) & (15 Months) & (24 Months) & (13 Months) & (25 Months) & (7 Months) & (17 Monthy) \\
\hline ED\&I (4 Yrs) & $1 / 1 / 11-1 / 1 / 15$ & & & & & & & \\
\hline Management & $1 / 1 / 11-3 / 1 / 34$ & & & & & & & \\
\hline Construction & & $1 / 1 / 15-4 / 1 / 15$ & $3 / 1 / 19-6 / 1 / 20$ & $8 / 1 / 23-8 / 1 / 25$ & $2 / 1 / 28-3 / 1 / 29$ & $2 / 1 / 28-3 / 1 / 30$ & $2 / 1 / 30-9 / 1 / 30$ & $10 / 1 / 32-3 / 1 / 34$ \\
\hline Clean Bin Floors with Robot & & (3 Months) & (15 Months) & (24 Months) & (13 Months) & (25 Months) & (7 Months) & (17 Months) \\
\hline ED\&I (4 Yrs) & $4 / 1 / 11-4 / 1 / 16$ & & & & & & & \\
\hline Management & $4 / 1 / 11-8 / 1 / 35$ & & & & & & & \\
\hline Construction & & $4 / 1 / 15-7 / 1 / 15$ & $6 / 1 / 20-9 / 1 / 21$ & $8 / 1 / 25-8 / 1 / 27$ & $3 / 1 / 29-4 / 1 / 30$ & $3 / 1 / 30-4 / 1 / 32$ & $9 / 1 / 30-4 / 1 / 31$ & $3 / 1 / 34-8 / 1 / 35$ \\
\hline \multicolumn{9}{|l|}{ RCAA CLOSUAE } \\
\hline Grout Bins "Cloan" & & (s Bonths) & (7 Months) & (9 Months) & (6 Months) & (9 Months) & (4 Months) & (7 Months) \\
\hline ED\&I (3 Yrs) & $7 / 1 / 12-7 / 1 / 15$ & & & & & & & \\
\hline Management & $7 / 1 / 12-8 / 1 / 36$ & & & & & & & \\
\hline Construotion & & $7 / 1 / 15-10 / 1 / 15$ & $9 / 1 / 21-4 / 1 / 22$ & $8 / 1 / 27-5 / 1 / 28$ & $4 / 1 / 30-10 / 1 / 30$ & $4 / 1 / 32-1 / 1 / 33$ & $4 / 1 / 31-8 / 1 / 31$ & $8 / 1 / 35-3 / 1 / 36$ \\
\hline
\end{tabular}

\section{ASSUMPTIONS:}

1) More than 1 crew could be utilized simultaneously for pouring the "clean" grout into the vaults.

2) More than 1 orew could be utllized simultaneously for cleaning the separate bins. However, cleaning of the floors will require scheduling after cleaning the walls/plping.

3) Installation of "clean" grout into vaults will average 1 year per vault.

4) Cleaning of bln floors are based on Individual bin pro-rated calcine retrieval volumes to total volume. These schedules assume mob/demob, installation of robotic units, and any modificatlons of bins will be completed and blns will be ready for retrieval.

5) Because of the difflculty of cleaning of bin walls/piping, it was assumed that it would take the same duration as the cleaning of the bin floors for each bln. These schedules assume mobilization/demobilization and any modificatlons of bins will be completed, and bins will be ready for retrleval.
Di $s$ for
'san' .
ng o:
ns ar id on
dual : sted b.. solur.
, the ... olum ... s the ..... Jule. 
Lockheed Martin Idaho Technologies Co. Rov. 696
PROJECT NAME: PermittingJDocumentation LOCATION 9: REQUESTOR: Risk Based - Clean INEELICPP Bryan Spaulding
COST ESTIMATE SUMMARY

TYPE OFESTMATE: PLANNING PROJECT NO: PREPARED BY: 2423-A1 D

S. L Coward REPORT NAME: Cost Estimate Summary
TOME

CHECKED BY

27 Jan-1998

19:00:52

APPRD BY:

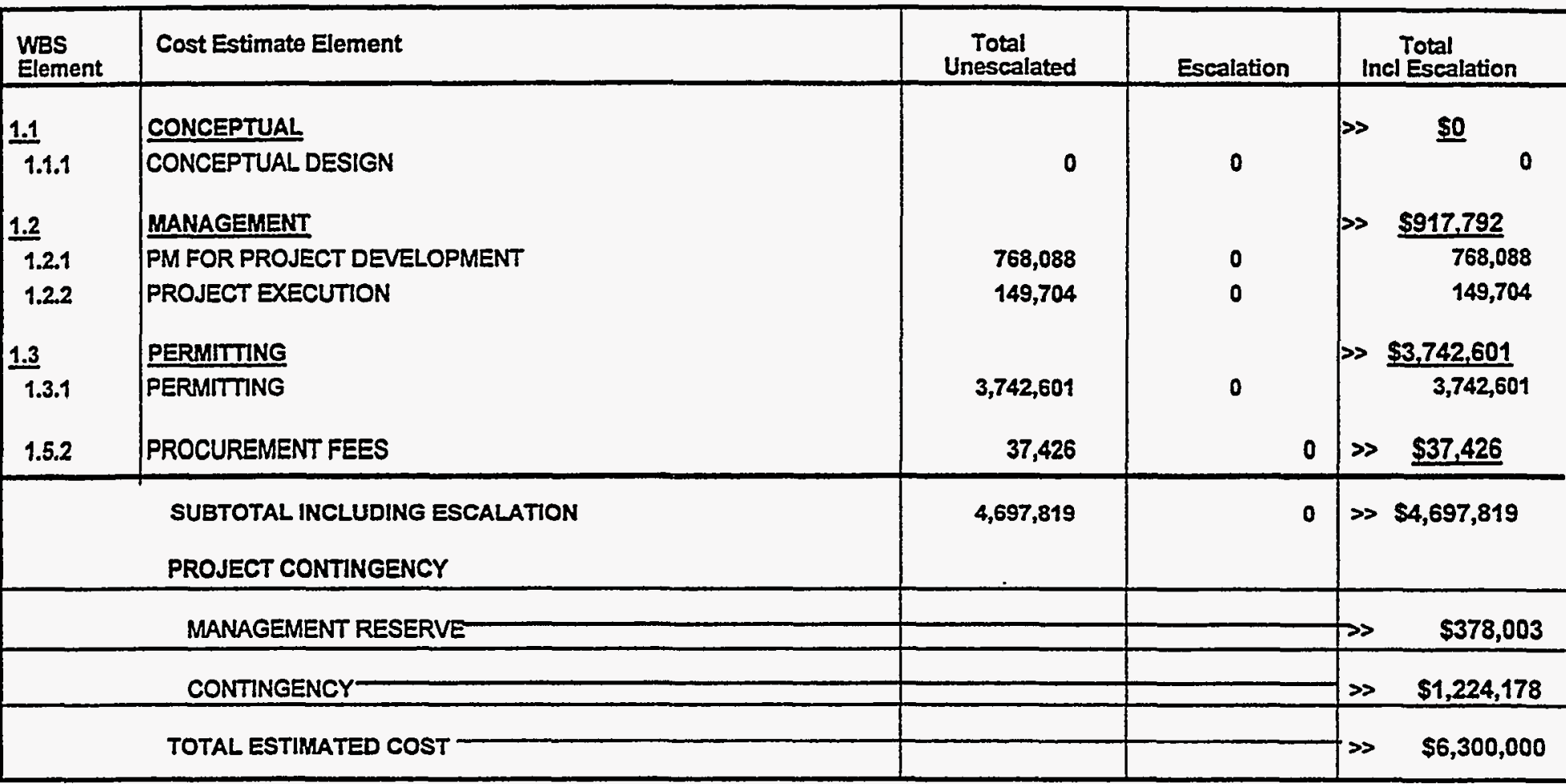

PROJECT COST PARAMETERS

EDI AS A $\%$ OF CONST. + GFE $=0.00 \%$

CONTINGENCY $=34.10 \%$ 
Lockheed Martin Idaho Technologles Co. Rev 6.96

PROJECT NAME: Permitting/Documentatlon Risk Based - Clean

LOCATION 1: INEELIICPP

Bryan Spaulding

REQUESTOR:
DETAILED COST ESTIMATE SHEET

TYPE OF ESTIMATE: PLANNING

PROJECT NO:: 2423-A1 D

PREPARED BY: S. L. Coward
PAGE 3

DATE 27-Jan-1998

TIME: 19:01:02

REPORT NAME: Detall Cost Estlmate Sheot

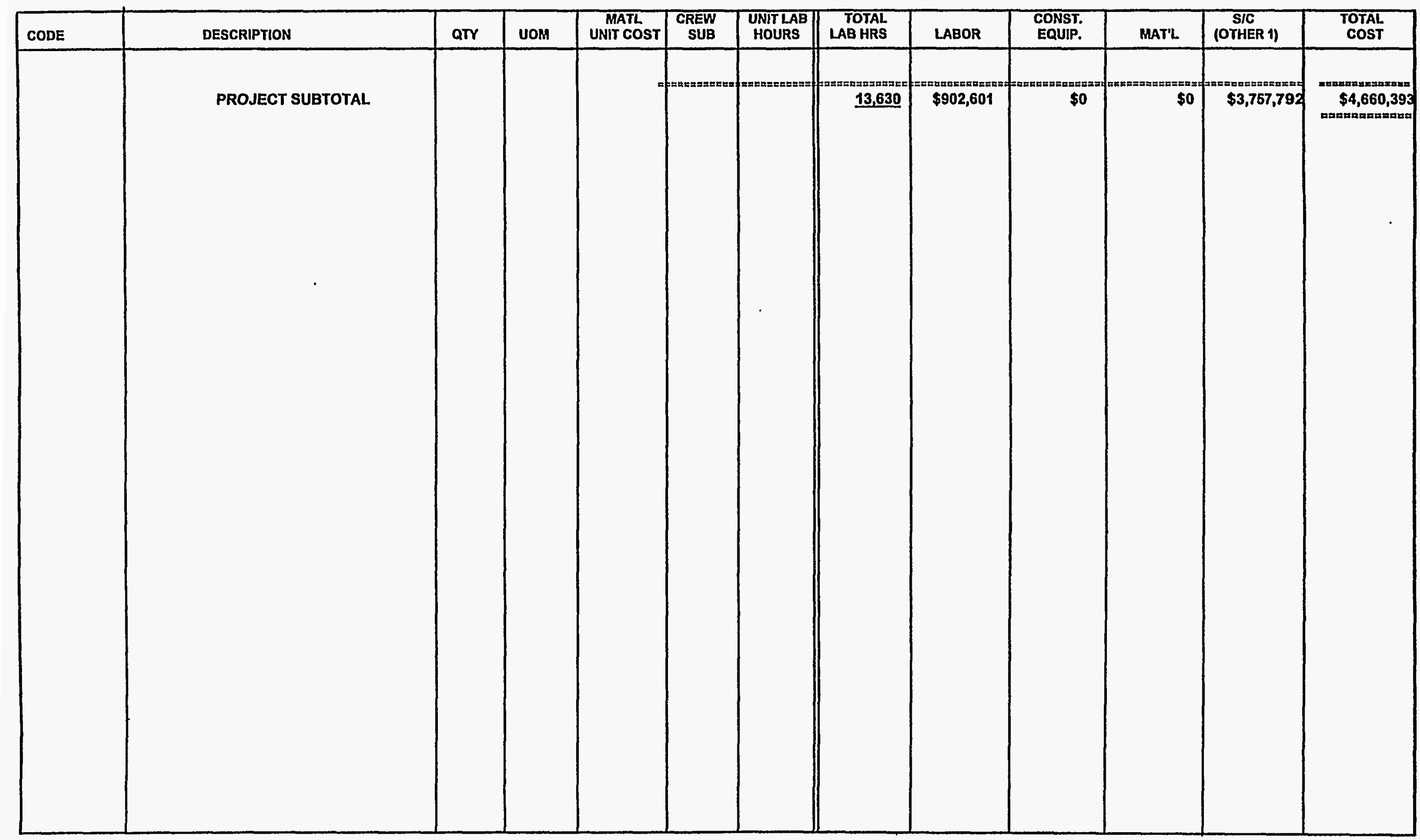


Lockheed Martin Idaho Technologies Co. Rev6-9S

PROJECT NAME: Permitting/Documentation

Risk Based - Clean

LOCATION 1: INEELICPP

REQUESTOR: Bryan Spaulding
CONTINGENCY ANALYSIS
TYPE OF ESTIMATE: PLANNING
PROJECT NO: 2423-A1 D

PROJECT NO:
PREPARED BY:
S. L. COWard
DATE: 27 Jan-1998

TME: 19:00:49
REPORT NAME: Contingency Analysis

\begin{tabular}{|c|c|c|c|c|c|c|c|c|c|c|c|}
\hline \multicolumn{9}{|c|}{ PROBABLE \% VARIATION } & \multicolumn{2}{|c|}{$\begin{array}{l}\text { PROJECT } \\
\text { CONTINGENCY }\end{array}$} & \multirow{3}{*}{$\begin{array}{l}\text { SUMMARY } \\
\text { Total Cost } \\
\text { by Element }\end{array}$} \\
\hline \multirow[t]{2}{*}{$\begin{array}{l}\text { WBS } \\
\text { Element }\end{array}$} & \multirow{2}{*}{ Cost Estimate Element } & \multirow{2}{*}{$\begin{array}{l}\text { Total Cost w/o } \\
\text { Contingency }\end{array}$} & \multirow[t]{2}{*}{$\begin{array}{l}\% \text { Total } \\
\text { Cost }\end{array}$} & \multicolumn{2}{|c|}{$\begin{array}{l}\text { Prob. \% Var. } \\
\text { From Est. }\end{array}$} & \multicolumn{2}{|c|}{ Wh \% of Prob. } & \multirow[t]{2}{*}{ Contingency } & \multirow[t]{2}{*}{$\%$} & \multirow[t]{2}{*}{ Cost } & \\
\hline & & & & - & 4 & - & + & & & & \\
\hline 1.1.1 & CONCEPTUAL DESIGN & 0 & 0.00 & 10 & 40 & 0.00 & 0.00 & $0.000 \%$ & $0.00 \%$ & 0 & 0 \\
\hline 1.21 & PM FOR PROJECT DEVELOPMENT & 768,088 & 16.35 & 10 & 40 & 1.63 & 6.54 & $5.722 \%$ & $16.35 \%$ & 261,955 & $1,030,043$ \\
\hline 1.22 & PROSECT EXECUTION & 149,704 & 3.19 & 10 & 40 & 0.32 & 1.27 & $1.115 \%$ & $3.19 \%$ & 51,056 & 200,760 \\
\hline 1.3 .1 & PERMTTING & $3,742,601$ & 79.57 & 10 & 40 & 797 & 31.87 & $27.883 \%$ & $79.67 \%$ & $1,276,406$ & $5,019,007$ \\
\hline \multirow[t]{2}{*}{1.5 .2} & PROCUREMENT FEES & 37,426 & 0.80 & 10 & 40 & 0.08 & 0.32 & $0.279 \%$ & $0.80 \%$ & 12,764 & 50,190 \\
\hline & ESCALATION & 0 & 0.00 & 0 & 0 & 0.00 & 0.00 & $0.000 \%$ & $0.00 \%$ & 0 & 0 \\
\hline & SUBTOTAL & $4,697,819$ & 100.00 & & & & & $35.000 \%$ & & & $\cdot$ \\
\hline & CALCULATED CONTINGENCY & $1,644,237$ & & & & & & & & & \\
\hline & RESULTANT TEC & $6,342,056$ & & & & & & & & & \\
\hline & ROUNDED TEC & $6,300,000$ & & & & & & & & & \\
\hline & PROJECT CONTINGENCY & $1,602,181$ & & & & & & $34.10 \%$ & & & \\
\hline & MANAGEMENT RESERVE & 378,003 & & & & & & & & & \\
\hline & CONTINGENCY & $1,224,178$ & & & & & & & & & \\
\hline & TOTAL ESTIMATED COST & $6,300,000$ & & & & & & & & $1,602,181$ & $6,300,000$ \\
\hline
\end{tabular}

\section{CONFIDENCE LEVEL AND ASSUMED RISKS:}

The Lockheed Idaho Technologies Co. Cost Estimate Contingency Analysis Model is based on the applied contingency and the assumptions upon which the estimate was predicated. The model is applied with a suggested risk level of $18 \%$ and a level of confidence of $90 \%$ the estimate will fall within the bid range. The Contingency Analysis is based on a weighted average to provide a

$90 \%$ probability of underrun and a $10 \%$ probability of overtun.
CONTINGENCY ANALYSIS GUIDE BY TYPE OF ESTIMATE

Guidelines established by DOE/FM 50, Cost Estimating Guide, Vol. 6 ,

Cost Guide, and as presented in the INEL Cost Estimating Guide. PLANNING Experimental/Special Conditions $20 \%-30 \%$ Conceptual

ceptual $\quad 15 \%-25 \%$ TITLE |

TITLE II

TITLE IUAFC
$10 \%-20 \%$

$5 \%-15 \%$

Market Conditions 


\section{G\&A/PIF ADDER CALCULATION SHEET ICPP BIN SET CLOSURE \\ RISK BASED CLEAN CLOSURE; CLEAN FILL; UNESCALATED \\ REGULATORY COMPLIANCE}

PROCUREMENT FEE:

CONSTRUCTION $=$

$\$ 3,742,601$

GFE =

Subtotal $\$ 3,742,601$

FEE @ 1\%=

$\$ 3,742,601 * 0.01=$

$\$ 37,426$

G\&A@ $@ 23 \%$ (with a ceiling of $\$ 500,000$ imposed per year, 3 yrs)

CONSTRUCTION OR

CEILING $\bullet 3$ YEARS $=\quad \$ 1,500,000$

GFE $=$

PROCUREMENT FEE $=$ $\$ 37,426$

Subtotal $\$ 1,537,426$

FEE @ 23\% =

$\$ 1,537,426 * 0.23=$

$\$ 353,608$

PIF @ 5.5\%

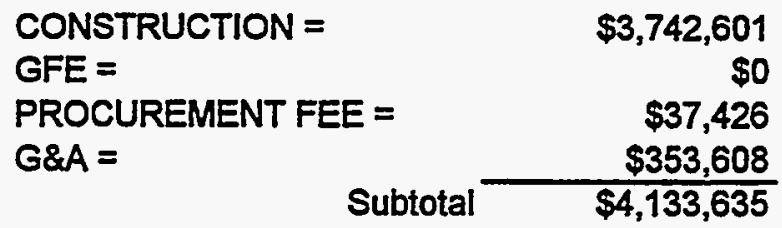

FEE @ 5.5\% =

$\$ 4,133,635 \cdot 0.055=\quad \$ 227,350$

TOTAL PROCUREMENT FEE:

$\$ 37,426$

TOTAL G\&A FEE:

$\$ 353,608$

TOTAL PIF:

$\$ 227,350$ 
Lockheed Martin Idaho Technologies Co. PROJECT NAME: ICPP Bin Set Closure (EIS Stud LOCATION 1: Place Clean Grout in Vault REQUESTOR:
TYPE OF ESTIMATE: Planning PROJECT NO: 2423-A2 PREPARED BY: S. L. Coward REPORT NAME: Cost Estimate Summary
DATE 27 Jan-1998

TIME: 19:06:11

CHECKED BY:

APPRD BY:

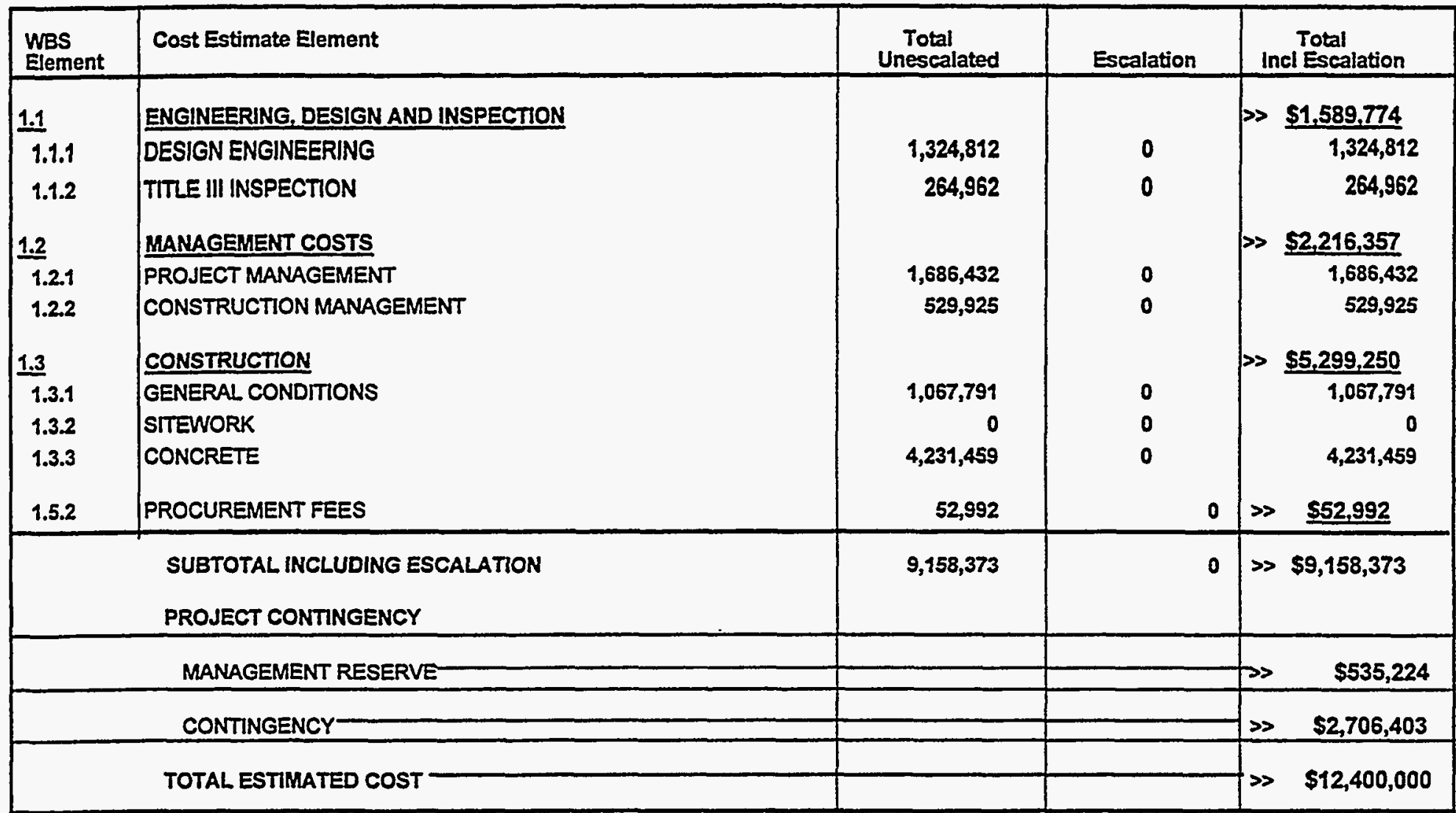

PROJECT COST PARAMETERS

EDI AS A $\%$ OF CONST. + GFE $=30.00 \%$ 


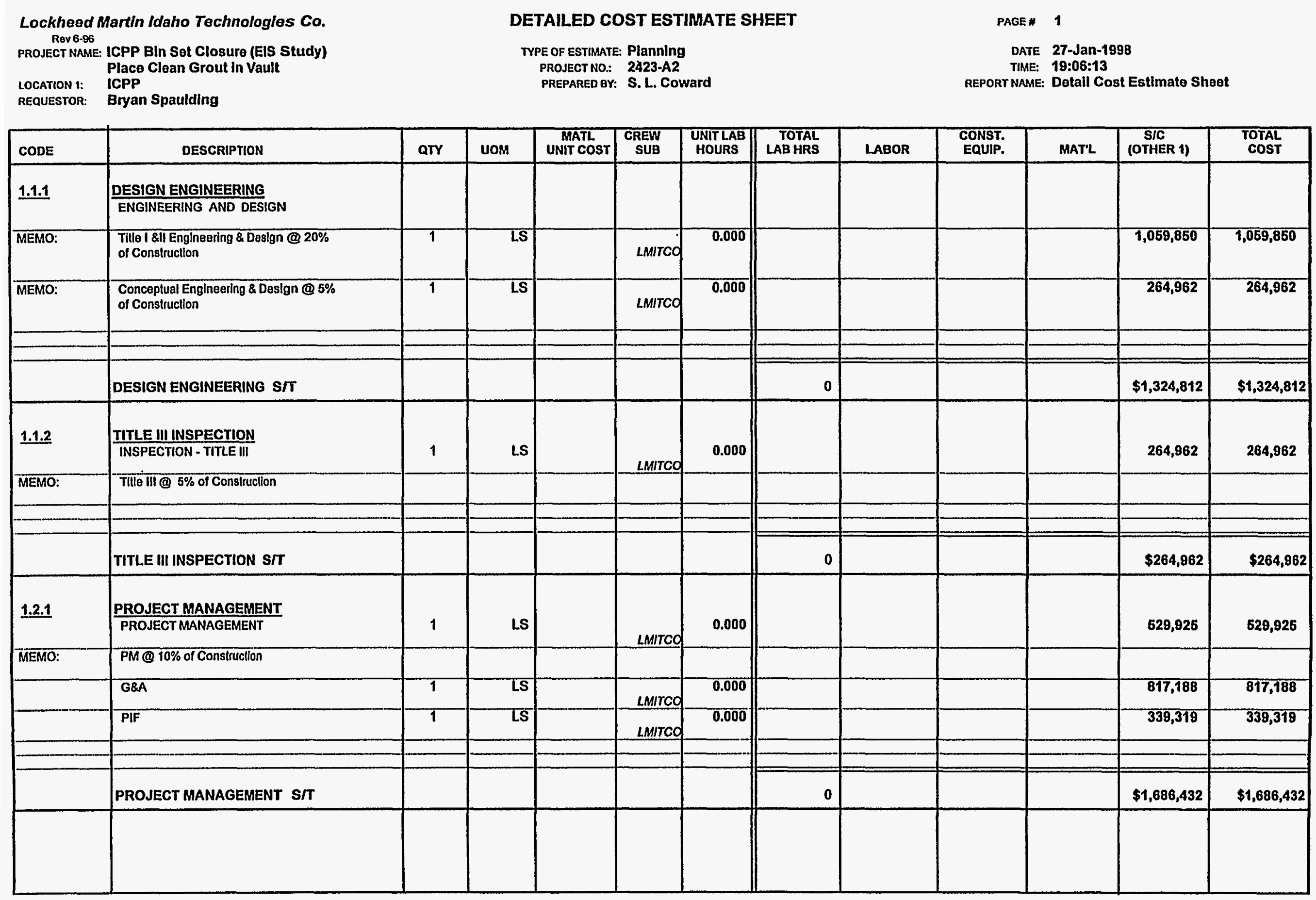


Lockheed Martin Idaho Technologles Co. Rov 6.96 PROJECT NAME: ICPP BIn Set Closure (EIS Study) Place Clean Grout in Vault LOCATION 1: ICPP REQUESTOR: Bryan Spaulding
DETAILED COST ESTIMATE SHEET

TYPE OF estimate: Planning

PROJECT NO.: 2423-A2

PREPARED BY: S. L. Coward
PAge* 3

DATE 27-Jan-1998

TIME: 19:06:13

REPORT NAME: Detall Cost Estimate Shoet

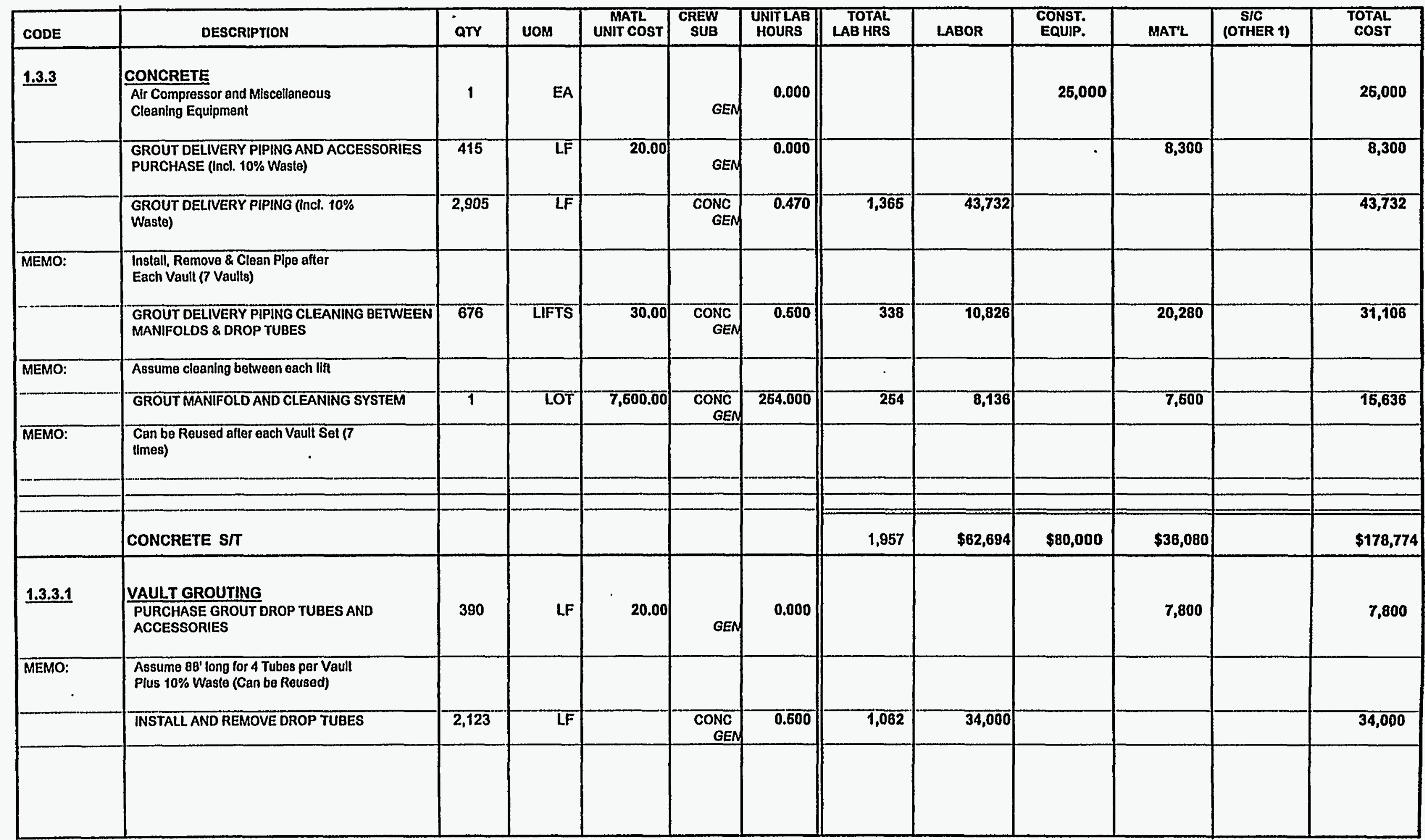


Lockheed Martin Idaho Technologies Co.

Rev $6-56$

PROJECT NAME: ICPP Bin Set Closure (EIS Stud

LOCATION 1: ICPP

REQUESTOR: Bryan Spaulding
CONTINGENCY ANALYSIS

$\begin{aligned} \text { TYPE OF ESTIMATE: } & \text { Planning } \\ \text { PROJECT NO: } & \text { 2423-A2 } \\ \text { PREPARED BY: } & \text { S.L. Coward }\end{aligned}$

DATE: 27 Jan-1998

TIME: 19:06:07

REPORT NAME: Contingency Analysis

\begin{tabular}{|c|c|c|c|c|c|c|c|c|c|c|c|}
\hline \multicolumn{9}{|c|}{ PROBABLE \% VARIATION } & \multicolumn{2}{|c|}{$\begin{array}{l}\text { PROJECT } \\
\text { CONTINGENCY }\end{array}$} & \multirow{3}{*}{$\begin{array}{l}\text { SUMMARY } \\
\text { Total Cost } \\
\text { by Element }\end{array}$} \\
\hline WBS & \multirow{2}{*}{ Cost Estimate Element } & \multirow{2}{*}{$\begin{array}{l}\text { Total Cost w/o } \\
\text { Contingency }\end{array}$} & \multirow{2}{*}{$\begin{array}{l}\% \text { Total } \\
\text { Cost }\end{array}$} & \multicolumn{2}{|c|}{$\begin{array}{l}\text { Prob. \% Var. } \\
\text { From Est }\end{array}$} & \multicolumn{2}{|c|}{ Wt \% of Prob. } & \multirow{2}{*}{ Contingency } & \multirow{2}{*}{$\%$} & \multirow{2}{*}{ Cost } & \\
\hline & & & & - & + & - & + & & & & \\
\hline 1.1 .1 & DESIGN ENGINEERING & $1,324,812$ & 14.47 & 10 & 40 & 1.45 & 5.79 & $5.063 \%$ & $14.47 \%$ & 468,920 & $1,793,732$ \\
\hline 1.1 .2 & TTLE III INSPECTION & 264,962 & 2.89 & 10 & 40 & 0.29 & 1.16 & $1.013 \%$ & $289 \%$ & 93,784 & 358,746 \\
\hline$\overline{1.21}$ & PROJECT MANAGEMENT & $1,686,432$ & 98.41 & 10 & 40 & 1.84 & 7.37 & $6.445 \%$ & $18.41 \%$ & 596,916 & $2,283,348$ \\
\hline 1.2 .2 & CONSTRUCTION MANAGEMENT & 529,925 & 5.79 & 10 & 40 & 0.58 & 231 & $2025 \%$ & $5.79 \%$ & 187,568 & 717,493 \\
\hline 1.3 .1 & GENERAL CONOITIONS & $1,067,791$ & 11.66 & 10 & 40 & 1.17 & 4.66 & $4.081 \%$ & $11.66 \%$ & $3 \pi, 947$ & $1,445,738$ \\
\hline 1.3 .2 & STIEWORK & 0 & 0.00 & 0 & 0 & 0.00 & 0.00 & $0.000 \%$ & $0.00 \%$ & 0 & 0 \\
\hline 1.3 .3 & CONCRETE & $4,231,459$ & 46.20 & 10 & 40 & 4.62 & 18.48 & $16.171 \%$ & $46.20 \%$ & $1,497,735$ & $5,729,194$ \\
\hline \multirow[t]{2}{*}{1.5 .2} & PROCUREMENT FEES & 52,992 & 0.58 & 10 & 40 & 0.06 & 0.23 & $0.203 \%$ & $0.58 \%$ & 18,757 & 71,749 \\
\hline & ESCALATION & 0 & 0.00 & 0 & 0 & 0.00 & 0.00 & $0.000 \%$ & $0.00 \%$ & 0 & 0 \\
\hline & SUBTOTAL & $9,158,373$ & 100.00 & & & & & $35.000 \%$ & & & \\
\hline & CALCULATED CONTINGENCY & $3,205,431$ & & & & & & & & & \\
\hline & RESULTANT TEC & $12,363,804$ & & & & & & & & & \\
\hline & ROUNDED TEC & $12,400,000$ & & & & & & & & & \\
\hline & PROJECT CONTINGENCY & $3,241,627$ & & & & & & $35.40 \%$ & & & \\
\hline & MANAGEMENT RESERVE & 535,224 & & & & & & & & & \\
\hline & CONTINGENCY & $2,706,403$ & & & & & & & & & \\
\hline & TOTAL ESTIMATED COST & $12,400,000$ & & & & & & & & $3,241,627$ & $12,400,000$ \\
\hline
\end{tabular}

\section{CONFIDENCE LEVEL AND ASSUMED RISKS:}

The Lockheed Idaho Technologies Co. Cost Estimate Contingency Analysis Model is based on the applied contingency and the assumptions upon which the estimate was predicated. The model is applied with a suggested risk level of $18 \%$ and a level of confidence of $90 \%$ the estimate will fall within the bid range. The Contingency Analysis is based on a weighted average to provide a $90 \%$ probability of undernun and a $10 \%$ probability of overrun.
CONTINGENCY ANALYSIS GUIDE BY TYPE OF ESTIMATE

Guidelines established by DOEJFM 50 , Cost Estimating Guide. Vol. 6 , Cost Guide, and as presented in the INEL Cost Estimating Guide. PLANNING ExperimentalSpecial Conditions. Conceptual

Experimentalspecial Conditions. TITLE I

TITLE II

TIILE IUAFC
$20 \%-30 \%$

$75 \%$ to $50 \%$

- $25 \%$

$10 \%-20 \%$

$5 \%-15 \%$

Market Conditions 


\section{G\&A/PIF ADDER CALCULATION SHEET \\ ICPP BIN SET CLOSURE \\ RISK BASED CLEAN CLOSURE; CLEAN FILL; UNESCALATED}

FILL VAULTS W/ CLEAN GROUT

PROCUREMENT FEE:

CONSTRUCTION $=\quad \$ 5,299,250$

GFE $=$

Subtotal $\$ 5,299,250$

FEE @ 1\% =

$\$ 5,299,250 * 0.01=$

$\$ 52,993$

G\&A @ 23\% (with a ceiling of $\$ 500,000$ imposed per year, 7 yrs)

\begin{tabular}{lr} 
CONSTRUCTION OR & \\
CEILING * 7 YEARS $=$ & $\$ 3,500,000$ \\
GFE = & $\$ 0$ \\
PROCUREMENT FEE $=$ & $\$ 52,993$ \\
\hline & Subtotal \\
\cline { 2 - 2 } & $\$ 3,552,993$
\end{tabular}

FEE @ 23\% =

$\$ 3,552,993 * 0.23=$

$\$ 817,188$

PIF @ 5.5\%

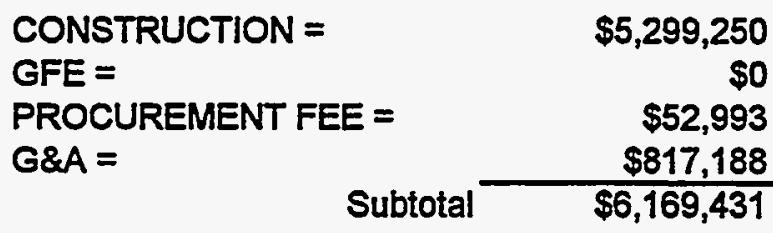

FEE @ $5.5 \%=$

$\$ 6,169,431 \cdot 0.055=$

$\$ 339,319$

TOTAL PROCUREMENT FEE:

$\$ 52,993$

TOTAL G\&A FEE:

$\$ 817,188$

TOTAL PIF:

$\$ 339,319$ 
Lockheed Martin Idaho Technologies Co. Rov. 6-96 PROJECT NAME: ICPP Bin Set Closure (EIS Stud -Tractor (Risk Based Estimates) LOCATION 1: ICPP REQUESTOR: Bryan Spaulding
COST ESTIMATE SUMMARY

$\begin{array}{cl}\text { TYPE OF ESTIMATE: } & \text { Planning } \\ \text { PROJECT NO: } & 2423-C 1 \\ \text { PREPARED BY: } & \text { S. L. Coward } \\ \text { REPORT NAME: } & \text { Cost Estimate Summary }\end{array}$

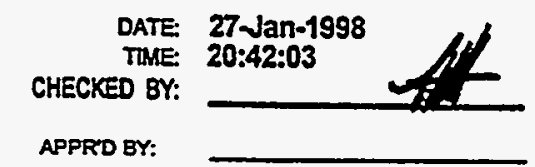

\begin{tabular}{|c|c|c|c|c|}
\hline $\begin{array}{l}\text { WBS } \\
\text { Element }\end{array}$ & Cost Estimate Element & $\begin{array}{c}\text { Total } \\
\text { Unescalated }\end{array}$ & Escalation & $\begin{array}{c}\text { Total } \\
\text { Incl Escalation }\end{array}$ \\
\hline $\begin{array}{l}\frac{1.1}{1.1 .1} \\
1.1 .2 \\
1.2 \\
1.2 .1 \\
1.2 .2 \\
\frac{1.3}{1.3 .1} \\
1.3 .13 \\
1.5 .2\end{array}$ & $\begin{array}{l}\text { ENGINEERING, DESIGN AND INSPECTION } \\
\text { DESIGN ENGINEERING TITLE I \& II } \\
\text { TITLE III INSPECTION } \\
\text { MANAGEMENT COSTS } \\
\text { PROJECT MANAGEMENT } \\
\text { CONSTRUCTION MANAGEMENT } \\
\text { CONSTRUCTION } \\
\text { GENERAL CONDITIONS } \\
\text { SPECIAL CONSTRUCTION } \\
\text { PROCUREMENT FEES }\end{array}$ & $\begin{array}{r}9,900,244 \\
3,300,081 \\
11,639,957 \\
6,600,163 \\
\\
6,840,414 \\
59,161,216 \\
660,016\end{array}$ & $\begin{array}{l}0 \\
0 \\
0 \\
0\end{array}$ & 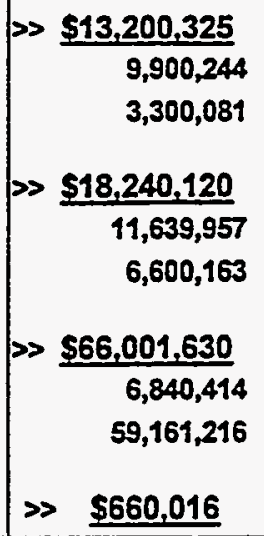 \\
\hline & $\begin{array}{l}\text { SUBTOTAL INCLUDING ESCALATION } \\
\text { PROJECT CONTINGENCY }\end{array}$ & $98,102,091$ & 0 & >> $\$ 98,102,091$ \\
\hline & MANAGEMENT RESERVE- & & & $\gg \quad \$ 6,666,165$ \\
\hline & CONTINGENCY- & & & $\gg \quad \$ 27,631,744$ \\
\hline & TOTAL ESTIMATED COST & & & $\gg \$ 132,400,000$ \\
\hline
\end{tabular}

PROJECT COST PARAMETERS

EDI AS A $\%$ OF CONST. + GFE $=20.00 \%$

CONTINGENCY $=34.96 \%$ 
Lockheed Martin Idaho Technologles Co. Rov 6.96

PROJECT NAME: ICPP Bin Set Closure.(EIS Study) Tractor (Rlsk Based Estimates)

LOCATION 1: ICPP

REQUESTOR: Byyan Spaulding
DETAILED COST ESTIMATE SHEET

TPE OF Estimate: Planning

PROJECT NO:: 2423-C1

PREPARED BY: S. L. Coward
PAgE: 1

DATE 27-Jan-1998

TIME: 20:42:06

REPORT NAME: Detall Cost Estlmate Shoot

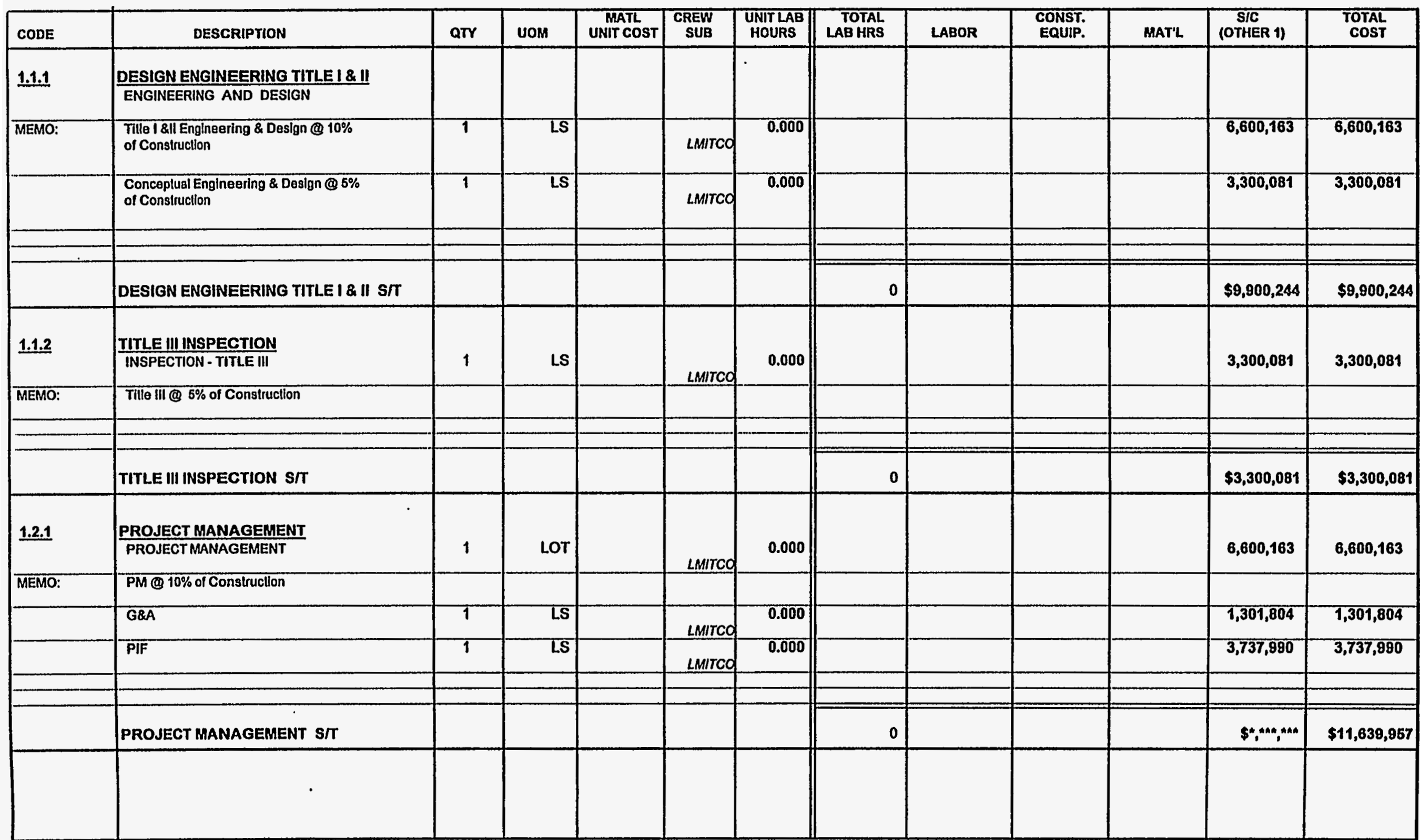


Lockheod Martin Idaho Technologies Co.

$$
\text { Rav 6.96 }
$$

ROJECT NAME: ICPP Bin Set Closure (EIS Study)

Tractor (RIsk Based Estimates)

Tractor

Bryan Spaulding

\section{DETAILED COST ESTIMATE SHEET}

TYPE OF ESTIMATE: Planning

PROJECT NO.: 2423-C1

PREPARED BY: S. L. Coward
PAGE \# 2

DATE 27 Jan-1898

TIME: 20:42:06

report name: Detall Cost Estlmate Sheot

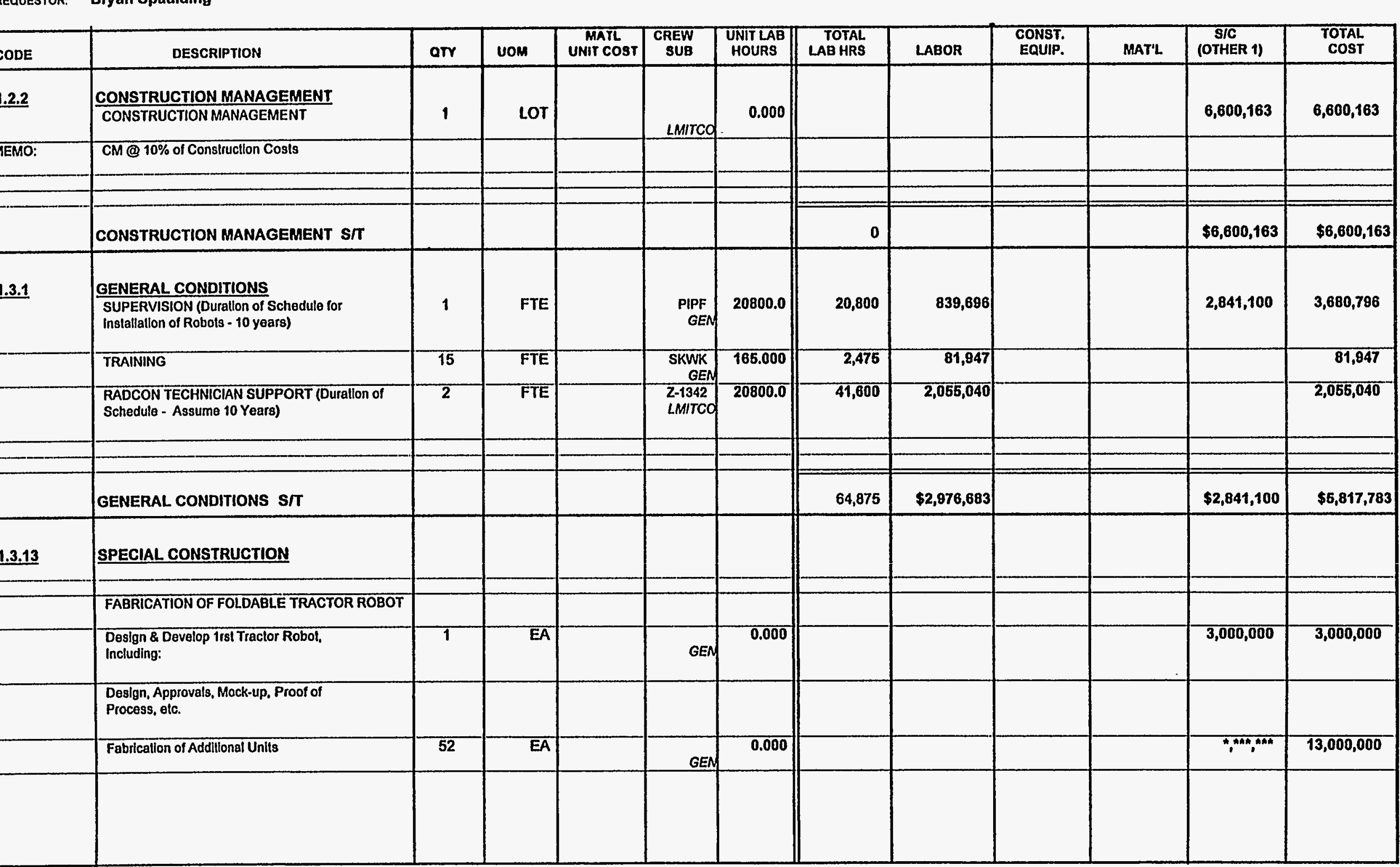


Lockheed Martin Idaho Technologles Co. Rov 6.96 PROJECT NAME: ICPP BIn Set Closure (ElS Study) Tractor (Risk Based EstImates) LOCATION 1: ICPP

REQUESTOR: Bryan Spaulding
DETAILED COST ESTIMATE SHEET

TYPE OF ESTIMATE: Planning

PROJECT NO:: 2423.C1

PREPARED BY: S. L. Coward
PAgE * 3

DATE 27-Jan-1998

TIME: 20:42:05

REPORT NAME: Detall Cost Estlmate Shoot

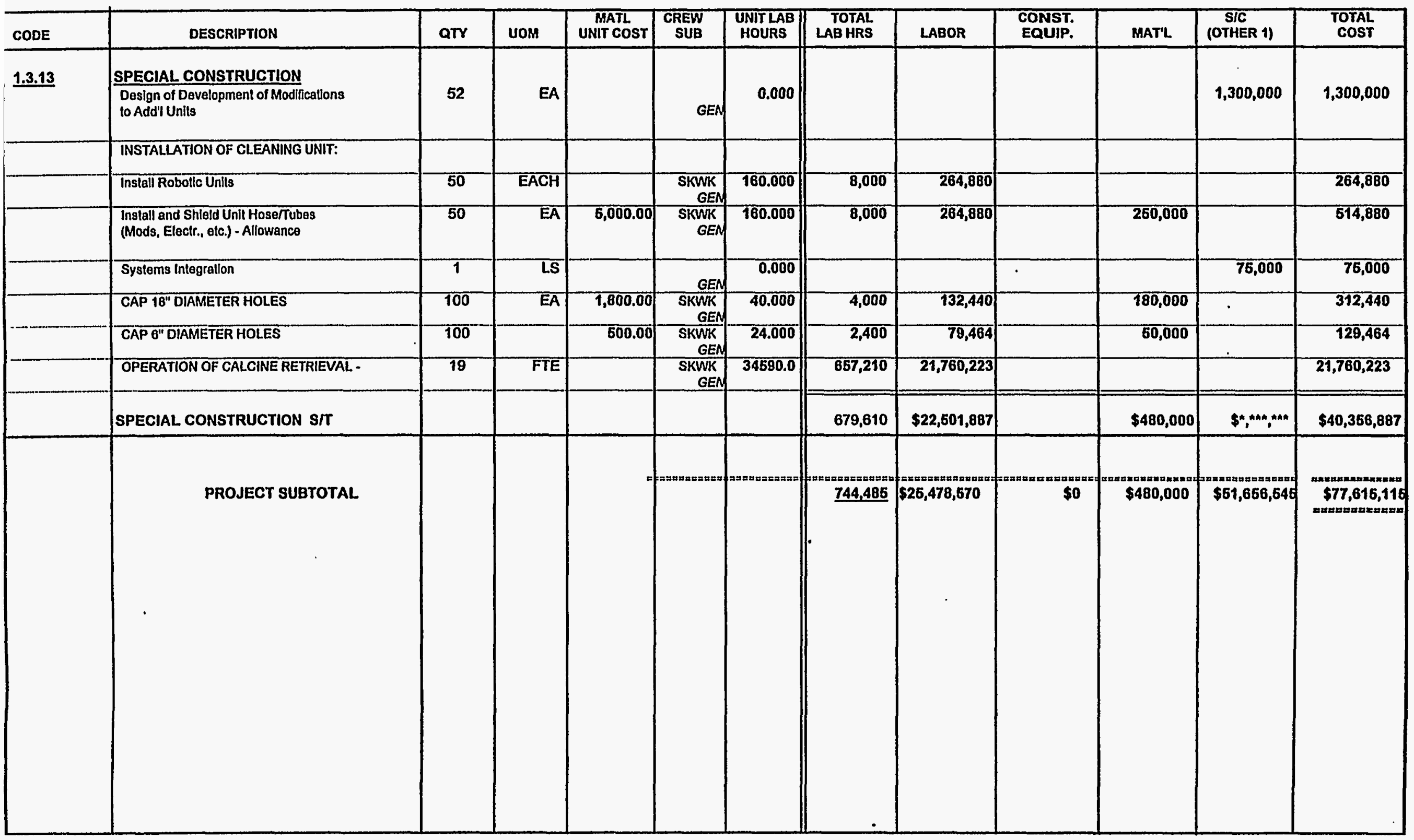


Lockheed Martin Idaho Technologies Co. PROJECT NAME: ICPP Bin Set Closure (EIS Stud Tractor (Risk Based Estimates) LOCATION 1: ICPP REQUESTOR: Bryan Spaulding
CONTINGENCY ANALYSIS
TYPE OFESTIMATE: Planning

PROJECT NO: 2423-C1

PREPARED BY: S. L. Cowat
DATE: 27-Jan-1998

TME: 20:42:00
REPORT NAME: Contingency Analysis

\begin{tabular}{|c|c|c|c|c|c|c|c|c|c|c|c|}
\hline & PROB & BLE \% VARIA & ION & & & & & & $\begin{array}{l}\text { PRC } \\
\text { CONT }\end{array}$ & $\begin{array}{l}\text { CT } \\
\text { JENCY }\end{array}$ & SUMMARY \\
\hline WBS & Cost Estimate Element & Total Cost wio & $\begin{array}{l}\% \text { Total } \\
\text { cost }\end{array}$ & & $\begin{array}{l}\% \text { Var. } \\
\text { m Est. }\end{array}$ & Wt \% & Prob. & Contingency & $\%$ & Cost & Tot 1 cost \\
\hline & & Contingency & & - & + & - & + & & & & by Eement \\
\hline 1.1 .1 & DESIGN ENGINEERING TITLE $\mid \& \|$ & $9,900,244$ & 10.09 & 10 & 40 & 1.01 & 4.04 & $3.532 \%$ & $10.09 \%$ & $3,461,268$ & $13,361,512$ \\
\hline 1.12 & TILE III INSPECTION & $3,300,081$ & 3.36 & 10 & 40 & 0.34 & 1.35 & $1.177 \%$ & $3.36 \%$ & $1,153,756$ & $4,453,837$ \\
\hline 1.21 & PROJECT MANAGEMENT & $11,639,957$ & 11.87 & 10 & 40 & 1.19 & 4.75 & $4.153 \%$ & $11.87 \%$ & $4,069,497$ & $15,709,454$ \\
\hline 122 & CONSTRUCTION MANAGEMENT & $6,600,163$ & 6.73 & 10 & 40 & 0.57 & 2.69 & $2355 \%$ & $6.73 \%$ & $2,307,512$ & $8,907,675$ \\
\hline 1.3 .1 & GENERAL CONDITONS & $6,840,414$ & 6.97 & 10 & 40. & 0.70 & 279 & $2440 \%$ & $6.97 \%$ & $2,391,508$ & $9,231,922$ \\
\hline 1.3 .13 & SPECLAL CONSTRUCTION & $59,161,216$ & 60.31 & 10 & 40 & 6.03 & 24.12 & $21.107 \%$ & $60.31 \%$ & $20,683,616$ & $79,844,832$ \\
\hline 1.5 .2 & PROCUREMENT FEES & 660,016 & 0.67 & 10 & 40 & 0.07 & 0.27 & $0.235 \%$ & $0.67 \%$ & 230,751 & 890,767 \\
\hline & ESCALATION & 0 & 0.00 & 0 & 0 & 0.00 & 0.00 & $0.000 \%$ & $0.00 \%$ & 1 & 1 \\
\hline & SUBTOTAL & $98,102,091$ & 100.00 & & & & & $35.000 \%$ & & & \\
\hline & CALCULATED CONTINGENCY & $34,335,732$ & & & & & & & & & \\
\hline & RESULTANT TEC & $132,437,823$ & & & & & & & & & \\
\hline & ROUNDED TEC & $132,400,000$ & & & & & & & & & \\
\hline & PROJECT CONTINGENCY & $34,297,909$ & & & & & & $34.96 \%$ & & & \\
\hline & MANAGEMENT RESERVE & $6,666,165$ & & & & & & & & & \\
\hline & CONTINGENCY & $27,631,744$ & & & & & & & & & \\
\hline & TOTAL ESTIMATED COST & $132,400,000$ & & & & & & & & $34,297,909$ & $132,400,000$ \\
\hline
\end{tabular}

\section{CONFIDENCE LEVEL AND ASSUMED RISKS:}

The Lockheed ldaho Technologies Co. Cost Estimate Contingency Analysis Model is based on the applied contingency and the assumptions upon which the estimate was predicated. The model is applied with a suggested risk level of $18 \%$ and a level of confidence of $90 \%$ the estimate will fall within the bid range.

The Contingency Analysis is based on a weighted average to provide a

$90 \%$ probability of underrun and a $10 \%$ probability of ovemun.
CONTINGENCY ANALYSIS GUIDE BY TYPE OF ESTIMATE

Guidelines established by DOE/FM 50 . Cost Estimating Guide, Vol. 6 , Cost Guide, and as presented in the INEl Cost Estimating Guide. PLANNING Experimental/Special Conditions

Conceptual

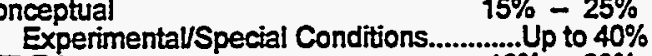
TITE I

TITLE II

TITLE IIJAFC .....Up to $50 \%$ $10 \%-20 \%$

$5 \%-15 \%$ Market Conditions 


\section{G\&APIF ADDER CALCULATION SHEET \\ ICPP BIN SET CLOSURE \\ RISK BASED CLEAN CLOSURE; CLEAN FILL; UNESCALATED \\ CLEAN BINS W/ ROBOTS - FLOOR}

PROCUREMENT FEE:

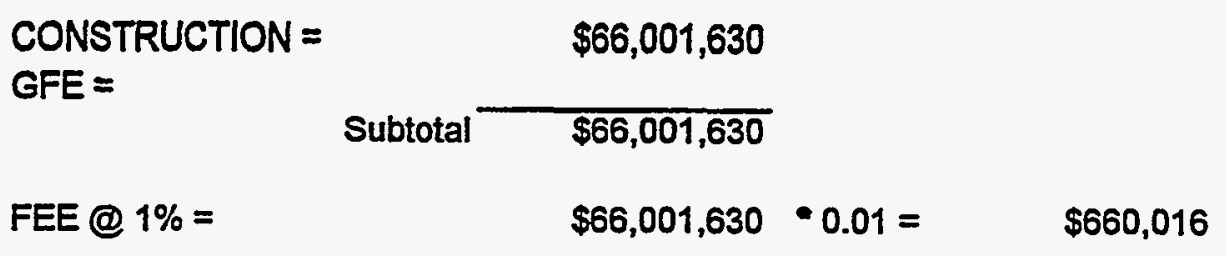

G\&A @ 23\% (with a ceiling of $\$ 500,000$ imposed per year, 10 yrs)

CONSTRUCTION OR

CEILING $\cdot 10$ YEARS $=\quad \$ 5,000,000$

GFE = \$O

PROCUREMENT FEE $=\quad \$ 660,016$

Subtotal $\$ 5,660,016$

FEE @ $23 \%=\quad \$ 5,660,016 * 0.23=\$ 1,301,804$

PIF @ 5.5\%

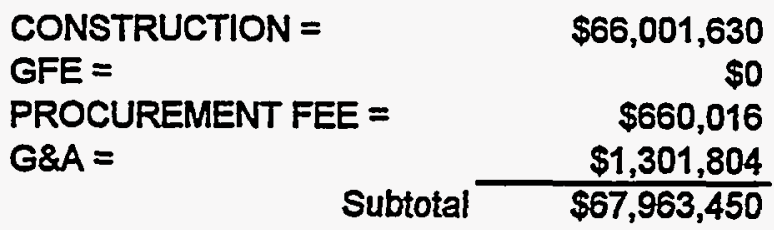

FEE @ $5.5 \%=\quad \$ 67,963,450 \cdot 0.055=\quad \$ 3,737,990$

$\begin{array}{ll}\text { TOTAL PROCUREMENT FEE: } & \$ 660,016\end{array}$

TOTAL G\&A FEE: $\quad \$ 1,301,804$

TOTAL PIF: $\quad \$ 3,737,990$ 
Lockheed Martin Idaho Technologies Co. Rov, $6-96$

PROJECT NAME: ICPP Bin Set Closure (EIS Stud

LOCATION 1: LDUA (Risk Based Estimates)

1: ICPP

REQUESTOR: Bryan Spaulding
COST ESTIMATE SUMMARY

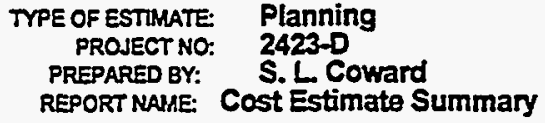

TIME

CHECKED BY

27-Jan-1998 20:47:33

APPRD BY:

\begin{tabular}{|c|c|c|c|c|}
\hline $\begin{array}{l}\text { WBS } \\
\text { Element }\end{array}$ & Cost Estimate Element & $\begin{array}{c}\text { Total } \\
\text { Unescalated }\end{array}$ & Escalation & $\begin{array}{c}\text { Total } \\
\text { Incl Escalation }\end{array}$ \\
\hline $\begin{array}{l}\frac{1.1}{1.1 .1} \\
1.1 .2 \\
\frac{1.2}{1.2 .1} \\
1.2 .2 \\
\frac{1.3}{1.3 .1} \\
1.3 .13 \\
1.5 .2\end{array}$ & $\begin{array}{l}\text { ENGINEERING, DESIGN AND INSPECTION } \\
\text { DESIGN ENGINEERING TITLE I \& } \| \\
\text { TILE III INSPECTION } \\
\text { MANAGEMENT COSTS } \\
\text { PROJECT MANAGEMENT } \\
\text { CONSTRUCTION MANAGEMENT } \\
\text { CONSTRUCTION } \\
\text { GENERAL CONDITIONS } \\
\text { SPECIAL CONSTRUCTION } \\
\text { PROCUREMENT FEES }\end{array}$ & $\begin{array}{r}2,083,513 \\
694,504 \\
\\
3,407,558 \\
2,463,481 \\
\\
1,831,085 \\
12,059,014 \\
138,901\end{array}$ & $\begin{array}{l}0 \\
0 \\
0 \\
0 \\
0 \\
0\end{array}$ & $\begin{array}{r}\text { >> } \begin{array}{r}\$ 2,778,017 \\
2,083,513 \\
694,504\end{array} \\
>\frac{\$ 5,871,039}{3,407,558} \\
2,463,481 \\
\\
>\frac{\$ 13,890,099}{1,831,085} \\
12,059,014\end{array}$ \\
\hline & $\begin{array}{l}\text { SUBTOTAL INCLUDING ESCALATION } \\
\text { PROJECT CONTINGENCY }\end{array}$ & $22,678,056$ & 0 & >> \$22,678,056 \\
\hline & MANAGEMENT RESERVE- & & & $\$ 1,402,900$ \\
\hline & CONTINGENCY - & & & $\$ 6,519,044$ \\
\hline & TOTAL ESTIMATED COST - & & & $\$ 30,600,000$ \\
\hline
\end{tabular}

PROJECT COST PARAMETERS

EDI AS A $\%$ OF CONST. + GFE $=20.00 \%$

CONTINGENCY $=34.93 \%$ 
Lockheed Martin Idaho Technologies Co.

Rev $6-96$

PROJECT NAME: ICPP Bin Set Closure (EIS Stud

LOCATION 1: LDUA (Risk Based Estimates)

ICPP

REQUESTOR: Bryan Spaulding

\section{CONTINGENCY ANALYSIS}

TYPE OF ESTRMATE: Planning

PROJECT NO: 2423-D

PREPARED BY: S. L. Coward
DATE: 27Jan-1998

TME: 20:47:30

REPORT NAME: Contingency Analysis

\begin{tabular}{|c|c|c|c|c|c|c|c|c|c|c|c|}
\hline \multicolumn{9}{|c|}{ PROBABLE \% VARIATION } & \multicolumn{2}{|c|}{$\begin{array}{l}\text { PROJECT } \\
\text { CONTINGENCY }\end{array}$} & \multirow{3}{*}{$\begin{array}{l}\text { SUMMARY } \\
\text { Total Cost } \\
\text { by Element }\end{array}$} \\
\hline \multirow[t]{2}{*}{$\begin{array}{l}\text { WBS } \\
\text { Element }\end{array}$} & \multirow{2}{*}{ Cost Estimate Element } & \multirow{2}{*}{$\begin{array}{l}\text { Total Cost w/o } \\
\text { Contingency }\end{array}$} & \multirow[t]{2}{*}{$\begin{array}{l}\text { \% Total } \\
\text { Cost }\end{array}$} & \multicolumn{2}{|c|}{$\begin{array}{c}\text { Prob. \% Var. } \\
\text { Erom Est. }\end{array}$} & \multicolumn{2}{|c|}{ Wt \% of Prob. } & \multirow[t]{2}{*}{ Contingency } & \multirow[t]{2}{*}{$\%$} & \multirow[t]{2}{*}{ Cost } & \\
\hline & & & & 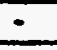 & + & - & \pm & & & & \\
\hline 1.1.1 & DESIGN ENGINEERING TILE $1 \& \|$ & $2,083,513$ & 9.19 & 10 & 40 & 0.92 & 3.67 & $3.216 \%$ & $9.19 \%$ & 727,817 & $2,811,330$ \\
\hline 1.1 .2 & TIRE III INSPECTION & 694,504 & 3.06 & 10 & 40 & 0.31 & 1.22 & 1.072\% & $3.06 \%$ & 242,605 & 937,110 \\
\hline 1.2 .1 & PROJECT MANAGEMENT & $3,407,558$ & $\mathbf{1 5 . 0 3}$ & 10 & 40 & 1.50 & 6.09 & $5.259 \%$ & $15.03 \%$ & $1,190,335$ & $4,597,893$ \\
\hline 1.22 & CONSTRUCTION MANAGEMENT & $2,463,489$ & 10.86 & 10 & 40 & 1.09 & 4.35 & $3.802 \%$ & $10.86 \%$ & 860,548 & $3,324,029$ \\
\hline 1.3 .1 & GENERAL CONDTIONS & $1,831,085$ & 8.07 & 80 & 40 & 0.81 & 3.23 & $2826 \%$ & $8.07 \%$ & 639,638 & $2,470,723$ \\
\hline 1.3 .13 & SPECLAL CONSTRUCTION & $12,059,014$ & 53.17 & 10 & 40 & 5.32 & 21.27 & $18.611 \%$ & $53.17 \%$ & $4,212,479$ & $16,271,493$ \\
\hline \multirow[t]{2}{*}{1.5 .2} & PROCUREMENT FEES & 138,901 & 0.61 & 10 & 40 & 0.06 & 0.24 & $0.214 \%$ & $0.61 \%$ & 48,521 & 187,422 \\
\hline & ESCALATION & 0 & 0.00 & 0 & 0 & 0.00 & 0.00 & $0.000 \%$ & $0.00 \%$ & 0 & 0 \\
\hline \multicolumn{2}{|r|}{ SUBTOTAL } & $22,678,056$ & 100.00 & & & & & $35.000 \%$ & & & \\
\hline \multicolumn{2}{|r|}{ CALCULATED CONTINGENCY } & $7,937,320$ & & & & & & & & & \\
\hline \multicolumn{2}{|r|}{ RESULTANT TEC } & $30,615,376$ & & & & & & & & & \\
\hline \multicolumn{2}{|r|}{ ROUNDED TEC } & $30,600,000$ & & & & & & & & & \\
\hline \multicolumn{2}{|r|}{ PROJECT CONTINGENCY } & $7,921,944$ & & & & & & $34.93 \%$ & & & \\
\hline \multicolumn{2}{|r|}{ MANAGEMENT RESERVE } & $1,402,900$ & & & & & & & & & \\
\hline \multicolumn{2}{|r|}{ CONTINGENCY } & $6,519,044$ & & & & & & & & & \\
\hline & TOTAL ESTIMATED COST & $30,600,000$ & & & & & & & & $7,921,944$ & $30,600,000$ \\
\hline
\end{tabular}

CONFIDENCE LEVEL AND ASSUMED RISKS:

The Lockheed Idaho Technologies Co. Cost Estimate Contingency Analysis

Model is based on the applied contingency and the assumptions upon which

the estimate was predicated. The model is applied with a suggested risk level

of $18 \%$ and a level of confidence of $90 \%$ the estimate will fall within the bid range.

The Contingency Analysis is based on a weighted average to provide a

$90 \%$ probability of undermun and a $10 \%$ probability of overun.
CONTINGENCY ANALYSIS GUIDE BY TYPE OF ESTIMATE

Guidelines established by DOEIFM 50 , Cost Estimating Guide, Vol. 6 ,

Cost Guide, and as presented in the INEL Cost Estimating Guide.

PLANNING

ExperimentalSpecial Conditions.

Conceptual

TIIE I

TITLE II

TITLE IIJAFC
$15 \%-25 \%$

$10 \%-20 \%$

$5 \%-15 \%$

Market Conditions 


\section{G\&APIF ADDER CALCULATION SHEET \\ ICPP BIN SET CLOSURE \\ RISK BASED CLEAN CLOSURE; CLEAN FILL; UNESCALATED}

CLEAN BINS W/ ROBOTS - WALLS

PROCUREMENT FEE:

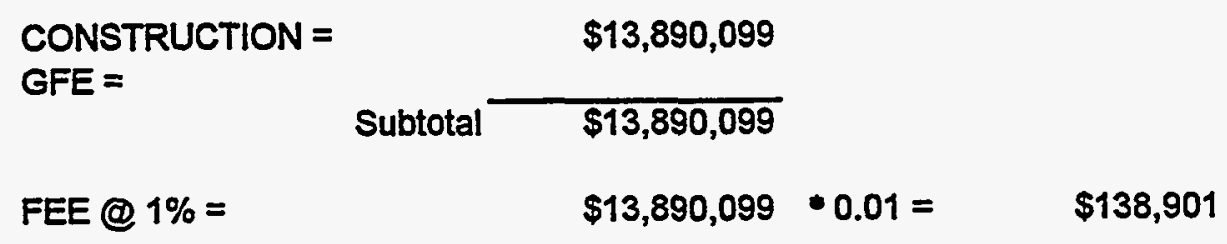

G\&A @ 23\% (with a ceiling of $\$ 500,000$ imposed per year, 10 yrs)

CONSTRUCTION OR

CEILING * 10 YEARS $=\quad \$ 5,000,000$

GFE = $\$$

PROCUREMENT FEE $=$

$\$ 138,901$

Subtotal $\$ 5,138,901$

FEE @ $23 \%=$

$\$ 5,138,901 * 0.23=\quad \$ 1,181,947$

PIF @ 5.5\%

CONSTRUCTION $=$

GFE =

PROCUREMENT FEE =

$G \& A=$

FEE @ 5.5\% =
Subtotal
$\$ 13,890,099$

$\$ 0$

$\$ 138,901$

$\$ 1,181,947$

$\$ 15,210,947$

TOTAL PROCUREMENT FEE:

$\$ 836,602$

TOTAL G\&A FEE:

$\$ 1,181,947$

TOTAL PIF:

$\$ 836,602$ 
Lockheed Martin Idaho Technologies Co. Rev, 6-96

PROJECT NAME: ICPP Bin Set Closure (EIS Stud LOCATION 1: REQUESTOR: Pipe Crawler (Risk Based Estim iCpP

Bryan Spaulding
COST ESTIMATE SUMMARY

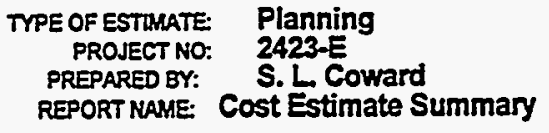

DATE:

TME: CHECKED BY: APPRO BY:
27 Jan-1998

20:52:33

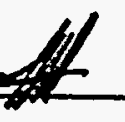

\begin{tabular}{|c|c|c|c|c|}
\hline $\begin{array}{l}\text { WBS } \\
\text { Element }\end{array}$ & Cost Estimate Element & $\begin{array}{c}\text { Total } \\
\text { Unescalated }\end{array}$ & Escalation & $\begin{array}{l}\text { Total } \\
\text { Inel Escalation }\end{array}$ \\
\hline $\begin{array}{l}\frac{1.1}{1.1 .1} \\
1.1 .2 \\
\frac{1.2}{1.2 .1} \\
1.2 .2 \\
\frac{1.3}{1.3 .1} \\
1.3 .13 \\
1.5 .2\end{array}$ & $\begin{array}{l}\text { ENGINEERING, DESIGN AND INSPECTION } \\
\text { DESIGN ENGINEERING } \\
\text { TILE III INSPECTION } \\
\text { MANAGEMENT COSTS } \\
\text { PROJECT MANAGEMENT } \\
\text { CONSTRUCTION MANAGEMENT } \\
\text { CONSTRUCTION } \\
\text { GENERAL CONDITIONS } \\
\text { SPECIAL CONSTRUCTION } \\
\text { PROCUREMENT FEES }\end{array}$ & $\begin{array}{r}2,149,702 \\
592,779 \\
\\
1,831,085 \\
4,096,709 \\
59,278\end{array}$ & $\begin{array}{l}0 \\
0 \\
0 \\
0\end{array}$ & 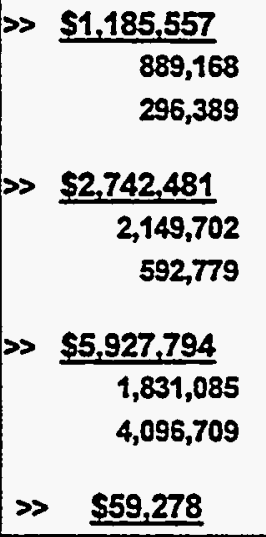 \\
\hline & $\begin{array}{l}\text { SUBTOTAL INCLUDING ESCALATION } \\
\text { PROJECT CONTINGENCY }\end{array}$ & $9,915,110$ & 0 & $\gg>\$ 9,9+5,110$ \\
\hline & MANAGEMENT RESERVE- & & & $\$ 598,707$ \\
\hline & CONTINGENCY - & & & $\$ 2,886,183$ \\
\hline & TOTAL ESTIMATED COST - & & & $\$ 13,400,000$ \\
\hline
\end{tabular}

PROJECT COST PARAMETERS

EDI AS A \% OF CONST. + GFE $=20.00 \%$

CONTINGENCY $=35.15 \%$ 


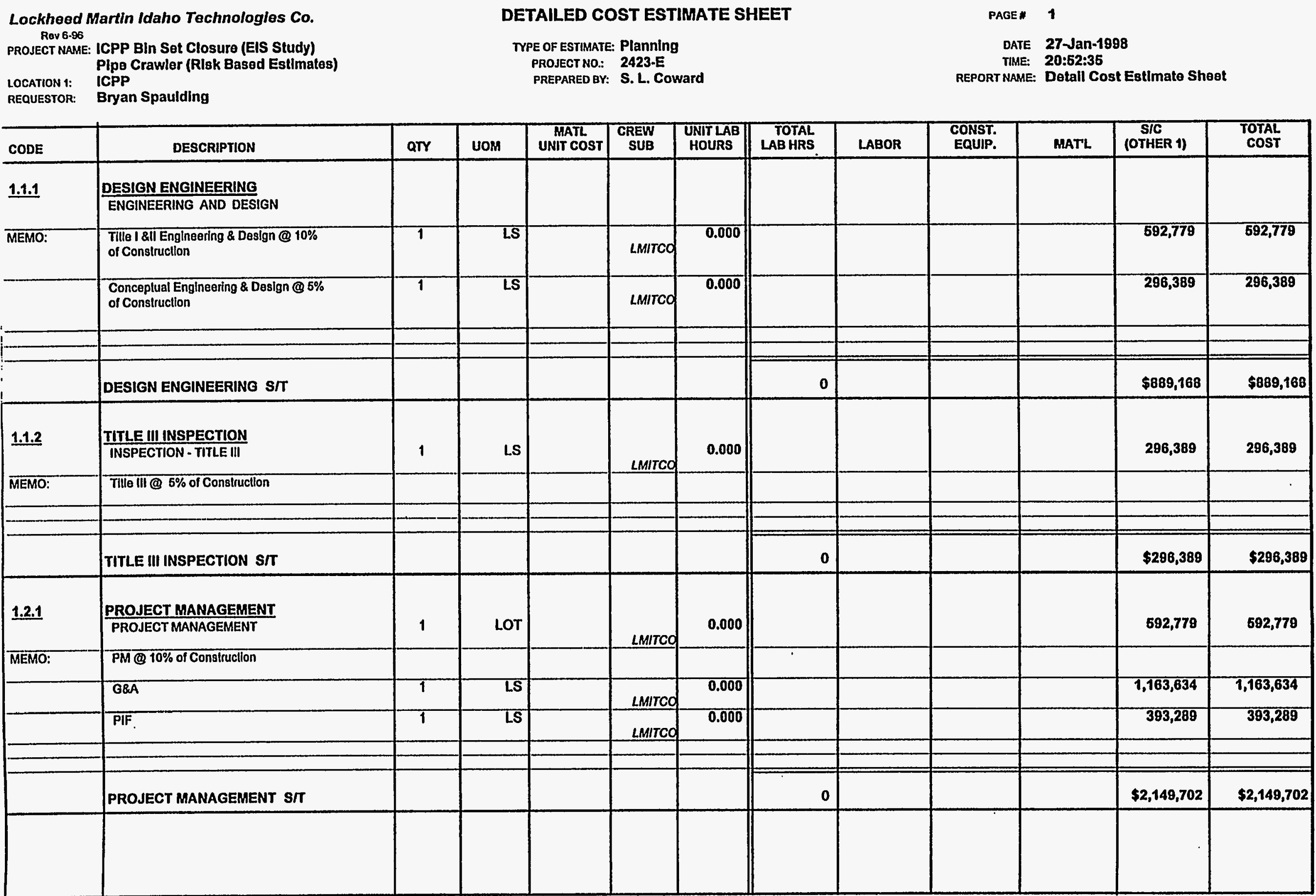


Lockheed Martin Idaho Tochnologles Co. Rov 6.96 PROJECT NAME: ICPP Bin Set Closure (EIS Study) Plpe Crawler (Rlsk Based Estimates) ICPP

LOCATION

Bryan Spaulding
DETAILED COST ESTIMATE SHEET

TYPE OF Estimate: Planning

PROJECT NO.: 2423-E

PREPARED BY: S. L. Coward
PAGE 3

DATE 27-Jan-1998

TIME: 20:52:35

REPORT NAME: Detall Cost Estimate Sheet

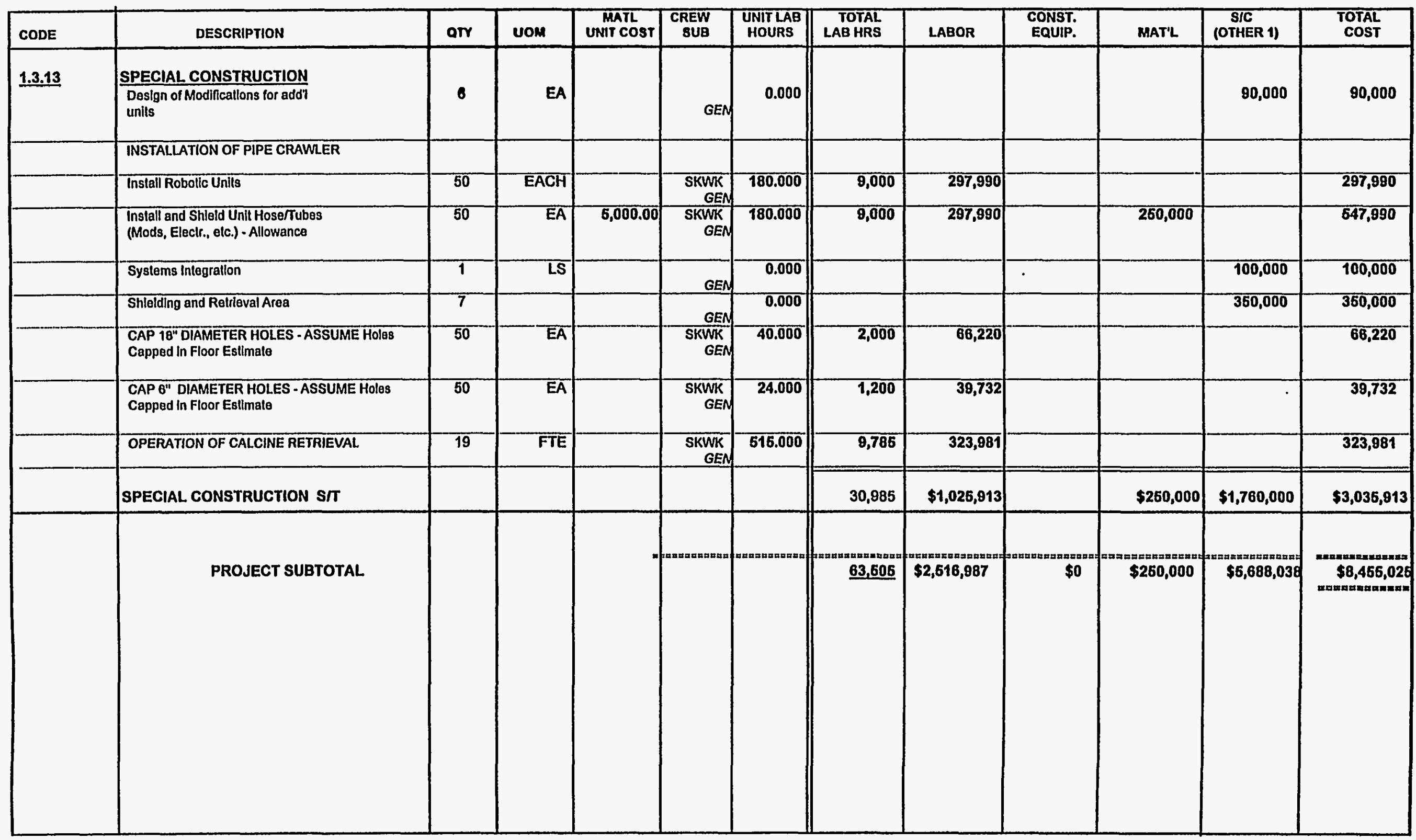


Lockheed Martin Idaho Technologies Co.

PROJECTNAME: ICPP Bin Set Closure (EIS Stud

LOCATION 1: Pipe Crawler (Risk Based Estim

REQUESTOR: Bryan Spaulding
CONTINGENCY ANALYSIS
TYPE OF ESTTMATE: Planning

PROJECT NO: 2423-E

PREPARED BY: S. L. Coward
DATE: 27-Jan-1998

TIME: 20:52:30
REPORT NAME: Contingency Analysis

\begin{tabular}{|c|c|c|c|c|c|c|c|c|c|c|c|}
\hline & PROE & BLE \% VARLA & ION & & & & & & $\begin{array}{l}\text { PRO } \\
\text { CONT }\end{array}$ & $\begin{array}{l}\text { CT } \\
\text { GENCY }\end{array}$ & SUMMARY \\
\hline $\begin{array}{l}\text { WBS } \\
\text { Element }\end{array}$ & Cont F-timnto Floment & Total Cost wh & $\%$ Total & & $\%$ Var. & Wt \% & f Prob. & Continonener & $\boldsymbol{\theta}$ & Cost +2, & Total Coet \\
\hline & & Contingency & & - & + & - & + & & & & by Element \\
\hline 1.1.1 & DESIGN ENGINEERING & 889,168 & 8.97 & 10 & 40 & 0.90 & 3.59 & $3.139 \%$ & $8.97 \%$ & 312,518 & $1,201,686$ \\
\hline 1.1 .2 & TITE III INSPECTION & 296,389 & 2.99 & 10 & 40 & 0.30 & 120 & 1.046\% & $2.99 \%$ & 104,173 & 400,562 \\
\hline 1.21 & PROJECT MANAGEMENT & $2,149,702$ & 21.68 & 10 & 40 & 2.17 & 8.57 & $7588 \%$ & $21.68 \%$ & 755,561 & $2,905,263$ \\
\hline 1.2 .2 & CONSTRUCTION MANAGEMENT & 592,779 & 5.98 & 10 & 40 & 0.60 & 239 & $2092 \%$ & $5.98 \%$ & 208,346 & 801,125 \\
\hline 1.3 .1 & GENERAL CONDITIONS & $1,831,085$ & 18.47 & 10 & 40 & 1.85 & 7.39 & $6.454 \%$ & $18.47 \%$ & 643,576 & $2,474,661$ \\
\hline 1.3 .13 & SPECLAL CONSTRUCTION & $4,096,709$ & 41.32 & 10 & 40 & 4.13 & 16.53 & $14.461 \%$ & $41.32 \%$ & $1,439,881$ & $5,536,590$ \\
\hline 1.5 .2 & PROCUREMENT FEES & 59,278 & 0.60 & 10 & 40 & 0.06 & 0.24 & $0.205 \%$ & $0.60 \%$ & 20,835 & 80,113 \\
\hline & ESCALATION & 0 & 0.00 & 0 & 0 & 0.00 & 0.00 & $0.000 \%$ & $0.00 \%$ & 0 & 0 \\
\hline & SUBTOTAL & $9,915,110$ & 100.00 & & & & & $35.000 \%$ & & & \\
\hline & CALCULATED CONTTNGENCY & $3,470,289$ & & & & & & & & & \\
\hline & RESULTANT TEC & $13,385,399$ & & & & & & & & & \\
\hline & ROUNDED TEC & $13,400,000$ & & & & & & & & & \\
\hline & PROJECT CONTINGENCY & $3,484,890$ & & & & & & $35.15 \%$ & & & \\
\hline & MANAGEMENT RESERVE & 598,707 & & & & & & & & & \\
\hline & CONTNGENCY & $2,886,183$ & & & & & & & & & \\
\hline & TOTAL ESTIMATED COST & $13,400,000$ & & & & & & & & $3,484,890$ & $13,400,000$ \\
\hline
\end{tabular}

CONFIDENCE LEVEL AND ASSUMED RISKS:

The Lockheed Idaho Technologies Co. Cost Estimate Contingency Analysis Model is based on the applied contingency and the assumptions upon which the estimate was predicated. The model is applied with a suggested risk level of $18 \%$ and a level of confidence of $90 \%$ the estimate will fall within the bid range. The Contingency Analysis is based on a weighted average to provide a $90 \%$ probability of undertun and a $10 \%$ probability of overrun.
CONTINGENCY ANALYSIS GUIDE BY TYPE OF ESTIMATE

Guidelines established by DOE/FM 50, Cost Estimating Guide, Vol. 6 ,

Cost Guide, and as presented in the INEL Cost Estimating Guide. PLANNING Experiment

Conceptual

$15 \%-25 \%$

TITLE

TITLE II

TITLE IIIAFC
$20 \%-30 \%$

Up to $40 \%$

$10 \%-20 \%$

$5 \%-15 \%$

Market Conditions 


\section{G\&A/PIF ADDER CALCULATION SHEET \\ ICPP BIN SET CLOSURE \\ RISK BASED CLEAN CLOSURE; CLEAN FILL; UNESCALATED}

CLEAN BINS W/ ROBOTS - PIPING

PROCUREMENT FEE:

CONSTRUCTION =

$\$ 5,927,794$

GFE =

Subtotal $\longdiv { \$ 5 , 9 2 7 , 7 9 4 }$

FEE @ 1\% =

$\$ 5,927,794 * 0.01=$

$\$ 59,278$

G\&A @ 23\% (with a ceiling of $\$ 500,000$ imposed per year, 10 yrs)

CONSTRUCTION OR

CEILING * 10 YEARS =

GFE =

$\$ 5,000,000$

PROCUREMENT FEE =

Subtotal $\$ 5,059,278$

FEE @ 23\% =

$\$ 5,059,278 \cdot 0.23=$

$\$ 1,163,634$

PIF @ 5.5\%

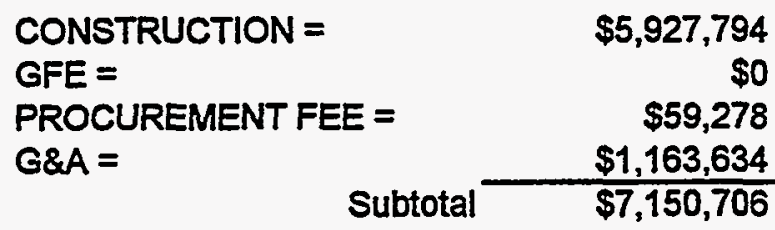

FEE @ 5.5\% =

$\$ 7,150,706 * 0.055=$

$\$ 393,289$

TOTAL PROCUREMENT FEE:

$\$ 59,278$

TOTAL G\&A FEE:

$\$ 1,163,634$

TOTAL PIF:

$\$ 393,289$ 
Lockheed Martin Idaho Technologies Co. Rev. 6-96 PROJECT NAME: ICPP Bin Set Closure (EIS Stud Place Clean Grout in Bins LOCATION 1: ICPP REQUESTOR: Bryan Spaulding
COST ESTIMATE SUMMARY

TYPE OF ESTIMATE: Planning PROJECT NO: 2423-A1 PREPARED BY: S.L. Coward REPORT NAME: Cost Estimate Summary

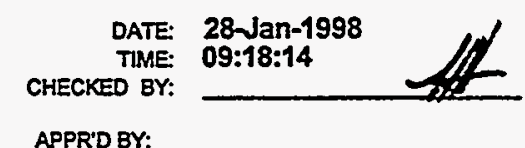

\begin{tabular}{|c|c|c|c|c|}
\hline $\begin{array}{l}\text { WBS } \\
\text { Element }\end{array}$ & Cost Estimate Element & $\begin{array}{c}\text { Total } \\
\text { Unescalated }\end{array}$ & Escalation & $\begin{array}{c}\text { Total } \\
\text { Incl Escalation } \\
\end{array}$ \\
\hline $\begin{array}{l}\frac{1.1}{1.1 .1} \\
1.1 .2 \\
\frac{1.2}{1.2 .1} \\
1.2 .2 \\
\frac{1.3}{1.3 .1} \\
1.3 .2 \\
1.3 .3 \\
1.5 .2\end{array}$ & $\begin{array}{l}\text { ENGINEERING, DESIGN AND INSPECTION } \\
\text { DESIGN ENGINEERING } \\
\text { TITLE III INSPECTION } \\
\text { MANAGEMENT COSTS } \\
\text { PROJECT MANAGEMENT } \\
\text { CONSTRUCTION MANAGEMENT } \\
\text { CONSTRUCTION } \\
\text { GENERAL CONDITIONS } \\
\text { SITEWORK } \\
\text { CONCRETE } \\
\text { PROCUREMENT FEES }\end{array}$ & $\begin{array}{r}1,646,982 \\
329,396 \\
\\
1,526,038 \\
658,793 \\
\\
1,067,791 \\
229,119 \\
5,291,021 \\
65,879\end{array}$ & $\begin{array}{l}0 \\
0 \\
0 \\
0 \\
0\end{array}$ & 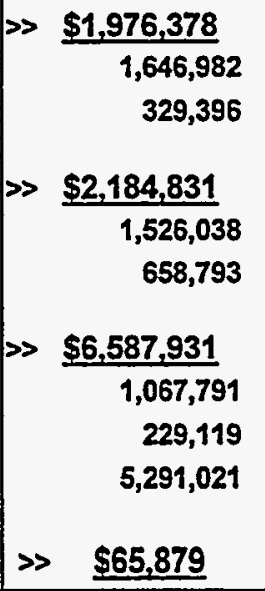 \\
\hline & $\begin{array}{l}\text { SUBTOTAL INCLUDING ESCALATION } \\
\text { PROJECT CONTINGENCY }\end{array}$ & $10,815,019$ & 0 & $\gg \$ 10,815,019$ \\
\hline & MANAGEMENT RESERVE- & & & $\$ 665,381$ \\
\hline & CONTINGENCY- & & & $\$ 3,119,600$ \\
\hline & TOTAL ESTIMATED COST - & & & $\$ 14,600,000$ \\
\hline
\end{tabular}

PROJECT COST PARAMETERS

EDI AS A \% OF CONST. + GFE $=30.00 \%$ 
Lockheed Martin Idaho Technologies Co. PROJECT NAME: ICPP Bin Set Closure (EIS Stud Place Clean Grout in Bins

Bryan Spaulding

LOCATION 1: REQUESTOR:
CONTINGENCY ANALYSIS
TYPE OF ESTIMATE:

PROJECT NO:

PREPARED BY:
Planning

2423-A1

S. L. Coward
DATE: 28-Jan-1998

TIME: 09:18:11
REPORT NAME: Contingency Analysis

\begin{tabular}{|c|c|c|c|c|c|c|c|c|c|c|c|}
\hline & PROB & BLE \% VARIAT & ON & & & & & & $\begin{array}{l}\text { PRO } \\
\text { CONT }\end{array}$ & $\begin{array}{l}\text { CT } \\
\text { SENCY }\end{array}$ & SUMMARY \\
\hline \begin{tabular}{l|} 
WBS \\
Element
\end{tabular} & Cost Estimate Element & Total Cost w/o & $\begin{array}{l}\text { \% Total } \\
\text { Cost }\end{array}$ & & $\begin{array}{l}\% \text { Var. } \\
\text { nest. }\end{array}$ & Wt. \% & Prob. & Contingency & $\%$ & Cost & Total Cost \\
\hline & & Contingency & & - & $t$ & - & $\mp$ & & & & by Element \\
\hline 1.1.1 & DESIGN ENGINEERING & $1,646,982$ & 15.23 & 10 & 40 & 1.52 & 6.09 & $5.330 \%$ & $15.23 \%$ & 576,402 & $2,223,384$ \\
\hline 1.1 .2 & TITLE III INSPECTION & 329,396 & 3.05 & 10 & 40 & 0.30 & 1.22 & $1.066 \%$ & $3.05 \%$ & 115,280 & 444,676 \\
\hline 1.2 .1 & PROJECT MANAGEMENT & $1,526,038$ & 14.11 & 10 & 40 & 1.41 & 5.64 & $4.939 \%$ & $14.11 \%$ & 534,074 & $2,060,112$ \\
\hline 1.2 .2 & CONSTRUCTION MANAGEMENT & 658,793 & 6.09 & 10 & 40 & 0.61 & 2.44 & $2.132 \%$ & $6.09 \%$ & 230,561 & 889,354 \\
\hline 1.3 .1 & GENERAL CONDITIONS & $1,067,791$ & 9.87 & 90 & 40 & 0.99 & 3.95 & $3.456 \%$ & $9.87 \%$ & 373,700 & $1,441,491$ \\
\hline 1.3 .2 & STEWORK & 229,119 & 2.12 & 10 & 40 & 0.21 & 0.85 & $0.741 \%$ & $2.12 \%$ & 80,186 & 309,305 \\
\hline 1.3 .3 & CONCRETE & $5,291,021$ & 48.92 & 10 & 40 & 4.89 & 19.57 & $17.123 \%$ & $48.92 \%$ & $1,851,722$ & $7,142,743$ \\
\hline 1.5 .2 & PROCUREMENT FEES & 65,879 & 0.61 & 10 & 40 & 0.06 & 0.24 & $0.213 \%$ & $0.61 \%$ & 23,056 & 88,935 \\
\hline & ESCALATTON & 0 & 0.00 & 0 & 0 & 0.00 & 0.00 & $0.000 \%$ & $0.00 \%$ & 0 & 0 \\
\hline & SUBTOTAL & $10,815,019$ & 100.00 & & & & & $35.000 \%$ & & & \\
\hline & CALCULATED CONTINGENCY & $3,785,257$ & & & & & & & & & \\
\hline & RESULTANT TEC & $14,600,276$ & & & & & & & & & \\
\hline & ROUNDED TEC & $14,600,000$ & & & & & & & & & \\
\hline & PROJECT CONTINGENCY & $3,784,981$ & & & & & & $35.00 \%$ & & & \\
\hline & MANAGEMENT RESERVE & 665,381 & & & & & & & & & \\
\hline & CONTINGENCY & $3,119,600$ & & & & & & & & & \\
\hline & TOTAL ESTIMATED COST & $14,600,000$ & & & & & & & & $3,784,981$ & $14,600,000$ \\
\hline
\end{tabular}

CONFIDENCE LEVEL AND ASSUMED RISKS:

The Lockheed ldaho Technologies Co. Cost Estimate Contingency Analysis Model is based on the applied contingency and the assumptions upon which the estimate was predicated. The model is applied with a suggested risk level of $18 \%$ and a level of confidence of $90 \%$ the estimate will fall within the bid range. The Contingency Analysis is based on a weighted average to provide a

$90 \%$ probability of undertun and a $10 \%$ probability of overmun.
CONTINGENCY ANALYSIS GUIDE BY TYPE OF ESTIMATE

Guidelines established by DOEIFM 50 , Cost Estimating Guide, Vol. 6 , Cost Guide, and as presented in the INEL Cost Estimating Guide.

PLANNING Conceptual $15 \%-25 \%$

TITLE I

TITLE II

TITLE IUAFC
$20 \%$ - $30 \%$

$10 \%-20 \%$

$5 \%-15 \%$

Market Conditions 


\section{G\&A/PIF ADDER CALCULATION SHEET ICPP BIN SET CLOSURE \\ RISK BASED CLEAN CLOSURE; CLEAN FILL; UNESCALATED}

FILL BINS W/ NRC CLEAN GROUT

PROCUREMENT FEE:

CONSTRUCTION $=$ GFE =

Subtotal $\$ \$ 6,587,931$

FEE @ $1 \%=$

$\$ 6,587,931 * 0.01=$

$\$ 65,879$

G\&A @ 23\% (with a ceiling of $\$ 500,000$ imposed per year, 4 yrs)

CONSTRUCTION OR

CEILING * 4 YEARS =

GFE =

$\$ 2,000,000$

PROCUREMENT FEE $=$ $\$ 0$

$\$ 65,879$

Subtotal $\$ 2,065,879$

FEE @ 23\%=

$\$ 2,065,879 * 0.23=$

$\$ 475,152$

PIF @ 5.5\%

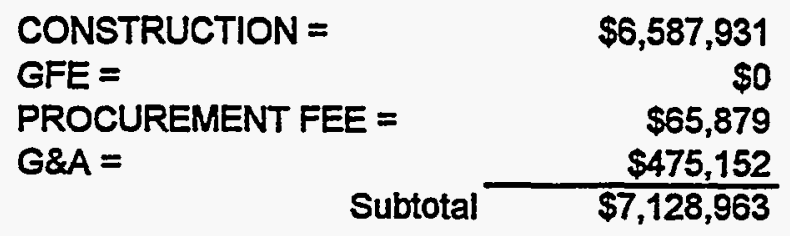

FEE @ 5.5\%=

TOTAL PROCUREMENT FEE:

$\$ 65,879$

TOTAL G\&A FEE:

$\$ 475,152$

TOTAL PIF: 
Lockheed Martin Idaho Technologies Co.

PROJECT NAME: ICPP Bin Set Closure

D\&D of Equipment

INEEL / ICPP

LOCATION 1:

REQUESTOR:
COST ESTIMATE SUMMARY

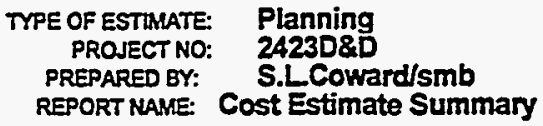

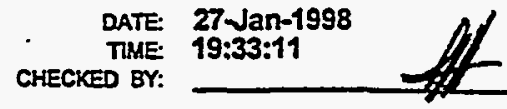

APPROBY:

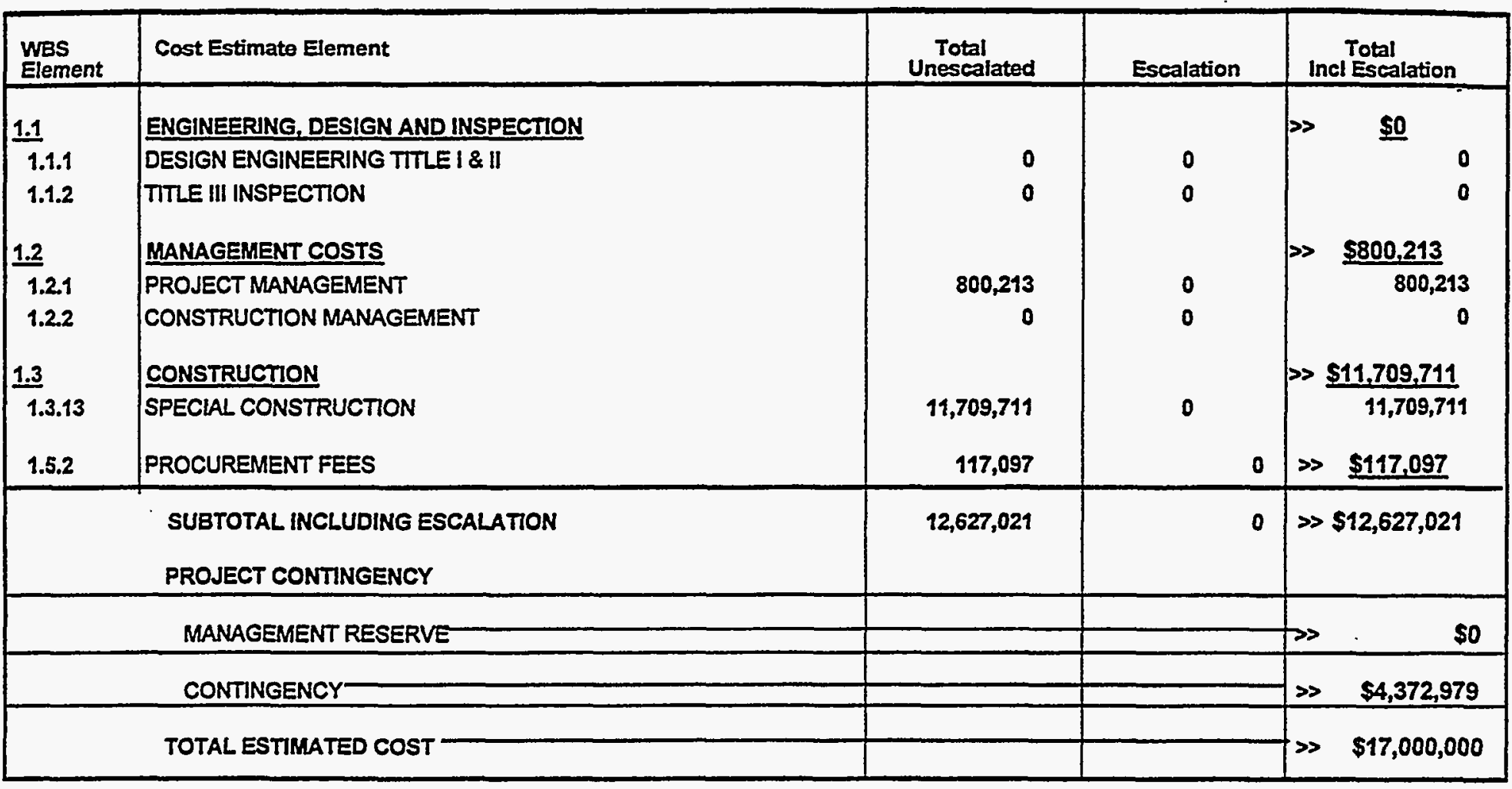

\section{PROJECT COST PARAMETERS}

EDI AS A \% OF CONST. + GFE $=0.00 \%$ 


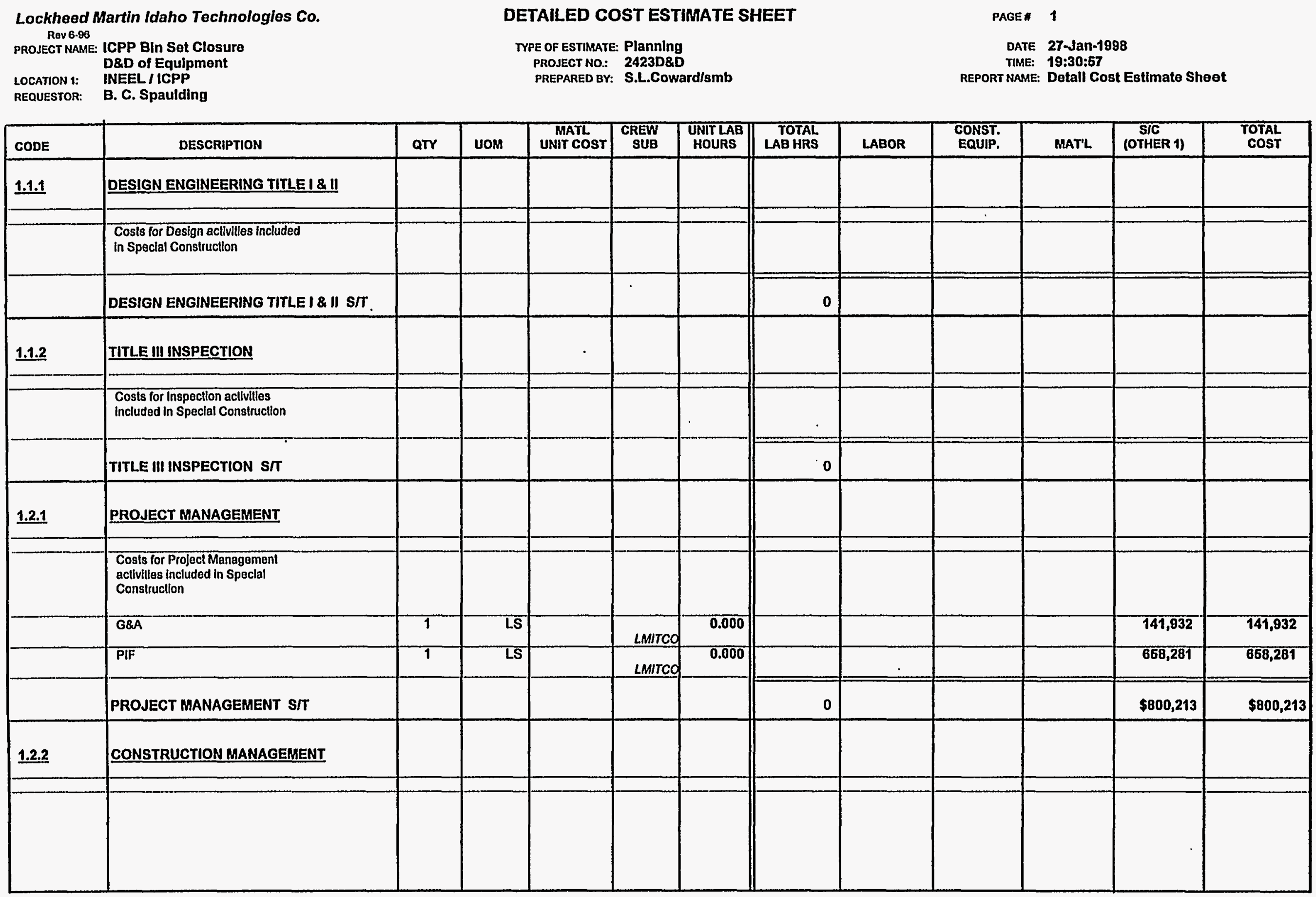


Lockheed Martin Idaho Technologies Co.

Rov 6.96

DROJECT NAME: ICPP BIn Set Closure

D\&D of Equipment

OCATION 1:

REQUESTOR:

B. C. Spaulding

\section{DETAILED COST ESTIMATE SHEET}

TYPE OF ESTIMATE: Planning

PROJECT NO.: 2423D\&D

PREPARED OY: S.L.Coward/smb
PAGE \# 4

DATE 27-Jan-1998

TIME: 19:30:67

REPORT NAME: Detall Cost Estimate Sheot

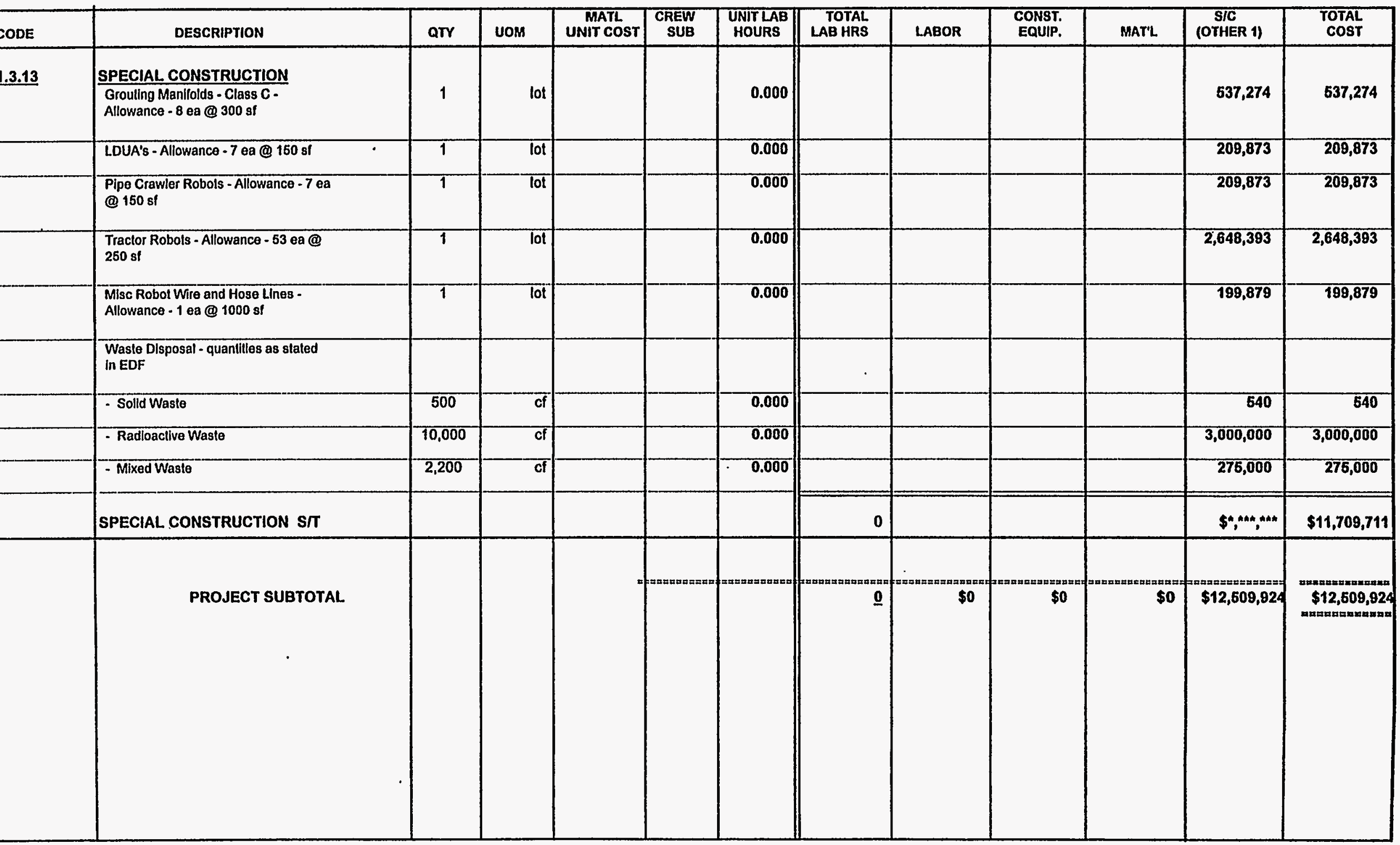


Lockheed Martin Idaho Technologies Co. PROJECT NAME: ICPP Bin Set Closure D\&D of Equipment

LOCATION $\%$ REQUESTOR:
INEEL / ICPP

B. C. Spaulding

CONTINGENCY ANALYSIS

\author{
TYPE OF ESTMATE \\ PROJECT NO: \\ PREPARED BY:
}

\section{Planning}

2423D\&D

S.LCoward/smb
DATE: 27-Jan-1998

TIME: 19:33:04

\begin{tabular}{|c|c|c|c|c|c|c|c|c|c|c|c|}
\hline & PROB & BLE \% VARIA & ION & & & & & & $\begin{array}{r}\text { PRO } \\
\text { CONT }\end{array}$ & $\begin{array}{l}\text { ST } \\
\text { IENCY }\end{array}$ & SUMMARY \\
\hline $\begin{array}{l}\text { WBS } \\
\text { Element }\end{array}$ & Cost Estimate Element & Total Cost w/o & $\begin{array}{l}\text { \% Total } \\
\text { Cost }\end{array}$ & & $\begin{array}{l}\% \text { Var. } \\
\text { n Est. }\end{array}$ & Wt \% & Prob. & Contingeney & $\%$ & Cost & Total Cost \\
\hline & & Contingency & & - & + & - & + & & & & by Element \\
\hline 1.1 .1 & DESIGN ENGINEERING TTLE I \& I & 0 & 0.00 & 0 & 0 & 0.00 & 0.00 & $0.000 \%$ & $0.00 \%$ & 0 & 0 \\
\hline 1.1 .2 & TIRE III INSPECTION & 0 & 0.00 & 0 & 0 & 0.00 & 0.00 & $0.000 \%$ & $0.00 \%$ & 0 & 0 \\
\hline 1.2 .1 & PROJECT MANAGEMENT & 800,213 & 6.34 & 10 & 40 & 0.63 & 2.53 & $2218 \%$ & $6.34 \%$ & 277,129 & $1,077,342$ \\
\hline 1.22 & CONSTRUCTION MANAGEMENT & 0 & 0.00 & 0 & 0 & 0.00 & 0.00 & $0.000 \%$ & $0.00 \%$ & 0 & 0 \\
\hline 1.3 .13 & SPECLAL CONSTRUCTION & $11,709,711$ & 9274 & 10 & 40 & 9.27 & 37.09 & $32.457 \%$ & $92.74 \%$ & $4,055,297$ & $15,765,008$ \\
\hline 1.5 .2 & PROCUREMENT FEES & 117,097 & 0.93 & 10 & 40 & 0.09 & 0.37 & $0.325 \%$ & $0.93 \%$ & 40,553 & 157,650 \\
\hline & ESCALATION & 0 & 0.00 & 0 & 0 & 0.00 & 0.00 & $0.000 \%$ & $0.00 \%$ & 0 & 0 \\
\hline & SUBTOTAL & $12,627,021$ & 100.00 & & & & & $35.000 \%$ & & & \\
\hline & CALCULATED CONTINGENCY & $4,419,457$ & & & & & & & & & \\
\hline & RESULTANT TEC & $17,046,478$ & & & & & & & & & \\
\hline & ROUNDED TEC & $17,000,000$ & & & & & & & & & \\
\hline & PROJECT CONTINGENCY & $4,372,979$ & & & & & & $34.63 \%$ & & & \\
\hline & MANAGEMENT RESERVE & 0 & & & & & & & & & \\
\hline & CONTINGENCY & $4,372,979$ & & & & & & & & & \\
\hline & TOTAL ESTIMATED COST & $17,000,000$ & & & & & & & & $4,372,979$ & $17,000,000$ \\
\hline
\end{tabular}

CONFIDENCE LEVEL AND ASSUMED RISKS:

The Lockheed Idaho Technologies Co. Cost Estimate Contingency Analysis Model is based on the applied contingency and the assumptions upon which

the estimate was predicated. The model is applied with a suggested risk level of $18 \%$ and a level of confidence of $90 \%$ the estimate will fall within the bid range. The Contingency Analysis is based on a weighted average to provide a

$90 \%$ probability of underrun and a $10 \%$ probability of overnun.
CONTINGENCY ANALYSIS GUIDE BY TYPE OF ESTIMATE

Guidelines established by DOE/FM 50, Cost Estimating Guide, Vol. 6 , Cost Guide, and as presented in the INEL Cost Estimating Guide. PLÁNNING ExperimentalSpecial Conditions. Conceptual TITE

TITLE II

TITLE II/AFC
.....Up to $50 \%$ $5 \%-25 \%$ $10 \%-20 \%$ $5 \%-15 \%$ Market Conditions 
INEL RAD Contaminuted Surplus Fucillties Cost Model $\Rightarrow$ D\&D Cost

CPP Bin set Closure

Piopact Fita 2013720

\begin{tabular}{|c|c|c|c|c|c|c|c|c|c|c|}
\hline & \multirow{2}{*}{\multicolumn{2}{|c|}{$\begin{array}{c}\text { Fuslltoy } \\
\text { Number } \\
\text { Descrlption }\end{array}$}} & \multirow{2}{*}{$\begin{array}{l}\text { Tolal Usable } \\
\text { Fluor Space }\end{array}$} & \multirow[b]{2}{*}{$\begin{array}{l}\text { Bilds } \\
\text { Lws }\end{array}$} & \multirow{2}{*}{\multicolumn{3}{|c|}{ Facilly Rathng }} & & & \multirow{2}{*}{$\begin{array}{l}\text { DED } \\
\text { ROAs } \\
\text { Cost (s) }\end{array}$} \\
\hline & & & & & & & & Bldg & CIRTZ & \\
\hline
\end{tabular}

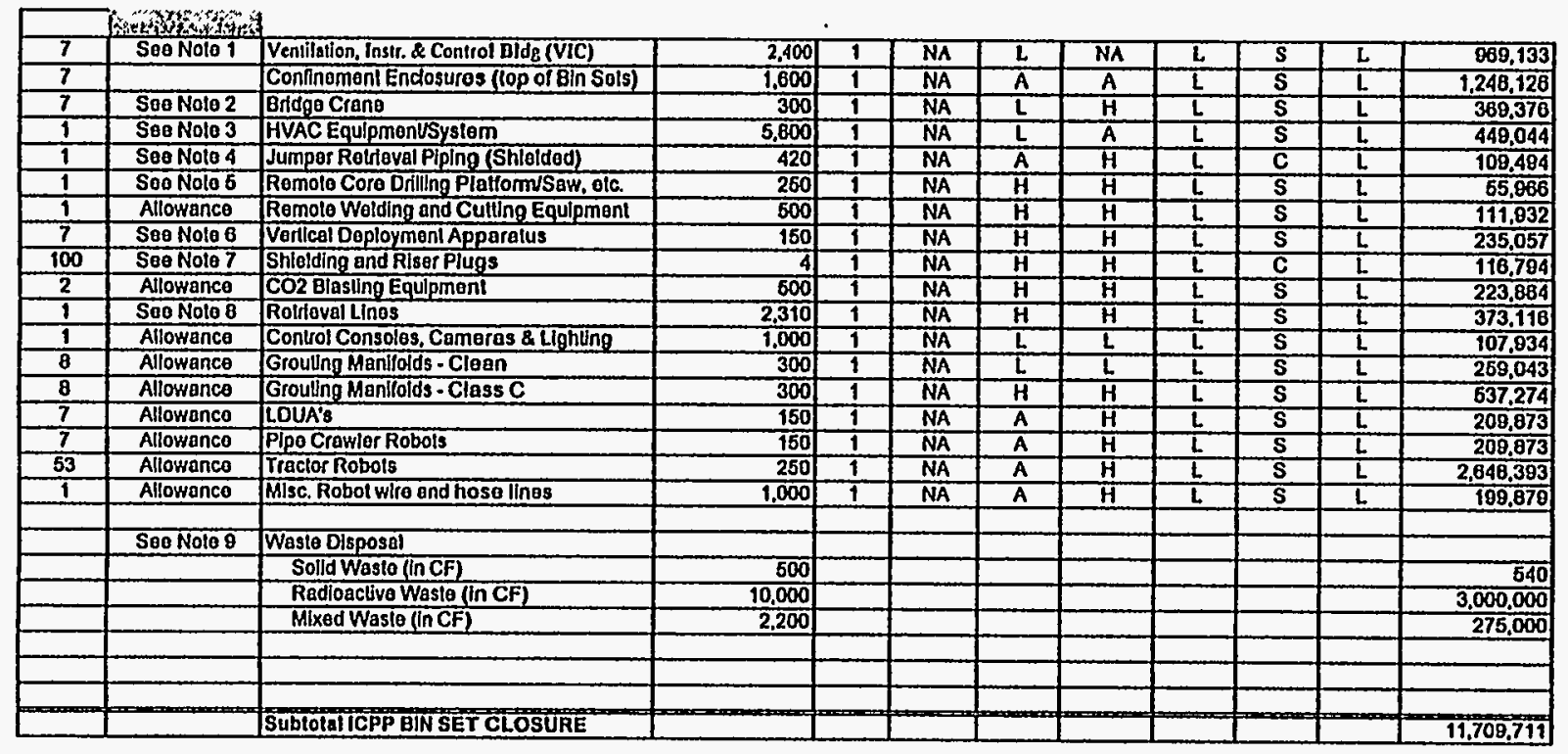

Notes:

1 - Assumed that faclifity will havo tow lovals of hazardous material, no rad conlamination, and no asbestos

2 - Assume $40^{\prime}$ accoss by $45^{\circ}$ high $\times 2^{\prime} \mathrm{w}$ for ralls plus trolley $\left(5^{\prime} \times 5^{\circ} \times 5^{\prime}\right)$

3 - Assume 7 Alr handlers $\left(5^{\prime} \times 5^{\prime} \times 6^{\prime}\right)$, 3 Exhaust Fans $\left(4^{\prime} \times 4^{\prime} \times 3^{\prime}\right)$, and 83 HEPA fillors $\left(8^{\prime} \times 0^{\prime} \times 2^{\prime}\right)$

4 -Assumo 210 LF $\times 2$ wido concrolo

5 - Assume plattorm $\left(5^{\circ} \times 5^{\prime} \times 6\right)$ plus saw (100 3)

6-Assume $5^{\circ} \times 5^{\circ} \times 6^{\prime}$

7-Assume $2^{2} \times 2^{\prime}$ plugs
8 - Assume $2310^{\prime} \times 1^{\prime}$ wido

8 - Assume $2310^{\prime} \times 11^{\prime}$ wide
9 - Waste Disposat Quantilios woro providad by EDF

Assume tho following Wasto Disposal Unil Costs:

$\begin{array}{ll}\text { Indusirfal Landfill } & \$ 1.08 / \mathrm{cl} \\ \text { Low Lovol Wasto Ropository } & \$ 125 / \mathrm{cl}\end{array}$

$\begin{array}{ll}\text { Low Lovel Wasto Ropository } & \$ 125 / \mathrm{cl} \\ \text { Rad Waslo } & \$ 300 / \mathrm{ct}\end{array}$ 


\section{G\&APIF ADDER CALCULATION SHEET ICPP BIN SET CLOSURE \\ RISK BASED CLEAN CLOSURE; CLEAN FILL; UNESCALATED \\ D\&D OF EQUIPMENT}

PROCUREMENT FEE:

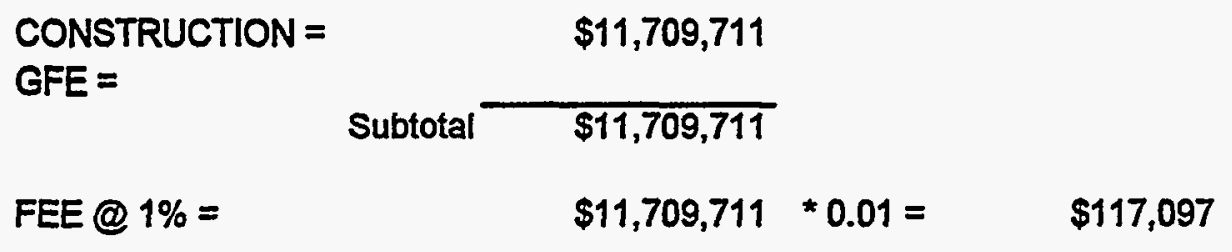

G\&A @ 23\% (with a ceiling of $\$ 500,000$ imposed per year, 1 yr)

CONSTRUCTION OR

CEILING * 1 YEARS $=\quad \$ 500,000$

GFE =

$\$ 0$

PROCUREMENT FEE =

$\$ 117,097$

Subtotal $\$ \$ 617,097$

FEE @ 23\% =

$\$ 617,097 \cdot 0.23=\quad \$ 141,932$

PIF @ 5.5\%

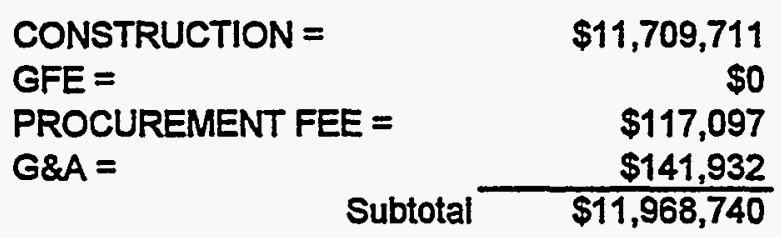

FEE @ $5.5 \%=$

TOTAL G\&A FEE:

$\$ 141,932$

TOTAL PIF: 
COST ESTIMATE SUMMARY

ESCALATED

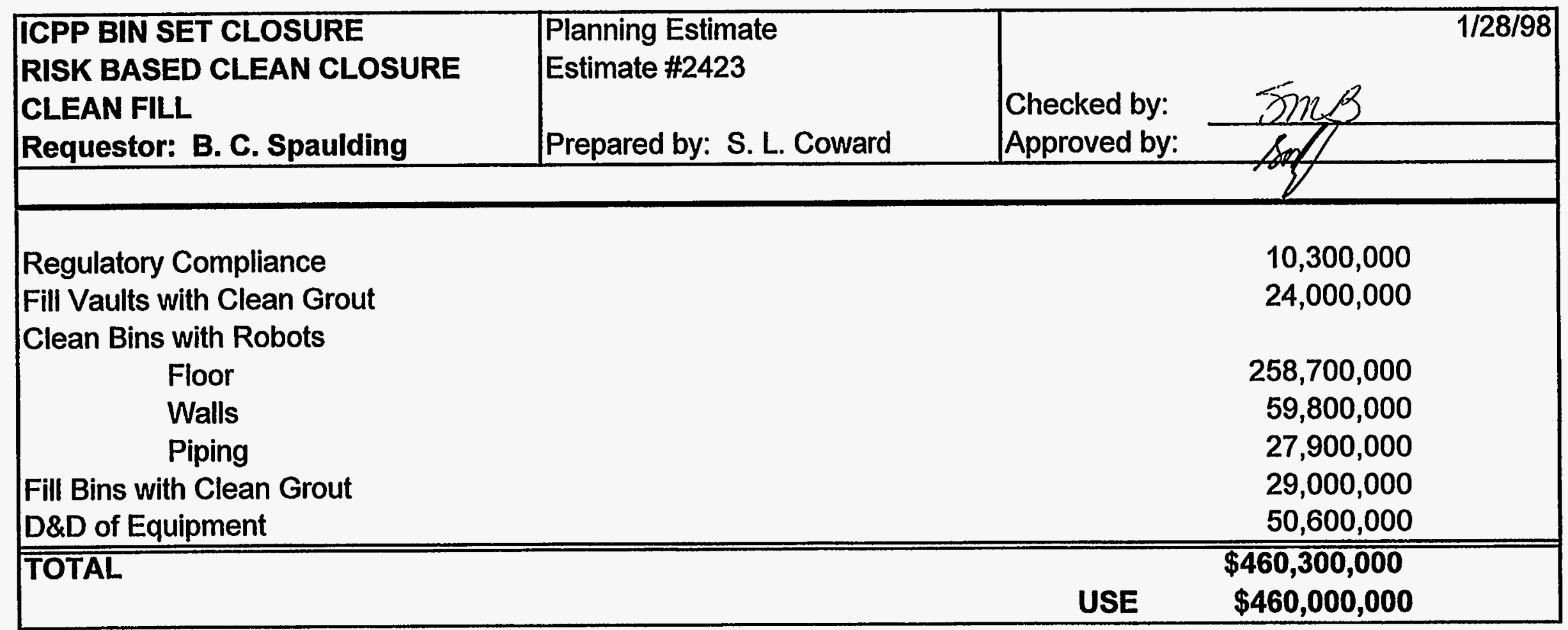


Lockheed Martin Idaho Technologies Co. Rov. G-96

PROJECT NAME: Permitting/Documentation

Risk Based - Clean

LOCATION 1: INEELICPP

REQUESTOR: Bryan Spaulding
COST ESTIMATE SUMMARY

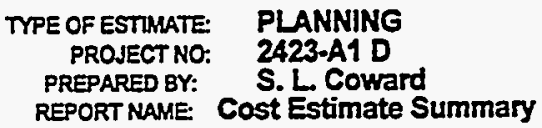

28-Jan-1998

CHECKED BY: 09:48:36

APPRD BY:

\begin{tabular}{|c|c|c|c|c|}
\hline $\begin{array}{l}\text { WBS } \\
\text { Element }\end{array}$ & Cost Estimate Element & $\begin{array}{c}\text { Total } \\
\text { Unescalated }\end{array}$ & Escalation & $\begin{array}{l}\text { Total } \\
\text { Incl Escalation }\end{array}$ \\
\hline $\begin{array}{l}\frac{1.1}{1.1 .1} \\
\frac{1.2}{1.2 .1} \\
1.2 .2 \\
\frac{1.3}{1.3 .1} \\
1.5 .2\end{array}$ & $\begin{array}{l}\text { CONCEPTUAL } \\
\text { CONCEPTUAL DESIGN } \\
\text { MANAGEMENT } \\
\text { PM FOR PROJECT DEVELOPMENT } \\
\text { PROJECT EXECUTION } \\
\text { PERMITTING } \\
\text { PERMITTING } \\
\text { PROCUREMENT FEES }\end{array}$ & $\begin{array}{r}0 \\
774,995 \\
149,704 \\
3,742,601 \\
37,426\end{array}$ & $\begin{array}{r}0 \\
480,497 \\
92,816 \\
2,320,412 \\
23,204\end{array}$ & $\begin{array}{l}\gg \quad 0 \quad \frac{\$ 0}{1,255,492} \\
242,520\end{array}$ \\
\hline & $\begin{array}{l}\text { SUBTOTAL INCLUDING ESCALATION } \\
\text { PROJECT CONTINGENCY }\end{array}$ & $4,704,726$ & $2,916,929$ & $\gg \$ 7,621,655$ \\
\hline & MANAGEMENT RESERVE- & & & $\$ 612,364$ \\
\hline & CONTINGENCY - & & & $\$ 2,065,981$ \\
\hline & TOTAL ESTIMATED COST - & & & $\gg \quad \$ 10,300,000$ \\
\hline
\end{tabular}

PROJECT COST PARAMETERS

EDI AS A \% OF CONST. + GFE $=0.00 \%$ 


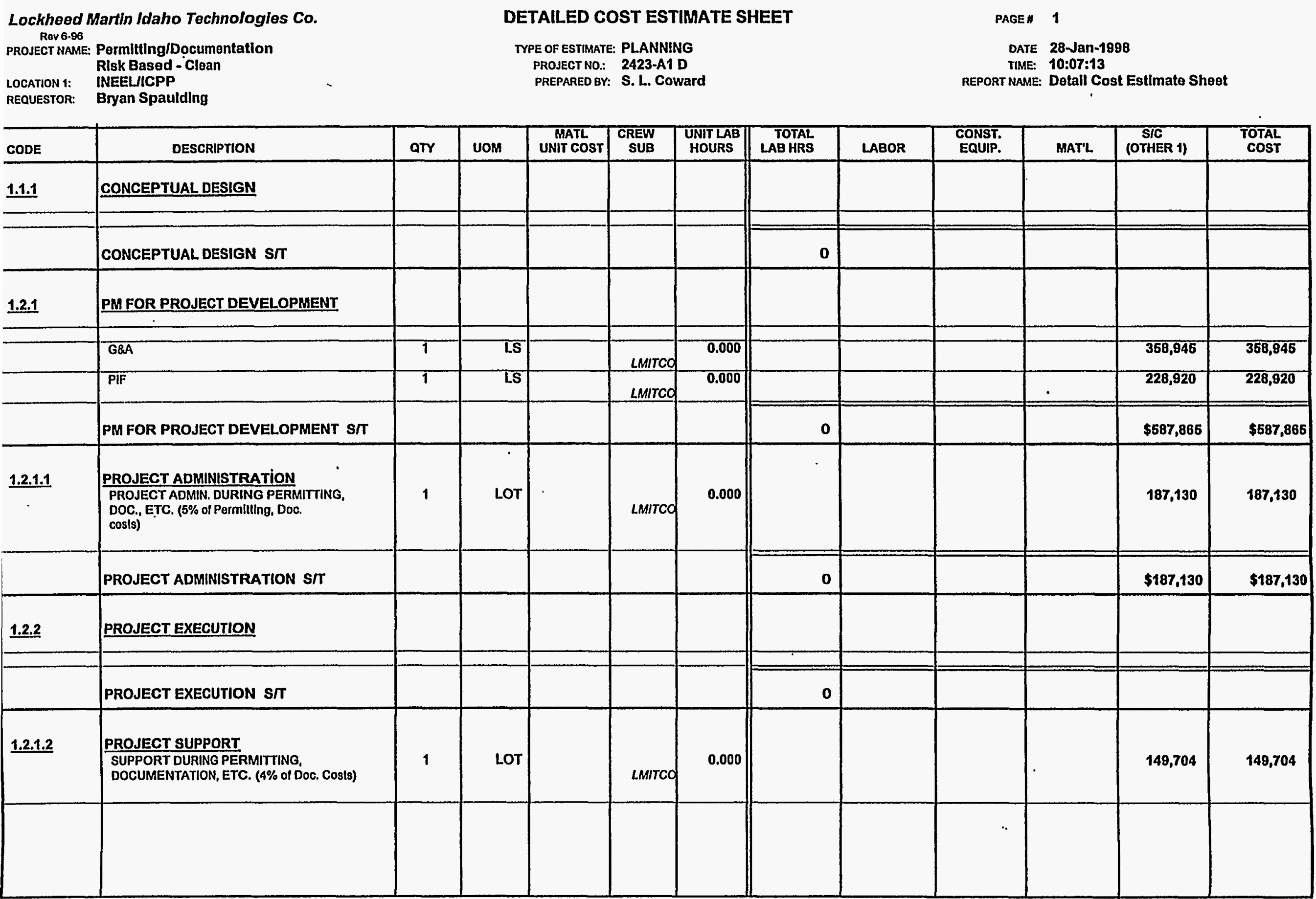


Lockheed Martin Idaho Technologies Co.

Rev $6-96$

PROJECT NAME: Permitting/Documentation

Risk Based - Clean

LOCATION 1: INEEL/CPP
REQUESTOR: $\quad$ Bryan Spaulding
CONTINGENCY ANALYSIS

TYPE OF ESTIMATE: PLANNING

PROJECT NO: 2423-A1D

PREPARED BY: S. L. Coward
DATE: 28-Jan-1998

TIME: 09:48:12

REPORT NAME: Contingency Analysis

\begin{tabular}{|c|c|c|c|c|c|c|c|c|c|c|c|}
\hline \multicolumn{9}{|c|}{ PROBABLE \% VARIATION } & \multicolumn{2}{|c|}{$\begin{array}{l}\text { PROJECT } \\
\text { CONTINGENCY }\end{array}$} & \multirow{3}{*}{$\begin{array}{l}\text { SUMMARY } \\
\text { Total Cost } \\
\text { by Element }\end{array}$} \\
\hline \multirow[t]{2}{*}{$\begin{array}{l}\text { WBS } \\
\text { Element }\end{array}$} & \multirow[t]{2}{*}{ Cost Estimate Element } & \multirow{2}{*}{$\begin{array}{l}\text { Total Cost w/o } \\
\text { Contingency }\end{array}$} & \multirow[t]{2}{*}{$\begin{array}{l}\text { \% Total } \\
\text { Cost }\end{array}$} & \multicolumn{2}{|c|}{$\begin{array}{c}\text { Prob. \% Var. } \\
\text { Erom Est }\end{array}$} & \multicolumn{2}{|c|}{ Wt. $\%$ of Prob. } & \multirow[t]{2}{*}{ Contingency } & \multirow[t]{2}{*}{$\%$} & \multirow[t]{2}{*}{ Cost } & \\
\hline & & & & - & + & - & + & & & & \\
\hline 1.1.1 & CONCEPTUAL DESIGN & 0 & 0.00 & 10 & 40 & 0.00 & 0.00 & $0.000 \%$ & $0.00 \%$ & 0 & 0 \\
\hline 1.2 .1 & PM FOR PROJECT DEVELOPMENT & 774,995 & 10.17 & 10 & 40 & 1.02 & 4.07 & $3.559 \%$ & $10.17 \%$ & 272,343 & $1,047,338$ \\
\hline 1.2 .2 & PROJECT EXECUTION & 149,704 & 1.96 & 10 & 40 & 0.20 & 0.79 & $0.687 \%$ & $1.96 \%$ & 52,508 & 202,312 \\
\hline 1.3 .1 & PERMITTING & $3,742,601$ & 49.10 & 10 & 40 & 4.91 & 19.64 & $17.187 \%$ & $49.10 \%$ & $1,315,197$ & $5,057,798$ \\
\hline \multirow[t]{2}{*}{1.5 .2} & PROCUREMENT FEES & 37,426 & 0.49 & 10 & 40 & 0.05 & 0.20 & $0.172 \%$ & $0.49 \%$ & 13,152 & 50,578 \\
\hline & ESCALATION & $2,916,929$ & 38.27 & 10 & 40 & 3.83 & 15.31 & $13.395 \%$ & $38.27 \%$ & $1,025,045$ & $3,941,974$ \\
\hline & SUBTOTAL & $7,621,655$ & 100.00 & & & & & $35.000 \%$ & & & \\
\hline & CALCULATED CONTINGENCY & $2,657,579$ & & & & & & & & & \\
\hline & RESULTANT TEC & $10,289,234$ & & & & & & & & & \\
\hline & ROUNDED TEC & $10,300,000$ & & & & & & & & & \\
\hline & PROJECT CONTINGENCY & $2,678,345$ & & & & & & $35.14 \%$ & & & \\
\hline & MANAGEMENT RESERVE & 612,364 & & & & & & & & & \\
\hline & CONTINGENCY & $2.065,981$ & & & & & & & & & \\
\hline & TOTAL ESTIMATEO COST & $10,300,000$ & & & & & & & & $2,678,345$ & $10,300,000$ \\
\hline
\end{tabular}

\section{CONFIDENCE LEVEL AND ASSUMED RISKS:}

The Lockheed Idaho Technologies Co. Cost Estimate Contingency Analysis Model is based on the applied contingency and the assumptions upon which the estimate was predicated. The model is applied with a suggested risk level of $18 \%$ and a level of confidence of $90 \%$ the estimate will fall within the bid range. The Contingency Analysis is based on a weighted average to provide a $90 \%$ probability of underrun and a $10 \%$ probability of overnun.
CONTINGENCY ANALYSIS GUIDE BY TYPE OF ESTIMATE

Guidelines established by DOE/FM 50, Cost Estimating Guide, Vol. 6 , Cost Guide, and as presented in the INEL Cost Estimating Guide. PLANNING

Experimental/Special Conditions.

Conceptual

Experimentalspecial Conditions............. Up to $40 \%$

TITLE!

TITLE II

TITLE IIJAFC

$5 \%-15 \%$ Market Conditions 


\section{G\&A/PIF ADDER CALCULATION SHEET \\ ICPP BIN SET CLOSURE \\ RISK BASED CLEAN CLOSURE; CLEAN FILL; ESCALATED \\ REGULATORY COMPLIANCE}

PROCUREMENT FEE:

CONSTRUCTION $=$
GFE $=$$\quad \begin{aligned} & \$ 6,063,013 \\ & \text { Subtotal }\end{aligned}$

FEE @ $1 \%=$

$\$ 6,063,013 \cdot 0.01=$

$\$ 60,630$

G\&A @ 23\% (with a ceiling of $\$ 500,000$ imposed per year, 3 yrs)

CONSTRUCTION OR

CEILING * 3 YEARS $=\quad \$ 1,500,000$

GFE $=$

$\$ 0$

PROCUREMENT FEE $=$ $\$ 60,630$

Subtotal $\$ 1,560,630$

FEE @ $23 \%=$

$\$ 1,560,630 * 0.23=$

$\$ 358,945$

PIF @ 5.5\%

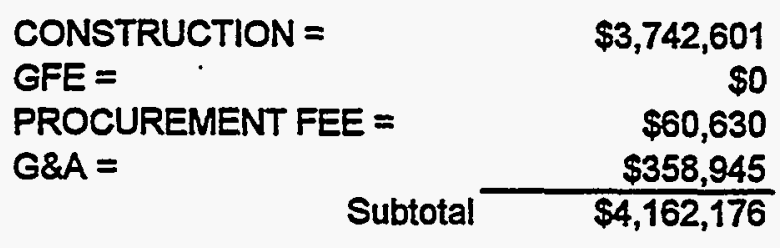

FEE @ 5.5\% =

$\$ 228,920$

TOTAL PROCUREMENT FEE:

$\$ 60,630$

TOTAL G\&A FEE:

$\$ 358,945$

TOTAL PIF:

$\$ 228,920$ 
Lockheed Martin Idaho Technologies Co. Rov. 6-96
PROJECT NAME: ICPP Bin Set Closure (EIS Stud LOCATION 1: REQUESTOR:
Place Clean Grout in Vault

ICPP

Bryan Spaulding

\section{COST ESTIMATE SUMMARY}

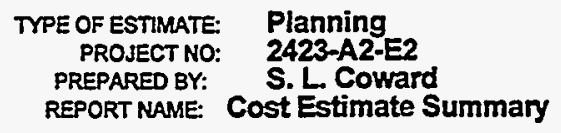

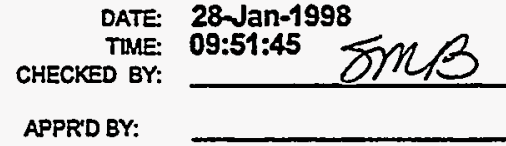

\begin{tabular}{|c|c|c|c|c|}
\hline $\begin{array}{l}\text { WBS } \\
\text { Element }\end{array}$ & Cost Estimate Element & $\begin{array}{c}\text { Total } \\
\text { Unescalated }\end{array}$ & Escalation & $\begin{array}{c}\text { Total } \\
\text { incl Escalation }\end{array}$ \\
\hline $\begin{array}{l}\frac{1.1}{1.1 .1} \\
1.1 .2 \\
\frac{1.2}{1.2 .1} \\
1.2 .2 \\
\frac{1.3}{1.3 .1} \\
1.3 .2 \\
1.3 .3 \\
1.5 .2\end{array}$ & $\begin{array}{l}\text { ENGINEERING, DESIGN AND INSPECTION } \\
\text { DESIGN ENGINEERING } \\
\text { TITLE IIIINSPECTION } \\
\text { MANAGEMENT COSTS } \\
\text { PROJECT MANAGEMENT } \\
\text { CONSTRUCTION MANAGEMENT } \\
\text { CONSTRUCTION } \\
\text { GENERAL CONDITIONS } \\
\text { SITEWORK } \\
\text { CONCRETE } \\
\text { PROCUREMENT FEES }\end{array}$ & $\begin{array}{r}1,702,520 \\
529,925 \\
\\
1,067,791 \\
0 \\
4,231,459 \\
52,992\end{array}$ & $\begin{array}{c}1,634,419 \\
540,524 \\
\\
1,089,146 \\
0 \\
4,316,088 \\
54,052\end{array}$ & $\begin{array}{r}\gg>\begin{array}{r}\$ 2,562,185 \\
2,026,962 \\
535,223\end{array} \\
>>\frac{\$ 4,407,388}{3,336,939} \\
1,070,449 \\
>>\frac{\$ 10,704,484}{2,156,937} \\
0 \\
8,547,547\end{array}$ \\
\hline & $\begin{array}{l}\text { SUBTOTAL INCLUDING ESCALATION } \\
\text { PROJECT CONTINGENCY }\end{array}$ & $9,174,461$ & $8,606,640$ & $\gg \$ 17,781,101$ \\
\hline & MANAGEMENT RESERVE- & & & $\$ 1,081,153$ \\
\hline & CONTINGENCY- & & & $\$ 5,137,746$ \\
\hline & TOTAL ESTIMATED COST - & & & $\$ 24,000,000$ \\
\hline
\end{tabular}

PROJECT COST PARAMETERS

EDI AS A $\%$ OF CONST. + GFE $=24.00 \%$ CONTINGENCY $=34.97 \%$ 
Lockheed Martin Idaho Technologies Co.

$$
\text { Rev6-96 }
$$

PROJECT NAME: ICPP Bin Set Closure (EIS Study) Place Clean Grout In Vault

ICPP

LOCATION t:

Bryan Spaulding

\section{DETAILED COST ESTIMATE SHEET}

TYPE OF Estimate: Planning

PROJECT NO:: 2423-A2-E2

PREPARED BY: S. L. Coward
PAGE \# 1

DATE 28-Jan-1998

TIME: 10:10:46

REPORT NAME: Detall Cost Estimate Sheet

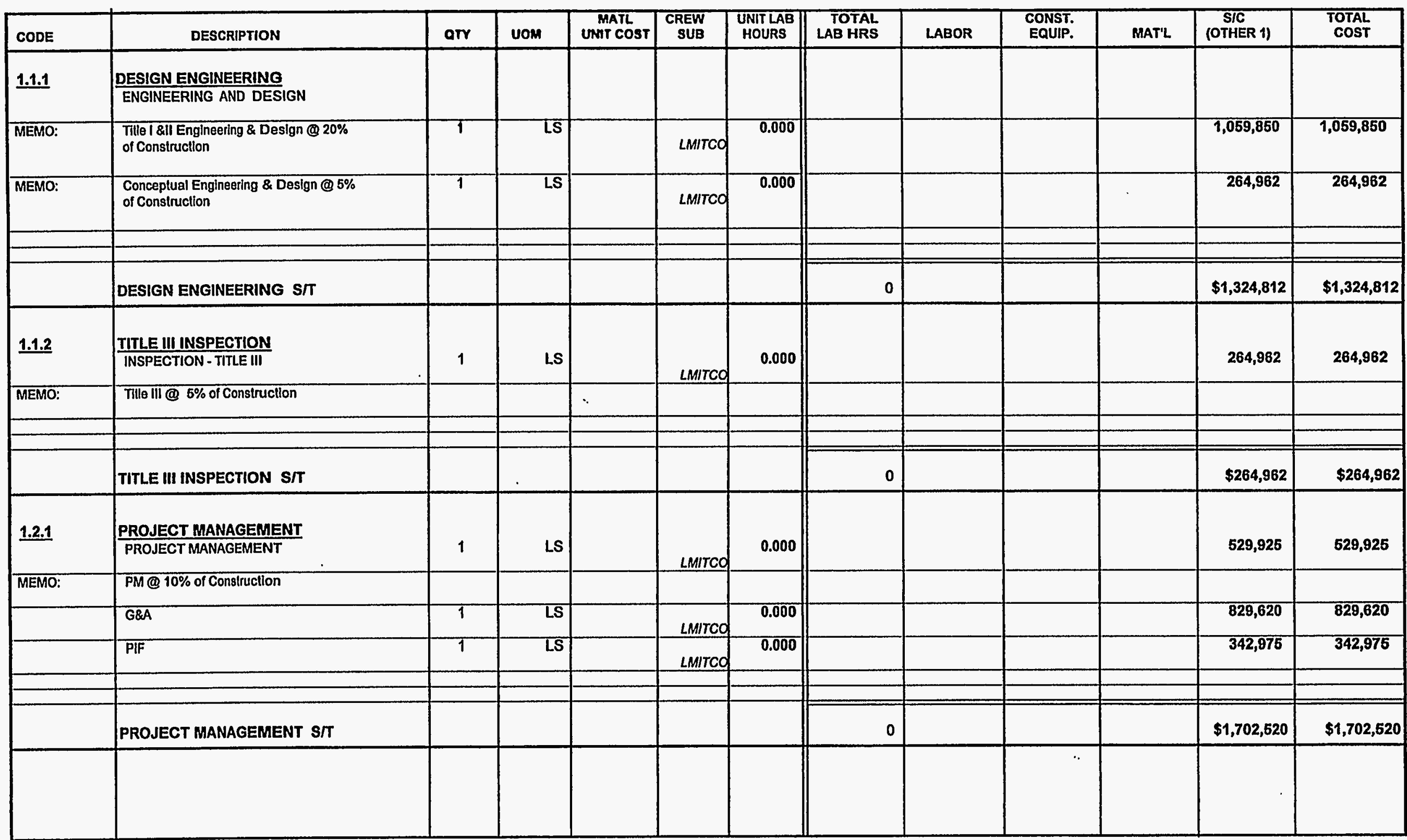


Lockheed Martin Idaho Technologles Co.

Rav 6.96

PROJECT NAME: ICPP Bin Set Closure (EIS Study)

Place Clean Grout in Vault

ICPP

Bryan Spaulding
DETAILED COST ESTIMATE SHEET

TYPE OF ESTIMATE: Planning

PROJECT NO.: 2423-A2-E2

PREPARED BY: S. L. Coward
PAGE $\# \mathbf{2}$

DATE 28-Jan-1998

TIME: 10:10:46

REPORT NAME: Detall Cost Estimate Sheet

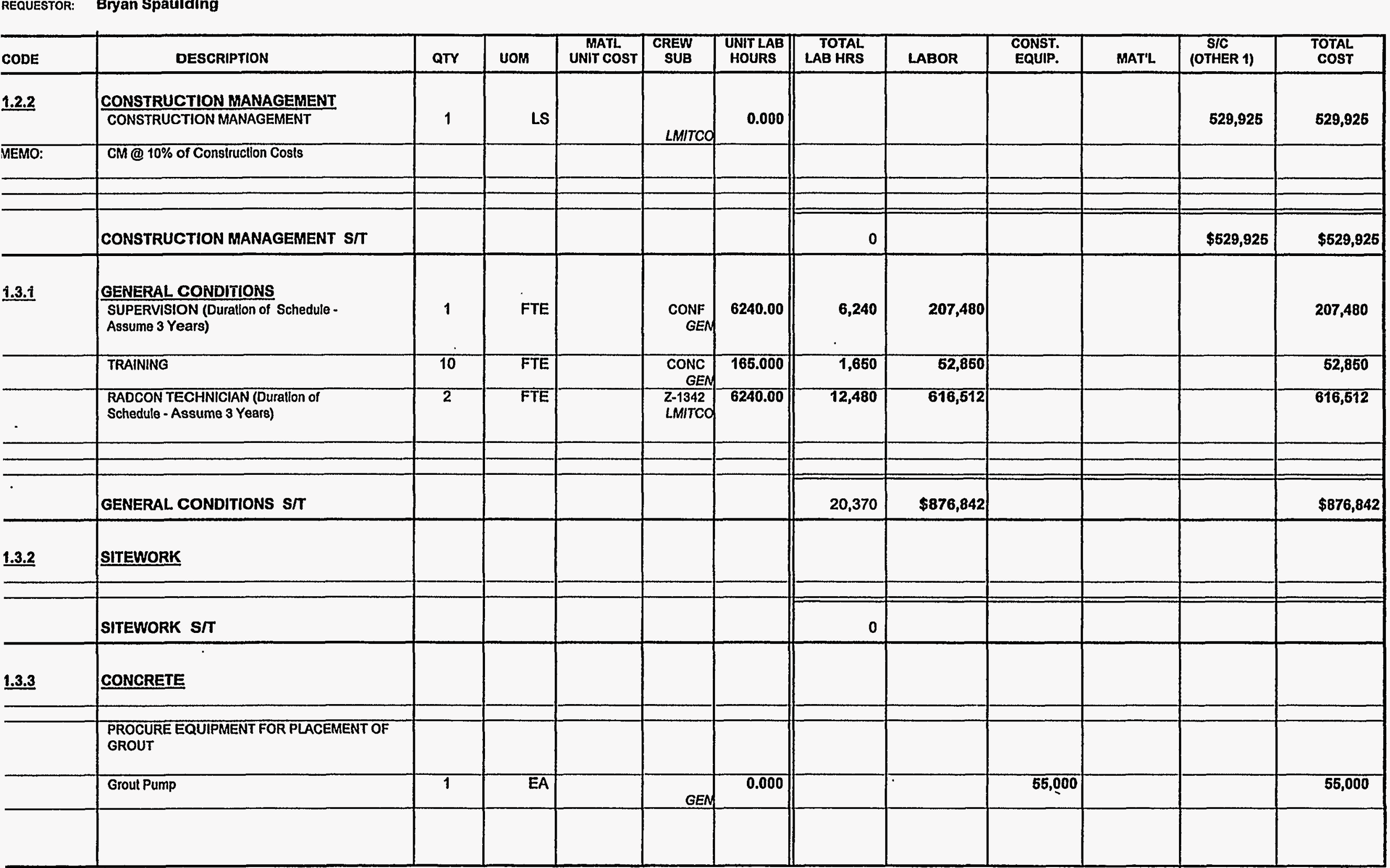


Lockhieed Martin Idaho Technologies Co. Rov 6-96

PROJECT NAME: ICPP Bin Set Closure (EIS Study) Place Clean Grout in Vault

LOCATION 1:

ICPP

REQUESTOR: Bryan Spaulding

\section{DETAILED COST ESTIMATE SHEET}

TYPE OF ESTIMATE: Planning

PROJECT NO:: 2423-A2-E2

PREPARED BY: S. L. Coward
PAGE \# 3

DATE 28-Jan-1998

TIME: 10:08:07

REPORT NAME: Detail Cost Estimate Sheot

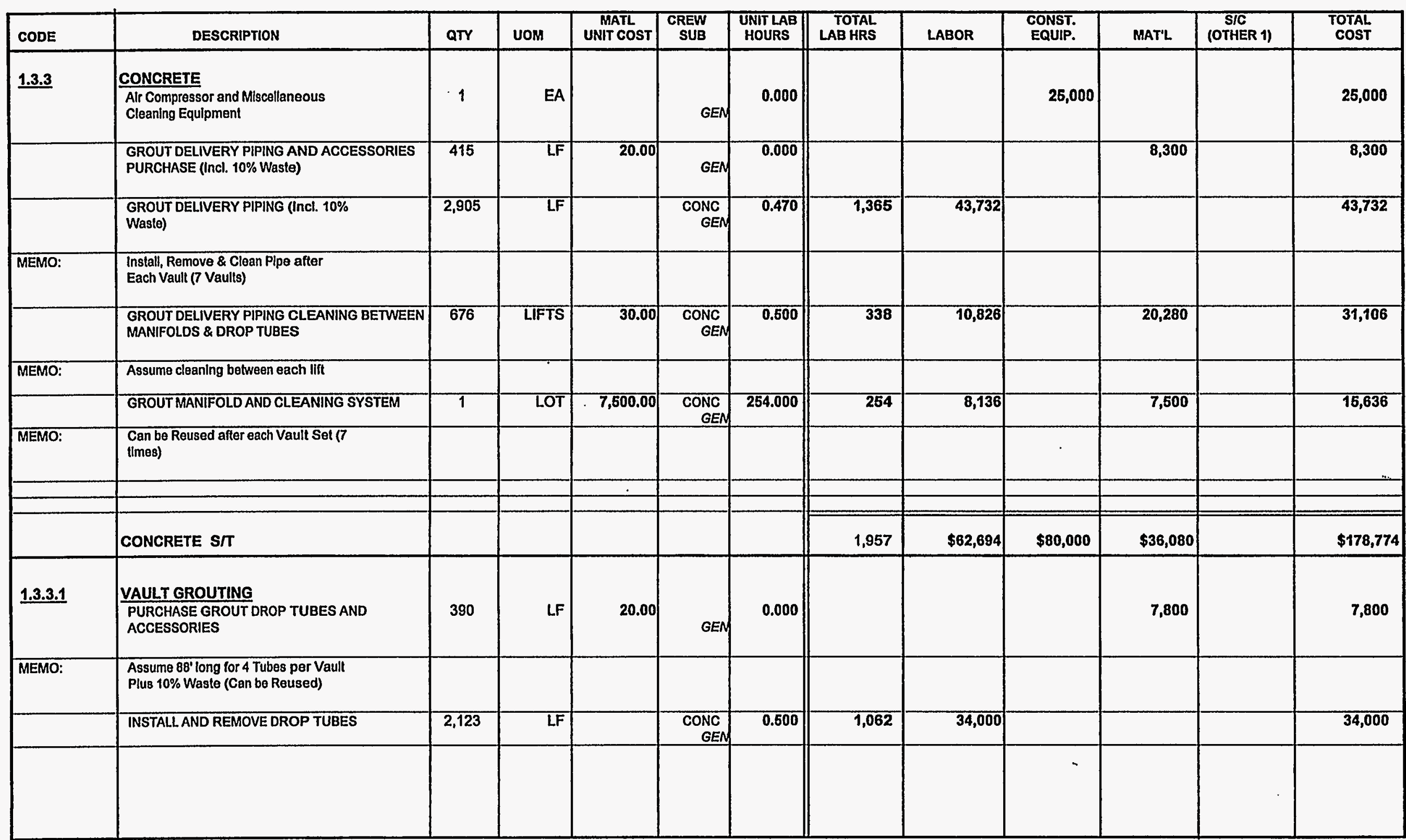


Lockheed Martin Idaho Technologies Co.

PROJECT NAME: ICPP Bin Set Closure (EIS Stud Place Clean Grout in Vault ICPP

LOCATION 1 REQUESTOR:
CONTINGENCY ANALYSIS

TYPE OF ESTIMATE: PROJECT NO: PREPARED BY:
Planning

2423-A2-E2

S. L. Coward
DATE: 28-Jan-1998

TIME: 09:51:35

REPORT NAME: Contingency Analysis

\begin{tabular}{|c|c|c|c|c|c|c|c|c|c|c|c|}
\hline \multicolumn{9}{|c|}{ PROBABLE \% VARIATION } & \multicolumn{2}{|c|}{$\begin{array}{c}\text { PROJECT } \\
\text { CONTINGENCY }\end{array}$} & \multirow{3}{*}{$\begin{array}{l}\text { SUMMARY } \\
\begin{array}{l}\text { Total Cost } \\
\text { by Element }\end{array}\end{array}$} \\
\hline \multirow[t]{2}{*}{$\begin{array}{l}\text { WBS } \\
\text { Element }\end{array}$} & \multirow{2}{*}{ Cost Estimate Element } & \multirow{2}{*}{$\begin{array}{l}\text { Total Cost w/o } \\
\text { Contingency }\end{array}$} & \multirow[t]{2}{*}{$\begin{array}{l}\text { \% Total } \\
\text { Cost }\end{array}$} & \multicolumn{2}{|c|}{$\begin{array}{c}\text { Prob. \% Var. } \\
\text { From Est. }\end{array}$} & \multicolumn{2}{|c|}{ Wt. $\%$ of Prob. } & \multirow[t]{2}{*}{ Contingency } & \multirow[t]{2}{*}{$\%$} & \multirow[t]{2}{*}{ Cost } & \\
\hline & & & & - & + & - & + & & & & \\
\hline 1.1.1 & DESIGN ENGINEERING & $1,324,812$ & 7.45 & 10 & 40 & 0.75 & 2.98 & $2.608 \%$ & $7.45 \%$ & 463,350 & $1,788,162$ \\
\hline 1.1 .2 & TILE III INSPECTION & 264,962 & 1.49 & 10 & 40 & 0.15 & 0.60 & $0.522 \%$ & $1.49 \%$ & 92,670 & 357,632 \\
\hline 1.21 & PROJECT MANAGEMENT & $1,702,520$ & 9.57 & 10 & 40 & 0.96 & 3.83 & $3.351 \%$ & $9.57 \%$ & 595,452 & $2,297,972$ \\
\hline 1.2 .2 & CONSTRUCTION MANAGEMENT & 529,925 & 2.98 & 10 & 40 & 0.30 & 1.19 & $1.043 \%$ & $2.98 \%$ & 185,340 & 715,265 \\
\hline 1.3 .1 & GENERAL CONDITONS & $1,057,791$ & 6.01 & 10 & 40 & 0.60 & 2.40 & $2.102 \%$ & $6.01 \%$ & 373,457 & $1,441,248$ \\
\hline 1.3 .2 & SITEWORK & 0 & 0.00 & 0 & 0 & 0.00 & 0.00 & $0.000 \%$ & $0.00 \%$ & 0 & 0 \\
\hline 1.3 .3 & CONCRETE & $4,231,459$ & 23.80 & 10 & 40 & 2.38 & 9.52 & $8.329 \%$ & $23.80 \%$ & $1,479,943$ & $5,711,402$ \\
\hline \multirow[t]{2}{*}{1.5 .2} & PROCUREMENT FEES & 52,992 & 0.30 & 10 & 40 & 0.03 & 0.12 & $0.104 \%$ & $0.30 \%$ & 18,534 & 71,526 \\
\hline & ESCALATION & $8,606,640$ & 48.40 & 10 & 40 & 4.84 & 19.36 & $16.941 \%$ & $48.40 \%$ & $3,010,153$ & $11,616,793$ \\
\hline & SUBTOTAL & $17,781,101$ & 100.00 & & & & & $35.000 \%$ & & & \\
\hline & CALCULATED CONTINGENCY & $6,223,385$ & & & & & & & & & \\
\hline & RESULTANT TEC & $24,004,486$ & & & & & & & & & \\
\hline & ROUNDED TEC & $24,000,000$ & & & & & & & & & \\
\hline & PROJECT CONTINGENCY & $6,218,899$ & & & & & & $34.97 \%$ & & & \\
\hline & MANAGEMENT RESERVE & $1,081,153$ & & & & & & & & & \\
\hline & CONTINGENCY & $5,137,746$ & & & & & & & & & \\
\hline & TOTAL ESTIMATED COST & $24,000,000$ & & & & & & & & $6,218,899$ & $24,000,000$ \\
\hline
\end{tabular}

CONFIDENCE LEVEL AND ASSUMED RISKS:

The Lockheed Idaho Technologies Co. Cost Estimate Contingency Analysis Model is based on the applied contingency and the assumptions upon which the estimate was predicated. The model is applied with a suggested risk level of $18 \%$ and a level of confidence of $90 \%$ the estimate will fall within the bid range. The Contingency Analysis is based on a weighted average to provide a $90 \%$ probability of underrun and a $10 \%$ probability of overrun.
CONTINGENCY ANALYSIS GUIDE BY TYPE OF ESTIMATE

Guidelines established by DOE/FM 50 , Cost Estimating Guide, Vol. 6,

Cost Guide, and as presented in the INEL Cost Estimating Guide.

PLANNING

Experimental/Special Conditions.

$20 \%-30 \%$

Conceptual

Experimentalspecial Conditions $\quad 15 \%-25 \%$

TITLE I

TITLE II

TITLE IIIAFC
$10 \%-20 \%$

$5 \%-15 \%$

Market Conditions 


\section{G\&APIF ADDER CALCULATION SHEET \\ ICPP BIN SET CLOSURE \\ RISK BASED CLEAN CLOSURE; CLEAN FILL; ESCALATED}

FILL VAULTS W/ CLEAN GROUT

PROCUREMENT FEE:

CONSTRUCTION $=$
GFE $=$ Subtotal $\frac{\$ 10,704,484}{\$ 10,704,484}$

FEE @ 1\%=

$\$ 10,704,484 \cdot 0.01=$

$\$ 107,045$

G\&A @ 23\% (with a ceiling of $\$ 500,000$ imposed per year, 7 yrs)

CONSTRUCTION OR

CEILING * 7 YEARS $=\quad \$ 3,500,000$

GFE $=$

PROCUREMENT FEE $=$

Subtotal $\$ 107,045$

$\$ 3,607,045$

FEE @ 23\% =

$\$ 3,607,045 \cdot 0.23=$

$\$ 829,620$

PIF @ 5.5\%

CONSTRUCTION =

GFE =

PROCUREMENT FEE =

G\&A =

FEE @ $5.5 \%=$
$\$ 5,299,250$

$\$ 0$

$\$ 107,045$

$\$ 829,620$

Subtotal

\begin{tabular}{|c|c|c|}
\hline FEE @ $5.5 \%=$ & $\$ 6,235,915 \cdot 0.055=$ & $\$ 342,975$ \\
\hline TOTAL PROCUREMENT FEE: & & $\$ 107,045$ \\
\hline TOTAL G\&A FEE: & & $\$ 829,620$ \\
\hline TOTAL PIF: & & $\$ 342,975$ \\
\hline
\end{tabular}


Lockheed Martin Idaho Technologies Co. Rev, 6-96 PROJECT NAME: ICPP Bin Set Closure (EIS Stud LOCATION 1: Tractor (Risk Based Estimates) REQUESTOR

Bryan Spaulding
COST ESTIMATE SUMMARY

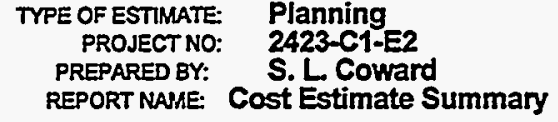

ECKED BY:

DATE. 28-Jan-1998

TIME: 12:02:53

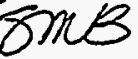

APPR'D BY:

\begin{tabular}{|c|c|c|c|c|}
\hline $\begin{array}{l}\text { WBS } \\
\text { Element }\end{array}$ & Cost Estimate Element & $\begin{array}{c}\text { Total } \\
\text { Unescalated }\end{array}$ & Escalation & $\begin{array}{c}\text { Total } \\
\text { Incl Escalation }\end{array}$ \\
\hline $\begin{array}{l}\frac{1.1}{1.1 .1} \\
1.1 .2 \\
1.2 \\
1.2 .1 \\
1.2 .2 \\
1.3 \\
1.3 .1 \\
1.3 .13 \\
1.5 .2\end{array}$ & $\begin{array}{l}\text { ENGINEERING, DESIGN AND INSPECTION } \\
\text { DESIGN ENGINEERING TITLE I \& II } \\
\text { TITLE III INSPECTION } \\
\text { MANAGEMENT COSTS } \\
\text { PROJECT MANAGEMENT } \\
\text { CONSTRUCTION MANAGEMENT } \\
\text { CONSTRUCTION } \\
\text { GENERAL CONDITIONS } \\
\text { SPECIAL CONSTRUCTION } \\
\text { PROCUREMENT FEES }\end{array}$ & $\begin{array}{r}9,900,244 \\
3,300,081 \\
\\
11,840,340 \\
6,600,163 \\
\\
6,840,414 \\
59,161,216 \\
660,016\end{array}$ & $\begin{array}{r}10,774,709 \\
6,732,166 \\
6,977,222 \\
60,344,441 \\
673,217\end{array}$ & $\begin{array}{r}>>\frac{\$ 21,021,518}{14,355,354} \\
6,666,164 \\
\\
>\frac{\$ 35,947,378}{22,615,049} \\
13,332,329 \\
\\
>>\frac{\$ 133,323,293}{13,817,636} \\
119,505,657 \\
\\
>>\$ 1,333,233\end{array}$ \\
\hline & $\begin{array}{l}\text { SUBTOTAL INCLUDING ESCALATION } \\
\text { PROJECT CONTINGENCY }\end{array}$ & $98,302,474$ & $93,322,948$ & $\gg \$ 191,625,422$ \\
\hline & MANAGEMENT RESERVE- & & & $\gg \quad \$ 13,465,653$ \\
\hline & CONTINGENCY & & & $\gg \quad \$ 53,608,925$ \\
\hline & TOTAL ESTIMATED COST - & & & $\gg \$ 258,700,000$ \\
\hline
\end{tabular}

PROJECT COST PARAMETERS

EDI AS A \% OF CONST. + GFE $=16.00 \%$ 
Lockheed Martin Idaho Technologies Co. PROJECT NAME: ICPP Bin Set Closure (EIS Stud Tractor (Risk Based Estimates) LOCATION 1: ICPP REQUESTOR: Bryan Spaulding
CONTINGENCY ANALYSIS

$\begin{array}{cl}\text { TYPE OF ESTIMATE: } & \text { Planning } \\ \text { PROJECT NO: } & 2423-C 1-E 2 \\ \text { PREPARED BY: } & \text { S. L. Coward }\end{array}$

DATE: $28-$ Jan-1998

TIME: 12:02:49

REPORT NAME: Contingency Analysis

\begin{tabular}{|c|c|c|c|c|c|c|c|c|c|c|c|}
\hline \multicolumn{9}{|c|}{ PROBABLE \% VARIATION } & \multicolumn{2}{|c|}{$\begin{array}{c}\text { PROJECT } \\
\text { CONTINGENCY }\end{array}$} & \multirow{3}{*}{$\begin{array}{l}\text { SUMMARY } \\
\begin{array}{l}\text { Total Cost } \\
\text { by Element }\end{array}\end{array}$} \\
\hline \multirow[t]{2}{*}{$\begin{array}{l}\text { WBS } \\
\text { Element }\end{array}$} & \multirow{2}{*}{ Cost Estimate Element } & \multirow{2}{*}{$\begin{array}{l}\text { Total Cost w/o } \\
\text { Contingency }\end{array}$} & \multirow[t]{2}{*}{$\begin{array}{l}\text { \% Total } \\
\text { Cost }\end{array}$} & \multicolumn{2}{|c|}{$\begin{array}{c}\text { Prob. \% Var. } \\
\text { From Est. }\end{array}$} & \multicolumn{2}{|c|}{ Wt $\%$ of Prob. } & \multirow[t]{2}{*}{ Contingency } & \multirow[t]{2}{*}{$\%$} & \multirow[t]{2}{*}{ Cost } & \\
\hline & & & & - & + & - & + & & & & \\
\hline 1.1 .1 & DESIGN ENGINEERING TITLE I\&\| & $9,900,244$ & 5.17 & 10 & 40 & 0.52 & 2.07 & $1.808 \%$ & $5.17 \%$ & $3,465,379$ & $13,365,623$ \\
\hline 1.1 .2 & TITLE III INSPECTION & $3,300,081$ & 1.72 & 10 & 40 & 0.17 & 0.69 & $0.603 \%$ & $1.72 \%$ & $1,155,126$ & $4,455,207$ \\
\hline 1.2 .1 & PROJECT MANAGEMENT & $11,840,340$ & 6.18 & 10 & 40 & 0.62 & 247 & $2.163 \%$ & $6.18 \%$ & $4,144,470$ & $15,984,810$ \\
\hline 1.22 & CONSTRUCTION MANAGEMENT & $6,600,163$ & 3.44 & 10 & 40 & 0.34 & 1.38 & $1.206 \%$ & $3.44 \%$ & $2,310,253$ & $8,910,416$ \\
\hline 1.3 .1 & GENERAL CONDITIONS & $6,840,414$ & 3.57 & 10 & 40 & 0.36 & 1.43 & $1.249 \%$ & $3.57 \%$ & $2,394,348$ & $9,234,762$ \\
\hline 1.3 .13 & SPECIAL CONSTRUCTION & $59,161,216$ & 30.87 & 10 & 40 & 3.09 & 1235 & $10.806 \%$ & $30.87 \%$ & $20,708,179$ & $79,869,395$ \\
\hline \multirow[t]{2}{*}{1.5 .2} & PROCUREMENT FEES & 660,016 & 0.34 & 10 & 40 & 0.03 & 0.14 & $0.121 \%$ & $0.34 \%$ & 231,025 & 891,041 \\
\hline & ESCALATION & $93,322,948$ & 48.70 & 10 & 40 & 4.87 & 19.48 & $17.045 \%$ & $48.70 \%$ & $32,665,798$ & $125,988,746$ \\
\hline & SUBTOTAL & $191,625,422$ & 100.00 & & & & & $35.000 \%$ & & & \\
\hline & CALCULATED CONTINGENCY & $67,068,898$ & & & & & & & & & \\
\hline & RESULTANT TEC & $258,694,320$ & & & & & & & & & \\
\hline & ROUNDED TEC & $258,700,000$ & & & & & & & & & \\
\hline & PROJECT CONTINGENCY & $67,074,578$ & & & & & & $35.00 \%$ & & & \\
\hline & MANAGEMENT RESERVE & $13,465,653$ & & & & & & & & & \\
\hline & CONTINGENCY & $53,608,925$ & & & & & & & & & \\
\hline & TOTAL ESTIMATED COST & $258,700,000$ & & & & & & & & $67,074,578$ & $258,700,000$ \\
\hline
\end{tabular}

CONFIDENCE LEVEL AND ASSUMED RISKS:

The Lockheed Idaho Technologies Co. Cost Estimate Contingency Analysis Model is based on the applied contingency and the assumptions upon which the estimate was predicated. The model is applied with a suggested risk level of $18 \%$ and a level of confidence of $90 \%$ the estimate will fall within the bid range. The Contingency Analysis is based on a weighted average to provide a $90 \%$ probability of underrun and a $10 \%$ probability of overrun.
CONTINGENCY ANALYSIS GUIDE BY TYPE OF ESTIMATE

Guidelines established by DOE/FM 50 , Cost Estimating Guide, Vol. 6 , Cost Guide, and as presented in the INEL Cost Estimating Guide. PLANNING Experimen $20 \%-30 \%$ Conceptual TITLE ITLE

TITLE IIVAFC
.....Up to $50 \%$

$15 \%-25 \%$

...Up to $40 \%$

$10 \%-20 \%$

$5 \%-15 \%$

Market Conditions 


\section{G\&APIF ADDER CALCULATION SHEET ICPP BIN SET CLOSURE \\ RISK BASED CLEAN CLOSURE; CLEAN FILL; ESCALATED \\ CLEAN BINS W/ ROBOTS - FLOOR}

PROCUREMENT FEE:

CONSTRUCTION $=$ $\$ 133,323,293$

GFE =

Subtotal $\longdiv { \$ 1 3 3 , 3 2 3 , 2 9 3 }$

FEE @ 1\%=

$\$ 133,323,293 * 0.01=$

$\$ 1,333,233$

G\&A @ 23\% (with a ceiling of $\$ 500,000$ imposed per year, 10 yrs)

CONSTRUCTION OR

CEILING $\bullet 10$ YEARS =

GFE =

PROCUREMENT FEE $=$

Subtotal
$\$ 5,000,000$

$01,333,233$

$\$ 6,333,233$

$\$ 6,333,233 \cdot 0.23=\quad \$ 1,456,644$

FEE @ 23\%=

$\$ 6,333,233 \cdot 0.23=$

PIF@ $0.5 \%$

CONSTRUCTION =
GFE =
PROCUREMENT FEE =
G\&A =

Subtotal
$\$ 66,001,630$

\$o

$\$ 1,333,233$

$\$ 1,456,644$

$\$ 68,791,507$

$\$ 68,791,507 \cdot 0.055=$

$\$ 3,783,533$

FEE @ 5.5\% =

$\$ 68,791,507 \cdot 0.055=$

$\$ 1,333,233$

TOTAL PROCUREMENT FEE:

$\$ 1,456,644$

TOTAL G\&A FEE:

$\$ 3,783,533$

TOTAL PIF: 
Lockheed Martin Idaho Technologies Co. PROJECT NAME: ICPP Bin Set Closure (EIS Stud LDUA (Risk Based Estimates) LOCATION 1: ICPP REQUESTOR: Bryan Spaulding
COST ESTIMATE SUMMARY

$\begin{array}{cl}\text { TYPE OF ESTIMATE: } & \text { Planning } \\ \text { PROJECT NO: } & \text { 2423-D-E2 } \\ \text { PREPARED BY: } & \text { S. L. Coward } \\ \text { REPORT NAME: } & \text { Cost Estimate Summary }\end{array}$

DATE: 28-Jan-1998

TIME: 12:15:23 CHECKED BY: APPRD BY:

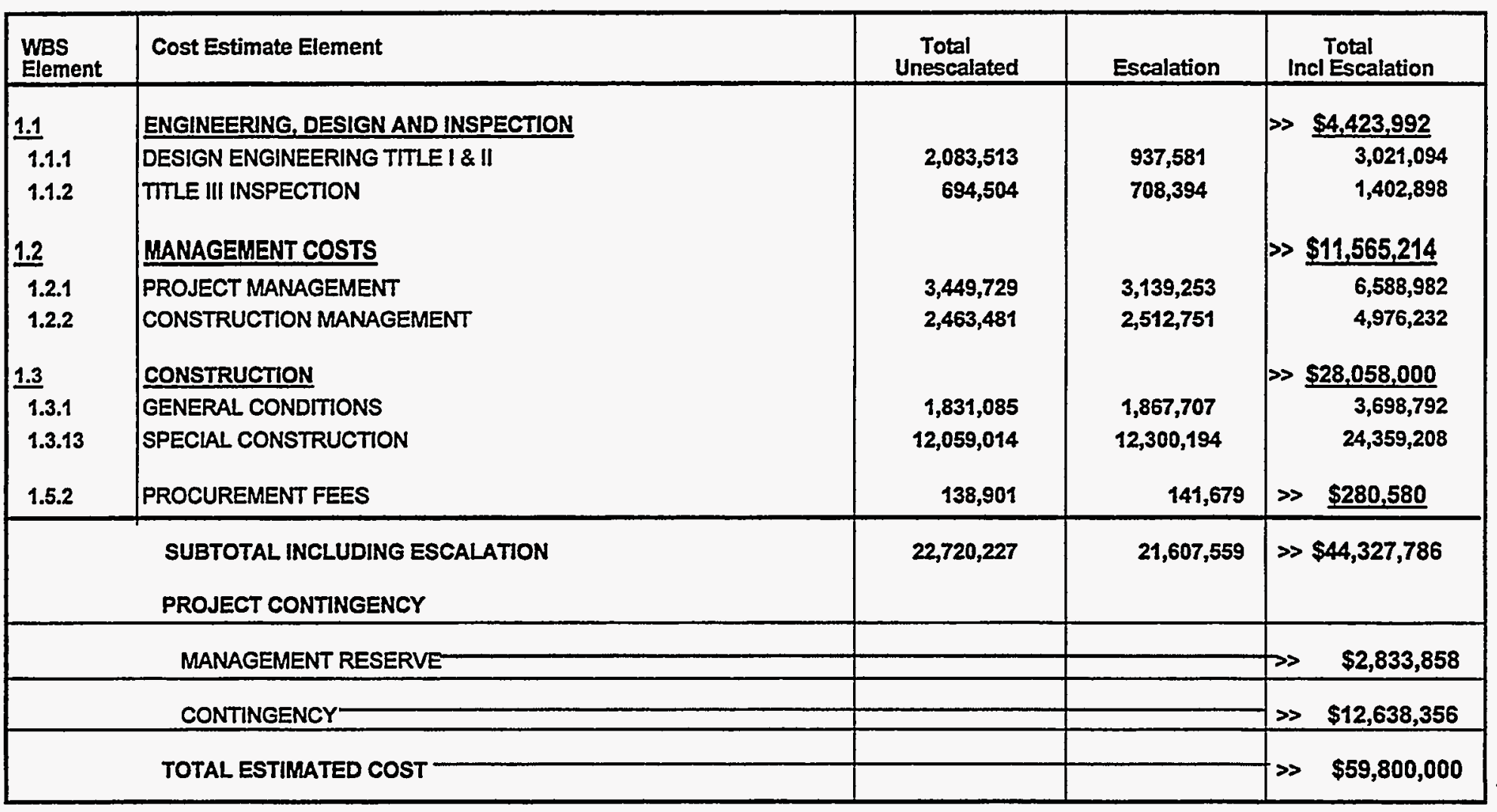

PROJECT COST PARAMETERS

EDI AS A \% OF CONST. + GFE $=16.00 \%$ 


\section{Lockheed Martin Idaho Technologies Co.} Rev 6.96 PROJECT NAME: ICPP Bin Set Closure (EIS Study) LDUA (RIsk Based Estimates) LOCATION 1: ICPP REQUESTOR: Bryan Spaulding

\section{DETAILED COST ESTIMATE SHEET}

TYPE OF ESTIMATE: Planning

PROJECT NO.: 2423-D-E2

PREPARED BY: S. L. Coward
PAGE \# 1

DATE 28-Jan-1998

TIME: 12:16:25

REPORT NAME: Detall Cost Estimate Sheet

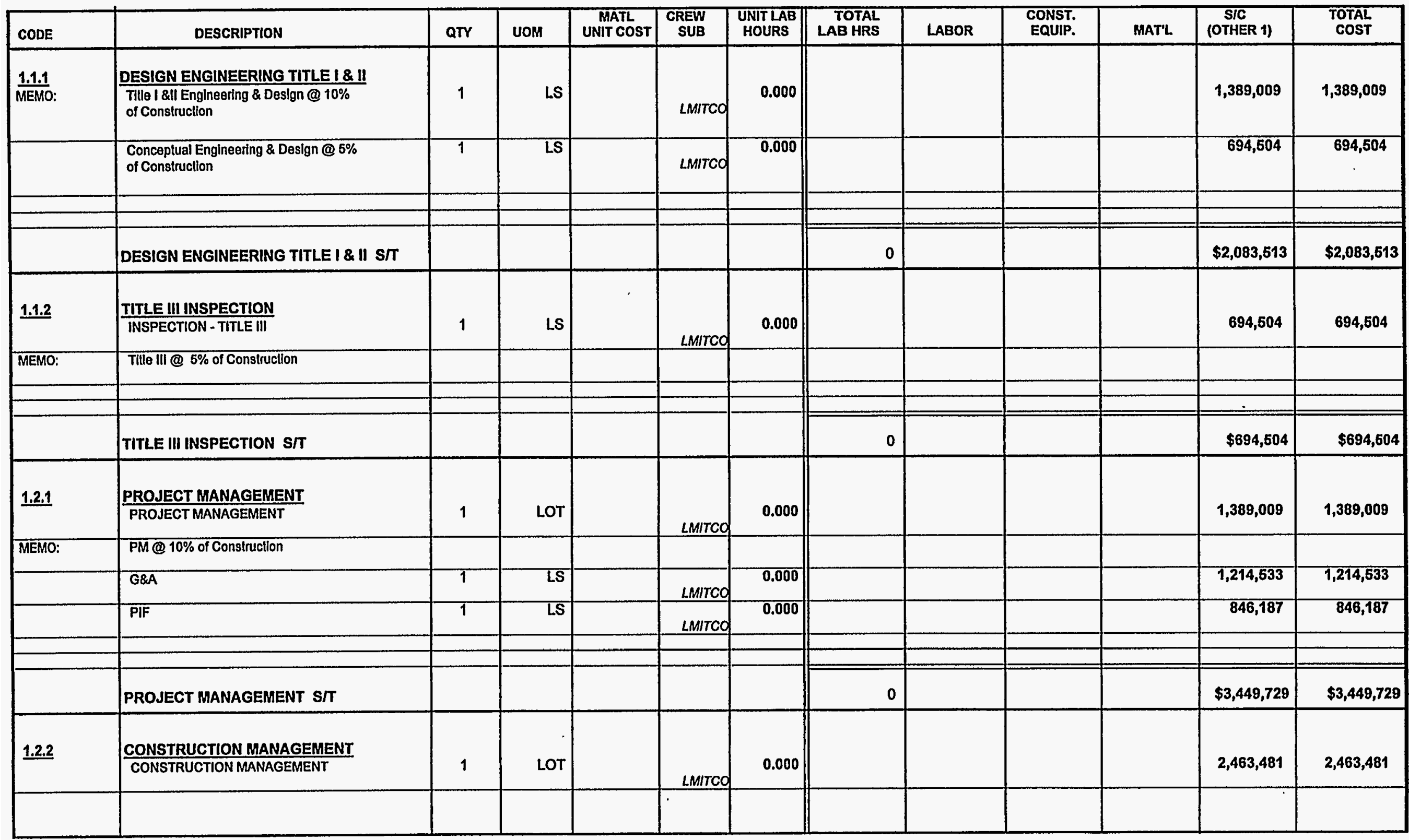




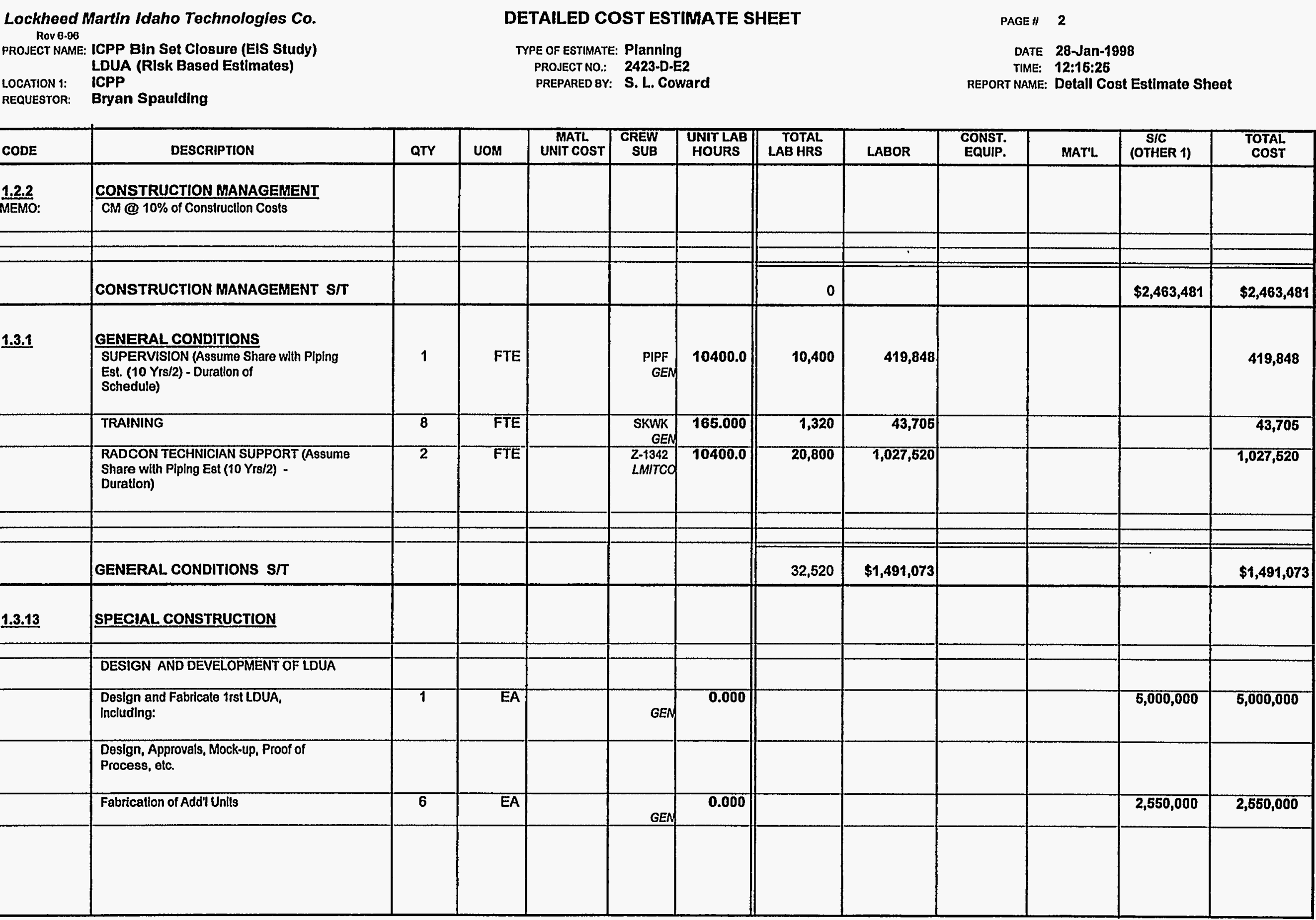


Lockheed Martin Idaho Technologies Co. Rov 6.96

PROJECT NAME: ICPP Bin Set Closure (EIS Study) LDUA (Risk Based Estimates)

LOCATION $:$ I: ICPP

REQUESTOR: Bryan Spaulding

\section{DETAILED-COST ESTIMATE SHEET}

TYPE OF estimate: Planning

PROJECT NO: 2423-D-E2

PREPARED BY: S. L. Coward
PAgE\# 3

DATE 28-Jan-1998

TIME: 12:16:25

REPORT NAME: Detall Cost Estimate Sheet

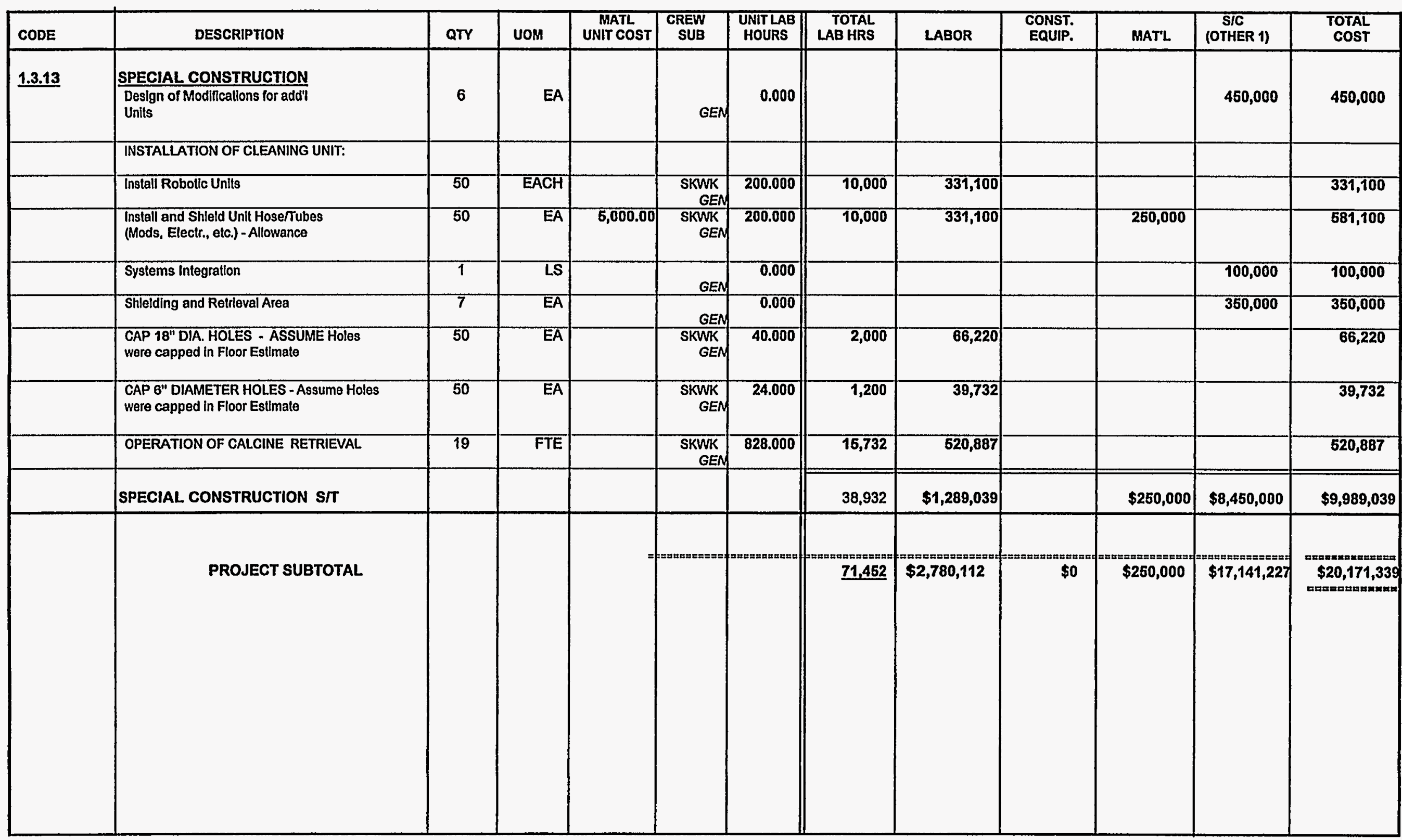


Lockheed Martin Idaho Technologies Co.

PROJECT NAME: ICPP Bin Set Closure (EIS Stud

IDUA (Risk Based Estimates)

LOCATION 1:

REQUESTOR:
Bryan Spaulding
CONTINGENCY ANALYSIS
TYPE OF ESTMMATE:
PROJECT NO: PROJECT NO:

PREPARED BY:
Planning

2423-D-E2

S. L. Coward
DATE: 28-Jan-1998

TIME: 12:15:18
REPORT NAME: Contingency Analysis

\begin{tabular}{|c|c|c|c|c|c|c|c|c|c|c|c|}
\hline \multicolumn{9}{|c|}{ PROBABLE \% VARIATION } & \multicolumn{2}{|c|}{$\begin{array}{l}\text { PROJECT } \\
\text { CONTINGENCY }\end{array}$} & \multirow{3}{*}{$\begin{array}{l}\text { SUMMARY } \\
\text { Total Cost } \\
\text { by Element }\end{array}$} \\
\hline \multirow{2}{*}{$\begin{array}{l}\text { WBS } \\
\text { Element }\end{array}$} & \multirow{2}{*}{ Cost Estimate Element } & \multirow{2}{*}{$\begin{array}{l}\text { Total Cost w/o } \\
\text { Contingency }\end{array}$} & \multirow[t]{2}{*}{$\begin{array}{l}\text { \% Total } \\
\text { Cost }\end{array}$} & \multicolumn{2}{|c|}{$\begin{array}{l}\text { Prob. \% Var. } \\
\text { From Est }\end{array}$} & \multicolumn{2}{|c|}{ Wt. \% of Prob. } & \multirow{2}{*}{ Contingency } & \multirow{2}{*}{$\%$} & \multirow{2}{*}{ Cost } & \\
\hline & & & & - & + & - & + & & & & \\
\hline 1.1.1.1 & DESIGN ENGINEERING TITLE $1 \& \|$ & $2,083,513$ & 4.70 & 10 & 40 & 0.47 & 1.88 & $1.645 \%$ & $4.70 \%$ & 727,231 & $2,810,744$ \\
\hline 1.1 .2 & TITLE III INSPECTION & 694,504 & 1.57 & 10 & 40 & 0.16 & 0.63 & $0.548 \%$ & $1.57 \%$ & 242,410 & 936,914 \\
\hline 1.2 .1 & PROJECT MANAGEMENT & $3,449,729$ & 7.78 & 10 & 40 & 0.78 & 3.11 & $2.724 \%$ & $\mathbf{7 . 7 8 \%}$ & 1,204,097 & $4,653,826$ \\
\hline 1.22 & CONSTRUCTION MANAGEMENT & $2,463,481$ & 5.56 & 10 & 40 & 0.56 & 222 & $1.945 \%$ & $5.55 \%$ & 859,856 & $3,323,337$ \\
\hline 1.3 .1 & GENERAL CONDMTONS & $1,831,085$ & 4.13 & 10 & 40 & 0.41 & 1.65 & $1.446 \%$ & 4.13\% & 639,124 & $2,470,209$ \\
\hline 1.3 .13 & SPECIAL CONSTRUCTION & $12,059,014$ & 27.20 & 10 & 40 & 2.72 & 10.88 & 9.521\% & $27.20 \%$ & $4,209,090$ & $16,268,104$ \\
\hline \multirow[t]{2}{*}{ 1.5.2 } & PROCUREMENT FEES & 138,904 & 0.31 & 10 & 40 & 0.03 & 0.13 & $0.110 \%$ & $0.31 \%$ & 48,482 & 187,383 \\
\hline & ESCALATION & $21,607,559$ & 48.74 & 10 & 40 & 4.87 & 19.50 & $17.054 \%$ & $48.74 \%$ & $7,541,924$ & $29,149,483$ \\
\hline & SUBTOTAL & $44,327,786$ & 100.00 & & & & & $35.000 \%$ & & & \\
\hline & CALCULATED CONTINGENCY & $15,514,725$ & & & & & & & & & \\
\hline & RESULTANT TEC & $59,842,511$ & & & & & & & & & \\
\hline & ROUNDED TEC & $59,800,000$ & & & & & & & & & \\
\hline & PROJECT CONTINGENCY & $15,472,214$ & & & & & & $34.90 \%$ & & & \\
\hline & MANAGEMENT RESERVE & $2,833,858$ & & & & & & & & & \\
\hline & CONTINGENCY & $12,638,356$ & & & & & & & & & \\
\hline & TOTAL ESTIMATED COST & $59,800,000$ & & & & & & & & $15,472,214$ & $59,800,000$ \\
\hline
\end{tabular}

CONFIDENCE LEVEL AND ASSUMED RISKS:

The Lockheed Idaho Technologies Co. Cost Estimate Contingency Analysis Model is based on the applied contingency and the assumptions upon which the estimate was predicated. The model is applied with a suggested risk level of $18 \%$ and a level of confidence of $90 \%$ the estimate will fall within the bid range. The Contingency Analysis is based on a weighted average to provide a $90 \%$ probability of underrun and a $10 \%$ probability of overrun.
CONTINGENCY ANALYSIS GUIDE BY TYPE OF ESTIMATE

Guidelines established by DOE/FM 50 , Cost Estimating Guide, Vol. 6 , Cost Guide, and as presented in the INEL Cost Estimating Guide.

$$
\text { PLANNING }
$$

Experimental/Special Conditions.

$20 \%-30 \%$

Conceptual

Experimental/Special Conditions.

TITLEI

TITLE II

TITLE IVIAFC
$15 \%-25 \%$

U... to $40 \%$

$10 \%-20 \%$
$5 \%-15 \%$

Market Conditions 


\section{G\&APIF ADDER CALCULATION SHEET \\ ICPP BIN SET CLOSURE \\ RISK BASED CLEAN CLOSURE; CLEAN FILL; ESCALATED \\ CLEAN BINS W/ ROBOTS - WALLS}

PROCUREMENT FEE:

$\begin{aligned} & \text { CONSTRUCTION }= \\ & \text { GFE }= \\ & \text { FEE @ } 1 \%= \\ & \text { Subtotal }\end{aligned} \quad \begin{aligned} & \$ 28,058,000 \\ & \$ 28,058,000\end{aligned}$

G\&A @ 23\% (with a ceiling of $\$ 500,000$ imposed per year, 10 yrs)

CONSTRUCTION OR

CEILING $\cdot 10$ YEARS $=\quad \$ 5,000,000$

GFE =

PROCUREMENT FEE $=$

$\$ 280,580$

Subtotal $\$ 5,280,580$

FEE @ 23\% =

$\$ 5,280,580 * 0.23=$

$\$ 1,214,533$

PIF @ 5.5\%

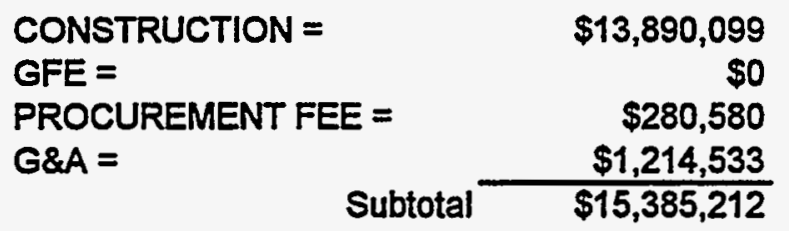

FEE @ 5.5\% =

$\$ 15,385,212 * 0.055=$

$\$ 846,187$

TOTAL PROCUREMENT FEE:

$\$ 280,580$

TOTAL G\&A FEE:

$\$ 1,214,533$

TOTAL PIF:

$\$ 846,187$ 
Lockheed Martin Idaho Technologies Co. PROJECT NAME: ICPP Bin Set Closure (EIS Stud Pipe Crawler (Risk Based Estim LOCATION 1: ICPP REQUESTOR: Bryan Spaulding
COST ESTIMATE SUMMARY

TYPE OF ESTIMATE: Planning PROJECT NO:

PREPARED BY:

2423-E-E2

S. L. Coward REPORT NAME: Cost Estimate Summary
CHECKED BY

DATE: 28-Jan-1998

TIME: 12:18:20

APPR'D BY:

\begin{tabular}{|c|c|c|c|c|}
\hline $\begin{array}{l}\text { WBS } \\
\text { Element }\end{array}$ & Cost Estimate Element & $\begin{array}{c}\text { Total } \\
\text { Unescalated }\end{array}$ & Escalation & $\begin{array}{c}\text { Total } \\
\text { Incl Escalation }\end{array}$ \\
\hline $\begin{array}{l}\frac{1.1}{1.1 .1} \\
1.1 .2 \\
\frac{1.2}{1.2 .1} \\
1.2 .2 \\
\frac{1.3}{1.3 .1} \\
1.3 .13 \\
1.5 .2\end{array}$ & $\begin{array}{l}\text { ENGINEERING, DESIGN AND INSPECTION } \\
\text { DESIGN ENGINEERING } \\
\text { TITLE III INSPECTION } \\
\text { MANAGEMENT COSTS } \\
\text { PROJECT MANAGEMENT } \\
\text { CONSTRUCTION MANAGEMENT } \\
\text { CONSTRUCTION } \\
\text { GENERAL CONDITIONS } \\
\text { SPECIAL CONSTRUCTION } \\
\text { PROCUREMENT FEES }\end{array}$ & $\begin{array}{r}1,778,336 \\
296,389 \\
2,167,699 \\
592,779 \\
\\
1,831,085 \\
4,096,709 \\
59,278\end{array}$ & $\begin{array}{r}871,385 \\
302,317 \\
1,972,606 \\
604,635 \\
\\
1,867,707 \\
4,178,643 \\
60,463\end{array}$ & 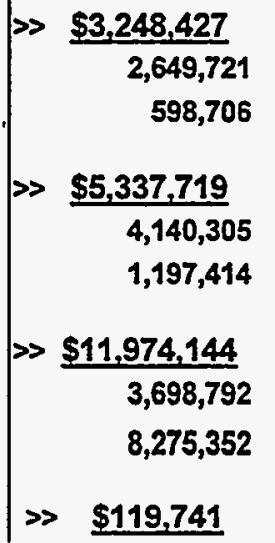 \\
\hline & $\begin{array}{l}\text { SUBTOTAL INCLUDING ESCALATION } \\
\text { PROJECT CONTINGENCY }\end{array}$ & $10,822,275$ & $9,857,756$ & $\gg \$ \$ 20,680,031$ \\
\hline & MANAGEMENT RESERVE- & & & $\$ 1,209,388$ \\
\hline & CONTINGENCY- & & & $\gg \quad \$ 6,010,581$ \\
\hline & TOTAL ESTIMATED COST - & & & $\$ 27,900,000$ \\
\hline
\end{tabular}

PROJECT COST PARAMETERS

EDI AS A \% OF CONST. + GFE $=27.00 \%$

CONTINGENCY $=34.91 \%$ 
Lockheed Martin Idaho Technologies Co.

\section{Rov 8-96}

PROJECT NAME: ICPP Bin Set Closure (EIS Study)

Plpe Crawler (Rlsk Based Estimates)

ICPP

Bryan Spaulding

\section{DETAILED COST ESTIMATE SHEET}

TYPE OF ESTIMATE: Planning

PROJECTNO:: 2423-E-E2

PREPARED BY: S. L. Coward
PAGE \# 1

DATE 28-Jan-1998

TIME: 12:18:22

REPORT NAME: Detail Cost Estimate Sheet

\begin{tabular}{|c|c|c|c|c|c|c|c|c|c|c|c|c|}
\hline CODE & DESCRIPTION & QTY & vom & $\begin{array}{c}\text { MATL } \\
\text { UNIT COST }\end{array}$ & $\begin{array}{l}\text { CREW } \\
\text { SUB }\end{array}$ & \begin{tabular}{|l} 
UNIT LAB \\
HOURS
\end{tabular} & $\begin{array}{l}\text { TOTAL } \\
\text { LAB HRS }\end{array}$ & LABOR & $\begin{array}{l}\text { CONST. } \\
\text { EQUIP. }\end{array}$ & MAT'L & $\begin{array}{c}\text { SIC } \\
\text { (OTHER 1) }\end{array}$ & $\begin{array}{l}\text { TOTAL } \\
\text { COST }\end{array}$ \\
\hline \multirow[t]{2}{*}{$\frac{1.1 .1}{\text { MEMO: }}$} & $\begin{array}{l}\text { DESIGN ENGINEERING } \\
\begin{array}{l}\text { Ttlie I \&ll Engineering \& Design @ 1 10\% } \\
\text { of Construction }\end{array}\end{array}$ & 1 & LS & & LMITCO & 0.000 & & & & & 592,779 & 592,779 \\
\hline & $\begin{array}{l}\text { Conceptual Englneering \& Deslgn @ } 5 \% \\
\text { of Construction }\end{array}$ & 1 & LS & & LMITCO & 0.000 & & & & & 296,389 & 296,389 \\
\hline \multirow[t]{4}{*}{ MEMO: } & $\begin{array}{l}\text { Tille I \&ill Engineering \& Deslgn @ 10\% } \\
\text { of Constructlon }\end{array}$ & 1 & LS & & LMITCO & 0.000 & & & & & 592,779 & 692,779 \\
\hline & $\begin{array}{l}\text { Conceptual Englineering \& Design @ 5\% } \\
\text { of Construction }\end{array}$ & 1 & $\overline{\text { LS }}$ & & LMITCO & 0.000 & & & & & 296,389 & 296,389 \\
\hline & 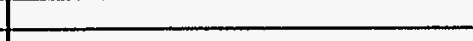 & & & & & & & & & & & 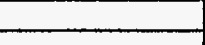 \\
\hline & DESIGN ENGINEERING S/T & & & & & & 0 & & & & $\$ 1,778,336$ & $\$ 1,778,336$ \\
\hline 1.1 .2 & $\frac{\text { TITLE III INSPECTION }}{\text { INSPECTION - TITLE III }}$ & 1 & LS & & LMITCO & 0.000 & & & & & 296,389 & 296,389 \\
\hline \multirow[t]{3}{*}{ MEMO: } & Tille III @ 5\% of Construclion & & & & & & & & & & & \\
\hline & & & & & & & & & & & & -2 \\
\hline & TITLE III INSPECTION SIT & & & & & & 0 & & & & $\$ 296,389$ & $\$ 296,389$ \\
\hline 1.2 .1 & $\begin{array}{l}\text { PROJECT MANAGEMENT } \\
\text { PROJECT MANAGEMENT }\end{array}$ & 1 & LOT & & LMITCO & 0.000 & & & & & 692,779 & 592,779 \\
\hline \multirow[t]{3}{*}{ MEMO: } & PM @ $10 \%$ of Construction & & & & & & & & & & & \\
\hline & G\&A & 1 & $\mathbf{L S}$ & & LMITCO & 0.000 & & & & & $1,177,541$ & $1,177,641$ \\
\hline & PIF & 1 & LS & & LMITCO & 0.000 & & & & & 397,379 & 397,379 \\
\hline & & & & & & & & & & & & \\
\hline & & & & & & & & & & & & \\
\hline
\end{tabular}


Lockheed Martin Idaho Technologies Co.

PROJECT NAME: ICPP Bin Set Closure (EIS Stud

LOCATION 1: ICPP

REQUESTOR: Bryan Spaulding
CONTINGENCY ANALYSIS

TYPE OF ESTIMATE:

Planning

2423-E-E2

S. L. Coward
DATE: 28-Jan-1998

TIME: 12:18:16

REPORT NAME: Contingency Analysis

\begin{tabular}{|c|c|c|c|c|c|c|c|c|c|c|c|}
\hline \multicolumn{9}{|c|}{ PROBABLE \% VARIATION } & \multicolumn{2}{|c|}{$\begin{array}{c}\text { PROJECT } \\
\text { CONTINGENCY }\end{array}$} & \multirow{3}{*}{$\begin{array}{l}\text { SUMMARY } \\
\text { Total Cost } \\
\text { by Element }\end{array}$} \\
\hline \multirow[t]{2}{*}{$\begin{array}{l}\text { WBS } \\
\text { Element }\end{array}$} & \multirow[t]{2}{*}{ Cost Estimate Element } & \multirow{2}{*}{$\begin{array}{c}\text { Total Cost w/o } \\
\text { Contingency }\end{array}$} & \multirow[t]{2}{*}{$\begin{array}{l}\text { \% Total } \\
\text { Cost }\end{array}$} & \multicolumn{2}{|c|}{$\begin{array}{c}\text { Prob. } \% \text { Var. } \\
\text { From Est. }\end{array}$} & \multicolumn{2}{|c|}{ Wt. \% of Prob. } & \multirow{2}{*}{ Contingency } & \multirow[t]{2}{*}{$\%$} & \multirow{2}{*}{ Cost } & \\
\hline & & & & - & + & - & + & & & & \\
\hline 1.1 .1 & DESIGN ENGINEERING & $1,778,336-$ & 8.60 & 10 & 40 & 0.86 & 3.44 & $3.010 \%$ & $8.60 \%$ & 620,866 & $2,399,202$ \\
\hline 1.1 .2 & TITLE III INSPECTION & 296,389 & 1.43 & 10 & 40 & 0.14 & 0.57 & $0.502 \%$ & $1.43 \%$ & 103,478 & 399,867 \\
\hline 1.2 .1 & PROJECT MANAGEMENT & $2,167,699$ & 10.48 & 10 & 40 & 1.05 & 4.19 & $3.669 \%$ & $10.48 \%$ & 756,803 & $2,924,502$ \\
\hline 1.22 & CONSTRUCTION MANAGEMENT & 592,779 & 2.87 & 10 & 40 & 0.29 & 1.15 & $1.003 \%$ & $2.87 \%$ & 206,955 & 799,734 \\
\hline 1.3 .1 & GENERAL CONDITONS & $1,831,085$ & 8.85 & 10 & 40 & 0.89 & 3.54 & $3.099 \%$ & $8.85 \%$ & 639,282 & $2,470,367$ \\
\hline 1.3 .13 & SPECIAL CONSTRUCTION & $4,096,709$ & 19.81 & 10 & 40 & 1.98 & 7.92 & $6.933 \%$ & $19.81 \%$ & $1,430,274$ & $5,526,983$ \\
\hline \multirow[t]{2}{*}{1.5 .2} & PROCUREMENT FEES & 59,278 & 0.29 & 10 & 40 & 0.03 & 0.11 & $0.100 \%$ & $0.29 \%$ & 20,696 & 79,974 \\
\hline & ESCALATION & $9,857,756$ & 47.67 & 10 & 40 & 4.77 & 19.07 & $16.684 \%$ & $47.67 \%$ & $3,441,615$ & $13,299,371$ \\
\hline \multicolumn{2}{|r|}{ SUBTOTAL } & $20,680,031$ & 100.00 & & & & & $35.000 \%$ & & & \\
\hline \multicolumn{2}{|r|}{ CALCULATED CONTINGENCY } & $7,238,011$ & & & & & & & & & \\
\hline \multicolumn{2}{|r|}{ RESULTANT TEC } & $27,918,042$ & & & & & & & & & \\
\hline \multicolumn{2}{|r|}{ ROUNDED TEC } & $27,900,000$ & & & & & & & & & \\
\hline \multicolumn{2}{|r|}{ PROJECT CONTINGENCY } & $7,219,969$ & & & & & & $34.91 \%$ & & & \\
\hline \multicolumn{2}{|r|}{ MANAGEMENT RESERVE } & $1,209,388$ & & & & & & & & & \\
\hline \multicolumn{2}{|r|}{ CONTINGENCY } & $6,010,581$ & & & & & & & & & \\
\hline \multicolumn{2}{|r|}{ TOTAL ESTIMATED COST } & $27,900,000$ & & & & & & & & $7,219,969$ & $27,900,000$ \\
\hline
\end{tabular}

CONFIDENCE LEVEL AND ASSUMED RISKS:

The Lockheed Idaho Technologies Co. Cost Estimate Contingency Analysis Model is based on the applied contingency and the assumptions upon which the estimate was predicated. The model is applied with a suggested risk level of $18 \%$ and a level of confidence of $90 \%$ the estimate will fall within the bid range. The Contingency Analysis is based on a weighted average to provide a

$90 \%$ probability of underrun and a $10 \%$ probability of overrun.
CONTINGENCY ANALYSIS GUIDE BY TYPE OF ESTIMATE

Guidelines established by DOE/FM 50 . Cost Estimating Guide, Vol. 6 , Cost Guide, and as presented in the INEL Cost Estimating Guide. PLANNING $20 \%-30 \%$ Experimental/Special Conditions............. Up to $50 \%$ Conceptual ExperimentalSpecial Conditions............Up to $40 \%$ TITLE I

TITLE ॥

TITLE IUAFC
$10 \%-20 \%$

$5 \%-15 \%$

Market Conditions 


\section{G\&APIF ADDER CALCULATION SHEET \\ ICPP BIN SET CLOSURE \\ RISK BASED CLEAN CLOSURE; CLEAN FILL; ESCALATED \\ CLEAN BINS WI ROBOTS - PIPING}

PROCUREMENT FEE:

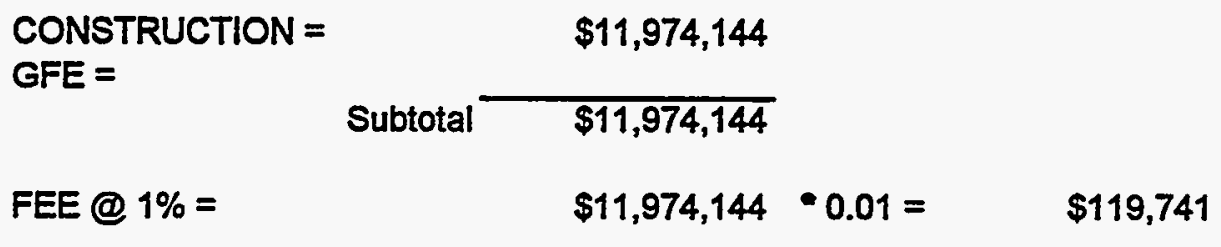

G\&A @ 23\% (with a ceiling of $\$ 500,000$ imposed per year, 10 yrs)

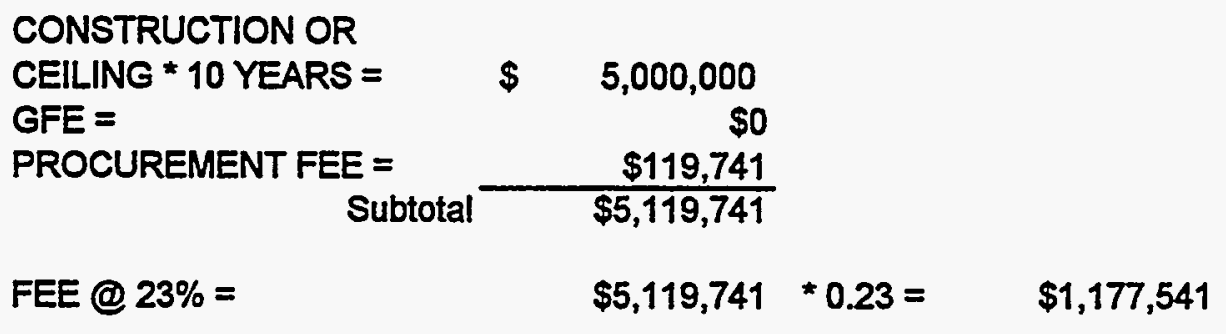

PIF @ 5.5\%

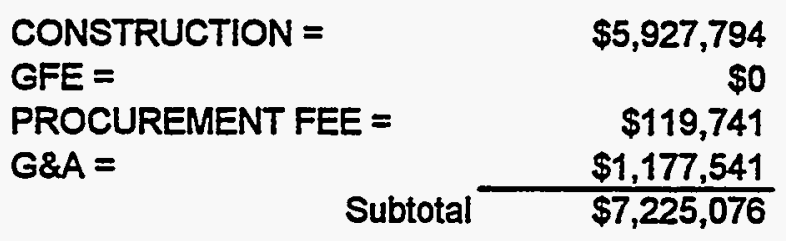

FEE @ 5.5\% =

$\$ 7,225,076 \bullet 0.055=$

$\$ 397,379$

TOTAL PROCUREMENT FEE:

$\$ 119,741$

TOTAL G\&A FEE:

$\$ 1,177,541$

TOTAL PIF:

$\$ 397,379$ 
Lockheed Martin Idaho Technologies Co. Rev. 6-96 PROJECT NAME: ICPP Bin Set Closure (EIS Stud LOCATION 1: REQUESTOR: Place Clean Grout in Bins ICPP

Bryan Spaulding

COST ESTIMATE SUMMARY

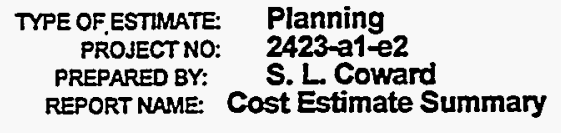

APPR'D BY:

\begin{tabular}{|c|c|c|c|c|}
\hline $\begin{array}{l}\text { WBS } \\
\text { Element }\end{array}$ & Cost Estimate Element & $\begin{array}{c}\text { Total } \\
\text { Unescalated }\end{array}$ & Escalation & $\begin{array}{c}\text { Total } \\
\text { Incl Escalation } \\
\end{array}$ \\
\hline $\begin{array}{l}\frac{1.1}{1.1 .1} \\
1.1 .2 \\
1.2 \\
1.2 .1 \\
1.2 .2 \\
1.3 \\
1.3 .1 \\
1.3 .2 \\
1.3 .3 \\
1.5 .2\end{array}$ & $\begin{array}{l}\text { ENGINEERING, DESIGN AND INSPECTION } \\
\text { DESIGN ENGINEERING } \\
\text { TITLE III INSPECTION } \\
\text { MANAGEMENT COSTS } \\
\text { PROJECT MANAGEMENT } \\
\text { CONSTRUCTION MANAGEMENT } \\
\text { CONSTRUCTION } \\
\text { GENERAL CONDITIONS } \\
\text { SITEWORK } \\
\text { CONCRETE } \\
\text { PROCUREMENT FEES }\end{array}$ & $\begin{array}{r}1,547,020 \\
658,793 \\
\\
1,067,791 \\
229,119 \\
5,291,021 \\
65,879\end{array}$ & $\begin{array}{c}1,577,960 \\
704,909 \\
\\
1,142,536 \\
245,157 \\
5,661,393 \\
70,491\end{array}$ & 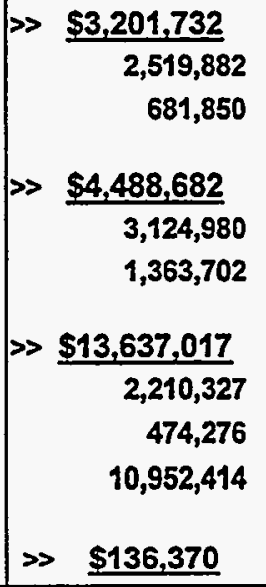 \\
\hline & $\begin{array}{l}\text { SUBTOTAL INCLUDING ESCALATION } \\
\text { PROJECT CONTINGENCY }\end{array}$ & $10,836,001$ & $10,627,800$ & $\gg \$ \$ 21,463,801$ \\
\hline & MANAGEMENT RESERVE- & & & $\$ 1,377,339$ \\
\hline & CONTINGENCY - & & & $\$ 6,158,860$ \\
\hline & TOTAL ESTIMATED COST - & & & $\gg \$ 29,000,000$ \\
\hline
\end{tabular}

\section{PROJECT COST PARAMETERS}

EDI AS A \% OF CONST. + GFE $=23.00 \%$ 
Lockheed Martin Idaho Technologies Co.

Rav 6-90

PROJECT NAME: ICPP BIn Sot Closure (EIS Study)

Place Clean Grout in Bins

LOCATION 1: ICPP

REQUESTOR: Bryan Spaulding

\section{DETAILED COST ESTIMATE SHEET}

TYPE OF ESTIMATE: Planning PROJECT NO.: 2423-a1-e2 PREPARED BY: S. L. Coward
PAGE \# 3

DATE 28-Jan-1998

TIME: 12:05:06

REPORT NAME: Detall Cost Estimate She日t

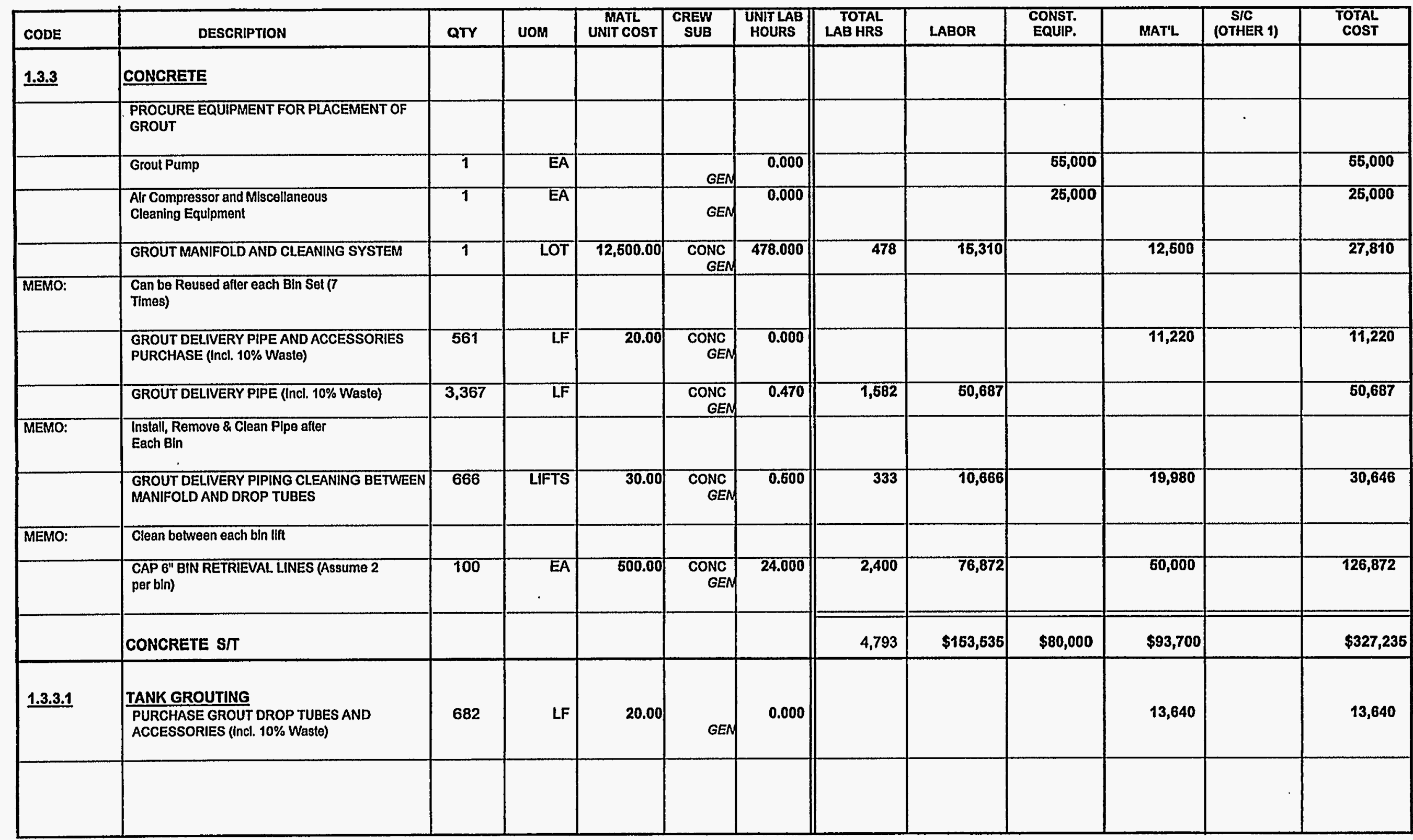


Lockheed Martin Idaho Technologies Co. Rev6-96 PROJECT NAME: ICPP Bin Set Closure (EIS Stud Place Clean Grout in Bins

LOCATION 1: REQUESTOR:
ICPP

Bryan Spaulding
CONTINGENCY ANALYSIS
DATE: 28-Jan-1998

TIME: 12:05:01

REPORT NAME: Contingency Analysis

\begin{tabular}{|c|c|c|c|c|c|c|c|c|c|c|c|}
\hline \multirow{3}{*}{$\begin{array}{l}\text { WBS } \\
\text { Element }\end{array}$} & \multicolumn{8}{|c|}{ PROBABLE \% VARIATION } & \multicolumn{2}{|c|}{$\begin{array}{l}\text { PROJECT } \\
\text { CONTINGENCY }\end{array}$} & \multirow{3}{*}{$\begin{array}{l}\text { SUMMARY } \\
\begin{array}{l}\text { Total Cost } \\
\text { by Element }\end{array}\end{array}$} \\
\hline & \multirow{2}{*}{ Cost Estimate Element } & \multirow{2}{*}{$\begin{array}{l}\text { Total Cost w/o } \\
\text { Contingency }\end{array}$} & \multirow{2}{*}{$\begin{array}{l}\% \text { Total } \\
\text { Cost }\end{array}$} & \multicolumn{2}{|c|}{$\begin{array}{c}\text { Prob. \% Var. } \\
\text { From Est. }\end{array}$} & \multicolumn{2}{|c|}{ Wt \% of Prob. } & \multirow{2}{*}{ Contingency } & \multirow{2}{*}{$\%$} & \multirow{2}{*}{ Cost } & \\
\hline & & & & - & + & - & + & & & & \\
\hline 1.1.1 & DESIGN ENGINEERING & $1,646,982$ & 7.67 & 10 & 40 & 0.77 & 3.07 & $2.686 \%$ & $7.67 \%$ & 578,275 & $2,225,257$ \\
\hline 1.1 .2 & TITLE III INSPECTION & 329,396 & 1.53 & 10 & 40 & 0.15 & 0.61 & $0.537 \%$ & $1.53 \%$ & 115,655 & 445,051 \\
\hline 1.2 .1 & PROJECT MANAGEMENT & $1,547,020$ & 7.21 & 10 & 40 & 0.72 & 2.88 & $2.523 \%$ & $7.21 \%$ & 543,177 & $2,090,197$ \\
\hline 1.2 .2 & CONSTRUCTION MANAGEMENT & 658,793 & 3.07 & 10 & 40 & 0.31 & 1.23 & $1.074 \%$ & $3.07 \%$ & 231,310 & 890,103 \\
\hline 1.3 .1 & GENERAL CONDITIONS & $1,067,791$ & 4.97 & 10 & 40 & 0.50 & 1.99 & $1.741 \%$ & $4.97 \%$ & 374,914 & $1,442,705$ \\
\hline 1.3 .2 & SITEWORK & 229,119 & 1.07 & 10 & 40 & 0.11 & 0.43 & $0.374 \%$ & $1.07 \%$ & 80,446 & 309,565 \\
\hline 1.3 .3 & CONCRETE & $5,291,021$ & 24.65 & 10 & 40 & 2.47 & 9.86 & $8.628 \%$ & $24.65 \%$ & $1,857,741$ & $7,148,762$ \\
\hline \multirow[t]{2}{*}{1.5 .2} & PROCUREMENT FEES & 65,879 & 0.31 & 10 & 40 & 0.03 & 0.12 & $0.107 \%$ & $0.31 \%$ & 23,131 & 89,010 \\
\hline & ESCALATION & $10,627,800$ & 49.51 & 10 & 40 & 4.95 & 19.81 & $17.330 \%$ & $49.51 \%$ & $3,731,550$ & $14,359,350$ \\
\hline & SUBTOTAL & $21,463,801$ & 100.00 & & & & & $35.000 \%$ & & & \\
\hline & CALCULATED CONTINGENCY & $7,512,330$ & & & & & & & & & \\
\hline & RESULTANT TEC & $28,976,131$ & & & & & & & & & \\
\hline & ROUNDED TEC & $29,000,000$ & & & & & & & & & \\
\hline & PROJECT CONTINGENCY & $7,536,199$ & & & & & & $35.11 \%$ & & & \\
\hline & MANAGEMENT RESERVE & $1,377,339$ & & & & & & & & & \\
\hline & CONTINGENCY & $6,158,860$ & & & & & & & & & \\
\hline & TOTAL ESTIMATED COST & $29,000,000$ & & & & & & & & $7,536,199$ & $29,000,000$ \\
\hline
\end{tabular}

CONFIDENCE LEVEL AND ASSUMED RISKS:

The Lockheed Idaho Technologies Co. Cost Estimate Contingency Analysis Model is based on the applied contingency and the assumptions upon which the estimate was predicated. The model is applied with a suggested risk level of $18 \%$ and a level of confidence of $90 \%$ the estimate will fall within the bid range. The Contingency Analysis is based on a weighted average to provide a $90 \%$ probability of undertun and a $10 \%$ probability of ovemun.
CONTINGENCY ANALYSIS GUIDE BY TYPE OF ESTIMATE

Guidelines established by DOE/FM 50, Cost Estimating Guide, Vol. 6, Cost Guide, and as presented in the INEL Cost Estimating Guide. PLANNING

Experimental/Special Conditions Conceptual

Experimental/Special Conditions............. Up to $40 \%$ TITLE I

TITLE UI

TITLE IIAFC
$15 \%-25 \%$

$10 \%-20 \%$

$5 \%-15 \%$

Market Conditions 


\section{G\&A/PIF ADDER CALCULATION SHEET ICPP BIN SET CLOSURE \\ RISK BASED CLEAN CLOSURE; CLEAN FILL; ESCALATED}

FILL BINS WI NRC CLEAN GROUT

PROCUREMENT FEE:

CONSTRUCTION $=\quad \$ 13,637,017$

GFE =

Subtotal $\$ \$ 13,637,017$

FEE @ $1 \%=$

$\$ 13,637,017 * 0.01=$

$\$ 136,370$

G\&A @ 23\% (with a ceiling of $\$ 500,000$ imposed per year, 4 yrs)

\begin{tabular}{lr} 
CONSTRUCTION OR & \\
CEILING * 4 YEARS $=$ & $\$ 2,000,000$ \\
GFE $=$ & $\$ 0$ \\
PROCUREMENT FEE $=$ & $\$ 136,370$ \\
\hline & Subtotal
\end{tabular}

FEE @ $23 \%=$

$\$ 2,136,370 \cdot 0.23=$

$\$ 491,365$

PIF @ 5.5\%

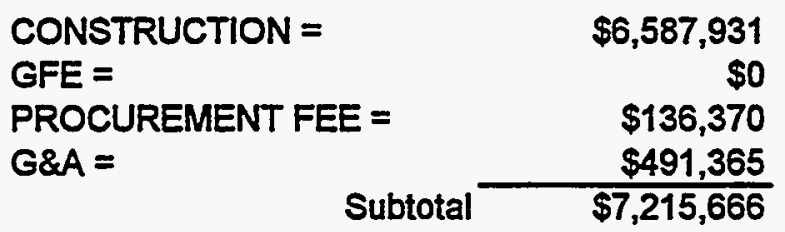

FEE @ 5.5\% =

$\$ 7,215,666 * 0.055=$

$\$ 396,862$

TOTAL PROCUREMENT FEE:

$\$ 136,370$

TOTAL G\&A FEE:

$\$ 491,365$

TOTAL PIF:

$\$ 396,862$ 
Lockheed Martin Idaho Technologies Co. PROJECT NAME: ICPP Bin Set Closure D\&D of Equipment

INEEL /ICPP

REQUESTOR: B. C. Spaulding

\section{COST ESTIMATE SUMMARY}

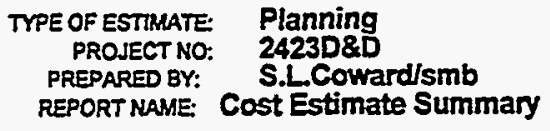

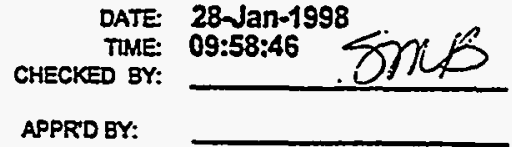

\begin{tabular}{|c|c|c|c|c|}
\hline $\begin{array}{l}\text { WBS } \\
\text { Element }\end{array}$ & Cost Estimate Element & $\begin{array}{c}\text { Total } \\
\text { Unescalated }\end{array}$ & Escalation & $\begin{array}{c}\text { Total } \\
\text { Incl Escalation }\end{array}$ \\
\hline 1.1 & ENGINEERING, DESIGN AND INSPECTION & & & so \\
\hline$\overline{1.1 .1}$ & DESIGN ENGINEERING TITLE I \& II & 0 & 0 & 0 \\
\hline 1.1.2 & TITLE III INSPECTION & & 0 & 0 \\
\hline 1.2 & MANAGEMENT COSTS & & & $\gg \$ 2,561,125$ \\
\hline 1.2 .1 & PROJECT MANAGEMENT & 868,178 & $1,692,947$ & $2,2,561,125$ \\
\hline 1.2 .2 & CONSTRUCTION MANAGEMENT & 0 & 0 & 0 \\
\hline 1.3 & CONSTRUCTION & & & $\gg \$ \$ 34,543,647$ \\
\hline$\overline{1.3 .13}$ & SPECIAL CONSTRUCTION & $11,709,711$ & $22,833,936$ & $34,543,647$ \\
\hline \multirow[t]{2}{*}{ 1.5.2 } & PROCUREMENT FEES & 117,097 & 228,339 & $\$ 345,436$ \\
\hline & $\begin{array}{l}\text { SUBTOTAL INCLUDING ESCALATION } \\
\text { PROJECT CONTINGENCY }\end{array}$ & $12,694,986$ & $24,755,222$ & $>\$ 37,450,208$ \\
\hline & MANAGEMENT RESERVE- & & & $\gg$ \\
\hline & CONTINGENCY- & & & $\gg \quad \$ 13,149,792$ \\
\hline & TOTAL ESTIMATED COST - & & & $\gg \quad \$ 50,600,000$ \\
\hline
\end{tabular}

PROJECT COST PARAMETERS

EDI AS A \% OF CONST. + GFE $=0.00 \%$ CONTINGENCY $=35.11 \%$ 

Lockheed Martin Idaho Technologies Co.

PROJECT NAME: ICPP Bin Set Closure

D\&D of Equipment

INEEL IICPP

LOCATION 1:

B. C. Spaulding
CONTINGENCY ANALYSIS

TYPE OF ESTIMATE:

PREPARED BY: S.L.Coward/smb
DATE: 28-Jan-1998

TWME: 09:58:43

REPORT NAME: Contingency Analysis

\begin{tabular}{|c|c|c|c|c|c|c|c|c|c|c|c|}
\hline \multicolumn{9}{|c|}{ PROBABLE \% VARIATION } & \multicolumn{2}{|c|}{$\begin{array}{c}\text { PROJECT } \\
\text { CONTINGENCY }\end{array}$} & \multirow{3}{*}{$\begin{array}{l}\text { SUMMARY } \\
\begin{array}{l}\text { Total Cost } \\
\text { by Eement }\end{array}\end{array}$} \\
\hline \multirow{2}{*}{$\begin{array}{l}\text { WBS } \\
\text { Element }\end{array}$} & \multirow{2}{*}{ Cost Estimate Element } & \multirow{2}{*}{$\begin{array}{l}\text { Total Cost w/o } \\
\text { Contingency }\end{array}$} & \multirow[t]{2}{*}{$\begin{array}{l}\text { \% Total } \\
\text { Cost }\end{array}$} & \multicolumn{2}{|c|}{$\begin{array}{c}\text { Prob. \% Var. } \\
\text { From Est. }\end{array}$} & \multicolumn{2}{|c|}{ Wt. $\%$ of Prob. } & \multirow{2}{*}{ Contingency } & \multirow{2}{*}{$\%$} & \multirow{2}{*}{ Cost } & \\
\hline & & & & - & + & - & + & & & & \\
\hline 1.1 .1 & DESIGN ENGINEERING TITLE 1 \& \| & 0 & 0.00 & 0 & 0 & 0.00 & 0.00 & $0.000 \%$ & $0.00 \%$ & 0 & 0 \\
\hline 1.12 & TITLE III INSPECTION & 0 & 0.00 & 0 & 0 & 0.00 & 0.00 & $0.000 \%$ & $0.00 \%$ & 0 & 0 \\
\hline 1.21 & PROJECT MANAGEMENT & 868,178 & 232 & 10 & 40 & 0.23 & 0.93 & $0.811 \%$ & $232 \%$ & 304,841 & $1,173,019$ \\
\hline 1.2 .2 & CONSTRUCTON MANAGEMENT & 0 & 0.00 & 0 & 0 & 0.00 & 0.00 & $0.000 \%$ & $0.00 \%$ & 0 & 0 \\
\hline$\overline{1.3 .13}$ & SPECLAL CONSTRUCTION & $11,709,711$ & 31.27 & 10 & 40 & 3.13 & 12.51 & $10.944 \%$ & $31.27 \%$ & $4,111,680$ & $15,821,311$ \\
\hline \multirow[t]{2}{*}{1.5 .2} & PROCUREMENT FEES & 117,097 & 0.31 & 10 & 40 & 0.03 & 0.13 & $0.109 \%$ & $0.31 \%$ & 41,116 & 158,213 \\
\hline & ESCALATION & $24,755,222$ & 66.10 & 10 & 40 & 6.61 & 26.44 & $23.136 \%$ & $66.10 \%$ & $8,692,235$ & $33,447,457$ \\
\hline \multicolumn{2}{|r|}{ SUBTOTAL } & $37,450,208$ & 100.00 & & & & & $35.000 \%$ & & & \\
\hline \multicolumn{2}{|r|}{ CALCULATED CONTINGENCY } & $13,107,573$ & & & & & & & & & \\
\hline \multicolumn{2}{|r|}{ RESULTANT TEC } & $50,557,781$ & & & & & & & & & \\
\hline \multicolumn{2}{|r|}{ ROUNDED TEC } & $50,600,000$ & & & & & & & & & \\
\hline \multicolumn{2}{|r|}{ PROJECT CONTINGENCY } & $13,149,792$ & & & & & & $35.11 \%$ & & & \\
\hline \multicolumn{2}{|r|}{ MANAGEMENT RESERVE } & 0 & & & & & & . & & & \\
\hline \multicolumn{2}{|r|}{ CONTINGENCY } & $13,149,792$ & & & & & & & & & \\
\hline \multicolumn{2}{|r|}{ TOTAL ESTIMATED COST } & $50,600,000$ & & & & & & & & $13,149,792$ & $50,600,000$ \\
\hline
\end{tabular}

CONFIDENCE LEVEL AND ASSUMED RISKS:

The Lockheed Idaho Technologies Co. Cost Estimate Contingency Analysis Model is based on the applied contingency and the assumptions upon which the estimate was predicated. The model is applied with a suggested risk level of $18 \%$ and a level of confidence of $90 \%$ the estimate will fall within the bid range. The Contingency Analysis is based on a weighted average to provide a $90 \%$ probability of undermun and a $10 \%$ probability of overnun.
CONTINGENCY ANALYSIS GUIDE BY TYPE OF ESTIMATE

Guidelines established by DOE/FM 50 , Cost Estimating Guide, Vol. 6 ,

Cost Guide, and as presented in the INEL Cost Estimating Guide. PLANNING

Conceptual

Experimentalspecial Conditions

TITLE I

TITLE II

TITLE IIIAFC
$5 \%-25 \%$ Up to $40 \%$ $10 \%-20 \%$

$5 \%-15 \%$ Market Conditions 


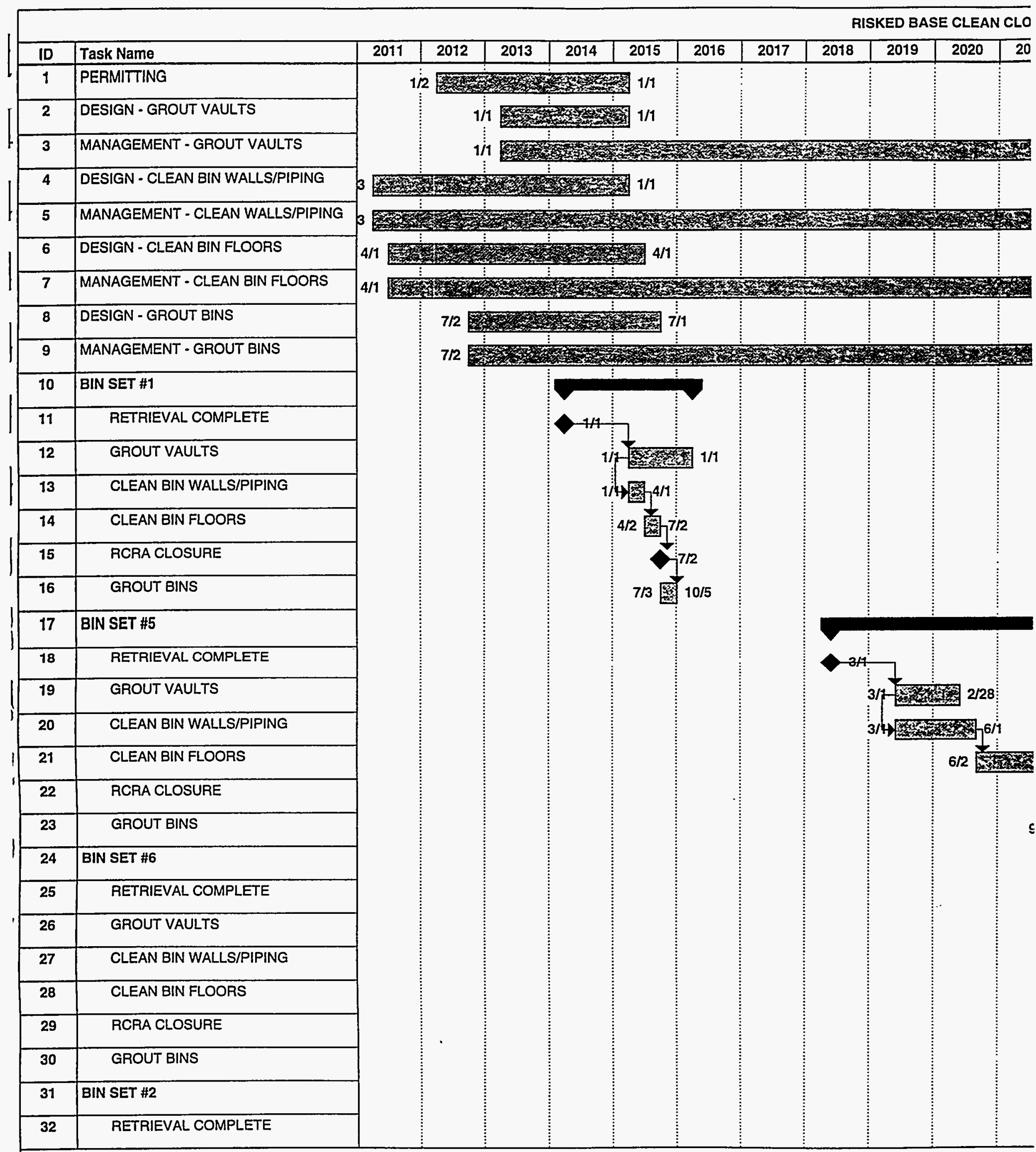




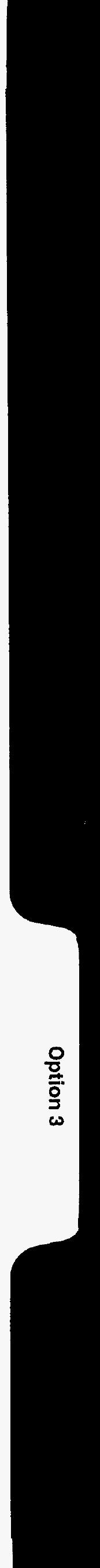


COST ESTIMATE SUMMARY

UNESCALATED

ICPP BIN SET CLOSURE CLOSURE TO RCRA LANDFILL STANDARDS; CLASS C FILL

Requestor: B. C. Spaulding
Planning Estimate

Estimate \#2423

Prepared by: S. L. Coward

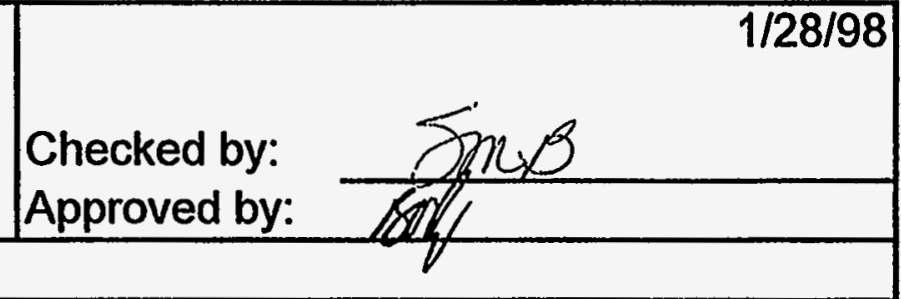

$19,500,000$

$12,400,000$

$82,600,000$

$30,100,000$

$17,000,000$

D\&D of Equipment
TOTAL

Floor

Fill Vaults with Clean Grout

Clean Bins with Robots

Fill Bins with NRC Class C Grout

-

\begin{tabular}{cc|} 
& $19,500,000$ \\
& $12,400,000$ \\
& \\
& $82,600,000$ \\
& $30,100,000$ \\
& $17,000,000$ \\
\hline & $\$ 161,600,000$ \\
USE & $\$ 162,000,000$ \\
\hline
\end{tabular}


RCRA CLOSURE TO LANDFILL STANDARDS - NRC CLASS C GROUT

DESCRIPTION

BIN SET \#1

BIN SET *5

BIN SET \#7

BIN SET \#6

BIN SET \#3

BIN SET \#4 BIN SET \#2

Schoduled Complotion

$(1 / 1 / 14)$

(4/1/18)

$(12 / 1 / 22)$

$(11 / 1 / 26)$

$(8 / 1 / 28)$

$(2 / 1 / 31)$

$(5 / 1 / 31)$

ASSUME: Walt 6 Months until Start of Closure

\section{Pormitting (5 Years)}

$7 / 1 / 9-7 / 1 / 14$

Grout Vaults "Clean" (Assumo 1 Yoar)

\begin{tabular}{ll}
\hline ED\&I (2 Yrs) & $7 / 1 / 12-7 / 1 / 14$ \\
Management & $7 / 1 / 12-11 / 1 / 32$
\end{tabular}

Construction

$7 / 1 / 14-7 / 1 / 15$

$10 / 1 / 18-10 / 1 / 19 \quad 6 / 1 / 23-6 / 1 / 24$

$5 / 1 / 27-5 / 1 / 28$

$2 / 1 / 29-2 / 1 / 30$

$8 / 1 / 31-8 / 1 / 32 \quad 11 / 1 / 31-11 / 1 / 32$

Clean Bin Floors with Robot

ED\&l (4 Yrs)

(3 Months)

(15 Months)

(25 Months)

(24 Months)

(17 Months)

(7 Months)

(19 Months)

Management $7 / 1 / 10-12 / 1 / 32$

Construction

$7 / 1 / 14-10 / 1 / 14$

$10 / 1 / 18-1 / 1 / 20$

$6 / 1 / 23-7 / 1 / 25$

$5 / 1 / 27-5 / 1 / 29$

$2 / 1 / 29-7 / 1 / 30$

$8 / 1 / 31-3 / 1 / 32$

$11 / 1 / 31-12 / 1 / 32$

\section{RCRA CLOSURE}

\begin{tabular}{|c|c|c|c|c|c|c|c|c|}
\hline Grout Bins "NAC Class C" & & (3 Months) & (11 Months) & (18 Months) & (17 Months) & (12 Months) & (6 Months) & (10 Months) \\
\hline ED\&l (3 Yrs) & $1 / 1 / 21-1 / 1 / 24$ & & & & & & & \\
\hline Management & $1 / 1 / 21-10 / 1 / 33$ & & & & & & & \\
\hline Construction & & $1 / 1 / 24-4 / 1 / 24$ & $4 / 1 / 24-3 / 1 / 25$ & $7 / 1 / 25-1 / 1 / 27$ & $5 / 1 / 29-10 / 1 / 30$ & $10 / 1 / 30-10 / 1 / 31$ & $3 / 1 / 32-9 / 1 / 32$ & $12 / 1 / 32-10 / 1 / 33$ \\
\hline
\end{tabular}

\section{ASSUMPTIONS:}

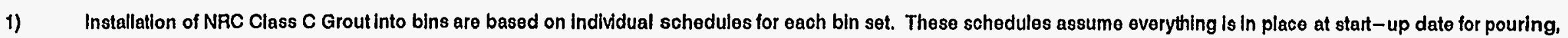
and allows no flexiblity for error or downtime.

2) More than 1 crew could be utllized slmultaneously for pouring the "clean" grout into the vaults.

3) More than 1 crew could be utllized simultaneously for cleaning the separate bins.

4) Installation of "clean" grout into vaults will average 1 year per vault.

5) Cleaning of bin floors are based on individual bln pro-rated calcine retrieval volumes to total volume. These schedules assume mob/demob, Installatlon of robotlo. units, and any modifications of bins will be completed and blns will be ready for retrieval. 
Lockheed Martin Idaho Technologies Co. Rov, 6-96 PROJECT NAME: Permitting/Documentation Risk Based - NRC Class C INEELICPP

LOCATION 1: REQUESTOR:

Bryan Spaulding
COST ESTIMATE SUMMARY

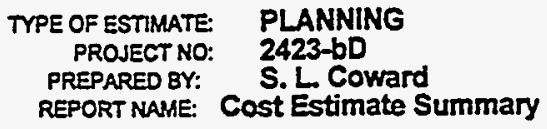

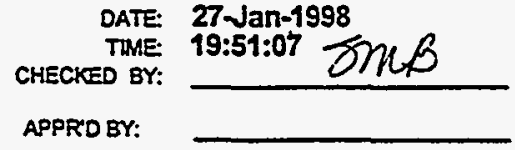

DATE: 27 Jan-1998

APPROD BY:

\begin{tabular}{|c|c|c|c|c|}
\hline $\begin{array}{l}\text { WBS } \\
\text { Element }\end{array}$ & Cost Estimate Element & $\begin{array}{l}\text { Total } \\
\text { Unescalated }\end{array}$ & Escalation & $\begin{array}{c}\text { Total } \\
\text { Incl Escalation }\end{array}$ \\
\hline$\frac{1.1}{1.1}$ & CONCEPTUAL & 0 & 0 & $\gg \quad$ \$O \\
\hline & MANAGEMENT & & & \multirow{6}{*}{$\begin{array}{r}\gg>\frac{\$ 2,374,382}{1,896,534} \\
477,848 \\
\end{array}$} \\
\hline$\overline{1.2 .1}$ & PM FOR PROJECT DEVELOPMENT & $1,896,534$ & 0 & \\
\hline 1.2 .2 & PROJECT EXECUTION & 477,848 & 0 & \\
\hline 1.3 & PERMITIING & & & \\
\hline$\overline{1.3 .1}$ & PERMITTING & $11,946,206$ & 0 & \\
\hline 1.5.2 & PROCUREMENT FEES & 119,462 & $\mathbf{0}$ & \\
\hline & $\begin{array}{l}\text { SUBTOTAL INCLUDING ESCALATION } \\
\text { PROJECT CONTINGENCY }\end{array}$ & $14,440,050$ & 0 & $\gg \$ \$ 14,440,050$ \\
\hline & MANAGEMENT RESERVE- & & & $\$ 1,206,567$ \\
\hline & CONTINGENCY- & & & $\gg \quad \$ 3,853,383$ \\
\hline & TOTAL ESTIMATED COST - & & & $\gg \$ 19,500,000$ \\
\hline
\end{tabular}

\section{PROJECT COST PARAMETERS}

EDI AS A \% OF CONST. + GFE $=0.00 \%$ 
Lockheed Martin Idaho Technologies Co. Rev 6.96

PROJECT NAME: Pormitting/Documentation

Risk Based - NRC Class C

LOCATION 1: INEELICPP

REQUESTOR: Bryan Spaulding
DETAILED COST ESTIMATE SHEET

TYPE OF ESTIMATE: PLANNING

PROJECT NO.: 2423-bD

PREPARED BY: S. L. Coward
PAgE \# 3

DATE 27-Jan-1998

TIME: 19:51:09

REPORT NAME: DetaIl Cost Estimate Sheet

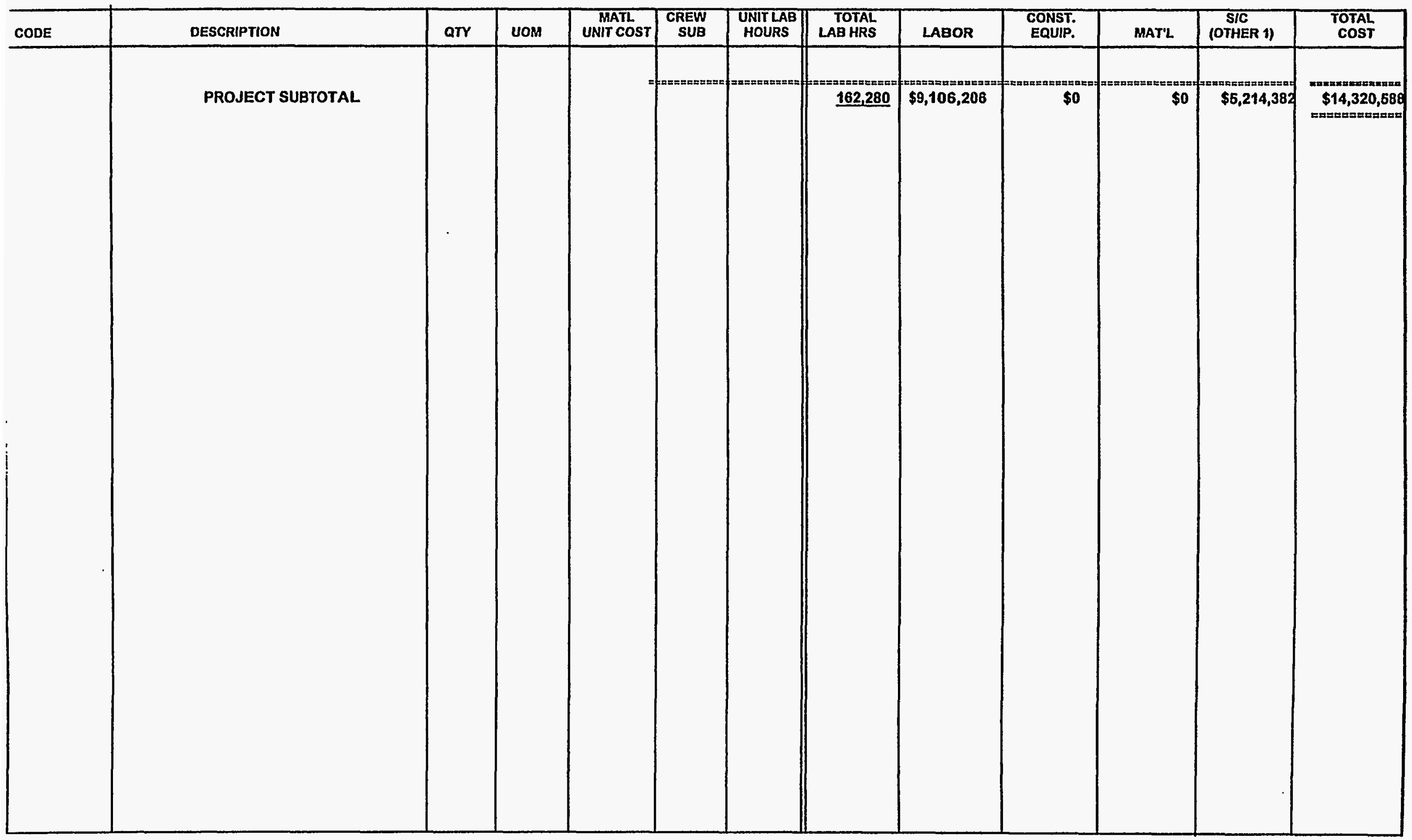


Lockheed Martin Idaho Technologies Co.

Rev $6-96$

PROJECT NAME: Permitting/Documentation

Risk Based - NRC Class C

LOCATION 1:

REOUESTOR:
CONTINGENCY ANALYSIS
PLANNING

2423-bD

S. L Coward
DATE. 27-Jan-1998

TIME: 19:51:03

REPORT NAME: Contingency Analysis

\begin{tabular}{|c|c|c|c|c|c|c|c|c|c|c|c|}
\hline & PROE & BLE \% VARIA & $10 N$ & & & & & & $\begin{array}{l}\text { PRC } \\
\text { CONT }\end{array}$ & $\begin{array}{l}\text { OT } \\
\text { IENCY }\end{array}$ & SUMMARY \\
\hline $\begin{array}{l}\text { WBS } \\
\text { Element }\end{array}$ & Cost Estimate Element & Total Cost w/o & $\begin{array}{l}\% \text { Total } \\
\text { Cost }\end{array}$ & & $\begin{array}{l}\% \text { Var. } \\
\text { m Est. }\end{array}$ & Wt $\%$ & Prob. & Contingency & $\%$ & Cost & Total Cost \\
\hline & & Contingency & & - & + & - & + & & & & by Element \\
\hline 1.1.1 & CONCEPTUAL DESIGN & $\mathbf{0}$ & 0.00 & 10 & 40 & 0.00 & 0.00 & $0.000 \%$ & $0.00 \%$ & 0 & 0 \\
\hline 1.2 .1 & PM FOR PROJECT DEVELOPMENT & $1,896,534$ & 13.13 & 10 & 40 & 1.31 & 5.25 & $4.597 \%$ & $13.13 \%$ & 684,566 & $2,561,100$ \\
\hline 1.22 & PROJECT EXECUTION & 477,848 & 3.31 & 10 & 40 & 0.33 & 1.32 & $1.158 \%$ & $3.31 \%$ & 167,443 & 645,291 \\
\hline 1.3 .1 & PERMITIING & $11,946,206$ & 82.73 & 10 & 40 & 8.27 & 33.09 & $28.955 \%$ & $82.73 \%$ & $4,186,080$ & $16,132,286$ \\
\hline 1.5 .2 & PROCUREMENT FEES & 119,462 & 0.83 & 10 & 40 & 0.08 & 0.33 & $0.290 \%$ & $0.83 \%$ & 41,861 & 161,323 \\
\hline & ESCALATTON & 0 & 0.00 & 0 & 0 & 0.00 & 0.00 & $0.000 \%$ & $0.00 \%$ & 0 & 0 \\
\hline & SUBTOTAL & $14,440,050$ & 100.00 & & & & & $35.000 \%$ & & & \\
\hline & CALCULATED CONTINGENCY & $5,054,017$ & & & & & & & & & \\
\hline & RESULTANT TEC & $19,494,057$ & & & & & & & & & - \\
\hline & ROUNDED TEC & $19,500,000$ & & & & & & & & & \\
\hline & PROJECT CONTINGENCY & $5,059,950$ & & & & & & $35.04 \%$ & & & \\
\hline & MANAGEMENT RESERVE & $1,206,567$ & & & & & & & & & \\
\hline & CONTINGENCY & $3,853,383$ & & & & & & & & & \\
\hline & TOTAL ESTIMATED COST & $19,500,000$ & & & & & & & & $5,059,950$ & $19,500,000$ \\
\hline
\end{tabular}

CONFIDENCE LEVEL AND ASSUMED RISKS:

The Lockheed Idaho Technologies Co. Cost Estimate Contingency Analysis

Model is based on the applied contingency and the assumptions upon which

the estimate was predicated. The model is applied with a suggested risk level

of $18 \%$ and a level of confidence of $90 \%$ the estimate will fall within the bid range.

The Contingency Analysis is based on a weighted average to provide a

$90 \%$ probability of underrun and a $10 \%$ probability of overrun.
CONTINGENCY ANALYSIS GUIDE BY TYPE OF ESTIMATE

Guidelines established by DOEIFM 50, Cost Estimating Guide, Vol. 6 ,

Cost Guide, and as presented in the INEL Cost Estimating Guide.

PLANNING

ExperimentauSpecial Conditions.

$20 \%-30 \%$

Conceptual

$15 \%-25 \%$

TITLE

TITLE II

TITLE IIIAFC
....Up to $40 \%$

$10 \%-20 \%$

Market Conditions 


\section{G\&A/PIF ADDER CALCULATION SHEET \\ ICPP BIN SET CLOSURE \\ CLOSURE TO RCRA LANDFILL STANDARDS; CLASS C FILL; UNESCALATED \\ REGULATORY COMPLIANCE}

PROCUREMENT FEE:

CONSTRUCTION $=$
GFE $=$ Subtotal $\frac{\$ 11,946,206}{\$ 11,946,206}$

FEE @ $1 \%=\quad \$ 11,946,206 \cdot 0.01=\quad \$ 119,462$

G\&A @ 23\% (with a ceiling of $\$ 500,000$ imposed per year , 5 Years)

CONSTRUCTION OR

CEILING * \# OF YEARS $=\quad \$ 2,500,000$

GFE =

PROCUREMENT FEE $=$ $\$ 119,462$

Subtotal $\$ 2,619,462$

FEE @ $23 \%=$

$\$ 2,619,462 \cdot 0.23=$

$\$ 602,476$

PIF @ 5.5\%

CONSTRUCTION =

GFE =

PROCUREMENT FEE =

G\&A =

FEE @ $5.5 \%=$
$\$ 11,946,206$

$\$ 0$

$\$ 119,462$

$\$ 602,476$

$\$ 12,668,144$

$\$ 12,668,144 * 0.055=$

$\$ 696,748$

$\$ 119,462$

$\$ 602,476$

TOTAL G\&A FEE:

$\$ 696,748$

TOTAL PIF:

$\begin{array}{ll}\text { Subtotal } & \$ 12,668,144 \\ & \$ 12,668,144 * 0.055=\quad \$ 696,748\end{array}$

TOTAL PROCUREMENT FEE:

$\$ 69,748$ 
Lockheed Martin Idaho Technologies Co. Rov. 6 -s6

PROJECT NAME: ICPP Bin Set Closure (EIS Stud

LOCATION 4: Place Clean Grout in Vault ICPP

REQUESTOR: Bryan Spaulding

\section{COST ESTIMATE SUMMARY}

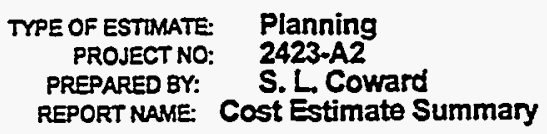

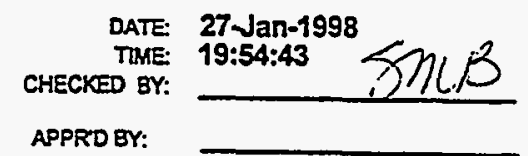

\begin{tabular}{|c|c|c|c|c|}
\hline $\begin{array}{l}\text { WBS } \\
\text { Element }\end{array}$ & Cost Estimate Element & $\begin{array}{c}\text { Total } \\
\text { Unescalated }\end{array}$ & Escalation & $\begin{array}{c}\text { Total } \\
\text { Incl Escalation }\end{array}$ \\
\hline $\begin{array}{l}\frac{1.1}{1.1 .1} \\
1.1 .2 \\
\frac{1.2}{1.2 .1} \\
1.2 .2 \\
1.3 \\
1.3 .1 \\
1.3 .2 \\
1.3 .3 \\
1.5 .2\end{array}$ & $\begin{array}{l}\text { ENGINEERING, DESIGN AND INSPECTION } \\
\text { DESIGN ENGINEERING } \\
\text { TITLE III INSPECTION } \\
\text { MANAGEMENT COSTS } \\
\text { PROJECT MANAGEMENT } \\
\text { CONSTRUCTION MANAGEMENT } \\
\text { CONSTRUCTION } \\
\text { GENERAL CONDITIONS } \\
\text { SITEWORK } \\
\text { CONCRETE } \\
\text { PROCUREMENT FEES }\end{array}$ & $\begin{array}{r}1,324,812 \\
264,962 \\
\\
\\
1,686,432 \\
529,925 \\
\\
\\
1,067,791 \\
0 \\
0 \\
4,231,459 \\
52,992\end{array}$ & $\begin{array}{l}0 \\
0 \\
0 \\
0 \\
0\end{array}$ & $\begin{array}{r}\gg \frac{\$ 1,589,774}{1,324,812} \\
264,962 \\
\\
\gg \frac{\$ 2,216,357}{1,686,432} \\
529,925 \\
\\
>\frac{\$ 5,299,250}{1,067,791} \\
0 \\
4,231,459\end{array}$ \\
\hline & $\begin{array}{l}\text { SUBTOTAL INCLUDING ESCALATION } \\
\text { PROJECT CONTINGENCY }\end{array}$ & $9,158,373$ & 0 & $\gg>\$ 9,158,373$ \\
\hline & MANAGEMENT RESERVE- & & & $\$ 535,224$ \\
\hline & CONTINGENCY - & & & $\$ 2,706,403$ \\
\hline & TOTAL ESTIMATED COST - & & & $\gg \quad \$ 12,400,000$ \\
\hline
\end{tabular}

\section{PROJECT COST PARAMETERS}

EDI AS A $\%$ OF CONST. + GFE $=30.00 \%$ 


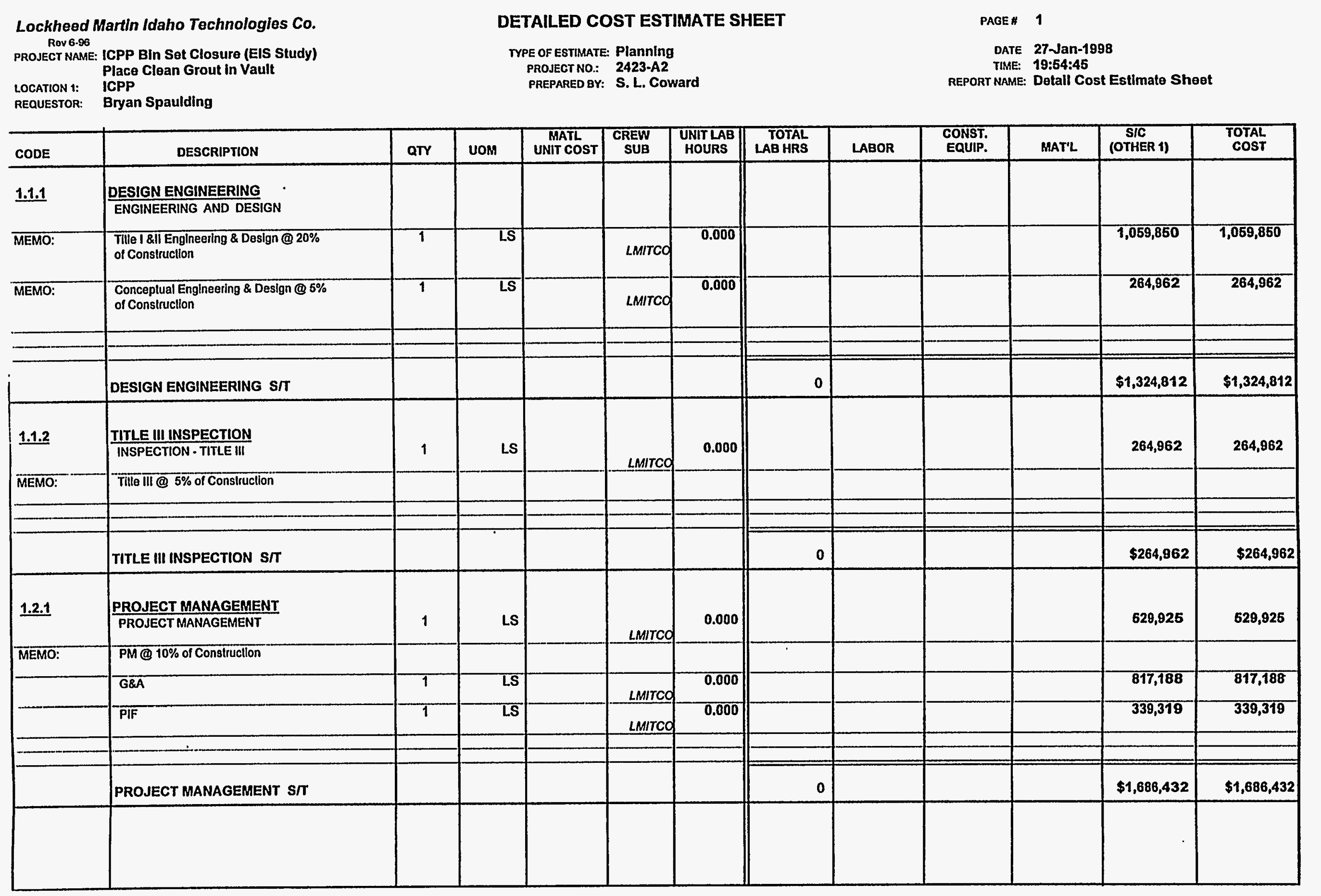


Lockheed Martin Idaho Tochnologles Co.

Rov 6.06

IROJECT NAME: ICPP Bin Sot Closure (EIS Study)

Place Clean Grout in Vault

OCATION 1: ICPP

REQUESTOR: Bryan Spaulding

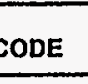

22

1.2 .2

IEMO:

1.3 .1

DESCRIPTIO

CONSTRUCTION MANAGEMENT CONSTRUCTION MANAGEMENT

CM @ 10\% of Construction Costs

\section{CONSTRUCTION MANAGEMENT SIT}

GENERAL CONDITIONS

SUPERVISION (Duration of Schedule -

Assume 3 Years)

TRAINING

RADCON TECHNICIAN (Duration o

Schedule - Assume 3 Years)

\section{GENERAL CONDITIONS $\mathrm{S} / \mathrm{T}$}

1.3 .2

SITEWORK

\section{SITEWORK SIT}

1.3 .3

\section{CONCRETE}

PROCURE EQUIPMENT FOR PLACEMENT OF GROUT

Grout Pump

\section{DETAILED COST ESTIMATE SHEET}

TYPE OF estimate: Planning

PROJECT NO.: 2423-A2

PREPARED BY: S. L. Coward
DATE 27-Jan-1998

TIME: 19:64:46

REPORT NAME: Dotall Cost Estlmate Sheot

\begin{tabular}{c|c|c|}
\hline MATL & CREW & UNIT LAB \\
\hline UNIT COST & SUB & UOU \\
\hline
\end{tabular}

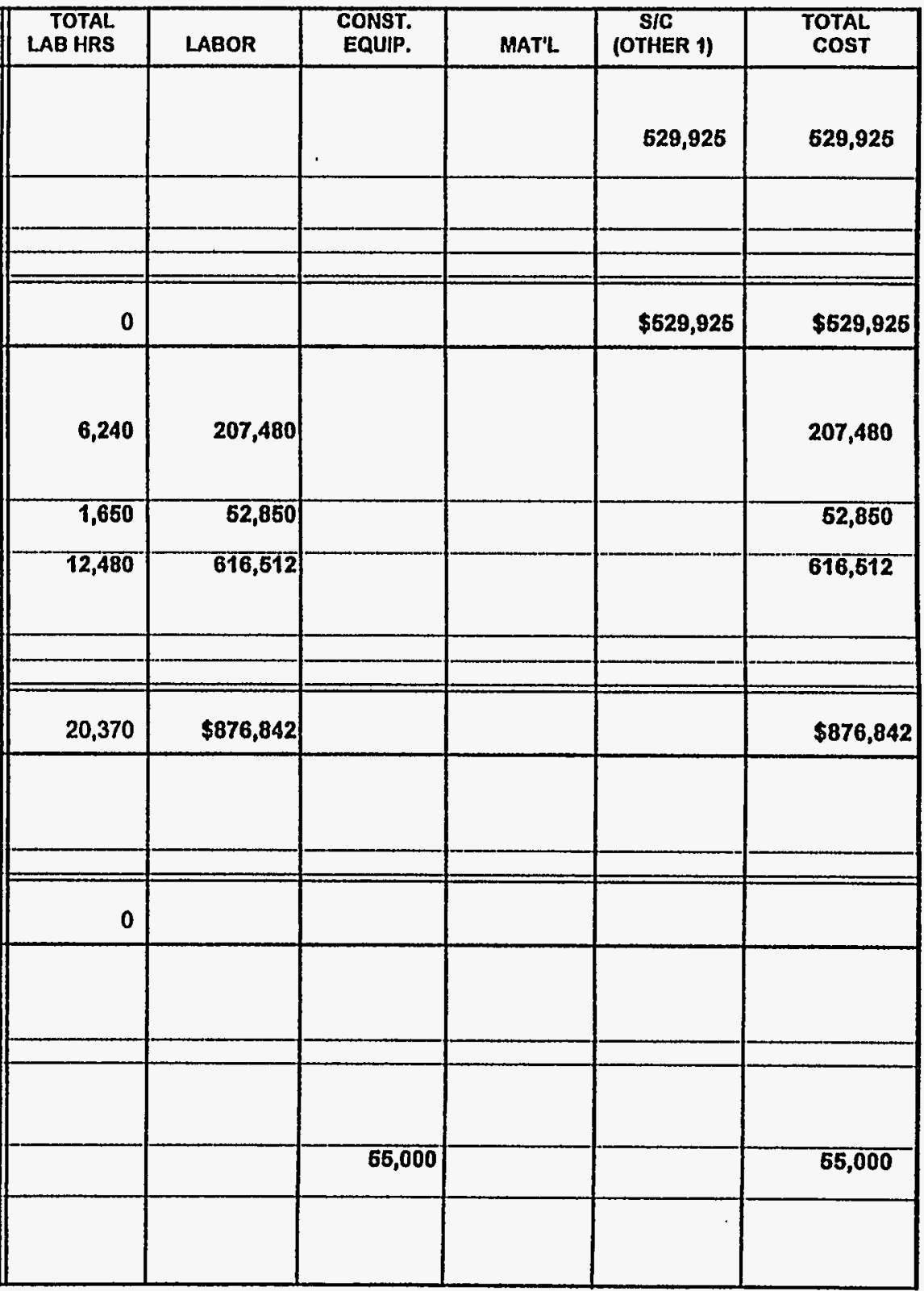


Lockheed Martin Idaho Technologies Co.

Rev 6-96 PROJECT NAME: ICPP Bin Set Closure (EIS Stud LOCATION 1: ICPP REQUESTOR: Bryan Spaulding
CONTINGENCY ANALYSIS
DATE: 27 Jan-1998

TME: 19:54:39

REPORT NAME: Contingency Analysis

\begin{tabular}{|c|c|c|c|c|c|c|c|c|c|c|c|}
\hline \multicolumn{9}{|c|}{ PROBABLE \% VARIATTON } & \multicolumn{2}{|c|}{$\begin{array}{l}\text { PROJECT } \\
\text { CONTINGENCY }\end{array}$} & \multirow{3}{*}{$\begin{array}{l}\text { SUMMARY } \\
\begin{array}{l}\text { Total Cost } \\
\text { by Element }\end{array}\end{array}$} \\
\hline \multirow[t]{2}{*}{$\begin{array}{l}\text { WBS } \\
\text { Element }\end{array}$} & \multirow{2}{*}{ Cost Estimate Element } & \multirow{2}{*}{$\begin{array}{l}\text { Total Cost w/o } \\
\text { Contingeney }\end{array}$} & \multirow[t]{2}{*}{$\begin{array}{l}\text { \% Total } \\
\text { Cost }\end{array}$} & \multicolumn{2}{|c|}{$\begin{array}{c}\text { Prob. \% Var. } \\
\text { From Est. }\end{array}$} & \multicolumn{2}{|c|}{ Wt. \% of Prob. } & \multirow[t]{2}{*}{ Contingency } & \multirow[t]{2}{*}{$\%$} & \multirow{2}{*}{ Cost } & \\
\hline & & & & - & + & - & \pm & & & & \\
\hline 1.4.1 & DESIGN ENGINEERING & $1,324,812$ & 14.47 & 10 & 40 & 1.45 & 5.79 & $5.063 \%$ & $14,47 \%$ & 468,920 & $1,793,732$ \\
\hline 1.1 .2 & TIILE III INSPECTION & 264,962 & 2.89 & 10 & 40 & 0.29 & 9.16 & 1.013\% & $2.89 \%$ & 93,784 & 358,746 \\
\hline 1.2 .1 & PROJECT MANAGEMENT & $1,686,432$ & 18.41 & 10 & 40 & 9.84 & 7.37 & $6.445 \%$ & 18.41\% & 596,916 & $2,283,348$ \\
\hline 1.22 & CONSTRUCTION MANAGEMENT & 529,925 & 5.79 & 10 & 40 & 0.58 & 231 & $2025 \%$ & $5.79 \%$ & 187,568 & 717,493 \\
\hline 1.3 .1 & GENERAL CONDIIONS & $1,067,791$ & 11.66 & 10 & 40 & 1.17 & 4.66 & $4.081 \%$ & $11.66 \%$ & 37,947 & $1,445,738$ \\
\hline 1.3 .2 & SIREWORK & 0 & 0.00 & 0 & 0 & 0.00 & 0.00 & $0.000 \%$ & $0.00 \%$ & 0 & 0 \\
\hline 1.3 .3 & CONCRETE & $4,231,459$ & 46.20 & 10 & 40 & 4.62 & 18.48 & 16.179\% & $46.20 \%$ & 4,497,735 & $5,729,194$ \\
\hline \multirow[t]{2}{*}{1.5 .2} & PROCUREMENT FEES & 52,992 & 0.58 & 10 & 40 & 0.06 & 0.23 & $0.203 \%$ & $0.58 \%$ & 18,757 & 74,749 \\
\hline & ESCALATION & 0 & 0.00 & 0 & 0 & 0.00 & 0.00 & $0.000 \%$ & $0.00 \%$ & 0 & 0 \\
\hline & SUBTOTAL & $9,158,373$ & 100.00 & & & & & $35.000 \%$ & & & \\
\hline & CALCULATED CONTINGENCY & $3,205,431$ & & & & & & & & & \\
\hline & RESULTANT TEC & $12,363,804$ & & & & & & & & & \\
\hline & ROUNDED TEC & $12,400,000$ & & & & & & & & & \\
\hline & PROJECT CONTINGENCY & $3,241,627$ & & & & & & $35.40 \%$ & & & \\
\hline & MANAGEMENT RESERVE & 535,224 & & & & & & & & & \\
\hline & CONTINGENCY & $2,706,403$ & & & & & & & & & \\
\hline & TOTAL ESTIMATED COST & $12,400,000$ & & & & & & & - & $3,241,627$ & $12,400,000$ \\
\hline
\end{tabular}

CONFIDENCE LEVEL AND ASSUMED RISKS:

The Lockheed Idaho Technologies Co. Cost Estimate Contingency Analysis Model is based on the applied contingency and the assumptions upon which

the estimate was predicated. The model is applied with a suggested risk level of $18 \%$ and a level of confidence of $90 \%$ the estimate will fall within the bid range. The Contingency Analysis is based on a weighted average to provide a

$90 \%$ probability of underrun and a $10 \%$ probability of overtun.
CONTINGENCY ANALYSIS GUIDE BY TYPE OF ESTIMATE

Guidelines estabiished by DOEJFM 50, Cost Estimating Guide, Vol. 6 , Cost Guide, and as presented in the INEL Cost Estimating Guide.

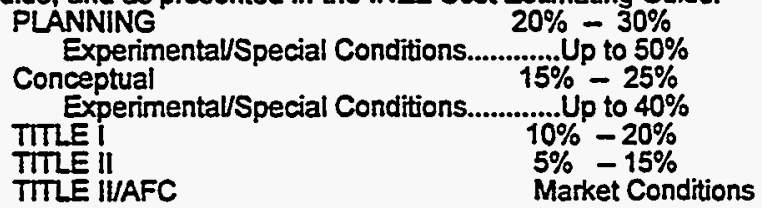




\section{G\&A/PIF ADDER CALCULATION SHEET \\ ICPP BIN SET CLOSURE \\ CLOSURE TO RCRA LANDFILL STANDARDS; CLASS C FILL; UNESCALATED}

FILL VAULTS W/ CLEAN GROUT

PROCUREMENT FEE:

CONSTRUCTION $=$

$\$ 5,299,250$

GFE =

Subtotal $\$ \$, 299,250$

FEE @ $1 \%=$

$\$ 5,299,250 \cdot 0.01=$

$\$ 52,993$

G\&A @ 23\% (with a ceiling of $\$ 500,000$ imposed per year , 7 Years)

CONSTRUCTION OR

CEILING * \# OF YEARS =

GFE =

$\$ 3,500,000$

PROCUREMENT FEE =

Subtotal

$\$ 52,993$

$\$ 3,552,993$

FEE @ 23\% =

$\$ 3,552,993 \cdot 0.23=$

$\$ 817,188$

PIF @ 5.5\%

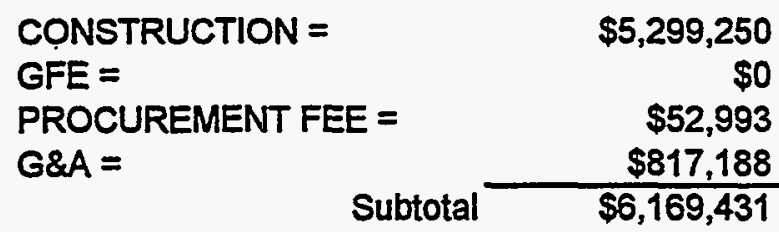

FEE @ 5.5\% =

$\$ 6,169,431 \cdot 0.055=$

$\$ 339,319$

TOTAL PROCUREMENT FEE:

$\$ 52,993$

TOTAL G\&A FEE:

$\$ 817,188$

TOTAL PIF:

$\$ 339,319$ 
Lockheed Martin Idaho Technologies Co. Rev, 6-56

PROJECT NAME: ICPP Bin Set Closure (EIS Stud Tractor (RCRA Estimates)

LOCATION 1: ICPP REQUESTOR:
Bryan Spaulding
COST ESTIMATE SUMMARY

TYPE OF ESTIMATE: $\quad$ Planning
PROJECT NO: $2423-C$
PREPARED BY:
S. L Coward
REPORT NAME: COSt EStimate Summary

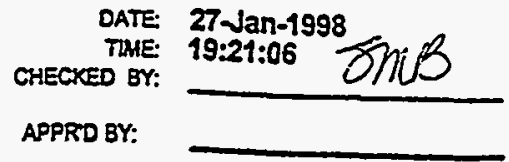

\begin{tabular}{|c|c|c|c|c|}
\hline $\begin{array}{l}\text { WBS } \\
\text { Element }\end{array}$ & Cost Estimate Element & $\begin{array}{c}\text { Total } \\
\text { Unescalated }\end{array}$ & Escalation & $\begin{array}{c}\text { Total } \\
\text { Incl Escalation }\end{array}$ \\
\hline $\begin{array}{l}\frac{1.1}{1.1 .1} \\
1.1 .2 \\
\frac{1.2}{1.2 .1} \\
1.2 .2 \\
\frac{1.3}{1.3 .1} \\
1.3 .13 \\
1.5 .2\end{array}$ & $\begin{array}{l}\text { ENGINEERING, DESIGN AND INSPECTION } \\
\text { DESIGN ENGINEERING TTILE I \& II } \\
\text { TITLE III INSPECTION } \\
\text { MANAGEMENT COSTS } \\
\text { PROJECT MANAGEMENT } \\
\text { CONSTRUCTION MANAGEMENT } \\
\text { CONSTRUCTION } \\
\text { GENERAL CONDITIONS } \\
\text { SPECIAL CONSTRUCTION } \\
\text { PROCUREMENT FEES }\end{array}$ & $\begin{array}{r}6,128,131 \\
2,042,710 \\
7,657,255 \\
4,085,421 \\
\\
6,840,414 \\
34,013,797 \\
408,542\end{array}$ & $\begin{array}{l}0 \\
0\end{array}$ & $\begin{array}{r}\gg>\begin{array}{r}\frac{\$ 8,170,841}{6,128,131} \\
2,042,710\end{array} \\
\gg>\frac{\$ 11,752,676}{7,667,255} \\
4,085,421 \\
\\
\gg \frac{\$ 40,854,211}{6,840,414} \\
34,013,797\end{array}$ \\
\hline & $\begin{array}{l}\text { SUBTOTAL INCLUDING ESCALATION } \\
\text { PROJECT CONTINGENCY }\end{array}$ & $61,186,270$ & 0 & $\gg \$ 61,186,270$ \\
\hline & MANAGEMENT RESERVE- & & & $\gg \quad \$ 4,126,275$ \\
\hline & CONTINGENCY - & & & $\gg \quad \$ 17,287,455$ \\
\hline & TOTAL ESTIMATED COST - & & & $\$ 82,600,000$ \\
\hline
\end{tabular}

\section{PROJECT COST PARAMETERS}

EDI AS A $\%$ OF CONST. + GFE $=20.00 \%$ CONTINGENCY $=35.00 \%$ 
ockheed Martin Idaho Technologles Co. Hov 6.26

ZOJECT NAME: ICPP Bin Set Closure (EIS Study)

Tractor (RCRA Estimates)

CAYION 1: ICPP

:QUESTOR: Bryan Spaulding

\section{DETAILED COST ESTIMATE SHEET}

TYPE OF ESTIMATE: Planning

PROJECT NO.: 2423-C

PREPARED 日Y: S. L. Coward
PAGE * 2

DATE 27 Jan-1898

TIME: 19:21:08

RePort NaME: Detail Cost Estlmate Sheot

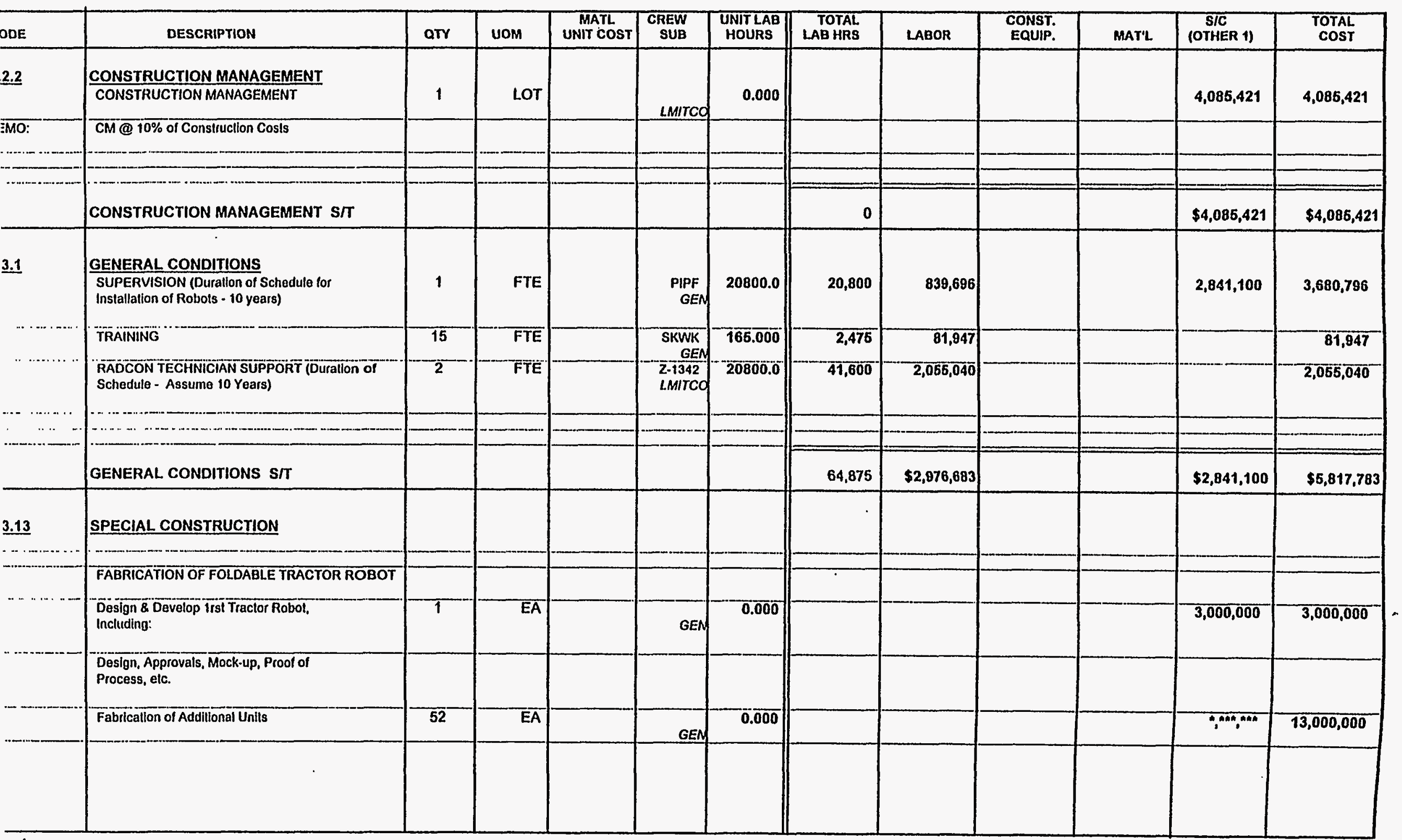


Lockheed Martin Idaho Technologles Co.

Rov 6.96

IDIECT NAME: ICPP Bin Set Closure (EIS Study) Tractor (RCRA Estimates)

ICATION 1: ICPP

il:OUESTOn Bryan Spaulding

\section{DETAILED COST ESTIMATE SHEET}

TYPE OF ESTIMATE: Planning

PROJECT NO: : 2423-C

PREPARED BY: S. L. Coward
PAgE 3

DATE 27 Jan-1998

TIME: 19:21:08

REPORT NAME: Dotall Cost Estimate Sheot

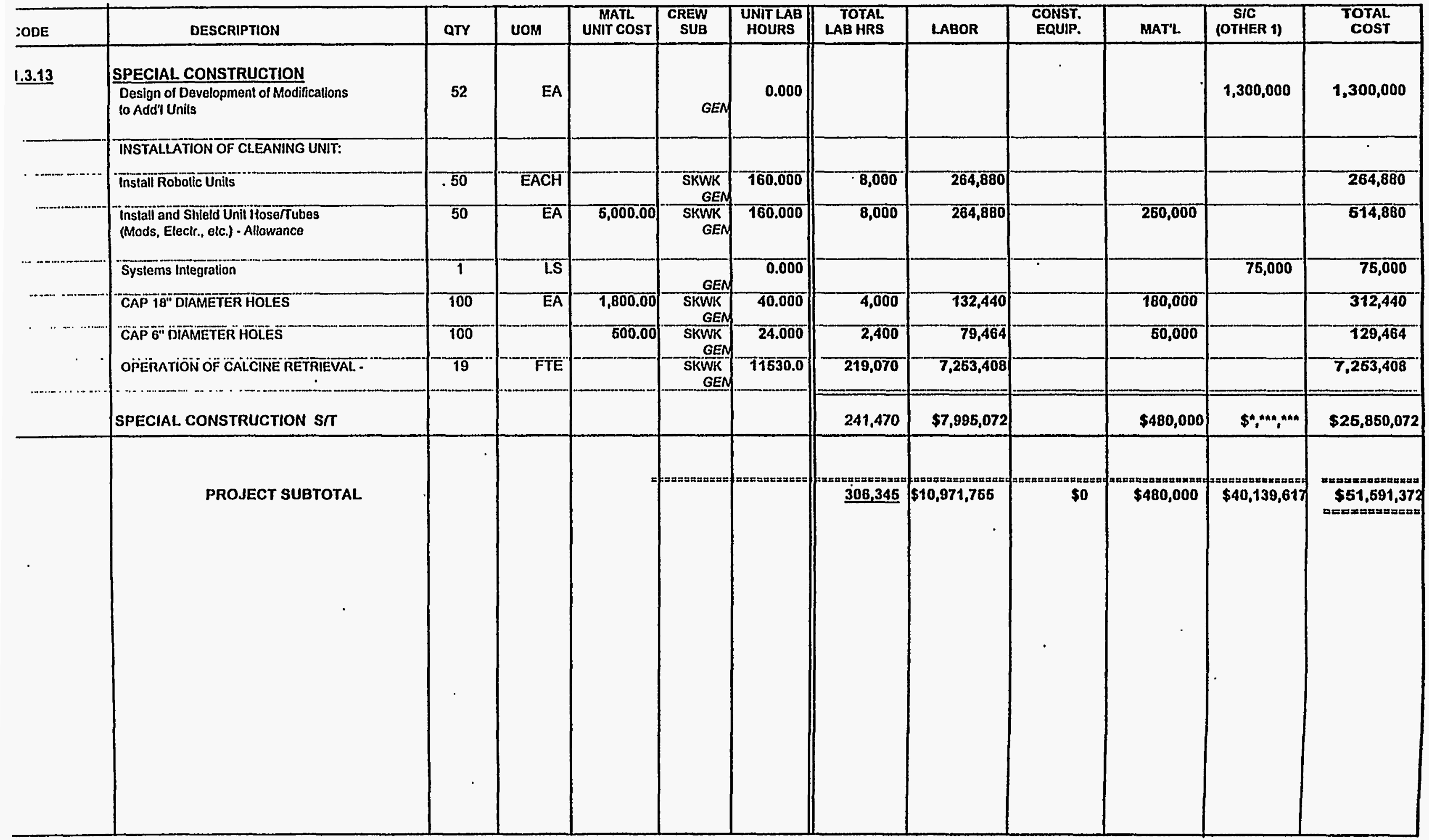



Lockheed Martin Idaho Technologies Co.

PROJECT NAME: ICPP Bin Set Closure (EIS Stud Tractor (RCRA Estimates)

LOCATION 1: ICPP

REQUESTOR: Bryan Spaulding
CONTINGENCY ANALYSIS
TYPE OF ESTHAATE: Planning

PROJECTNO: 2423-C

PREPARED BY: S. L Coward
DATE: 27-Jan-1998

TIME: 19:21:02
REPORT NAME: Contingency Analysis

\begin{tabular}{|c|c|c|c|c|c|c|c|c|c|c|c|}
\hline \multicolumn{9}{|c|}{ PROBABLE \% VARIATION } & \multicolumn{2}{|c|}{$\begin{array}{l}\text { PROJECT } \\
\text { CONTINGENCY }\end{array}$} & \multirow{3}{*}{$\begin{array}{l}\text { SUMMARY } \\
\text { Total Cost } \\
\text { by घement }\end{array}$} \\
\hline $\begin{array}{l}\text { WBS } \\
\text { Element }\end{array}$ & \multirow{2}{*}{ Cost Estimate Element } & \multirow{2}{*}{$\begin{array}{l}\text { Total Cost w/o } \\
\text { Comtingency }\end{array}$} & \multirow[t]{2}{*}{$\begin{array}{l}\text { \% Total } \\
\text { cost }\end{array}$} & \multicolumn{2}{|c|}{$\begin{array}{l}\text { Prob. \% Var. } \\
\text { From Est. }\end{array}$} & \multicolumn{2}{|c|}{ We. \% of Prob. } & \multirow{2}{*}{ Contingency } & \multirow[t]{2}{*}{$\%$} & \multirow{2}{*}{ Cost } & \\
\hline & & & & - & + & $=$ & \pm & & & & \\
\hline 1.4 .1 & DESIGN ENGINEERING TILE I \& \| & $6,128,131$ & 10.02 & 10 & 40 & 1.00 & 4.01 & $3.505 \%$ & $10.02 \%$ & $2,144,699$ & $8,272,830$ \\
\hline 4.1 .2 & TIRE III INSPECTION & $2,042,710$ & 3.34 & 10 & 40 & 0.33 & 1.34 & $1.168 \%$ & $3.34 \%$ & 714,900 & $2,757,610$ \\
\hline 1.21 & PROJECT MANAGEMENT & $7,667,255$ & 12.53 & 10 & 40 & 1.25 & 5.01 & $4.386 \%$ & $12.53 \%$ & $2,683,356$ & $10,350,611$ \\
\hline 1.22 & CONSTRUCTION MANAGEMENT & $4,085,429$ & 6.68 & 10 & 40 & 0.67 & 2.57 & $2337 \%$ & $6.68 \%$ & $1,429,800$ & $5,515,221$ \\
\hline 1.3 .1 & GENERAL CONDITTONS & $6,840,414$ & 11.18 & 10 & 40 & 1.12 & 4.47 & $3.913 \%$ & $19.18 \%$ & $2,393,981$ & $9,234,395$ \\
\hline 13.13 & SPECLAL CONSTRUCTION & $34,013,797$ & 55.59 & 10 & 40 & 5.56 & 22.24 & $19.457 \%$ & $55.59 \%$ & $11,904,015$ & $45,917,812$ \\
\hline \multirow[t]{2}{*}{1.5 .2} & PROCUREMENT FEES & 408,542 & 0.67 & 10 & 40 & 0.07 & 0.27 & $0.234 \%$ & $0.67 \%$ & 142,980 & 551,522 \\
\hline & ESCALATION & 0 & 0.00 & 10 & 40 & 0.00 & 0.00 & $0.000 \%$ & $0.00 \%$ & (4) & (1) \\
\hline \multicolumn{2}{|r|}{ SUBTOTAL } & $61,186,270$ & 100.00 & & & & & $35.000 \%$ & & & \\
\hline \multicolumn{2}{|r|}{ CALCULATED CONTINGENCY } & $21,415,195$ & & & & & & & & & \\
\hline \multicolumn{2}{|r|}{ RESULTANT TEC } & $82,601,465$ & & & & & & & & & \\
\hline \multicolumn{2}{|r|}{ ROUNDED TEC } & $82,600,000$ & & & & & & & & & \\
\hline \multicolumn{2}{|r|}{ PROJECT CONTINGENCY } & $21,413,730$ & & & & & & $35.00 \%$ & & & \\
\hline \multicolumn{2}{|r|}{ MANAGEMENT RESERVE } & $4,126,275$ & & & & & & & & & \\
\hline \multicolumn{2}{|r|}{ CONTINGENCY } & $17,287,455$ & & & & & & & & & \\
\hline & TOTAL ESTIMATED COST & $82,600,000$ & & & & & & & & $21,413,730$ & $82.600,000$ \\
\hline
\end{tabular}

The Lockheed Idaho Technologies Co. Cost Estimate Contingency Analysis

Model is based on the applied contingency and the assumptions upon which

the estimate was predicated. The model is applied with a suggested risk level

of $18 \%$ and a level of confidence of $90 \%$ the estimate will fall within the bid range.

The Contingency Analysis is based on a weighted average to provide a

$90 \%$ probability of undermun and a $10 \%$ probability of overnun.
CONFIOENCE LEVEL AND ASSUMED RISKS:
CONTINGENCY ANALYSIS GUIDE BY TYPE OF ESTIMATE

Guidelines established by DOEIFM 50. Cost Estimating Guide. Vol. 6 . Cost Guide, and as presented in the INEL Cost Estimating Guide.

PLANNING $20 \%-30 \%$ ExperimentalSpecial Conditions............ Up to $50 \%$ Conceptual

Experimental/Special Conditions.............Up to $40 \%$

TITLE I

TITLE II

TITLE IIIAFC

$10 \%-20 \%$

$5 \%-15 \%$

Market Conditions 


\section{G\&AVPIF ADDER CALCULATION SHEET \\ ICPP BIN SET CLOSURE \\ CLOSURE TO RCRA LANDFILL STANDARDS; CLASS C FILL; UNESCALATED \\ CLEAN BINS W/ ROBOTS FLOOR}

PROCUREMENT FEE:

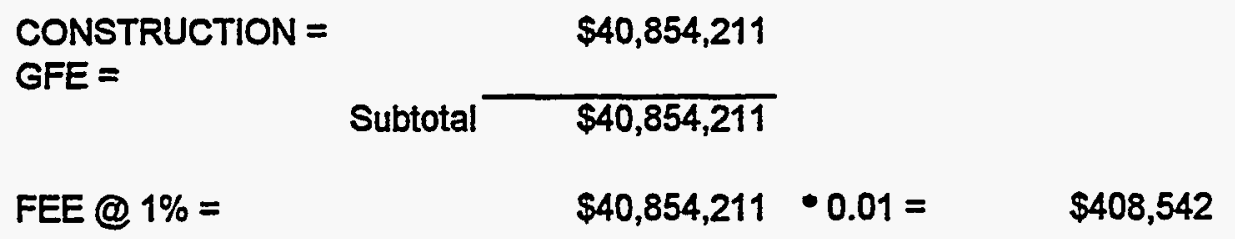

G\&A @ 23\% (with a ceiling of $\$ 500,000$ imposed per year , 10 Years)

CONSTRUCTION OR

CEILING ${ }^{\bullet}$ \# OF YEARS $=\quad \$ 5,000,000$

GFE =

PROCUREMENT FEE $=$ $\$ 408,542$

Subtotal $\$ 5,408,542$

FEE @ $23 \%=$

$\$ 5,408,542 \cdot 0.23=$

$\$ 1,243,965$

PIF @ 5.5\%

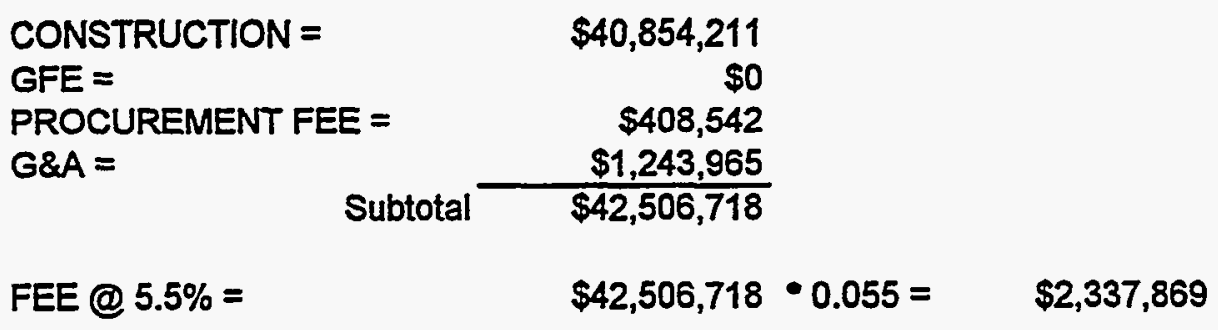

TOTAL PROCUREMENT FEE:

$\$ 408,542$

TOTAL G\&A FEE:

$\$ 1,243,965$

TOTAL PIF:

$\$ 2,337,869$ 
Lockheed Martin Idaho Technologies Co.

PROJECT NAME: ICPP Bin Set Closure (EIS Stud Place NRC Class C Grout LOCATION 1: ICPP REQUESTOR: Bryan Spaulding
COST ESTIMATE SUMMARY

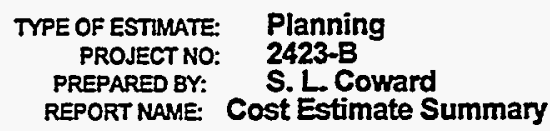

DATE: $27-$ Jan-1998

TIME: 20:26:45

CHECKED BY:

APPR'D BY:

\begin{tabular}{|c|c|c|c|c|}
\hline $\begin{array}{l}\text { WBS } \\
\text { Element }\end{array}$ & Cost Estimate Element & $\begin{array}{c}\text { Total } \\
\text { Unescalated }\end{array}$ & Escalation & $\begin{array}{c}\text { Total } \\
\text { Incl Escalation }\end{array}$ \\
\hline 1.1 & ENGINEERING, DESIGN AND INSPECTION & & & $\gg \$ \$ 3,939,045$ \\
\hline$\overline{1.1 .1}$ & DESIGN ENGINEERING TITLE I \& II & $3,282,538$ & 0 & $3,282,538$ \\
\hline 1.1.2 & TITLE III INSPECTION & 656,507 & 0 & 656,507 \\
\hline 1.2 & MANAGEMENT COSTS & & & $\gg \$ 4,225,021$ \\
\hline 1.2 .1 & PROJECT MANAGEMENT & $2,923,531$ & 0 & $2,923,531$ \\
\hline 1.2 .2 & CONSTRUCTION MANAGEMENT & $1,301,490$ & 0 & $1,301,490$ \\
\hline 1.3 & CONSTRUCTION & & & $\gg \$ 13,130,159$ \\
\hline 7.3 .1 & GENERAL CONDITIONS & $3,437,150$ & 0 & $3,437,150$ \\
\hline 1.3 .2 & SITEWORK & 0 & 0 & $\mathbf{0}$ \\
\hline 1.3 .3 & CONCRETE & $3,876,603$ & 0 & $3,876,603$ \\
\hline 1.3 .15 & MECHANICAL . & $3,365,584$ & 0 & $3,365,584$ \\
\hline 1.3.16 & ELECTRICAL & $2,450,822$ & 0 & $2,450,822$ \\
\hline \multirow[t]{3}{*}{1.5 .2} & PROCUREMENT FEES & 131,302 & 0 & $\$ 131,302$ \\
\hline & SUBTOTAL INCLUDING ESCALATION & $21,425,527$ & 0 & \multirow[t]{2}{*}{$\gg \$ 21,425,527$} \\
\hline & PROJECT CONTINGENCY & & & \\
\hline \multicolumn{2}{|r|}{ MANAGEMENT RESERVE- } & & & $\$ 1,326,146$ \\
\hline \multicolumn{2}{|r|}{ CONTINGENCY- } & & & $\gg \quad \$ 7,348,327$ \\
\hline \multicolumn{2}{|r|}{ TOTAL ESTIMATED COST - } & & & $\gg \quad \$ 30,100,000$ \\
\hline
\end{tabular}

\section{PROJECT COST PARAMETERS}

EDI AS A \% OF CONST. + GFE $=30.00 \%$ CONTINGENCY $=40.49 \%$ 
DETAILED COST ESTIMATE SHEET

TYPE OF estimate: Planning

PROJECT NO.: 2423-B

PREPARED BY: S. L. Coward

Place NRC Class C Grout

ICPP

LOCATION 1:

Bryan Spaulding
PAgE H 2

DATE 27-Jan-1998

TIME: 20:26:47

REPORT NAME: Detail Cost Estimate Sheot

\begin{tabular}{|c|c|c|c|c|c|c|c|c|c|c|c|c|}
\hline CODE & DESCRIPTION & QTY & UOM & $\begin{array}{l}\text { MATL } \\
\text { UNIT COST }\end{array}$ & $\begin{array}{l}\text { CREW } \\
\text { SUB }\end{array}$ & $\begin{array}{l}\text { UNIT LAB } \\
\text { HOURS }\end{array}$ & $\begin{array}{l}\text { TOTAL } \\
\text { LAB HRS }\end{array}$ & LABOR & $\begin{array}{l}\text { CONST. } \\
\text { EQUIP. }\end{array}$ & MAT'L & $\begin{array}{l}\text { SIC } \\
\text { (OTHER 1) }\end{array}$ & $\begin{array}{l}\text { TOTAL } \\
\text { COST }\end{array}$ \\
\hline$\underline{1.2 .2}$ & $\frac{\text { CONSTRUCTION MANAGEMENT }}{\text { CONSTRUCTION MANAGEMENT }}$ & 1 & LS & & LMITCO & 0.000 & & & & & $1,301,490$ & $1,301,490$ \\
\hline \multirow[t]{4}{*}{ MEMO: } & CM @ 10\% of Construction Costs & & & & & & & & & & & \\
\hline & & & & & & & & & & & & \\
\hline & & & & & & & & & & & & \\
\hline & CONSTRUCTION MANAGEMENT SIT & & & & & & 0 & & & & $\$ 1,301,490$ & $\$ 1,301,490$ \\
\hline \multirow[t]{6}{*}{1.3 .1} & $\begin{array}{l}\text { GENERAL CONDITIONS } \\
\text { SUPERVISION (Durallon of Project - } \\
\text { Assume } 10 \text { Years) }\end{array}$ & 1 & FTE & & $\begin{array}{c}\text { CONF } \\
\text { GEN }\end{array}$ & 20800.0 & 20,800 & 691,600 & & & & 691,600 \\
\hline & TRAINING & 20 & FTE & & $\begin{array}{c}\text { CONC } \\
\text { GEN }\end{array}$ & 165.000 & 3,300 & 105,699 & & & & 105,699 \\
\hline & $\begin{array}{l}\text { RADCON TECHNICIAN SUPPORT (Duration of } \\
\text { Project - Assume } 10 \text { Years) }\end{array}$ & 2 & FTE & & $\begin{array}{l}\text { Z-1342 } \\
\text { LMTCO }\end{array}$ & 20800.0 & 41,600 & $2,055,040$ & & & & $2,055,040$ \\
\hline & & & & & & & & & & & & \\
\hline & & & & & & & & & & & & \\
\hline & GENERAL CONDITIONS S/T & & & & & & 65,700 & $\$ 2,852,339$ & & & & $\$ 2,852,339$ \\
\hline \multirow[t]{5}{*}{$\underline{1.3 .2}$} & SITEWORK & & & & & & & & & & & \\
\hline & & & & & & & & & & & & \\
\hline & $\begin{array}{l}\text { ASSUME NO EXCAVATION WILL BE REQUIRED } \\
\text { FOR MONITORS }\end{array}$ & & & & & & & & & & & \\
\hline & & & & & & & & & & & & \\
\hline & SITEWORK SIT & & & & & & 0 & & & & & \\
\hline 1.3 .3 & CONCRETE & & & & & & & & & & & \\
\hline & & & & & & & & & & & & \\
\hline & & & & & & & & & & & & \\
\hline & & & & & & & & & & & & \\
\hline
\end{tabular}


Lockheed Martin Idaho Technologies Co.

Rev 6.96

PROJECT NAME: ICPP Bin Set Closure (EIS Study)

Place NRC Class C Grout

LOCATION 1: ICPP

REQUESTOR: Bryan Spaulding
DETAILED COST ESTIMATE SHEET

TYPE OF EsTIMATE: Planning

PROJECT NO:: 2423-B

PREPARED BY: S. L. Coward
PAGE\# 3

DATE 27-Jan-1998

TIME: 20:26:47

REPORT NAME: Detall Cost Estimate Sheet

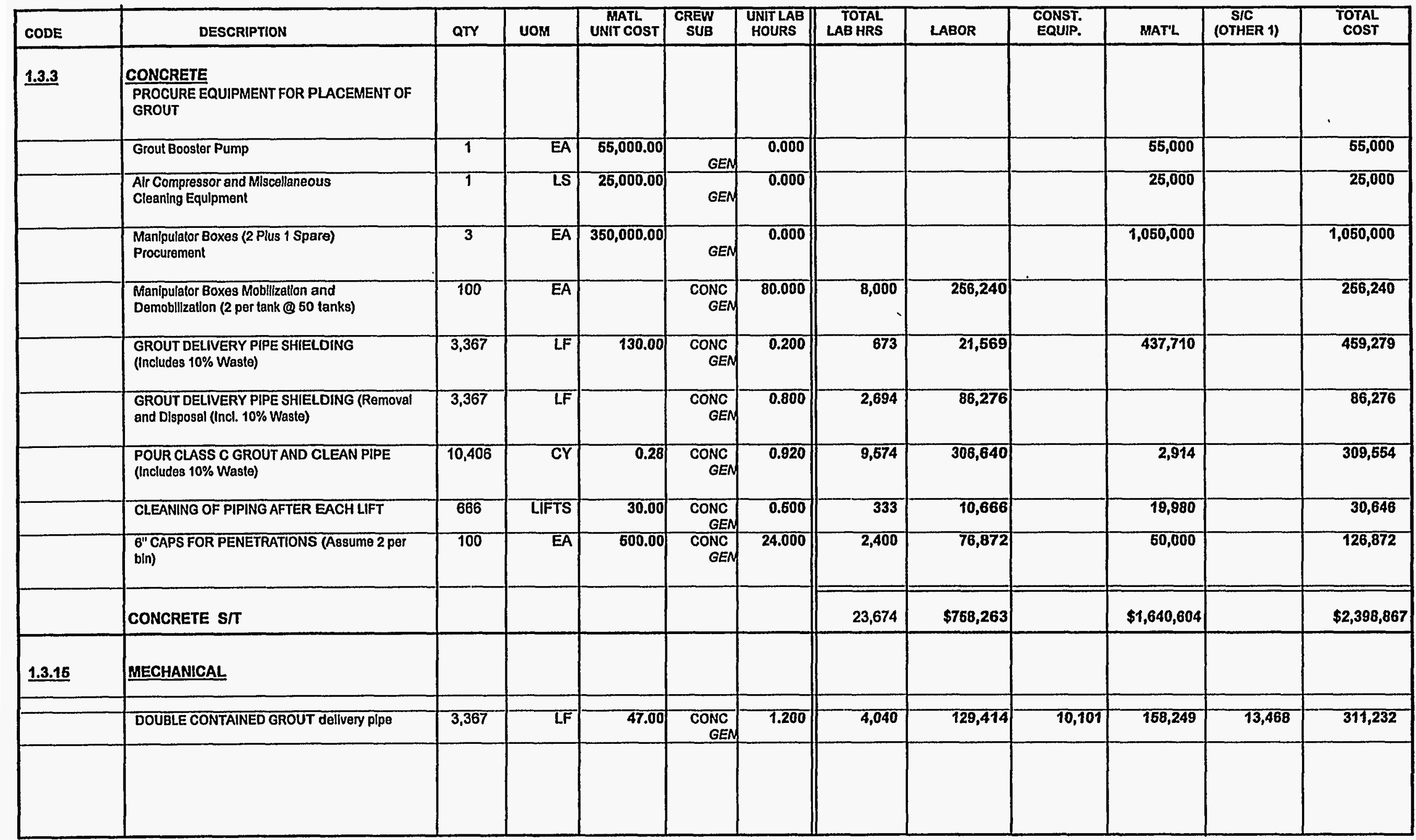


Lockheed Martin Idaho Technologies Co.

Rev G-96 PROJECT NAME: ICPP Bin Set Closure (EIS Stud

LOCATION 1: REQUESTOR: Place NRC Class C Grout ICPP Bryan Spaulding

\section{CONTINGENCY ANALYSIS}

$\begin{array}{cl}\text { TYPE OF ESTIMATE: } & \text { Planning } \\ \text { PROJECT NO: } & 2423-B \\ \text { PREPARED BY: } & \text { S. L. Coward }\end{array}$

DATE: 27 Jan-1998

TIME: 20:26:41

REPORT NAME: Contingency Analysis

\begin{tabular}{|c|c|c|c|c|c|c|c|c|c|c|c|}
\hline \multicolumn{9}{|c|}{ PROBABLE \% VARIATION } & \multicolumn{2}{|c|}{$\begin{array}{l}\text { PROJECT } \\
\text { CONTINGENCY }\end{array}$} & \multirow{3}{*}{$\begin{array}{l}\text { SUMMARY } \\
\begin{array}{l}\text { Total Cost } \\
\text { by Element }\end{array}\end{array}$} \\
\hline \multirow[t]{2}{*}{$\begin{array}{l}\text { WBS } \\
\text { Element }\end{array}$} & \multirow{2}{*}{ Cost Estimate Element } & \multirow{2}{*}{$\begin{array}{l}\text { Total Cost w/o } \\
\text { Contingency }\end{array}$} & \multirow[t]{2}{*}{$\begin{array}{l}\text { \% Total } \\
\text { Cost }\end{array}$} & \multicolumn{2}{|c|}{$\begin{array}{c}\text { Prob. \% Var. } \\
\text { From Est. }\end{array}$} & \multicolumn{2}{|c|}{ Wt. \% of Prob. } & \multirow[t]{2}{*}{ Contingency } & \multirow[t]{2}{*}{$\%$} & \multirow[t]{2}{*}{ Cost } & \\
\hline & & & & - & + & - & \pm & & & & \\
\hline 1.1.1 & DESIGN ENGINEERING TILE $\mid \& \|$ & $3,282,538$ & 15.32 & 10 & 40 & 1.53 & 6.13 & $5.362 \%$ & $13.08 \%$ & $1,134,503$ & $4,417,041$ \\
\hline 1.1 .2 & TIRE III INSPECTION & 656,507 & 3.06 & 10 & 40 & 0.31 & 1.23 & $1.072 \%$ & $2.62 \%$ & 226,900 & 883,407 \\
\hline 1.2 .1 & PROJECT MANAGEMENT & $2,923,531$ & 93.65 & 10 & 40 & 1.36 & 5.46 & $4.776 \%$ & $11.65 \%$ & $1,010,424$ & $3,933,955$ \\
\hline 1.2 .2 & CONSTRUCTION MANAGEMENT & $1,301,490$ & 6.07 & 10 & 40 & 0.61 & 243 & $2.126 \%$ & $5.19 \%$ & 449,818 & $1,751,308$ \\
\hline 1.3 .1 & GENERAL CONDITIONS & $3,437,150$ & 16.04 & 10 & 50 & 1.60 & 8.02 & $7.059 \%$ & $17.22 \%$ & $1,493,409$ & $4,930,559$ \\
\hline 1.3 .2 & SITEWORK & 0 & 0.00 & 0 & $\overline{0}$ & 0.00 & 0.00 & $0.000 \%$ & $0.00 \%$ & 0 & 0 \\
\hline 1.3 .3 & CONCRETE & $3,876,603$ & 18.09 & 10 & 50 & 1.81 & 9.05 & $7.961 \%$ & $19.42 \%$ & $1,684,348$ & $5,560,951$ \\
\hline 1.3 .15 & MECHANICAL & $3,365,584$ & 15.71 & 10 & 50 & 1.57 & 7.85 & $6.912 \%$ & $16.86 \%$ & $1,462,315$ & $4,827,899$ \\
\hline 1.3 .16 & ELECTRICAL & $2,450,822$ & 11.44 & 10 & 50 & 1.14 & 5.72 & $5.033 \%$ & $12.28 \%$ & 1,064,859 & $3,515,681$ \\
\hline \multirow[t]{2}{*}{1.5 .2} & PROCUREMENT FEES & 131,302 & 0.61 & 10 & 40 & 0.06 & 0.25 & $0.214 \%$ & $0.52 \%$ & 45,380 & 176,682 \\
\hline & ESCALATION & 0 & 0.00 & 0 & 0 & 0.00 & 0.00 & $0.000 \%$ & $0.00 \%$ & 102,517 & 102,517 \\
\hline & SUBTOTAL & $21,425,527$ & 100.00 & & & & & $40.515 \%$ & & & \\
\hline & CALCULATED CONTINGENCY & $8,680,649$ & & & & & & & & & \\
\hline & RESULTANT TEC & $30,106,176$ & & & & & & & & & \\
\hline & ROUNDED TEC & $30,100,000$ & & & & & & & & & \\
\hline & PROJECT CONTINGENCY & $8,674,473$ & & & & & & $40.49 \%$ & & & \\
\hline & MANAGEMENT RESERVE & $1,326,146$ & & & & & & & & & \\
\hline & CONTINGENCY & $7,348,327$ & & & & & & & & & \\
\hline & TOTAL ESTIMATED COST & $30,100,000$ & & & & & & & & $8,674,473$ & $30,100,000$ \\
\hline
\end{tabular}

\section{CONFIDENCE LEVEL AND ASSUMED RISKS:}

The Lockheed Idaho Technologies Co. Cost Estimate Contingency Analysis Model is based on the applied contingency and the assumptions upon which the estimate was predicated. The model is applied with a suggested risk level of $18 \%$ and a level of confidence of $90 \%$ the estimate will fall within the bid range. The Contingency Analysis is based on a weighted average to provide a $90 \%$ probability of underun and a $10 \%$ probability of overnun.
CONTINGENCY ANALYSIS GUIDE BY TYPE OF ESTIMATE

Guidelines established by DOE/FM 50, Cost Estimating Guide, Vol. 6. Cost Guide, and as presented in the INEL Cost Estimating Guide.
PLANNING
$20 \%-30 \%$
Conceptual
ExperimentaUSpecial Conditions
...... Up to $50 \%$
TITLE I
TITLE U
TITLE IIJAFC
$15 \%-25 \%$
Up to $40 \%$
$10 \%-20 \%$
$5 \%-15 \%$
Market Conditions 


\section{G\&APIF ADDER CALCULATION SHEET \\ ICPP BIN SET CLOSURE}

CLOSURE TO RCRA LANDFILLL STANDARDS; CLASS C FILL; UNESCALATED

FILL BINS W/ NRC CLASS C GROUT

PROCUREMENT FEE:

CONSTRUCTION $=$
GFE $=$ Subtotal $\frac{\$ 13,130,159}{\$ 13,130,159}$

FEE @ 1\% =

$\$ 13,130,159 * 0.01=$

$\$ 131,302$

G\&A @ 23\% (with a ceiling of $\$ 500,000$ imposed per year , 7 Years)

CONSTRUCTION OR

CEILING * \# OF YEARS $=\quad \$ 3,500,000$

GFE $=$

PROCUREMENT FEE $=$ $\$ 131,302$

Subtotal $\$ 3,631,302$

FEE @ 23\% =

$\$ 3,631,302 \cdot 0.23=$

$\$ 835,199$

PIF @ 5.5\%

\begin{tabular}{lr} 
CONSTRUCTION = & $\begin{array}{r}\$ 13,130,159 \\
\text { GFE = }\end{array}$ \\
PROCUREMENT FEE = & $\$ 0$ \\
G\&A $=\quad \begin{array}{r}\$ 131,302 \\
\end{array} \quad$ Subtotal & $\$ 14,096,199$ \\
\hline & $\$ 14,660$
\end{tabular}

FEE @ $5.5 \%=$

$\$ 14,096,660 \cdot 0.055=$

$\$ 775,316$

TOTAL PROCUREMENT FEE:

$\$ 131,302$

TOTAL G\&A FEE:

$\$ 835,199$

TOTAL PIF:

$\$ 775,316$ 
Lockheed Martin Idaho Technologies Co. PROJECT NAME: ICPP Bin Set Closure D\&D of Equipment

LOCATION 1: REQUESTOR:
COST ESTIMATE SUMMARY

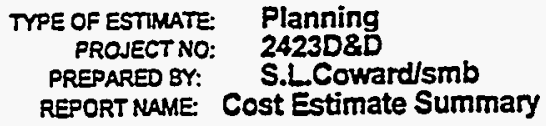

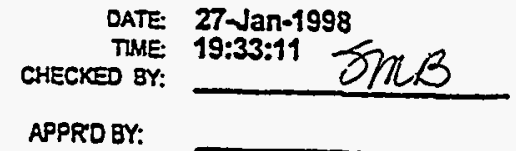

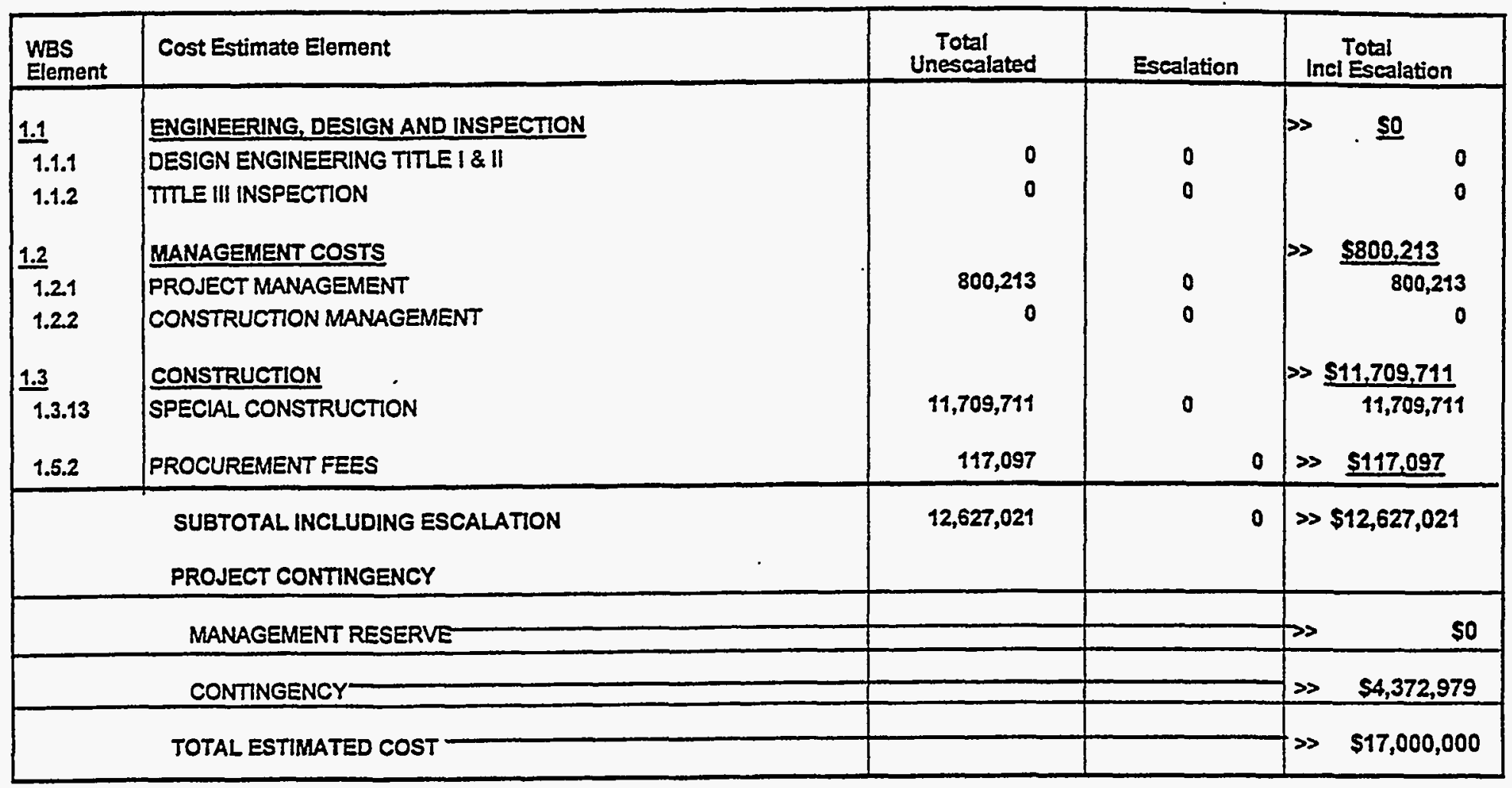

PROJECT COST PARAMETERS

EDI AS A \% OF CONST. + GFE $=0.00 \%$ 
.ockheed Martin Idaho Technologies Co.

$$
\text { Rov } 6.96
$$

ROJECT NAME: ICPP BIn Set Closure

D\&D of Equlpment

INEEL / ICPP

OCATION 1:

EQUESTOR

B. C. Spaulding

\section{DETAILED COST ESTIMATE SHEET}

TYPE OF Estimate: Planning

PROJECT NO:: 2423D\&D

PREPARED BY: S.L.Coward/smb
PAGE 1

DATE 27 Jan-1998

TIME: 19:30:67

report naMe: Detall Cost Estlmate Sheet

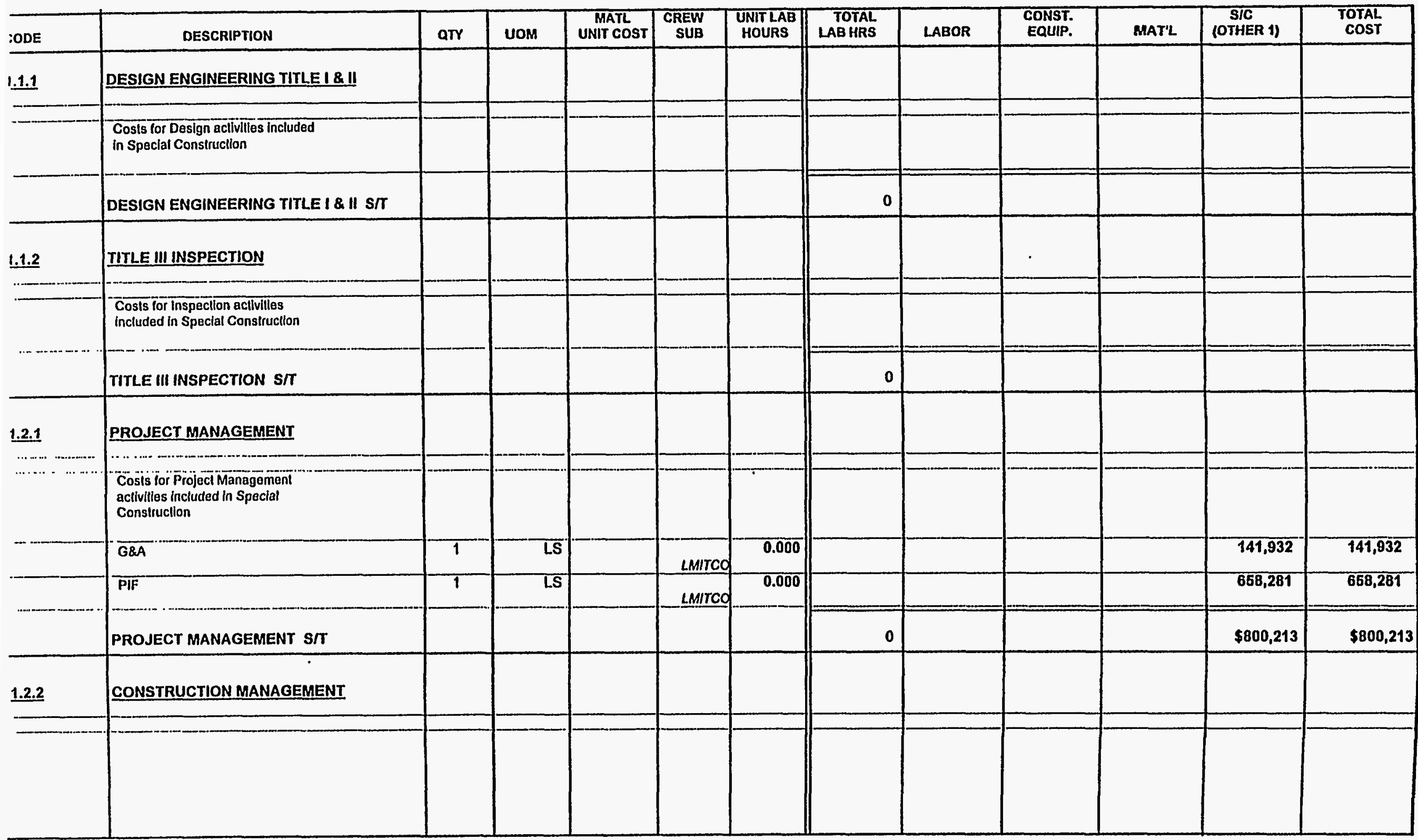


Lockheed Martin Idaho Technologles Co.

Rev 6.96

ROAECT NAME. ICPP Bin Sel Closure

D\&D of Equipment

$\begin{array}{ll}\text { OCATION I } & \text { INEEL / ICPP } \\ \text { IEQUESTOR: } & \text { B. C. Spaulding }\end{array}$

\section{DETAILED COST ESTIMATE SHEET}

TYPE OF ESTIMATE: Planning

PROJECT NO.: 2423D\&D

PREPARED BY: S.L.Coward/smb
PAgE * 3

DATE 27-Jan-1998

TIME: 19:30:67

RePort name: Detall Cost EstImate Sheot

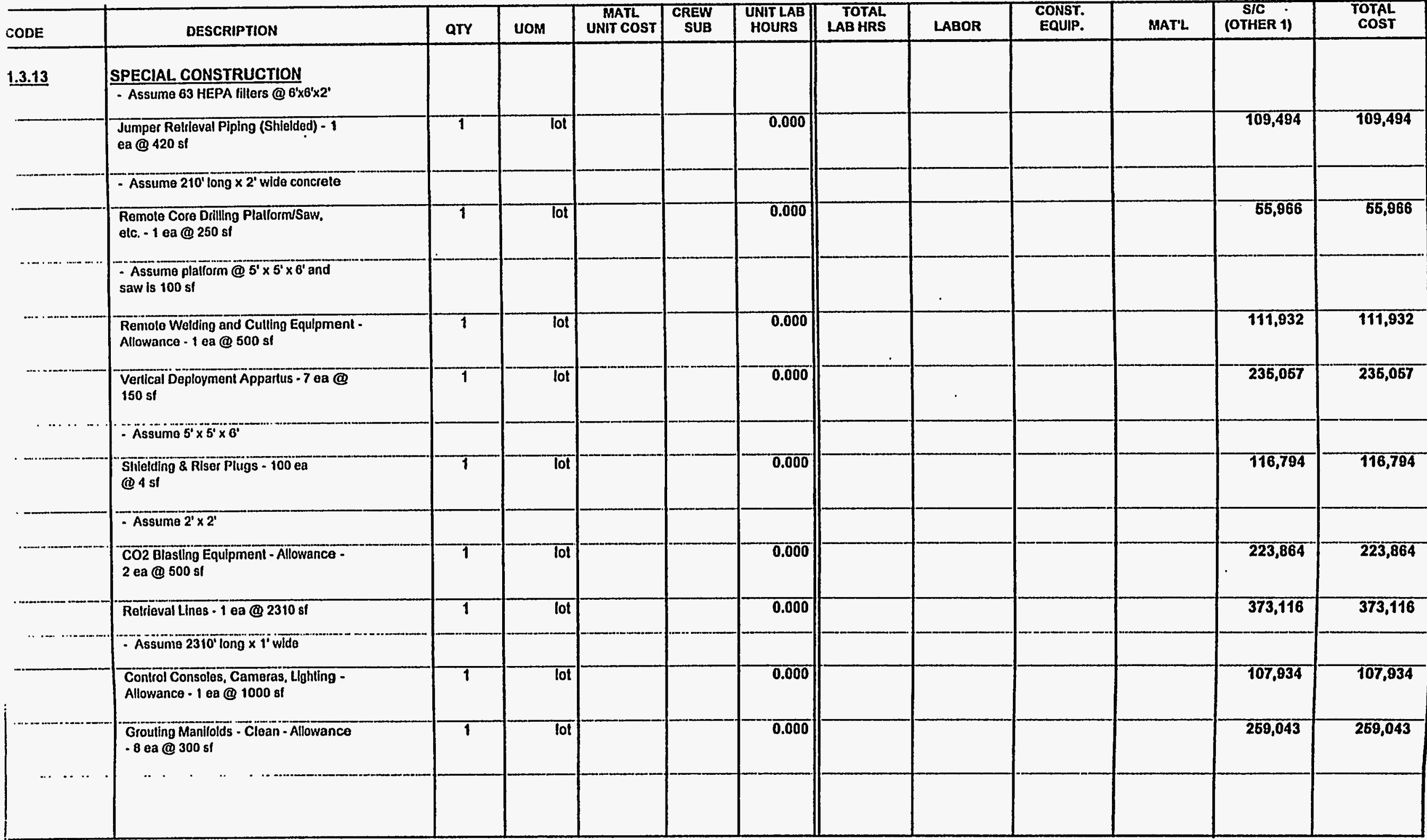


ockheed Martin Idaho Technologles Co. Rov 6.06

OJECT NAME. ICPP BIn Set Closure D\&D of Equipment

INEEL / ICPP

B. C. Spaulding
DETAILED COST ESTIMATE SHEET

TYPE Of Estimate: Planning

PROJECT NO.: 2423D\&D

PREPARED oY: S.L.Coward/smb
PAGE \# 4

DATE 27-Jan-1998

TIME: 19:30:67

heport name: Detall Cost Estimate Sheot

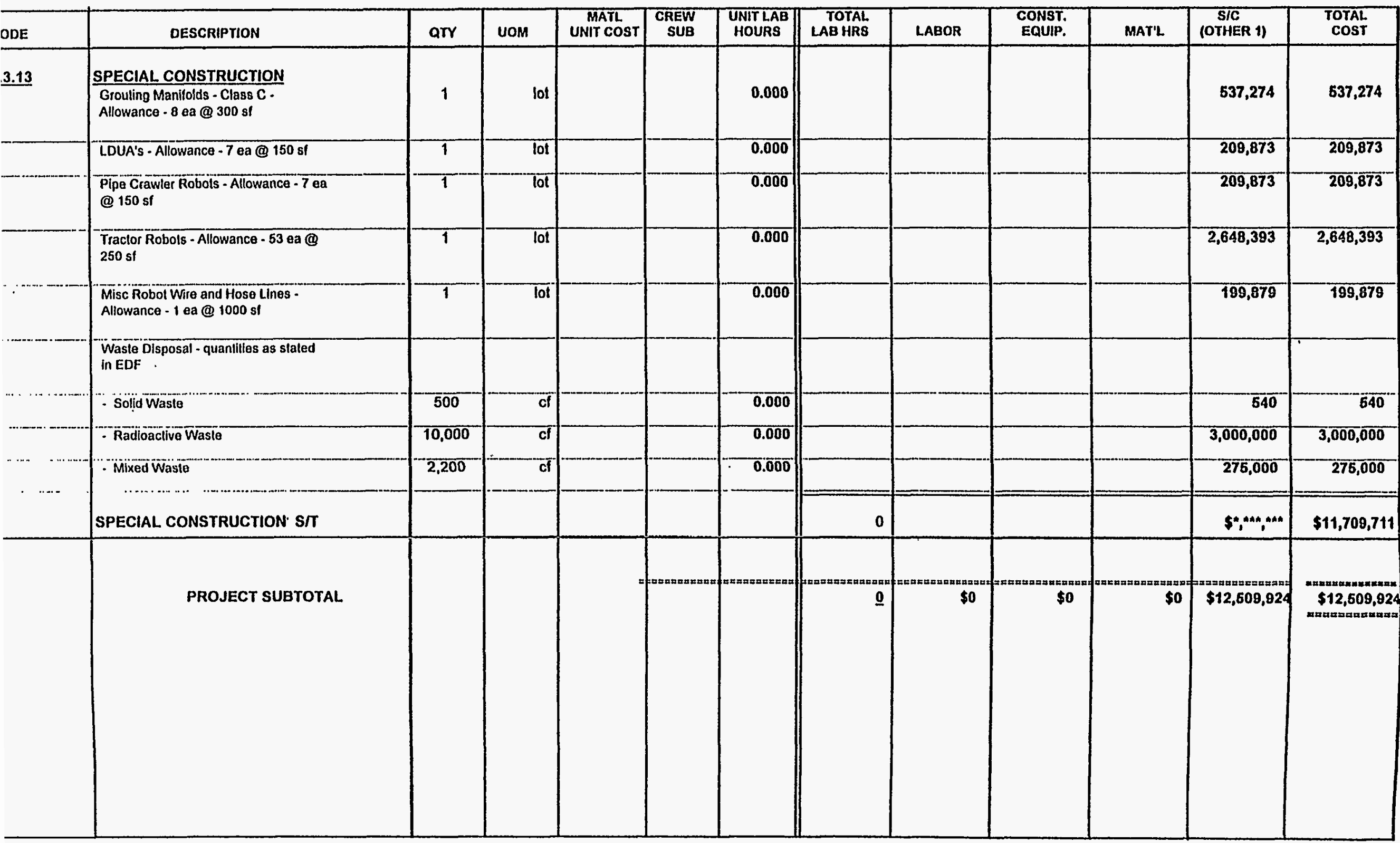


Lockheed Martin Idaho Technologies Co.

Rov 6.96

PROJECT MAME: ICPP Bin Sot Closuro

D\&D of Equipment

REQUESTOR:

INEEL / ICPP

B. C. Spaulding
CONTINGENCY ANALYSIS

TYPE OF ESTMMATE:

PROJECT NO: Planning

PREPARED BY: S.LCOWard/smb
OATE: 27 Jan-1998

TRE: 19:33:04

REPORT NAME: Contingency Analysis

\begin{tabular}{|c|c|c|c|c|c|c|c|c|c|c|c|}
\hline \multicolumn{9}{|c|}{ PROBABLE \% VARIATION } & \multicolumn{2}{|c|}{$\begin{array}{l}\text { PROJECT } \\
\text { CONTINGENCY }\end{array}$} & \multirow{3}{*}{$\begin{array}{l}\text { SUMMARY } \\
\text { Total Cost } \\
\text { by Eement }\end{array}$} \\
\hline \multirow{2}{*}{$\begin{array}{l}\text { WBS } \\
\text { Element }\end{array}$} & \multirow{2}{*}{ Cost Estimate Element } & \multirow{2}{*}{$\begin{array}{l}\text { Total Cost wio } \\
\text { Contingency }\end{array}$} & \multirow[t]{2}{*}{$\begin{array}{l}\% \text { Total } \\
\text { Cost }\end{array}$} & \multicolumn{2}{|c|}{$\begin{array}{c}\text { Prob. \% Var. } \\
\text { From Est. }\end{array}$} & \multicolumn{2}{|c|}{ Wh $\%$ of Prob. } & \multirow{2}{*}{ Contingency } & \multirow{2}{*}{$\%$} & \multirow{2}{*}{ Cost } & \\
\hline & & & & - & + & - & + & & & & \\
\hline 1.1.1 & DESIGN ENGINEERING TITLEI\&\| & 0 & 0.00 & 0 & 0 & 0.00 & 0.00 & $0.000 \%$ & $0.00 \%$ & 0 & 0 \\
\hline 1.1 .2 & TIRE III INSPECTION & 0 & 0.00 & 0 & 0 & 0.00 & 0.00 & $0.000 \%$ & $0.00 \%$ & $\overline{0}$ & 0 \\
\hline 1.21 & PROJECT MANAGEMENT & 800,213 & 6.34 & 10 & 40 & 0.63 & 253 & $2218 \%$ & $6.34 \%$ & $2 \pi, 129$ & $9,077,342$ \\
\hline 1.22 & CONSTRUCTION MANAGEMENT & 0 & 0.00 & 0 & 0 & 0.00 & 0.00 & $0.000 \%$ & $0.00 \%$ & 0 & 0 \\
\hline 9.3 .13 & SPECLAL CONSTRUCTION & $11,709,711$ & 9274 & 10 & 40 & 9.27 & 37.09 & $32.457 \%$ & $92.74 \%$ & $4,055,297$ & $15,765,008$ \\
\hline \multirow[t]{2}{*}{1.5 .2} & PROCUREMENT FEES & 147,097 & 0.93 & 10 & 40 & 0.09 & 0.37 & $0.325 \%$ & $0.93 \%$ & 40,553 & 157,650 \\
\hline & ESCALATION & 0 & 0.00 & 0 & 0 & 0.00 & 0.00 & $0.000 \%$ & $0.00 \%$ & 0 & 0 \\
\hline & SUBTOTAL & $12,627,021$ & 100.00 & & & & & $35.000 \%$ & & : & \\
\hline & CALCULATED CONTINGENCY & $4,419,457$ & & & & & & & & & \\
\hline & RESULTANT TEC & $17,046,478$ & & & & & & & & & \\
\hline & ROUNDED TEC & $17,000,000$ & & & & & & & & & \\
\hline & PROJECT CONTINGENCY & $4,372,979$ & & & & & & $34.63 \%$ & & & \\
\hline & MANAGEMENT RESERVE & 0 & & & & & & & & & \\
\hline & CONTINGENCY & $4,372.979$ & & & & & & & & & \\
\hline & TOTALESTIMATED COST & $17,000,000$ & & & & & & & & $4,372,979$ & $17,000,000$ \\
\hline
\end{tabular}

CONFIDENCE LEVEL AND ASSUMED RISKS:

The Lockheed ldaho Tectnologies Co. Cost Estimate Contingency Anatysis Model is based on the applied contingency and the assumptions upon which the estimate was predicated. The model is applied with a suggested risk level of $18 \%$ and a level of confidence of $90 \%$ the estimate will fall within the bid range. The Contingency Analysis is based on a weighted average to provide a

$90 \%$ probability of undernun and a $10 \%$ probability of overrun.
CONTINGENCY ANALYSIS GUIDE BY TYPE OF ESTIMATE

Guidelines established by DOEFM 50, Cost Estimating Guide. Vol. 6 ,

Cost Guide, and as presented in the INEL Cost Estimating Guide.

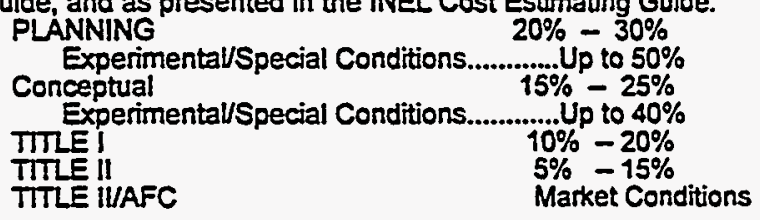


INEL RAD Contaminated Surplus Fucillues Cost Mollel $\Rightarrow D \& D$ Cost

CIP Bin sier Closire

Pioject fine cols120

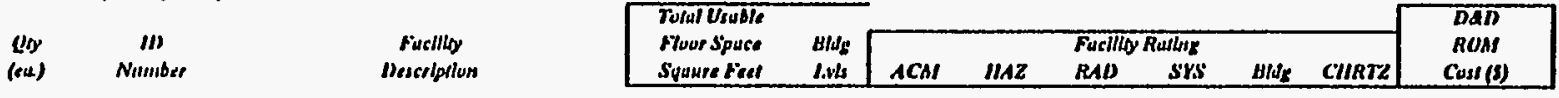

\begin{tabular}{|c|c|c|c|c|c|c|c|c|c|c|c|}
\hline 7 & Seokolo T & Ventilition, Instr. \& Control Bldg (VIC) & 2,400 & 1 & NA & $\mathbf{L}$ & NA & $\mathbf{L}$ & $\bar{S}$ & t & 069,133 \\
\hline 7 & & Confinemmonl Enclosures (lop of $\operatorname{lin} 5$ Sols) & 1,600 & $i$ & NA & $A$ & $A$ & L & S & $\frac{G}{L}$ & $0,248,128$ \\
\hline 7 & SoONolo 2 & gindge Crane & $\frac{300}{300}$ & $i$ & NA & L & H & L & s & L & $\frac{0,160,1,00}{360,370}$ \\
\hline$T$ & SooNolo 3 & HVAC EquipmonUSystem & 5.800 & 1 & NA & L & $\bar{A}$ & $\mathbf{L}$ & $\mathbf{S}$ & $\mathbf{L}$ & 440.049 \\
\hline$T$ & SOQNOLO 4 & Jumper Rourioval piphog (shelded) & 420 & $T$ & NA & A & H & $\bar{L}$ & c & $\bar{L}$ & 100,484 \\
\hline 1 & SooNoto 6 & Remola Core Drilling Platom/Saw, elc. & 260 & 1 & NA & H & $\mathrm{H}$ & $\mathbf{L}$ & $\mathbf{S}$ & $\bar{L}$ & 65.966 \\
\hline 1 & Allowance & Femolo Wolding and Cuitting Equipmont & हक & 1 & NA & H & H & $\bar{L}$ & $\mathbf{s}$ & $\bar{L}$ & 111.932 \\
\hline 7 & Sod Noto 6 & Vortical Doployment Apparalus & 160 & 1 & NA & H & H & $\bar{L}$ & s & $\mathrm{L}$ & 235.057 \\
\hline 100 & Soo Note 7 & Shiolding and Ribor Plugs & 4 & $\frac{1}{1}$ & NA & H & $\frac{\pi}{H}$ & $\frac{L}{L}$ & $\frac{\mathrm{c}}{\mathrm{c}}$ & $\frac{L}{L}$ & $\frac{1}{118,784}$ \\
\hline 2 & Allowanco & CO2 Elasulng Equigmont & 600 & 1 & NA & H & H & $\bar{L}$ & $\mathbf{s}$ & L & 223,684 \\
\hline T & So0 Noto 8 & Rolfioval Linos & 2.310 & $\frac{1}{1}$ & NA & H & $\bar{H}$ & L & $\mathbf{S}$ & $\mathbf{L}$ & 373,116 \\
\hline 9 & Allowanco & Control Consoles, Cameras \& LIghuing & 1,000 & 1 & NA & $\mathrm{L}$ & $L$ & L & $\mathbf{S}$ & $L$ & 107.934 \\
\hline 8 & Allowance & Groutung Manilolds - Cloan & 300 & $i$ & NA & L & L & ti & $\overline{8}$ & $\bar{L}$ & 259,043 \\
\hline 8 & Allowanco & Grouling Manilolds -Class C & 300 & 1 & NA & H & $\bar{H}$ & I & $\mathbf{s}$ & $\mathrm{L}$ & 637,274 \\
\hline 7 & Allowance & LOUA's & 150 & 1 & NA & A & H & t. & $\mathbf{s}$ & $\bar{L}$ & 2000,873 \\
\hline 7 & Allowanco & Plpo Crowlor Robols & 150 & 1 & NA & A & H & ti & s & L & 209.073 \\
\hline 53 & Allowance & Iraclor Robols & 260 & i & NA & $\bar{A}$ & H & $\mathrm{L}$ & $\frac{5}{s}$ & $\frac{L}{L}$ & $2,648,393$ \\
\hline 1 & Allowance & Misc. Robol whro and hose lines & 1.000 & 1 & NA & $A$ & H & I & $\mathbf{s}$ & $L$ & 109,070 \\
\hline & S00 Nolog & Wasto Disasol & & & & & & & & & \\
\hline & & Sollawasto (n CF) & 500 & & & & & & & & 6.60 \\
\hline & & Radioaclivo Waslo (In CF) & 10,000 & & & & & & & & $3,000,000$ \\
\hline & & Mlxed Wasto (in CF) & 2.200 & & & & & & & & $\frac{0,0,000}{275,000}$ \\
\hline & & & & & & & & & & & \\
\hline & & & & & & & & & & & \\
\hline & & & & & & & & & & & \\
\hline & & SUbTOLIICP B BIN SETCLOSUAE & & & & & & & & & $\{1,700,711\}$ \\
\hline
\end{tabular}

\section{NoTES:}

1. Assumed Ihal lacilly will havo low lovols of hazardous material, no rad conlaminallon, and no asbostos

2. Assune $40^{\circ}$ accoss by $45^{\circ}$ high $\times 2^{\prime}$ w tor ralls plus trolloy $\left(5^{\circ} \times 5^{\prime} \times 6^{\prime}\right)$

3 - Assumo 7 Alr handlors $\left(5^{\circ} \times 5^{\circ} \times 6^{\prime}\right)$, 3 Exhsusl Fans $\left(4^{\circ} \times 4^{\prime} \times 3^{\prime}\right)$, and 63 HEPA fillers $\left(6^{\prime} \times 8^{\prime} \times 2^{\prime \prime}\right)$

4 - Assume $210 \mathrm{LF} \times 2$ ' wide concrete

5. Assume plattorm $\left(6^{6} \times 6^{\circ} \times 6\right)$ plus saw (100 8D)

6. Assume $5^{\circ} \times 5^{\prime} \times 6^{\prime}$

7. Assume $2 \times 2 \times 2^{\prime}$ plugs

9. Wastu Disposal Quentitios wero provided by EDF

Assumbo tho following Wasto Disposal Unit Costs:

$\begin{array}{ll}\text { Industial Landfill } & \$ 1.08 / d \\ \text { Low Lovel Wasto Reposilory } & \$ 1251 / 1 \\ \text { Rad Wasto } & \end{array}$




\section{G\&A/PIF ADDER CALCULATION SHEET \\ ICPP BIN SET CLOSURE \\ CLOSURE TO RCRA LANDFILL STANDARDS; CLASS C FILL; UNESCALATED \\ D\&D OF EQUIPMENT}

PROCUREMENT FEE:

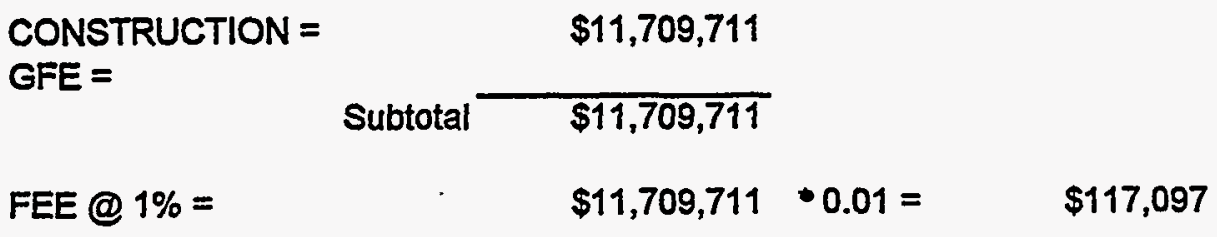

G\&A @ 23\% (with a ceiling of $\$ 500,000$ imposed per year , 1 Year)

\begin{tabular}{lr} 
CONSTRUCTION OR & \\
CEILING * \# OF YEARS $=$ & $\$ 500,000$ \\
GFE = & $\$ 0$ \\
PROCUREMENT FEE = & $\$ 117,097$ \\
\cline { 2 - 2 } & Subtotal
\end{tabular}

FEE @ 23\% = $\quad \$ 617,097 * 0.23=\quad \$ 141,932$

PIF @ 5.5\%

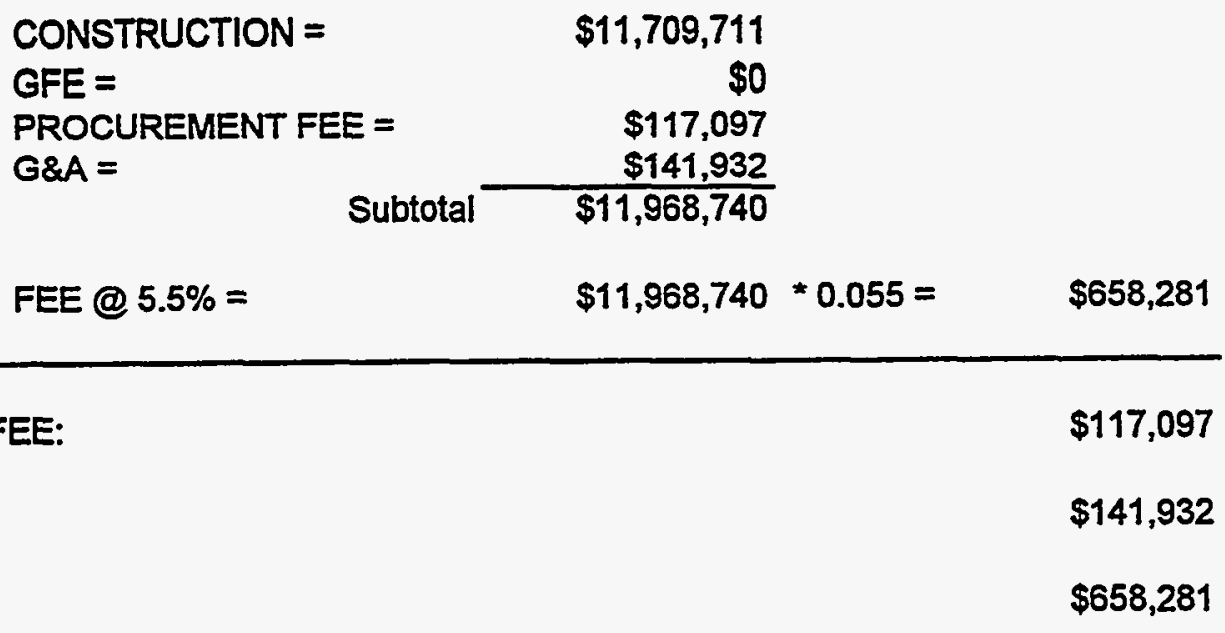

TOTAL PIF: 


\section{COST ESTIMATE SUMMARY}

\section{ESCALATED}

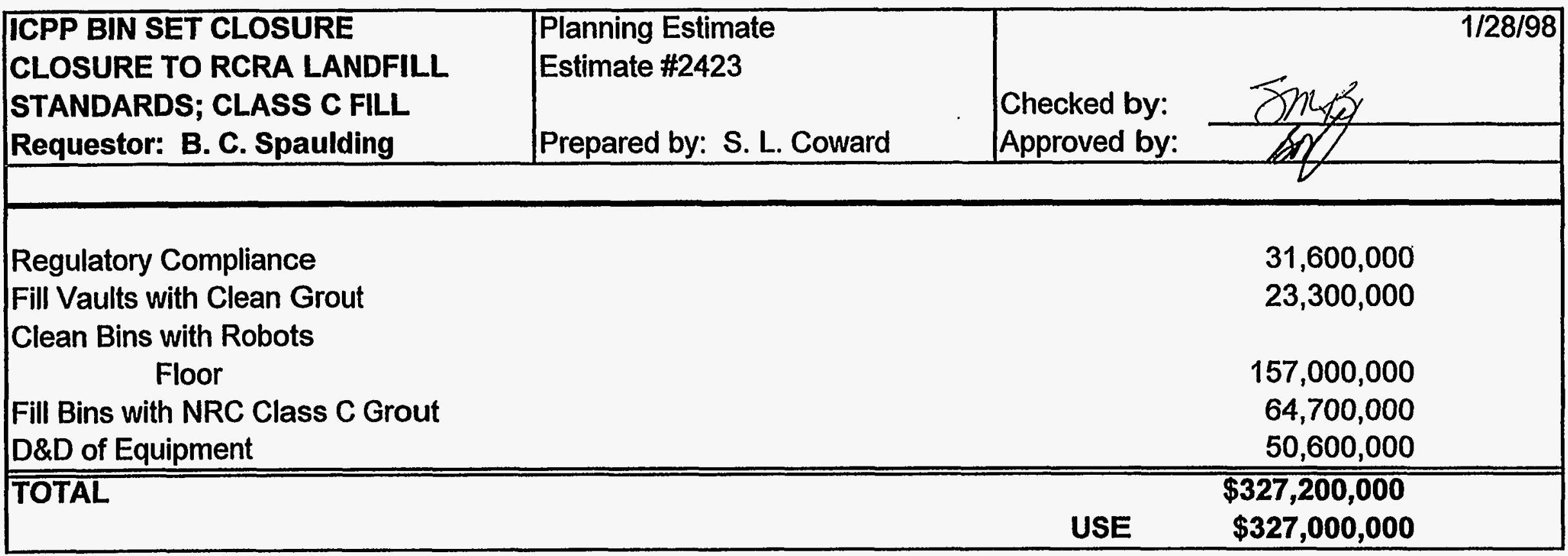


Lockheed Martin Idaho Technologies Co. Rey, 6-96

PROJect naME: Permitting/Documentation

Risk Based - NRC Class C

INEEL ICPP

LOCATION 1:

REQUESTOR:
COST ESTIMATE SUMMARY

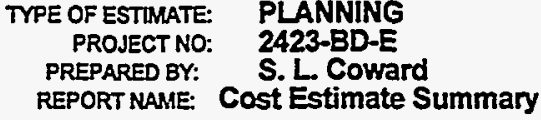

TIME: 11:02:58

CHECKED BY:

APPR'D BY:

\begin{tabular}{|c|c|c|c|c|}
\hline $\begin{array}{l}\text { WBS } \\
\text { Element }\end{array}$ & Cost Estimate Element & $\begin{array}{c}\text { Total } \\
\text { Unescalated }\end{array}$ & Escalation & $\begin{array}{l}\text { Total } \\
\text { Incl Escalation }\end{array}$ \\
\hline $\begin{array}{l}\frac{1.1}{1.1 .1} \\
\frac{1.2}{1.2 .1} \\
1.2 .2 \\
\frac{1.3}{1.3 .1} \\
1.5 .2\end{array}$ & $\begin{array}{l}\text { CONCEPTUAL } \\
\text { CONCEPTUAL DESIGN } \\
\text { MANAGEMENT } \\
\text { PM FOR PROJECT DEVELOPMENT } \\
\text { PROJECT EXECUTION } \\
\text { PERMITTING } \\
\text { PERMITTING } \\
\text { PROCUREMENT FEES }\end{array}$ & $\begin{array}{r}1,942,993 \\
477,848 \\
\\
12,385,106 \\
123,851\end{array}$ & $\begin{array}{c}0 \\
1,107,506 \\
272,373 \\
7,059,510 \\
70,595\end{array}$ & $\begin{array}{l}\gg>\quad \text { s0 } \\
\gg \frac{\$ 3,800,720}{3,050,499} \\
750,221 \\
\gg \frac{\$ 19,444,616}{19,444,616} \\
\gg \quad \underline{\$ 194,446}\end{array}$ \\
\hline & $\begin{array}{l}\text { SUBTOTAL INCLUDING ESCALATION } \\
\text { PROJECT CONTINGENCY }\end{array}$ & $14,929,798$ & $8,509,984$ & $\gg \$ 23,439,782$ \\
\hline & MANAGEMENT RESERVE- & & & $\$ 1,963,906$ \\
\hline & CONTINGENCY - & & & $\$ 6,196,312$ \\
\hline & TOTAL ESTIMATED COST - & & & $\$ 31,600,000$ \\
\hline
\end{tabular}

PROJECT COST PARAMETERS

EDI AS A $\%$ OF CONST. + GFE $=0.00 \%$ 


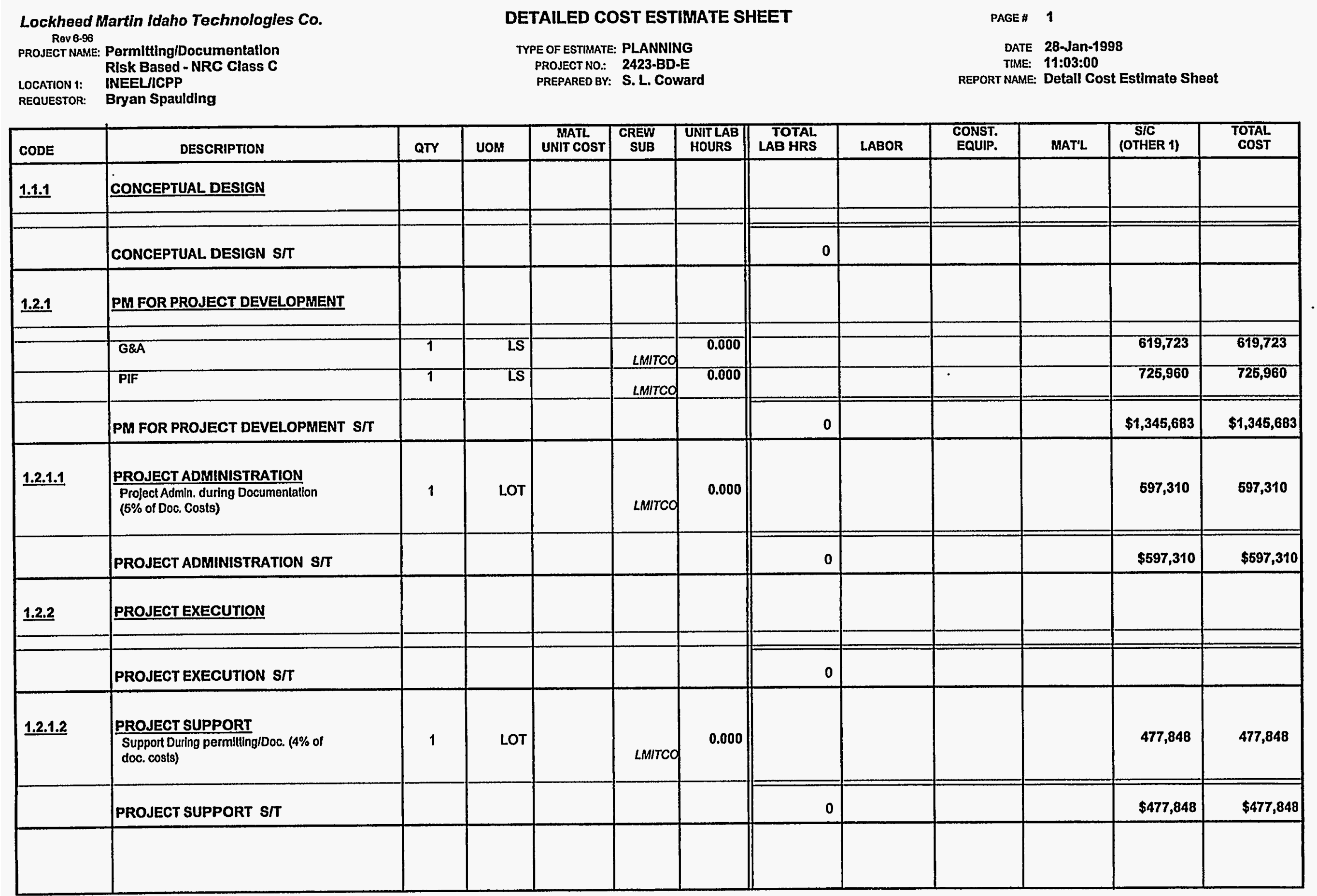


Lockheed Martin Idaho Technologies Co.

Rev6-96

PROJECT NAME: Permitting/Documentation

Risk Based - NRC Class C

LOCATION 1: INEELICPP

REQUESTOR: Bryan Spaulding
CONTINGENCY ANALYSIS

TYPE OF ESTIMATE:

PROJECT NO:

PREPARED BY:
DATE: 28-Jan-1998

TME: 11:02:47

REPORT NAME: Contingency Analysis

\begin{tabular}{|c|c|c|c|c|c|c|c|c|c|c|c|}
\hline \multicolumn{9}{|c|}{ PROBABLE \% VARIATION } & \multicolumn{2}{|c|}{$\begin{array}{c}\text { PROJECT } \\
\text { CONTINGENCY }\end{array}$} & \multirow{3}{*}{$\begin{array}{l}\text { SUMMARY } \\
\begin{array}{l}\text { Total Cost } \\
\text { by Element }\end{array}\end{array}$} \\
\hline $\begin{array}{l}\text { WBS } \\
\text { Element }\end{array}$ & \multirow{2}{*}{ Cost Estimate Element } & \multirow{2}{*}{$\begin{array}{c}\text { Total Cost w/o } \\
\text { Contingency }\end{array}$} & \multirow[t]{2}{*}{$\begin{array}{l}\% \text { Total } \\
\text { Cost }\end{array}$} & \multicolumn{2}{|c|}{$\begin{array}{l}\text { Prob. \% Var. } \\
\text { From Est. }\end{array}$} & \multicolumn{2}{|c|}{ Wt \% of Prob. } & \multirow{2}{*}{ Contingency } & \multirow{2}{*}{$\%$} & \multirow{2}{*}{ Cost } & \\
\hline & & & & - & + & - & 4 & & & & \\
\hline 1.1 .1 & CONCEPTUAL DESIGN & 0 & 0.00 & 10 & 40 & 0.00 & 0.00 & $0.000 \%$ & $0.00 \%$ & 0 & $\mathbf{0}$ \\
\hline 1.2 .1 & PM FOR PROJECT DEVELOPMENT & $1,942,993$ & 8.29 & 10 & 40 & 0.83 & 3.32 & $2901 \%$ & $8.29 \%$ & 676,425 & $2,619,418$ \\
\hline 1.2 .2 & PROJECT EXECUTION & 477,848 & 2.04 & 10 & 40 & 0.20 & 0.82 & $0.714 \%$ & $2.04 \%$ & 166,356 & 644,204 \\
\hline 1.3 .1 & PERMITING & $12,385,106$ & 52.84 & 10 & 40 & 5.28 & 21.14 & $18.493 \%$ & $52.84 \%$ & $4,311,694$ & $16,696,800$ \\
\hline \multirow[t]{2}{*}{1.5 .2} & PROCUREMENT FEES & 123,851 & 0.53 & 10 & 40 & 0.05 & 0.21 & $0.185 \%$ & $0.53 \%$ & 43,117 & 166,968 \\
\hline & ESCALATION & $8,509,984$ & 36.31 & 10 & 40 & 3.63 & 14.52 & $12.707 \%$ & $36.31 \%$ & $2,962,626$ & $11,472,610$ \\
\hline & SUBTOTAL & $23,439,782$ & 100.00 & & & & & $35.000 \%$ & & & \\
\hline & CALCULATED CONTINGENCY & $8,203,924$ & & & & & & & & & \\
\hline & RESULTANT TEC & $31,643,706$ & & & & & & & & & \\
\hline & ROUNDED TEC & $31,600,000$ & & & & & & & & & \\
\hline & PROJECT CONTINGENCY & $8,160,218$ & & & & & & $34.81 \%$ & & & \\
\hline & MANAGEMENT RESERVE & $1,963,906$ & & & & & & & & & \\
\hline & CONTINGENCY & $6,196,312$ & & & & & & & & & \\
\hline & TOTAL ESTIMATED COST & $31,600,000$ & & & & & & & & $8,160,218$ & $31,600,000$ \\
\hline
\end{tabular}

CONFIDENCE LEVEL AND ASSUMED RISKS:

The Lockheed Idaho Technologies Co. Cost Estimate Contingency Analysis Model is based on the applied contingency and the assumptions upon which the estimate was predicated. The model is applied with a suggested risk level of $18 \%$ and a level of confidence of $90 \%$ the estimate will fall within the bid range. The Contingency Analysis is based on a weighted average to provide a $90 \%$ probability of underrun and a $10 \%$ probability of overun.
CONTINGENCY ANALYSIS GUIDE BY TYPE OF ESTIMATE

Guidelines established by DOE/FM 50, Cost Estimating Guide, Vol. 6 , cost Guide, and as presented in the INEL Cost Estimating Guide. PLANNING

Experimental/Special Conditions............. Up to $50 \%$ Conceptual Experimental/Special Conditions............. - to to $40 \%$ TITLE I

TITLE II

TITLE IVIAFC $10 \%-20 \%$

$5 \%-15 \%$ Market Conditions 


\section{G\&APIF ADDER CALCULATION SHEET \\ ICPP BIN SET CLOSURE \\ CLOSURE TO RCRA LANDFILL STANDARDS; CLASS C FILL; ESCALATED \\ REGULATORY COMPLIANCE}

PROCUREMENT FEE:

$\begin{aligned} & \text { CONSTRUCTION = } \\ & \text { GFE }=\end{aligned} \quad \begin{aligned} & \$ 19,444,616 \\ & \text { Subtotal }\end{aligned} \quad \begin{aligned} & \$ 19,444,616 \\ & \text { FEE @ } 1 \%=\end{aligned} \quad \$ 19,444,616 * 0.01=\quad \$ 194,446$

G\&A @ $23 \%$ (with a ceiling of $\$ 500,000$ imposed per year, 5 yrs)

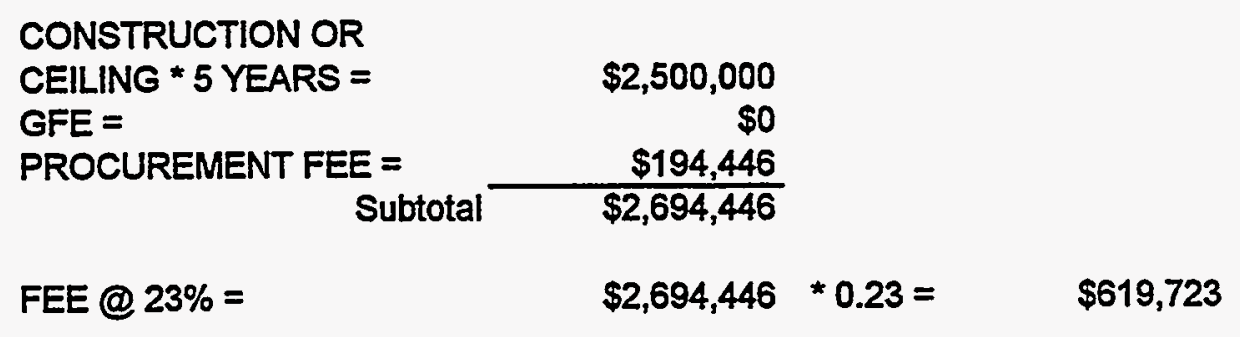

PIF @ 5.5\%

CONSTRUCTION =
GFE =
PROCUREMENT FEE =
G\&A =

Subtotal

FEE @ $5.5 \%=$
$\$ 12,385,106$

$\$ 0$

$\$ 194,446$

$\$ 619,723$

$\$ 13,199,275$

TOTAL PROCUREMENT FEE:

$\$ 13,199,275 \cdot 0.055=$

$\$ 725,960$

TOTAL G\&A FEE:

$\$ 194,446$

$\$ 619,723$

TOTAL PIF:

$\$ 725,960$ 
Lockheed Martin Idaho Technologies Co. Rev, 6-96 PROJECT NAME: ICPP Bin Set Closure (EIS Stud PROJET LOCATION 1: REQUESTOR: Place Clean Grout in Vault ICPP

Bryan Spaulding
COST ESTIMATE SUMMARY

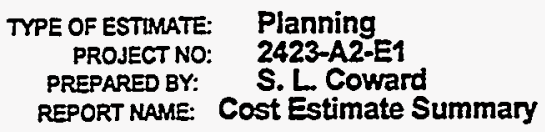

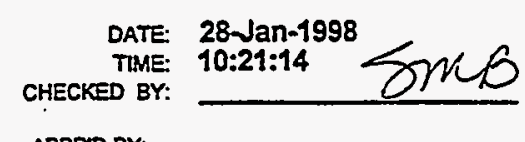

APPRD BY:

\begin{tabular}{|c|c|c|c|c|}
\hline $\begin{array}{l}\text { WBS } \\
\text { Element }\end{array}$ & Cost Estimate Element & $\begin{array}{c}\text { Total } \\
\text { Unescalated }\end{array}$ & Escalation & $\begin{array}{c}\text { Total } \\
\text { Incl Escalation }\end{array}$ \\
\hline 1.1 & ENGINEERING, DESIGN AND INSPECTION & & & \multirow{11}{*}{ 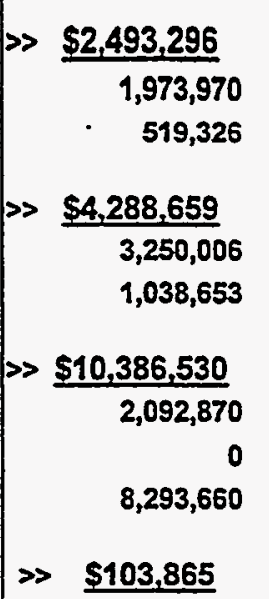 } \\
\hline 1.1.1 & DESIGN ENGINEERING & $1,324,812$ & 649,158 & \\
\hline 1.1 .2 & TITLE III INSPECTION & 264,962 & 254,364 & \\
\hline 1.2 & MANAGEMENT COSTS & & & \\
\hline 1.2 .1 & PROJECT MANAGEMENT & $1,701,574$ & $1,548,432$ & \\
\hline 1.2 .2 & CONSTRUCTION MANAGEMENT & 529,925 & 508,728 & \\
\hline 1.3 & CONSTRUCTION & & & \\
\hline 1.3.1 & GENERAL CONDITIONS & $1,067,791$ & $1,025,079$ & \\
\hline 1.3.2 & SITEWORK & & 0 & \\
\hline 1.3.3 & CONCRETE & $4,231,459$ & $4,062,201$ & \\
\hline \multirow[t]{3}{*}{ 1.5.2 } & PROCUREMENT FEES & 52,992 & 50,873 & \\
\hline & SUBTOTAL INCLUDING ESCALATION & $9,173,515$ & $8,098,835$ & \multirow[t]{2}{*}{ >> $\$ 17,272,350$} \\
\hline & PROJECT CONTINGENCY & & & \\
\hline \multicolumn{2}{|r|}{ MANAGEMENT RESERVE- } & & & $\gg \quad \$ 1,049,039$ \\
\hline \multicolumn{2}{|r|}{ CONTINGENCY- } & & & $\gg \quad \$ 4,978,611$ \\
\hline \multicolumn{2}{|r|}{ TOTAL ESTIMATED COST - } & & & $\gg \$ 23,300,000$ \\
\hline
\end{tabular}

\section{PROJECT COST PARAMETERS}

EDI AS A \% OF CONST. + GFE $=24.00 \%$ 
Lockheed Martin Idaho Technologles Co. Rav 6.98

PROJECT NAME: ICPP Bin Set Closure (EIS Study)

LOCATION 1: Place Clean Grout in Vault

REQUESTOR: Bryan Spaulding

\section{DETAILED COST ESTIMATE SHEET}

TYPE OF EstimATE: Planning

PROJECT NO.: 2423-A2-E1

PREPARED BY: S. L. Coward
PAGE* 1

DATE 28-Jan-1998

TIME: 10:21:16

REPORT NAME: Detall Cost Estimate Shee

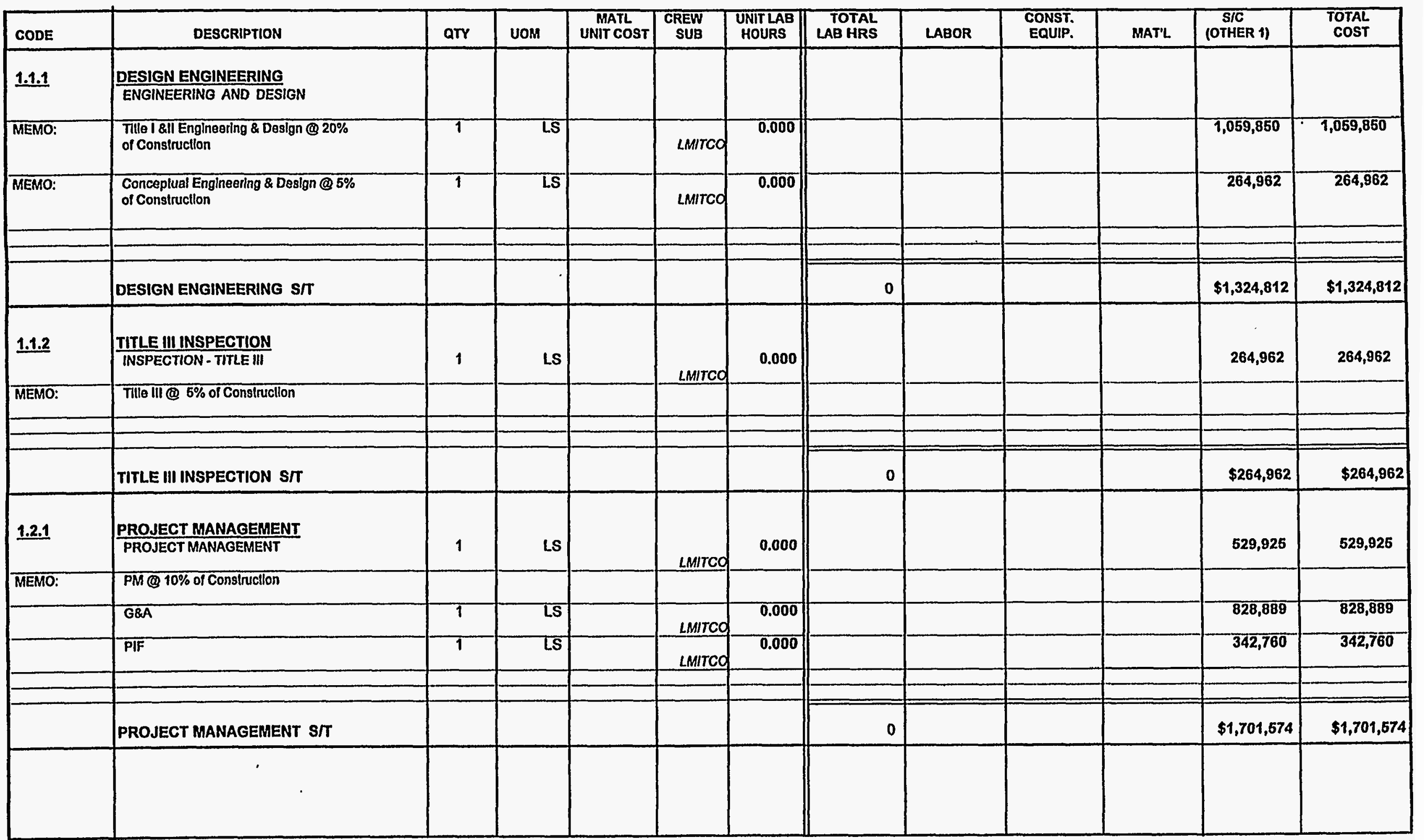


Lockheed Martin Idaho Technologies Co. Rev 6.93

PROJeCt NAME: ICPP Bin Set Closure (EIS Study)

Place Clean Grout in Vault

LOCATION 1: ICPP

REQUESTOR: Bryan Spaulding

\section{DETAILED COST ESTIMATE SHEET}

TYPE OF ESTIMATE: Planning

PROJECT NO.: 2423-A2-E1

PREPARED BY: S. L. Coward
PAGE * 3

DATE 28-Jan-1998

TIME: 10:21:16

report NAME: Detail Cost Estimate Sheet

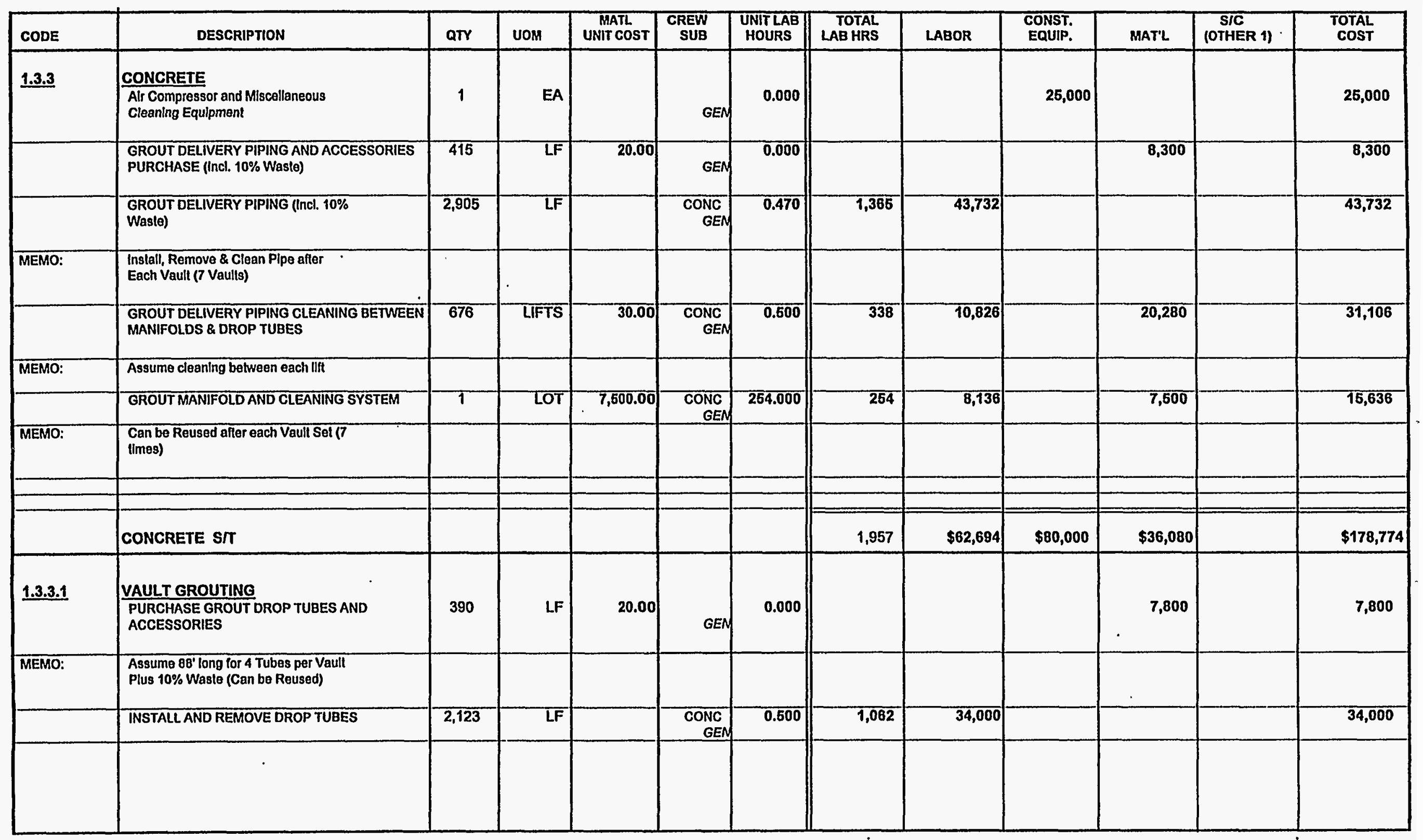




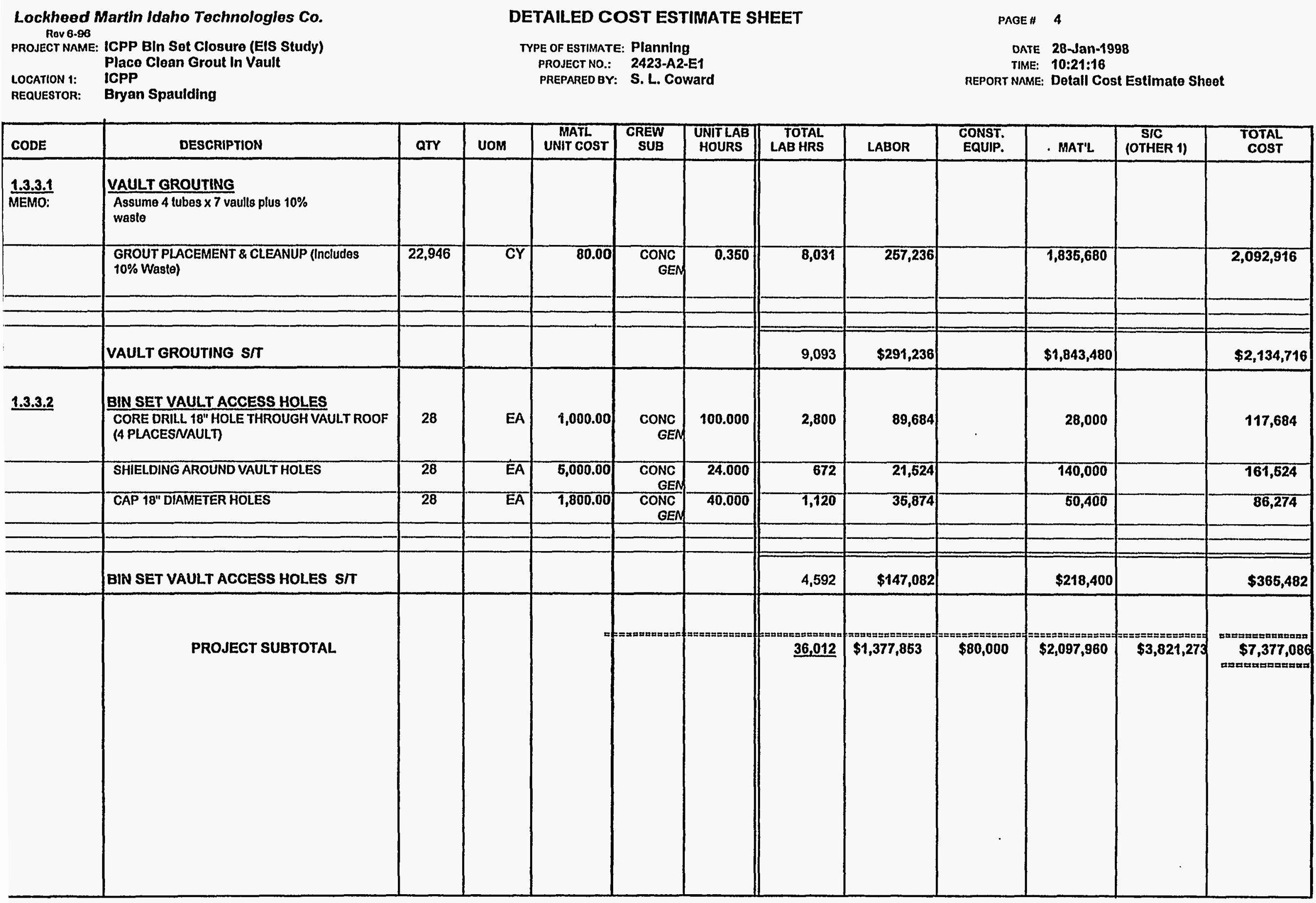


Lockheed Martin Idaho Technologies Co.

Rev 6-96

PROJECT NAME: ICPP Bin Set Closure (EIS Stud Place Clean Grout in Vault

I ICATION 1: ICPP

:QUESTOR: Bryan Spaulding
CONTINGENCY ANALYSIS

TYPE OF ESTIMATE:

PROJECT NO:

PREPARED BY:

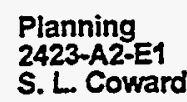

DATE: 28 -Jan-1998

TIME: 10:20:01

REPORT NAME: Contingency Analysis

\begin{tabular}{|c|c|c|c|c|c|c|c|c|c|c|c|}
\hline \multicolumn{9}{|c|}{ PROBABLE \% VARIATION } & \multicolumn{2}{|c|}{$\begin{array}{l}\text { PROJECT } \\
\text { CONTINGENCY }\end{array}$} & \multirow{3}{*}{$\begin{array}{l}\text { SUMMARY } \\
\begin{array}{l}\text { Total Cost } \\
\text { by Element }\end{array}\end{array}$} \\
\hline \multirow[t]{2}{*}{$\begin{array}{l}\text { WBS } \\
\text { Element }\end{array}$} & \multirow{2}{*}{ Cost Estimate Element } & \multirow{2}{*}{$\begin{array}{l}\text { Total Cost w/o } \\
\text { Contingency }\end{array}$} & \multirow[t]{2}{*}{$\begin{array}{l}\% \text { Total } \\
\text { Cost }\end{array}$} & \multicolumn{2}{|c|}{$\begin{array}{l}\text { Prob. \% Var. } \\
\text { From Est. }\end{array}$} & \multicolumn{2}{|c|}{ Wt \% of Prob. } & \multirow{2}{*}{ Contingency } & \multirow{2}{*}{$\%$} & \multirow{2}{*}{ Cost } & \\
\hline & & & & - & + & $=$ & + & & & & \\
\hline .1 .1 & DESIGN ENGINEERING & $1,324,812$ & 7.67 & 10 & 40 & 0.77 & 3.07 & $2.685 \%$ & $7.67 \%$ & 462,329 & $1,787,141$ \\
\hline 1.1 .2 & TITLE III INSPECTION & 264,962 & 1.53 & 10 & 40 & 0.15 & 0.61 & $0.537 \%$ & $1.53 \%$ & 92,465 & 357,428 \\
\hline 1.2 .1 & PROJECT MANAGEMENT & $1,701,574$ & 9.85 & 10 & 40 & 0.99 & 3.94 & $3.448 \%$ & $9.85 \%$ & 593,810 & $2,295,384$ \\
\hline .22 & CONSTRUCTION MANAGEMENT & 529,925 & 3.07 & 10 & 40 & 0.39 & 1.23 & 1.074\% & $3.07 \%$ & 184,932 & 714,857 \\
\hline .3 .1 & GENERAL CONDMINS & $1,067,791$ & 6.18 & 10 & 40 & 0.62 & 247 & $2.164 \%$ & $6.18 \%$ & 372,634 & $1,440,425$ \\
\hline 1.3 .2 & STIEWORK & 0 & 0.00 & 0 & 0 & 0.00 & 0.00 & $0.000 \%$ & $0.00 \%$ & 0 & 0 \\
\hline .3 .3 & CONCREIE & $4,231,459$ & 24.50 & 10 & 40 & 2.45 & 9.80 & $8.574 \%$ & $24.50 \%$ & $1,476,681$ & $5,708,140$ \\
\hline 5.5 & PROCUREMENT FEES & 52,992 & 0.31 & 10 & 40 & 0.03 & 0.12 & $0.907 \%$ & $0.31 \%$ & 18,493 & 71,485 \\
\hline & ESCALATION & $8,098,835$ & 46.89 & 10 & 40 & 4.69 & 18.76 & $16.411 \%$ & $46.89 \%$ & $2,826,305$ & $10,925,140$ \\
\hline & SUBTOTAL & $17,272,350$ & 100.00 & & & & & $35.000 \%$ & & & \\
\hline & CALCULATED CONTINGENCY & $6,045,323$ & & & & & & & & & \\
\hline & RESULTANT TEC & $23,317,673$ & & & & & & & & & \\
\hline & ROUNDED TEC & $23,300,000$ & & & & & & & & & \\
\hline & PROJECT CONTINGENCY & $6,027,650$ & & & & & & $34.90 \%$ & & & \\
\hline & MANAGEMENT RESERVE & $1,049,039$ & & & & & & & & & \\
\hline & CONTINGENCY & $4,978,611$ & & & & & & & & & \\
\hline & TOTAL ESTIMATED COST & $23,300,000$ & & & & & & & & $6,027,650$ & $23,300,000$ \\
\hline
\end{tabular}

CONFIDENCE LEVEL AND ASSUMED RISKS:

The Lockheed Idaho Technologies Co. Cost Estimate Contingency Analysis Model is based on the applied contingency and the assumptions upon which the estimate was predicated. The model is applied with a suggested risk level of $18 \%$ and a level of confidence of $90 \%$ the estimate will fall within the bid range. The Contingency Analysis is based on a weighted average to provide a $90 \%$ probability of underrun and a $10 \%$ probability of ovemun.
CONTINGENCY ANALYSIS GUIDE BY TYPE OF ESTIMATE

Guidelines established by DOEJFM 50, Cost Estimating Guide, Vol. 6, Cost Guide, and as presented in the INEL Cost Estimating Guide. PLANNING

Conceptual

TITLE I

TITLE II

TITLE IUAFC
$20 \%-30 \%$

$15 \%-25 \%$

...

$5 \%-15 \%$

Market Conditions 


\section{G\&APIF ADDER CALCULATION SHEET \\ ICPP BIN SET CLOSURE \\ CLOSURE TO RCRA LANDFILL STANDARDS; CLASS C FILL; ESCALATED}

FILL VAULTS W/ CLEAN GROUT

PROCUREMENT FEE:

CONSTRUCTION $=$
GFE $=$$\quad \begin{aligned} & \$ 10,386,530 \\ & \end{aligned}$

FEE @ 1\% =

$\$ 10,386,530 \cdot 0.01=$

$\$ 103,865$

G\&A @ 23\% (with a ceiling of $\$ 500,000$ imposed per year, 7 yrs)

CONSTRUCTION OR

CEILING * 7 YEARS =

$\$ 3,500,000$

GFE =

PROCUREMENT FEE $=$ $\$ 103,865$

Subtotal $\$ 3,603,865$

FEE @ 23\% =

$\$ 3,603,865 \cdot 0.23=$

$\$ 828,889$

PIF @ 5.5\%

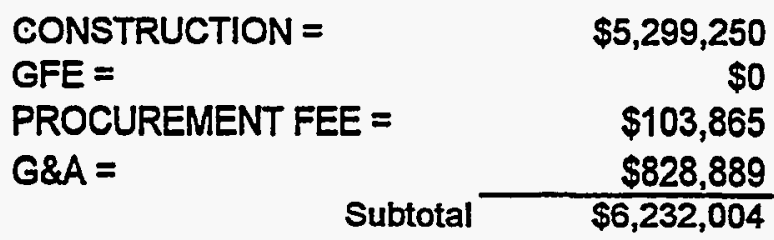

FEE @ $5.5 \%=$

$\$ 6,232,004 \cdot 0.055=$

$\$ 342,760$

TOTAL PROCUREMENT FEE:

$\$ 103,865$

TOTAL G\&A FEE:

$\$ 828,889$

TOTAL PIF:

$\$ 342,760$ 
Lockheed Martin Idaho Technologies Co. Rov. 6-96 PROJECT NAME: ICPP Bin Set Closure (EIS Stud Tractor (RCRA Estimates) LOCATION 1: ICPP REQUESTOR: Bryan Spaulding
COST ESTIMATE SUMMARY

TYPE OF ESTIMATE: Planning PROJECT NO: 2423-C-E1 PREPARED BY: S. L Coward REPORT NAME: Cost Estimate Summary
CHECKED BY

DATE: 28-Jan-1998

TTME. 09:54:26

APPRD BY:

\begin{tabular}{|c|c|c|c|c|}
\hline $\begin{array}{l}\text { WBS } \\
\text { Element }\end{array}$ & Cost Estimate Element & $\begin{array}{c}\text { Total } \\
\text { Unescalated }\end{array}$ & Escalation & $\begin{array}{c}\text { Total } \\
\text { Incl Escalation }\end{array}$ \\
\hline $\begin{array}{l}\frac{1.1}{1.1 .1} \\
1.1 .2 \\
\frac{1.2}{1.2 .1} \\
1.2 .2 \\
\frac{1.3}{1.3 .1} \\
1.3 .13 \\
1.5 .2\end{array}$ & $\begin{array}{l}\text { ENGINEERING, DESIGN AND INSPECTION } \\
\text { DESIGN ENGINEERING TITLE I \& II } \\
\text { TITLE III INSPECTION } \\
\text { MANAGEMENT COSTS } \\
\text { PROJECT MANAGEMENT } \\
\text { CONSTRUCTION MANAGEMENT } \\
\text { CONSTRUCTION } \\
\text { GENERAL CONDITIONS } \\
\text { SPECIAL CONSTRUCTION } \\
\text { PROCUREMENT FEES }\end{array}$ & $\begin{array}{r}6,128,131 \\
2,042,710 \\
7,783,994 \\
4,085,421 \\
\\
6,840,414 \\
34,013,797 \\
408,542\end{array}$ & $\begin{array}{r}2,757,659 \\
1,961,002 \\
6,694,235 \\
3,922,004 \\
\\
6,566,797 \\
32,653,245 \\
392,200\end{array}$ & $\begin{array}{r}\text { P12,889,502 } \\
8,885,790 \\
4,003,712\end{array}$ \\
\hline & $\begin{array}{l}\text { SUBTOTAL INCLUDING ESCALATION } \\
\text { PROJECT CONTINGENCY }\end{array}$ & $61,303,009^{\circ}$ & $54,947,142$ & $\gg \$ 116,250,151$ \\
\hline & MANAGEMENT RESERVE- & & & $\$ 8,087,499$ \\
\hline & CONTINGENCY & & & $\gg \quad \$ 32,662,350$ \\
\hline & TOTAL ESTIMATED COST & & & $\gg \$ 157,000,000$ \\
\hline
\end{tabular}

\section{PROJECT COST PARAMETERS}


Lockhoed Martin Idaho Technologies Co. Rov 6.96

PROJECT NAME: ICPP Bin Set Closuro (EIS Study) Tractor (RCRA Estimates)

ICPP

REQUESTOR: Bryan Spaulding

\section{DETAILED COST ESTIMATE SHEET}

TYPE OF estimate: Planning

PROJECT NO: $\quad$ 2423-C-E1

PREPARED BY: $\mathbf{S}$. L. Coward
PAGE \# 2

DATE 28-Jan-1998

TIME: 10:09:30

REPORT NAME: Detall Cost Estimate Sheet

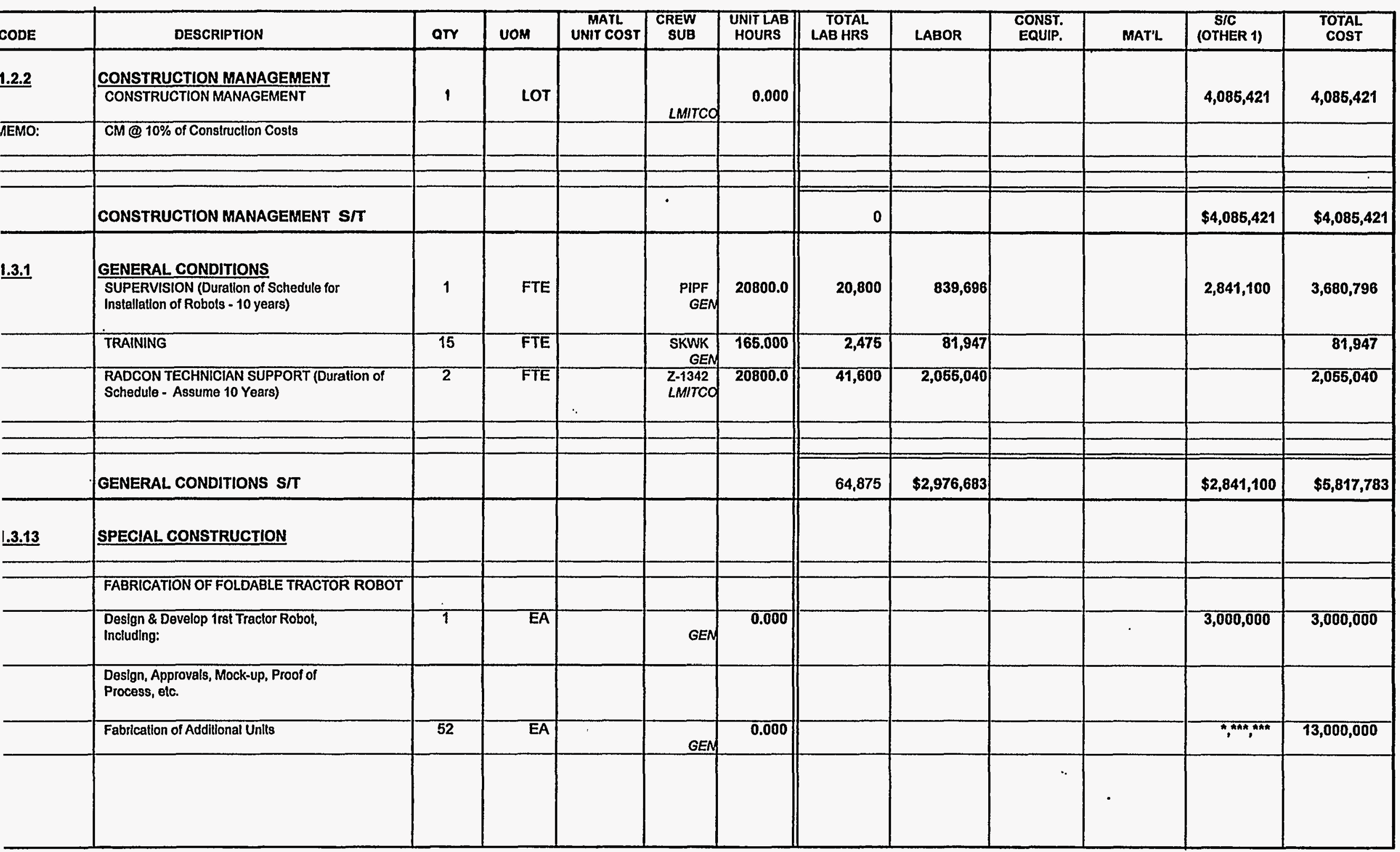


Lockheed Martin Idaho Technologies Co.

PROJECT NAME: ICPP Bin Set Closure (EIS Stud Tractor (RCRA Estimates)

LOCATION 1: REQUESTOR:
ICPP

Bryan Spaulding

\section{CONTINGENCY ANALYSIS}

$\begin{array}{cl}\text { TYPE OF ESTMMATE: } & \text { Planning } \\ \text { PROJECT NO: } & 2423-C-E 1 \\ \text { PREPARED BY: } & \text { S. L. Coward }\end{array}$

DATE: 28-Jan-1998

TMEE: 09:54:36

REPORT NAME: Contingency Analysis

\begin{tabular}{|c|c|c|c|c|c|c|c|c|c|c|c|}
\hline \multicolumn{9}{|c|}{ PROBABLE \% VARIATION } & \multicolumn{2}{|c|}{$\begin{array}{l}\text { PROJECT } \\
\text { CONTINGENCY }\end{array}$} & \multirow{3}{*}{$\begin{array}{l}\text { SUMMARY } \\
\begin{array}{l}\text { Total cost } \\
\text { by Element }\end{array}\end{array}$} \\
\hline \multirow[t]{2}{*}{$\begin{array}{l}\text { WBS } \\
\text { Element }\end{array}$} & \multirow{2}{*}{ Cost Estimate Element } & \multirow{2}{*}{$\begin{array}{l}\text { Total Cost w/o } \\
\text { Contingency }\end{array}$} & \multirow[t]{2}{*}{$\begin{array}{l}\text { \% Total } \\
\text { Cost }\end{array}$} & \multicolumn{2}{|c|}{$\begin{array}{l}\text { Prob. \% Var. } \\
\text { From Est }\end{array}$} & \multicolumn{2}{|c|}{ Wt \% of Prob. } & \multirow[t]{2}{*}{ Contingency } & \multirow{2}{*}{$\%$} & \multirow{2}{*}{ Cost } & \\
\hline & & & & - & + & - & + & & & & \\
\hline 1.1.1 & DESIGN ENGINEERING TILE I\& $\|$ & $6,128,131$ & 5.27 & 10 & 40 & 0.53 & 2.11 & $1.845 \%$ & $527 \%$ & $2,148,130$ & $8,276,269$ \\
\hline 1.1 .2 & TILE III INSPECTION & $2,042,710$ & 4.76 & 10 & 40 & 0.18 & 0.70 & $0.645 \%$ & $1.76 \%$ & 716,043 & $2,758,753$ \\
\hline$\overline{1.2 .1}$ & PROJECT MANAGEMENT & $7,783,994$ & 6.70 & 10 & 40 & 0.67 & 2.68 & $2344 \%$ & $6.70 \%$ & $2,728,569$ & $10,512,563$ \\
\hline 1.22 & CONSTRUCTON MANAGEMENT & $4,085,421$ & 3.51 & 10 & 40 & 0.35 & 1.41 & $1.230 \%$ & $3.51 \%$ & $1,432,087$ & $5,517,508$ \\
\hline 1.3 .1 & GENERAL CONDMONS & $6,840,414$ & 5.88 & 10 & 40 & 0.59 & 2.35 & $2059 \%$ & $5.88 \%$ & $2,397,811$ & $9,238,225$ \\
\hline 1.3 .13 & SPECIAL CONSTRUCTION & $34,013,797$ & 29.26 & 10 & 40 & 293 & 11.70 & $10.241 \%$ & $29.26 \%$ & $11,923,056$ & $45,936,853$ \\
\hline \multirow[t]{2}{*}{ 1.5.2. } & PROCUREMENT FEES & 408,542 & 0.35 & 10 & 40 & 0.04 & 0.14 & $0.123 \%$ & $0.35 \%$ & 143,209 & 551,751 \\
\hline & ESCALATION & $54,947,142$ & 47.27 & 10 & 40 & 4.73 & 18.91 & $16.543 \%$ & $47.27 \%$ & $19,260,944$ & $74,208,086$ \\
\hline \multicolumn{2}{|r|}{ SUBTOTAL } & $116,250,151$ & 100.00 & & & & & $35.000 \%$ & & & \\
\hline \multicolumn{2}{|r|}{ CALCULATED CONTINGENCY } & $40,687,553$ & & & & & & & & & \\
\hline \multicolumn{2}{|r|}{ RESULTANT TEC } & $156,937,704$ & & & & & & & & & \\
\hline \multicolumn{2}{|r|}{ ROUNDED TEC } & $157,000,000$ & & & & & & & & & \\
\hline \multicolumn{2}{|r|}{ PROJECT CONTINGENCY } & $40,749,849$ & & & & & & $35.05 \%$ & & & \\
\hline \multicolumn{2}{|r|}{ MANAGEMENT RESERVE } & $8,087,499$ & & & & & & & & & \\
\hline \multicolumn{2}{|r|}{ CONTINGENCY } & $32,662,350$ & & & & & & & & & \\
\hline \multicolumn{2}{|r|}{ TOTAL ESTIMATED COST } & $157,000,000$ & & & & & & & & $40,749,849$ & $157,000,000$ \\
\hline
\end{tabular}

CONFIDENCE LEVEL AND ASSUMED RISKS:

The Lockheed Idaho Technologies Co. Cost Estimate Contingency Analysis

Model is based on the applied contingency and the assumptions upon which

the estimate was predicated. The model is applied with a suggested risk level

of $18 \%$ and a level of confidence of $90 \%$ the estimate will fall within the bid range.

The Contingency Analysis is based on a weighted average to provide a

$90 \%$ probability of underrun and a $10 \%$ probability of overnu.
CONTINGENCY ANALYSIS GUIDE BY TYPE OF ESTIMATE

Guidelines established by DOEIFM 50 , Cost Estimating Guide, Vol. 6 ,

Cost Guide, and as presented in the INEL Cost Estimating Guide.

PLANNING

Experimental/Special Conditions

$20 \%-30 \%$

Conceptual

Experimental/Special Conditions $\quad 15 \%-25 \%$

TITLE

TITLE II

TITLE IUAFC

.... Up to $40 \%$

$10 \%-20 \%$
$5 \%-15 \%$

Market Conditions 


\section{G\&AVPIF ADDER CALCULATION SHEET \\ ICPP BIN SET CLOSURE \\ CLOSURE TO RCRA LANDFILL STANDARDS; CLASS C FILL; ESCALATED \\ CLEAN BINS WI ROBOTS - FLOOR}

PROCUREMENT FEE:

$\begin{aligned} & \text { CONSTRUCTION }= \\ & \text { GFE }=\end{aligned}$
Subtotal
FEE @ $1 \%=$

G\&A @ 23\% (with a ceiling of $\$ 500,000$ imposed per year, 10 yrs)

\begin{tabular}{|c|c|}
\hline CEILING * 10 YEARS = & $\$ 5,000,000$ \\
\hline GFE $=$ & $\$ 0$ \\
\hline $\begin{array}{r}\text { PROCUREMENT FEE }= \\
\text { Subtotal }\end{array}$ & $\frac{\$ 800,743}{\$ 5,800,743}$ \\
\hline
\end{tabular}

FEE @ $23 \%=$

$\$ 5,800,743 * 0.23=\$ 1,334,171$

PIF @ 5.5\%

\begin{tabular}{lr} 
CONSTRUCTION $=$ & $\begin{array}{r}\$ 40,854,211 \\
\text { GFE }=\end{array}$ \\
PROCUREMENT FEE $=$ & $\$ 0$ \\
G\&A $=\quad \begin{array}{r}\$ 800,743 \\
\end{array} \quad$ Subtotal & $\$ 1,334,171$ \\
\cline { 2 - 2 } & $\$ 42,989,124$
\end{tabular}

FEE @ $5.5 \%=$

$\$ 42,989,124 * 0.055=\quad \$ 2,364,402$

TOTAL PROCUREMENT FEE:

$\$ 800,743$

TOTAL G\&A FEE:

$\$ 1,334,171$

TOTAL PIF:

$\$ 2,364,402$ 
Lockheed Martin Idaho Technologies Co. Rev. 6-96

PROJECT NAME: ICPP Bin Set Closure (EIS Stud

LOCATION 1: Place NRC Class C Grout ICPP

REQUESTOR: Bryan Spaulding
COST ESTIMATE SUMMARY

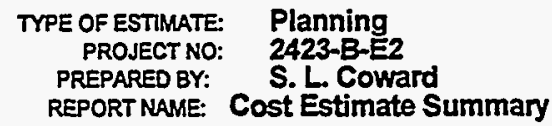

DATE: 28-Jan-1998

TIME: 10:46:06

CHECKED BY:

APPR'D BY:

\begin{tabular}{|c|c|c|c|c|}
\hline $\begin{array}{l}\text { WBS } \\
\text { Element }\end{array}$ & Cost Estimate Element & $\begin{array}{c}\text { Total } \\
\text { Unescalated }\end{array}$ & Escalation & $\begin{array}{c}\text { Total } \\
\text { Incl Escalation }\end{array}$ \\
\hline 1.1 & ENGINEERING, DESIGN AND INSPECTION & & & $\gg>\$ 7,746,789$ \\
\hline 7.1 .1 & DESIGN ENGINEERING TITLE I \& II & $3,282,538$ & $2,987,110$ & $6,269,648$ \\
\hline 1.1 .2 & TITLE III INSPECTION & 656,507 & 820,634 & $1,477,141$ \\
\hline 1.2 & MANAGEMENT COSTS & & & $\gg \$ 9,437,874$ \\
\hline 1.2 .1 & PROJECT MANAGEMENT & $2,972,384$ & $3,537,137$ & $6,509,521$ \\
\hline 1.2 .2 & CONSTRUCTION MANAGEMENT & $1,301,490$ & $1,626,863$ & $2,928,353$ \\
\hline 1.3 & CONSTRUCTION & & & $\gg \underline{\$ 29,542,856}$ \\
\hline 1.3.1 & GENERAL CONDITIONS & $3,437,150$ & $4,296,437$ & $7,733,587$ \\
\hline 1.3 .2 & SITEWORK & 0 & o & 0 \\
\hline 1.3.3 & CONCRETE & $3,876,603$ & $4,845,753$ & $8,722,356$ \\
\hline 1.3.15 & MECHANICAL & $3,365,584$ & $4,206,980$ & $7,572,564$ \\
\hline 1.3.16 & ELECTRICAL & $2,450,822$ & $3,063,527$ & $5,514,349$ \\
\hline \multirow[t]{2}{*}{ 1.5.2 } & PROCUREMENT FEES & 131,302 & 164,127 & $\gg \$ 295,429$ \\
\hline & $\begin{array}{l}\text { SUBTOTAL INCLUDING ESCALATION } \\
\text { PROJECT CONTINGENCY }\end{array}$ & $21,474,380$ & $25,548,568$ & $\gg>\$ 47,022,948$ \\
\hline & MANAGEMENT RESERVE- & & & $\$ 2,983,829$ \\
\hline & CONTINGENCY- & & & $\gg \quad \$ 14,693,223$ \\
\hline & TOTAL ESTIMATED COST - & & & $\gg \quad \$ 64,700,000$ \\
\hline
\end{tabular}

PROJECT COST PARAMETERS

EDI AS A \% OF CONST. + GFE $=26.00 \%$

CONTINGENCY $=37.59 \%$ 
Lockhoed Martin Idaho Technologles Co. Rev6-96 PROJECT NAME: ICPP Bin Sot Closuro (ElS Study) Place NRC Class C Grout Place
ICPP

LOCATION 1: REQUESTOR:

Bryan Spaulding

\section{DETAILED COST ESTIMATE SHEET}

TYPE OF ESTIMATE: Planning PROJECT NO:: 2423-B-E2

PREPARED BY: S. L. Coward
PAGE \# 1

DATE 28-Jan-1998

TIME: 10:46:08

REPORT NAME: Detall Cost Estimate Sheet

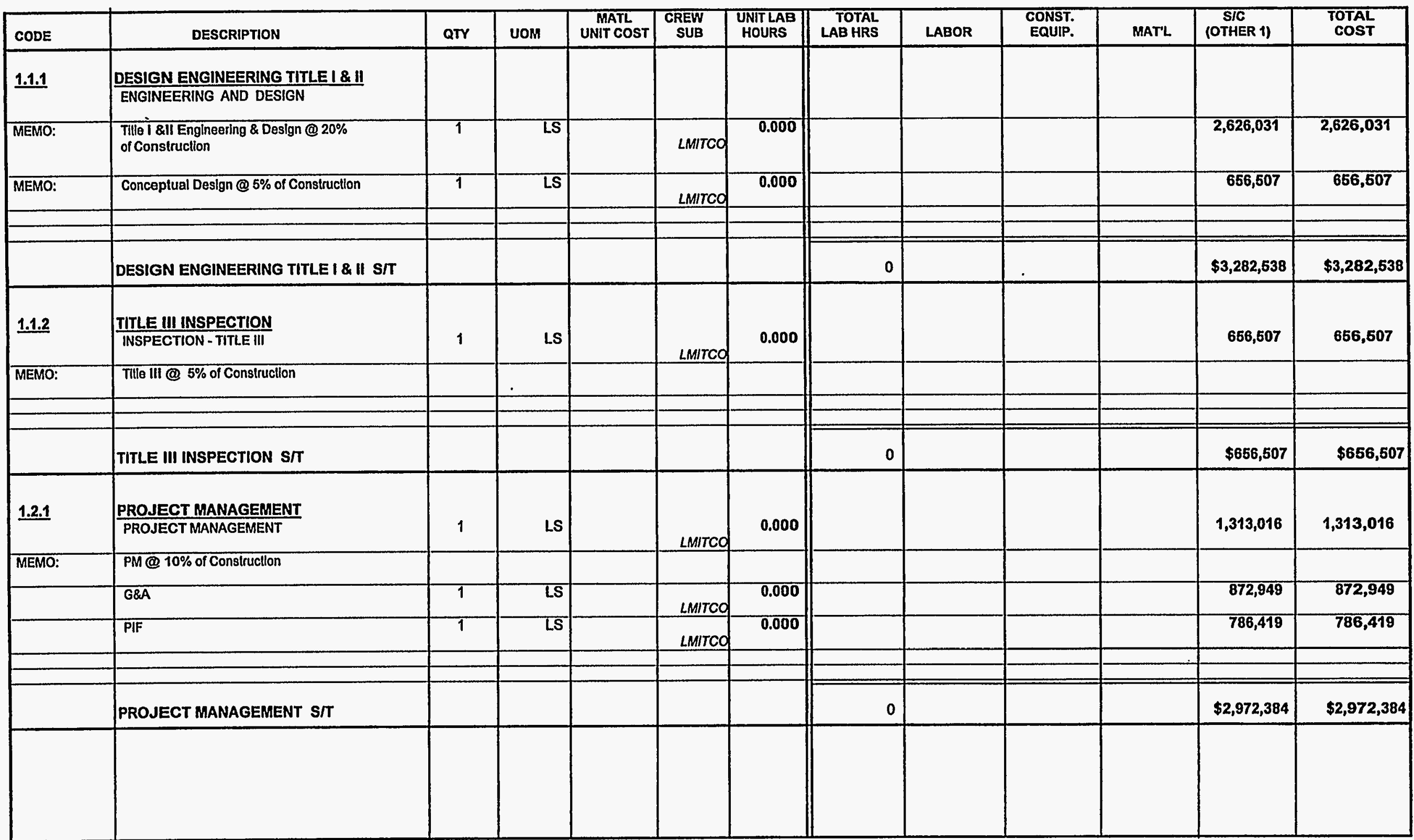


Lockheed Martin Idaho TechnoJogies Co.

PROJECT NAME: ICPP Bin Set Closure (EIS Stud

Place NRC Class $C$ Grout

ICPP

OCATION 1:

Bryan Spaulding
CONTINGENCY ANALYSIS

TYPE OF ESTIMATE:

PROJECT NO:

PREPARED BY:
Planning

S. L Coward
DATE: 28-Jan-1998

TIME: 10:46:03

REPORT NAME: Contingency Analysis

\begin{tabular}{|c|c|c|c|c|c|c|c|c|c|c|c|}
\hline \multirow{3}{*}{\begin{tabular}{|l|} 
WBS \\
Element
\end{tabular}} & \multicolumn{8}{|c|}{ PROBABLE \% VARIATION } & \multicolumn{2}{|c|}{$\begin{array}{c}\text { PROJECT } \\
\text { CONTINGENCY }\end{array}$} & \multirow{3}{*}{$\begin{array}{l}\text { SUMMARY } \\
\begin{array}{l}\text { Total Cost } \\
\text { by Element }\end{array}\end{array}$} \\
\hline & \multirow{2}{*}{ Cost Estimate Element } & \multirow{2}{*}{$\begin{array}{l}\text { Total Cost w/o } \\
\text { Contingency }\end{array}$} & \multirow{2}{*}{$\begin{array}{l}\text { \% Total } \\
\text { Cost }\end{array}$} & \multicolumn{2}{|c|}{$\begin{array}{c}\text { Prob. \% Var. } \\
\text { From Est. }\end{array}$} & \multicolumn{2}{|c|}{ Wt \% of Prob. } & \multirow{2}{*}{ Contingency } & \multirow{2}{*}{$\%$} & \multirow{2}{*}{ Cost } & \\
\hline & & & & - & + & - & \pm & & & & \\
\hline 1.1 .1 & DESIGN ENGINEERING TITLE | \& $\|$ & $3,282,538$ & 6.98 & 10 & 40 & 0.70 & 2.79 & $2.443 \%$ & $5.96 \%$ & $1,053,402$ & $4,335,940$ \\
\hline 1.1 .2 & TITLE III INSPECTION & 656,507 & 1.40 & 10 & 40 & 0.14 & 0.56 & $0.489 \%$ & $1.19 \%$ & 210,680 & 867,187 \\
\hline 1.2 .1 & PROJECT MANAGEMENT & $2,972,384$ & 6.32 & 10 & 40 & 0.63 & 253 & $2.212 \%$ & $5.40 \%$ & 953,870 & $3,926,254$ \\
\hline$\overline{1.2 .2}$ & CONSTRUCTION MANAGEMENT & $1,301,490$ & 2.77 & 10 & 40 & 0.28 & 1.11 & $0.969 \%$ & $236 \%$ & 417,662 & $1,719,152$ \\
\hline 1.3 .1 & GENERAL CONDITIONS & $3,437,150$ & 7.31 & 10 & 50 & 0.73 & 3.65 & $3.216 \%$ & $7.84 \%$ & $1,386,651$ & $4,823,801$ \\
\hline 1.3 .2 & SITEWORK & 0 & 0.00 & 0 & 0 & 0.00 & 0.00 & $0.000 \%$ & $0.00 \%$ & 0 & 0 \\
\hline 1.3 .3 & CONCRETE & $3,876,603$ & 8.24 & 10 & 50 & 0.82 & 4.12 & $3.627 \%$ & $8.85 \%$ & $1,563,940$ & $5,440,543$ \\
\hline 1.3 .15 & MECHANICAL & $3,365,584$ & 7.16 & 10 & 50 & 0.72 & 3.58 & $3.149 \%$ & $7.68 \%$ & $9,357,779$ & $4,723,363$ \\
\hline 1.3 .16 & ELECTRICAL & $2,450,822$ & 5.21 & 10 & 50 & 0.52 & 2.61 & $2293 \%$ & $5.59 \%$ & 988,737 & $3,439,559$ \\
\hline \multirow[t]{2}{*}{1.5 .2} & PROCUREMENT FEES & 131,302 & 0.28 & 10 & 40 & 0.03 & 0.11 & $0.098 \%$ & $0.24 \%$ & 42,136 & 173,438 \\
\hline & ESCALATTON & $25,548,568$ & 54.33 & 10 & 40 & 5.43 & 21.73 & $19.016 \%$ & $46.38 \%$ & $9,702,195$ & $35,250,763$ \\
\hline & SUBTOTAL & $47,022,948$ & 100.00 & & & & & $37.513 \%$ & & & \\
\hline & CALCULATED CONTINGENCY & $17,639,746$ & & & & & & & & & \\
\hline & RESULTANT TEC & $64,662,694$ & & & & & & & & & \\
\hline & ROUNDED TEC & $64,700,000$ & & & & & & & & & \\
\hline & PROJECT CONTINGENCY & $17,677,052$ & & & & & & $37.59 \%$ & & & \\
\hline & MANAGEMENT RESERVE & $2,983,829$ & & & & & & & & & \\
\hline & CONTINGENCY & $14,693,223$ & & & & & & & & & \\
\hline & TOTAL ESTIMATED COST & $64,700,000$ & & & & & & & & $17,677,052$ & $64,700,000$ \\
\hline
\end{tabular}

CONFIDENCE LEVEL AND ASSUMED RISKS:

The Lockheed Idaho Technologies Co. Cost Estimate Contingency Analysis Model is based on the applied contingency and the assumptions upon which the estimate was predicated. The model is applied with a suggested risk level of $18 \%$ and a level of confidence of $90 \%$ the estimate will fall within the bid range. The Contingency Analysis is based on a weighted average to provide a $90 \%$ probability of undemun and a $10 \%$ probability of overrun.
CONTINGENCY ANALYSIS GUIDE BY TYPE OF ESTIMATE

Guidelines established by DOE/FM 50, Cost Estimating Guide, Vol. 6 , Cost Guide, and as presented in the INEL Cost Estimating Guide.
PLANNING $20 \%-30 \%$ Experimental/Special Conditions............Up to 50\%

Conceptual $15 \%-25 \%$ TITLE I Experimental/Special Conditions............. Up to $40 \%$

TITLE II

TITLE II/AFC
$10 \%-20 \%$

$5 \%-15 \%$

Market Conditions 


\section{G\&APIF ADDER CALCULATION SHEET \\ ICPP BIN SET CLOSURE \\ CLOSURE TO RCRA LANDFILL STANDARDS; CLASS C FILL; ESCALATED}

FILL BINS W/ NRC CLASS C GROUT

PROCUREMENT FEE:

CONSTRUCTION $=$
GFE $=$

FEE @ 1\%=
$\$ 29,542,856$

Subtotal $\longdiv { \$ 2 9 , 5 4 2 , 8 5 6 }$

$\$ 29,542,856 * 0.01=$

G\&A @ 23\% (with a ceiling of $\$ 500,000$ imposed per year, 7 yrs)

CONSTRUCTION OR

CEILING * 7 YEARS $=$

GFE =

$\$ 3,500,000$

PROCUREMENT FEE =

FEE @ 23\% =

PIF @ 5.5\%

\begin{tabular}{|c|c|}
\hline $\begin{array}{l}\text { CONSTRUCTION }= \\
\text { GFE }= \\
\text { PROCUREMENT FEE }= \\
\text { G\&A }=\end{array}$ & $\begin{array}{r}\$ 13,130,159 \\
\$ 0 \\
\$ 295,429 \\
\$ 872,949 \\
\end{array}$ \\
\hline Subtotal & $\$ 14,298,536$ \\
\hline
\end{tabular}

FEE @ $5.5 \%=$ 
Lockheed Martin Idaho Technologies Co. Rov, 696 PROJECT MAME: ICPP Bin Set Closure D\&D of Equipment

LOCATION 1:

INEEL / ICPP

B. C. Spaulding

\section{COST ESTIMATE SUMMARY}

TYPE OF ESTMATE: Planning PROJECT NO: 2423D\&D PREPARED BY: S.LCoWard/smb REPORT NAME: Cost Estimate Summary

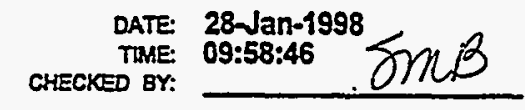

APPRD BY:

\begin{tabular}{|c|c|c|c|c|}
\hline $\begin{array}{l}\text { WBS } \\
\text { Element }\end{array}$ & Cost Estimate Element & $\begin{array}{c}\text { Total } \\
\text { Unescalated }\end{array}$ & Escalation & $\begin{array}{c}\text { Total } \\
\text { Inel Escalation }\end{array}$ \\
\hline $\begin{array}{l}\frac{1.1}{1.1 .1} \\
1.1 .2 \\
\frac{1.2}{1.2 .1} \\
1.2 .2 \\
\frac{1.3}{1.3 .13} \\
1.5 .2\end{array}$ & $\begin{array}{l}\text { ENGINEERING, DESIGN AND INSPECTION } \\
\text { DESIGN ENGINEERING TITLE I \& II } \\
\text { TITLE III INSPECTION } \\
\text { MANAGEMENT COSTS } \\
\text { PROJECT MANAGEMENT } \\
\text { CONSTRUCTION MANAGEMENT } \\
\text { CONSTRUCTION } \\
\text { SPECIAL CONSTRUCTION } \\
\text { PROCUREMENT FEES }\end{array}$ & $\begin{array}{r}868,178 \\
0 \\
11,709,711 \\
117,097\end{array}$ & $\begin{array}{c}1,692,947 \\
0 \\
\\
22,833,936 \\
228,339\end{array}$ & $\begin{array}{r}\gg \quad \text { S0 } \\
0 \\
\gg>\frac{\$ 2,561,125}{2,561,125} \\
0 \\
\gg>\frac{\$ 34,543,647}{34,543,647} \\
\gg>\quad \$ 345,436\end{array}$ \\
\hline & $\begin{array}{l}\text { SUBTOTAL INCLUDING ESCALATION } \\
\text { PROJECT CONTINGENCY }\end{array}$ & $12,694,986$ & $24,755,222$ & 〉 $\$ 37,450,208$ \\
\hline & MANAGEMENT RESERVE & & & $\gg$ \\
\hline & CONTINGENCY- & & & $\gg \quad \$ 13,149,792$ \\
\hline & TOTAL ESTIMATED COST - & & & $\$ 50,600,000$ \\
\hline
\end{tabular}

\section{PROJECT COST PARAMETERS}

EDI AS A $\%$ OF CONST. + GFE $=0.00 \%$ 


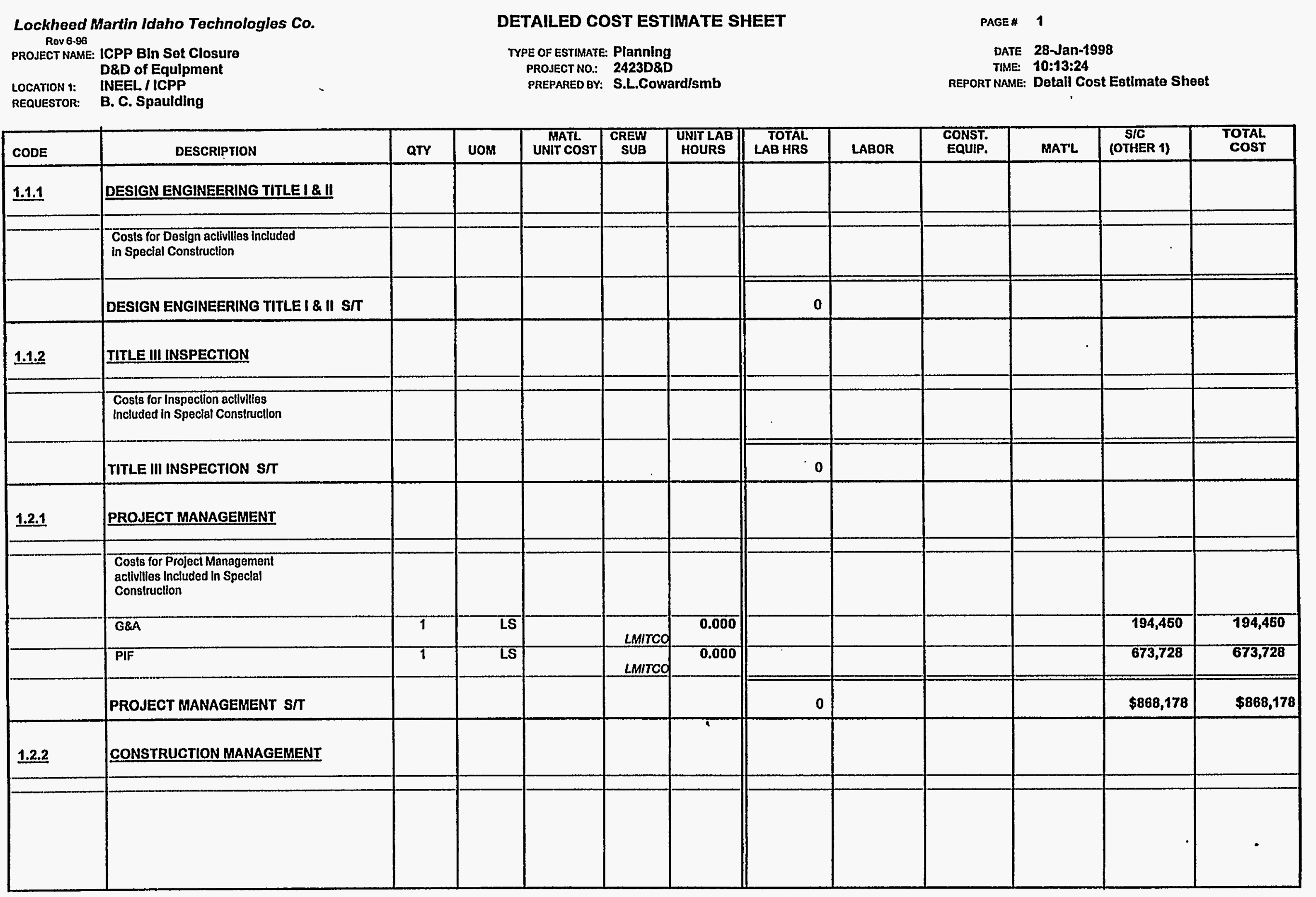


Lockheed Martin Idaho Technologies Co.

Rav 6.96

DJECT NAME: ICPP Bin Set Closure

D\&D of Equlpment

INEEL / ICPP

B. C. Spaulding

REQUESTOR:

\section{DETAILED COST ESTIMATE SHEET}

TYPE OF estimate: Planning

PROJECT NO: 2423D\&D

PREPARED BY: S.L.Coward/smb
PAGE \# $\mathbf{3}$

DATE 28-Jan-1998

TIME: 10:13:24

REPort name: Detall Cost Estimate Sheot

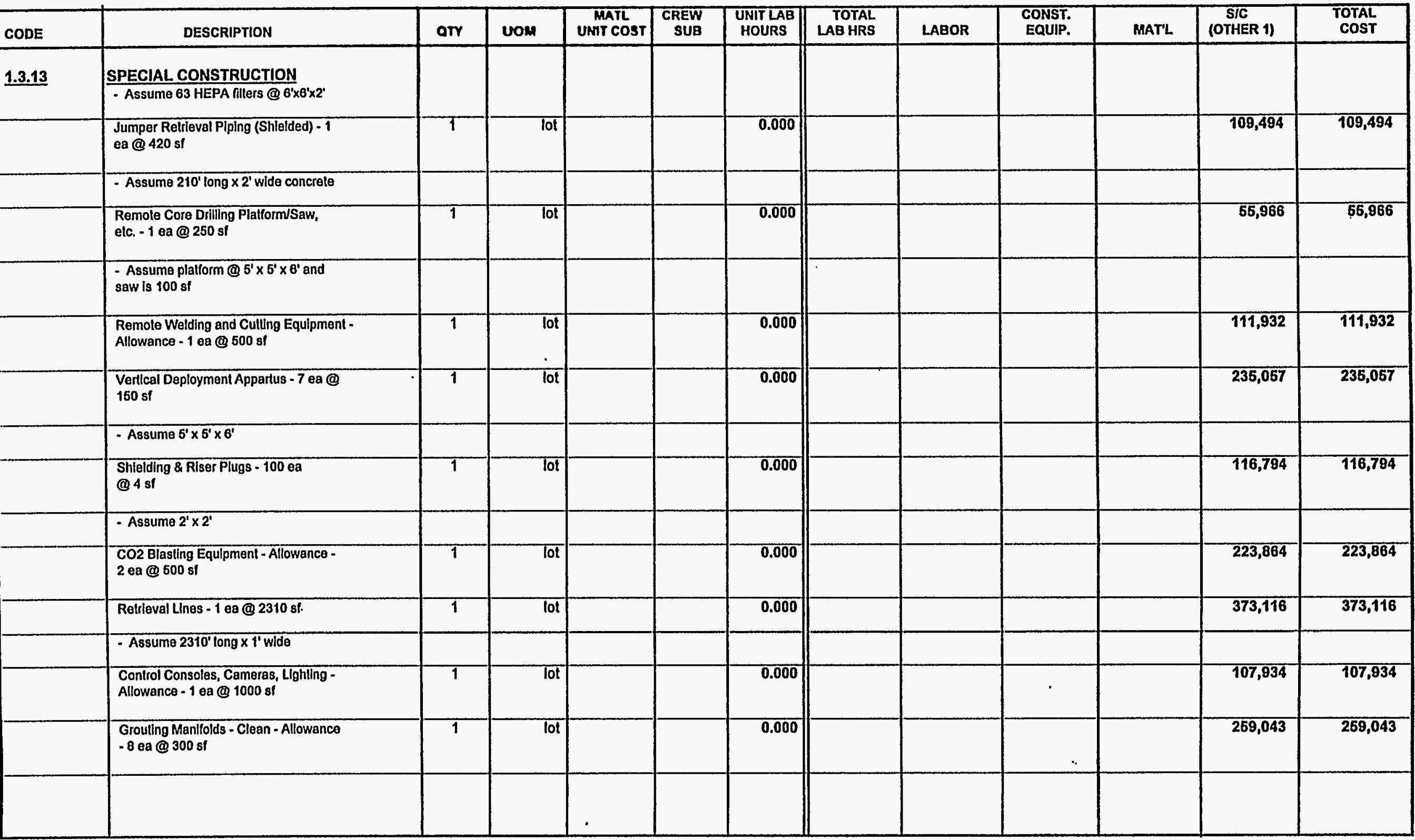


:kheed Martin Idaho Technologles Co. Rov 6.98

ECT NAME: ICPP Bin Sot Closure

D\&D of Equlpment

INEEL / ICPP

IESTOR: B. C. Spaulding

\section{DETAILED COST ESTIMATE SHEET}

TYPE OF ESTIMATE: Planning

PREPARED BY: S.L.Coward/smb
PAGE \# 4

DATE 28-Jan-1998

TIME: 10:13:24

REPORT name: Dotall Cost Estlmate Sheot

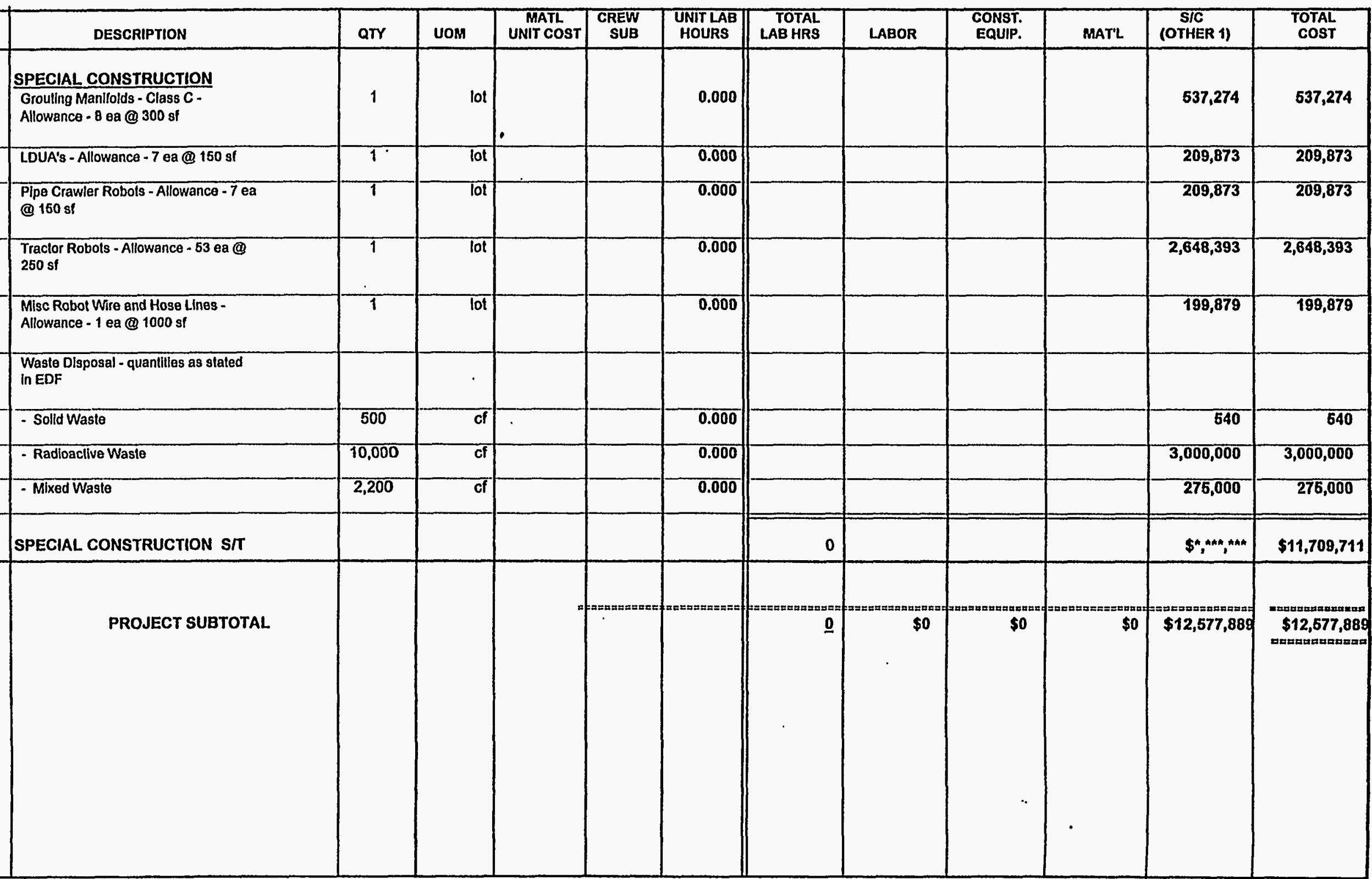


Lockheed Martin Idaho Technologies Co.

Rov $6-96$

PROJECT NAME: ICPP Bin Set Closure

D\&D of Equipment

LOCATION 1: INEEL / ICPP

REQUESTOR: B. C. Spaulding
CONTINGENCY ANALYSIS

TYPE OF ESTIMATE:

PREPARED BY: S.LCOWard/smb
OATE: 28-Jan-1998

TMME: 09:58:43

REPORT NAME: Contingency Analysis

\begin{tabular}{|c|c|c|c|c|c|c|c|c|c|c|c|}
\hline & PROB & BLE \% VARIA & ION & & & & & & $\begin{array}{l}\text { PRO } \\
\text { CONT }\end{array}$ & $\begin{array}{l}\text { CT } \\
\text { SENCY }\end{array}$ & SUMMARY \\
\hline $\begin{array}{l}\text { WBS } \\
\text { Element }\end{array}$ & Cost Estimate Element & Totai Cost w/o & $\begin{array}{l}\% \text { Total } \\
\text { Cost }\end{array}$ & & $\%$ Var. & Wt. \% & f Prob. & Contingener & $\%$ & Cost & \\
\hline & & Contingency & & - & + & - & + & & & & by Element \\
\hline 1.1 .1 & DESIGN ENGINEERING TITE $\mid \& \|$ & $\mathbf{0}$ & 0.00 & 0 & $\mathbf{0}$ & 0.00 & 0.00 & $0.000 \%$ & $0.00 \%$ & 0 & 0 \\
\hline 1.1 .2 & TIILE III INSPECTION & 0 & 0.00 & 0 & 0 & 0.00 & 0.00 & $0.000 \%$ & $0.00 \%$ & 0 & 0 \\
\hline 1.2 .1 & PROJECT MANAGEMENT & 868,178 & 232 & 10 & 40 & 0.23 & 0.93 & $0.811 \%$ & $232 \%$ & 304,841 & $1,173,019$ \\
\hline 1.22 & CONSTRUCTION MANAGEMENT & 0 & 0.00 & 0 & 0 & 0.00 & 0.00 & $0.000 \%$ & $0.00 \%$ & 0 & 0 \\
\hline 1.3 .13 & SPECIAL CONSTRUCTION & $11,709,711$ & 31.27 & 10 & 40 & 3.13 & 12.51 & $10.944 \%$ & $31.27 \%$ & $4,111,600$ & $15,821,311$ \\
\hline 1.5 .2 & PROCUREMENT FEES & 117,097 & 0.31 & 10 & 40 & 0.03 & 0.13 & $0.109 \%$ & $0.31 \%$ & 41,116 & 158,213 \\
\hline & ESCALATION & $24,755,222$ & 66.10 & 10 & 40 & 6.61 & 26.44 & $23.136 \%$ & $66.10 \%$ & $8,692,235$ & $33,447,457$ \\
\hline & SUBTOTAL & $37,450,208$ & 100.00 & & & & & $35.000 \%$ & & & \\
\hline & CALCULATED CONTINGENCY & $13,107,573$ & & & & & & & & & \\
\hline & RESULTANT TEC & $50,557,781$ & & & & & & & & & \\
\hline & ROUNDED TEC & $50,600,000$ & & & & & & & & & \\
\hline & PROJECT CONTINGENCY & $13,149,792$ & & & & & & $35.11 \%$ & & & \\
\hline & MANAGEMENT RESERVE & 0 & & & & & & . & & & \\
\hline & CONTINGENCY & $13,149,792$ & & & & & & & & & \\
\hline & TOTAL ESTIMATED COST & $50,600,000$ & & & & & & & & $13,149,792$ & $50,600,000$ \\
\hline
\end{tabular}

\section{CONFIDENCE LEVEL AND ASSUMED RISKS:}

The Lockheed Idaho Technologies Co. Cost Estimate Contingency Analysis Model is based on the applied contingency and the assumptions upon which the estimate was predicated. The model is applied with a suggested risk level of $18 \%$ and a level of confidence of $90 \%$ the estimate will fall within the bid range. The Contingency Analysis is based on a weighted average to provide a $90 \%$ probability of undermun and a $10 \%$ probability of ovemun.
CONTINGENCY ANALYSIS GUIDE BY TYPE OF ESTIMATE

Guidelines established by DOE/FM 50, Cost Estimating Guide, Vol. 6,

Cost Guide, and as presented in the INEL Cost Estimating Guide.

PLANNING $20 \%-30 \%$ Experimental/Special Conditions............. Up to $50 \%$

Conceptual ExperimentaVSpecial Conditions.............Up to $40 \%$ TITLE 1

TITLE II

TITLE IUAFC $10 \%-20 \%$

$5 \%-15 \%$ Market Conditions 


\section{G\&A/PIF ADDER CALCULATION SHEET \\ ICPP BIN SET CLOSURE \\ CLOSURE TO RCRA LANDFILL STANDARDS; CLASS C FILL; ESCALATED \\ D\&D OF EQUIPMENT}

PROCUREMENT FEE:

CONSTRUCTION $=$
GFE $=$

FEE @ $1 \% \doteq$

$\$ 34,543,647 * 0.01=$

$\$ 345,436$

G\&A @ 23\% (with a ceiling of $\$ 500,000$ imposed per year, 1 yrs)

CONSTRUCTION OR

CEILING 1 YEARS $=\quad \$ 500,000$

GFE $=$

PROCUREMENT FEE $=$

$\$ 345,436$

Subtotal $\$ 845,436$

FEE @ 23\% =

$\$ 845,436 \cdot 0.23=$

$\$ 194,450$

PIF @ 5.5\%

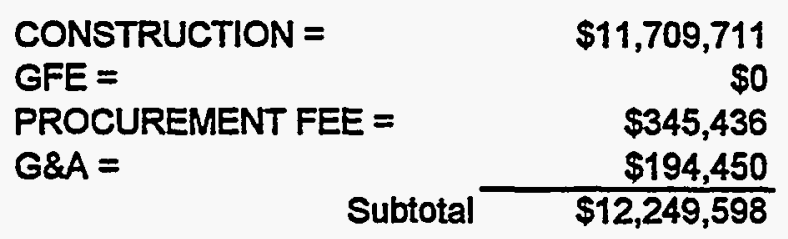

FEE @ $5.5 \%=$

$\$ 673,728$

TOTAL PROCUREMENT FEE:

$\$ 345,436$

TOTAL G\&A FEE:

$\$ 194,450$

TOTAL PIF:

$\$ 673,728$ 


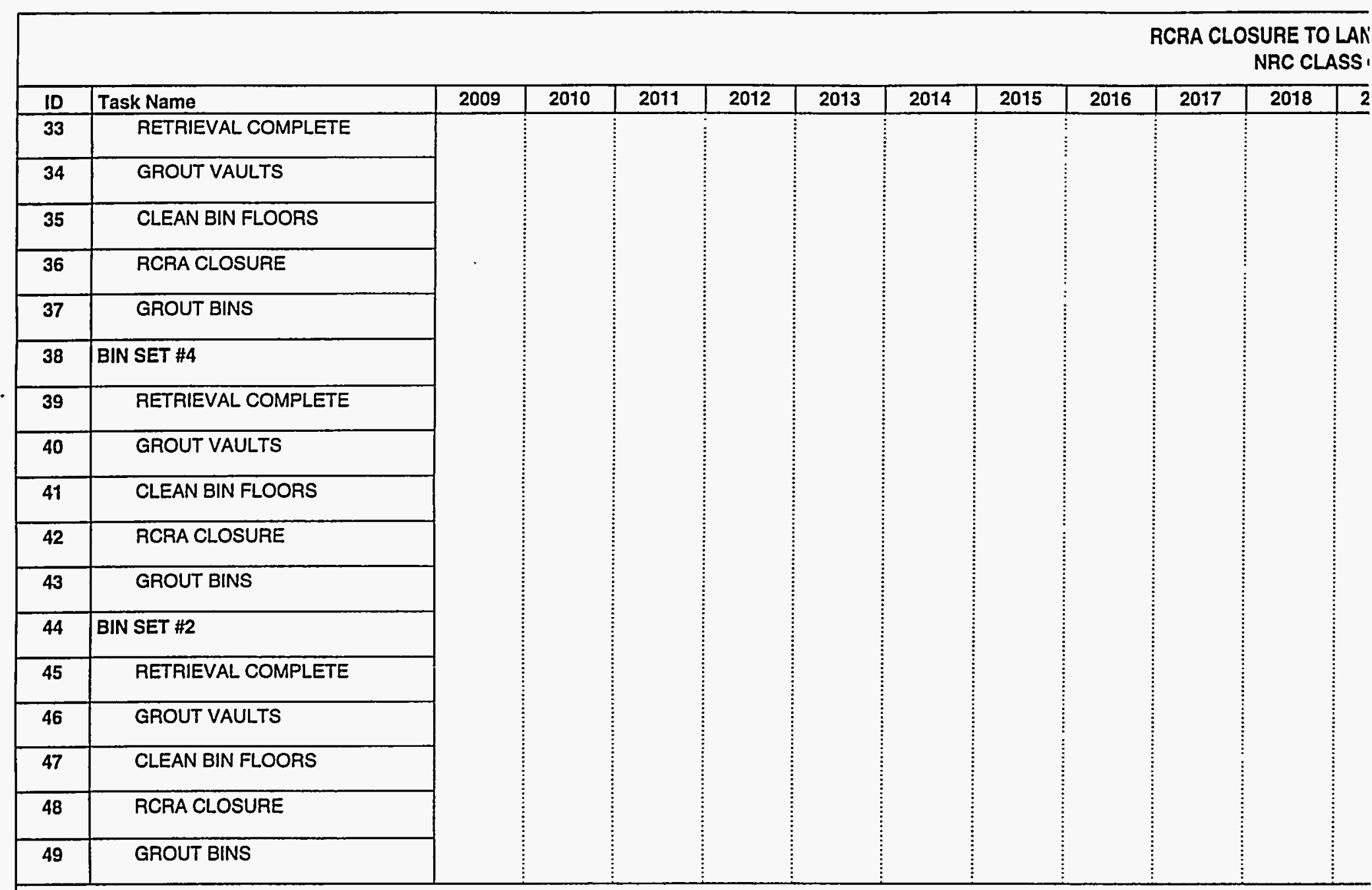

\begin{tabular}{|l|l|l|l|}
\hline $\begin{array}{l}\text { Project: 2423-4.MPP } \\
\text { Date: Tue 1/6/98 }\end{array}$ & Task & Rolled Up Task \\
\hline
\end{tabular}


ILL STANDARDS -

iROUT

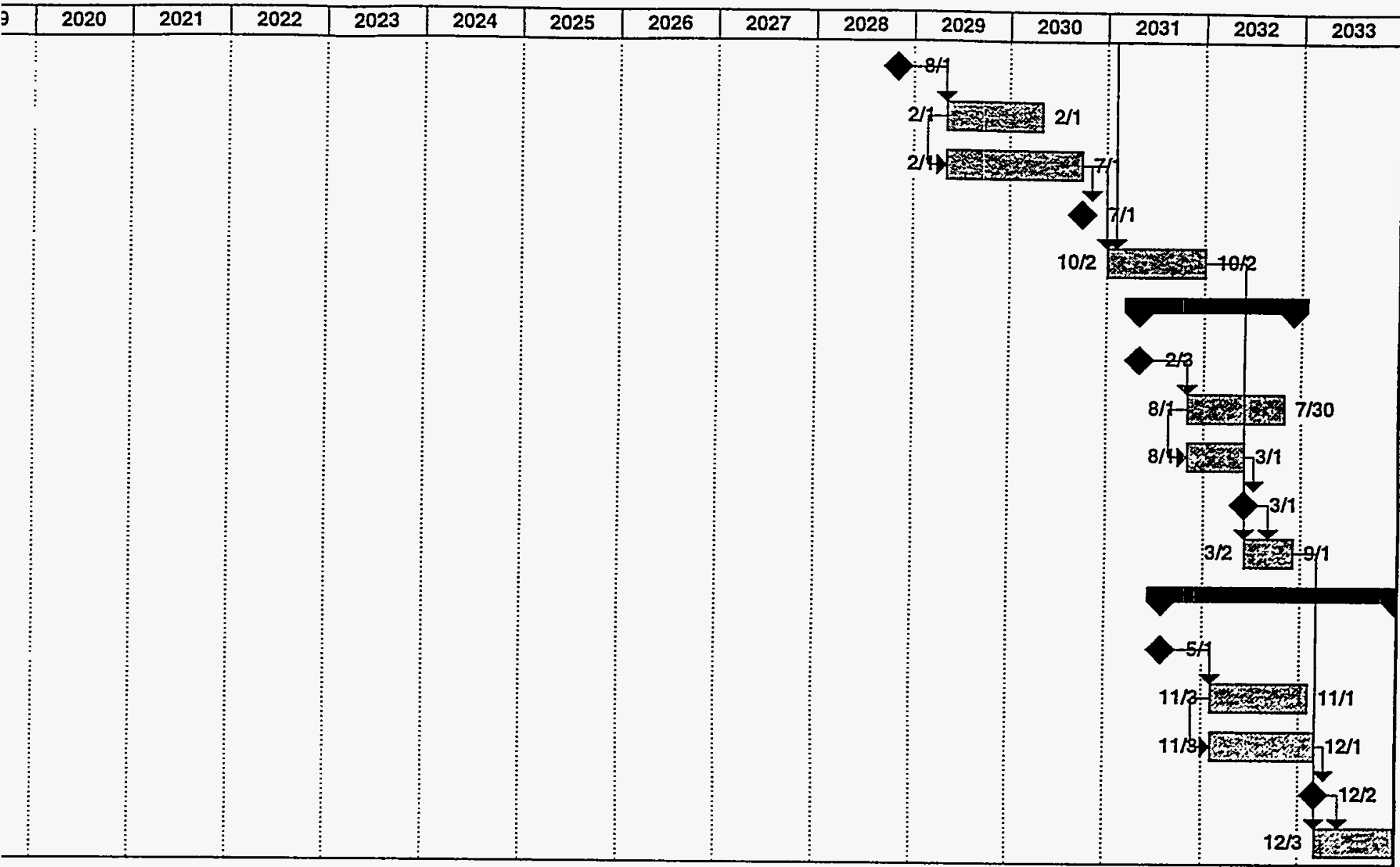





\section{COST ESTIMATE SUMMARY}

\section{UNESCALATED}

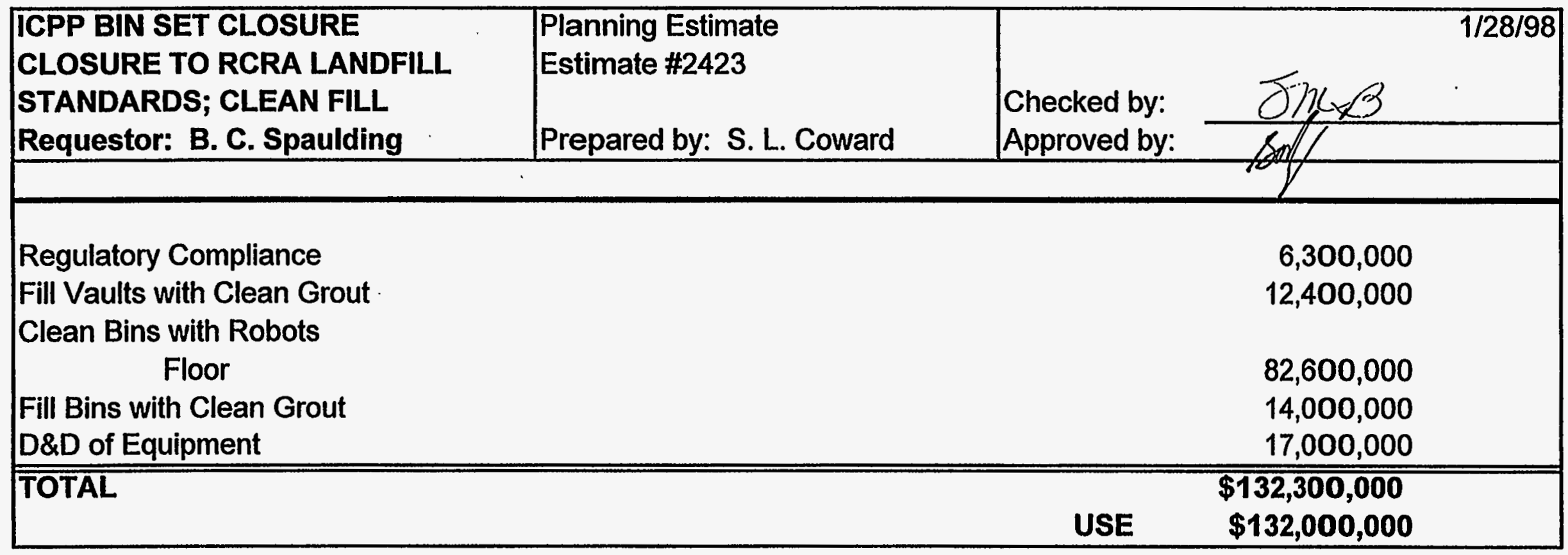


RCRA CLOSURE TO LANDFILL STANDARDS - "CLEAN" GROUT

\section{DESCRIPTION}

Schoduled Retrioval Completion

BIN SET \#1

$(1 / 1 / 14)$

BIN SET \#5

$(3 / 1 / 18)$

BIN SET \#6

$(8 / 1 / 22)$

BIN

BIN SET

$(2 / 1 / 27)$

BIN SET \#7

$(2 / 1 / 27)$

BIN SET *4

(2/1/29)

BIN SET \#3

ASSUME: Wait 1 Yoar until Start of Closuro

Permitting (3 Years)

$1 / 1 / 12-1 / 1 / 15$

Grout Vaults "Clean" (Assume 1 Year)

\begin{tabular}{ll}
\hline ED\&l (2 Yrs) & $1 / 1 / 13-1 / 1 / 15$ \\
Management & $1 / 1 / 13-10 / 1 / 33$
\end{tabular}

Construction

Months) (15 Months)

$2 / 1 / 28-2 / 1 / 29$

$2 / 1 / 28-2 / 1 / 29$

$2 / 1 / 30-2 / 1 / 31$

$10 / 1 / 32-10 / 1 / 33$

\section{Clean Bin Floors with Robot}

\begin{tabular}{ll}
\hline ED\&l (4 Yrs) & $1 / 1 / 11-1 / 1 / 15$ \\
Management & $1 / 1 / 11-3 / 1 / 34$
\end{tabular}

$1 / 1 / 11-3 / 1 / 34$

Construction

(3 Months)

(24 Months)

(13 Months)

(25 Months)

(7 Months)

(17 Months)

\section{RCAA ClosURE}

\begin{tabular}{|c|c|c|c|c|c|c|c|c|}
\hline Grout Bins "Cloan" & & (3 Months) & (7 Months) & (9 Months) & (6 Months) & (9 Months) & (4 Months) & (7 Months) \\
\hline ED\&l (3 Yrs) & $4 / 1 / 12-4 / 1 / 15$ & & & & & & & \\
\hline Management & $4 / 1 / 12-3 / 1 / 35$ & & & & & & & \\
\hline Construction & & $4 / 1 / 15-7 / 1 / 15$ & $6 / 1 / 20-12 / 1 / 20$ & $8 / 1 / 25-5 / 1 / 26$ & $3 / 1 / 29-9 / 1 / 29$ & $3 / 1 / 30-12 / 1 / 30$ & $9 / 1 / 30-1 / 1 / 31$ & $3 / 1 / 34-10 / 1 / 34$ \\
\hline
\end{tabular}

\section{ASSUMPTIONS:}

1) More than 1 crew could be utllized simultaneously for pouring the "clean" grout into the vaults.

2) More than 1 crew could be utilized simultaneously for cleaning the separate bins.

3) Installation of "olean" grout into vaults will average 1 year per vault.

4) Cleaning of bin floors are based on individual bin pro-rated calcine retrieval volumes to total volume. These schedules assume mob/demob, installatlon of robotic units, and any modiflcations of bins will be completed and bins will be ready for retrieval.

5) Durations for the "clean" grouting of the blns are based on individual pro-rated grout volumes to the total volume times the schedule. 

Lockheed Martin Idaho Technologies Co.

PROJECT NAME: Permitting/Documentation

Risk Based - Clean

LOCATION 1: INEELICPP

REQUESTOR: Bryan Spaulding
COST ESTIMATE SUMMARY

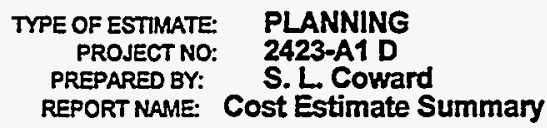

DATE: 27 Jan-1998

TIME: CHECKED BY APPR'D BY:

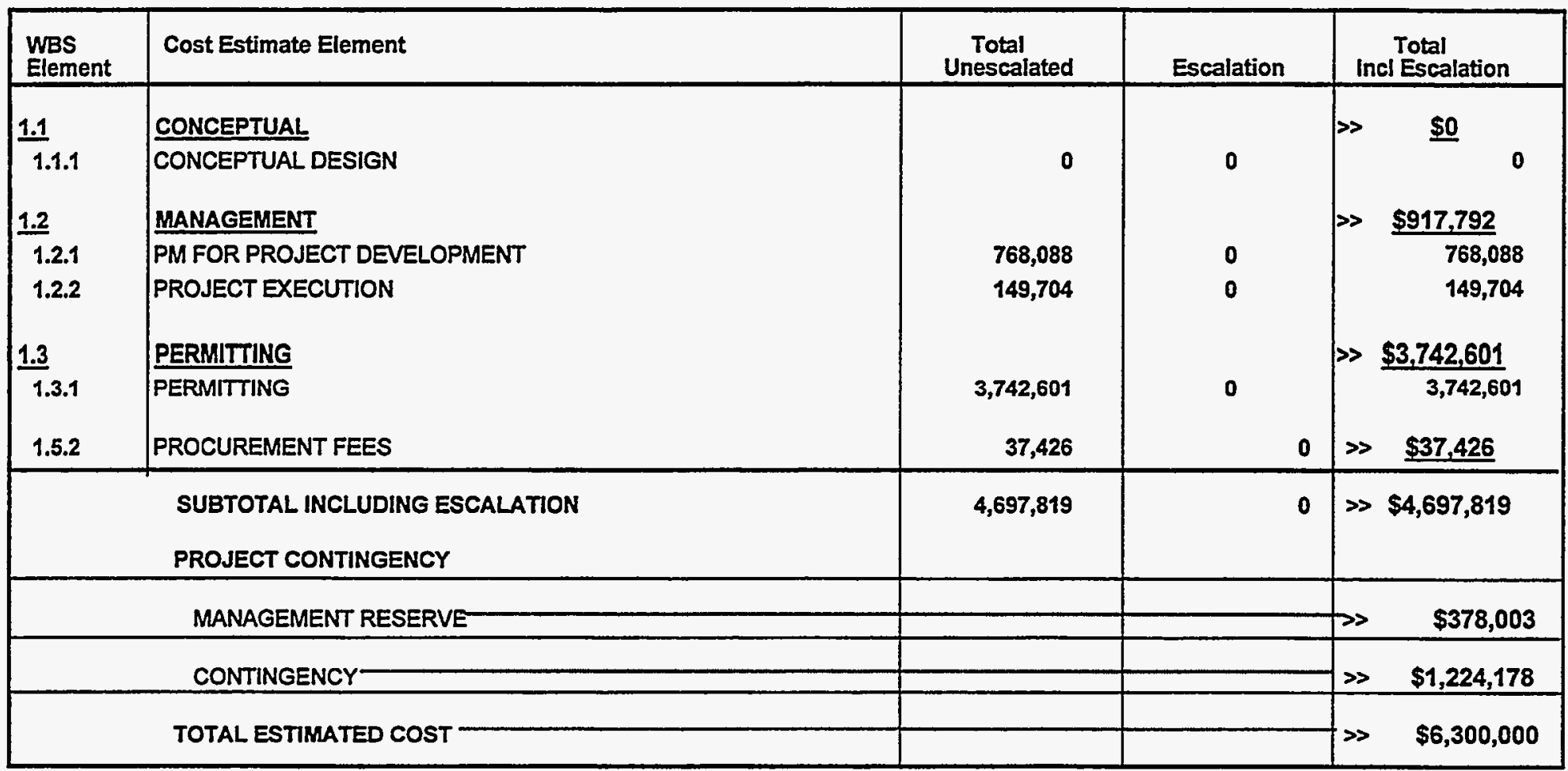

\section{PROJECT COST PARAMETERS}

EDI AS A $\%$ OF CONST. + GFE $=\quad 0.00 \%$ 
Lockheed Martin Idaho Technologies Co.

Rev 6-96

PROJECT NAME: Permitting/Documentation

Risk Based - Clean

LOCATION 1: INEELICPP

REQUESTOR: Bryan Spaulding

\section{CONTINGENCY ANALYSIS}

TYPE OFESTIMATE: PLANNING

PROJECT NO: 2423-A1 D

PREPARED BY: S.L Coward
DATE: 27-Jan-1998

TIME: 19:00:49

REPORT NAME: Contingency Analysis

\begin{tabular}{|c|c|c|c|c|c|c|c|c|c|c|c|}
\hline \multicolumn{9}{|c|}{ PROBABLE \% VARIATION } & \multicolumn{2}{|c|}{$\begin{array}{c}\text { PROJECT } \\
\text { CONTINGENCY }\end{array}$} & \multirow{3}{*}{$\begin{array}{l}\text { SUMMARY } \\
\text { Total Cost } \\
\text { by Element }\end{array}$} \\
\hline \multirow[t]{2}{*}{$\begin{array}{l}\text { WBS } \\
\text { Element }\end{array}$} & \multirow[t]{2}{*}{ Cost Estimate Element } & \multirow{2}{*}{$\begin{array}{l}\text { Total Cost w/o } \\
\text { Contingency }\end{array}$} & \multirow[t]{2}{*}{$\begin{array}{l}\text { \% Total } \\
\text { Cost }\end{array}$} & \multicolumn{2}{|c|}{$\begin{array}{c}\text { Prob. \% Var. } \\
\text { From Est. }\end{array}$} & \multicolumn{2}{|c|}{ Wt. \% of Prob. } & \multirow[t]{2}{*}{ Contingency } & \multirow[t]{2}{*}{$\%$} & \multirow[t]{2}{*}{ Cost } & \\
\hline & & & & - & + & - & \pm & & & & \\
\hline 1.1.1 & CONCEPTUAL DESIGN & 0 & 0.00 & 10 & 40 & 0.00 & 0.00 & $0.000 \%$ & $0.00 \%$ & 0 & 0 \\
\hline 1.2 .1 & PM FOR PROJECT DEVELOPMENT & 768,088 & 16.35 & 10 & 40 & 1.63 & 6.54 & $5.722 \%$ & $16.35 \%$ & 261,955 & $1,030,043$ \\
\hline 1.2 .2 & PROJECT EXECUTION & 149,704 & 3.19 & 10 & 40 & 0.32 & 1.27 & $1.115 \%$ & $3.19 \%$ & 51,056 & 200,760 \\
\hline 1.3 .1 & PERMITTING & $3,742,601$ & 79.67 & 10 & 40 & 7.97 & 31.87 & $27.883 \%$ & $79.67 \%$ & $1,276,406$ & $5,019,007$ \\
\hline \multirow[t]{2}{*}{1.5 .2} & PROCUREMENT FEES & 37,426 & 0.80 & 10 & 40 & 0.08 & 0.32 & $0.279 \%$ & $0.80 \%$ & 12,764 & 50,190 \\
\hline & ESCALATION & 0 & 0.00 & 0 & 0 & 0.00 & 0.00 & $0.000 \%$ & $0.00 \%$ & 0 & 0 \\
\hline & SUBTOTAL & $4,697,819$ & 100.00 & & & & & $35.000 \%$ & & & \\
\hline & CALCULATED CONTINGENCY & $1,644,237$ & & & & & & & & & \\
\hline & RESULTANT TEC & $6,342,056$ & & & & & & & & & \\
\hline & ROUNDED TEC & $6,300,000$ & & & & & & & & & \\
\hline & PROJECT CONTINGENCY & $1,602,181$ & & & & & & $34.10 \%$ & & & \\
\hline & MANAGEMENT RESERVE & 378,003 & & & & & & & & & \\
\hline & CONTINGENCY & $1,224,178$ & & & & & & & & & \\
\hline & TOTAL ESTIMATED COST & $6,300,000$ & & & & & & & & $1,602,181$ & $6,300,000$ \\
\hline
\end{tabular}

\section{CONFIDENCE LEVEL AND ASSUMED RISKS:}

The Lockheed Idaho Technologies Co. Cost Estimate Contingency Analysis Model is based on the applied contingency and the assumptions upon which the estimate was predicated. The model is applied with a suggested risk level of $18 \%$ and a level of confidence of $90 \%$ the estimate will fall within the bid range. The Contingency Analysis is based on a weighted average to provide a $90 \%$ probability of underrun and a $10 \%$ probability of overrun.
CONTINGENCY ANALYSIS GUIDE BY TYPE OF ESTIMATE

Guidelines established by DOE/FM 50, Cost Estimating Guide, Vol. 6 ,

Cost Guide, and as presented in the INEL Cost Estimating Guide. PLANNING Experimental/Spe

Conceptual TITLE I

Experimental/Special Conditions............Up to $40 \%$

TITLE II

$10 \%-20 \%$

TITLE IUAFC 


\section{G\&APIF ADDER CALCULATION SHEET \\ ICPP BIN SET CLOSURE \\ CLOSURE TO RCRA LANDFILL STANDARDS; CLEAN FILL; UNESCALATED \\ REGULATORY COMPLIANCE}

PROCUREMENT FEE:

CONSTRUCTION $=$

$\$ 3,742,601$

GFE =

Subtotal $\$ \$ 3,742,601$

FEE @ 1\%=

$\$ 3,742,601 * 0.01=$

$\$ 37,426$

G\&A @ 23\% (with a ceiling of $\$ 500,000$ imposed per year, 3 yrs)

CONSTRUCTION OR

CEILING $\bullet 3$ YEARS $=\quad \$ 1,500,000$

GFE =

PROCUREMENT FEE $=$

$\$ 37,426$

Subtotal

$\$ 1,537,426$

FEE @ 23\% =

$\$ 1,537,426 \cdot 0.23=$

$\$ 353,608$

PIF @ 5.5\%

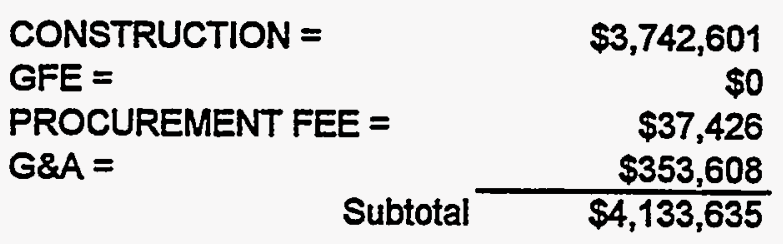

FEE @ $5.5 \%=$

$\$ 4,133,635 * 0.055=$

$\$ 227,350$

TOTAL PROCUREMENT FEE:

$\$ 37,426$

TOTAL G\&A FEE:

$\$ 353,608$

TOTAL PIF:

$\$ 227,350$ 
Lockheed Martin Idaho Technologies Co. PROJECT NAME: ICPP Bin Set Closure (EIS Stud

LOCATION 1: Place Clean Grout in Vault ICPP

REQUESTOR: Bryan Spaulding
COST ESTIMATE SUMMARY

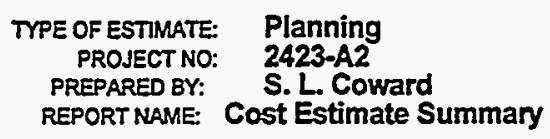

DATE: 27-Jan-1998

TIME: 19:06:11 CHECKED BY: APPRO BY:

\begin{tabular}{|c|c|c|c|c|}
\hline $\begin{array}{l}\text { WBS } \\
\text { Element }\end{array}$ & Cost Estimate Element & $\begin{array}{c}\text { Total } \\
\text { Unescalated }\end{array}$ & Escalation & $\begin{array}{l}\text { Total } \\
\text { Incl Escalation }\end{array}$ \\
\hline $\begin{array}{l}\frac{1.1}{1.1 .1} \\
1.1 .2 \\
\frac{1.2}{1.2 .1} \\
1.2 .2 \\
\frac{1.3}{1.3 .1} \\
1.3 .2 \\
1.3 .3 \\
1.5 .2\end{array}$ & $\begin{array}{l}\text { ENGINEERING, DESIGN AND INSPECTION } \\
\text { DESIGN ENGINEERING } \\
\text { TITLE III INSPECTION } \\
\text { MANAGEMENT COSTS } \\
\text { PROJECT MANAGEMENT } \\
\text { CONSTRUCTION MANAGEMENT } \\
\text { CONSTRUCTION } \\
\text { GENERAL CONDITIONS } \\
\text { SITEWORK } \\
\text { CONCRETE } \\
\text { PROCUREMENT FEES }\end{array}$ & $\begin{array}{r}1,324,812 \\
264,962 \\
\\
\\
1,686,432 \\
529,925 \\
\\
\\
1,067,791 \\
0 \\
4,231,459 \\
52,992\end{array}$ & $\begin{array}{l}0 \\
0 \\
0\end{array}$ & 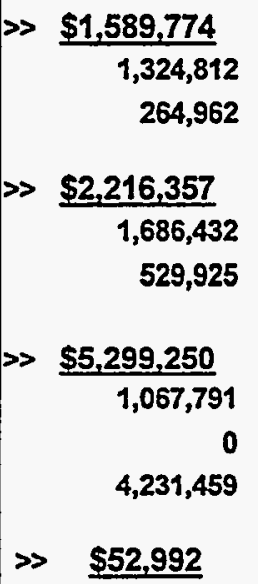 \\
\hline & $\begin{array}{l}\text { SUBTOTAL INCLUDING ESCALATION } \\
\text { PROJECT CONTINGENCY }\end{array}$ & $9,158,373$ & 0 & >> $\$ 9,158,373$ \\
\hline & MANAGEMENT RESERVE- & & & $\$ 535,224$ \\
\hline & CONTINGENCY- & & & $\$ 2,706,403$ \\
\hline & TOTAL ESTIMATED COST - & & & $\$ 12,400,000$ \\
\hline
\end{tabular}

\section{PROJECT COST PARAMETERS}

EDI AS A \% OF CONST. + GFE $=30.00 \%$ 
Lockheod Martin Idaho Technologies Co.

$$
\text { Rov } 6.98
$$

PROJECT NAME: ICPP BIn Set Closure (EIS Study)

Place Clean Grout in Vault

ICPP

Bryan Spaulding
DETAILED COST ESTIMATE SHEET

TYPE OF ESTIMATE: Planning

PROJECT NO.: 2423-A2

PREPARED BY: S. L. Coward
PAGE \# 2

DATE 27-Jan-1998

TIME: 19:06:13

REPORT NAME: Detall Cost Estimate Sheot

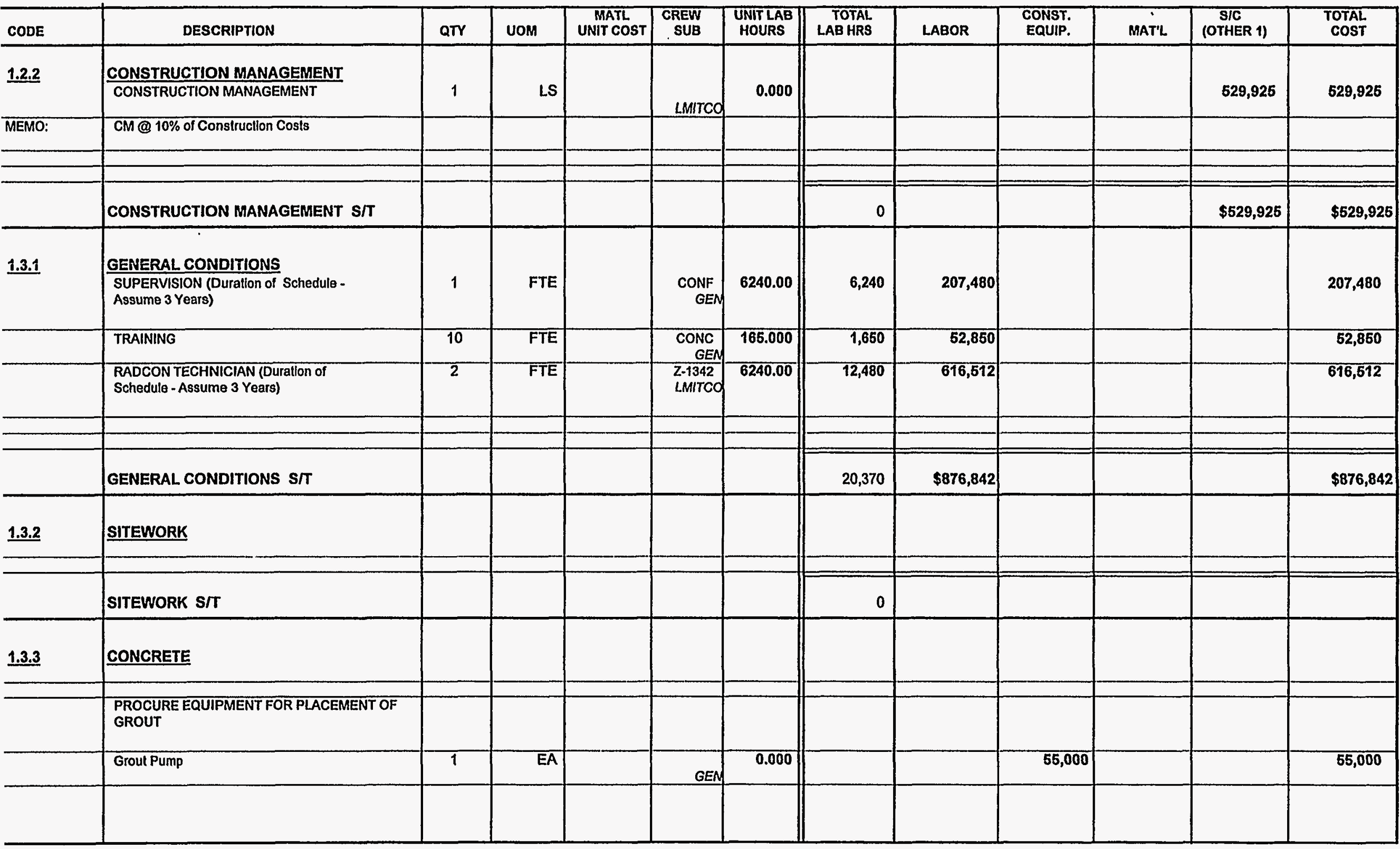


Lockhoed Martin Idaho Technologles Co. Rov 6.96 PROJECT NAME: ICPP Bin Set Closure (EIS Study) Place Clean Grout in Vault

ICPP

REQUESTOR: Bryan Spaulding
DETAILED COST ESTIMATE SHEET

TYPE OF estimate: Planning

PROJECT NO.: 2423-A2

PREPARED BY: S. L. Coward
PAGE\# 4

DATE 27-Jan-1998

TIME: 19:06:13

REPORT NAME: Detail Cost Estimate Sheet

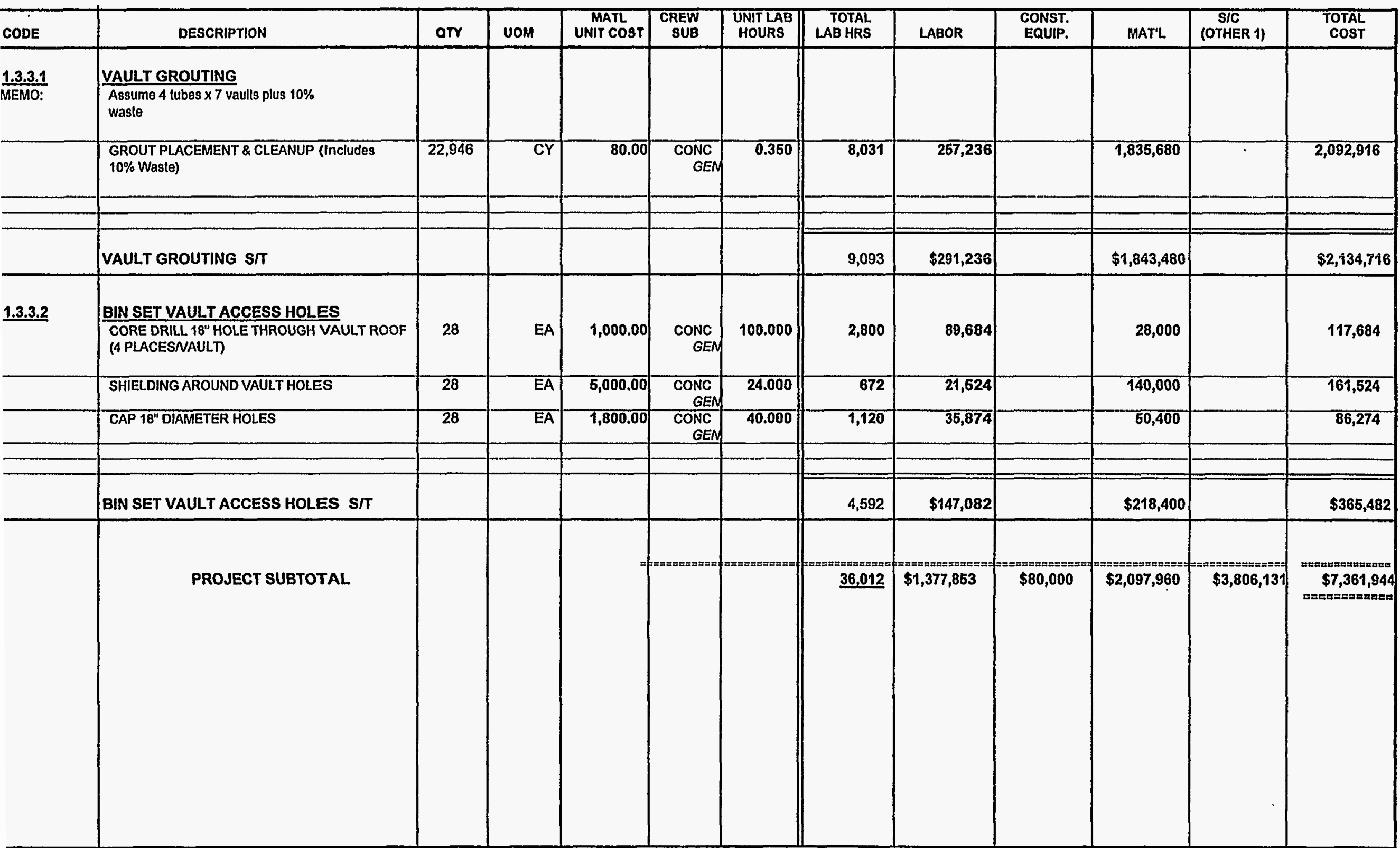


Lockheed Martin Idaho Technologies Co.

$$
\text { Rov } 6 \text {-S6 }
$$
PROJECT NAME: ICPP Bin Set Closure (EIS Stud

LOCATION 1: Place Clean Grout in Vault REQUESTOR:
ICPP

Bryan Spaulding
CONTINGENCY ANALYSIS TYPE OF ESTIMATE:
PROJECT NO: PREPARED BY:
2423-A2
Planning
DATE: 27 Jan-1998

TME: 19:06:07
REPORT NAME: Contingency Analysis

\begin{tabular}{|c|c|c|c|c|c|c|c|c|c|c|c|}
\hline \multicolumn{9}{|c|}{ PROBABLE \% VARIATION } & \multicolumn{2}{|c|}{$\begin{array}{l}\text { PROJECT } \\
\text { CONTINGENCY }\end{array}$} & \multirow{3}{*}{$\begin{array}{l}\text { SUMMARY } \\
\begin{array}{l}\text { Total Cost } \\
\text { by Element }\end{array}\end{array}$} \\
\hline \multirow[t]{2}{*}{$\begin{array}{l}\text { WBS } \\
\text { Element }\end{array}$} & \multirow[t]{2}{*}{ Cost Estimate Element } & \multirow{2}{*}{$\begin{array}{l}\text { Total Cost w/o } \\
\text { Contingency }\end{array}$} & \multirow[t]{2}{*}{$\begin{array}{l}\text { \% Total } \\
\text { Cost }\end{array}$} & \multicolumn{2}{|c|}{$\begin{array}{c}\text { Prob. \% Var. } \\
\text { From Est }\end{array}$} & \multicolumn{2}{|c|}{ Wt \% of Prob. } & \multirow[t]{2}{*}{ Contingency } & \multirow[t]{2}{*}{$\%$} & \multirow[t]{2}{*}{ Cost } & \\
\hline & & & & - & + & $=$ & + & & & & \\
\hline 1.1 .1 & DESIGN ENGINEERING & $1,324,812$ & 14.47 & 10 & 40 & 1.45 & 5.79 & $5.063 \%$ & $14.47 \%$ & 468,920 & $1,793,732$ \\
\hline 1.1 .2 & TTTLE III INSPECTION & 264,962 & 2.89 & 10 & 40 & 0.29 & 1.16 & $1.013 \%$ & $2.89 \%$ & \begin{tabular}{|l|}
93,784 \\
\end{tabular} & 358,746 \\
\hline 1.2 .1 & PROJECT MANAGEMENT & $1,686,432$ & 18.41 & 10 & 40 & 1.84 & 7.37 & $6.445 \%$ & $18.41 \%$ & 596,916 & $2,283,348$ \\
\hline 1.2 .2 & CONSTRUCTION MANAGEMENT & 529,925 & 5.79 & 10 & 40 & 0.58 & 231 & $2.025 \%$ & $5.79 \%$ & 187,568 & 717,493 \\
\hline 1.3 .1 & GENERAL CONDITIONS & $1,067,791$ & 11.66 & 10 & 40 & 1.17 & 4.66 & $4.081 \%$ & $11.66 \%$ & 377,947 & $1,445,738$ \\
\hline 1.3 .2 & SITEWORK & 0 & 0.00 & 0 & 0 & 0.00 & 0.00 & $0.000 \%$ & $0.00 \%$ & 0 & 0 \\
\hline 1.3 .3 & CONCRETE & $4,231,459$ & 46.20 & 90 & 40 & 4.62 & 18.48 & $16.174 \%$ & $46.20 \%$ & $1,497,735$ & $5,729,194$ \\
\hline \multirow[t]{2}{*}{1.5 .2} & PROCUREMENT FEES & 52,992 & 0.58 & 10 & 40 & 0.06 & 0.23 & $0.203 \%$ & $0.58 \%$ & 18,757 & 71,749 \\
\hline & ESCALATION & 0 & 0.00 & 0 & 0 & 0.00 & 0.00 & $0.000 \%$ & $0.00 \%$ & 0 & 0 \\
\hline \multicolumn{2}{|r|}{ SUBTOTAL } & $9,158,373$ & 100.00 & & & & & $35.000 \%$ & & & \\
\hline \multicolumn{2}{|r|}{ CALCULATED CONTINGENCY } & $3,205,431$ & & & & & & & & & \\
\hline \multicolumn{2}{|r|}{ RESULTANT TEC } & $12,363,804$ & & & & & & & & & \\
\hline \multicolumn{2}{|r|}{ ROUNDED TEC } & $12,400,000$ & & & & & & & & & \\
\hline \multicolumn{2}{|r|}{ PROJECT CONTINGENCY } & $3,241,627$ & & & & & & $35.40 \%$ & & & \\
\hline \multicolumn{2}{|r|}{ MANAGEMENT RESERVE } & 535,224 & & & & & & & & & \\
\hline \multicolumn{2}{|r|}{ CONTINGENCY } & $2,706,403$ & & & & & & & & & \\
\hline \multicolumn{2}{|r|}{ TOTAL ESTIMATED COST } & $12,400,000$ & & & & & & & & $3,241,627$ & $12,400,000$ \\
\hline
\end{tabular}

\section{CONFIDENCE LEVEL AND ASSUMED RISKS:}

The Lockheed Idaho Technologies Co. Cost Estimate Contingency Analysis Model is based on the applied contingency and the assumptions upon which the estimate was predicated. The model is applied with a suggested risk level of $18 \%$ and a level of confidence of $90 \%$ the estimate will fall within the bid range. The Contingency Analysis is based on a weighted average to provide a $90 \%$ probability of underrun and a $10 \%$ probability of overnun.
CONTINGENCY ANALYSIS GUIDE BY TYPE OF ESTIMATE

Guidelines established by DOE/FM 50, Cost Estimating Guide, Vol. 6, Cost Guide, and as presented in the INEL Cost Estimating Guide. PLANNING Conceptual Experimental/Special Conditions. $20 \%-30 \%$

TITLE

TITLE II

TITLE IIVAFC
$15 \%-25 \%$ U.... Up to $40 \%$ $10 \%-20 \%$ $5 \%-15 \%$ Market Conditions 


\section{G\&APIF ADDER CALCULATION SHEET \\ ICPP BIN SET CLOSURE \\ CLOSURE TO RCRA LANDFILL STANDARDS; CLEAN FILL; UNESCALATED}

FILL VAULTS W/ CLEAN GROUT

PROCUREMENT FEE:

CONSTRUCTION $=$
GFE $=$$\quad$\begin{tabular}{l}
$\$ 5,299,250$ \\
\cline { 2 - 2 }
\end{tabular}

FEE @ 1\%=

$\$ 5,299,250 * 0.01=$

$\$ 52,993$

G\&A @ 23\% (with a ceiling of $\$ 500,000$ imposed per year, 7 yrs)

CONSTRUCTION OR

CEILING * 7 YEARS $=\quad \$ 3,500,000$

GFE =

$\$ 0$

PROCUREMENT FEE $=$

Subtotal

$\$ 52993$

$\$ 3,552,993$

FEE @ $23 \%=$

$\$ 3,552,993 * 0.23=$

$\$ 817,188$

PIF @ 5.5\%

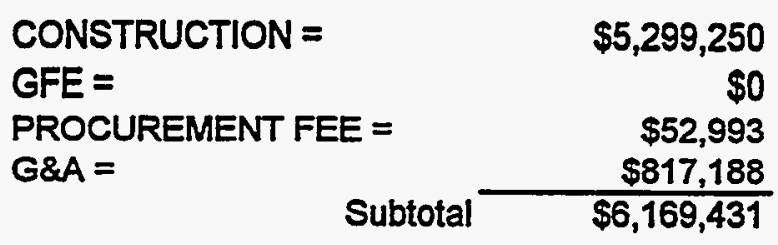

FEE @ 5.5\%=

$\$ 6,169,431 * 0.055=$

$\$ 339,319$

TOTAL PROCUREMENT FEE:

$\$ 52,993$

TOTAL G\&A FEE:

$\$ 817,188$

TOTAL PIF:

$\$ 339,319$ 
Lockheed Martin Idaho Technologies Co. Rev. 6-95 PROJECT NAME: ICPP Bin Set Closure (EIS Stud LOCATION 1: Tractor (RCRA Estimates) REQUESTOR: ICPP

Bryan Spaulding
COST ESTIMATE SUMMARY

TYPE OF ESTIMATE: Planning

PROJECT NO: 2423-C

PREPARED BY: S. L. Coward

REPORT NAME: Cost Estimate Summary

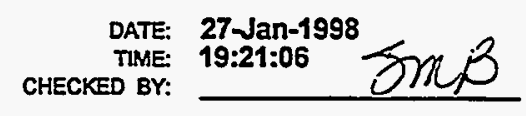

APPR'D BY:

\begin{tabular}{|c|c|c|c|c|}
\hline $\begin{array}{l}\text { WBS } \\
\text { Element }\end{array}$ & Cost Estimate Element & $\begin{array}{l}\text { Total } \\
\text { Unescalated }\end{array}$ & Escalation & $\begin{array}{c}\text { Total } \\
\text { Incl Escalation }\end{array}$ \\
\hline $\begin{array}{l}\frac{1.1}{1.1 .1} \\
1.1 .2 \\
1.2 \\
1.2 .1 \\
1.2 .2 \\
\frac{1.3}{1.3 .1} \\
1.3 .13 \\
1.5 .2\end{array}$ & $\begin{array}{l}\text { ENGINEERING, DESIGN AND INSPECTION } \\
\text { DESIGN ENGINEERING TITLE I \& } \| \\
\text { TITLE III INSPECTION } \\
\text { MANAGEMENT COSTS } \\
\text { PROJECT MANAGEMENT } \\
\text { CONSTRUCTION MANAGEMENT } \\
\text { CONSTRUCTION } \\
\text { GENERAL CONDITIONS } \\
\text { SPECIAL CONSTRUCTION } \\
\text { PROCUREMENT FEES }\end{array}$ & $\begin{array}{r}6,128,131 \\
2,042,710 \\
7,667,255 \\
4,085,421 \\
\\
6,840,414 \\
34,013,797 \\
408,542\end{array}$ & $\begin{array}{l}0 \\
0 \\
0 \\
0\end{array}$ & $\begin{array}{r}\gg>\begin{array}{r}\frac{\$ 8,170,841}{6,128,131} \\
2,042,710\end{array} \\
>>\frac{\$ 11,752,676}{7,667,255} \\
4,085,421 \\
\\
>\frac{\$ 40,854,211}{6,840,414} \\
34,013,797\end{array}$ \\
\hline & $\begin{array}{l}\text { SUBTOTAL INCLUDING ESCALATION } \\
\text { PROJECT CONTINGENCY }\end{array}$ & $61,186,270$ & 0 & $\gg \$ \$ 61,186,270$ \\
\hline & MANAGEMENT RESERVE- & & & $\$ 4,126,275$ \\
\hline & CONTINGENCY - & & & $\gg>\$ 17,287,455$ \\
\hline & TOTAL ESTIMATED COST - & & & $\$ 82,600,000$ \\
\hline
\end{tabular}

PROJECT COST PARAMETERS

EDI AS A $\%$ OF CONST. + GFE $=20.00 \%$ 
Lockheed Martin Idaho Technologies Co. Rov 6.96

PROJECT NAME: ICPP BIn Set Closure (EIS Study) Tractor (RCRA Estimates)

LOCATION 1: ICPP

REQUESTOR: Bryan Spaulding

\section{DETAILED COST ESTIMATE SHEET}

TYPE OF ESTIMATE: Planning

PROJECT NO: 2423-C

PREPARED BY: S. L. Coward
PAGE 3

DATE 27-Jan-1998

TIME: 19:21:08

REPORT NAME: Detail Cost Estimate Sheet

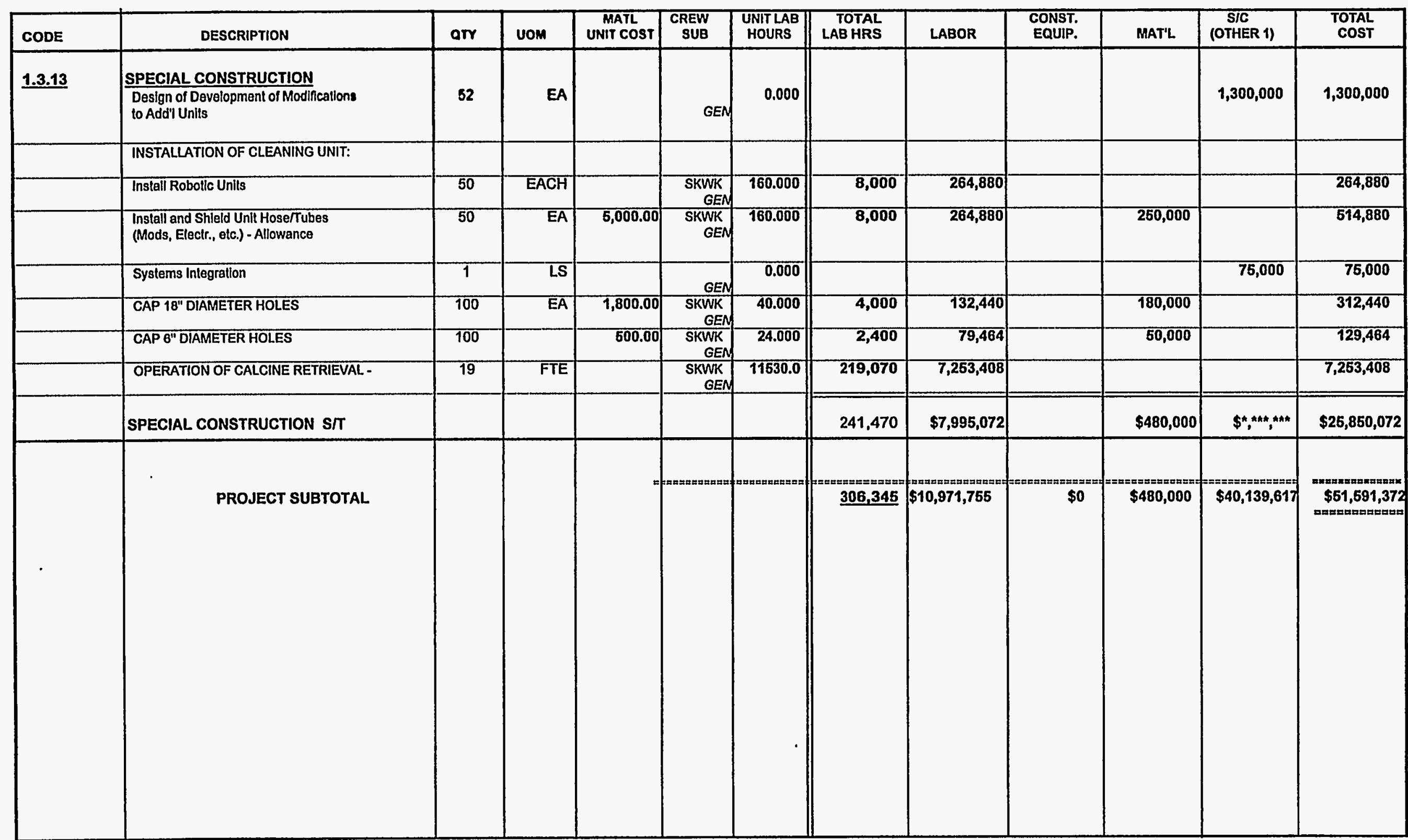


Lockheed Martin Idaho Technologies Co.

Rev 6-96

PROJECT NAME: ICPP Bin Set Closure (EIS Stud Tractor (RCRA Estimates)

LOCATION : ICPP REQUESTOR
CONTINGENCY ANALYSIS

TYPE OF ESTIMATE: PROJECT NO: PREPARED BY:
Planning

2423-C

S. L. Coward
DATE: 27-Jan-1998

TIME: 19:21:02

REPORT NAME: Contingency Analysis

\begin{tabular}{|c|c|c|c|c|c|c|c|c|c|c|c|}
\hline \multicolumn{9}{|c|}{ PROBABLE \% VARIATION } & \multicolumn{2}{|c|}{$\begin{array}{c}\text { PROJECT } \\
\text { CONTINGENCY }\end{array}$} & \multirow{3}{*}{$\begin{array}{l}\text { SUMMARY } \\
\begin{array}{l}\text { Total Cost } \\
\text { by Element }\end{array}\end{array}$} \\
\hline \multirow[t]{2}{*}{$\begin{array}{l}\text { WBS } \\
\text { Element }\end{array}$} & \multirow[t]{2}{*}{ Cost Estimate Element } & \multirow{2}{*}{$\begin{array}{l}\text { Total Cost w/o } \\
\text { Contingency }\end{array}$} & \multirow[t]{2}{*}{$\begin{array}{l}\text { \% Total } \\
\text { Cost }\end{array}$} & \multicolumn{2}{|c|}{$\begin{array}{c}\text { Prob. \% Var. } \\
\text { From Est. }\end{array}$} & \multicolumn{2}{|c|}{ Wt. $\%$ of Prob. } & \multirow[t]{2}{*}{ Contingency } & \multirow[t]{2}{*}{$\%$} & \multirow[t]{2}{*}{ Cost } & \\
\hline & & & & - & + & - & + & & & & \\
\hline 1.1.1 & DESIGN ENGINEERING TITLE I \& I| & $6,128,131$ & 10.02 & 10 & 40 & 1.00 & 4.01 & $3.505 \%$ & $10.02 \%$ & $2,144,699$ & $8,272,830$ \\
\hline 1.1 .2 & TITLE III INSPECTION & $2,042,710$ & 3.34 & 10 & 40 & 0.33 & 1.34 & $1.168 \%$ & $3.34 \%$ & 714,900 & $2,757,610$ \\
\hline 1.2 .1 & PROJECT MANAGEMENT & $7,667,255$ & 12.53 & 10 & 40 & 1.25 & 5.01 & $4.386 \%$ & $12.53 \%$ & $2,683,356$ & $10,350,611$ \\
\hline 1.2 .2 & CONSTRUCTION MANAGEMENT & $4,085,421$ & 6.68 & 10 & 40 & 0.67 & 2.67 & $2.337 \%$ & $6.68 \%$ & $1,429,800$ & $5,515,221$ \\
\hline 1.3 .1 & GENERAL CONDMIONS & $6,840,414$ & 11.18 & 10 & 40 & 1.12 & 4.47 & $3.913 \%$ & $11.18 \%$ & $2,393,981$ & $9,234,395$ \\
\hline 1.3 .13 & SPECIAL CONSTRUCTION & $34,013,797$ & 55.59 & 10 & 40 & 5.56 & 22.24 & $19.457 \%$ & $55.59 \%$ & $11,904,015$ & $45,917,812$ \\
\hline 1.5 .2 & PROCUREMENT FEES & 408,542 & 0.67 & 10 & 40 & 0.07 & 0.27 & $0.234 \%$ & $0.67 \%$ & 142,980 & 551,522 \\
\hline. & ESCALATION & 0 & 0.00 & 10 & 40 & 0.00 & 0.00 & $0.000 \%$ & $0.00 \%$ & (1) & (1) \\
\hline & SUBTOTAL & $61,186,270$ & 100.00 & & & & & $35.000 \%$ & & & \\
\hline & CALCULATED CONTINGENCY & $21,415,195$ & & & & & & & & & \\
\hline & RESULTANT TEC & $82,601,465$ & & & & & & & & & \\
\hline & ROUNDED TEC & $82,600,000$ & & & & & & & & & \\
\hline & PROJECT CONTINGENCY & $21,413,730$ & & & & & & $35.00 \%$ & & & \\
\hline & MANAGEMENT RESERVE & $4,126,275$ & & & & & & & & & \\
\hline & CONTINGENCY & $17,287,455$ & & & & & & & & & \\
\hline & TOTALESTIMATED COST & $82,600,000$ & & & & & & & & $21,413,730$ & $82,600,000$ \\
\hline
\end{tabular}

CONFIDENCE LEVEL AND ASSUMED RISKS:

The Lockheed Idaho Technologies Co. Cost Estimate Contingency Analysis Model is based on the applied contingency and the assumptions upon which the estimate was predicated. The model is applied with a suggested risk level of $18 \%$ and a level of confidence of $90 \%$ the estimate will fall within the bid range. The Contingency Analysis is based on a weighted average to provide a

$90 \%$ probability of underrun and a $10 \%$ probability of overtun.
CONTINGENCY ANALYSIS GUIDE BY TYPE OF ESTIMATE

Guidelines established by DOE/FM 50, Cost Estimating Guide, Vol. 6 , Cost Guide, and as presented in the INEL Cost Estimating Guide. PLANNING Experimen $20 \%-30 \%$ Conceptual TITLE I

TITLE II

TITLE IUAFC
Up to $40 \%$

$10 \%-20 \%$
$5 \%-15 \%$

Market Conditions
$15 \%-25 \%$ 


\section{G\&APIF ADDER CALCULATION SHEET \\ ICPP BIN SET CLOSURE \\ CLOSURE TO RCRA LANDFILL STANDARDS; CLEAN FILL; UNESCALATED \\ CLEAN BINS W/ ROBOTS FLOOR}

PROCUREMENT FEE:

CONSTRUCTION $=$

$\$ 40,854,211$

GFE $=$

Subtotal $\$ \$ 40,854,211$

FEE @ 1\%=

$\$ 40,854,211 * 0.01=\quad \$ 408,542$

G\&A @ 23\% (with a ceiling of $\$ 500,000$ imposed per year, 10 yrs)

CONSTRUCTION OR

CEILING * 10 YEARS =

$\$ 5,000,000$

GFE $=$

\$0

PROCUREMENT FEE $=$ $\$ 408,542$

Subtotal $\$ 5,408,542$

FEE @ 23\% =

$\$ 5,408,542 \cdot 0.23=\quad \$ 1,243,965$

PIF @ 5.5\%

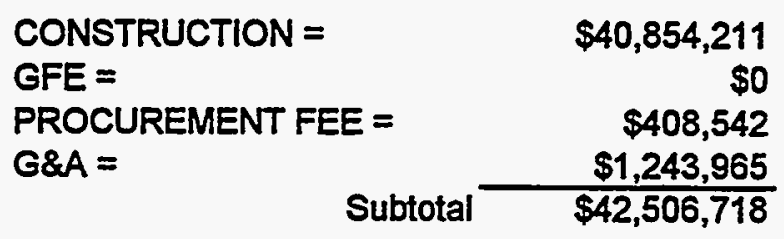

FEE @ 5.5\% =

$\$ 42,506,718 \cdot 0.055=$

$\$ 2,337,869$

TOTAL PROCUREMENT FEE:

$\$ 408,542$

TOTAL G\&A FEE:

$\$ 1,243,965$

TOTAL PIF:

$\$ 2,337,869$ 
Lockheed Martin Idaho Technologies Co. PROJECT NAME: ICPP Bin Set Closure (ElS Stud LOCATION 1: REQUESTOR: Place Clean Grout in Bins ICPP Bryan Spaulding
COST ESTIMATE SUMMARY

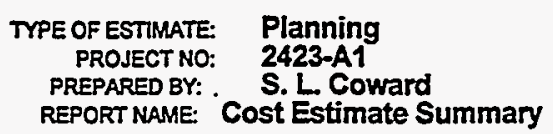

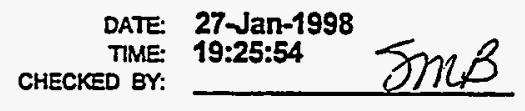

APPR'D BY:

\begin{tabular}{|c|c|c|c|c|}
\hline $\begin{array}{l}\text { WBS } \\
\text { Element }\end{array}$ & Cost Estimate Element & $\begin{array}{c}\text { Total } \\
\text { Unescalated }\end{array}$ & Escalation & $\begin{array}{c}\text { Total } \\
\text { Incl Escalation }\end{array}$ \\
\hline $\begin{array}{l}\frac{1.1}{1.1 .1} \\
1.1 .2 \\
1.2 \\
1.2 .1 \\
1.2 .2 \\
1.3 \\
1.3 .1 \\
1.3 .2 \\
1.3 .3 \\
1.5 .2\end{array}$ & $\begin{array}{l}\text { ENGINEERING, DESIGN AND INSPECTION } \\
\text { DESIGN ENGINEERING } \\
\text { TITLE III INSPECTION } \\
\text { MANAGEMENT COSTS } \\
\text { PROJECT MANAGEMENT } \\
\text { CONSTRUCTION MANAGEMENT } \\
\text { CONSTRUCTION } \\
\text { GENERAL CONDITIONS } \\
\text { SITEWORK } \\
\text { CONCRETE } \\
\text { PROCUREMENT FEES }\end{array}$ & $\begin{array}{r}1,646,982 \\
329,396 \\
\\
1,089,268 \\
658,793 \\
\\
1,067,791 \\
229,119 \\
5,291,021 \\
65,879\end{array}$ & $\begin{array}{l}0 \\
0 \\
0 \\
0 \\
0 \\
0 \\
0\end{array}$ & $\begin{array}{r}\gg \frac{\$ \$ 1,976,378}{1,646,982} \\
329,396\end{array}$ \\
\hline & $\begin{array}{l}\text { SUBTOTAL INCLUDING ESCALATION } \\
\text { PROJECT CONTINGENCY }\end{array}$ & $10,378,249$ & 0 & $\gg>\$ 10,378,249$ \\
\hline & MANAGEMENT RESERVE- & & & $\$ 665,381$ \\
\hline & & & & $\gg \quad \$ 2,956,370$ \\
\hline & TOTAL ESTIMATED COST & & & $\$ 14,000,000$ \\
\hline
\end{tabular}

\section{PROJECT COST PARAMETERS}

EDI AS A $\%$ OF CONST. + GFE $=30.00 \%$ 


\section{DETAILED COST ESTIMATE SHEET}

TYPE OF ESTIMATE: Planning

PROJECT NO.: 2423-A1

PREPARED BY: S. L. Coward
PAGE \# 2

DATE 27-Jan-1998

TIME: 19:25:56

REPORT NAME: Detail Cost Estlmate Sheet

LOCATION 1: ICPP

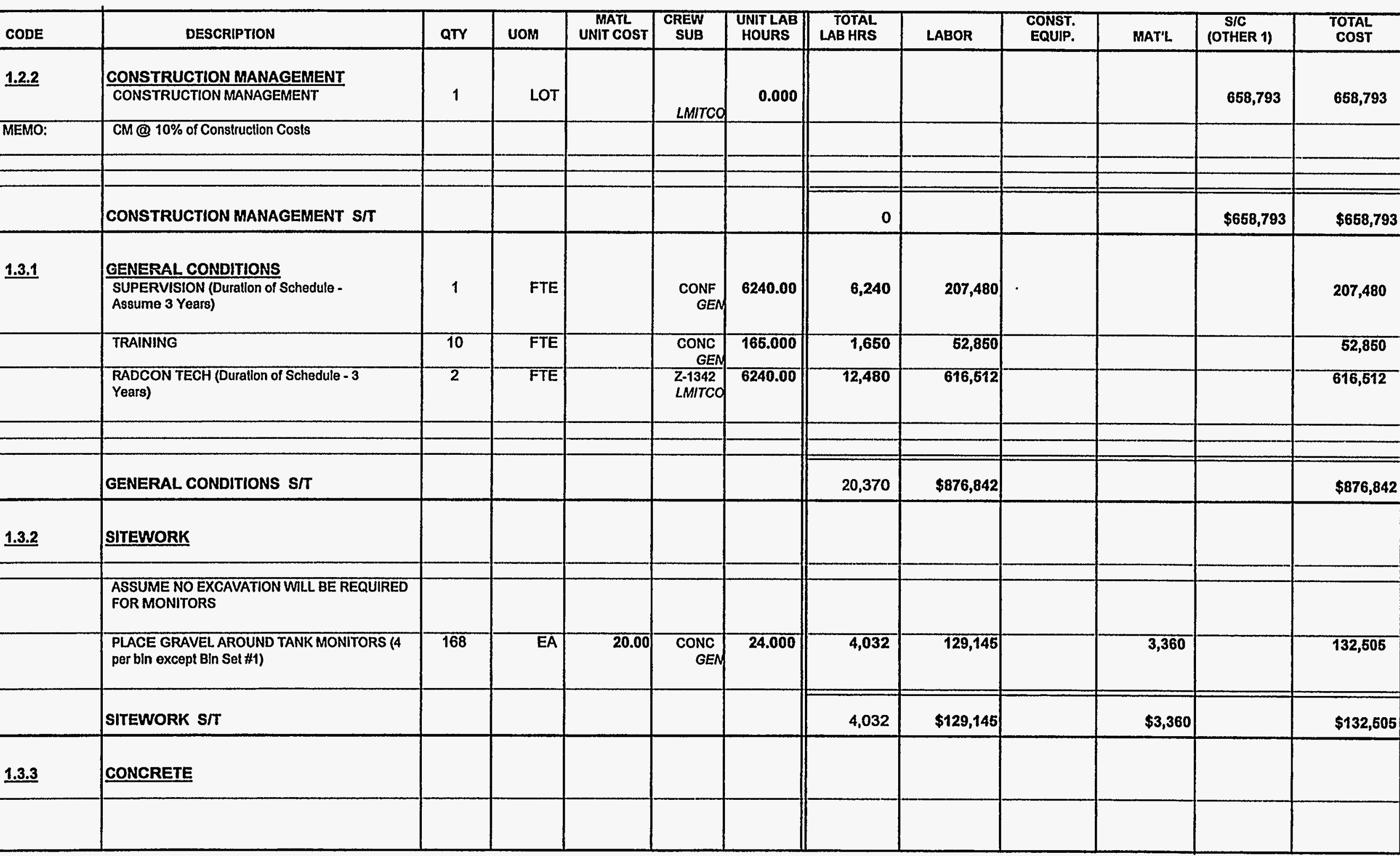


Lockheed Martin Idaho Technologies Co. Rev 6-96 PROJECT NAME: ICPP Bin Set Closure (EIS Stud LOCATION 1: REQUESTOR: Place Clean Grout in Bins ICPP Bryan Spaulding
CONTINGENCY ANALYSIS

$\begin{array}{cl}\text { TYPE OF ESTIMATE: } & \text { Planning } \\ \text { PROJECT NO: } & \text { 2423-A1 } \\ \text { PREPARED BY: } & \text { S. L. Coward }\end{array}$

DATE: 27-Jan-1998

TIME: 19:25:50

\begin{tabular}{|c|c|c|c|c|c|c|c|c|c|c|c|}
\hline \multirow{3}{*}{$\begin{array}{l}\text { WBS } \\
\text { Element }\end{array}$} & \multicolumn{8}{|c|}{ PROBABLE \% VARIATION } & \multicolumn{2}{|c|}{$\begin{array}{c}\text { PROJECT } \\
\text { CONTINGENCY }\end{array}$} & \multirow{3}{*}{$\begin{array}{l}\text { SUMMARY } \\
\text { Total Cost } \\
\text { by Element }\end{array}$} \\
\hline & \multirow{2}{*}{ Cost Estimate Element } & \multirow{2}{*}{$\begin{array}{l}\text { Total Cost w/o } \\
\text { Contingency }\end{array}$} & \multirow[t]{2}{*}{$\begin{array}{l}\% \text { Total } \\
\text { Cost }\end{array}$} & \multicolumn{2}{|c|}{$\begin{array}{c}\text { Prob. \% Var. } \\
\text { From Est. }\end{array}$} & \multicolumn{2}{|c|}{ Wh. $\%$ of Prob. } & \multirow[t]{2}{*}{ Contingency } & \multirow[t]{2}{*}{$\%$} & \multirow[t]{2}{*}{ Cost } & \\
\hline & & & & - & + & - & \pm & & & & \\
\hline 1.1 .1 & DESIGN ENGINEERING & $1,646,982$ & 15.87 & 10 & 40 & 1.59 & 6.35 & $5.554 \%$ & $15.87 \%$ & 574,756 & $2,221,738$ \\
\hline 1.1 .2 & TILE III INSPECTION & 329,396 & 3.17 & 10 & 40 & 0.32 & 1.27 & $1.111 \%$ & $3.17 \%$ & 114,951 & 444,347 \\
\hline 1.2 .1 & PROJECT MANAGEMENT & $1,089,268$ & 10.50 & 10 & 40 & 1.05 & 4.20 & $3.673 \%$ & $10.50 \%$ & 380,127 & $1,469,395$ \\
\hline 1.2 .2 & CONSTRUCTION MANAGEMENT & 658,793 & 6.35 & 10 & 40 & 0.63 & 2.54 & $2222 \%$ & $6.35 \%$ & 229,902 & 888,695 \\
\hline 1.3 .1 & GENERAL CONDITIONS & $1,067,791$ & 10.29 & 10 & 40 & 1.03 & 4.12 & $3.601 \%$ & $10.29 \%$ & 372,633 & $1,440,424$ \\
\hline 1.3 .2 & SITEWORK & 229,119 & 2.21 & 10 & 40 & 022 & 0.88 & $0.773 \%$ & $2.21 \%$ & 79,957 & 309,076 \\
\hline 1.3 .3 & CONCRETE & $5,291,021$ & 50.98 & 10 & 40 & 5.10 & 20.39 & $17.844 \%$ & $50.98 \%$ & $1,846,435$ & $7,137,456$ \\
\hline \multirow[t]{2}{*}{1.5 .2} & PROCUREMENT FEES & 65,879 & 0.63 & 10 & 40 & 0.06 & 0.25 & $0.222 \%$ & $0.63 \%$ & 22,990 & 88,869 \\
\hline & ESCALATION & 0 & 0.00 & 0 & 0 & 0.00 & 0.00 & $0.000 \%$ & $0.00 \%$ & 0 & 0 \\
\hline \multicolumn{2}{|r|}{ SUBTOTAL } & $10,378,249$ & 100.00 & & & & & $35.000 \%$ & & & \\
\hline \multicolumn{2}{|r|}{ CALCULATED CONTINGENCY } & $3,632,387$ & & & & & & & & & \\
\hline \multicolumn{2}{|r|}{ RESULTANT TEC } & $14,010,636$ & & & & & & & & & \\
\hline \multicolumn{2}{|r|}{ ROUNDED TEC } & $14,000,000$ & & & & & & & & & \\
\hline \multicolumn{2}{|r|}{ PROJECT CONTINGENCY } & $3,621,751$ & & & & & & $34.90 \%$ & & & \\
\hline \multicolumn{2}{|r|}{ MANAGEMENT RESERVE } & 665,381 & & & & & & & & & \\
\hline \multicolumn{2}{|r|}{ CONTINGENCY } & $2,956,370$ & & & & & & & & & \\
\hline \multicolumn{2}{|r|}{ TOTAL ESTIMATED COST } & $14,000,000$ & & & & & & & & $3,621,751$ & $14,000,000$ \\
\hline
\end{tabular}

CONFIDENCE LEVEL AND ASSUMED RISKS:

The Lockheed Idaho Technologres Co. Cost Estimate Contingency Analysis Model is based on the applied contingency and the assumptions upon which the estimate was predicated. The model is applied with a suggested risk level of $18 \%$ and a level of confidence of $90 \%$ the estimate will fall within the bid range. The Contingency Analysis is based on a weighted average to provide a $90 \%$ probability of undermu and a $10 \%$ probability of overrun.

\section{CONTINGENCY ANALYSIS GUIDE BY TYPE OF ESTIMATE}

Guidelines established by DOE/FM 50, Cost Estimating Guide, Vol. 6, Cost Guide, and as presented in the INEL Cost Estimating Guide. PLANNING Conceptual $15 \%-25 \%$ TITLE ! TITLE II TITLE II/AFC ....Up to $40 \%$ $10 \%-20 \%$ $5 \%-15 \%$ Market Conditions 


\section{G\&APIF ADDER CALCULATION SHEET \\ ICPP BIN SET CLOSURE \\ CLOSURE TO RCRA LANDFILL STANDARDS; CLEAN FILL; UNESCALATED \\ FILL BINS WI NRC CLEAN GROUT}

PROCUREMENT FEE:

CONSTRUCTION $=$
GFE $=$ Subtotal $\frac{\$ 6,587,931}{\$ 6,587,931}$

FEE @ 1\%=

$\$ 6,587,931 * 0.01=$

$\$ 65,879$

G\&A @ 23\% (with a ceiling of $\$ 500,000$ imposed per year, 4 yrs)

CONSTRUCTION OR

CEILING * 4 OF YEARS =

GFE =

$\$ 200,000$

PROCUREMENT FEE =

$\$ 65,879$

Subtotal

$\$ 265,879$

FEE @ 23\% =

$\$ 265,879 \cdot 0.23=$

$\$ 61,152$

PIF @ 5.5\%

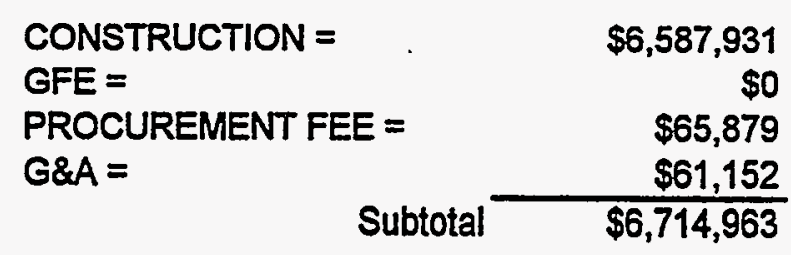

FEE @ $5.5 \%=$

$\$ 6,714,963 * 0.055=$

$\$ 369,323$

TOTAL PROCUREMENT FEE:

$\$ 65,879$

TOTAL G\&A FEE:

$\$ 61,152$

TOTAL PIF:

$\$ 369,323$ 
Lockheed Martin Idaho Technologies Co. PROJECT NAME: ICPP Bin Set Closure D\&D of Equipment LOCATION 1: INEEL / ICPP REQUESTOR: B. C. Spaulding
COST ESTIMATE SUMMARY

\begin{tabular}{|c|c|c|c|}
\hline $\begin{array}{l}\text { TYPE OF ESTIMATE: } \\
\text { PROJECT NO: } \\
\text { PRFPARED BY: }\end{array}$ & $\begin{array}{l}\text { Planning } \\
\text { 2423D\&D } \\
\text { S.LCoward/smb }\end{array}$ & $\begin{array}{r}\text { DATE: } \\
\text { TIME: } \\
\text { CHECKED BY: }\end{array}$ & 19:33:11 \\
\hline
\end{tabular}

APPRD BY:

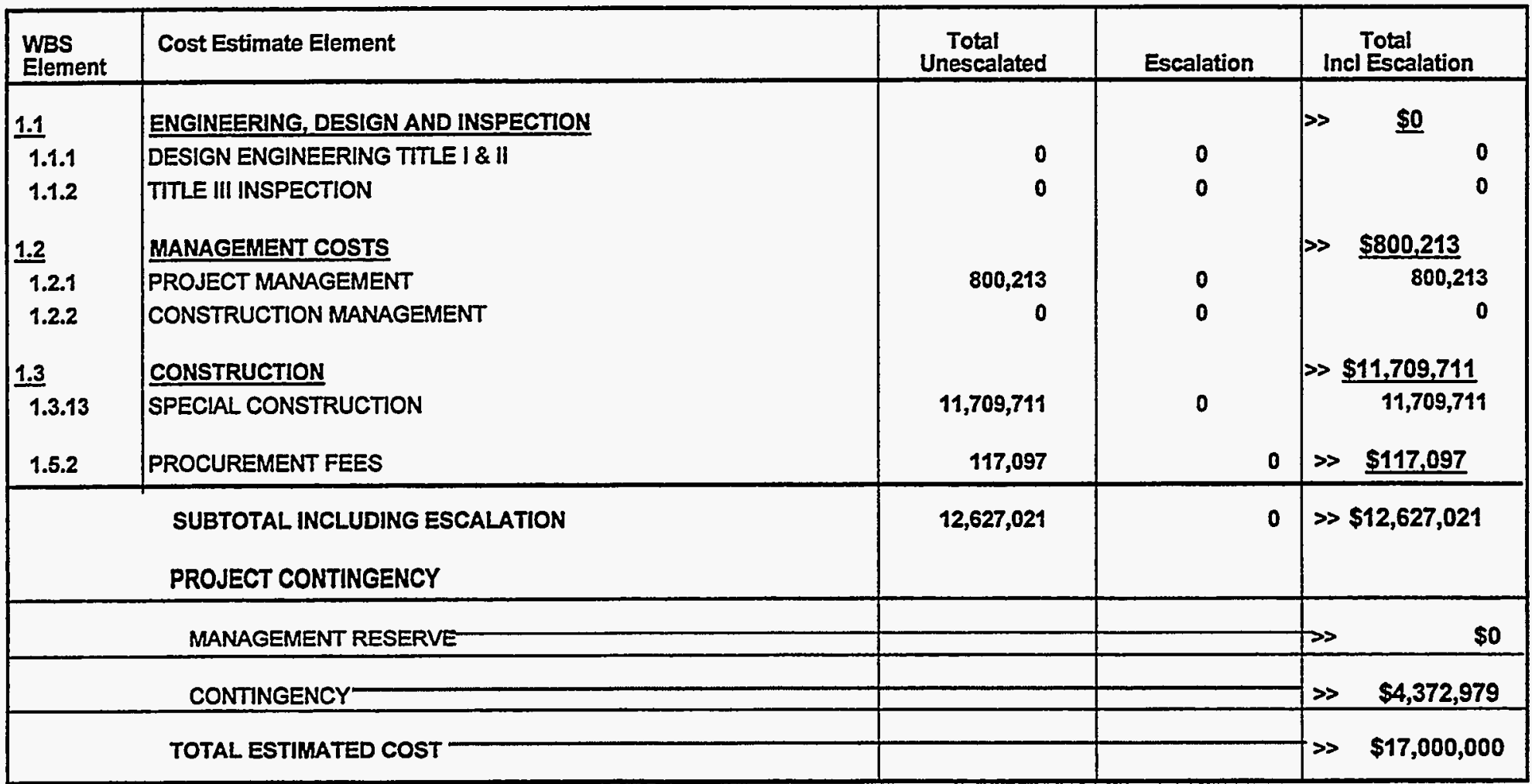

PROJECT COST PARAMETERS

EDI AS A \% OF CONST. + GFE $=0.00 \%$ 
Lockhoed Martin Idaho Technologles Co.

PROJECT NAME: ICPP Bin Set Closure

D\&D of Equipment

LOCATION 1: INEEL/ICPP

REQUESTOR: B. C. Spaulding

\section{DETAILED COST ESTIMATE SHEET}

TYPE OF ESTIMATE: Planning

PROJECT NO.: 2423D\&D

PREPARED BY: S.L.Coward/smb
PAGE \# 1

DATE 27-Jan-1998

TIME: 19:30:57

REPORT NAME: Detail Cost Estimate Sheet

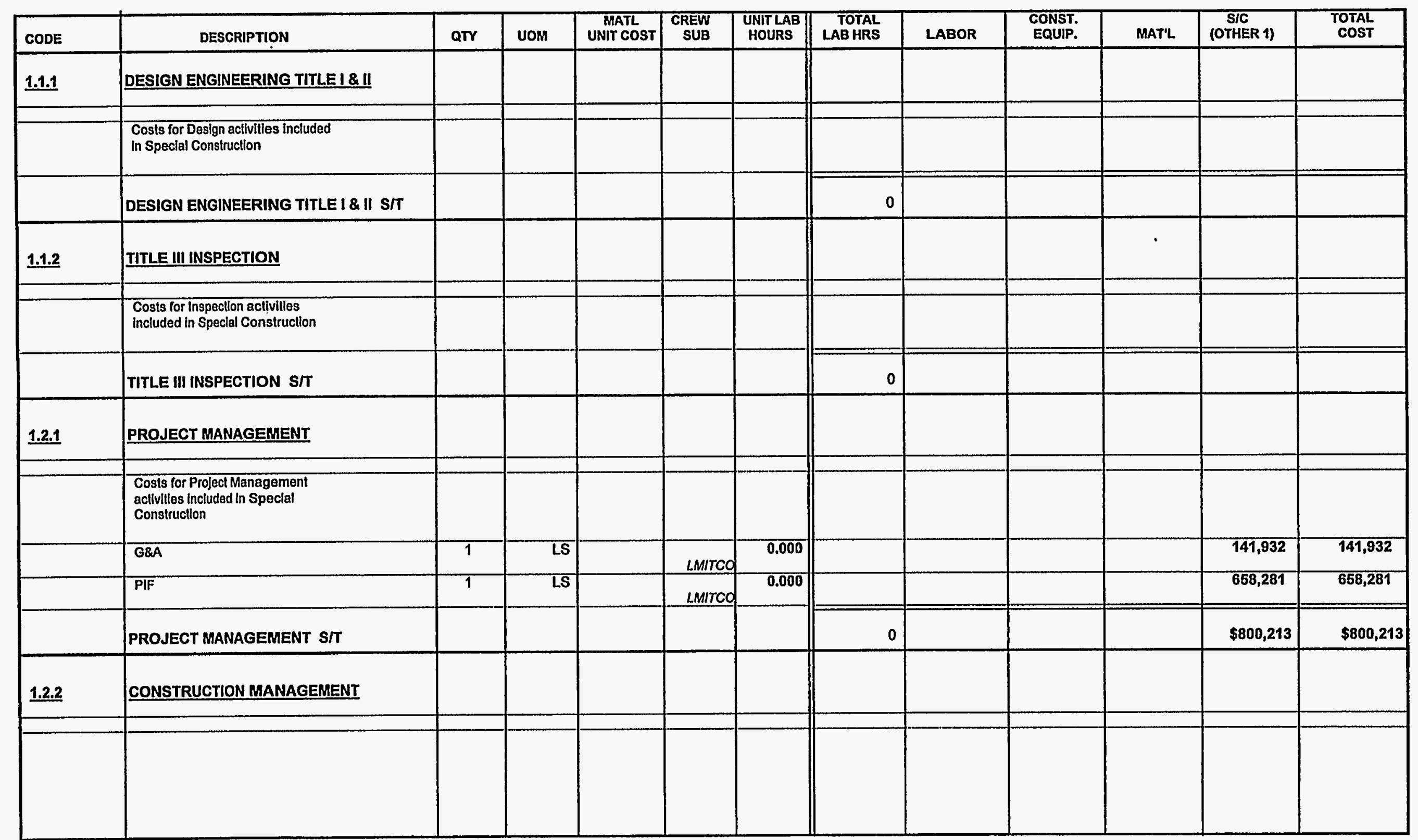


Lockheed Martin Idaho Technologies Co.

Rov 6-95

PROJECT NAME: ICPP Bin Sot Closure

D\&D of Equipment

LOCATION 1: INEEL / ICPP

REQUESTOR: B.C. Spaulding
DETAILED COST ESTIMATE SHEET

TYPE OF ESTIMATE: Planning

PROJECT NO: 2423D\&D

PREPARED BY: S.L.Coward/smb
PAGE \# 3

DATE 27-Jan-1998

TIME: 19:30:57

REPORT NAME: Detail Cost Estimate Sheot

\begin{tabular}{|c|c|c|c|c|c|c|c|c|c|c|c|c|}
\hline CODE & DESCRIPTION & QTY & UOM & $\begin{array}{l}\text { MATL } \\
\text { UNIT COST }\end{array}$ & $\begin{array}{l}\text { CREW } \\
\text { SUB }\end{array}$ & $\begin{array}{l}\text { UNIT LAB } \\
\text { HOURS }\end{array}$ & $\begin{array}{l}\text { TOTAL } \\
\text { LAB HRS }\end{array}$ & LABOR & $\begin{array}{l}\text { CONST. } \\
\text { EQUIP. }\end{array}$ & MAT'L & $\begin{array}{c}\text { SIC } \\
\text { (OTHER 1) }\end{array}$ & $\begin{array}{l}\text { TOTAL } \\
\text { COST }\end{array}$ \\
\hline \multirow[t]{15}{*}{1.3 .13} & $\frac{\text { SPECIAL CONSTRUCTION }}{\text { - Assume } 63 \text { HEPA fillers @ 6 6 } \times 6^{\prime} \times 2^{\prime}}$ & & & & & & & & & & & \\
\hline & $\begin{array}{l}\text { Jumper Retrieval Piping (Shlelded) - } 1 \\
\text { ea @ } 420 \text { sf }\end{array}$ & 1 & 10t & & & 0.000 & & & & & 109,494 & 109,494 \\
\hline & - Assume $210^{\prime}$ long $\times 2$ ' wide concrete & & & & & & & & & & & \\
\hline & $\begin{array}{l}\text { Remote Core Drilling Platform/Saw, } \\
\text { etc. - } 1 \text { ea @ } 250 \text { sf }\end{array}$ & 1 & Tot & & & 0.000 & & & & & 56,966 & 65,966 \\
\hline & $\begin{array}{l}\text { - Assume platform @ } 5^{\prime} \times 5^{\prime} \times 6^{\prime} \text { and } \\
\text { saw is } 100 \text { sf }\end{array}$ & & & & & & & & & & & \\
\hline & $\begin{array}{l}\text { Remole Welding and Cutting Equipment - } \\
\text { Allowance- } 1 \text { ea @ } 500 \text { sf }\end{array}$ & 1 & $10 t$ & & & 0.000 & & & & & 111,932 & 111,932 \\
\hline & $\begin{array}{l}\text { Vertical Deployment Appartus - } 7 \text { ea @ } \\
150 \text { sf }\end{array}$ & 1 & Tot & & & 0.000 & & & & & 235,057 & 235,057 \\
\hline & - Assume $6^{\prime} \times 5^{\prime} \times 6^{\prime}$ & & & & & & & & & & & \\
\hline & $\begin{array}{l}\text { Shlelding \& Riser Plugs - } 100 \text { ea } \\
\text { @ } 4 \text { sf }\end{array}$ & 1 & Tot & & & 0.000 & & & & & 116,794 & 116,794 \\
\hline & - Assume 2' x 2' & & & & & & & & & & & \\
\hline & CO2 Blastlng Equlpment - Allowance - & 1 & lot & & & 0.000 & & & & & 223,864 & 223,864 \\
\hline & Retrleval LInes - 1 ea @ 2310 sf & 1 & Tol & & & 0.000 & & & & & 373,116 & 373,116 \\
\hline & - Assume $2310^{\prime}$ long $\times 11^{\prime}$ wide & & & & & & & & & & & \\
\hline & $\begin{array}{l}\text { Conirol Consoles, Cameras, Lighting - } \\
\text { Allowance - } 1 \text { ea @ } 1000 \text { sf }\end{array}$ & 1 & lot & & & 0.000 & & & & & 107,934 & 107,934 \\
\hline & $\begin{array}{l}\text { Grouling Manifolds - Clean - Allowance } \\
-8 \text { ea @ } 300 \text { si }\end{array}$ & 1 & lot & & & 0.000 & & & & & 259,043 & 259,043 \\
\hline & & & & & & & & & & & & \\
\hline & & & & & & & & & & & & \\
\hline
\end{tabular}


Lockheed Martin Idaho Technologies Co. Rev 6-96

PROJECT NAME: ICPP Bin Set Closure

D\&D of Equipment

LOCATION 1: INEEL / ICPP

REQUESTOR: B. C. Spaulding

CONTINGENCY ANALYSIS

TYPE OF ESTIMATE

PREPARED BY: $\quad$ S.LCCoward/smb
DATE: 27 Jan-1998

TIME: 19:33:04

REPORT NAME: Contingency Analysis

\begin{tabular}{|c|c|c|c|c|c|c|c|c|c|c|c|}
\hline & PROE & BLE \% VARIA & ION & & & & & & $\begin{array}{c}\text { PRR } \\
\text { CON }\end{array}$ & $\begin{array}{l}\text { CT } \\
\text { SENCY }\end{array}$ & SUMMARY \\
\hline $\begin{array}{l}\text { WBS } \\
\text { Element }\end{array}$ & Cost Estimate Element & Total Cost w/o & $\begin{array}{l}\% \text { Total } \\
\text { Cost }\end{array}$ & & $\begin{array}{l}\text { \% Var. } \\
\text { mEst. }\end{array}$ & Wt \% & Prob. & Contingency & $\%$ & Cost & Total Cost \\
\hline & & Contingency & & - & $t$ & - & + & & & & by Element \\
\hline 1.1.1 & DESIGN ENGINEERING TITLE $\mid \& \|$ & 0 & 0.00 & 0 & 0 & 0.00 & 0.00 & $0.000 \%$ & $0.00 \%$ & 0 & 0 \\
\hline 1.1 .2 & TITLE III INSPECTION & $\overline{0}$ & 0.00 & $\overline{0}$ & 0 & 0.00 & 0.00 & $0.000 \%$ & $0.00 \%$ & 0 & \\
\hline 1.2 .1 & PROJECT MANAGEMENT & 800,213 & 6.34 & 10 & 40 & 0.63 & 253 & $2.218 \%$ & $6.34 \%$ & 277,129 & $1,077,342$ \\
\hline 1.2 .2 & CONSTRUCTION MANAGEMENT & 0 & 0.00 & 0 & 0 & 0.00 & 0.00 & $0.000 \%$ & $0.00 \%$ & 0 & 0 \\
\hline 1.3 .13 & SPECIAL CONSTRUCTION & $11,709,711$ & 92.74 & 10 & 40 & 9.27 & 37.09 & $32.457 \%$ & $92.74 \%$ & $4,055,297$ & $15,765,008$ \\
\hline 1.5 .2 & PROCUREMENT FEES & 117,097 & 0.93 & 10 & 40 & 0.09 & 0.37 & $0.325 \%$ & $0.93 \%$ & 40,553 & 157,650 \\
\hline & ESCALATION & 0 & 0.00 & 0 & 0 & 0.00 & 0.00 & $0.000 \%$ & $0.00 \%$ & 0 & 0 \\
\hline & SUBTOTAL & $12,627,021$ & 100.00 & & & & & $35.000 \%$ & & & \\
\hline & CALCULATED CONTINGENCY & $4,419,457$ & & & & & & & & & \\
\hline & RESULTANT TEC & $17,046,478$ & & & & & & & & & \\
\hline & ROUNDED TEC & $17,000,000$ & & & & & & & & & \\
\hline & PROJECT CONTINGENCY & $4,372,979$ & & & & & & $34.63 \%$ & & & \\
\hline & MANAGEMENT RESERVE & 0 & & & & & & & & & \\
\hline & CONTINGENCY & $4,372,979$ & & & & & & & & & \\
\hline & TOTAL ESTIMATED COST & $17,000,000$ & & & & & & & & $4,372,979$ & $17,000,000$ \\
\hline
\end{tabular}

\section{CONFIDENCE LEVEL AND ASSUMED RISKS:}

The Lockheed idaho Technologies Co. Cost Estimate Contingency Analysis Model is based on the applied contingency and the assumptions upon which the estimate was predicated. The model is applied with a suggested risk level of $18 \%$ and a level of confidence of $90 \%$ the estimate will fall within the bid range. The Contingency Analysis is based on a weighted average to provide a $90 \%$ probability of underrun and a $10 \%$ probability of overrun.
CONTINGENCY ANALYSIS GUIDE BY TYPE OF ESTIMATE

Guidelines established by DOE/FM 50, Cost Estimating Guide, Vol. 6 Cost Guide, and as presented in the INEL Cost Estimating Guide. PLANNING Conceptual Up to $50 \%$ TITLE I

TITLE I TITLE II/AFC
$15 \%-25 \%$ $10 \%-20 \%$ $5 \%-15 \%$ Market Conditions .....Up to $40 \%$ 
INEI. RAD Contuminuted Surplus Fucilliles Cost Modlel $\Longrightarrow$ D\&D Cost

Crp blu seer Closure

Project Fut soist20

Piepared by Stietiy Coward

(2) (1)

sucilly

\begin{tabular}{|c|c|c|c|c|c|c|c|}
\hline Totul Usable & & & & & & & DEn \\
\hline $\begin{array}{l}\text { Plour Sporse } \\
\text { Syurre Fiets }\end{array}$ & $\begin{array}{l}\text { Bists } \\
\text { l.vis }\end{array}$ & $\mathrm{ACM}$ & $\mathbb{H A Z}$ & $\begin{array}{l}\text { Fuchlop Ruthes } \\
\text { RA1) SYS }\end{array}$ & 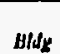 & CIIRTZ & $\begin{array}{l}\text { ROMS } \\
\text { Cour (s }\end{array}$ \\
\hline
\end{tabular}

Drations

\begin{tabular}{|c|c|c|c|c|c|c|c|c|c|c|c|}
\hline 7 & Soonolo i & Ventilation, Instr. \& Control Bildg (VIC) & 2,400 & $T$ & NA & $\mathbf{L}$ & तो & L. & $\mathbf{s}$ & L. & 869.133 \\
\hline 7 & & Conlinement Enclosures (lop of Bin Sols) & 1.600 & $I$ & NA & $\bar{A}$ & A & $I$ & $\mathbf{s}$ & $\mathbf{L}$ & $1,248,128$ \\
\hline 7 & SoON010 2 & Bridgo Crano & 300 & $i$ & NA & $L$ & $\mathrm{H}$ & L & $\mathbf{s}$ & $\underline{L}$ & 369,370 \\
\hline 1 & SeONolo 3 & HVAC Equipmenusyslem & 6,800 & $T$ & NA & L & $A$ & $t$ & $\mathbf{s}$ & 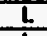 & 449,044 \\
\hline 1 & 500 Nolo 4 & Jumper Rotriovel Piplane (Shrolded) & 420 & 1 & NA & $\bar{A}$ & H & $\mathbf{L}$ & $\mathbf{C}$ & $I$ & 100,404 \\
\hline$i$ & So0 Noto 5 & Remolo Core Donling PlatiormS Saw, elc. & 260 & 1 & NA & H & $H$ & $L$ & $\mathbf{S}$ & $I$ & 56.960 \\
\hline 1 & Allowance & Remolo Wolding and Cutllng Equipment & 500 & $i$ & NA & H & H & L & s & $\mathbf{L}$ & 111,032 \\
\hline 7 & Soo Nolo 8 & Vortical Doploymont Apparalus & 150 & $I$ & NA & H & H & 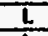 & $\mathbf{s}$ & $t$ & 235,057 \\
\hline 100 & Soo Nolo 7 & Shilotding and Riser Plugs & 4 & $\frac{1}{1}$ & NA & H & H & L & C & $\bar{L}$ & 116,784 \\
\hline 2 & Allowance & CO2 Bjasling Equipment & 500 & 1 & NA & $H$ & $\mathrm{H}$ & $L$ & $\mathbf{S}$ & $L$ & 223,064 \\
\hline$\frac{2}{1}$ & SOONO10 & Relriaval Lines & 2,310 & 1 & NA & $H$ & $H$ & $\bar{L}$ & $\mathbf{s}$ & $\bar{L}$ & 373.116 \\
\hline$i$ & Allowance & Control Consoles, Cameras \& Ulonungo & .0000 & 1 & NA & $L$ & 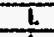 & L & $\mathbf{s}$ & L & 107.034 \\
\hline$\theta$ & Allowanco & Grouldng Manilolds - Cloan & 300 & 1 & NA & $t$ & $t$ & $\mathrm{~L}$ & s & $\bar{l}$ & 269,043 \\
\hline$\theta$ & Allowance & Groulling Manilolds - Class C & 300 & 1 & NA & H & $\mathrm{H}$ & $L$ & $\mathbf{S}$ & $I$ & 537.274 \\
\hline & Allowance & LOUA's & 160 & 1 & NA & $\bar{A}$ & $\mathrm{H}$ & $L$ & $\mathbf{S}$ & $L$ & 200,073 \\
\hline 7 & Allowanco & Plpo Crawlor Robols & 160 & 1 & NA & A & $\boldsymbol{H}$ & t & $\mathbf{s}$ & $t$ & 200,873 \\
\hline 53 & Allowanco & Tractor Robols & 250 & 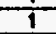 & NA & $A$ & H & L & s & $\bar{L}$ & $2,648,393$ \\
\hline \multirow[t]{6}{*}{1} & Allowance & Misc. Robol whro and hosa linos & 1.000 & 1 & NA & $A$ & H & $t$ & $\overline{\mathbf{s}}$ & 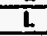 & 109.870 \\
\hline & & & & & & & & & & & \\
\hline & Soo Nolo 0 & Wasto Disposol & & & & & & & & & \\
\hline & Soures & Solld Wasto (In CF) & 600 & & & & & & & & .640 \\
\hline & & Radioaclivo Waste (n CF) & 10.000 & & & & & & & & $3.000,000$ \\
\hline & & Mixod Waslo (in CF) & 2.200 & & & & & & & & 275,000 \\
\hline & & & & & & & & & & & \\
\hline & & & & & & & & & & & \\
\hline & & & & & & & & & & & \\
\hline & & SUblolaIICPP BIN SET CLOSURE & & & & & & & & & 11.709 .711 \\
\hline
\end{tabular}

Nores:

1. Assunied that facilly will have low lovels of hazardous matorial, no rad conlamination, and no asbostos

2 - Assumio $40^{\circ}$ across by $45^{\circ}$ high $\times 2^{2}$ w lor rails plus trolley $\left(5^{\circ} \times 6^{\circ} \times 6^{\prime}\right)$

3. Assume 7 Alr handlors $\left(5^{\prime} \times 5^{\prime} \times 8^{\prime}\right)$, 3 Exhausi fans $\left(4^{\prime} \times 4^{\prime} \times 3^{\prime}\right)$, and 63 HEPA fillors $\left(8^{\prime} \times 6^{\circ} \times 2^{\prime}\right)$

4 - Assumo 210 LF $\times 2$ ' wido concreto

5. Assuma plattom $\left(5^{\circ} \times 6^{\circ} \times 6\right)$ plus saw (100 3i)

6. Assumo $6^{\circ} \times 6^{\circ} \times 6^{\circ}$

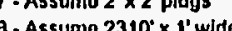

9 - Wasto Disposal Quantittlos wosa provlded by EDF

Assume lliu followitig Waslo Disposal Unill Costs:

$\begin{array}{ll}\text { Industrial Landfill } & \$ 1.08 / d 1 \\ \text { Low Lovol Wasto Repository } & \$ 125 / d 1 \\ \text { Rad Wasto } & \$ 300 / d\end{array}$




\section{G\&APIF ADDER CALCULATION SHEET \\ ICPP BIN SET CLOSURE \\ CLOSURE TO RCRA LANDFILL STANDARDS; CLEAN FILL; UNESCALATED \\ D\&D OF EQUIPMENT}

PROCUREMENT FEE:

CONSTRUCTION $=$
GFE $=$ Subtotal $\frac{\$ 11,709,711}{\$ 11,709,711}$

FEE @ 1\%=

$\$ 11,709,711 \cdot 0.01=$

$\$ 117,097$

G\&A@ $@ 23 \%$ (with a ceiling of $\$ 500,000$ imposed per year, 1 yr)

CONSTRUCTION OR

CEILING $\bullet 1$ YEARS $=\quad \$ 500,000$

GFE =

PROCUREMENT FEE $=$ $\$ 117,097$

Subtotal $\$ 617,097$

FEE @ $23 \%=$

$\$ 617,097 * 0.23=$

$\$ 141,932$

PIF @ 5.5\%

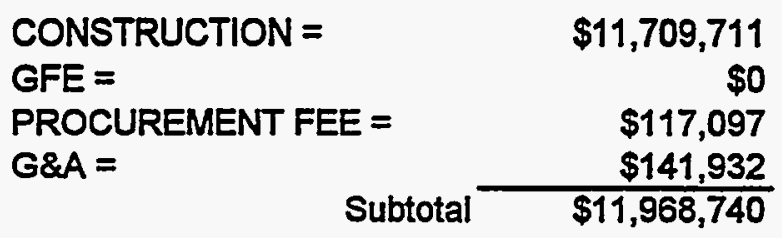

FEE @ 5.5\% =

$\$ 11,968,740 * 0.055=$

$\$ 658,281$

TOTAL PROCUREMENT FEE:

$\$ 117,097$

TOTAL G\&A FEE:

$\$ 141,932$

TOTAL PIF:

$\$ 658,281$ 
COST ESTIMATE SUMMARY

ESCALATED

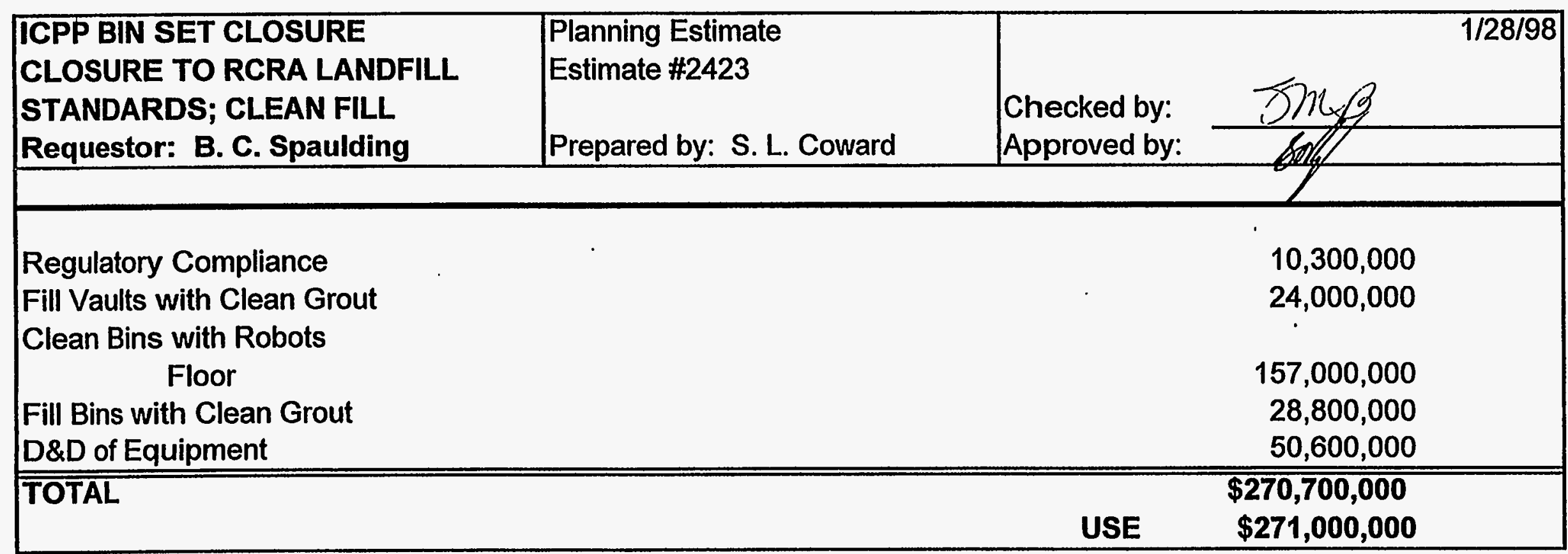


Lockheed Martin Idaho Technologies Co. Rov. 6-s

PROJECT NAME: Permitting/Documentation

Risk Based - Clean

INEELICPP

LOCATION 1:

REQUESTOR:

Bryan Spaulding
COST ESTIMATE SUMMARY

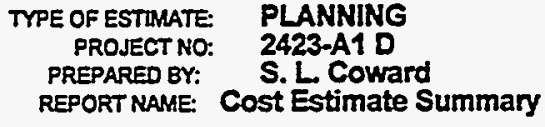

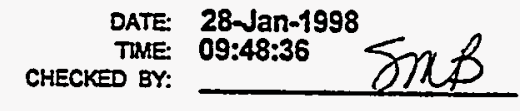

APPRD BY:

\begin{tabular}{|c|c|c|c|c|}
\hline $\begin{array}{l}\text { WBS } \\
\text { Element }\end{array}$ & Cost Estimate Element & $\begin{array}{c}\text { Total } \\
\text { Unescalated }\end{array}$ & Escalation & $\begin{array}{c}\text { Total } \\
\text { Incl Escalation }\end{array}$ \\
\hline $\begin{array}{l}\frac{1.1}{1.1 .1} \\
\frac{1.2}{1.2 .1} \\
1.2 .2 \\
\frac{1.3}{1.3 .1} \\
1.5 .2\end{array}$ & $\begin{array}{l}\text { CONCEPTUAL } \\
\text { CONCEPTUAL DESIGN } \\
\text { MANAGEMENT } \\
\text { PM FOR PROJECT DEVELOOMENT } \\
\text { PROJECT EXECUTION } \\
\text { PERMITTING } \\
\text { PERMITTING } \\
\text { PROCUREMENT FEES }\end{array}$ & $\begin{array}{r}0 \\
774,995 \\
149,704 \\
3,742,601 \\
37,426\end{array}$ & $\begin{array}{c}0 \\
480,497 \\
92,816 \\
2,320,412 \\
23,204\end{array}$ & $\begin{array}{r}\gg \frac{\$ 1,498,012}{1,255,492} \\
242,520\end{array}$ \\
\hline & $\begin{array}{l}\text { SUBTOTAL INCLUDING ESCALATION } \\
\text { PROJECT CONTINGENCY }\end{array}$ & $4,704,726$ & $2,916,929$ & $\gg>\$ 7,621,655$ \\
\hline & MANAGEMENT RESERVE- & & & $\$ 612,364$ \\
\hline & CONTINGENCY- & & & $\gg \quad \$ 2,065,981$ \\
\hline & TOTAL ESTIMATED COST & & & $\gg \quad \$ 10,300,000$ \\
\hline
\end{tabular}

PROJECT COST PARAMETERS

EDI AS A $\%$ OF CONST. + GFE $=\quad 0.00 \%$ 
Lockheed Martin Idaho Technologies Co. Rov6.96

PROJeCt NAME: Permitting/Documentation Risk Based - Clean

LOCATION 1: INEEUICPP
REQUESTOR:

\section{DETAILED COST ESTIMATE SHEET}

TYPE OF ESTIMATE: PLANNING

PROJECT NO.: 2423-A1 D

PREPARED BY: S. L. Coward
PAGE \# 1

DATE 28-Jan-1998

TIME: 10:07:13

REPORT NAME: Dotall Cost Estimate Sheot

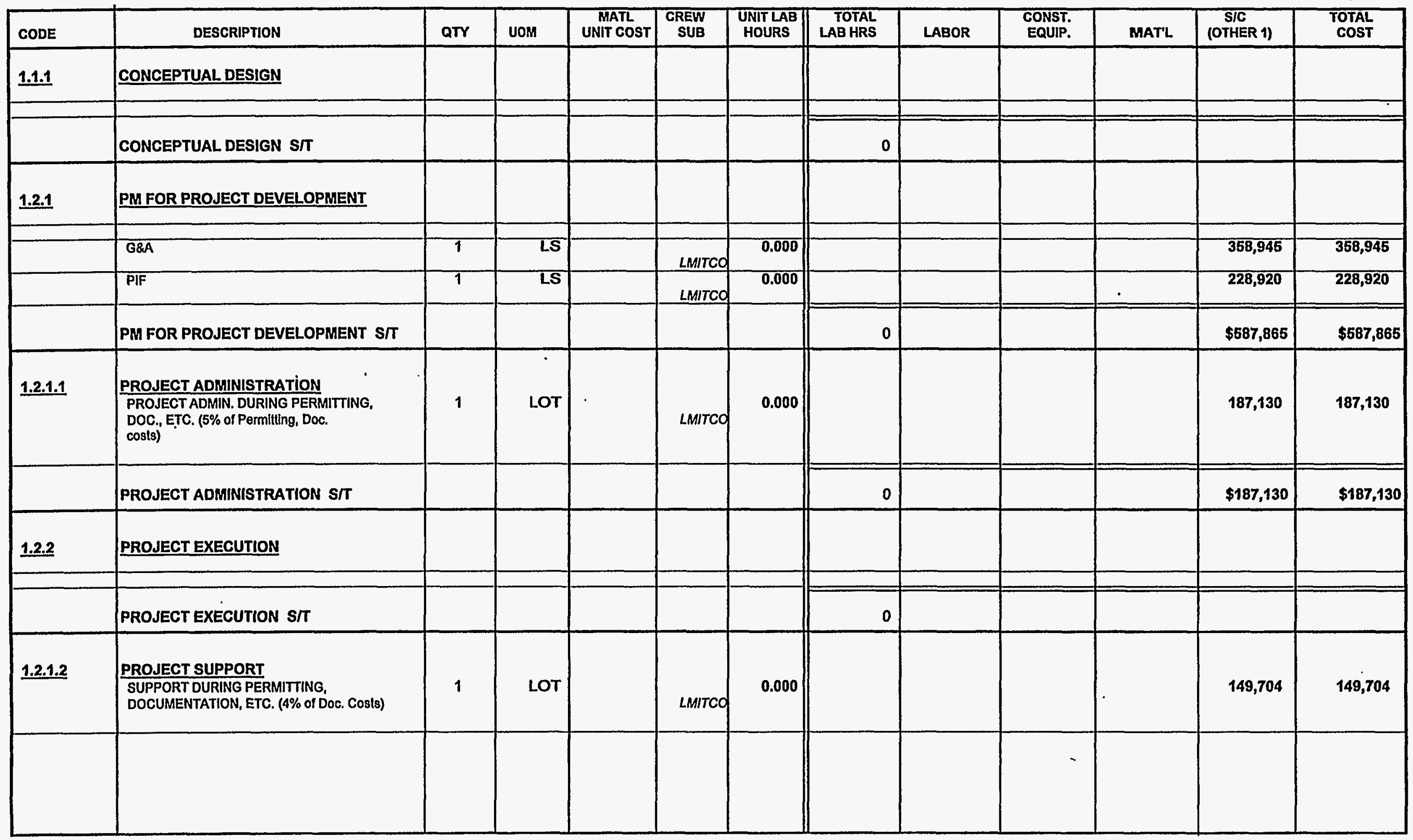


Lockheed Martin Idaho Technologles Co.

Rav 6.96

RACT NAME: Permltting/Documentation

Rlsk Based - Clean

INEEL/ICPP

DETAILED COST ESTIMATE SHEET

TYPE OF ESTIMATE: PLANNING

PROJECT NO.: 2423-A1 D

PREPARED BY: S. L. Coward
PAGE\# 2

DATE 28-Jan-1998

TIME: 10:07:13

REPORT NAME: Detall Cost Estimate Sheet

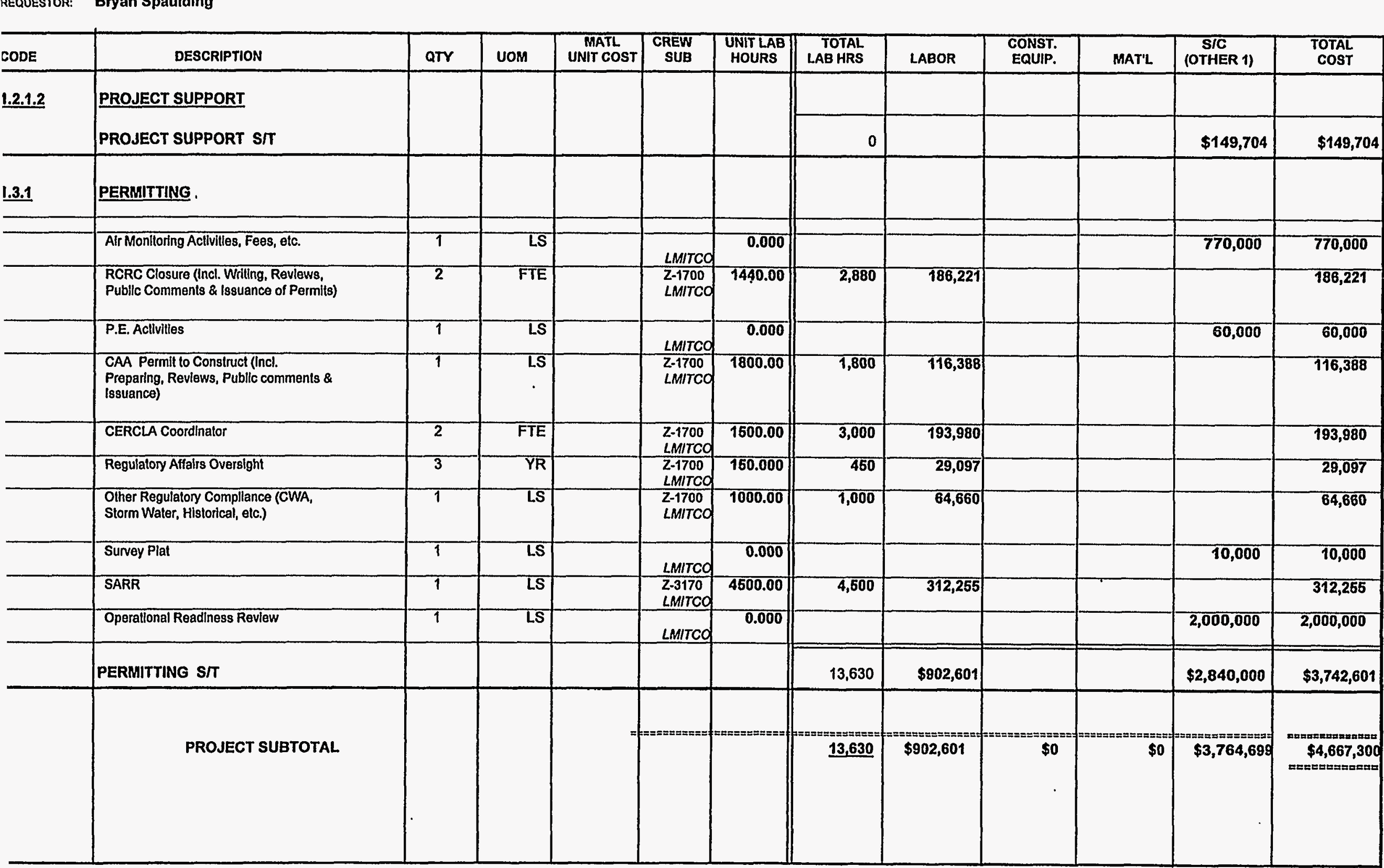




\section{Lockheed Martin Idaho Technologies Co.}

PROJECT NAME: Permitting/Documentation

Risk Based - Clean

INEELICPP

OCATION 1

REQUESTOR:

\section{CONTINGENCY ANALYSIS}

TYPE OF ESTIMATE: PLANNING
PROJECT NO: 2423-A1 D
PREPARED BY:

DATE: 28-Jan-1998

TIME: 09:48:12

REPORT NAME: Contingency Analysis

\begin{tabular}{|c|c|c|c|c|c|c|c|c|c|c|c|}
\hline \multirow{3}{*}{\begin{tabular}{|l|} 
WBS \\
Element
\end{tabular}} & \multicolumn{8}{|c|}{ PROBABLE \% VARIATION } & \multicolumn{2}{|c|}{$\begin{array}{c}\text { PROJECT } \\
\text { CONTINGENCY }\end{array}$} & \multirow{3}{*}{$\begin{array}{l}\text { SUMMARY } \\
\begin{array}{l}\text { Total Cost } \\
\text { by Element }\end{array}\end{array}$} \\
\hline & \multirow{2}{*}{ Cost Estimate Element } & \multirow{2}{*}{$\begin{array}{c}\text { Total Cost w/o } \\
\text { Contingency }\end{array}$} & \multirow{2}{*}{$\begin{array}{l}\% \text { Total } \\
\text { Cost }\end{array}$} & \multicolumn{2}{|c|}{$\begin{array}{c}\text { Prob. \% Var. } \\
\text { From Est. }\end{array}$} & \multicolumn{2}{|c|}{ Wh \% of Prob. } & \multirow{2}{*}{ Contingency } & \multirow{2}{*}{$\%$} & \multirow{2}{*}{ Cost } & \\
\hline & & & & - & + & - & + & & & & \\
\hline 1.1.1 & CONCEPTUAL DESIGN & 0 & 0.00 & 10 & 40 & 0.00 & 0.00 & $0.000 \%$ & $0.00 \%$ & 0 & 0 \\
\hline$\longdiv { 1 . 2 . 1 }$ & PM FOR PROJECT DEVELOPMENT & 774,995 & 10.17 & 10 & 40 & 1.02 & 4.07 & $3.559 \%$ & $10.17 \%$ & 272,343 & $1,047,338$ \\
\hline 1.2 .2 & PROJECT EXECUTION & 149,704 & 1.96 & 10 & 40 & 0.20 & 0.79 & $0.687 \%$ & $1.96 \%$ & 52,608 & 202,312 \\
\hline 1.3.1 & PERMITTING & $3,742,601$ & 49.10 & 10 & 40 & 4.91 & 19.64 & $17.187 \%$ & $49.10 \%$ & $1,315,197$ & $5,057,798$ \\
\hline \multirow[t]{2}{*}{1.5 .2} & PROCUREMENT FEES & 37,426 & 0.49 & 10 & 40 & 0.05 & 0.20 & $0.172 \%$ & $0.49 \%$ & 13,152 & 50,578 \\
\hline & ESCALATION & $2,916,929$ & 38.27 & 10 & 40 & 3.83 & 15.31 & $13.395 \%$ & $38.27 \%$ & $1,025,045$ & $3,941,974$ \\
\hline & SUBTOTAL & $7,621,655$ & 100.00 & & & & & $35.000 \%$ & & & \\
\hline & CALCULATED CONTINGENCY & $2,667,579$ & & & & & & & & & \\
\hline & RESULTANT TEC & $10,289,234$ & & & & & & & & & \\
\hline & ROUNDED TEC & $10,300,000$ & & & & & & & & & \\
\hline & PROJECT CONTINGENCY & $2,678,345$ & & & & & & $35.14 \%$ & & & \\
\hline & MANAGEMENT RESERVE & 612,364 & & & & & & & & & \\
\hline & CONTINGENCY & $2,065,981$ & & & & & & & & & \\
\hline & TOTALESTIMATED COST & $10,300,000$ & & & & & & & & $2,678,345$ & $10,300,000$ \\
\hline
\end{tabular}

\section{CONFIDENCE LEVEL AND ASSUMED RISKS:}

The Lockheed Idaho Technologies Co. Cost Estimate Contingency Analysis Model is based on the applied contingency and the assumptions upon which the estimate was predicated. The model is applied with a suggested risk level

of $18 \%$ and a level of confidence of $90 \%$ the estimate will fall within the bid range.

The Contingency Analysis is based on a weighted average to provide a

$90 \%$ probability of underrun and a $10 \%$ probability of overrun.
CONTINGENCY ANALYSIS GUIDE BY TYPE OF ESTIMATE

Guidelines established by DOE/FM 50, Cost Estimating Guide, Vol. 6,

Cost Guide, and as presented in the INEL Cost Estimating Guide. PLANNING Experimental/Special Conditions Conceptual TITLE I

TITLE II

TITLE IIVAFC Up to $50 \%$ ..Up to $40 \%$ $10 \%-20 \%$ $5 \%-15 \%$ Market Conditions 


\section{G\&APIF ADDER CALCULATION SHEET \\ ICPP BIN SET CLOSURE \\ CLOSURE TO RCRA LANDFILL STANDARDS; CLEAN FILL; ESCALATED}

REGULATORY COMPLIANCE

PROCUREMENT FEE:

CONSTRUCTION =

$\$ 6,063,013$

GFE =

Subtotal $\$ 6,063,013$

FEE @ 1\%=

$\$ 6,063,013 * 0.01=$

$\$ 60,630$

G\&A @ 23\% (with a ceiling of $\$ 500,000$ imposed per year, 3 yrs)

CONSTRUCTION OR

CEILING * 3 YEARS $=$

GFE $=$

$\$ 1,500,000$

PROCUREMENT FEE $=$

Subtotal

$\$ 60,630$

$\$ 1,560,630$

FEE @ 23\%=

$\$ 1,560,630 \cdot 0.23=$

$\$ 358,945$

PIF @ 5.5\%

\begin{tabular}{lr} 
CONSTRUCTION = & $\begin{array}{r}\$ 3,742,601 \\
\text { GFE }=\end{array}$ \\
PROCUREMENT FEE = & $\$ 0$ \\
G\&A $=\quad \begin{array}{r}\$ 60,630 \\
\end{array} \quad$ Subtotal & $\$ 358,945$ \\
\cline { 2 - 2 } & $\$ 4,162,176$
\end{tabular}

FEE @ 5.5\% =

$\$ 4,162,176 * 0.055=$

$\$ 228,920$

TOTAL PROCUREMENT FEE:

$\$ 60,630$

TOTAL G\&A FEE:

$\$ 358,945$

TOTAL PIF:

$\$ 228,920$ 

Lockheed Martin Idaho Technologies Co. PROJECT NAME: ICPP Bin Set Closure (EIS Stud

LOCATION 1: REQUESTOR: Place Clean Grout in Vauit ICPP

Bryan Spaulding
COST ESTIMATE SUMMARY

TYPE OF ESTMATE: Planning PROJECT NO: 2423-A2-E2 PREPARED BY: REPORT NAME: Cost Estimate Summary

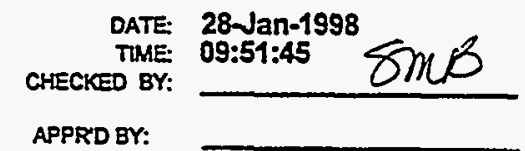

\begin{tabular}{|c|c|c|c|c|}
\hline $\begin{array}{l}\text { WBS } \\
\text { Element }\end{array}$ & Cost Estimate Element & $\begin{array}{c}\text { Total } \\
\text { Unescalated }\end{array}$ & Escalation & $\begin{array}{c}\text { Total } \\
\text { Incl Escalation }\end{array}$ \\
\hline $\begin{array}{l}\frac{1.1}{1.1 .1} \\
1.1 .2 \\
1.2 \\
1.2 .1 \\
1.2 .2 \\
1.3 \\
1.3 .1 \\
1.3 .2 \\
1.3 .3 \\
1.5 .2\end{array}$ & $\begin{array}{l}\text { ENGINEERING, DESIGN AND INSPECTION } \\
\text { DESIGN ENGINEERING } \\
\text { TITLE III INSPECTION } \\
\text { MANAGEMENT COSTS } \\
\text { PROJECT MANAGEMENT } \\
\text { CONSTRUCTION MANAGEMENT } \\
\text { CONSTRUCTION } \\
\text { GENERAL CONDITIONS } \\
\text { SITEWORK } \\
\text { CONCRETE } \\
\text { PROCUREMENT FEES }\end{array}$ & $\begin{array}{r}1,324,812 \\
264,962 \\
\\
1,702,520 \\
529,925 \\
\\
1,067,791 \\
0 \\
4,231,459 \\
52,992\end{array}$ & $\begin{array}{c}1,634,419 \\
540,524 \\
\\
1,089,146 \\
0 \\
4,316,088 \\
54,052\end{array}$ & 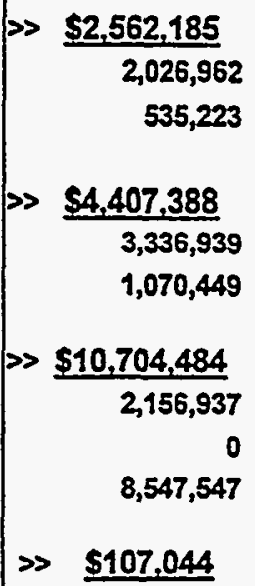 \\
\hline & $\begin{array}{l}\text { SUBTOTAL INCLUDING ESCALATION } \\
\text { PROJECT CONTINGENCY }\end{array}$ & $9,174,461$ & $8,606,640$ & $\gg>\$ 17,781,101$ \\
\hline & MANAGEMENT RESERVE- & & & $\$ 1,081,153$ \\
\hline & CONTINGENCY- & & & $\$ 5,137,746$ \\
\hline & TOTAL ESTIMATED COST - & & & $\$ 24,000,000$ \\
\hline
\end{tabular}

PROJECT COST PARAMETERS

EDI AS A \% OF CONST. + GFE $=24.00 \%$ 
Lockheed Martin Idaho Technologles Co.

Rev 6.96
PROJECT NAME: ICPP gin Set Closure (EIS Study) Place Clean Grout In Vault

ICPP

REQUESTOR: Bryan Spaulding
DETAILED COST ESTIMATE SHEET

TrPE OF Estimate: Planning

PROJECT NO: $\quad$ 2423-A2-E2

PREPARED aY: S. L. Coward

\author{
PAGE\# 1 \\ DATE 28-Jan-1998 \\ TIME: 10:10:46 \\ REPORT NAME: Detall Cost Estimato Sheot
}

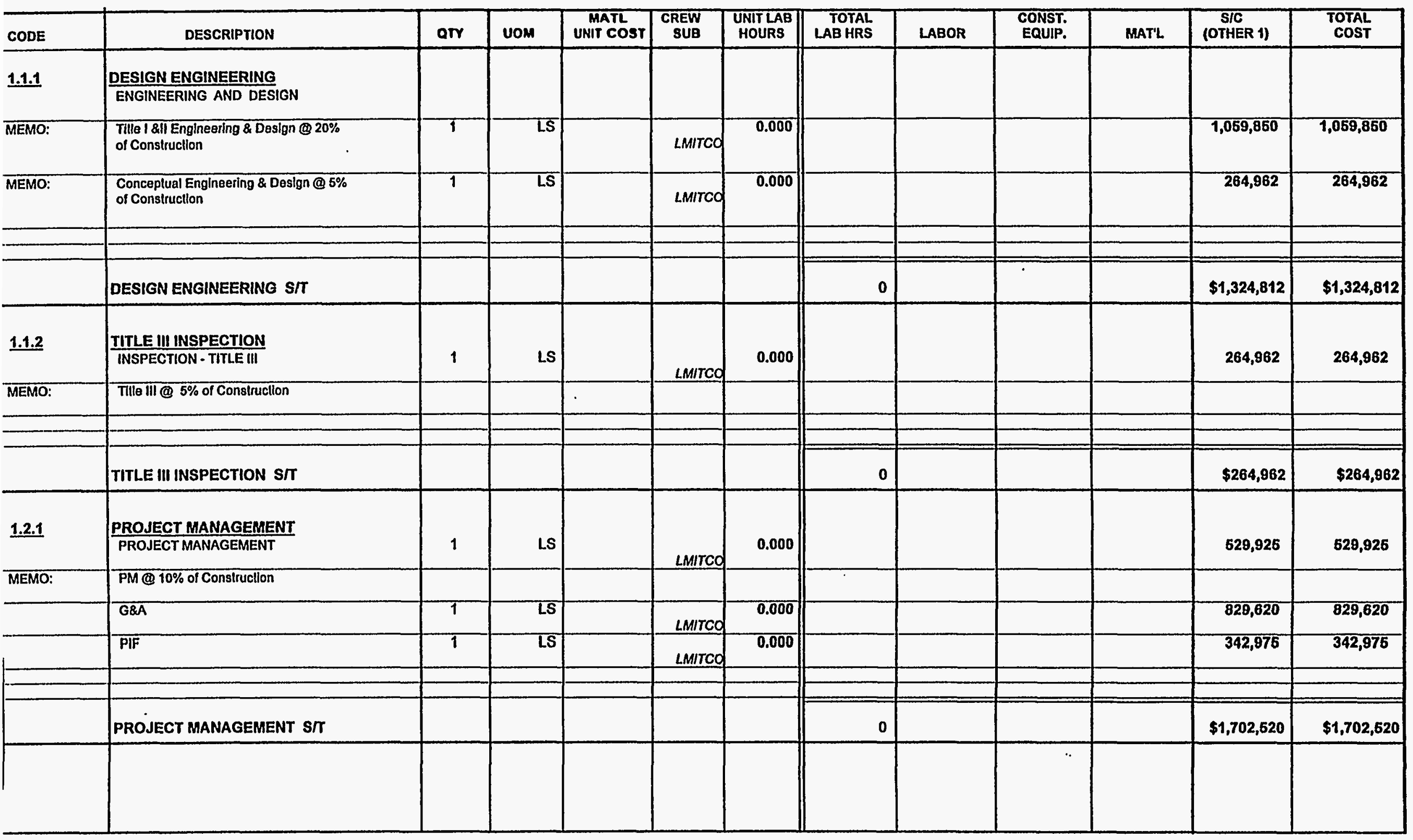


Lockhoed Martin Idaho Technologles Co.

$$
\text { Rev 6.96 }
$$

PROsECT NAME: ICPP Bin Set Closure (EIS Study)

Place Clean Grout in Vault

ICPP

LOCATION

REQUESTOR: Bryan Spaulding

\section{DETAILED COST ESTIMATE SHEET}

TPE OF EsTIMATE: Planning

PROJECT NO: : 2423-A2-E2

PREPARED BY: S. L. Coward
PAGE* 3

DATE 28-Jan-1998

TIME: 10:08:07

REPORT NAME: Dotail Cost Estimate Sheot

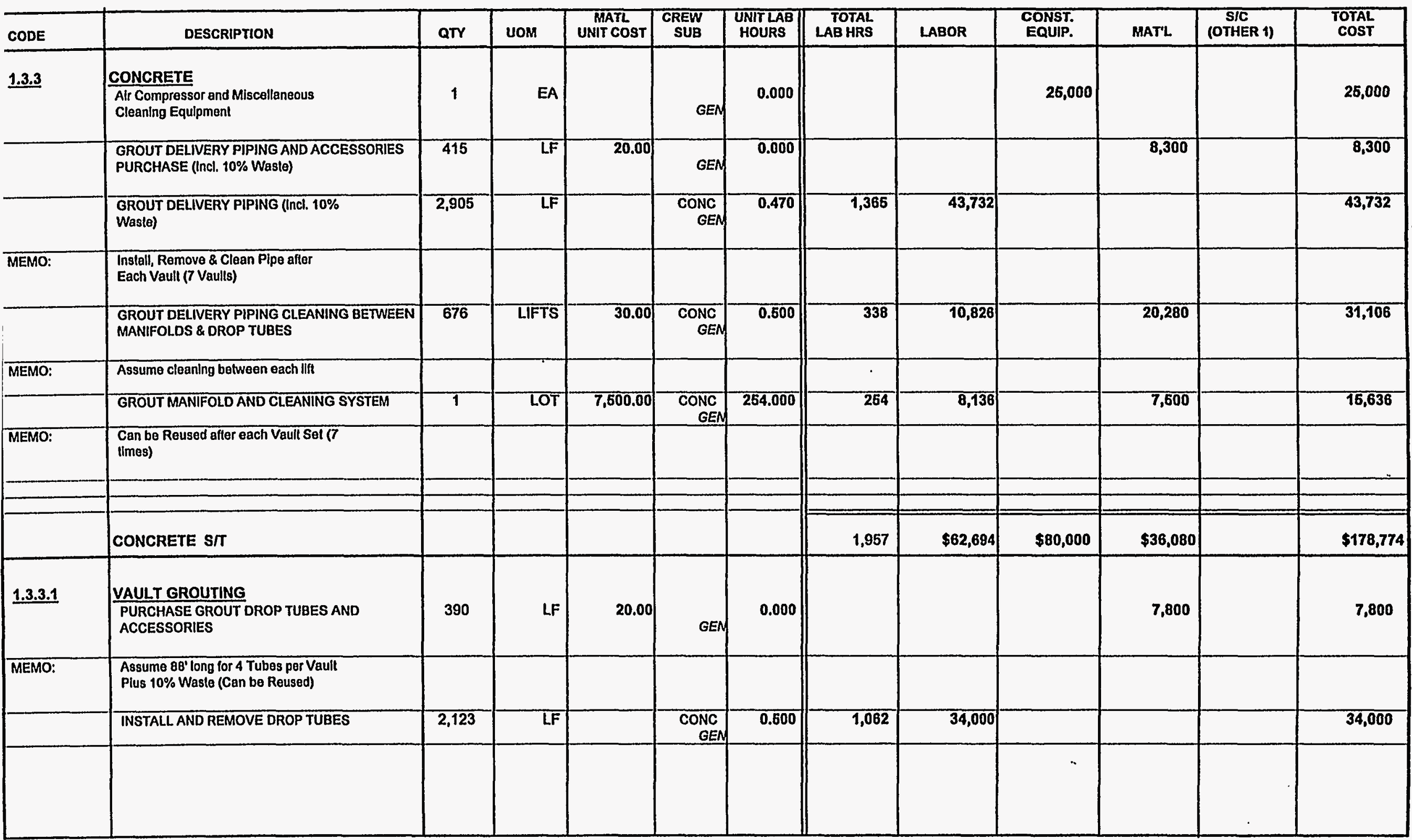


ckheed Martin Idaho Technologles Co.

Rev 0.98

JECT NAME: ICPP BIn Set Closure (EIS Study)

Place Clean Grout in Vault

ICPP

UESTOR: Bryan Spaulding

\section{DETAILED COST ESTIMATE SHEET}

TYPE OF ESTIMATE: Planning

PROJECT NO.: 2423-A2-E2

PREPARED BY: S. L. Coward
PAGE \# 4

DATE 28-Jan-1998

TIME: 10:10:46

REPORT NAME: Detall Cost Estlmate Sheot

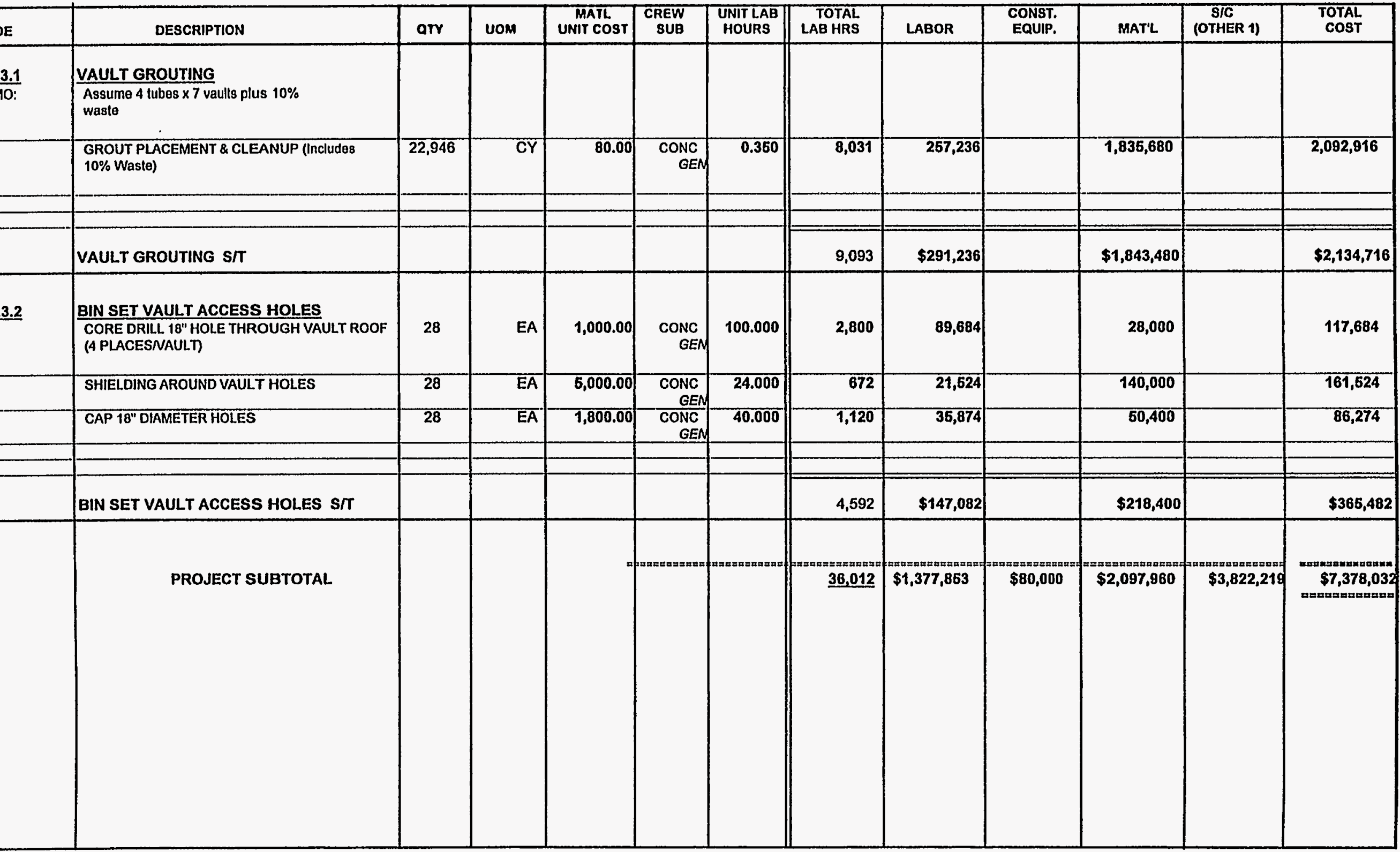



Lockheed Martin Idaho Technologies Co.

PROJECT NAME: ICPP Bin Set Closure (EIS Stud

LOCATION 1:

REQUESTOR
CONTINGENCY ANALYSIS

TYPE OF ESTIMATE:

PROJECTNO:

PREPARED BY: S.L. Coward
DATE: $28-J a n-1998$

TIME: 09:51:35

REPORT NAME: Contingency Analysis

\begin{tabular}{|c|c|c|c|c|c|c|c|c|c|c|c|}
\hline & PROB & BLE \% VARIA & ON & & & & & & $\begin{array}{l}\text { PRO } \\
\text { CONT }\end{array}$ & $\begin{array}{l}\text { CT } \\
\text { GENCY }\end{array}$ & SUMMARY \\
\hline $\begin{array}{l}\text { WBS } \\
\text { Element }\end{array}$ & Coet Fetimnto Flamant & & $\begin{array}{l}\% \text { Total } \\
\text { Cost }\end{array}$ & & \% Var. & Wt \% & Prob. & Continoency & $\%$ & Cost & Total Cost \\
\hline & (5) & Contingency & & - & + & - & \pm & & & & by Element \\
\hline 1.1.1 & DESIGN ENGINEERING & $1,324,812$ & 7.45 & 10 & 40 & 0.75 & 2.98 & $2.608 \%$ & $7.45 \%$ & 463,350 & $1,788,162$ \\
\hline 1.12 & TITLE III INSPECTION & 264,962 & 1.49 & 10 & 40 & 0.15 & 0.60 & $0.522 \%$ & $1.40 \%$ & 92,670 & 357,632 \\
\hline 121 & PROJECT MANAGEMENT & $1,702,520$ & 9.57 & 10 & 40 & 0.96 & 3.83 & $3.351 \%$ & $9.57 \%$ & 595,452 & $2,297,972$ \\
\hline 1.22 & CONSTRUCTION MANAGEMENT & 529,925 & 2.98 & 10 & 40 & 0.30 & 1.19 & $1.043 \%$ & $298 \%$ & 185,340 & 715,265 \\
\hline 1.3 .1 & GENERAL CONDITIONS & $1,067,791$ & 6.01 & 10 & 40 & 0.60 & 240 & $2.102 \%$ & $6.01 \%$ & 373,457 & $1,441,248$ \\
\hline 1.3 .2 & STTEWORK & 0 & 0.00 & 0 & 0 & 0.00 & 0.00 & $0.000 \%$ & $0.00 \%$ & 0 & 0 \\
\hline 1.3 .3 & CONCREIE & $4,231,459$ & 23.80 & 10 & 40 & 2.38 & 9.52 & $8.329 \%$ & $23.80 \%$ & $1,479,943$ & $5,711,402$ \\
\hline 1.5 .2 & PROCUREMENT FEES & 52,992 & 0.30 & 10 & 40 & 0.03 & 0.12 & $0.104 \%$ & $0.30 \%$ & 18,534 & 71,526 \\
\hline & ESCALATION & $8,606,640$ & 48,40 & 10 & 40 & 4.84 & 19.36 & $16.944 \%$ & $48.40 \%$ & $3,010,153$ & $11,616,793$ \\
\hline & SUBTOTAL & $17,781,101$ & 100.00 & & & & & $35.000 \%$ & & & \\
\hline & CALCULATED CONTINGENCY & $6,223,385$ & & & & & & & & & \\
\hline & RESULTANT TEC & $24,004,486$ & & & & & & & & & \\
\hline & ROUNDED TEC & $24,000,000$ & & & & & & & & & \\
\hline & PROJECT CONTINGENCY & $6,218,899$ & & & & & & $34.97 \%$ & & & \\
\hline & MANAGEMENT RESERVE & $1,081,153$ & & & & & & & & & \\
\hline & CONTINGENCY & $5,137,746$ & & & & & & & & & \\
\hline & TOTAL ESTIMATED COST & $24,000,000$ & & & & & & & & $6,218,899$ & $24,000,000$ \\
\hline
\end{tabular}

\section{CONFIDENCE LEVEL AND ASSUMED RISKS:}

The Lockheed Idaho Technologies Co. Cost Estimate Contingency Analysis Model is based on the applied contingency and the assumptions upon which the estimate was predicated. The model is applied with a suggested risk level of $18 \%$ and a level of confidence of $90 \%$ the estimate will fall within the bid range. The Contingency Analysis is based on a weighted average to provide a $90 \%$ probability of underun and a $10 \%$ probability of ovemun.

\section{CONTINGENCY ANALYSIS GUIDE BY TYPE OF ESTIMATE}

Guidelines established by DOE/FM 50, Cost Estimating Guide, Vol. 6 , Cost Guide, and as presented in the INEL Cost Estimating Guide. PLANNING

Conceptual

TITLE

TITLE II

TTILE IJAFC .....Up to $50 \%$

$15 \%-25 \%$

....Up to $40 \%$

$5 \%-15 \%$

Market Conditions 


\section{G\&A/PIF ADDER CALCULATION SHEET \\ ICPP BIN SET CLOSURE \\ CLOSURE TO RCRA LANDFILL STANDARDS; CLEAN FILL; ESCALATED}

FILL VAULTS W/ CLEAN GROUT

PROCUREMENT FEE:

$\begin{aligned} & \text { CONSTRUCTION }= \\ & \text { GFE }=\end{aligned}$
FEE @ $1 \%=$

G\&A @ 23\% (with a ceiling of $\$ 500,000$ imposed per year, 7 yrs)

CONSTRUCTION OR

CEILING * 7 YEARS =

GFE =

$\$ 3,500,000$

PROCUREMENT FEE =

Subtotal $\$ \$, 607,045$

FEE @ 23\% =

$\$ 3,607,045 \div 0.23=$

$\$ 829,620$

PIF @ 5.5\%

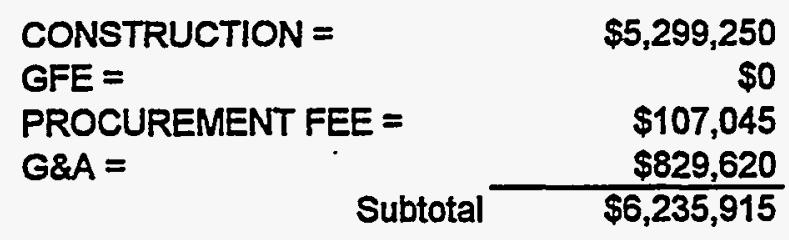

FEE @ 5.5\%=

$\$ 6,235,915 * 0.055=$

$\$ 342,975$

TOTAL PROCUREMENT FEE:

$\$ 107,045$

TOTAL G\&A FEE:

$\$ 829,620$

TOTAL PIF:

$\$ 342,975$ 
Lockheed Martin Idaho Technologies Co. Rov, 6-56 PROJECT NAME: ICPP Bin Set Closure (EIS Stud LOCATION 1: Tractor (RCRA Estimates) REQUESTOR:
COST ESTIMATE SUMMARY

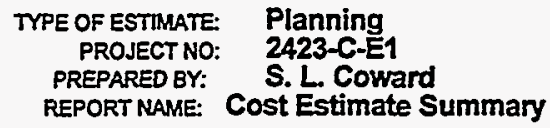

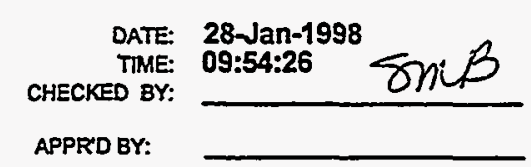

\begin{tabular}{|c|c|c|c|c|}
\hline $\begin{array}{l}\text { WBS } \\
\text { Element }\end{array}$ & Cost Estimate Element & $\begin{array}{c}\text { Total } \\
\text { Unescalated }\end{array}$ & Escalation & $\begin{array}{l}\text { Total } \\
\text { Incl Escalation }\end{array}$ \\
\hline $\begin{array}{l}\frac{1.1}{1.1 .1} \\
1.1 .2 \\
\frac{1.2}{1.2 .1} \\
1.2 .2 \\
\frac{1.3}{1.3 .1} \\
1.3 .13 \\
1.5 .2\end{array}$ & $\begin{array}{l}\text { ENGINEERING, DESIGN AND INSPECTION } \\
\text { DESIGN ENGINEERING TITLE I \& II } \\
\text { TITLE III INSPECTION } \\
\text { MANAGEMENT COSTS } \\
\text { PROJECT MANAGEMENT } \\
\text { CONSTRUCTION MANAGEMENT } \\
\text { CONSTRUCTION } \\
\text { GENERAL CONDITIONS } \\
\text { SPECIAL CONSTRUCTION } \\
\text { PROCUREMENT FEES }\end{array}$ & $\begin{array}{r}7,783,994 \\
4,085,421 \\
\\
6,840,414 \\
34,013,797 \\
408,542\end{array}$ & $\begin{array}{r}2,757,659 \\
1,961,002 \\
6,694,235 \\
3,922,004 \\
\\
6,566,797 \\
32,653,245 \\
392,200\end{array}$ & 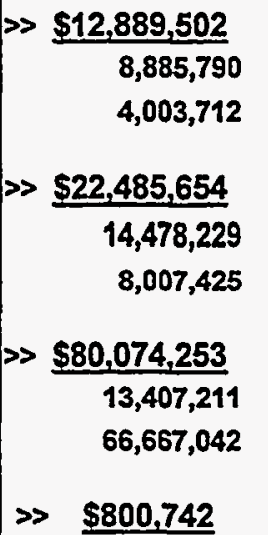 \\
\hline & $\begin{array}{l}\text { SUBTOTAL INCLUDING ESCALATION } \\
\text { PROJECT CONTINGENCY }\end{array}$ & $61,303,009$ & $54,947,142$ & $\gg \$ 116,250,151$ \\
\hline & MANAGEMENT RESERVE- & & & $\$ 8,087,499$ \\
\hline & CONTINGENCY- & & & $\gg \$ 32,662,350$ \\
\hline & TOTAL ESTIMATED COST & & & $\gg \$ 157,000,000$ \\
\hline
\end{tabular}

\section{PROJECT COST PARAMETERS}

EDI AS A $\%$ OF CONST. + GFE $=16.00 \%$ 

Lockheed Martin Idaho Technologies Co.

PROJECT NAME: ICPP Bin Set Closure (EIS Stud Tractor (RCRA Estimates)

LOCATION 1: ICPP

REQUESTOR: Bryan Spaulding
CONTINGENCY ANALYSIS
TYPE OF ESTIMATE:

PROJECT NO:

PREPARED BY:
Planning
2423-C-E1
S. L. Coward
DATE: 28-Jan-1998

TIME: 09:54:36
REPORT NAME: Contingency Analysis

\begin{tabular}{|c|c|c|c|c|c|c|c|c|c|c|c|}
\hline & PROB & BLE \% VARIAT & $10 N$ & & & & & & $\begin{array}{r}\text { PRO } \\
\text { CONT }\end{array}$ & $\begin{array}{l}\text { CT } \\
\text { jENCY }\end{array}$ & SUMMARY \\
\hline WBS & Cost Estimate Element & Total Costw/o & $\begin{array}{l}\% \text { Total } \\
\text { Cost }\end{array}$ & & $\begin{array}{l}\% \text { Var. } \\
\text { m Est. }\end{array}$ & Wt. \% & f Prob. & Contingency & $\%$ & Cost & Totrl Cost \\
\hline & $\because$ & Contingency & & - & + & - & + & & & & by Element \\
\hline 1.1 .1 & DESIGN ENGINEERING TILE $1 \& \|$ & $6,128,131$ & 5.27 & 10 & 40 & 0.53 & 2.11 & $1.845 \%$ & $5.27 \%$ & $2,148,130$ & $8,276,261$ \\
\hline 1.1 .2 & TIILE III INSPECTION & $2,042,710$ & 1.76 & 10 & 40 & 0.18 & 0.70 & $0.615 \%$ & $1.76 \%$ & 716,043 & $2,758,753$ \\
\hline 1.2 .1 & PROJECT MANAGEMENT & $7,783,994$ & 6.70 & 10 & 40 & 0.67 & 2.68 & $2344 \%$ & $6.70 \%$ & $2,728,569$ & $10,512,563$ \\
\hline 1.22 & CONSTRUCTION MANAGEMENT & $4,085,421$ & 3.51 & 10 & 40 & 0.35 & 1.41 & $1.230 \%$ & $3.54 \%$ & $1,432,087$ & $5,517,508$ \\
\hline 9.3 .1 & GENERAL CONDTTONS & $6,840,414$ & 5.88 & 10 & 40 & 0.59 & 2.35 & $2059 \%$ & $5.88 \%$ & $2,397,811$ & $9,238,225$ \\
\hline 1.3 .13 & SPECIAL CONSTRUCTION & $34,013,797$ & 29.26 & 10 & 40 & 2.93 & 11.70 & $10.241 \%$ & $29.26 \%$ & $11,923,056$ & $45,936,853$ \\
\hline 1.5 .2 & PROCUREMENT FEES & 408,542 & 0.35 & 10 & 40 & 0.04 & 0.14 & $0.123 \%$ & $0.35 \%$ & 143,209 & 551,751 \\
\hline & ESCALATION & $54,947,142$ & 47.27 & 10 & 40 & 4.73 & 18.91 & $16.543 \%$ & $47.27 \%$ & $19,260,944$ & $74,208,086$ \\
\hline & SUBTOTAL & $116,250,151$ & 100.00 & & & & & $35.000 \%$ & & & \\
\hline & CALCULATED CONTINGENCY & $40,687,553$ & & & & & & & & & \\
\hline & RESULTANT TEC & $156,937,704$ & & & & & & & & & \\
\hline & ROUNDED TEC & $157,000,000$ & & & & & & & & & \\
\hline & PROJECT CONTINGENCY & $40,749,849$ & & & & & & $35.05 \%$ & & & \\
\hline & MANAGEMENT RESERVE & $8,087,499$ & & & & & & & & & \\
\hline & CONTINGENCY & $32,652,350$ & & & & & & & & & \\
\hline & TOTAL ESTTMATED COST & $157,000,000$ & & & & & & & & $40,749,849$ & $157,000,000$ \\
\hline
\end{tabular}

CONFIDENCE LEVEL AND ASSUMED RISKS:

The Lockheed Idaho Technologies Co. Cost Estimate Contingency Analysis Model is based on the applied contingency and the assumptions upon which the estimate was predicated. The model is applied with a suggested risk level

of $18 \%$ and a level of confidence of $90 \%$ the estimate will fall within the bid range.

The Contingency Analysis is based on a weighted average to provide a

$90 \%$ probability of underun and a $10 \%$ probability of overmun.
CONTINGENCY ANALYSIS GUIDE BY TYPE OF ESTIMATE

Guidelines established by DOEIFM 50 , Cost Estimating Guide, Vol. 6 ,

Cost Guide, and as presented in the INEL Cost Estimating Guide. PLANNING $20 \%-30 \%$ ExperimentaVSpecial Conditions.............Up to 50\% Conceptual

ExperimentalSpecial Conditions.............. 15 - $25 \%$ TITLE!

TITLE II TITLE IUAFC $10 \%-20 \%$

$5 \%-15 \%$ Market Conditions 


\section{G\&APIF ADDER CALCULATION SHEET \\ ICPP BIN SET CLOSURE \\ CLOSURE TO RCRA LANDFILL STANDARDS; CLEAN FILL; ESCALATED \\ CLEAN BINS W/ ROBOTS - FLOOR}

PROCUREMENT FEE:

$$
\begin{aligned}
& \text { CONSTRUCTION = } \\
& \text { GFE = }
\end{aligned}
$$

FEE @ 1\% =
$\$ 80,074,253$

$\$ 80,074,253$

$\$ 80,074,253 \cdot 0.01=$

G\&A @ 23\% (with a ceiling of $\$ 500,000$ imposed per year, 10 yrs)

CONSTRUCTION OR

CEILING * 10 YEARS =

GFE =

$\$ 5,000,000$

PROCUREMENT FEE $=$

Subtotal $\$ 5,800,743$

FEE @ $23 \%=$

$\$ 5,800,743 \cdot 0.23=$

$\$ 1,334,171$

\begin{tabular}{|c|c|}
\hline $\begin{array}{l}\text { CONSTRUCTION = } \\
\text { GFE = } \\
\text { PROCUREMENT FEE = } \\
\text { G\&A = }\end{array}$ & $\begin{array}{r}\$ 40,854,211 \\
\$ 0 \\
\$ 800,743 \\
\$ 1,334,171 \\
\end{array}$ \\
\hline Subtotal & $\$ 42,989,124$ \\
\hline
\end{tabular}

PIF @ 5.5\%

FEE @ $5.5 \%=$

$\$ 42,989,124 * 0.055=$

$\$ 2,364,402$

TOTAL PROCUREMENT FEE:

$\$ 800,743$

TOTAL G\&A FEE:

$\$ 1,334,171$

TOTAL PIF:

$\$ 2,364,402$ 
Lockheed Martin Idaho Technologies Co. Rov. 6-96 PROJECT NAME: ICPP Bin Set Closure (EIS Stud LOCATION 1: REQUESTOR: Place Clean Grout in Bins ICPP

Bryan Spaulding

\section{COST ESTIMATE SUMMARY}

TYPE OF ESTIMATE: Planning PROJECT NO: PREPARED BY:

2423-a1-e1

S. L. Coward REPORT NAME: Cost Estimate Summary

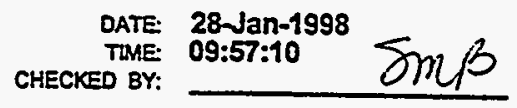

APPRD BY:

\begin{tabular}{|c|c|c|c|c|}
\hline $\begin{array}{l}\text { WBS } \\
\text { Element }\end{array}$ & Cost Estimate Element & $\begin{array}{c}\text { Total } \\
\text { Unescalated }\end{array}$ & Escalation & $\begin{array}{l}\text { Total } \\
\text { Incl Escalation }\end{array}$ \\
\hline $\begin{array}{l}\frac{1.1}{1.1 .1} \\
1.1 .2 \\
\frac{1.2}{1.2 .1} \\
1.2 .2 \\
\frac{1.3}{1.3 .1} \\
1.3 .2 \\
1.3 .3 \\
1.5 .2\end{array}$ & $\begin{array}{l}\text { ENGINEERING, DESIGN AND INSPECTION } \\
\text { DESIGN ENGINEERING } \\
\text { TITLE III INSPECTION } \\
\text { MANAGEMENT COSTS } \\
\text { PROJECT MANAGEMENT } \\
\text { CONSTRUCTION MANAGEMENT } \\
\text { CONSTRUCTION } \\
\text { GENERAL CONDITIONS } \\
\text { SITEWORK } \\
\text { CONCRETE } \\
\text { PROCUREMENT FEES }\end{array}$ & $\begin{array}{r}1,646,982 \\
329,396 \\
\\
1,547,020 \\
658,793 \\
\\
1,067,791 \\
229,119 \\
5,291,021 \\
65,879\end{array}$ & $\begin{array}{c}1,485,139 \\
704,909 \\
\\
1,142,536 \\
245,157 \\
5,661,393 \\
70,491\end{array}$ & $\begin{array}{r}\gg \frac{\$ 3,135,853}{2,454,003} \\
681,850 \\
\\
\gg \frac{\$ 4,395,861}{3,032,159} \\
1,363,702 \\
\gg \frac{\$ 13,637,017}{2,210,327} \\
474,276 \\
10,952,414\end{array}$ \\
\hline & $\begin{array}{l}\text { SUBTOTAL INCLUDING ESCALATION } \\
\text { PROJECT CONTINGENCY }\end{array}$ & $10,836,001$ & $10,469,100$ & $\gg>\$ 21,305,101$ \\
\hline & MANAGEMENT RESERVE- & & & $\$ 1,377,339$ \\
\hline & CONTINGENCY - & & & $\$ 6,117,560$ \\
\hline & TOTAL ESTIMATED COST - & & & $\$ 28,800,000$ \\
\hline
\end{tabular}

\section{PROJECT COST PARAMETERS}

EDI AS A \% OF CONST. + GFE $=23.00 \%$ 
Lockheed Martin Idaho Technologies Co.

Rov 6.96

PROJECT NAME: ICPP BIn Set Closure (EIS Study)

Place Clean Grout in Bins

LOCATION 1: ICPP

REQUESTOR: Bryan Spaulding

\section{DETAILED COST ESTIMATE SHEET}

TYPE OF ESTIMATE: Planning

PROJECT NO: 2423-a1-01

PREPARED BY: S. L. Coward
PAGE \# 1

DATE 28-Jan-1998

TIME: 10:11:32

REPORT NAME: Detail Cost Estimate Sheet

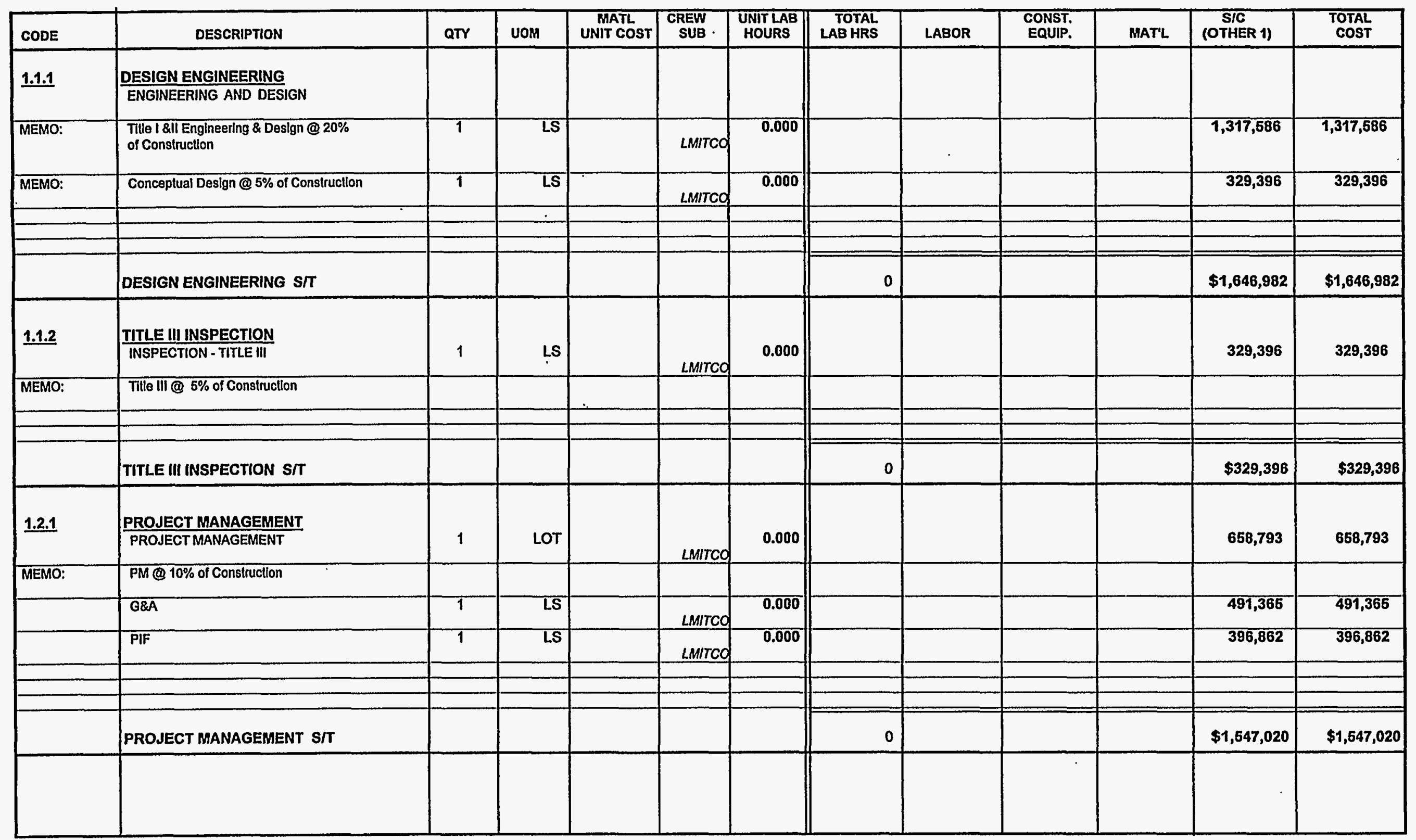




\begin{tabular}{|c|c|c|c|c|c|c|c|c|c|c|c|c|}
\hline $\begin{array}{l}\text { Lockheed } \\
\text { Rov 6.96 } \\
\text { PROJECT NAME } \\
\text { LOCATION 1: } \\
\text { REQUESTOR: }\end{array}$ & $\begin{array}{l}\text { Martin Idaho Technologies Co. } \\
\text { ICPP Bin Set Closure (EIS Study) } \\
\text { Place Clean Grout In Bins } \\
\text { ICPP } \\
\text { Bryan Spaulding }\end{array}$ & & & $\begin{array}{l}\text { TAILED CC } \\
\text { PE OF ESTIMATE } \\
\text { PROJECT NO.: } \\
\text { PREPARED BY: }\end{array}$ & $\begin{array}{l}\text { Planning } \\
2423-a 1- \\
\text { S. L. Coi }\end{array}$ & vard & HEET & & $\begin{array}{r}\text { PA } \\
\text { REPORT }\end{array}$ & $\begin{array}{l}2 \\
28-J a n-19 \\
10: 11: 32 \\
\text { Detall Cos }\end{array}$ & Estimate Sh & \\
\hline CODE & DESCRIPTION & QTY & vom & $\begin{array}{l}\text { MATL } \\
\text { UNIT COST }\end{array}$ & $\begin{array}{l}\text { CREW } \\
\text { SUB }\end{array}$ & $\begin{array}{l}\text { UNITLAB } \\
\text { HOURS }\end{array}$ & $\begin{array}{l}\text { TOTAL } \\
\text { LAB HRS }\end{array}$ & LABOR & $\begin{array}{l}\text { CONST. } \\
\text { EQUIP. }\end{array}$ & MAT'L & $\begin{array}{l}\text { S/C } \\
\text { (OTHER 1) }\end{array}$ & $\begin{array}{l}\text { TOTAL } \\
\text { COST }\end{array}$ \\
\hline$\underline{1.2 .2}$ & $\frac{\text { CONSTRUCTION MANAGEMENT }}{\text { CONSTRUCTION MANAGEMENT }}$ & 1 & LOT & & LMITCO & 0.000 & & & & & 658,793 & 658,793 \\
\hline MEMO: & CM@ $10 \%$ of Construcilon Costs & & & & & & & & & & & \\
\hline & & & & & & & & & & & & \\
\hline & CONSTRUCTION MANAGEMENT S/T & & & & & & 0 & & & & $\$ 668,793$ & $\$ 658,793$ \\
\hline 1.3 .1 & $\begin{array}{l}\text { GENERAL CONDITIONS } \\
\text { SUPERVISION (Duralion of Schedule - } \\
\text { Assume } 3 \text { Years) }\end{array}$ & 1 & FTE & & $\begin{array}{r}\text { CONF } \\
\text { GEN }\end{array}$ & 6240.00 & 6,240 & 207,480 & & & & 207,480 \\
\hline & TRAINING & 10 & FTE & & $\begin{array}{l}\text { CONC } \\
\text { GEN }\end{array}$ & 165.000 & 1,650 & 52,850 & & & & 52,850 \\
\hline & $\begin{array}{l}\text { RADCON TECH (Durallon of Schedule - } 3 \\
\text { Years) }\end{array}$ & 2 & FTE & & $\begin{array}{l}\text { Z-1342 } \\
\text { LMITCO }\end{array}$ & 6240.00 & 12,480 & 616,512 & & & & 616,512 \\
\hline & & & & & & & & & & & & \\
\hline & GENERAL CONDITIONS SIT & & & & & & 20,370 & $\$ 876,842$ & & & & $\$ 876,842$ \\
\hline 1.3 .2 & SITEWORK & & & & & & & & & & & \\
\hline & $\begin{array}{l}\text { ASSUME NO EXCAVATION WILL BE REQUIRED } \\
\text { FOR MONITORS }\end{array}$ & & & & & & & & & & & \\
\hline & $\begin{array}{l}\text { PLACE GRAVEL AROUND TANKMONITORS (4 } \\
\text { per bln except Bln Set } \# 1)\end{array}$ & 168 & EA & 20.00 & $\begin{array}{c}\text { CONC } \\
\text { GEN }\end{array}$ & 24.000 & 4,032 & 129,146 & & 3,360 & & 132,505 \\
\hline & SITEWORK SIT & & & & & & 4,032 & $\$ 129,145$ & & $\$ 3,360$ & & $\$ 132,605$ \\
\hline 1.3.3 & CONCRETE & & & & & & & & - & & & \\
\hline & & & & & & & & & & & & \\
\hline
\end{tabular}


Lockhoed Martin Idaho Technologies Co. Rov 6.96

PROJECT NAME: ICPP Bin Set Closure (EIS Study) Place Clean Grout in Bins

ICPP

REQUESTOR: Bryan Spaulding

\section{DETAILED COST ESTIMATE SHEET}

TYPE OF ESTIMATE: Planning

PROJECTNO:: 2423-a1-e1

PREPARED BY: S. L. Coward
PAGE \# 4

DATE 28-Jan-1998

TIME: 10:11:32

REPORT NAME: Detall Cost Estimate Sheet

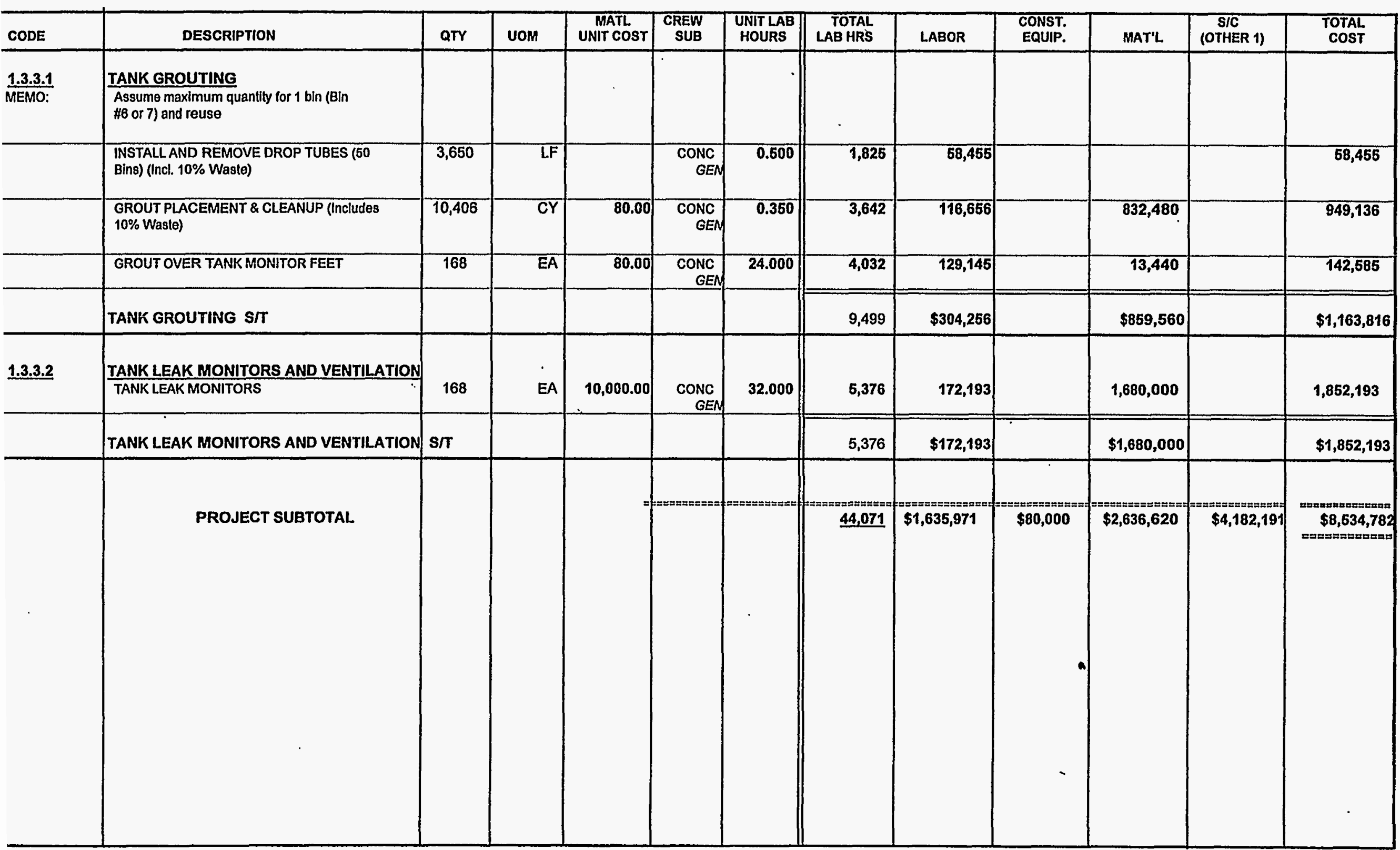


Lockheed Martin Idaho Technologies Co.

PROJECT NAME: ICPP Bin Set Closure (EIS Stud Place Clean Grout in Bins

LOCATION 1: ICPP

REQUESTOR: Bryan Spaulding

\section{CONTINGENCY ANALYSIS}

TYPE OF ESTIMATE: PROJECT NO: 2423-a1-1 PREPARED BY:
Planning
2423-a1-e1
S. L. Coward
DATE: 28-Jan-1998

TIME: 09:57:06

REPORT NAME: Contingency Analysis

\begin{tabular}{|c|c|c|c|c|c|c|c|c|c|c|c|}
\hline \multicolumn{9}{|c|}{ PROBABLE \% VARIATION } & \multicolumn{2}{|c|}{$\begin{array}{c}\text { PROJECT } \\
\text { CONTINGENCY }\end{array}$} & \multirow{3}{*}{$\begin{array}{l}\text { SUMMARY } \\
\text { Total Cost } \\
\text { by Element }\end{array}$} \\
\hline \multirow[t]{2}{*}{$\begin{array}{l}\text { WBS } \\
\text { Element }\end{array}$} & \multirow{2}{*}{ Cost Estimate Element } & \multirow{2}{*}{$\begin{array}{c}\text { Total Cost w/o } \\
\text { Contingency }\end{array}$} & \multirow[t]{2}{*}{$\begin{array}{l}\text { \% Total } \\
\text { Cost }\end{array}$} & \multicolumn{2}{|c|}{$\begin{array}{c}\text { Prob. \% Var. } \\
\text { From Est. }\end{array}$} & \multicolumn{2}{|c|}{ Wt $\%$ of Prob. } & \multirow{2}{*}{ Contingency } & \multirow[t]{2}{*}{$\%$} & \multirow{2}{*}{ Cost } & \\
\hline & & & & - & + & - & \pm & & & & \\
\hline 1.1.1 & DESIGN ENGINEERING & $1,646,982$ & 7.73 & 10 & 40 & 0.77 & 3.09 & $2.706 \%$ & $7.73 \%$ & 579,390 & $2,226,372$ \\
\hline 1.1 .2 & TIILE III INSPECTION & 329,396 & 1.55 & 10 & 40 & 0.15 & 0.62 & $0.541 \%$ & $1.55 \%$ & 115,878 & 445,274 \\
\hline 1.21 & PROJECT MANAGEMENT & $1,547,020$ & 7.26 & 10 & 40 & 0.73 & 2.90 & $2.544 \%$ & $7.26 \%$ & 544,225 & $2,091,245$ \\
\hline 1.2 .2 & CONSTRUCTION MANAGEMENT & 658,793 & 3.09 & 10 & 40 & 0.31 & 1.24 & $1.082 \%$ & $3.09 \%$ & 231,756 & 890,549 \\
\hline 1.3 .1 & GENERAL CONDITONS & $1,067,791$ & 5.04 & 10 & 40 & 0.50 & 200 & $1.754 \%$ & $5.01 \%$ & 375,637 & $1,443,428$ \\
\hline 1.3 .2 & SIIEWORK & 229,119 & 3.08 & 10 & 40 & 0.11 & 0.43 & $0.376 \%$ & $1.08 \%$ & 80,602 & 309,721 \\
\hline 1.3 .3 & CONCRETE & $5,291,021$ & 24.83 & 10 & 40 & 2.48 & 9.93 & $8.692 \%$ & $24.83 \%$ & $1,861,323$ & $7,152,344$ \\
\hline 1.5 .2 & PROCUREMENT FEES & 65,879 & 0.31 & 10 & 40 & 0.03 & 0.12 & $0.108 \%$ & $0.31 \%$ & 23,176 & 89,055 \\
\hline & ESCALATION & $10,469,100$ & 49.14 & 10 & 40 & 4.91 & 19.66 & $17.199 \%$ & $49.14 \%$ & $3,682,912$ & $14,152,012$ \\
\hline & SUBTOTAL & $21,305,101$ & 100.00 & & & & & $35.000 \%$ & & & \\
\hline & CALCULATED CONTINGENCY & $7,456,785$ & & & & & & & & & \\
\hline & RESULTANT TEC & $28,761,886$ & & & & & & & & & \\
\hline & ROUNDED TEC & $28,800,000$ & & & & & & & & & \\
\hline & PROJECT CONTINGENCY & $7,494,899$ & & & & & & $35.18 \%$ & & & \\
\hline & MANAGEMENT RESERVE & $1,377,339$ & & & & & & & & & \\
\hline & CONTINGENCY & $6,117,560$ & & & & & & & & & \\
\hline & TOTAL ESTIMATED COST & $28,800,000$ & & & & & & & & $7,494,899$ & $28,800,000$ \\
\hline
\end{tabular}

\section{CONFIDENCE LEVEL AND ASSUMED RISKS:}

The Lockheed Idaho Technologies Co. Cost Estimate Contingency Analysis Model is based on the applied contingency and the assumptions upon which the estimate was predicated. The model is applied with a suggested risk level of $18 \%$ and a level of confidence of $90 \%$ the estimate will fall within the bid range. The Contingency Anatysis is based on a weighted average to provide a $90 \%$ probability of undernun and a $10 \%$ probability of overrun.
CONTINGENCY ANALYSIS GUIDE BY TYPE OF ESTIMATE Guidelines established by DOEIFM 50, Cost Estimating Guide, Vol. 6 , Cost Guide, and as presented in the INEL Cost Estimating Guide. PLANNING

Experimental/Special Conditions............. Up to $50 \%$ Conceptual

Experimentalyspecial Conditions.............. TITLE I

TITLE II

TITLE IIIAFC $10 \%-20 \%$

$5 \%-15 \%$

Market Conditions 


\section{G\&APIF ADDER CALCULATION SHEET \\ ICPP BIN SET CLOSURE \\ CLOSURE TO RCRA LANDFILL STANDARDS; CLEAN FILL; ESCALATED}

FILL BINS WITH CLEAN GROUT

PROCUREMENT FEE:

CONSTRUCTION $=$
GFE $=$
Subtotal

FEE @ $1 \%=$

$\$ 13,637,017 * 0.01=$

$\$ 136,370$

G\&A @ 23\% (with a ceiling of $\$ 500,000$ imposed per year, 4 yrs)

CONSTRUCTION OR

CEILING * 4 YEARS $=$

GFE =

$\$ 2,000,000$

PROCUREMENT FEE $=$

Subtotal

$\$ 136,370$

FEE @ $23 \%=$

$\$ 2,136,370 * 0.23=$

$\$ 491,365$

PIF @ 5.5\%

\begin{tabular}{lr} 
CONSTRUCTION = & $\$ 6,587,931$ \\
GFE $=$ & $\$ 0$ \\
PROCUREMENT FEE = & $\$ 136,370$ \\
G\&A = & $\$ 491,365$ \\
\cline { 2 - 2 } & Subtotal
\end{tabular}

FEE @ $5.5 \%=$

$\$ 7,215,666 * 0.055=$

$\$ 396,862$

TOTAL PROCUREMENT FEE:

$\$ 136,370$

TOTAL G\&A FEE:

$\$ 491,365$

TOTAL PIF:

$\$ 396,862$ 
Lockheed Martin Idaho Technologies Co. Rev, 6-96

PROJECT NAME: ICPP Bin Set Closure

D\&D of Equipment

LOCATION 1: INEEL / ICPP

REQUESTOR: $\quad$ B. C. Spaulding
COST ESTIMATE SUMMARY

TYPE OF ESTIMATE: Planning

PROJECTNO: 2423D\&D

PREPARED BY: S.L.Coward/smb

REPORT NAME: Cost Estimate Summary

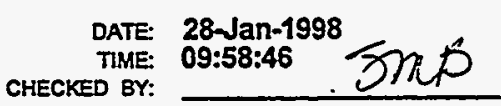

APPR'DBY:

\begin{tabular}{|c|c|c|c|c|}
\hline $\begin{array}{l}\text { WBS } \\
\text { Element }\end{array}$ & Cost Estimate Element & $\begin{array}{c}\text { Total } \\
\text { Unescalated }\end{array}$ & Escalation & $\begin{array}{c}\text { Total } \\
\text { Incl Escalation }\end{array}$ \\
\hline $\begin{array}{l}\frac{1.1}{1.1 .1} \\
1.1 .2 \\
\frac{1.2}{1.2 .1} \\
1.2 .2 \\
\frac{1.3}{1.3 .13} \\
1.5 .2\end{array}$ & $\begin{array}{l}\text { ENGINEERING, DESIGN AND INSPECTION } \\
\text { DESIGN ENGINEERING TITLE I \& II } \\
\text { TITLE III INSPECTION } \\
\text { MANAGEMENT COSTS } \\
\text { PROJECT MANAGEMENT } \\
\text { CONSTRUCTION MANAGEMENT } \\
\text { CONSTRUCTION } \\
\text { SPECIAL CONSTRUCTION } \\
\text { PROCUREMENT FEES }\end{array}$ & $\begin{array}{r}868,178 \\
0 \\
11,709,711 \\
117,097\end{array}$ & $\begin{array}{c}1,692,947 \\
0 \\
\\
22,833,936 \\
228,339\end{array}$ & $\begin{array}{r}\gg \quad \begin{array}{r}\$ 0 \\
0\end{array} \\
\gg \frac{\$ 2,561,125}{2,561,125} \\
0 \\
\gg \frac{\$ 34,543,647}{34,543,647} \\
\gg \frac{\$ 345,436}{}\end{array}$ \\
\hline & $\begin{array}{l}\text { SUBTOTAL INCLUDING ESCALATION } \\
\text { PROJECT CONTINGENCY }\end{array}$ & $12,694,986$ & $24,755,222$ & > $\$ 37,450,208$ \\
\hline & MANAGEMENT RESERVE- & & & $\gg$ \\
\hline & CONTINGENCY - & & & $\gg \quad \$ 13,149,792$ \\
\hline & TOTAL ESTIMATED COST & & & $\gg \quad \$ 50,600,000$ \\
\hline
\end{tabular}

\section{PROJECT COST PARAMETERS}

EDI AS A \% OF CONST. + GFE $=0.00 \%$ 
Lockheed Martin Idaho Technologles Co.

$$
\text { Rev 6.96 }
$$

PROJECT NAME: ICPP BIn Set Closure

D\&D of Equipment

$\begin{array}{ll} & \text { DED of Equipment } \\ \text { LOCATION :: } & \text { INEEL / ICPP } \\ \text { REQUESTOR: } & \text { B. C. Spaulding }\end{array}$
DETAILED COST ESTIMATE SHEET

TYPE OF EstiMATE: Planning

PROJECT NO:: 2423D\&D

PREPARED BY: S.L.Coward/smb
PAGE\# 1

DATE 28-Jan-1998

TIME. 10:13:24

REPORT NAME: Detail Cost Estimate Sheet

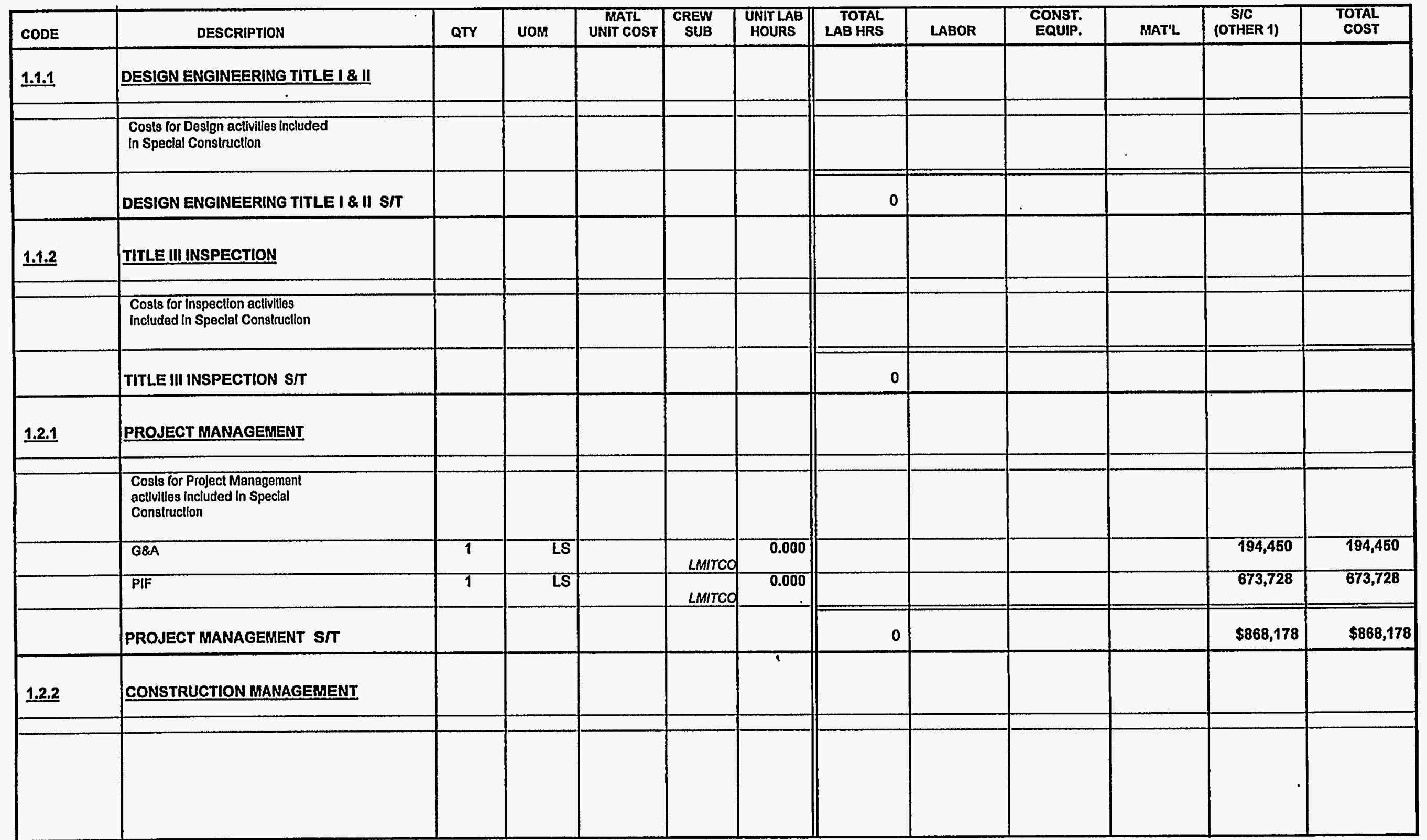


Lockheed Martin Idaho Technologies Co.

Rov 6-96

PROJECT NAME: ICPP Bin Sot Closure

D\&D of Equipment

LOCATION 9: INEEL / ICPP

REQUESTOR: $\quad$ B. C. Spaulding

\section{DETAILED COST ESTIMATE SHEET}

TYPE OF ESTIMATE: Planning

PROJECT NO:: 2423D\&D

PREPARED BY: S.L.Coward/smb
PAGE\# 3

DATE 28-Jan-1998

TIME: 10:13:24

REPORT NAME: Detall Cost Estimate Sheot

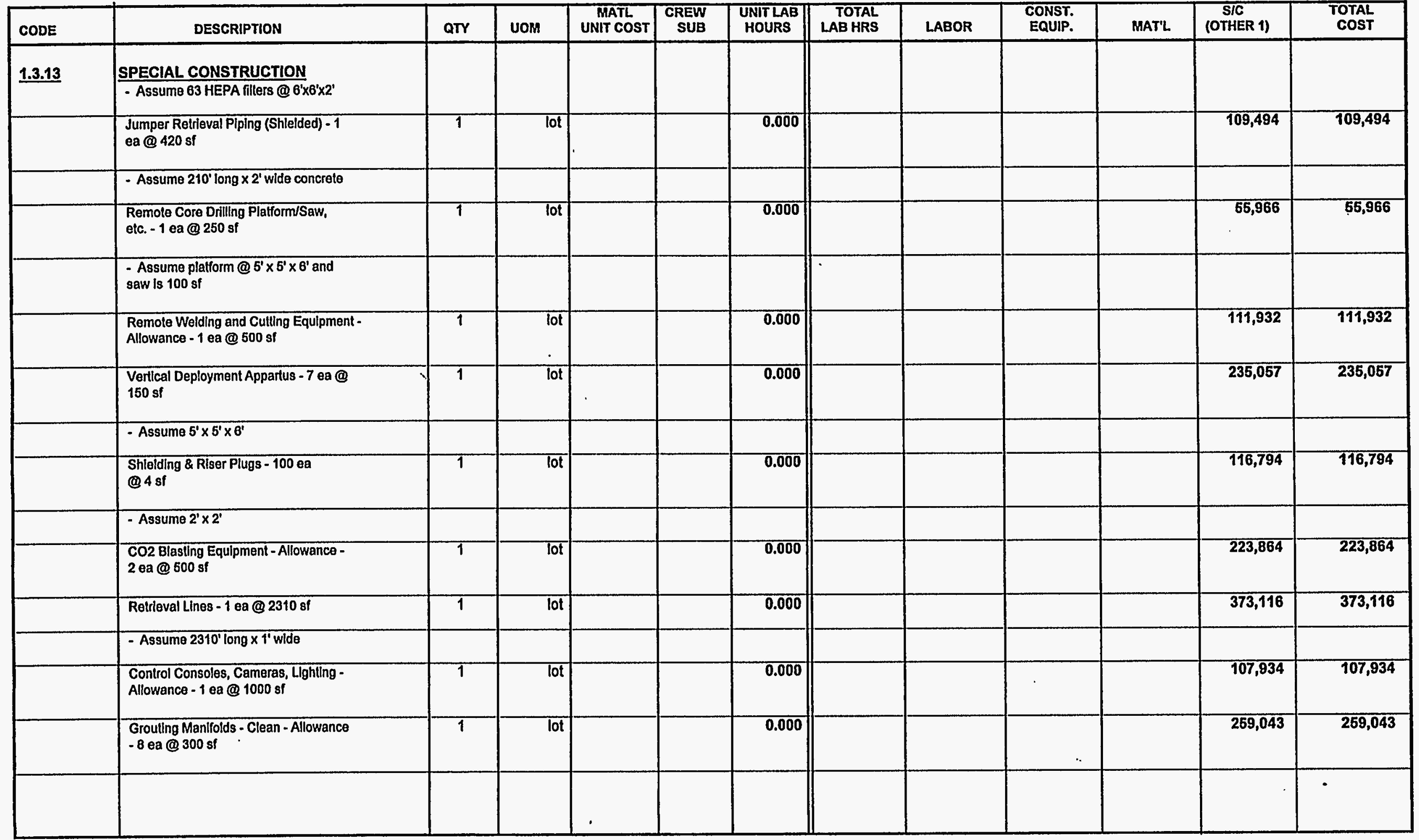


Lockheed Martin Idaho Technologles Co.

$$
\text { Rov 6.96 }
$$

PROJECT NAME: ICPP Bin Set Closure

D\&D of Equipment

INEEL / ICPP

B. C. Spaulding

\section{DETAILED COST ESTIMATE SHEET}

TYPE OF ESTIMATE: Planning

PROJECT NO.: 2423D\&D

PREPARED BY: S.L.Coward/smb
PAgE $\|$

DATE 28-Jan-1998

TIME: 10:13:24

REPORT NAME: Detall Cost Estimate Sheot

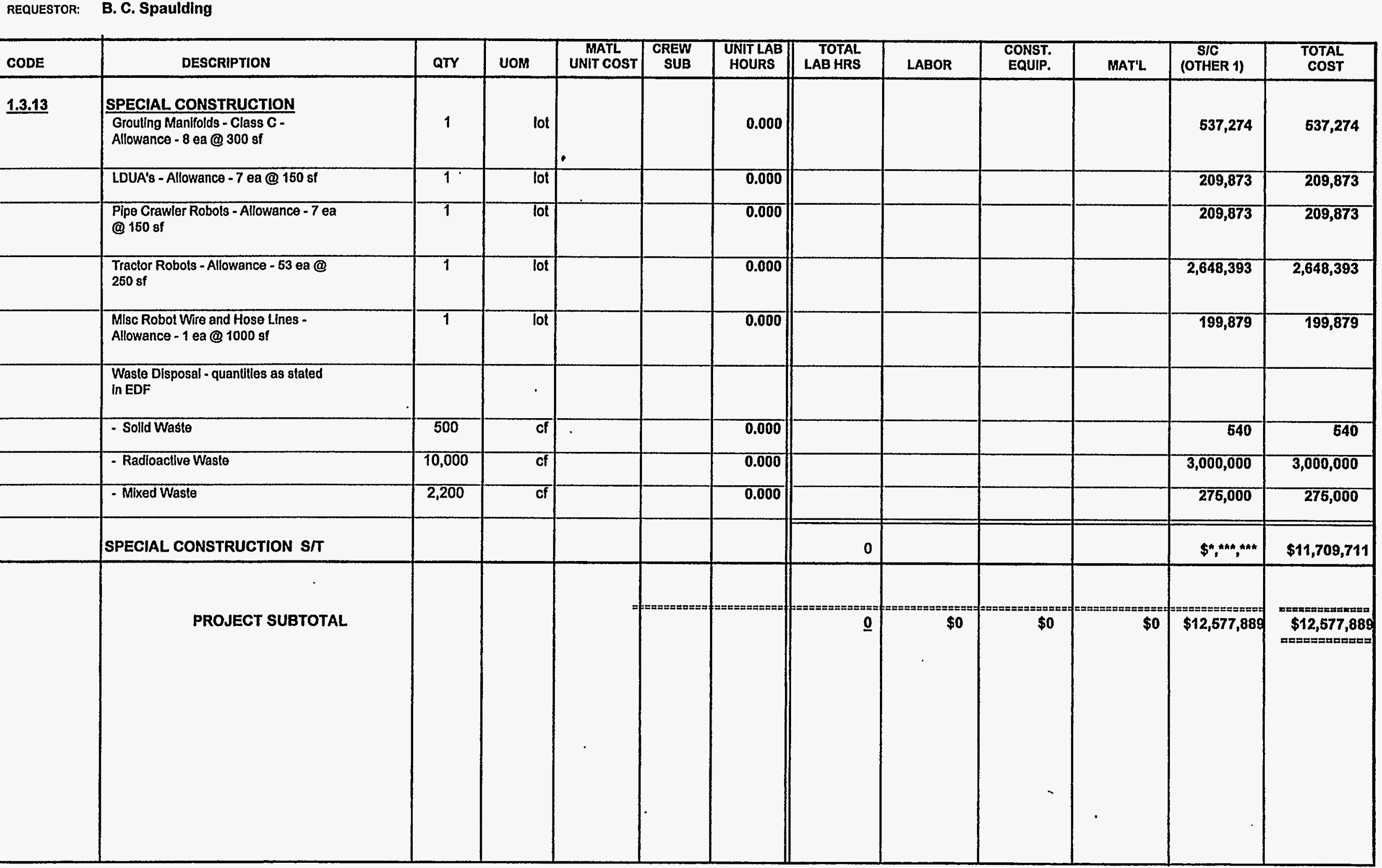


Lockheed Martin Idaho Technologies Co. Rev 6-S6

PROJECT NAME: ICPP Bin Set Closure

D\&D of Equipment

LOCATION 1: INEEL / ICPP

REQUESTOR: B. C. Spaulding
CONTINGENCY ANALYSIS

$\begin{array}{cl}\text { TYPE OF ESTIMATE: } & \text { Planning } \\ \text { PROJECT NO: } & \text { 2423D\&D } \\ \text { PREPARED BY: } & \text { S.LCoward/smb }\end{array}$

DATE. 28-Jan-1998

TIME: 09:58:43

REPORT NAME: Contingency Analysis

\begin{tabular}{|c|c|c|c|c|c|c|c|c|c|c|c|}
\hline \multicolumn{9}{|c|}{ PROBABLE \% VARIATION } & \multicolumn{2}{|c|}{$\begin{array}{c}\text { PROJECT } \\
\text { CONTINGENCY }\end{array}$} & \multirow{3}{*}{$\begin{array}{l}\text { SUMMARY } \\
\begin{array}{l}\text { Total Cost } \\
\text { by Element }\end{array}\end{array}$} \\
\hline \multirow[t]{2}{*}{$\begin{array}{l}\text { WBS } \\
\text { Element }\end{array}$} & \multirow{2}{*}{ Cost Estimate Element } & \multirow{2}{*}{$\begin{array}{c}\text { Total Cost w/o } \\
\text { Contingency }\end{array}$} & \multirow[t]{2}{*}{$\begin{array}{l}\% \text { Total } \\
\text { Cost }\end{array}$} & \multicolumn{2}{|c|}{$\begin{array}{c}\text { Prob. \% Var. } \\
\text { From Est. }\end{array}$} & \multicolumn{2}{|c|}{ Wt $\%$ of Prob. } & \multirow{2}{*}{ Contingency } & \multirow[t]{2}{*}{$\%$} & \multirow{2}{*}{ Cost } & \\
\hline & & & & - & + & - & $t$ & & & & \\
\hline 1.1 .1 & DESIGN ENGINEERING TITLE | \& II & 0 & 0.00 & 0 & 0 & 0.00 & 0.00 & $0.000 \%$ & $0.00 \%$ & 0 & 0 \\
\hline 1.1 .2 & TITLE III INSPECTION & 0 & 0.00 & 0 & 0 & 0.00 & 0.00 & $0.000 \%$ & $0.00 \%$ & 0 & 0 \\
\hline 1.2 .1 & PROJECT MANAGEMENT & 868,178 & 2.32 & 10 & 40 & 0.23 & 0.93 & $0.811 \%$ & $232 \%$ & 304,841 & $1,173,019$ \\
\hline 1.2 .2 & CONSTRUCTION MANAGEMENT & 0 & 0.00 & 0 & 0 & 0.00 & 0.00 & $0.000 \%$ & $0.00 \%$ & 0 & 0 \\
\hline 1.3 .13 & SPECIAL CONSTRUCTION & $11,709,711$ & 31.27 & 10 & 40 & 3.13 & 1251 & $10.944 \%$ & $31.27 \%$ & $4,111,600$ & $15,821,311$ \\
\hline \multirow[t]{2}{*}{1.5 .2} & PROCUREMENT FEES & 117,097 & 0.31 & 10 & 40 & 0.03 & 0.13 & $0.109 \%$ & $0.31 \%$ & 41,116 & 158,213 \\
\hline & ESCALATION & $24,755,222$ & 66.10 & 10 & 40 & 6.61 & 26.44 & $23.136 \%$ & $66.10 \%$ & $8,692,235$ & $33,447,457$ \\
\hline & SUBTOTAL & $37,450,208$ & 100.00 & & & & & $35.000 \%$ & & & \\
\hline & CALCULATED CONTINGENCY & $13,107,573$ & & & & & & & & & \\
\hline & RESULTANT TEC & $50,557,781$ & & & & & & & & & \\
\hline & ROUNDED TEC & $50,600,000$ & & & & & & & & & \\
\hline & PROJECT CONTINGENCY & $13,149,792$ & & & & & & $35.11 \%$ & & & \\
\hline & MANAGEMENT RESERVE & 0 & & & & & & & & & \\
\hline & CONTINGENCY & $13,149,792$ & & & & & & & & & \\
\hline & TOTAL ESTIMATED COST & $50,600,000$ & & & & & & & & $13,149,792$ & $50,600,000$ \\
\hline
\end{tabular}

\section{CONFIDENCE LEVEL AND ASSUMED RISKS:}

The Lockheed Idaho Technologies Co. Cost Estimate Contingency Analysis Model is based on the applied contingency and the assumptions upon which the estimate was predicated. The model is applied with a suggested risk level of $18 \%$ and a level of confidence of $90 \%$ the estimate will fall within the bid range. The Contingency Analysis is based on a weighted average to provide a

$90 \%$ probability of undemun and a $10 \%$ probability of overrun.
CONTINGENCY ANALYSIS GUIDE BY TYPE OF ESTIMATE

Guidelines established by DOEFM 50, Cost Estimating Guide, Vol. 6 , Cost Guide, and as presented in the INEL Cost Estimating Guide.
PLANNING
Experimental/Special Conditions..
$20 \%-30 \%$
Conceptual Up to $50 \%$ Experi
TITLE I
TITLE IIIAFC
$15 \%-25 \%$
....Up to $40 \%$
$10 \%-20 \%$
$5 \%-15 \%$
Market Conditions 


\section{G\&A/PIF ADDER CALCULATION SHEET \\ ICPP BIN SET CLOSURE \\ CLOSURE TO RCRA LANDFILL STANDARDS; CLEAN FILL; ESCALATED \\ D\&D OF EQUIPMENT}

PROCUREMENT FEE:

\begin{tabular}{|c|c|c|c|}
\hline CONSTRUCTION = & & $\$ 34,543,647$ & \\
\hline & Subtotal & $\$ 34,543,647$ & \\
\hline FEE @ $1 \%=$ & & $\$ 34,543,647$ & $* 0.01=$ \\
\hline
\end{tabular}

G\&A @ 23\% (with a ceiling of $\$ 500,000$ imposed per year, $1 \mathrm{yr}$ )

CONSTRUCTION OR

CEILING *1 YEARS $=\quad \$ 500,000$

GFE = $\$ 0$

PROCUREMENT FEE $=\quad \$ 345,436$

Subtotal $\$ 845,436$

FEE @ $23 \%=\quad \$ 845,436 * 0.23=\$ 194,450$

PIF @ 5.5\%

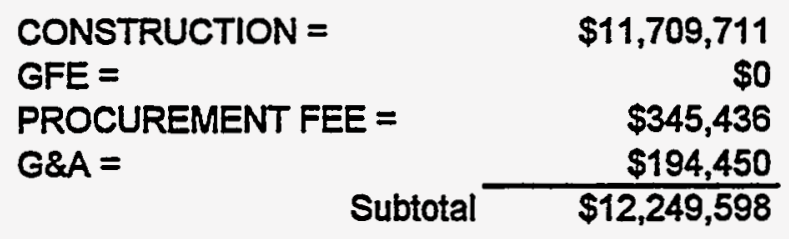

FEE @ $5.5 \%=$

TOTAL G\&A FEE:

$\$ 194,450$

TOTAL PIF:

$\$ 673,728$ 
Life cycle cost ana yeariy

\section{Cash Flow Estimates}


The options numbers in the Life-Cycle Costs (LCC) do not match the final option numbers in the report, as these estimates were prepared prior to determining that it is not feasible to create an NRC Class A landfill following Risk-Based Clean Closure (RBCC) or Closure to Landfill Standards (CLFS).

For the purposes of the LCC section only, the following key is provided:

Option 1: Risk-Based Clean Closure; NRC Class C fill (Option 1 in LCC) Option 2: Risk-Based Clean Closure; Clean fill (Option 3 in LCC)

Option 3: Closure to Landfill Standards; NRC Class C fill (Option 4 in LCC) Option 4: Closure to Landfill Standards; Clean fill (Option 6 in LCC) 


\section{Lockheed Martin Idaho Technologies Company \\ INTERDEPARTMENTAL COMMUNICATION}

Date: $\quad$ February 3, 1998

To:

From:

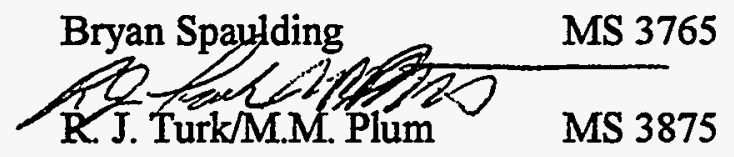

$6-8633$

$6-3611$

Subject: $\quad$ ECONOMIC AND LIFE CYCLE ANALYSIS CONDUCTED FOR THE CALCINE SOLIDS STORAGE CLOSURE STUDY OPTIONS - RJT-27-98

\section{Purpose:}

As requested an Economic and Life-Cycle Cost (LCC) has been conducted to evaluate the proposed CALCINE SOLIDS STORAGE CLOSURE STUDY OPTIONS . Each option proposes a different method for the closure of eight bins and the eight vaults at ICPP. At your direction Options 1,3,4 and 6 were evaluated in this model.

Option one: Risked Based Clean Closure; Class C fill.

Option three: Risked Based Clean Closure; Clean fill.

Option four: Close to RCRA Landfill Standards, Class C fill.

Option six: Close to RCRA Landfill Standards, Clean fill

The above options would then be turned over to CERCLA for monitoring after completion because contaminates will remain on the site.

This economic analysis is based on information provided by B. Spaulding, Lee Toutt, Max Christensen, Michelle Dahlmeir, and other team members. S. L. Coward provided cost estimates for the various options.

\section{Methodology:}

The Economic Evaluation assumed a maximum 29-year period, (2009-2037) since this is the estimated time required to complete all of the anticipated remediation activities. If monitoring will be required after closure, the analysis assumed CERCLA would provide this service at no cost. The LCC is identified for each alternative by evaluating the initial development, and other closure costs over the life-cycle. A discounted LCC analysis assumes a current 1998-dollar basis discounted at $6.30 \%$ per the Office of Management and Budget (OMB) Circular A-94. All costs are conservatively discounted assuming the end-of-year convention. 
Page 2

\section{Assumptions:}

The scope of work and requirements of all related activities are vague at this time. Facility and processing costs are typically taken from reference cost estimates of similar scope; however, projects of this magnitude are not available. All costs are assumed to be reasonable for the purposes of the estimate and reflect historical experience associated with DD\&D work at the INEEL.

The LCC analysis has been generated to match the division provided by cost estimating. These costs were allocated on an average monthly cost and duration of the activities which includes Permitting, Direct and Indirect Construction, Performance Incentive Fee (PIF), Procurement fee, Engineering, Inspection, Project Management, Construction Management, Escalation and Contingency costs.

\section{Results:}

Option one: Risked Based Clean Closure; Class C fill- $\$ 94.0$ million Option three: Risked Based Clean Closure; Clean fill. - $\$ 77.8$ million Option four: Close to RCRA Landfill Standards, Class C fill. - $\$ 64.4$ million Option six: Close to RCRA Landfill Standards, Clean fill-\$ 46.4 million

(All costs are reported as Discounted LCC)

Attachments:

cc: R. J. Turk/M.M. Plum File 



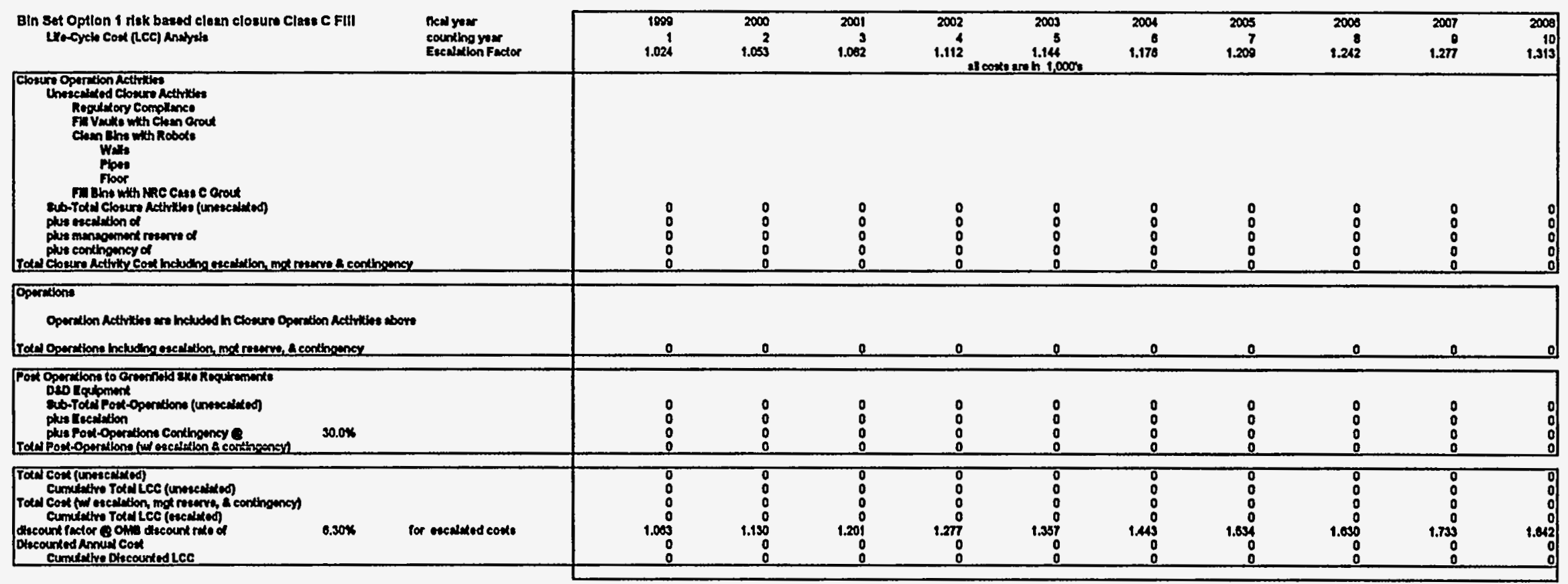




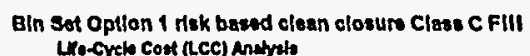

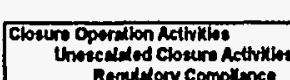

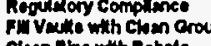
Cison wine wth Robote

Finger pher

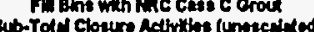

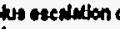

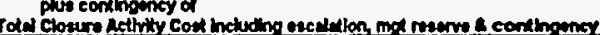

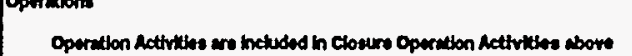

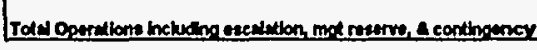

\begin{tabular}{|c|c|c|}
\hline 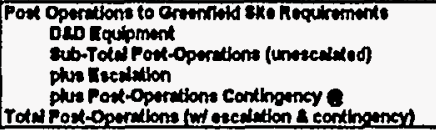 & $30.0 \%$ & \\
\hline 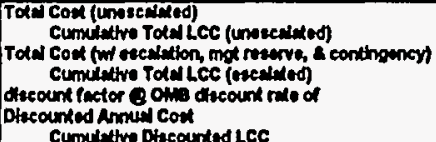 & $0.30 \%$ & for mesciked conte \\
\hline
\end{tabular}

new your

Countho year
Ecculution fuctor

\begin{tabular}{|c|c|c|c|c|c|c|c|c|c|}
\hline $\begin{array}{r}2009 \\
11 \\
1.350\end{array}$ & $\begin{array}{r}2010 \\
12 \\
1.287\end{array}$ & $\begin{array}{r}2011 \\
13 \\
1.428\end{array}$ & $\begin{array}{r}2012 \\
14 \\
1.460 \\
\end{array}$ & $\begin{array}{c}2013 \\
13 \\
1.507 \\
1 \text { conte ane h } 1,000 \% \\
\end{array}$ & $\begin{array}{r}2014 \\
10 \\
1.530\end{array}$ & $\begin{array}{r}2015 \\
17 \\
1.583\end{array}$ & $\begin{array}{r}2010 \\
18 \\
1.030\end{array}$ & $\begin{array}{r}2017 \\
10 \\
1.683\end{array}$ & $\begin{array}{c}2018 \\
20 \\
1.730\end{array}$ \\
\hline 140 & 2,000 & 2.000 & 2,006 & 2,000 & $\begin{array}{r}2,239 \\
327 \\
635 \\
312\end{array}$ & 2062 & & & \\
\hline $\begin{aligned} & 10 \\
& \infty \infty 10 \\
& \infty \\
& 411 \\
& 1,031\end{aligned}$ & $\begin{array}{l}2,000 \\
2, m 0 \\
j, 304 \\
i, 000\end{array}$ & $\begin{array}{l}2,000 \\
2,700 \\
1.34 \\
1,044 \\
7,000\end{array}$ & $\begin{array}{l}2,060 \\
2,770 \\
, 204 \\
1,044 \\
7,600\end{array}$ & $\begin{array}{l}2,000 \\
2,770 \\
1,94 \\
1,040 \\
7,003\end{array}$ & $\begin{array}{l}2,534 \\
3,2,200 \\
400 \\
1,0,40 \\
0,230 \\
0.230\end{array}$ & $\begin{array}{l}3,010 \\
3,503 \\
600 \\
2,102 \\
0,070\end{array}$ & $\begin{array}{l}0 \\
0 \\
0 \\
0 \\
0\end{array}$ & $\begin{array}{l}0 \\
: \\
: \\
0\end{array}$ & $\begin{array}{l}0 \\
0 \\
0 \\
0 \\
0\end{array}$ \\
\hline
\end{tabular}

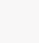
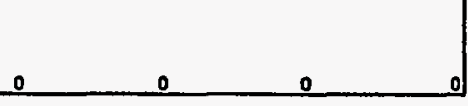

\begin{tabular}{|c|c|c|c|c|c|c|c|c|c|}
\hline $\begin{array}{l}0 \\
0 \\
0 \\
0\end{array}$ & $\begin{array}{l}0 \\
0 \\
0 \\
0 \\
\end{array}$ & $\begin{array}{l}0 \\
0 \\
0 \\
0\end{array}$ & $\begin{array}{l}0 \\
0 \\
0 \\
0 \\
\end{array}$ & $\begin{array}{r}0 \\
0 \\
0 \\
0\end{array}$ & $\begin{array}{l}0 \\
: \\
0 \\
0\end{array}$ & $\begin{array}{l}0 \\
0 \\
0 \\
0\end{array}$ & $\begin{array}{l}0 \\
0 \\
0 \\
0 \\
\end{array}$ & $\begin{array}{l}0 \\
0 \\
0 \\
0 \\
\end{array}$ & \\
\hline $\begin{array}{r}740 \\
710 \\
1,4081 \\
1,051 \\
1,058 \\
000 \\
0.06 \\
\end{array}$ & $\begin{array}{l}2,005 \\
3,792 \\
7,7003 \\
9,753 \\
2.002 \\
3,748 \\
1,745 \\
\end{array}$ & 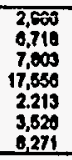 & $\begin{array}{r}2,0606 \\
0,704 \\
7,003 \\
25,358 \\
2,352 \\
3,317 \\
11,588 \\
\end{array}$ & $\begin{array}{r}2,000 \\
12,000 \\
7,7003 \\
33,162 \\
2,600 \\
3,121 \\
14,700 \\
\end{array}$ & $\begin{array}{r}3,534 \\
18,225 \\
0.2230 \\
42,397 \\
2.056 \\
3,475 \\
10,184 \\
\end{array}$ & 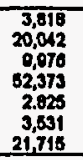 & $\begin{array}{r}0 \\
20,042 \\
0 \\
52,373 \\
3.003 \\
21,715 \\
\end{array}$ & $\begin{array}{r}0 \\
20,042 \\
0 \\
02,373 \\
3.190 \\
01,718 \\
\end{array}$ & $\begin{array}{r}20,042 \\
0 \\
32,379 \\
3,34 \\
21,715 \\
\end{array}$ \\
\hline
\end{tabular}




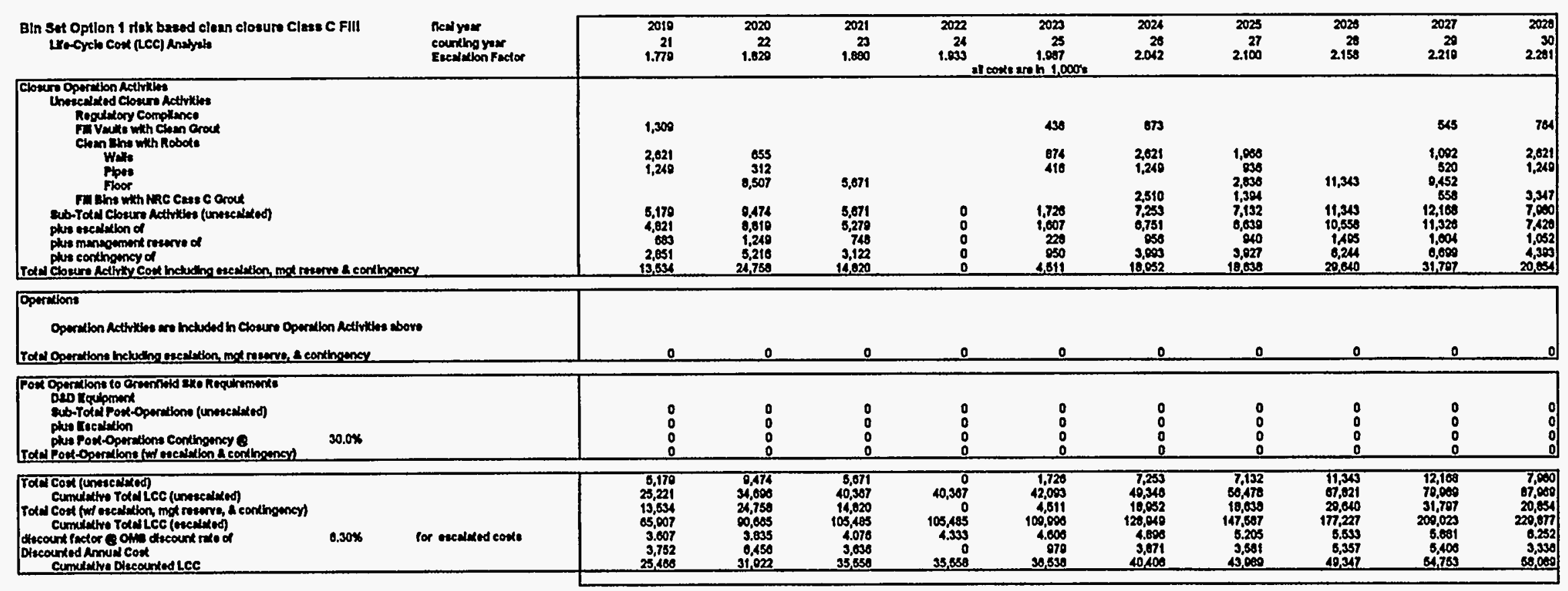




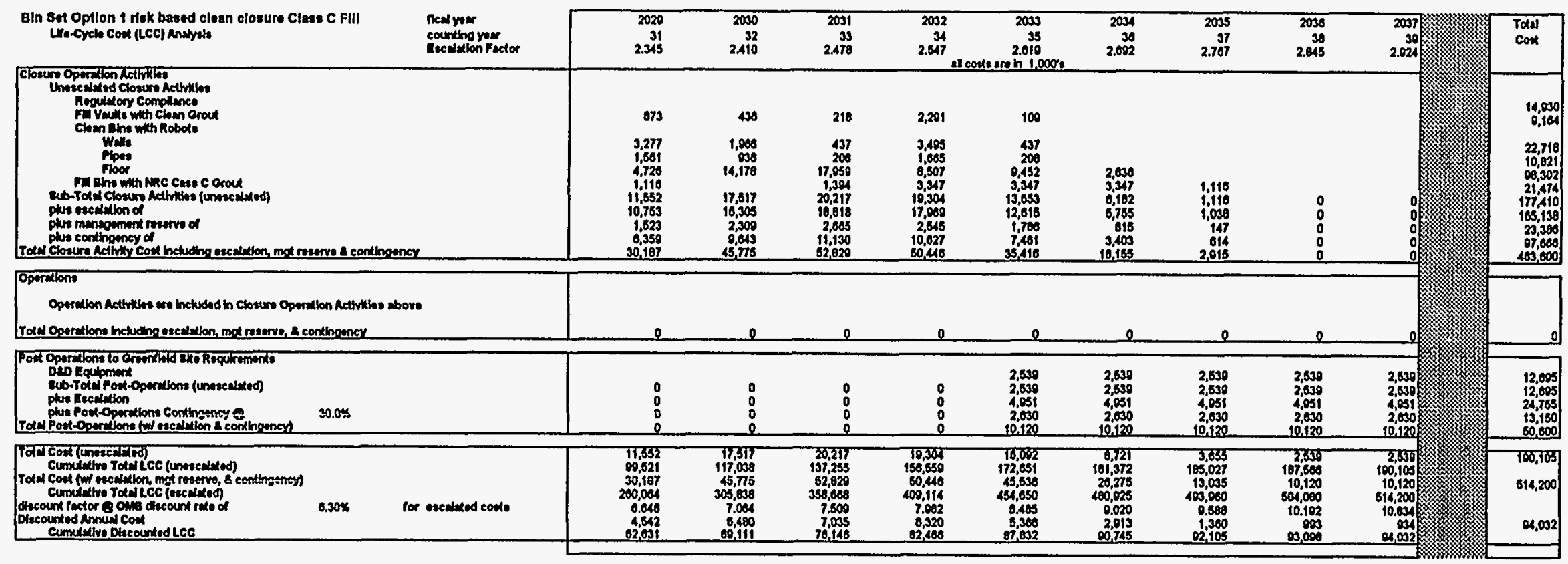




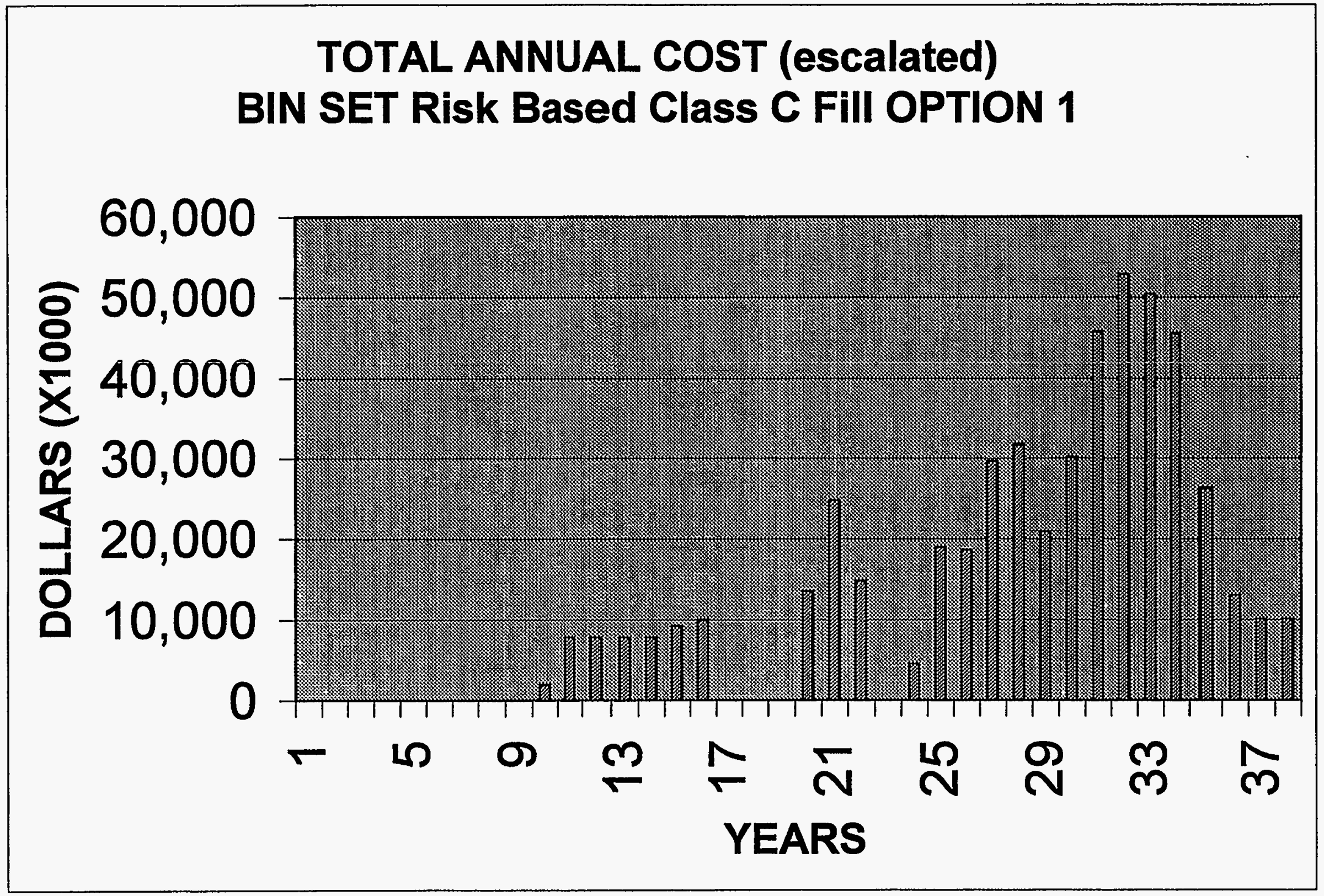




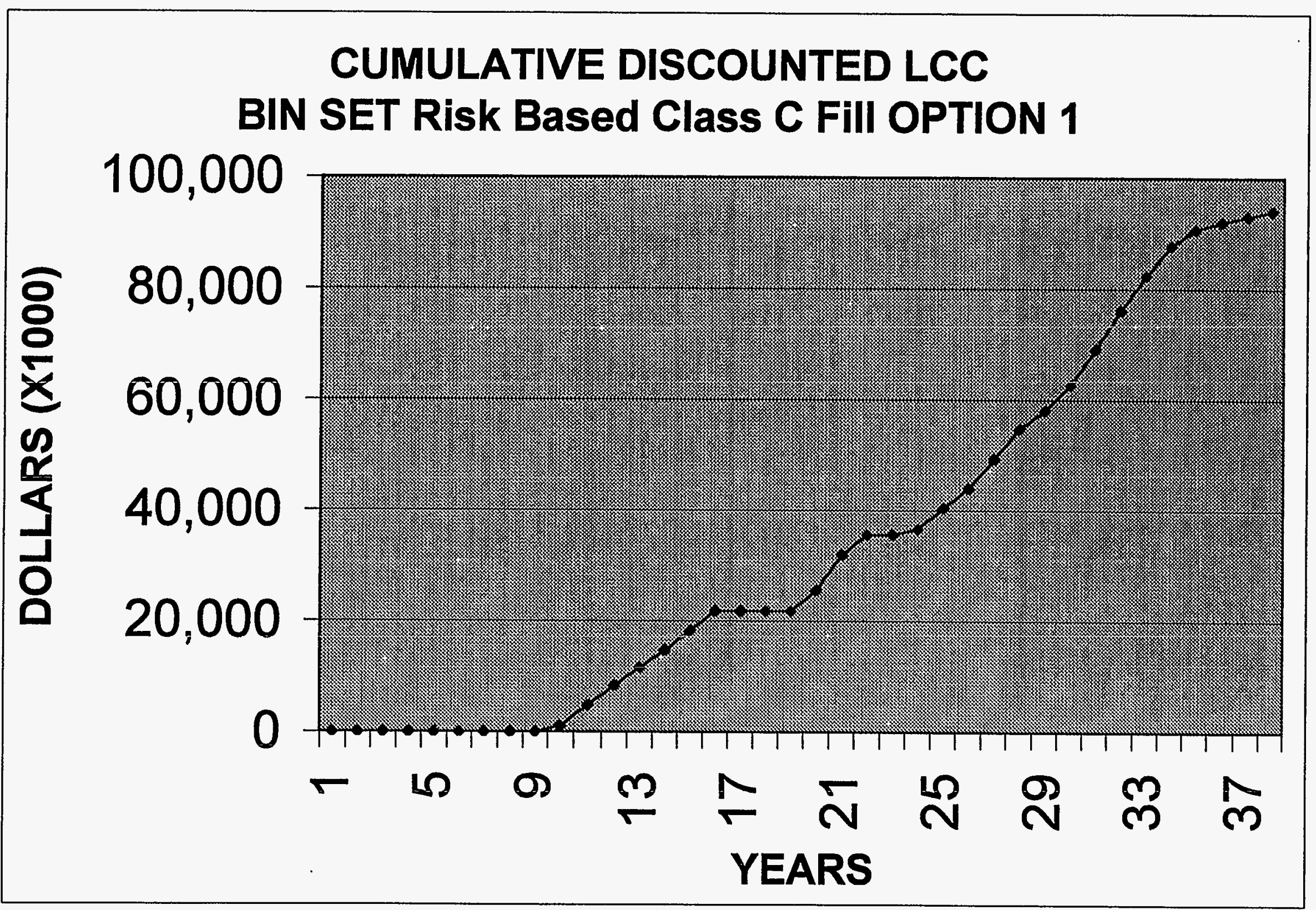




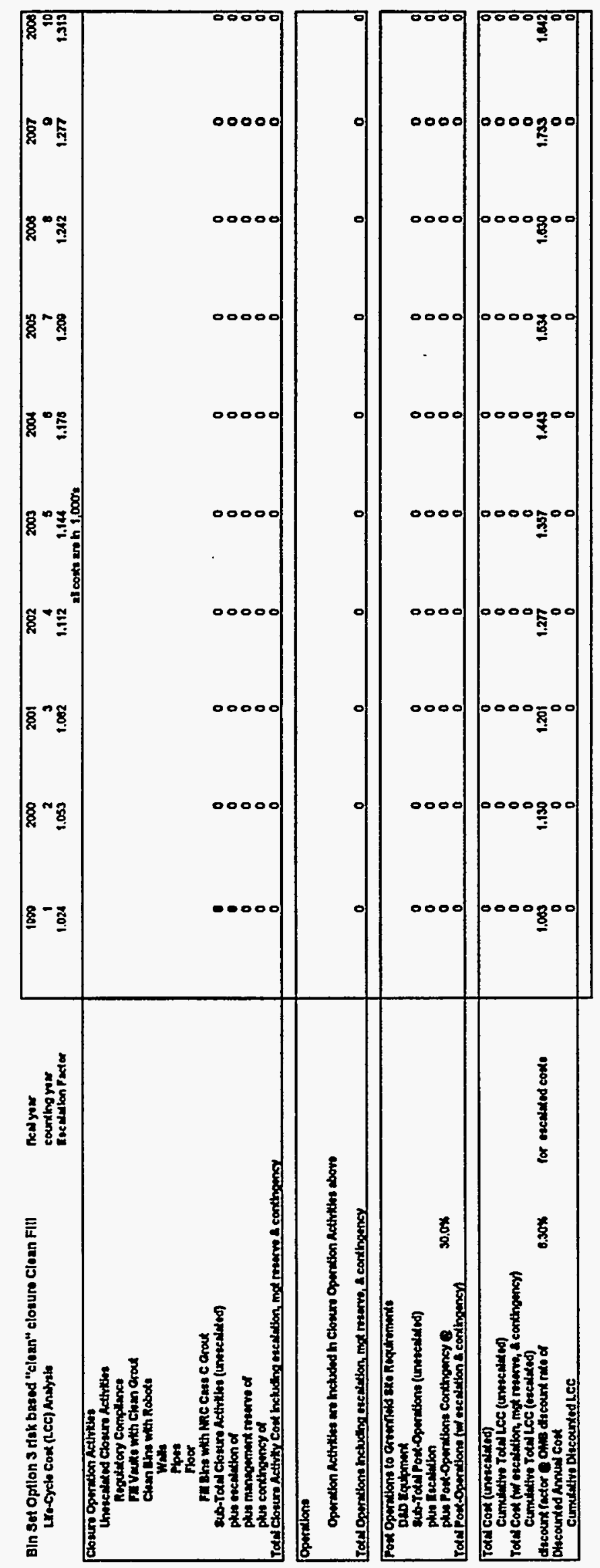


Bin Sot Option 3 Hisk based "oloan" eloaure Clean FIII Ho-Cyele Con (LCC) Anstiple

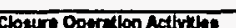

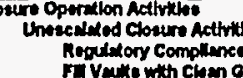

Foguldory Complimes amith

Proper

Proor

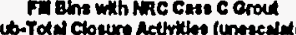
pus enextition a

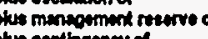

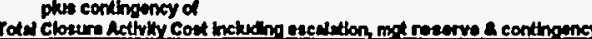

Rew you

courthoyer

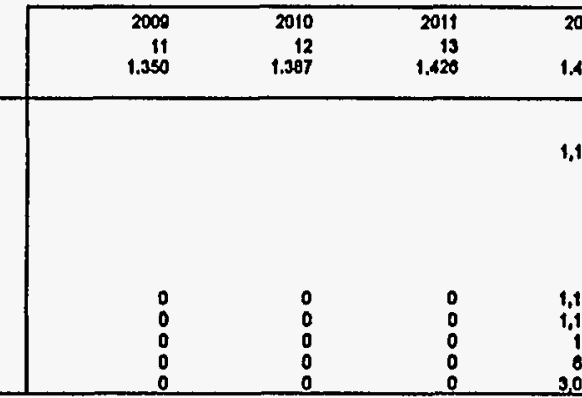

2012

2013
15
1.507

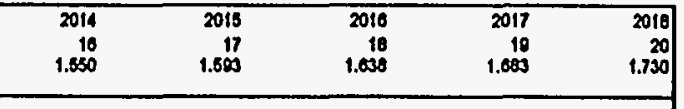

1,500

1,560

${ }_{2009}^{302}$

0.53
312
2,036

$\begin{array}{rrr}170 & 1,569 & 1,500 \\ 104 & 1,472 & 1,200 \\ 155 & 200 & 200 \\ 043 & 050 & 650\end{array}$

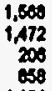

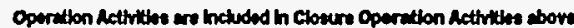

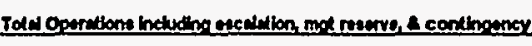

\begin{tabular}{|c|c|c|c|c|c|c|c|c|c|c|c|c|}
\hline 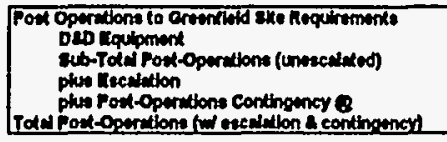 & $30.0 \%$ & & $\begin{array}{l}0 \\
0 \\
0 \\
0\end{array}$ & $\begin{array}{l}0 \\
0 \\
0 \\
0\end{array}$ & $\begin{array}{l}0 \\
0 \\
0 \\
0\end{array}$ & $\begin{array}{l}0 \\
0 \\
0 \\
0\end{array}$ & $\begin{array}{l}0 \\
: \\
0 \\
0\end{array}$ & $\begin{array}{l}0 \\
0 \\
0 \\
0 \\
\end{array}$ & $\begin{array}{l}0 \\
0 \\
0 \\
0 \\
\end{array}$ & $\begin{array}{l}0 \\
0 \\
0 \\
0\end{array}$ & $\begin{array}{l}0 \\
: \\
0 \\
0\end{array}$ & \\
\hline 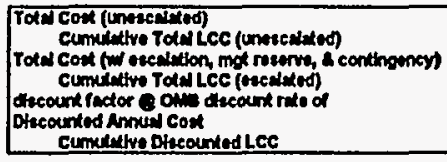 & $0.30 x$ & for recelled conts & $\begin{array}{r}0 \\
0 \\
0 \\
1.086 \\
0 \\
0\end{array}$ & $\begin{array}{r}0 \\
0 \\
0 \\
0 \\
2.002 \\
0 \\
0\end{array}$ & $\begin{array}{r}0 \\
0 \\
0 \\
2.213 \\
0 \\
0\end{array}$ & $\begin{array}{l}7,170 \\
1,170 \\
j, 070 \\
j, 070 \\
2,352 \\
1,300 \\
1,300\end{array}$ & 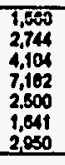 & 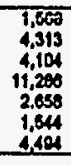 & $\begin{array}{l}, 0,034 \\
10,247 \\
15,620 \\
20,018 \\
2.025 \\
5,100 \\
0,000 \\
\end{array}$ & $\begin{array}{r}1329 \\
10,575 \\
557 \\
27,072 \\
0.003 \\
206 \\
10,276 \\
\end{array}$ & $\begin{array}{r}10 \\
10,575 \\
0 \\
27,672 \\
3.190 \\
10,270 \\
\end{array}$ & \\
\hline
\end{tabular}


BIn Set Optlon 3 riak baeed "cloan" clozure Cloan FIII

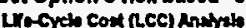

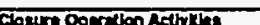

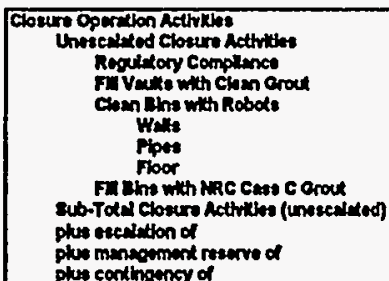

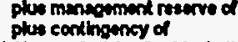

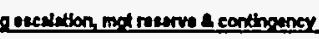

Tean your countho year
Erestuxtion Futor

2

$\begin{array}{rrrr}2019 & 2020 & 2021 & 202 \\ 21 & 22 & 23 & \\ 1.70 & 1.029 & 1.800 & 1.035\end{array}$

$\begin{array}{rr}2022 & 2023 \\ 24 & 2533\end{array}$ I coxtranh $1,000 \%$

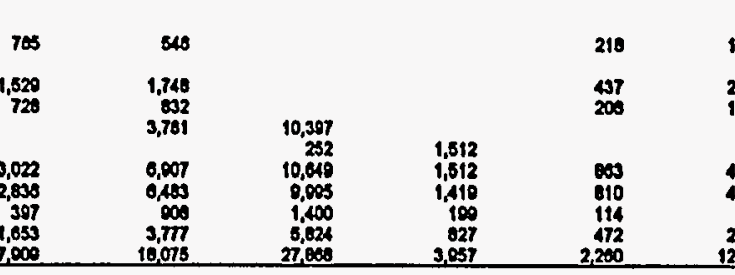

\begin{tabular}{|c|c|c|c|c|}
\hline $\begin{array}{l}2024 \\
2040 \\
2012\end{array}$ & $\begin{array}{r}2025 \\
27 \\
2.100\end{array}$ & $\begin{array}{r}2020 \\
28 \\
2.158\end{array}$ & $\begin{array}{r}2027 \\
29 \\
2.216\end{array}$ & $\begin{array}{r}2020 \\
30 \\
2.201\end{array}$ \\
\hline 1,002 & & & & 1,748 \\
\hline $\begin{array}{l}2,622 \\
1,240\end{array}$ & $\begin{array}{l}2,105 \\
1,041 \\
1,600\end{array}$ & 19,313 & 0,452 & $\begin{array}{l}3,4055 \\
1,065\end{array}$ \\
\hline 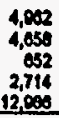 & 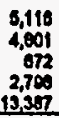 & 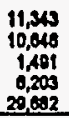 & 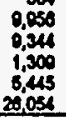 & 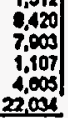 \\
\hline
\end{tabular}

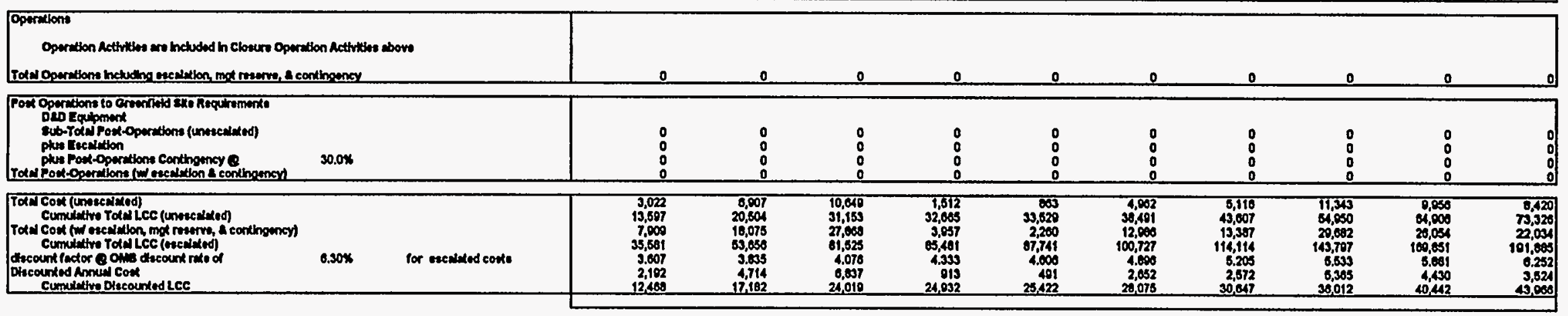




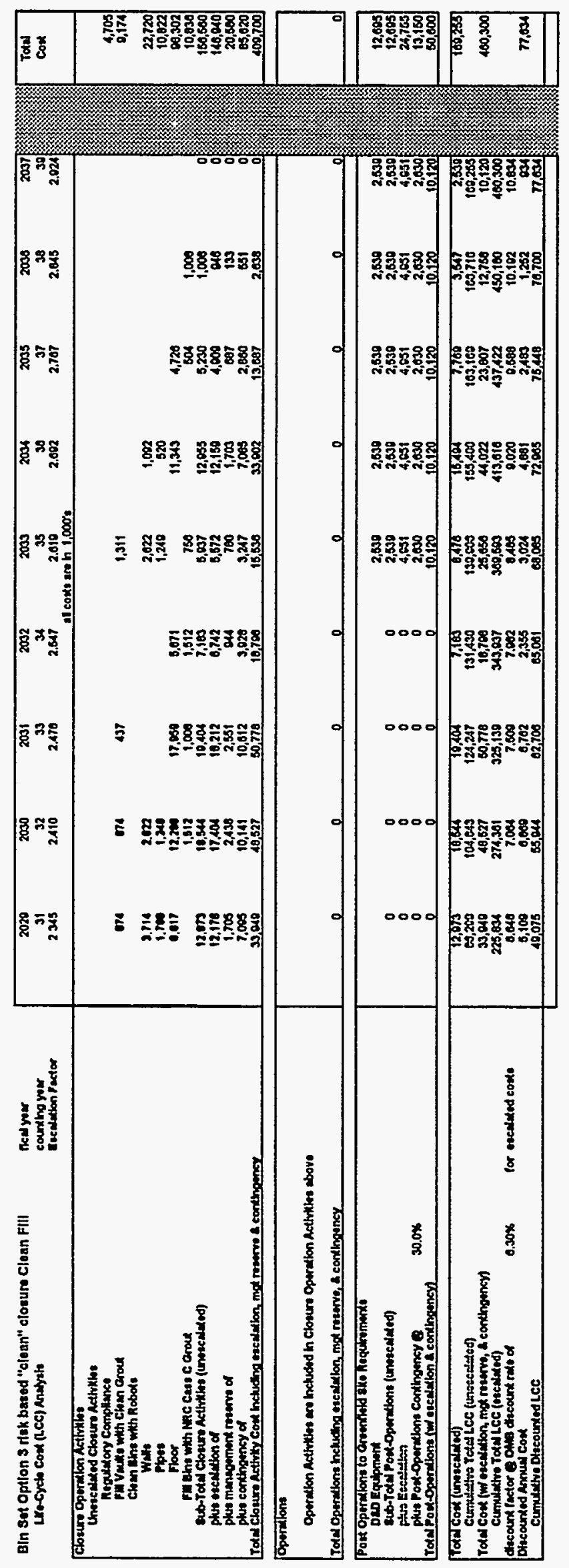




\section{TOTAL ANNUAL COST (escalated) BIN SET Risk Based "Clean" Fill OPTION 3}

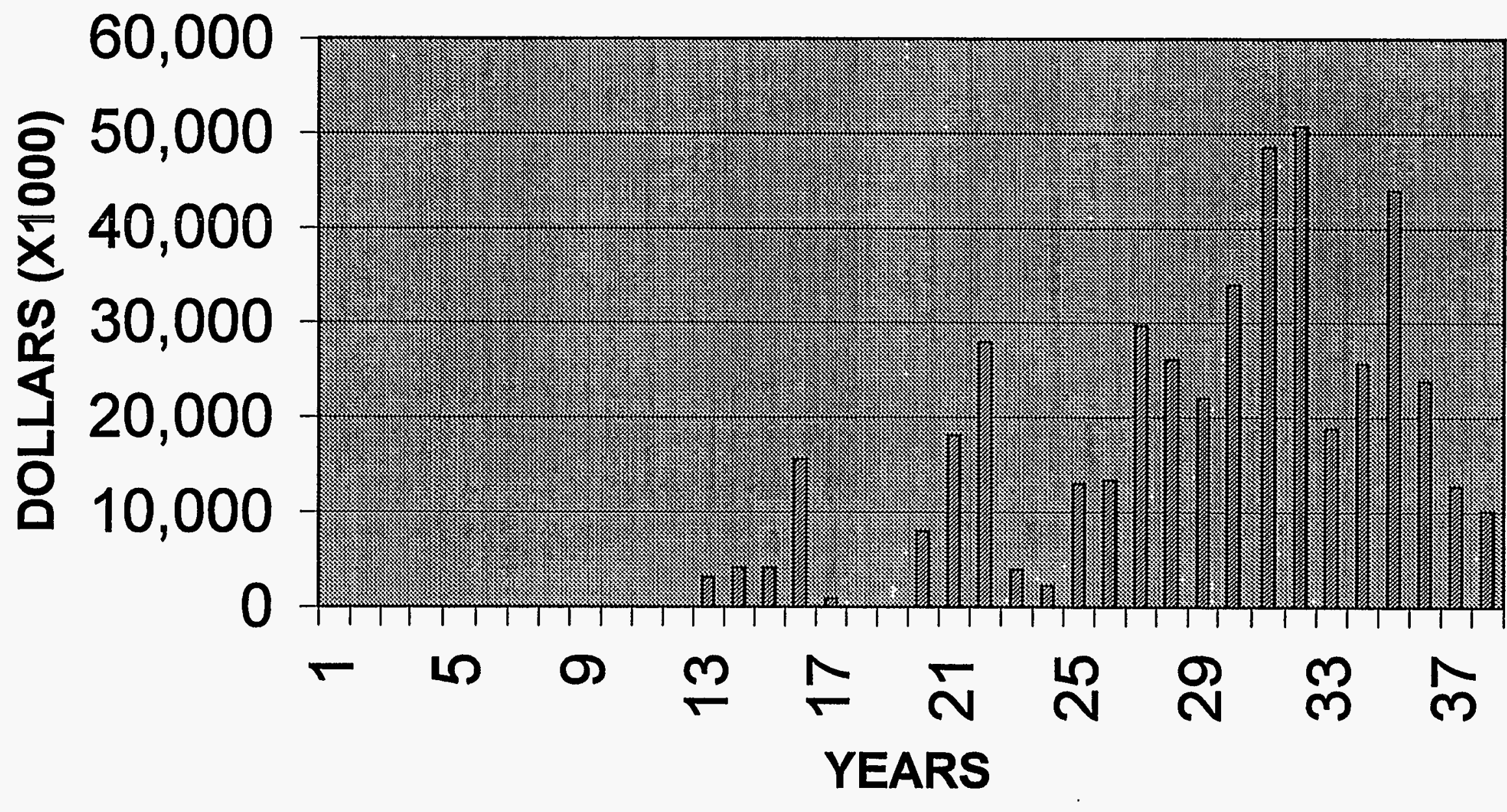




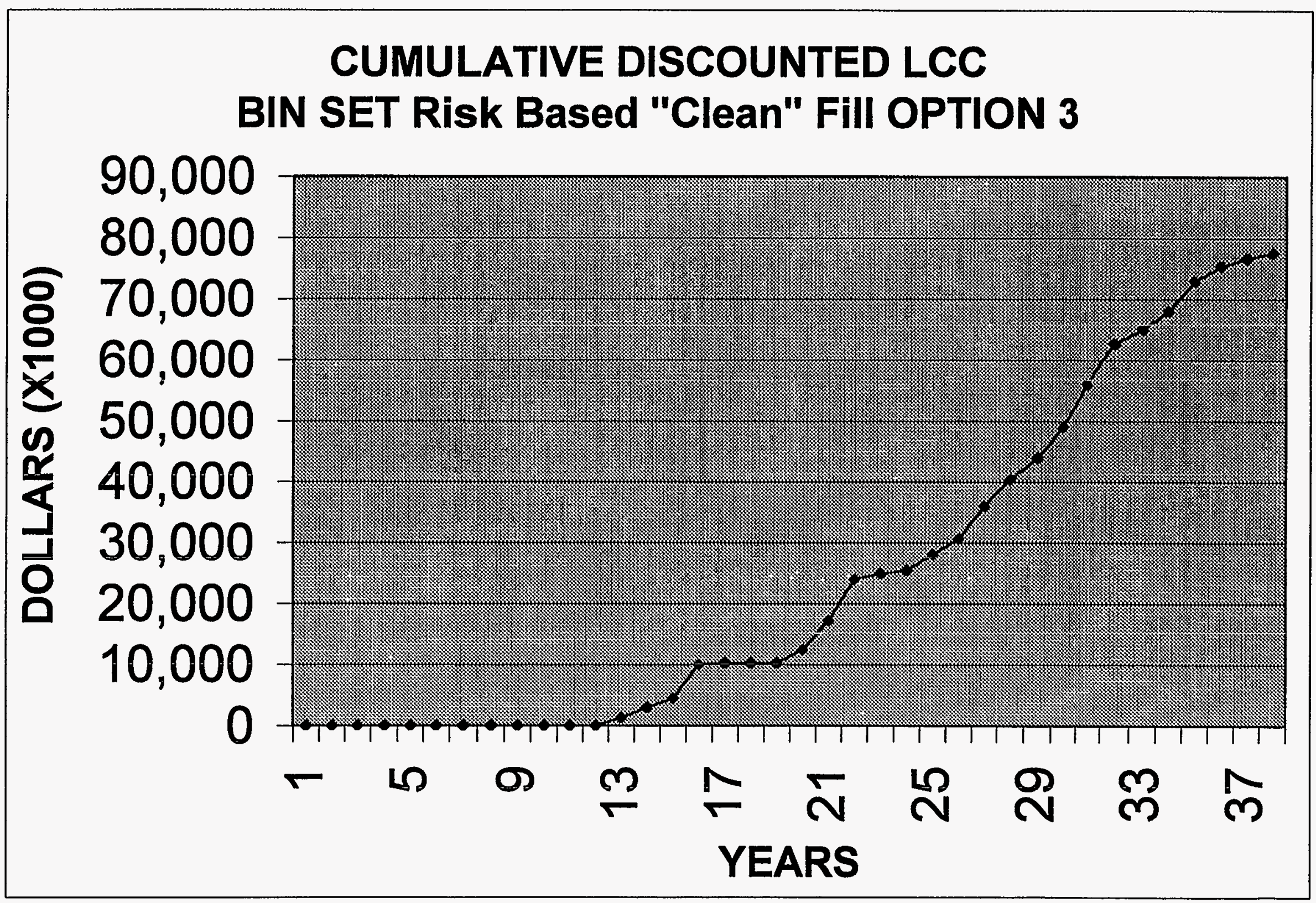





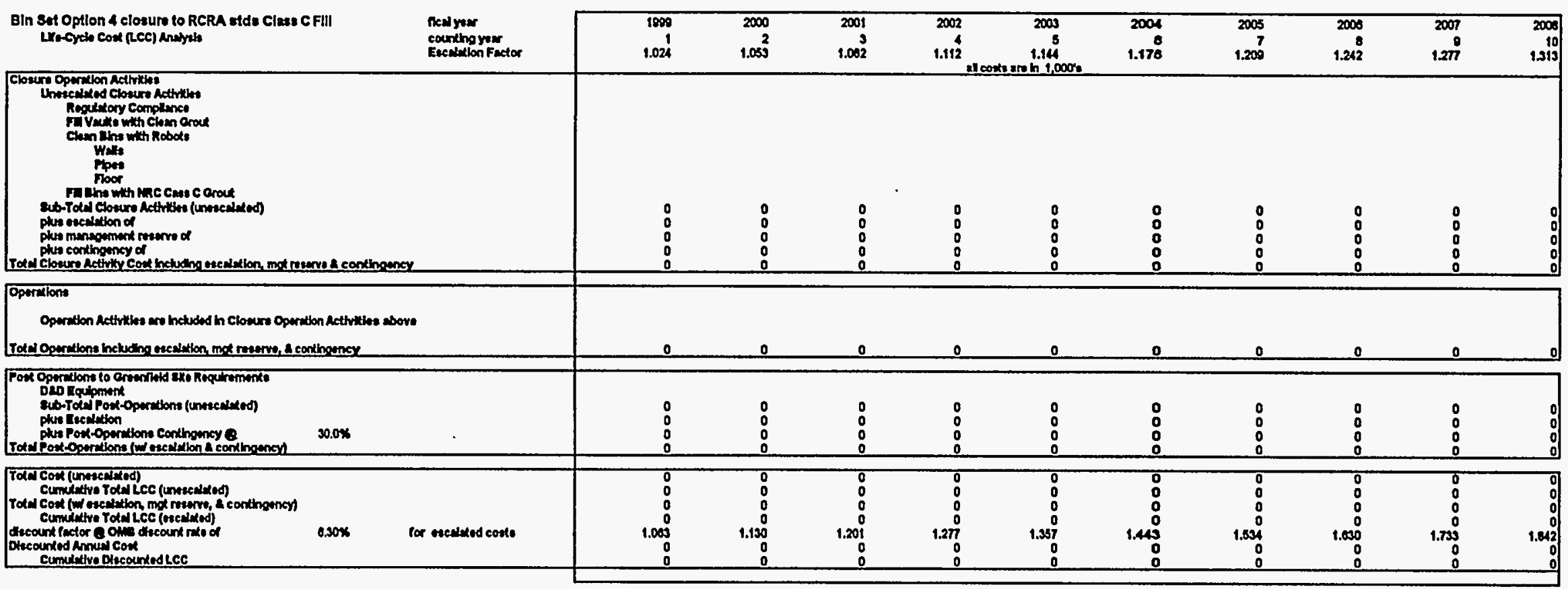


Oin set Optlon 4 closure to RCRA stds Clase C Fill

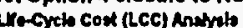

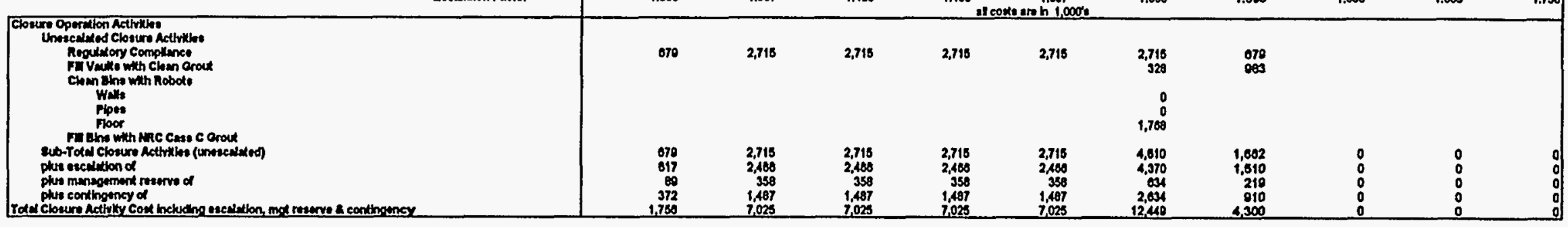

Ten your

counchan weer

Operalion

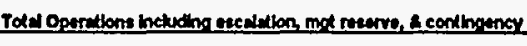

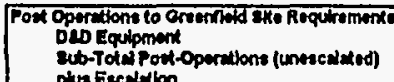

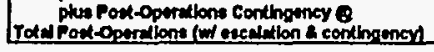

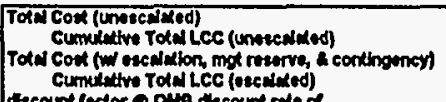

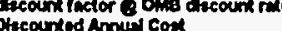
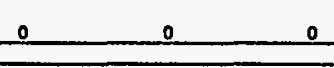

$30.0 \%$

\begin{tabular}{|c|c|c|}
\hline & $\begin{array}{l}0 \\
0 \\
0\end{array}$ & \\
\hline for encedixtod conte & $\begin{array}{r}978 \\
070 \\
1,750 \\
1,750 \\
1,058 \\
897 \\
897\end{array}$ & $\begin{array}{l}2,71 \\
3,302 \\
7,02 \\
0,70 \\
2,006 \\
3,37 \\
4,27\end{array}$ \\
\hline
\end{tabular}

\begin{tabular}{|c|c|c|c|c|c|c|c|c|}
\hline $\begin{array}{l}0 \\
\vdots \\
0 \\
0\end{array}$ & $\begin{array}{l}0 \\
0 \\
0 \\
0 \\
\end{array}$ & $\begin{array}{l}0 \\
0 \\
0 \\
0\end{array}$ & $\begin{array}{l}0 \\
0 \\
0 \\
0\end{array}$ & $\begin{array}{l}0 \\
0 \\
0 \\
0\end{array}$ & $\begin{array}{l}0 \\
0 \\
0 \\
0\end{array}$ & $\begin{array}{l}0 \\
0 \\
0 \\
0\end{array}$ & & \\
\hline 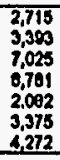 & $\begin{array}{r}2,716 \\
0,100 \\
7,0.025 \\
15,806 \\
2.213 \\
3,176 \\
7,466 \\
\end{array}$ & $\begin{array}{r}2,775 \\
8,022 \\
7,025 \\
2,, 031 \\
2,352 \\
2,097 \\
10,433 \\
\end{array}$ & $\begin{array}{r}2,71315 \\
11,507 \\
7,025 \\
20,050 \\
2.050 \\
2,010 \\
13,243 \\
\end{array}$ & $\begin{array}{r}4,010 \\
10,347 \\
12,440 \\
42,305 \\
2.059 \\
4,004 \\
17,027 \\
\end{array}$ & $\begin{array}{r}1,052 \\
10,000 \\
4,000 \\
40,005 \\
2.825 \\
1.522 \\
10,448 \\
\end{array}$ & $\begin{array}{r}10,000 \\
0 \\
40,005 \\
3,000 \\
0 \\
19,40 \\
\end{array}$ & $\begin{array}{r}10,000 \\
0 \\
40,605 \\
3.103 \\
10,48\end{array}$ & $\begin{array}{r}18,0 \\
48,6 \\
3.3 \\
10,4\end{array}$ \\
\hline
\end{tabular}




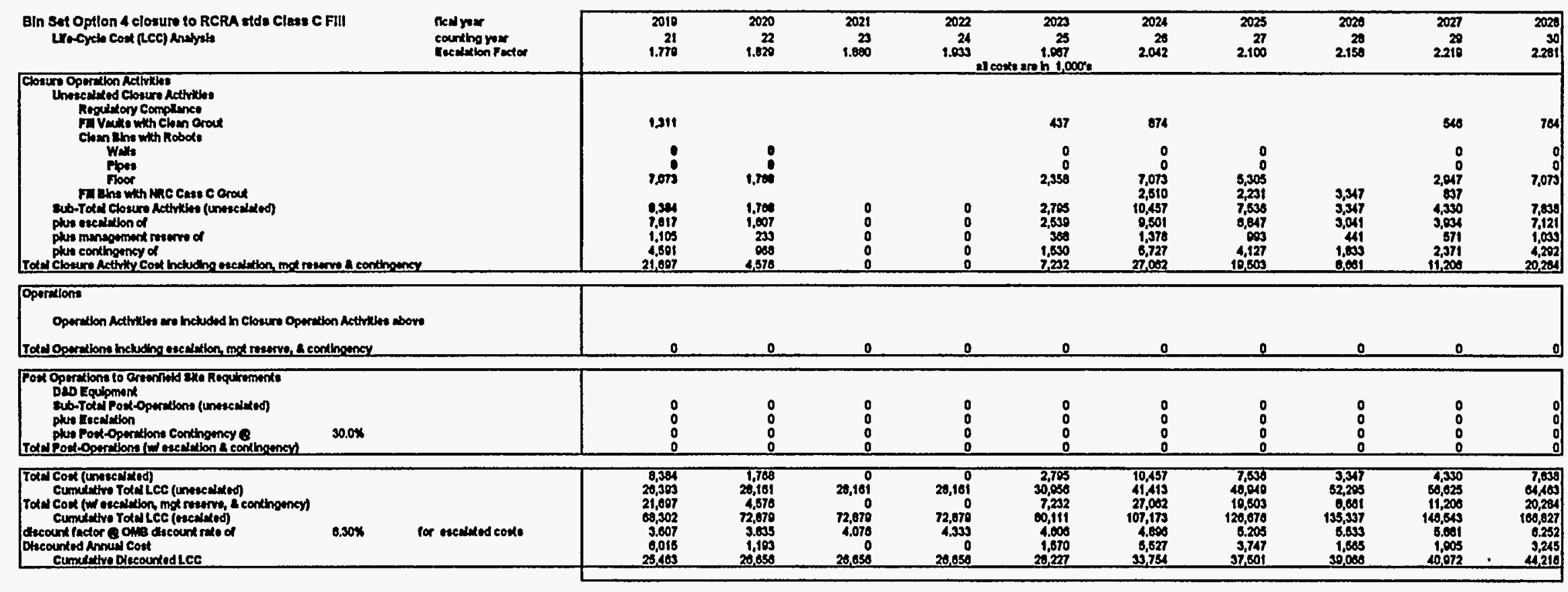




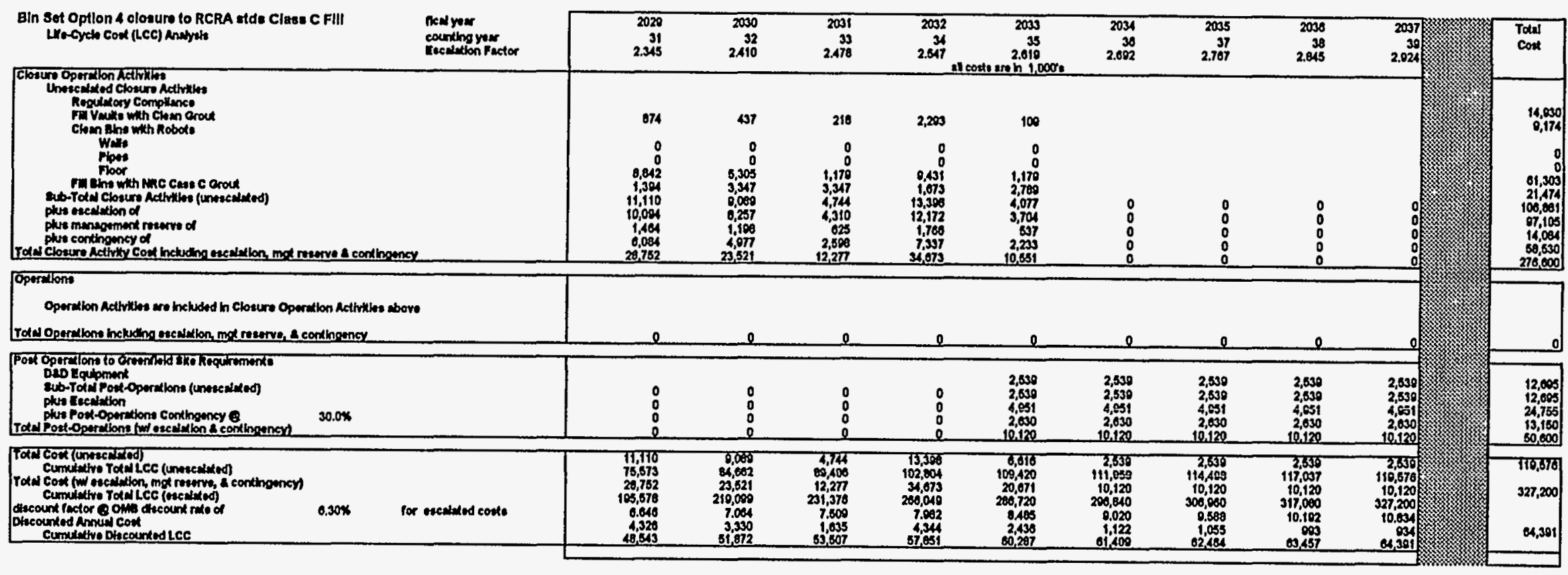




\section{TOTAL ANNUAL COST (escalated) BIN SET RCRA stds Class C Fill OPTION 4}

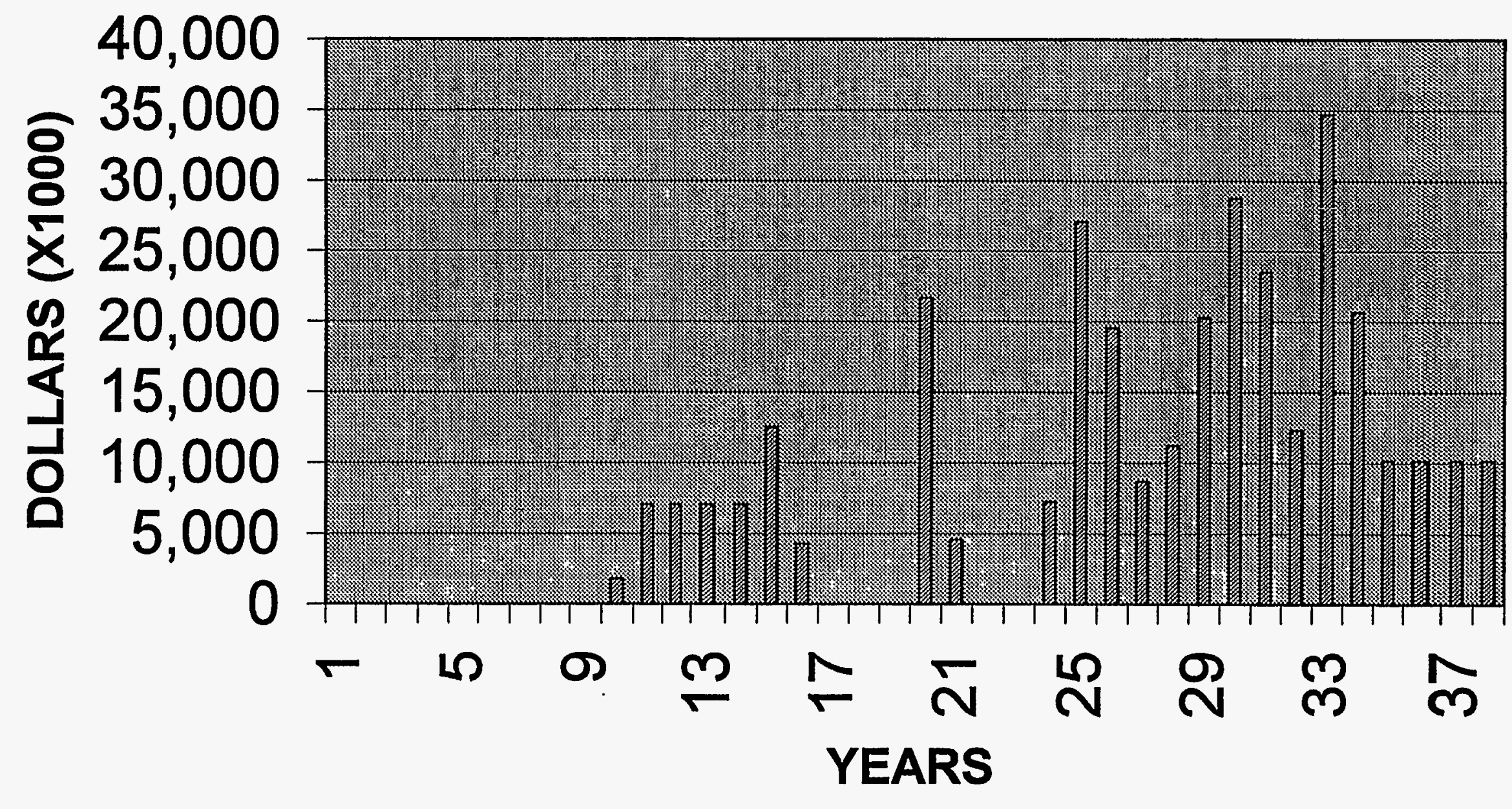




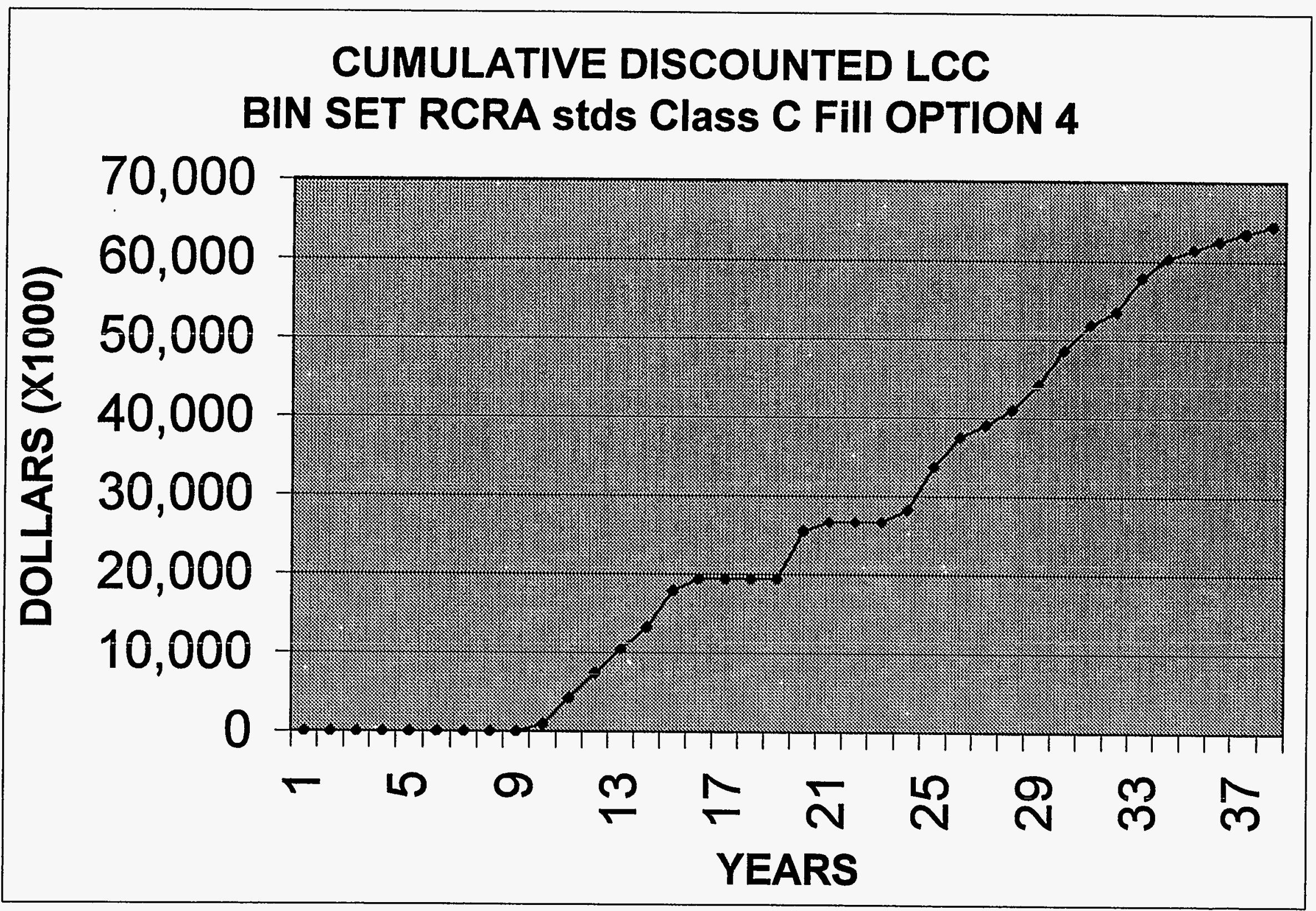


兽 


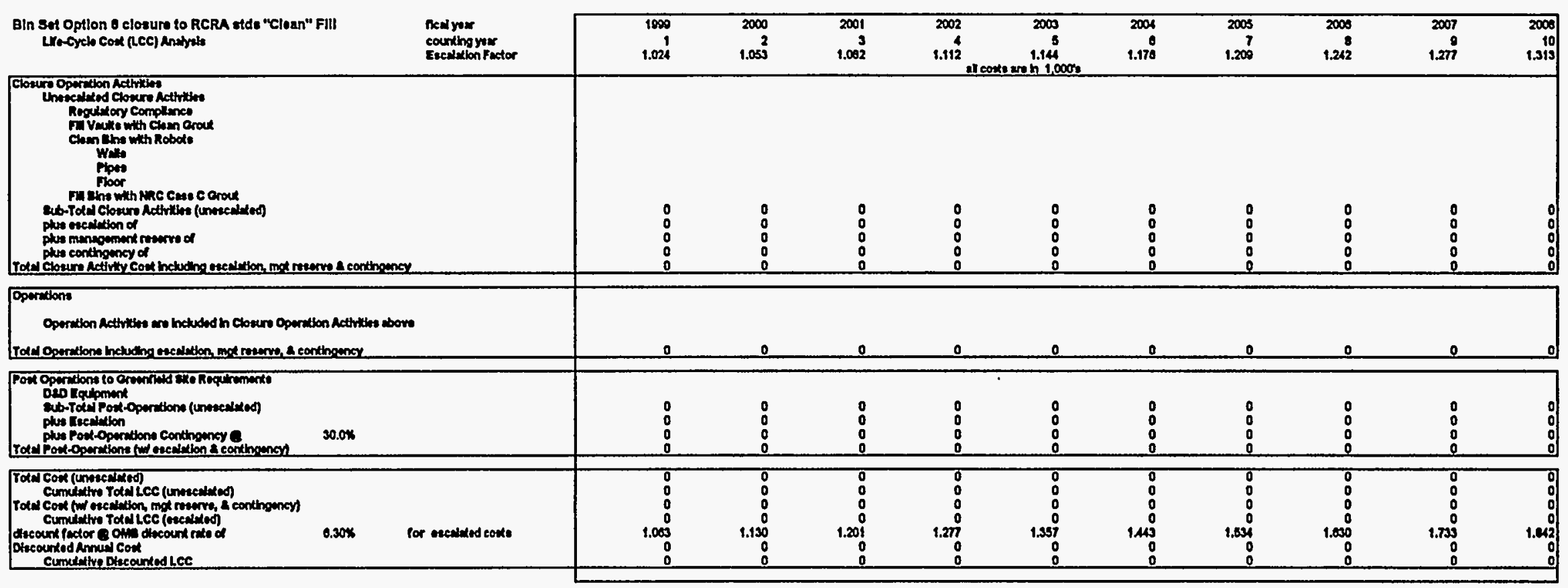




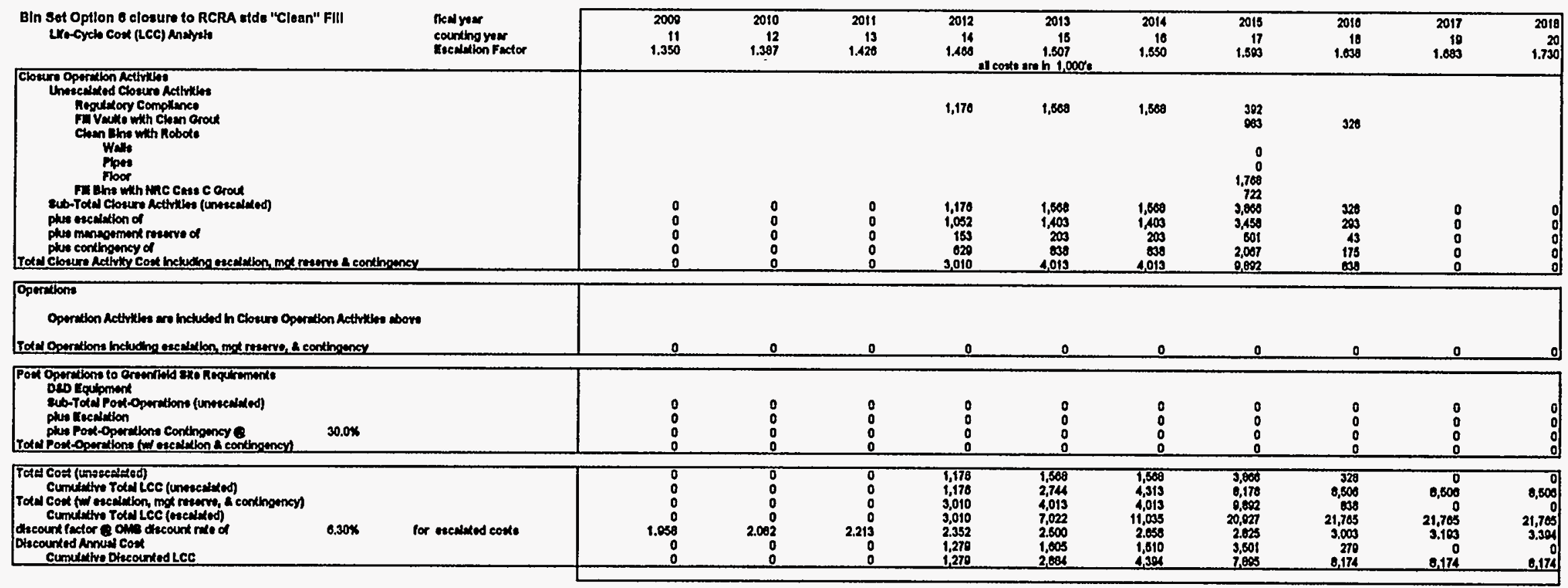




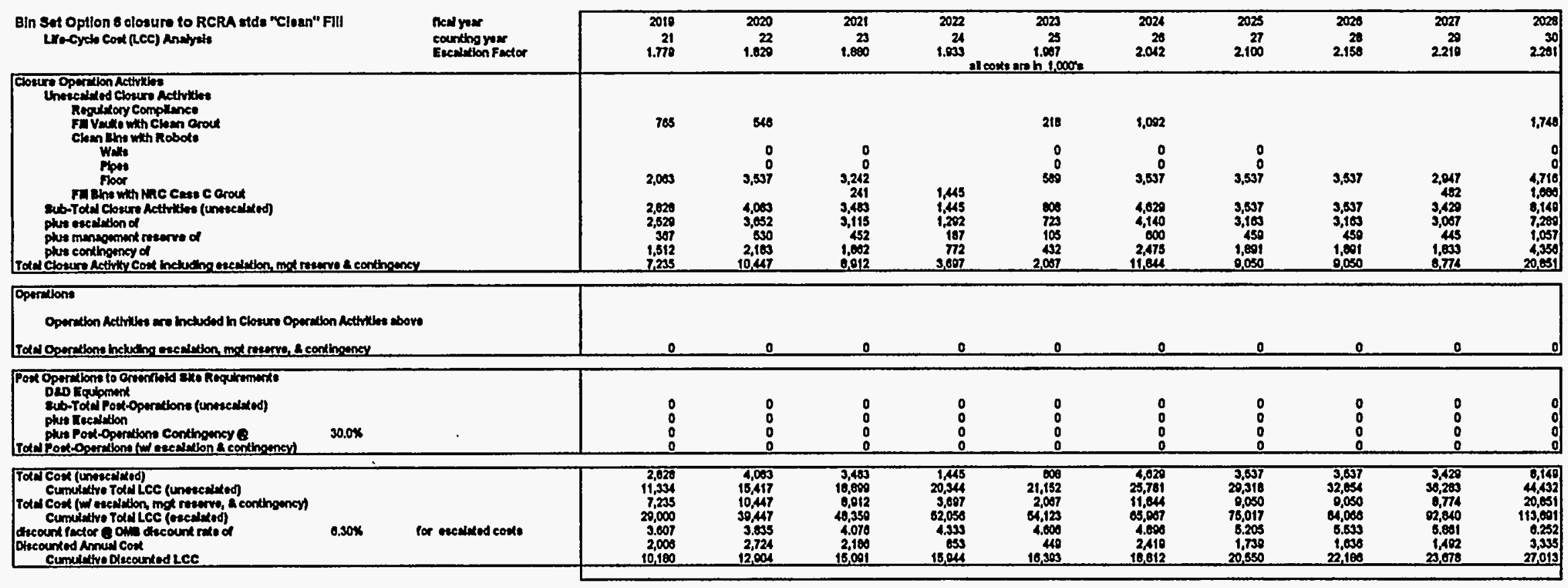




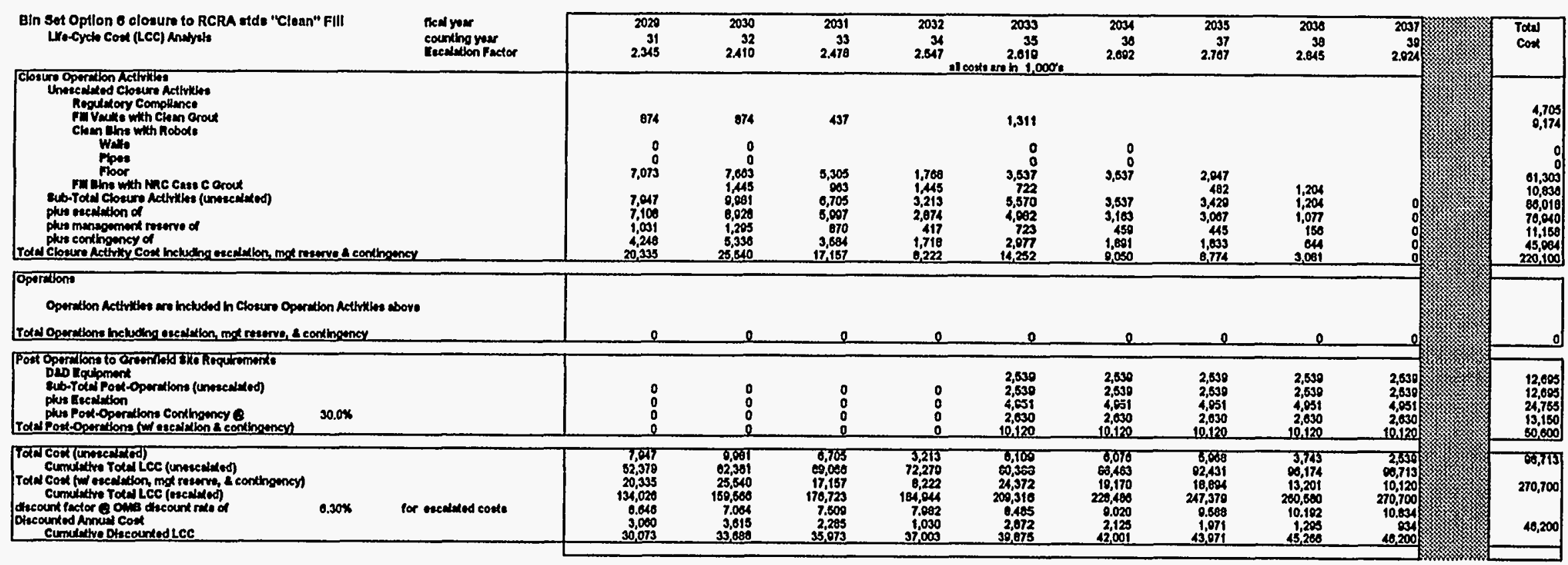




\section{TOTAL ANNUAL COST (escalated) BIN SET RCRA stds "Clean" Fill OPTION 6}

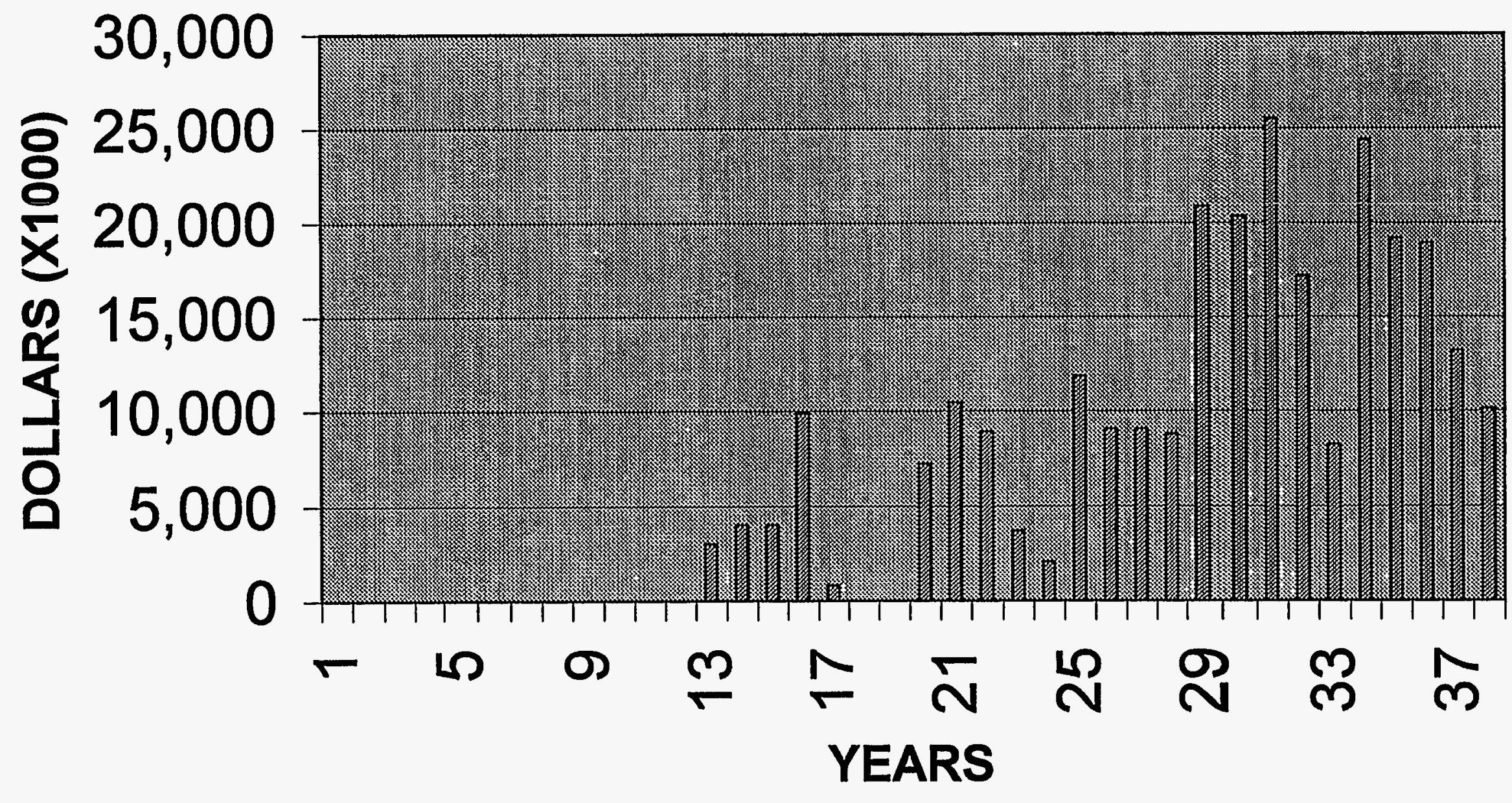




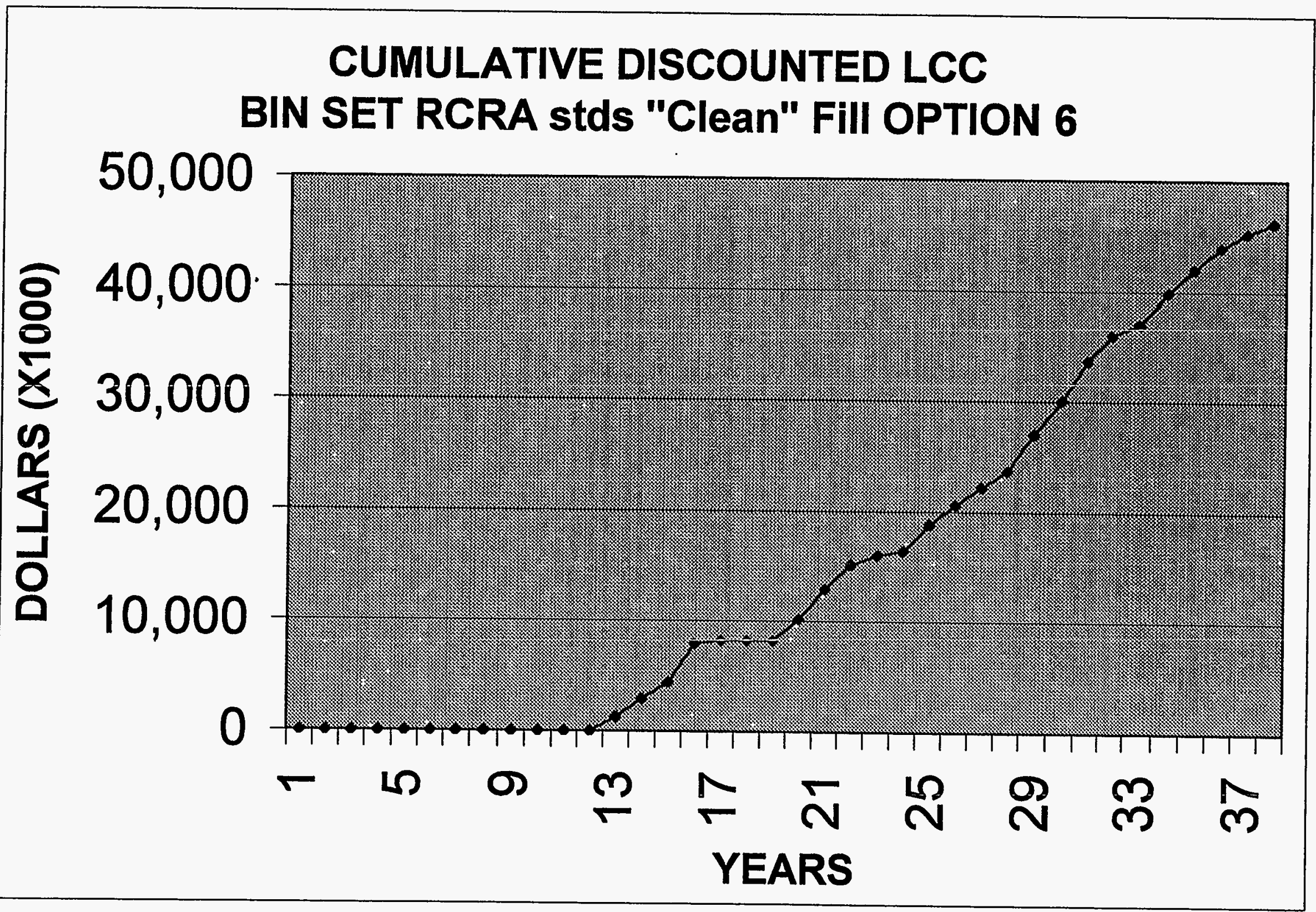

